UCRL-ID-136635

\title{
Signal and Imaging Sciences Workshop 1998 Proceedings
}

J.V. Candy

November 4, 1999

U.S. Department of Energy

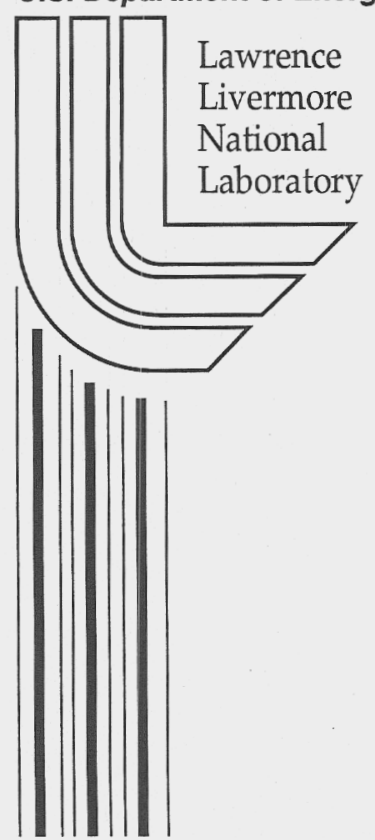

Approved for public release; further dissemination unlimited 


\section{DISCLAIMER}

This document was prepared as an account of work sponsored by an agency of the United States Government. Neither the United States Government nor the University of California nor any of their employees, makes any warranty, express or implied, or assumes any legal liability or responsibility for the accuracy, completeness, or usefulness of any information, apparatus, product, or process disclosed, or represents that its use would not infringe privately owned rights. Reference herein to any specific commercial product, process, or service by trade name, trademark, manufacturer, or otherwise, does not necessarily constitute or imply its endorsement, recommendation, or favoring by the United States Government or the University of California. The views and opinions of authors expressed herein do not necessarily state or reflect those of the United States Government or the University of California, and shall not be used for advertising or product endorsement purposes.

This work was performed under the auspices of the U. S. Department of Energy by the University of California, Lawrence Livermore National Laboratory under Contract No. W-7405-Eng-48. 


\section{Table of Contents}

\section{NOVEMBER 12 OPENING RRESENTAT10NS}

An Update on Engineering New Initiatives and Technology Development

Spiros Dimolitsas - Associate Director, Engineering ................................................................ 00

Diffraction Tomography - Anthony Devaney, Keynote Speaker .............................................. 00

\section{COMPULHA IOMOGRAPHY}

Land Mine Detection - Steve Azevedo

HERMES Bridge Deck Inspection System Current

Results and Future Research - Holger Jones

Active and Passive Computed Tomography with a Constrained

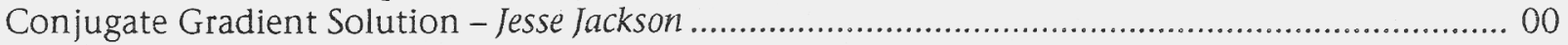

Applying Optimization Tomography Problems - Dennis Goodman ........................................ 00

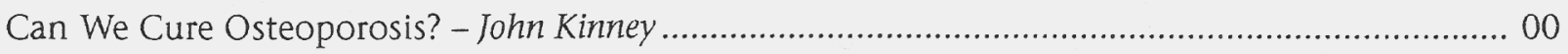

Spectral Partitioning of the Object Function in

Diffraction Tomography - Sean Lehman

\section{RAAAR-ACOUSTIC APRIICATIONS}

Introduction to Low Power Radars for Acoustics Applications:

Speech and Object Characterization - John Holzrichter 00

Excitation Function of Human Speech - Greg Burnett 00

Accurate EM/Acoustic Pitch Estimation - Larry Ng 00

EM/Acoustic Speaker Verification - Todd Gable 00

Object Identification Using Low Power Radars - Randy Roberts 00

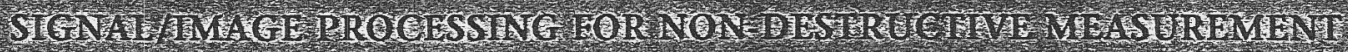

Overview of Center for NDE - Harry Martz 00

Ultrasonic Compound B-scan Imaging - Tyler Gomm, Jason A. Mauseth..... 00

Gigahertz Photodetector Modules: Faster, Smaller, Cheaper - Ken Froeschner 00

In GaAs Linescan Camera System for Paper Web

Moisture Measurements - Jose Hernandez 00

Evaluation of an Amorphous Selenium Array

for Industrial X-Ray Imaging - Clint Logan 00

Matched Field Imaging of Laser-Based

Ultrasound Data - Robert Huber. 
An introduction to the Information Sciences \& Technology Program:

Sheila Vaidya - Program Leader for IS\&T

Detection of Nonsolar Planets by Spinning Infrared

Interferometer - Ronald Bracewell, Keynote Speaker

\section{ASTRONOMIGAL IMAGING}

Novel Interferometer-Spectrometer for Sensitive Doppler Planet Detection - Dave Erskine 00

Resolution Enhancement of Adaptive Optics Corrected Astronomical Images - Jim Brase.... 00

LBNL/Lick Observatory Fully-Depleted CCD Image Sensor Development - Steve Holland. 00

Extrasolar Planet Search With Adaptive Optics Spectrometer with R =200,000 - Jian Ge 00

Micro-Satellite Technology for the 21st Century - Larry Ng. 00

Detecting Massed Troops with the French SPOT Satellites - Vipin Gupta 00

\section{ACOUSTICAI PROCESSING AND INAGING}

Combined Sensing and Simulation for Enhanced

Evaluation of Large Structures - Dave McCallen

Failure Detection for Mechanical Structures:

A State-Space Model-Based Approach - Greg Clark

Parametric Time-Frequency Estimation of Prosthetic

Heart Valve Sounds - Skip Perkins

Near Field / Far Field Transition Distance for

Broad Band Acoustic Pulses - Dave Chambers

Optimization of Array Generated Fields for

Broad Frequency Band Width - Kent Lewis

Automated Event Picking for Seismic Oil Exploration - Peter K-Z. Cheng.....

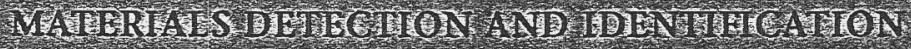

Explosive Detection and Discriminant Research for

Civil Aviation Security - Harry Martz

Grain Identification and Analysis of Shock Induced

Damage of Sandstone - Carl Hagelberg.... 00

Signal Design for Lithography - Jesse Kolman ....

Fluoro Immuno Sensors for Biological Warfare Agents

Detection and Identification - Kodumudi Venkateswaran 00 


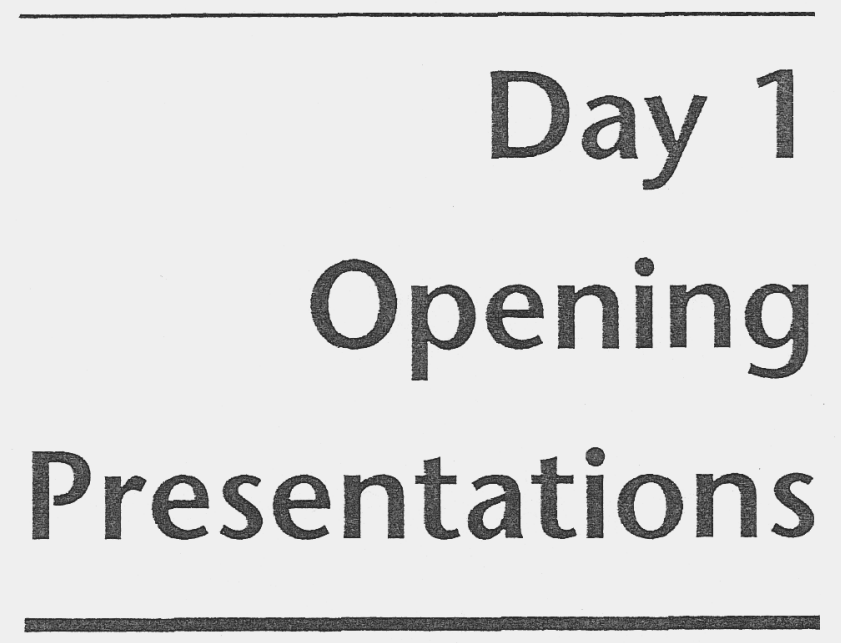




\section{Engineering: An Update on Engineering New Initiatives and Technology Development}

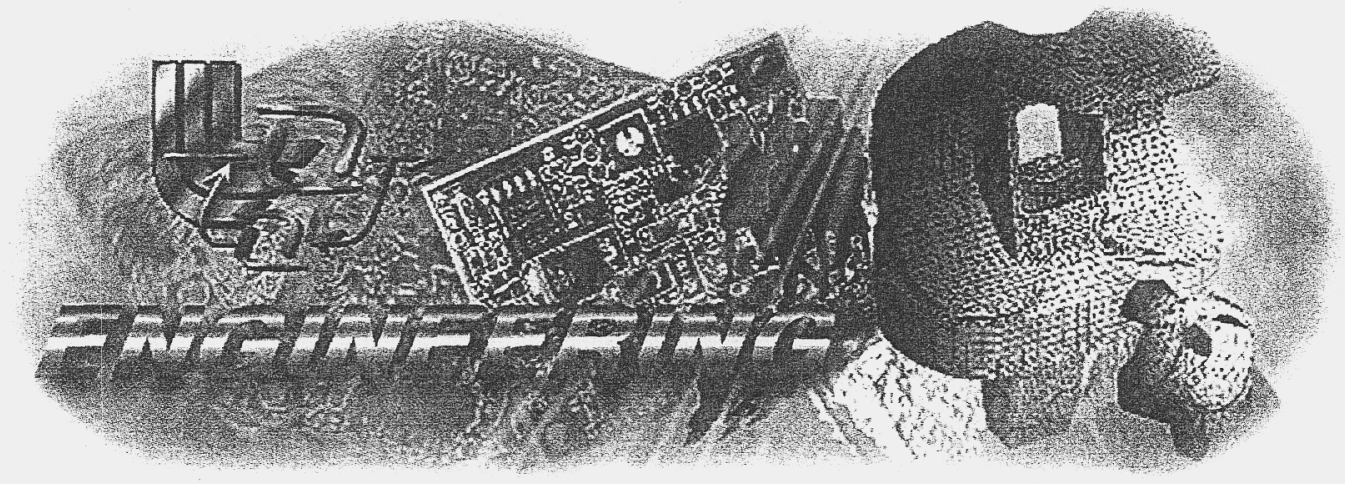

Spiros Dimolitsas Associate Director for Engineering

\section{LLNL Organization}

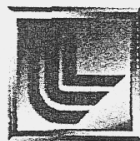

Ronald W. Cochran Laboratory Executive Officer

Ronald F. Lehman
Center for Global
SecurityResearch

Engineering

Spiros Dimolitsas Associate Director

Chemistry \& Materials Science

Harold C. Graboske Jr. Associate Director

\begin{tabular}{|c|c|}
\hline \multicolumn{2}{|c|}{ C. Bruce Tarter, Director } \\
\hline Science \& Technology & Operations \\
\hline $\begin{array}{l}\text { Jeffrey Wadsworth } \\
\text { Deputy Director }\end{array}$ & $\begin{array}{l}\text { Robert Kuckuck } \\
\text { Deputy Director }\end{array}$ \\
\hline & $\begin{array}{l}\text { Dennis K. Fisher } \\
\text { Associate Deputy Director }\end{array}$ \\
\hline $\begin{array}{l}\text { Defense \& Nuclear } \\
\text { Technologies }\end{array}$ & $\begin{array}{l}\text { Earth \& Environmental } \\
\text { Sciences }\end{array}$ \\
\hline $\begin{array}{l}\text { Michael R. Anastasio } \\
\text { Associate Director }\end{array}$ & $\begin{array}{l}\text { Leland W. Younker (acting) } \\
\text { Associate Director }\end{array}$ \\
\hline $\begin{array}{l}\text { Nonproliferation/Arms } \\
\text { Controlinternational Security }\end{array}$ & $\begin{array}{c}\text { Biology \& Biotechnology } \\
\text { Research }\end{array}$ \\
\hline $\begin{array}{l}\text { Wayne J. Shotts } \\
\text { Associate Director }\end{array}$ & $\begin{array}{l}\text { Anthony V. Carrano } \\
\text { Associate Director }\end{array}$ \\
\hline Laser Programs & Energy Programs \\
\hline $\begin{array}{l}\text { E. Michael Campbell } \\
\text { Associate Director }\end{array}$ & $\begin{array}{l}\text { Terrence G. Surles } \\
\text { Associate Director }\end{array}$ \\
\hline
\end{tabular}

National Security

George H. Miller

Associate Director

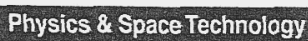

Richard J. Fortner Associate Director

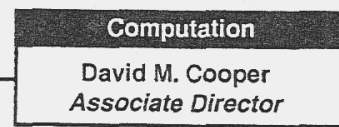

EMGNEEMING 

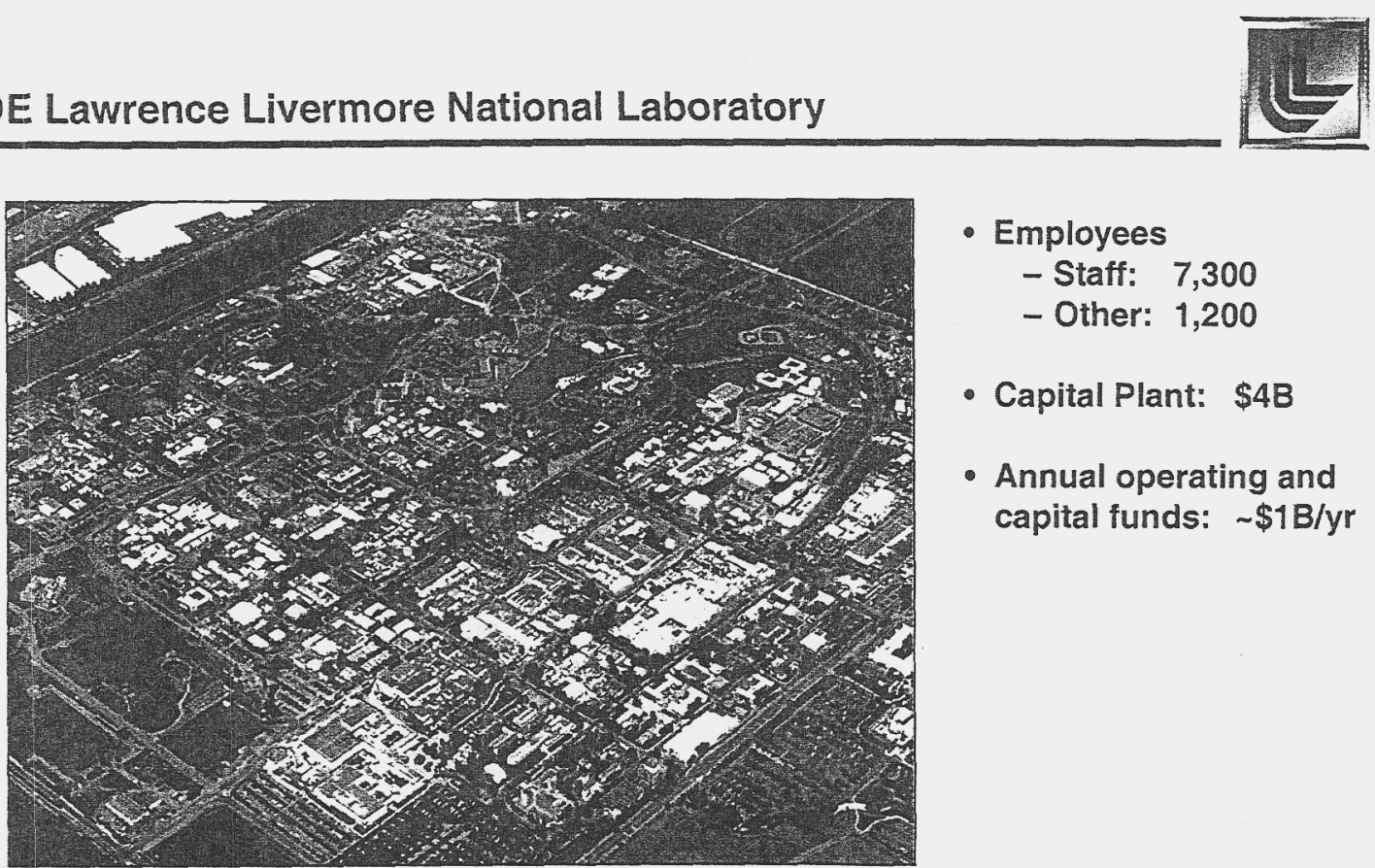

- Employees

- Staff: 7,300

- Other: 1,200

- Capital Plant: $\$ 48$

- Annual operating and capital funds: $\quad \$ 1 \mathrm{~B} / \mathrm{yr}$

Managed by the University of California since 1952

EMGINEEIMG D?

\section{Global Security}

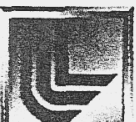

LLNL's defining mission is national security

- Dismantling the nuclear arsenals

- Stockpile stewardship

- Focused on the enduring stockpile

- Science-based (no underground testing)

- Production capability rather than capacity

- Dealing with the proliferation threat

- Detect

- Inhibit

- Respond

We have helped develop single, integrated multi-Lab programs in concert with DOE and others; execution of LLNL's part is through our Council on National Security

BMGINEERIMG 


\section{Characteristics of Lawrence Livermore National Laboratory}

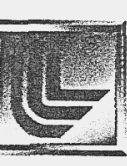

- Strong focus on problems of national importance

- Intentionally and intensely multi-disciplinary

- Science and technology are mostly programmatically driven

- Entrepreneurial, with an emphasis on high risk, high leverage technological solutions

- Utilizes teams on projects, not individuals on grants

Our Core Competencies allow us to solve problems involving multiple extreme dimensionalities simultaneously

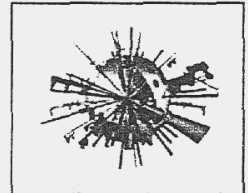

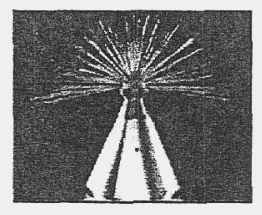

Measurement Science at Extreme Dimensionalities
Integrated Engineering of Large-Scale Complex Applied Physics Systems

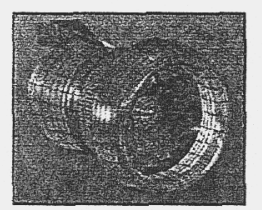

Large-Capacity Computational Modeling and Simulation

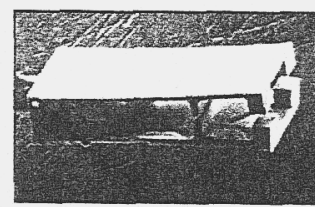

Microscale Engineering

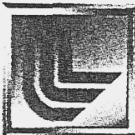

Integrated/Multidisciplinary $\bullet$ Couplex $\bullet$ Precise $\bullet$ Modelled

ENGMEERING 


\section{LLNL's Programmatic Evolution}
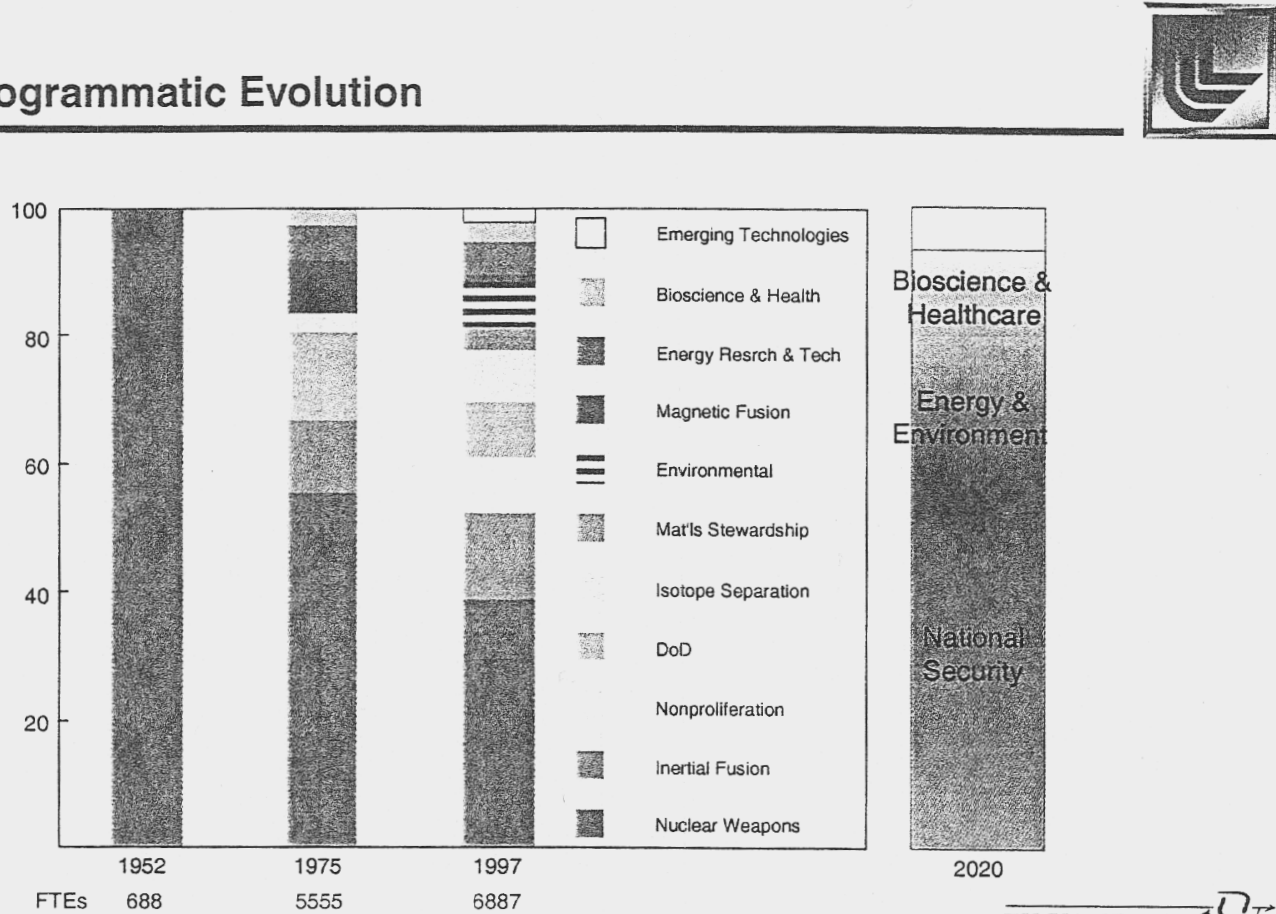

ENGINEERING TDT

A complex football-stadium-sized structure delivers 700 tera watts of laser power with $50 \mu \mathrm{m}(\mathrm{rms})$ accuracy

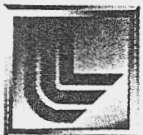

- 4000 tons of steel

- 2700 class-100 assemblies

- 35,000 control points

- 42,500 optical elements

- $18,000 \mathrm{ft}^{2}$ of optical surfaces

- 1.8 mega joules of energy

- 1 nanosecond event duration

- $<20 \mathrm{~nm}$ surface finish requirements

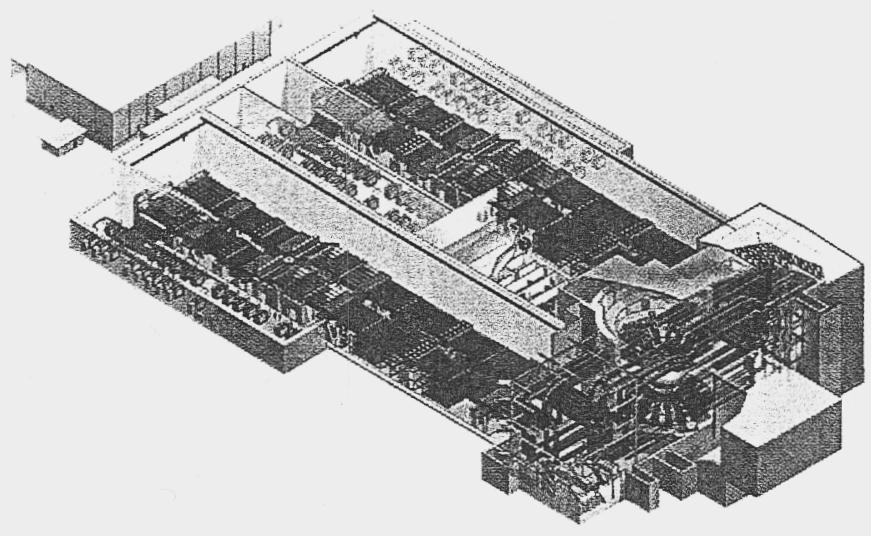

EMGINEERING TO 
DYNA3D simulations are critical to the process of design/analysis/experiment

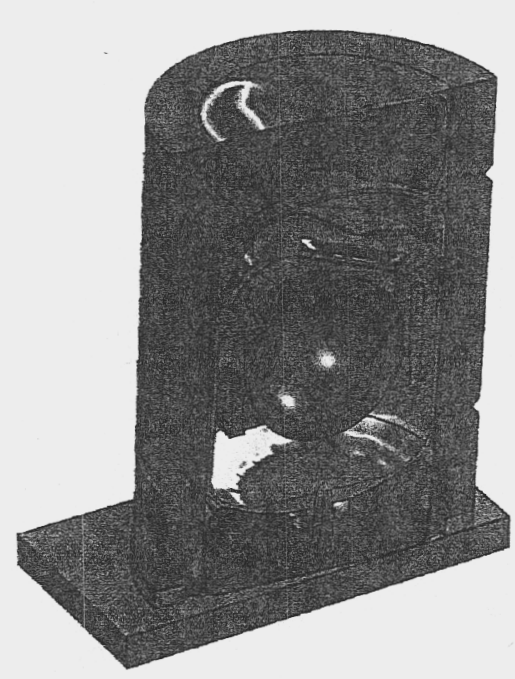

AT400A shipping container

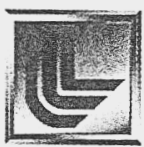

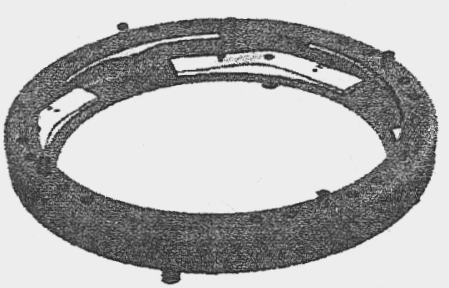

An energy absorption requirement demanded an analysis-driven flange design

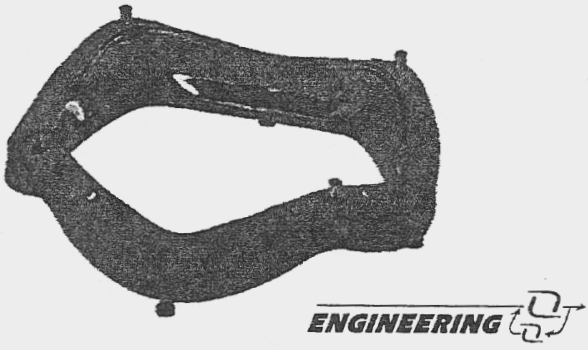

Our tiltmeter can detect 1-inch gap formations $10,000 \mathrm{ft}$. below the earth's surface and sense nanoradian earth-induced movement

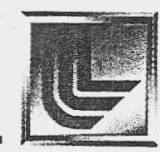

1997 R\&D-100 winner

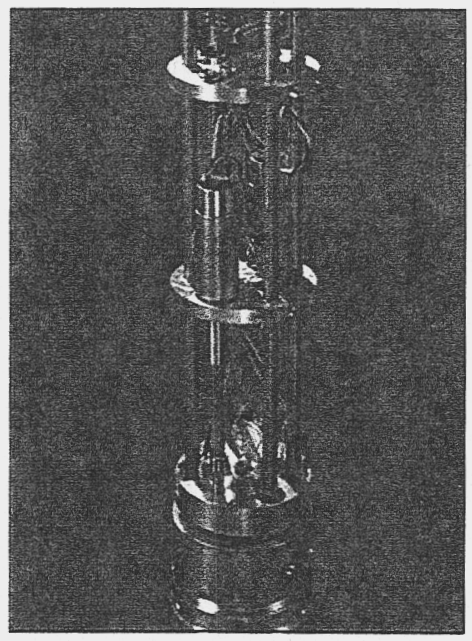


High-resolution, ultra-high speed, multi-frame stereoscopic imaging is a capability unique to LLNL

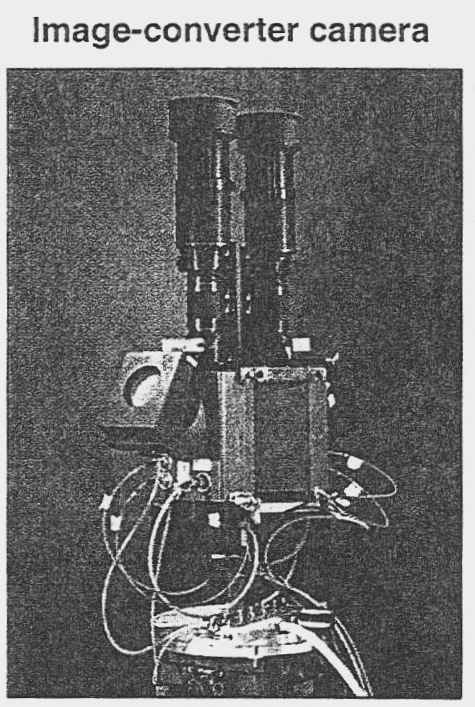

\section{Explosively-driven conical shell collapsing and} squirting out at $9.2 \mathrm{~km} / \mathrm{sec}$ (Mach number 27)

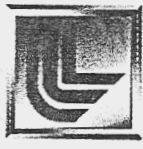

to +

17.1 usec
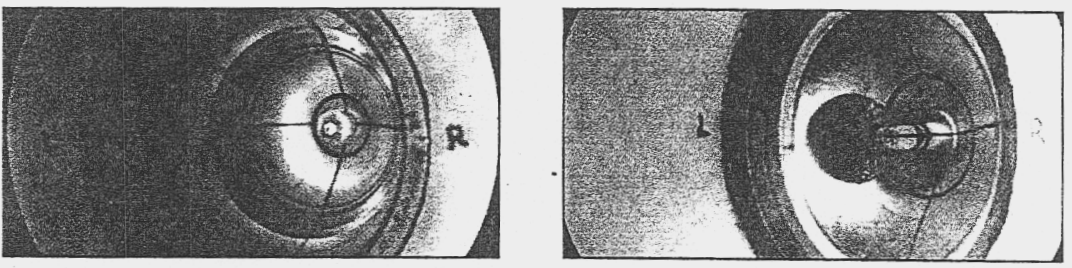

to +

$24.1 \mu \mathrm{sec}$

to + $40.8 \mu$ sec 


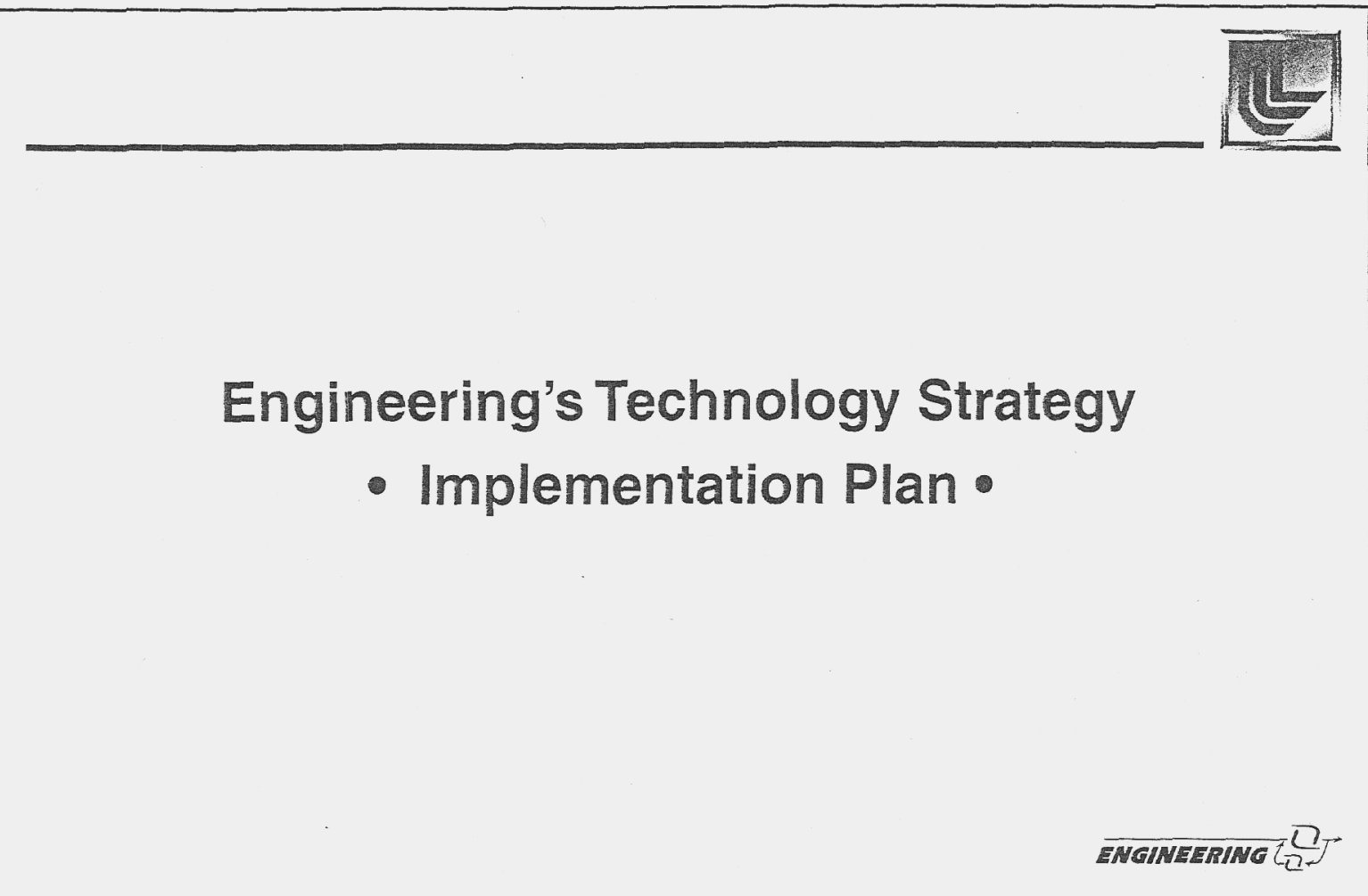

\section{Engineering's Technology Strategy \\ - Implementation Plan •}

EMGINEERING

A layered-structure can be defined to facilitate understanding and management of our core technologies and competencies

- Engineering competencies - definitions ( ...What we do)

- Engineering technologies - definitions (... How we do it) 
Engineering's Competencies as of August 1998

\begin{tabular}{|c|c|c|}
\hline Over-Arching Comperence & Corecampetencies & Application Comperencies \\
\hline \multirow{4}{*}{$\begin{array}{l}\text { Extreme Engineering: } \\
\text { Engineering of systems } \\
\text { involving the concurrent } \\
\text { integration of muitiple } \\
\text { technologies driven to } \\
\text { their extreme }\end{array}$} & $\begin{array}{l}\text { Integrated engineering of } \\
\text { large-scale, complex applied } \\
\text { physics systems }\end{array}$ & 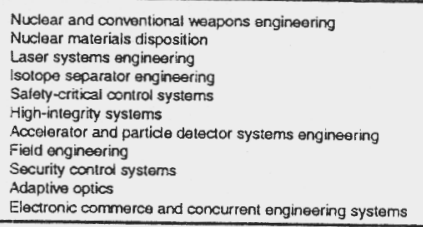 \\
\hline & $\begin{array}{l}\text { Large, complex } \\
\text { computational } \\
\text { modeling and } \\
\text { simulation }\end{array}$ & 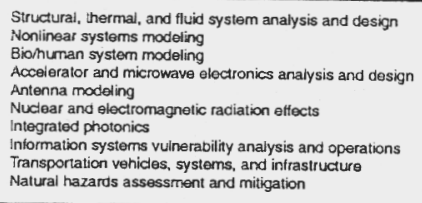 \\
\hline & Microscale engineering & 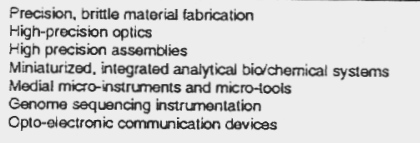 \\
\hline & $\begin{array}{l}\text { Measurement science at } \\
\text { extreme dimensionalities }\end{array}$ & 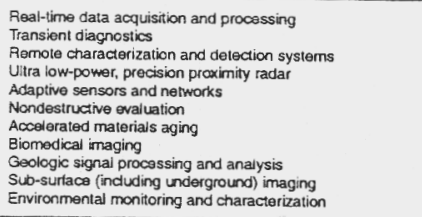 \\
\hline
\end{tabular}

DMGIMEERIMG T?

Engineering's Technologies as of August 1998

\begin{tabular}{|c|c|c|}
\hline Core Technologies & Enabling Technologies & Supporting Technologies \\
\hline Systems engineering & $\begin{array}{l}\text { Mochanical systerns design } \\
\text { Eloctronics and elsectrical systems design } \\
\text { Computer controls design } \\
\text { Optical enginoering } \\
\text { High power electro-optics } \\
\text { Soltware əngineering } \\
\text { Power conditioning ano pulse power } \\
\text { Reliability, avalability, and maintainability } \\
\text { analysis } \\
\text { Probabilistic risk analysis } \\
\text { Sparse data analysis }\end{array}$ & 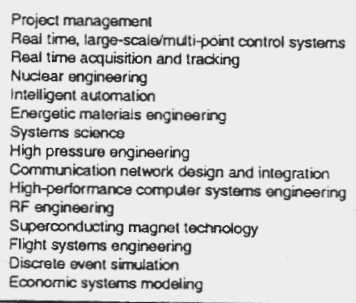 \\
\hline $\begin{array}{l}\text { Computational } \\
\text { engineering }\end{array}$ & 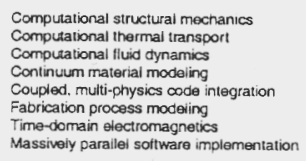 & $\begin{array}{l}\text { Computational toods for analysis and design } \\
\text { Mesh generation } \\
\text { Visumizzation } \\
\text { Numerical anaiysis } \\
\text { Large code architecture and design } \\
\text { Micro-scale mochanics and fluidics }\end{array}$ \\
\hline
\end{tabular}


Engineering's Technologies as of August 1998 (continued)

\begin{tabular}{|c|c|c|}
\hline Core Technologies & Enabling Technologies & Supporing Technologies \\
\hline Precision engineering & $\begin{array}{l}\text { Delerministic manuiacturing } \\
\text { Machine cesign and analysis } \\
\text { Precision postioning and control } \\
\text { rrittle materials processsing } \\
\text { Diamond turning } \\
\text { Precision grinding and polishing } \\
\text { Manufacturing process ceveropment and } \\
\text { control (casting. spin-forming welding, atc) }\end{array}$ & $\begin{array}{l}\text { Metal turning, shaping and torming } \\
\text { Composite and anisotropic materials fabrication } \\
\text { Metrology } \\
\text { Coatings and deposition } \\
\text { Multil-layer tabrication }\end{array}$ \\
\hline $\begin{array}{l}\text { Microsystems } \\
\text { technology }\end{array}$ & $\begin{array}{l}\text { Microsysterns integration and packaging } \\
\text { Opto-electronic materials processing } \\
\text { High-aspoct ratio micromactining } \\
\text { Controllad stress thin-fim tabrication } \\
\text { Microfluidics engineering } \\
\text { Material deposition } \\
\text { Integrated micro-actuators } \\
\text { Fusion bonding }\end{array}$ & $\begin{array}{l}\text { Microsystems procoss developpment } \\
\text { III-V material systems }\end{array}$ \\
\hline $\begin{array}{l}\text { Signal acquisition } \\
\text { and characterization }\end{array}$ & 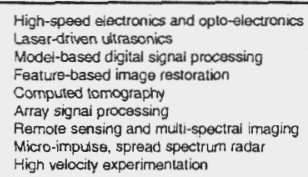 & 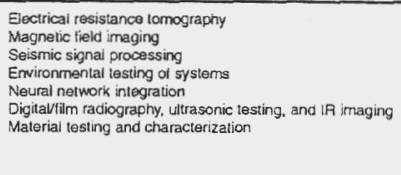 \\
\hline
\end{tabular}

RMAMEERMG DT

\section{Engineering Organization}

\begin{tabular}{|c|c|c|}
\hline & ENGINEERING & \\
\hline & 1 & \\
\hline $\begin{array}{l}\text { MECHANICAL } \\
\text { ENGINEERING }\end{array}$ & $\begin{array}{l}\text { ENGINEERING SCIENCE } \\
\text { ANDTECHNOLOGY } \\
\text { PROGRAM }\end{array}$ & $\begin{array}{l}\text { ELECTRONICS } \\
\text { ENGINEEAING }\end{array}$ \\
\hline $\begin{array}{l}\text { Applied Science } \\
\text { Engineering Division }\end{array}$ & $\begin{array}{l}\text { Center for } \\
\text { Computational } \\
\text { Engineering }\end{array}$ & $\begin{array}{l}\text { Electronics Engineering } \\
\text { Technologies Division }\end{array}$ \\
\hline $\begin{array}{l}\text { Manufacturing } \\
\text { and Materials } \\
\text { Engineering Division }\end{array}$ & $\begin{array}{l}\text { Center for } \\
\text { Precision } \\
\text { Engineering }\end{array}$ & $\begin{array}{l}\text { Defense Sciences } \\
\text { Engineering Division }\end{array}$ \\
\hline $\begin{array}{l}\text { New Technologies } \\
\text { Engineering Division }\end{array}$ & $\begin{array}{l}\text { Center for } \\
\text { Microtechnology }\end{array}$ & $\begin{array}{c}\text { Laser } \\
\text { Engineering Division }\end{array}$ \\
\hline $\begin{array}{l}\text { Laser Science } \\
\text { Engineering Division }\end{array}$ & $\begin{array}{l}\text { Center for } \\
\text { Non-Destructive } \\
\text { Evaluation }\end{array}$ & \\
\hline $\begin{array}{l}\text { Defense Technologies } \\
\text { Engineering Division }\end{array}$ & $\begin{array}{l}\text { Center for } \\
\text { Complex Distributed } \\
\text { Systems }\end{array}$ & $\overline{E N}$ \\
\hline
\end{tabular}




\section{Center mission}

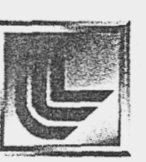

An Engineering Center is the focus at LLNL for the core technology represented.

- An Engineering Center has been delegated by the Directorate the responsibility for the vitality and growth of the core technology it represents. Hence, it focuses and guides investments in enabling technologies and supporting capabilities.

- A Center is an Engineering R\&D "program" whose output is technology.

- When a Center has a focus that is narrower than the core technology it is most closely related to, the Center will in addition provide a forum for the discussion of issues related to the broader core technology area (e.g. the Center on NDE will provide a forum for the discussion of issues related to "signal acquisition and characterization")

A successful Engineering Center...

- Solves compelling problems that require breakthrough innovation.

- Is world class, or is on a path to become world class, and put Engineering on the national map.

- Broadly impacts the Laboratory, enables new programs (one technology focus, many program applications).

- Requires and drives mechanical and electrical engineering enabling technologies to advance (is cross cutting).

- Is enabled and is critically dependent on unique LLNL capabilities (to create a sustainable differential advantage).

- Has close and/or significant University affiliations.

- Facilitates the introduction of new technology to applications (programs) and provides an avenue to facilitate access to engineering enabling technologies.

- Develops a significant intellectual property base.

- Can attract external sponsorship.

A robust Center solves compelling problems that require world-class innovation $\overline{\text { EMGINEERING }}$ ? 


\title{
Diffraction Tomography
}

\author{
By A.J. Devaney \\ Department of Electrical Engineering \\ Northeastern University \\ Boston, MA 02115
}

This talk will trace the evolution of diffraction tomography (DT) from its foundations in X-ray Crystallography to its current status as a rigorous solution to a host of linearized inverse problems involving acoustic, electromagnetic and optical wavefields. Special attention will be devoted to applications of DT to statistically based problems such as target detection and identification and parameter estimation from noisy wavefield data. The talk will review a number of example applications of modern DT that include imaging and parameter estimation from ground penetrating radar (GPR), ultrasound tomography, and optical diffraction microscopy. Current commercial ventures aimed at developing a high resolution optical microscope based on the concepts of optical diffraction microscopy and the development of a fast and efficient GPR system capable of mapping underground utility lines will be reviewed.

\section{Diffraction Tomography}

\author{
A.J. Devaney \\ Department of Electrical and Computer Engineering \\ Northeastern University \\ Boston, MA 02115 \\ tonydev2@aol.com \\ - Brief history \\ - Mathematical formulation \\ - Examples \\ - Summary and comments
}

Physics based signal processing 


\section{Problems addressed by DT}

\section{Geophysical}

Electromagnetic

Acoustic
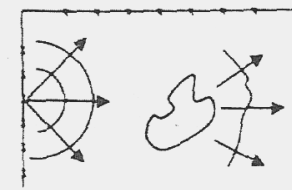

Medical

Ulirasonic

Optical

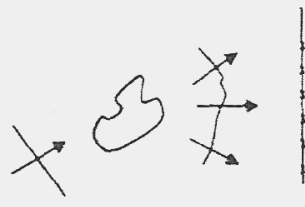

Ultrasound tomography opxical microscopy phoron imaging

Industrial

Electromagnetic Ultrasonic Optical

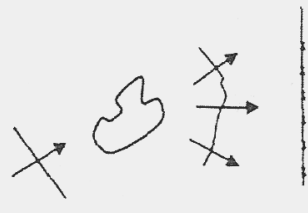

Ultrasound tomography optical microscopy induction imaging

\section{Historical survey}

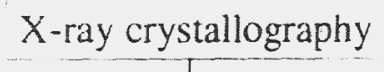

Fourier based

Born/Rytov inversion

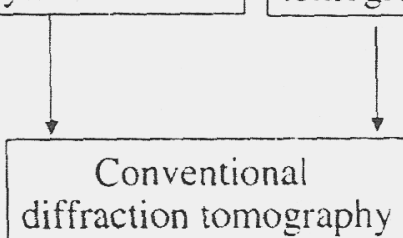

Computed

tomography

\section{Diffraction Tomography}




\section{Canonical scattering configuration}

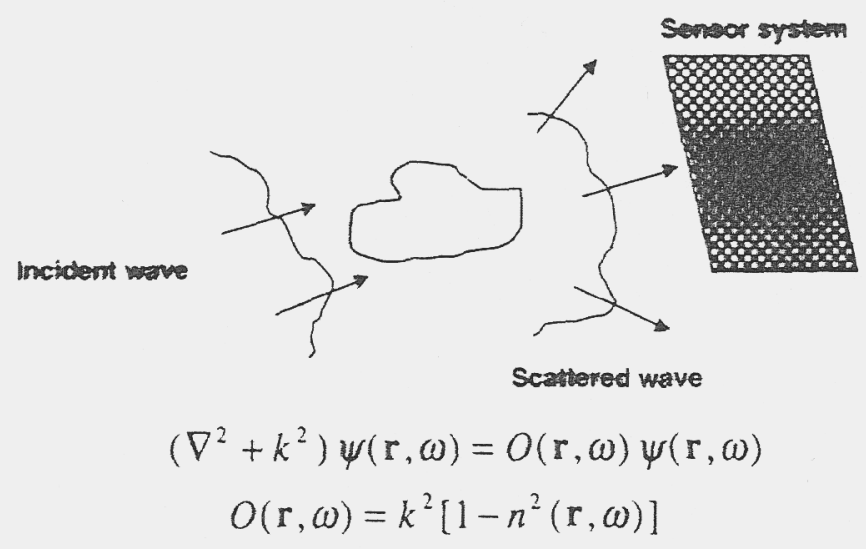

Inverse scattering problem: Given set of scattered field measurements determine object function

\section{Scattering models}

$\psi_{i n}(\mathbf{r}) \longrightarrow \underbrace{O(\mathbf{r})}_{\psi^{(n)}(\mathbf{r})=-\frac{1}{4 \pi} \int d^{i} r^{\prime} O\left(\mathbf{r}^{\prime}\right) \psi\left(\mathbf{r}^{\prime}\right) \frac{e^{\left.i k \mathbf{r}-\mathbf{r}^{\prime}\right)}}{\left|\mathbf{r}-\mathbf{r}^{\prime}\right|}}$

Born Model $\psi_{g}^{\prime(s)}(\mathbf{r})=-\frac{1}{4 \pi} \int d^{3} r^{\prime} O\left(r^{\prime}\right) \psi_{\text {in }}\left(r^{\prime}\right) \frac{e^{i k \mid r-r^{\prime}}}{\left|r-r^{\prime}\right|}$

Rytov Model $\quad \psi=e^{i k\left|W_{0}(r)+\delta W(r)\right|}$

$$
\begin{aligned}
& \delta W_{k}(\mathbf{r})=\frac{i}{4 \pi k} \int d^{3} r^{\prime} O\left(\mathbf{r}^{\prime}\right) e^{-i k\left|W_{n}(r)-W_{0}(\mathbf{r})\right|} \frac{e^{i k i \mathbf{r}-\mathbf{r}}}{|\mathbf{r}-\mathbf{r}|} \\
& \psi_{B}^{(s)}(\mathbf{r})=i k e^{i k W_{0}(\mathbf{r})} \delta W_{R}(\mathbf{r})
\end{aligned}
$$




\section{Accuracy of Rytov approximation}

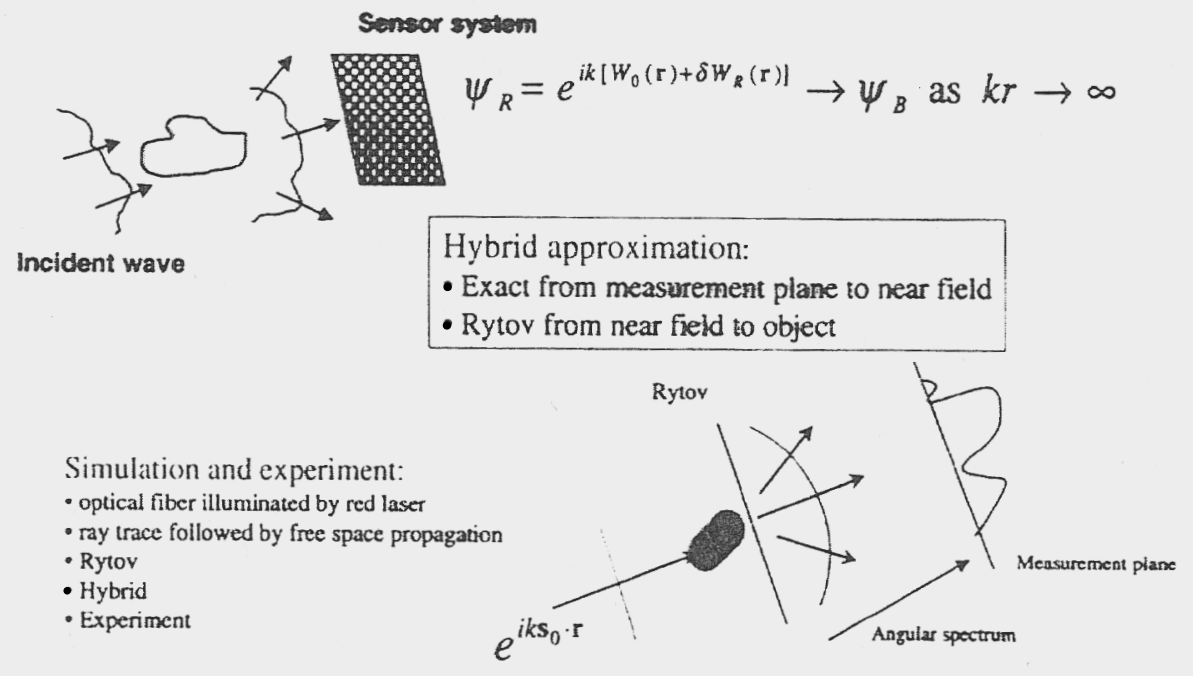

Ray trace, hybrid and Rytov
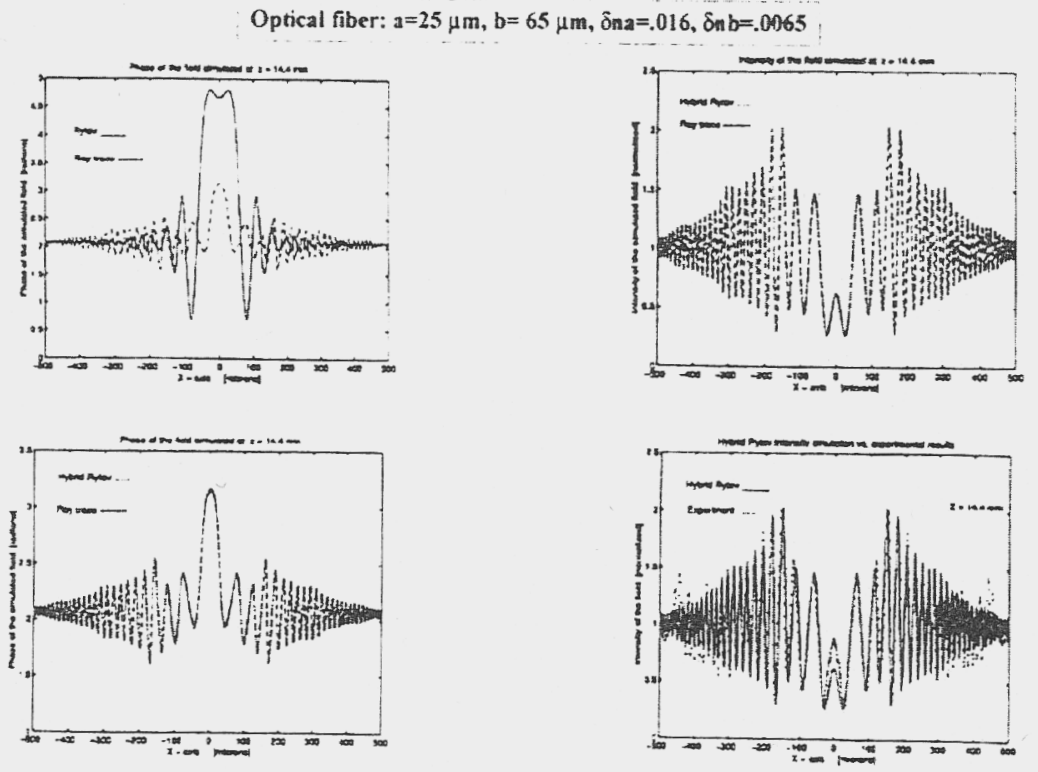


\section{Rytov vs hybrid model}
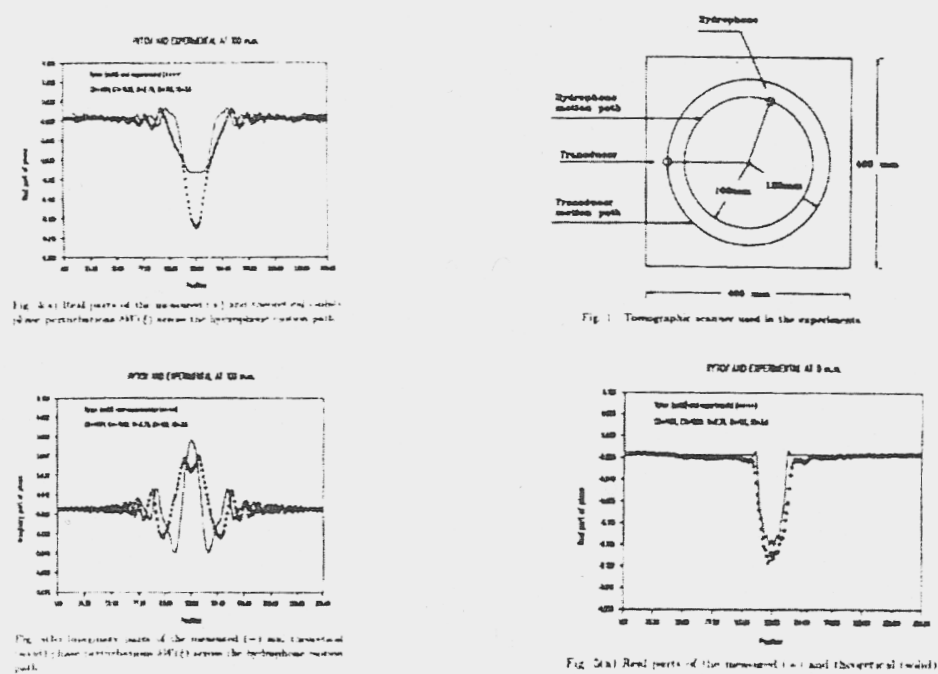

\ Sponheim, 1 Johansen. A J Devaney, Acoustical Imagning Fol 18 ed $\mathrm{H}$ Lee and $\mathrm{G}$ Wade, 1989

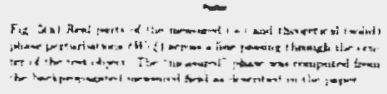

\section{Inverse problem}

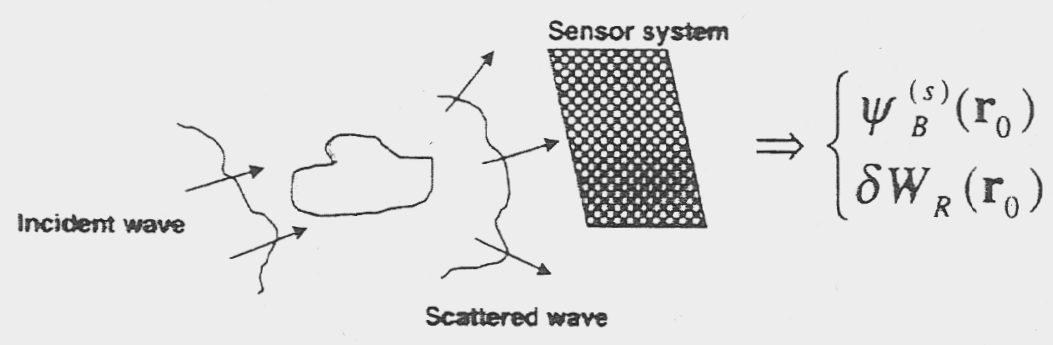

Inversion Algorithm

$$
\left.\begin{array}{l}
\psi_{B}^{(s)}\left(\mathbf{r}_{0}\right) \\
\delta W_{R}\left(\mathbf{r}_{0}\right)
\end{array}\right\} \Rightarrow O(\mathbf{r}, \omega)
$$

Problem reduces to solving set of coupled linear integral equations 


\section{Why tomography?}

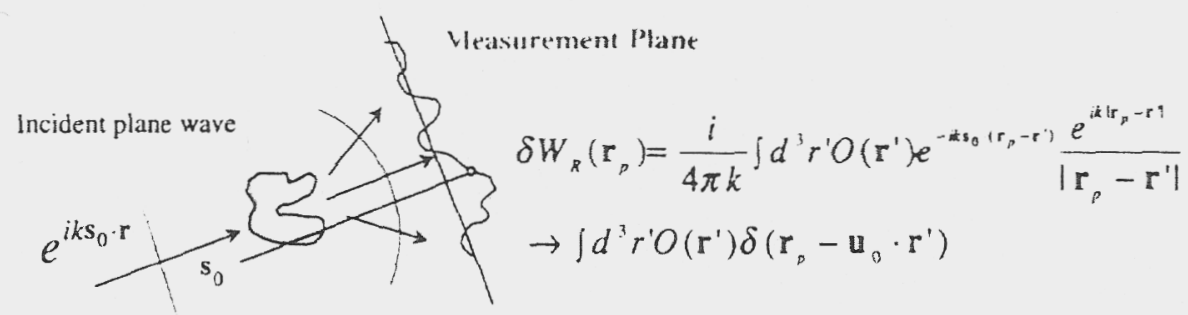

Integral along straightline ra! path: Inversion via CT

Diffraction tomography (DT) is generalization of CT to diffracting wavefields

- Filtered backpropagation

- Generalized ART and SIRT

- Various limited view algorithms

\section{Born approximation imaging}

$$
\psi_{b}^{s}(\mathbf{r}, \omega)=-\frac{1}{4 \pi} \int d^{3} r^{\prime} O\left(\mathbf{r}^{\prime}, \omega\right) \psi_{i n}\left(\mathbf{r}^{\prime}, \omega\right) \frac{e^{i k\left|\mathbf{r}-\mathbf{r}^{\prime}\right|}}{\left|\mathbf{r}-\mathbf{r}^{\prime}\right|}
$$

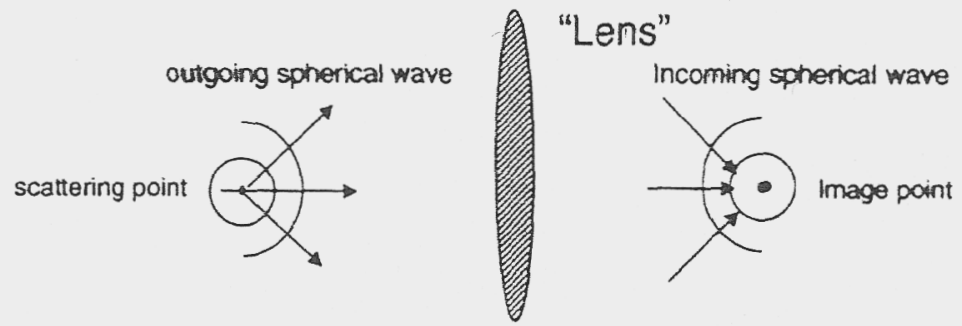

$$
\begin{gathered}
-\frac{1}{4 \pi} \frac{e^{i k\left|\mathbf{r}-\mathbf{r}^{\prime}\right|}}{\left|\mathbf{r}-\mathbf{r}^{\prime}\right|} \Rightarrow h\left(\mathbf{r}-\mathbf{r}^{\prime}, \omega\right) \\
\psi_{b}^{s}(\mathbf{r}, \omega) \Rightarrow \psi_{l}(\mathbf{r}, \omega)=\int d^{3} r^{\prime} O\left(\mathbf{r}^{\prime}, \omega\right) \psi_{\text {in }}\left(\mathbf{r}^{\prime}, \omega\right) h\left(\mathbf{r}-\mathbf{r}^{\prime}, \omega\right)
\end{gathered}
$$




\section{Backpropagation-the digital lens}

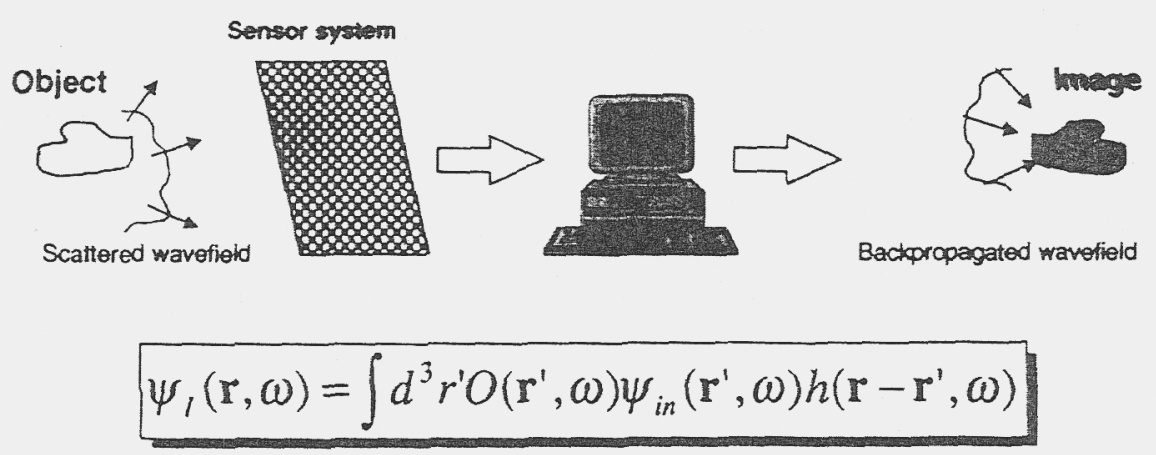

Single experiment generates mage of the product $O\left(\mathbf{r}^{\prime}, \omega\right) \psi_{\text {in }}\left(\mathbf{r}^{\prime}, \omega\right)$

\section{The backpropagation algorithm}

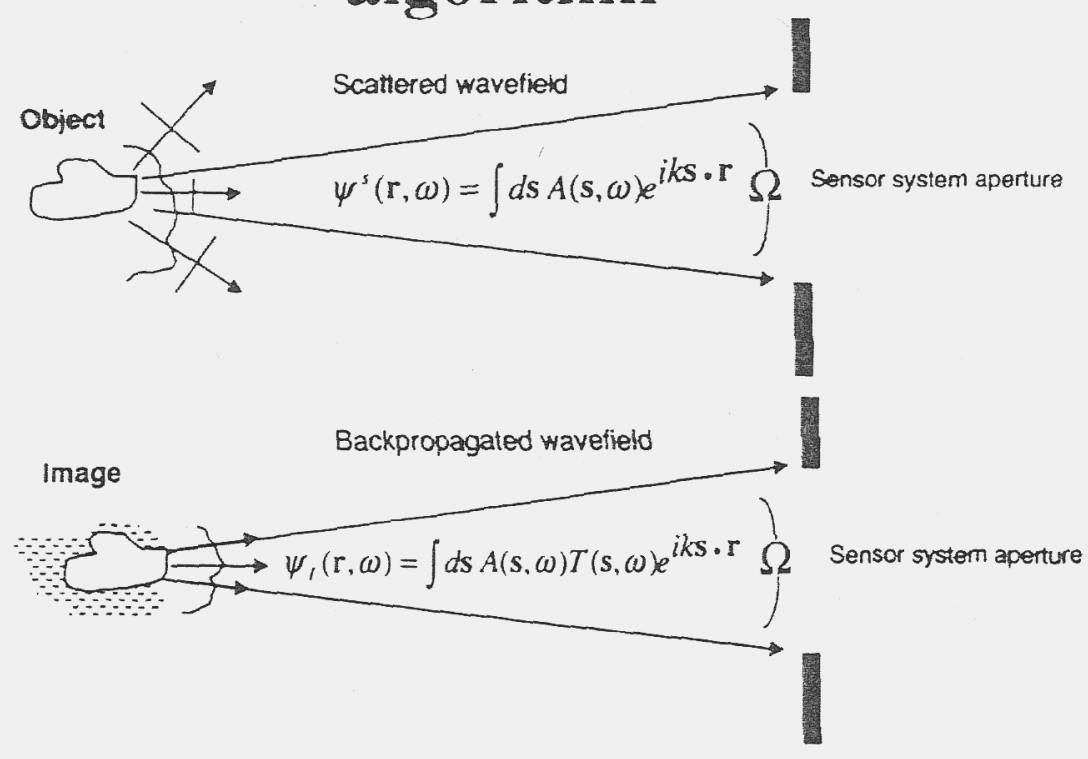




\section{The backpropagation point spread function}

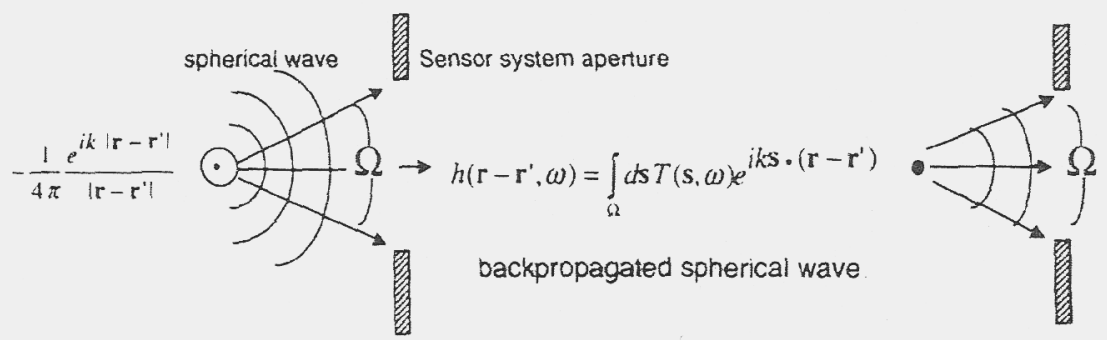

Point spread function is the image of a point (delta function) scatterer

Aperture function $T(s, \omega)$ models sensor and computational inaccuracies

\section{Point spread function}

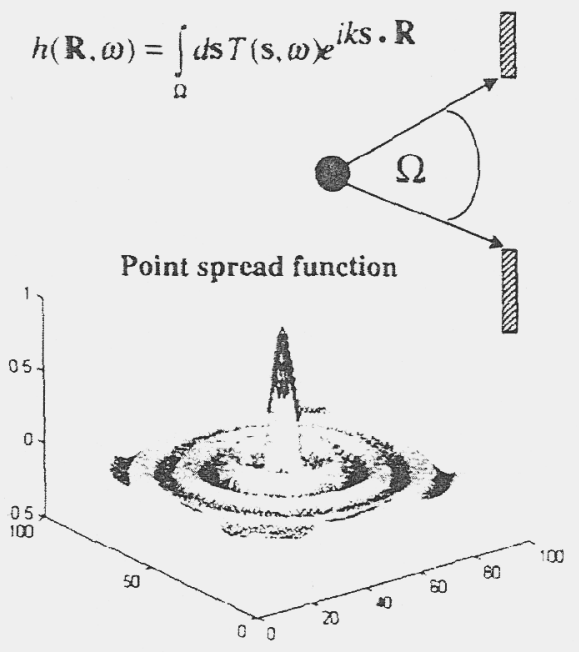

Leal Case :

Zero aberration and $\Omega=4 \pi$ steradians

$h(\mathbf{R}, \omega)=\frac{\sin k R}{k R}=\operatorname{sinc}(k R)$

Coberemt transfer function

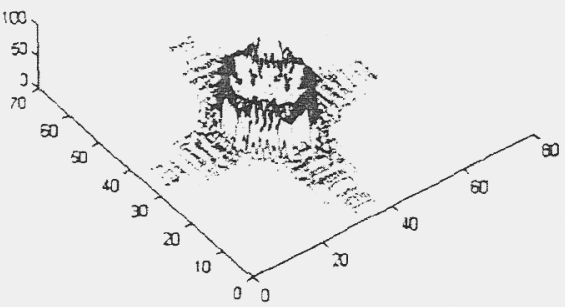




\section{Plane wave diffraction tomography}

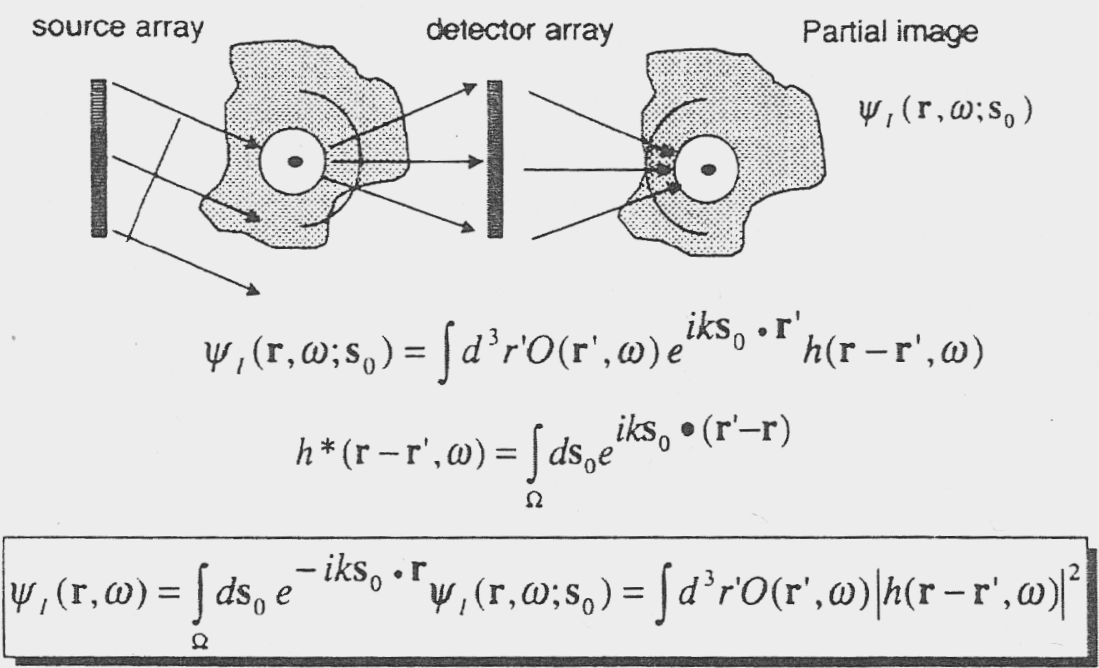

\section{Image quality}

$$
\begin{aligned}
& \psi_{l}(\mathbf{r}, \omega)=\int d^{\prime} r^{\prime} O\left(\mathbf{r}^{\prime}, \omega\right)\left|h\left(\mathbf{r}-\mathbf{r}^{\prime}, \omega\right)\right|^{2} \\
& |h(\mathbf{R}, \omega)|^{2}=\left(\frac{\sin (k R)}{k R}\right)^{2}
\end{aligned}
$$
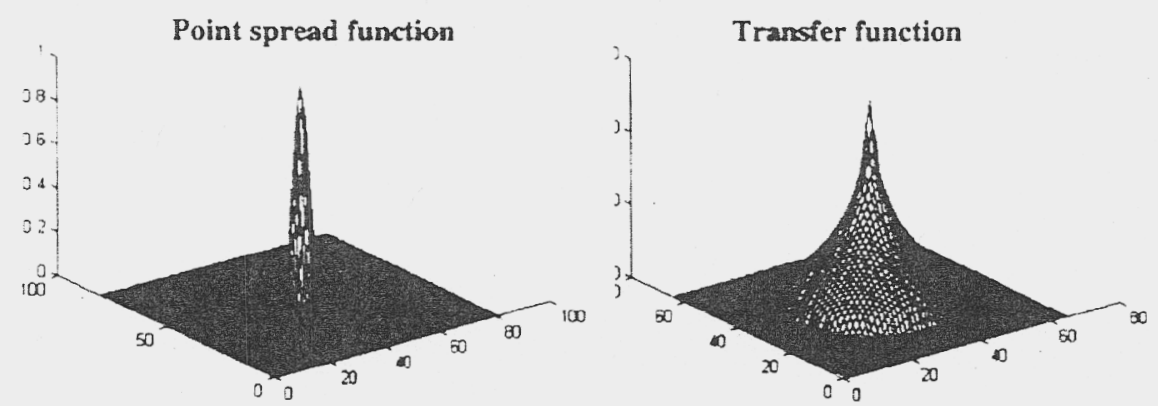


\section{Image processing}

$$
\begin{gathered}
|h(\mathbf{R}, \omega)|^{2}=\left(\frac{\sin (k R)}{k R}\right)^{2} \\
H(\mathbf{K})=\frac{1}{K} \text { for } K<2 k \Rightarrow H^{-1}(\mathbf{X})=K \\
\psi_{,}(\mathbf{r}, \omega)=\int d^{3} r^{\prime} O\left(\mathbf{r}^{\prime}, \omega\right)\left|h\left(\mathbf{r}-\mathbf{r}^{\prime}, \omega\right)\right|^{2} \\
\hat{O}(\mathbf{r}, \omega)=H^{-1} \otimes \psi_{1}(\mathbf{r}, \omega)=\int d^{3} r^{\prime} O\left(\mathbf{r}^{\prime}, \omega\right) \Delta\left(\mathbf{r}-\mathbf{r}^{\prime}, \omega\right)
\end{gathered}
$$

Filtered backpropagation algorithm

$$
\left.\hat{O}(\mathbf{r}, \omega)=\sum_{s_{0}} \mathrm{BP}\left\{Q_{\mathrm{s}_{0}} \otimes \psi_{\mathrm{s}_{0}}^{s}\right\} \mathbf{r}, \omega\right)
$$

\section{Filtered backpropagation algorithm}

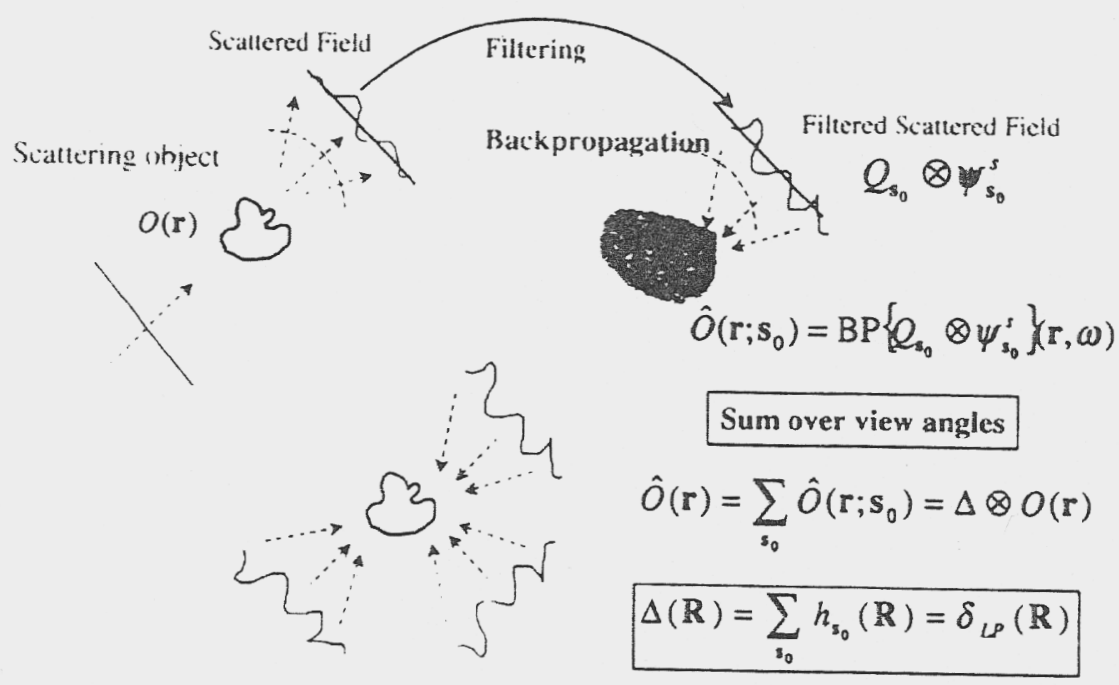




\section{Simulations}

2D objects: objects composed of superposition of cylivaders

0
00
- Single view as function of wavelength
- multiple view at fixed wavelength
- Comparison of (T versas DT with DT data
- muhipte vieu as function of war elength

Simulations test DT algorithms and not Rytov model

\section{Image quality as function of number of views}

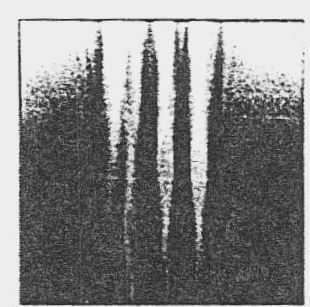

one view

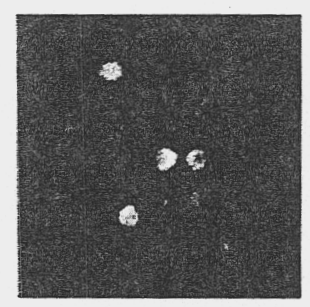

5 views

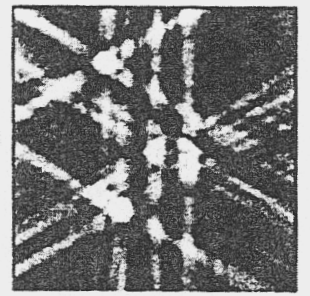

3 views

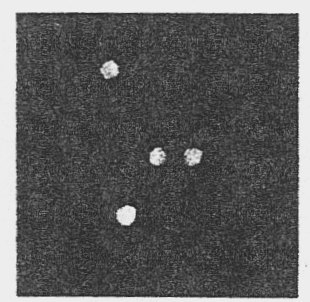

7 views 


\section{Backpropagation as a function of wavelength}

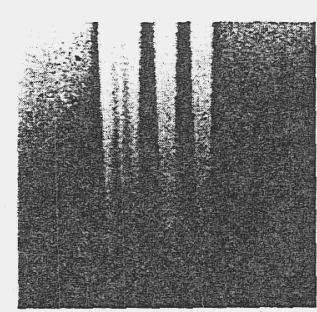

wavelength $=0.1$

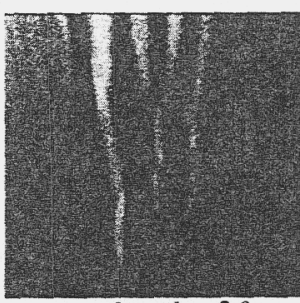

wavelength $=3.0$

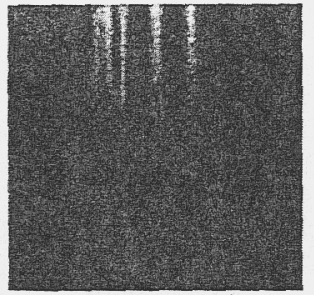

wavelength $=0.5$

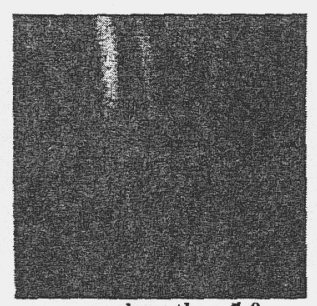

wavelength $=\mathbf{5 . 0}$

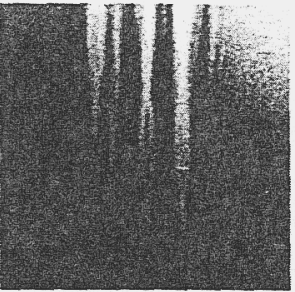

wavelength $=1.0$

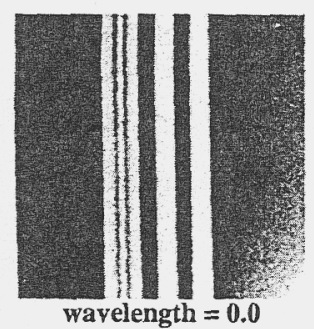

\section{Filtered backprojection and backpropagation} algorithms applied to scattered field data
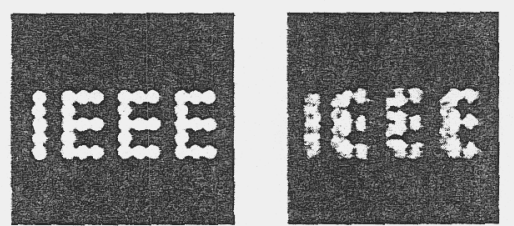

wavelength $=\mathbf{1 . 0}$

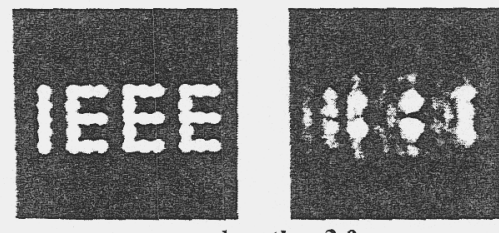

wavelength $=3.0$

Filtered backpropagation versus filtered backprojection
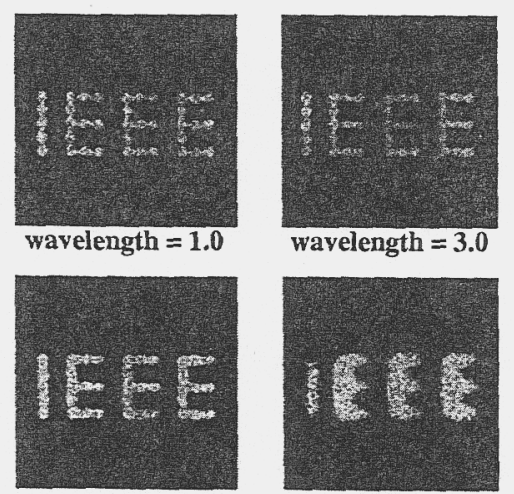

wavelength $=\mathbf{3 . 0}$

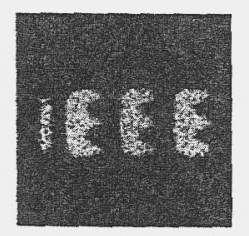

wavelength $=10.0$

Filtered backpropagation as function of wavelength 


\section{Norwegian ultrasound tomography system}

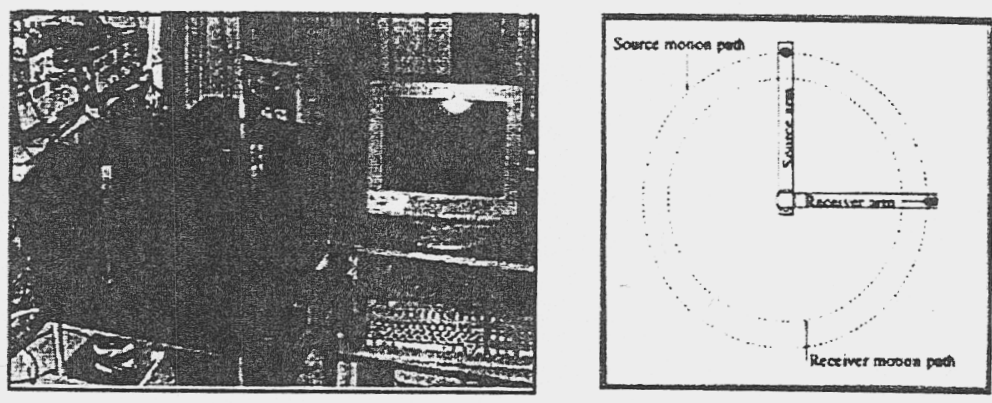

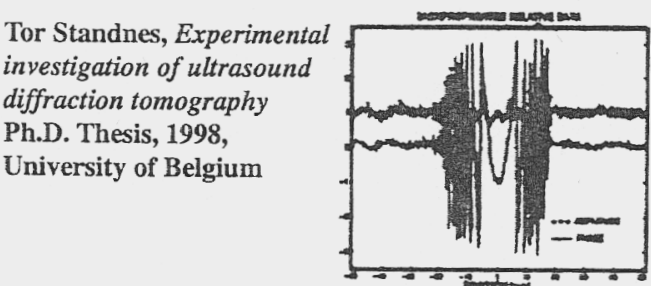

(a)

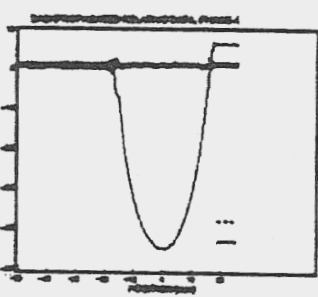

(b)

\section{Ultrasound simulations: Born vs Rytov}

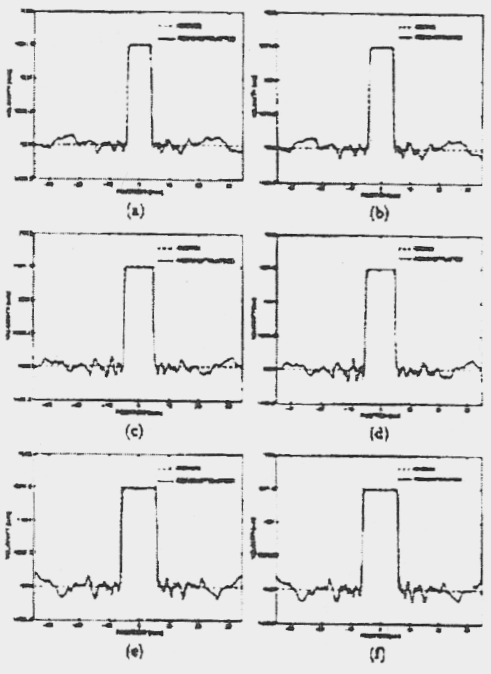

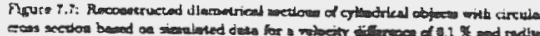

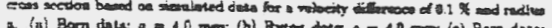

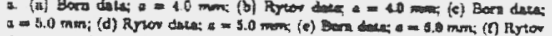
dats; $0=0.0 \mathrm{~mm}$
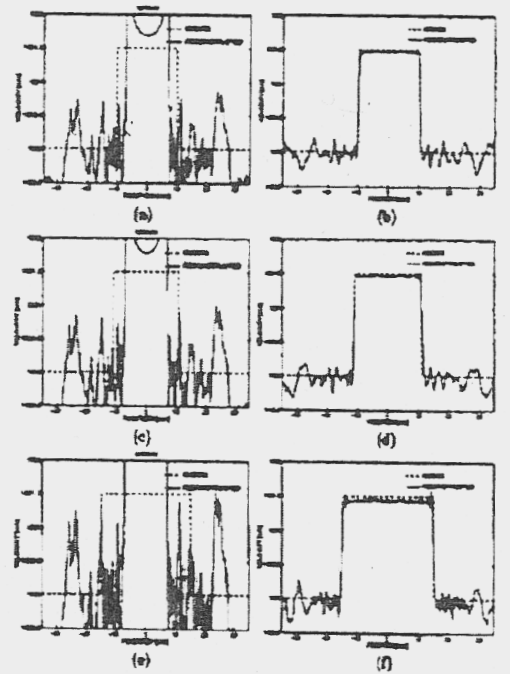

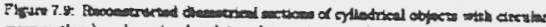

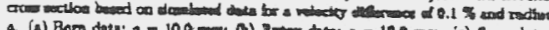

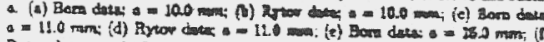

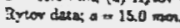


Ultrasound reconstructions from real data
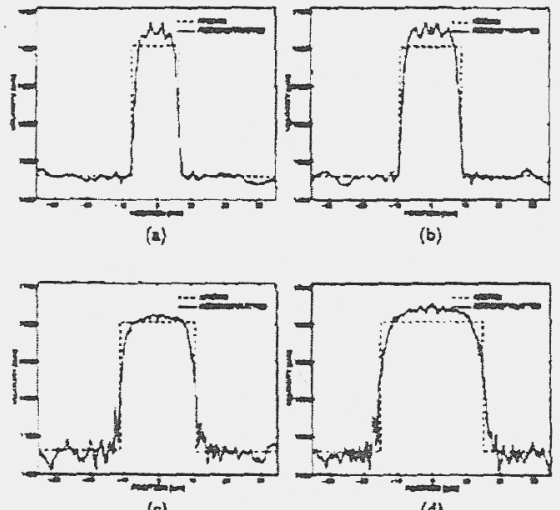

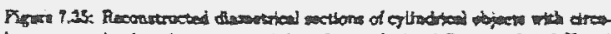

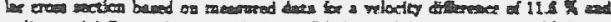

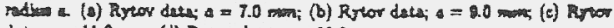

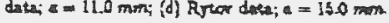

Tor Standnes, Experimental mestrgution

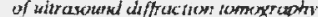

Ph. D Thesis, 1998. University of Bergen

\section{FBP vs ART reconstructions from ultrasound data}

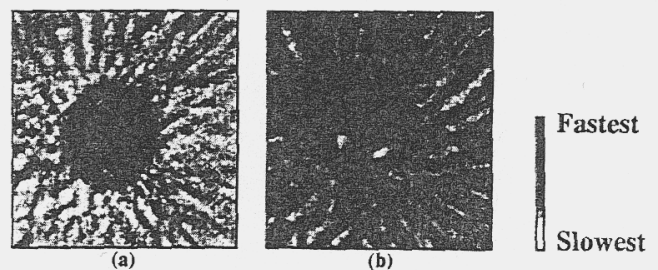

Fig. 5 (a) Real part of the reconstruction generated from the FBP algorithm from data taken at 26 equally spaced view angles. (b) Imaginary part of the reconstruction generated from the FBP algorithm from data taken at 26 equally spaced view angles.
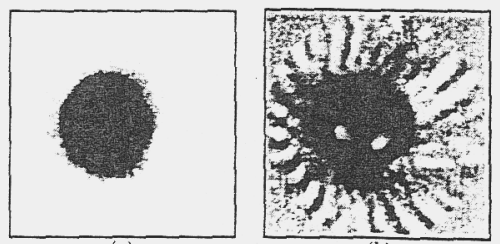

(b)

Fig. 6 (a) Real part of the reconstruction generated from the DT-ART algorithun from data taken at 26 equally spaced view angles. (b) Imaginary part of the reconstruction generated from the DT-ART algorithm from data taken at 26 equally spaced view angles. 


\section{What's Wrong with Optical Microscopy?}

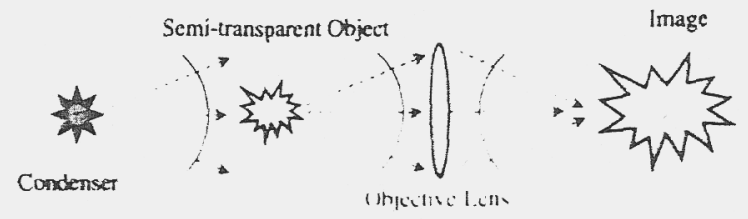

- Illuminating light spatially coherent over small scale:

- Poor image quality for 30 objects

- Need to thin slice

- Cannot image phase only ohjects:

- Need to stain

- Need to use special phase contrast methods

- Require high quahity optics

\section{Inverse Problem}

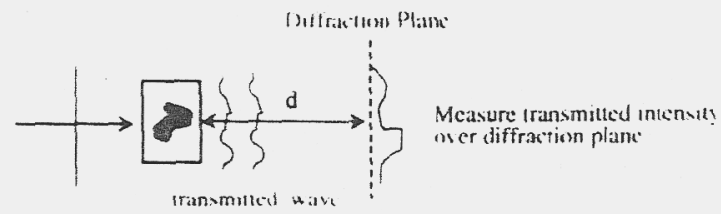

Inverse Problem: Given iuteusil of transmitted ware estimale the comples

inder of refraction distribution of the object.

Difficultien

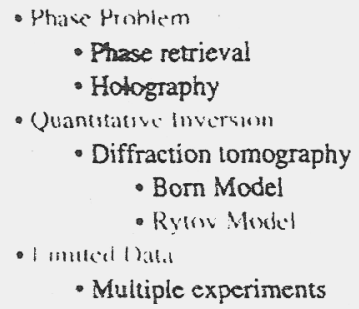




\section{Experimental systems for optical diffraction tomography}

Torolf Wiedberg, Quanututive phase microscopy by ho-dimenstonal optical diffraction tomograph

Ph. D. Thesis. 1994, University of Bergen

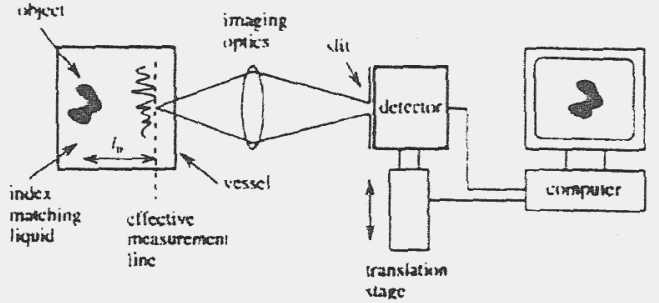

Yohammad Maleki, Optical diffruction tomograph Ph. D. Thesis. 1994, Northeastem University, Boston

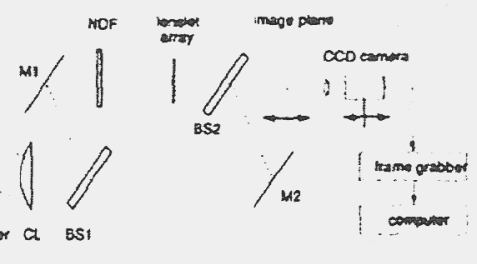

Fig. 6 Experimental setup where $\mathrm{CL}$ is the collimating lens, BS is the beam splitter, $M$ is the mirror, and NDF is the neutral-density tilter.

\section{ODT reconstructions directly from intensity}

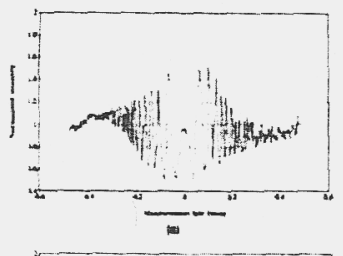

\section{Optical fiber: $=1.2 \mu \mathrm{m}, b=6.22 \mu \mathrm{m}, \delta \mathrm{n}=11.5 \times 10^{-3}$}

M Maleki, A.J. Devaney, A. Schatzberg, JOSA A, Vol. 9,1992
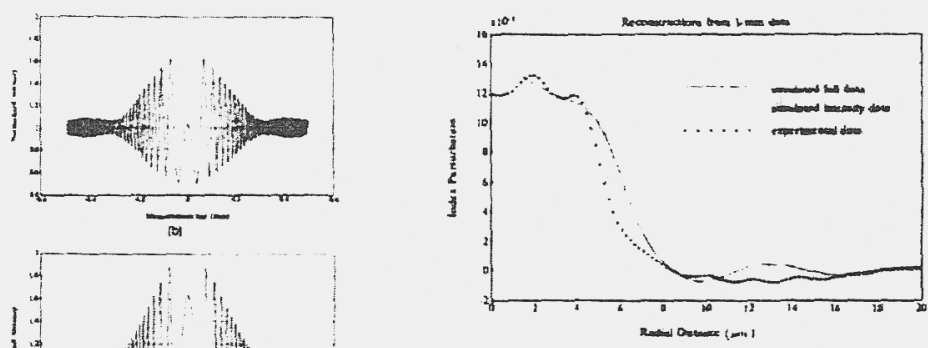

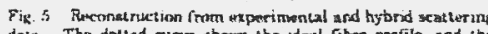
datu. The dotled eurve shows the deal fiber profile and the

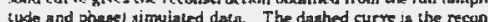
atruction obtuined from the hybrid intensity data, and the reconstruxtion from the rxperimsental intensity data is dimphayed with giusm: 1 . 


\section{Experimental results from ODT}
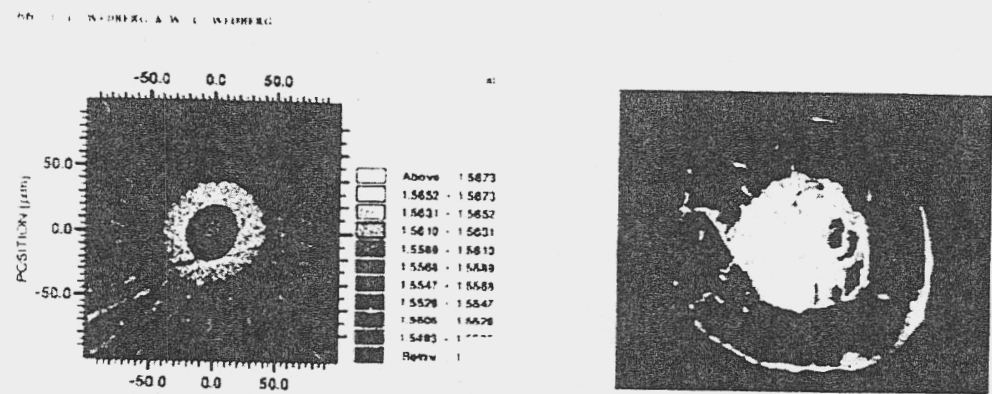

$500 \quad 00 \quad 580$

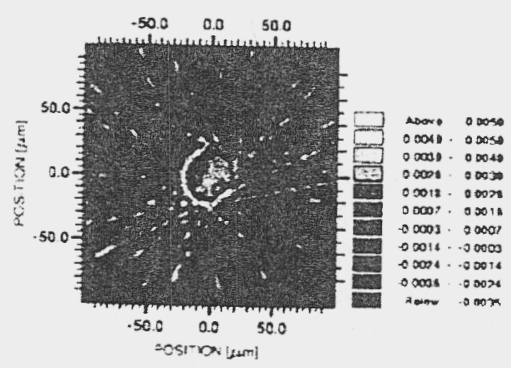

T. Wedberg and W. Wedberg, "Tomographic reconstruction of the cross-section refractive index distribution in semi-transparent, birefringent fibres." fontrit of Vicroscopy. Vol. 177, i 995

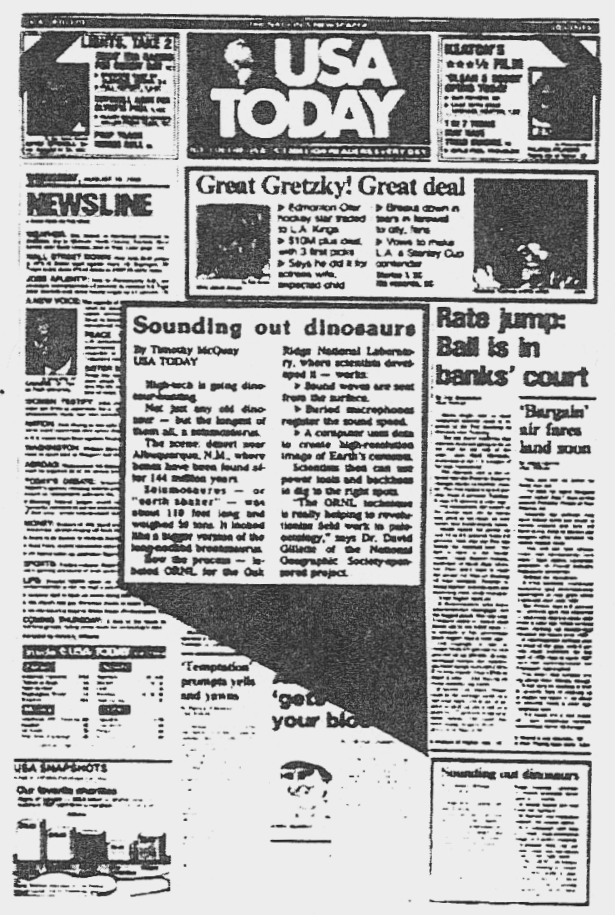




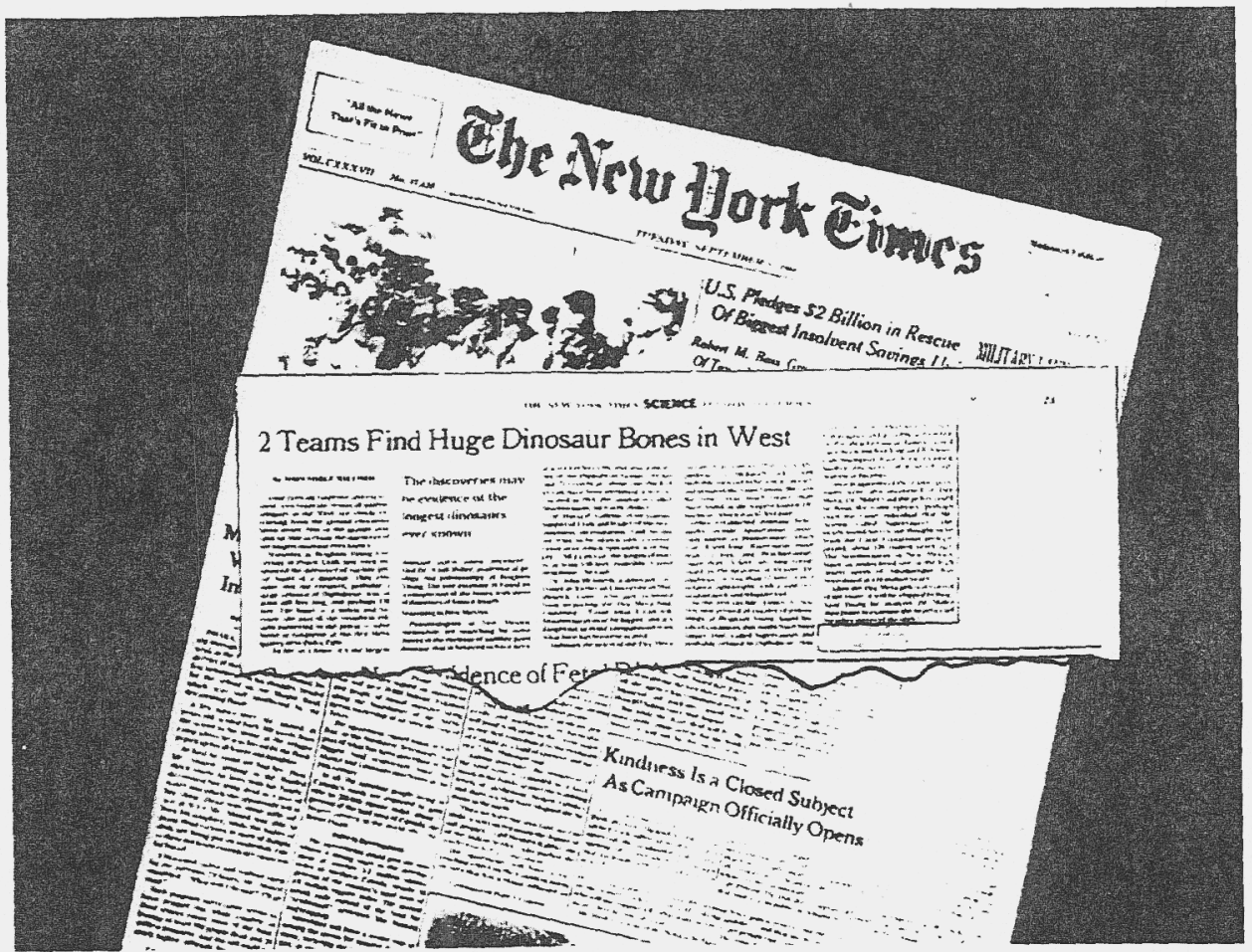

GDT survey lines and results
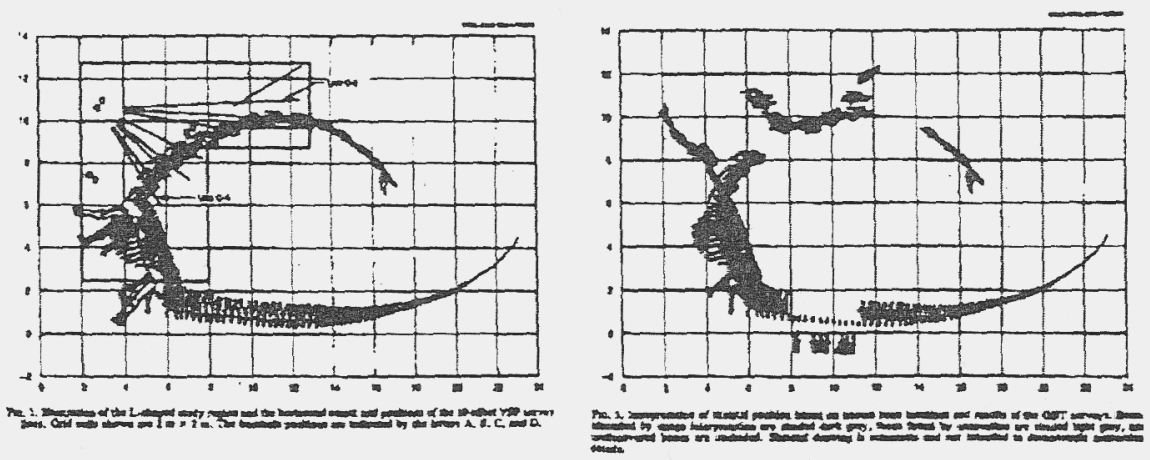

A. Witten, D. Gillette, J. Sypniewski, and W.C. King, "Geophysical diffraction tomography at a dinosaur site". Geophysics. Vol. 57, 1992 


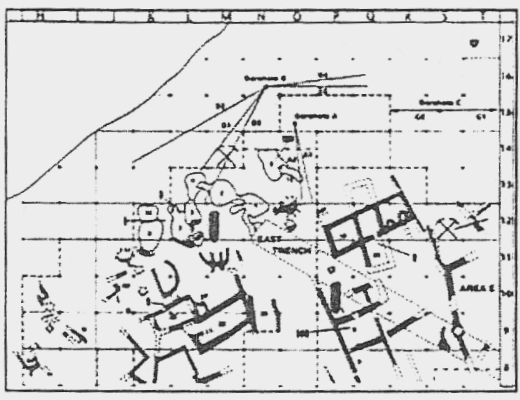

WITEN ET AL.

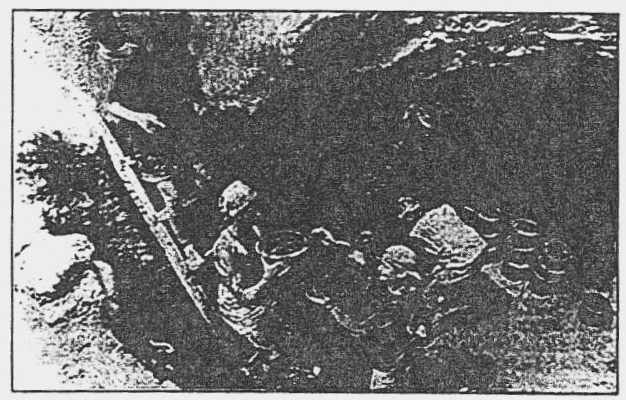

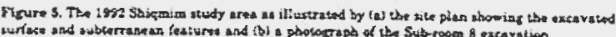

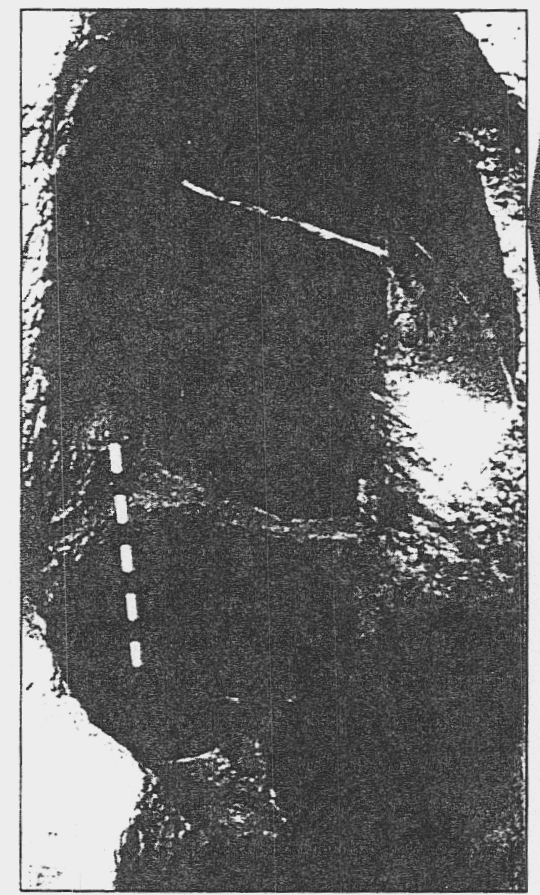

A photograph of a portion of an excavated subterranean room showing several small storage facilities dug into the floor (left) and a GDT image of a portion of an unexcavated room displaying a single storage pit in the floor (below).

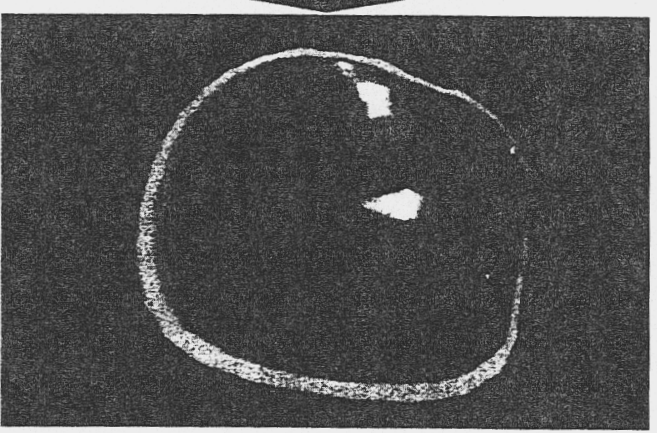




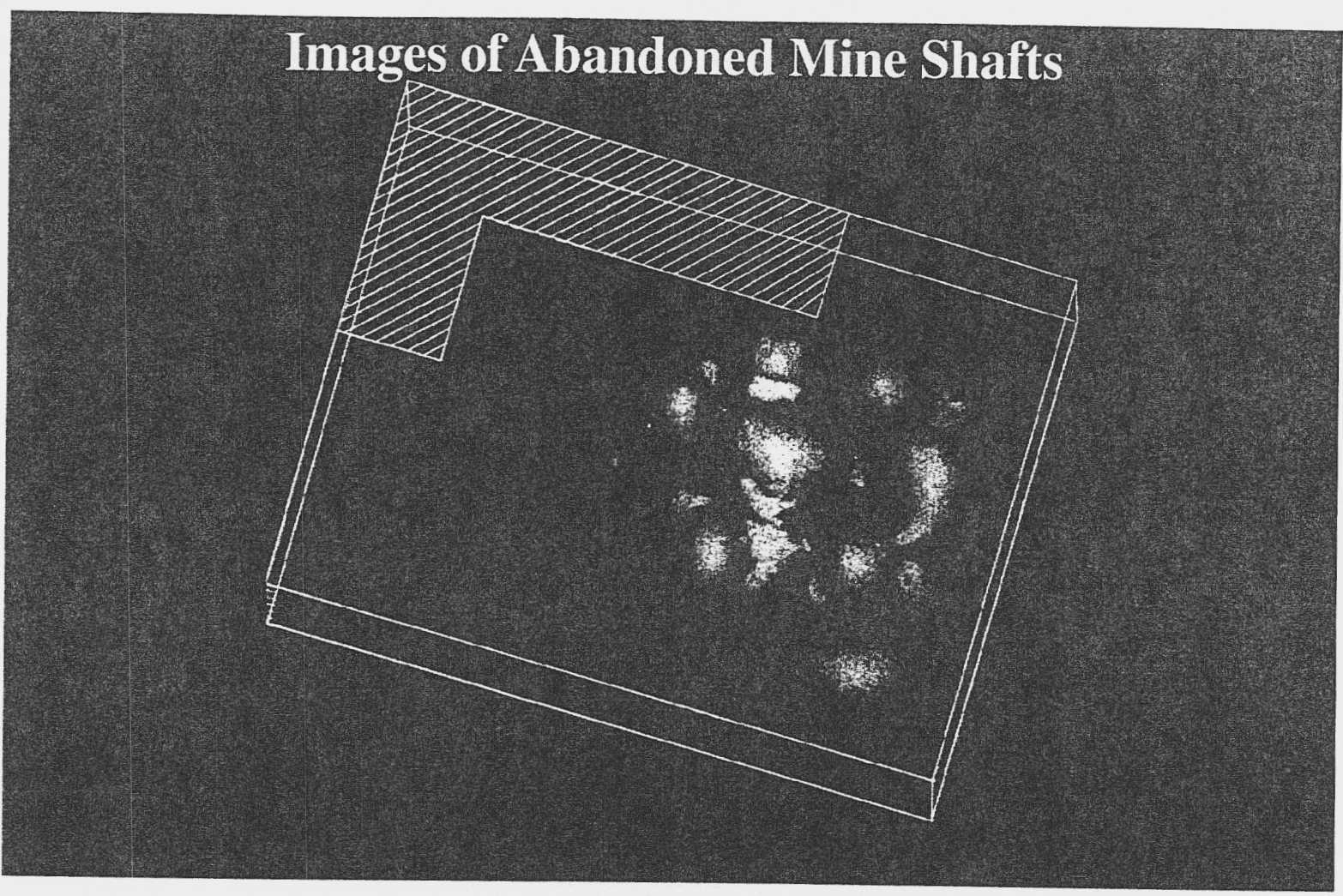

\section{Statistical based DT}

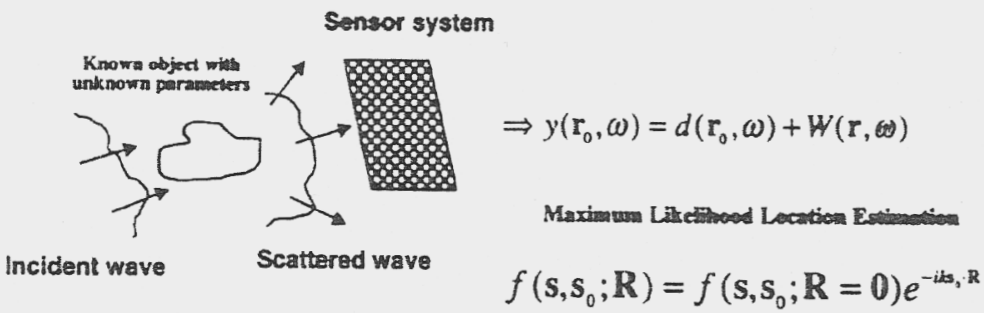

Algorithm generates image of log liketileod function

- Exact for p.r. illumuation and AWGN

- Solution in furm of FBP algorithm for

dala rich cases

- Can be employed for targea II) and

target damificaturl

- Appices fo limied dari

- Yiclds $C R$ trunds 


\section{DTMLE using multi-monostatic GPR}

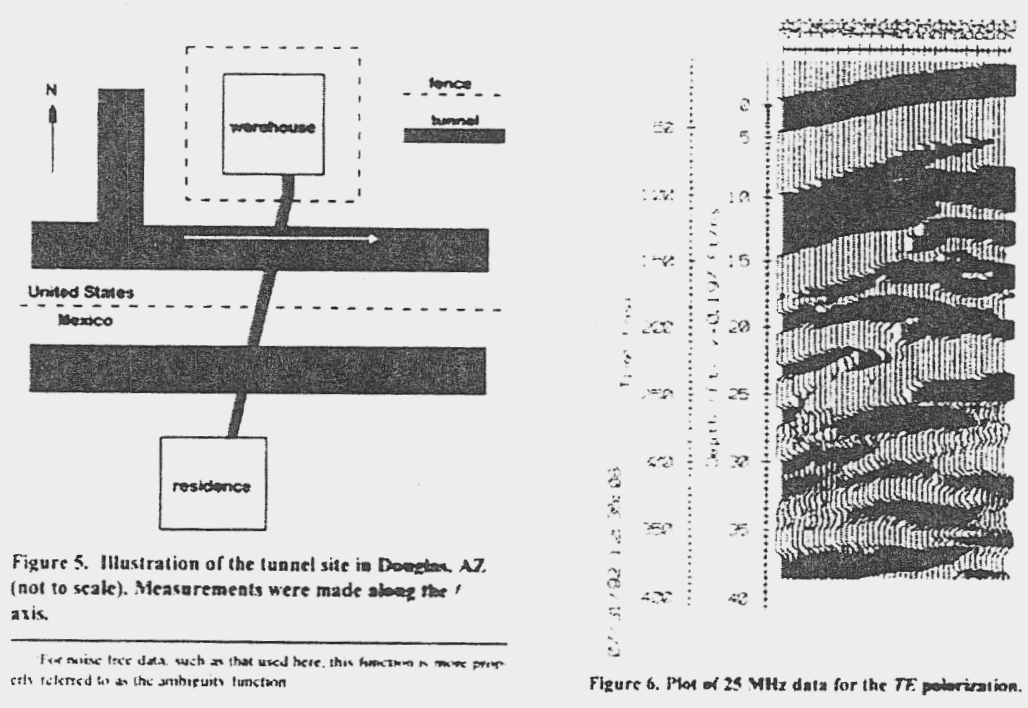

A. Wirten, A. Schsizberg, A.J Devency, J.Env. \& Eng. Geophssias, Val. 0, 1996

\section{MLE estimates of tunnel location}
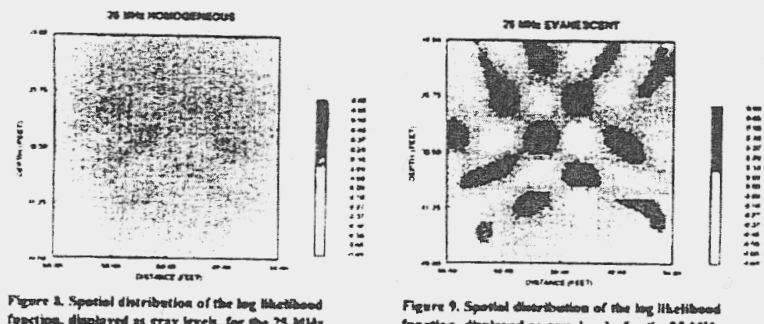

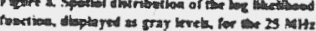

(1)

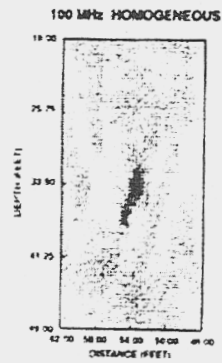

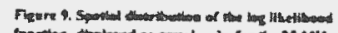

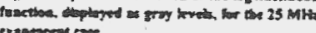

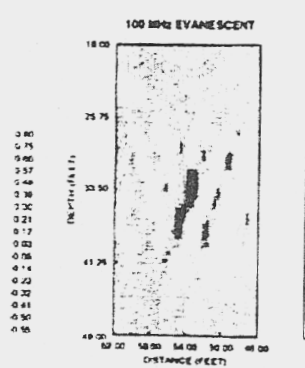

\section{范}

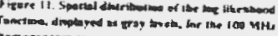

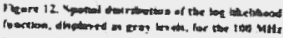




\section{Future}

- APPLICATIONS

- NON-LINEAR EXTENSIONS

- NOISE-INCLUDING CLUTTER

- ADAPTIVE SYSTEMS 


\section{Computed}

\section{Tomography}


The LANDMARC Project:

Land-mine Detection with Advanced Radar Systems

Stephen Azevedo

Laser Engineering Division, EE

Information Science and Technology Program

Laser Programs Directorate

CASIS Workshop

Lawrence Livermore National Laboratory

November 12-13, 1998

The problem we are addressing is radar detection of landmines

- Objective: Develop and demonstrate the key technologies to prove the efficacy of a landmine delection system based on radar

- Importance: Demining operations to reclaim land and save lives are currently slow and inefficient (estimated 1000 years to clear)

- Current methods are low-tech and dangerous

- New LLNL-technologies can be applied and can be cost-effective 


\section{The LANDMARC technical team has experience with} radar imaging

- Stephan G. Arevedo, Project Leader for MatR, Ph.O. in reconstructive imaging

- James M. Braee, Deputy Program Leader ALP, Image and signal processing

- E. Tom Rosenbury, Microwave hardware design

- Jeffrey E. Mast, Ph.D. Microwave diffraction tomographic imaging

- Sean Lohman, Pn.D. candidate at U.C. Davis

- Paul Walker, Ph.D. candidate at Purdue University

- Robert Greonwait (Lt.Col., U.S. Army, ret), Consultant

- Douglas Rake for Japan-US interactions, Consultant

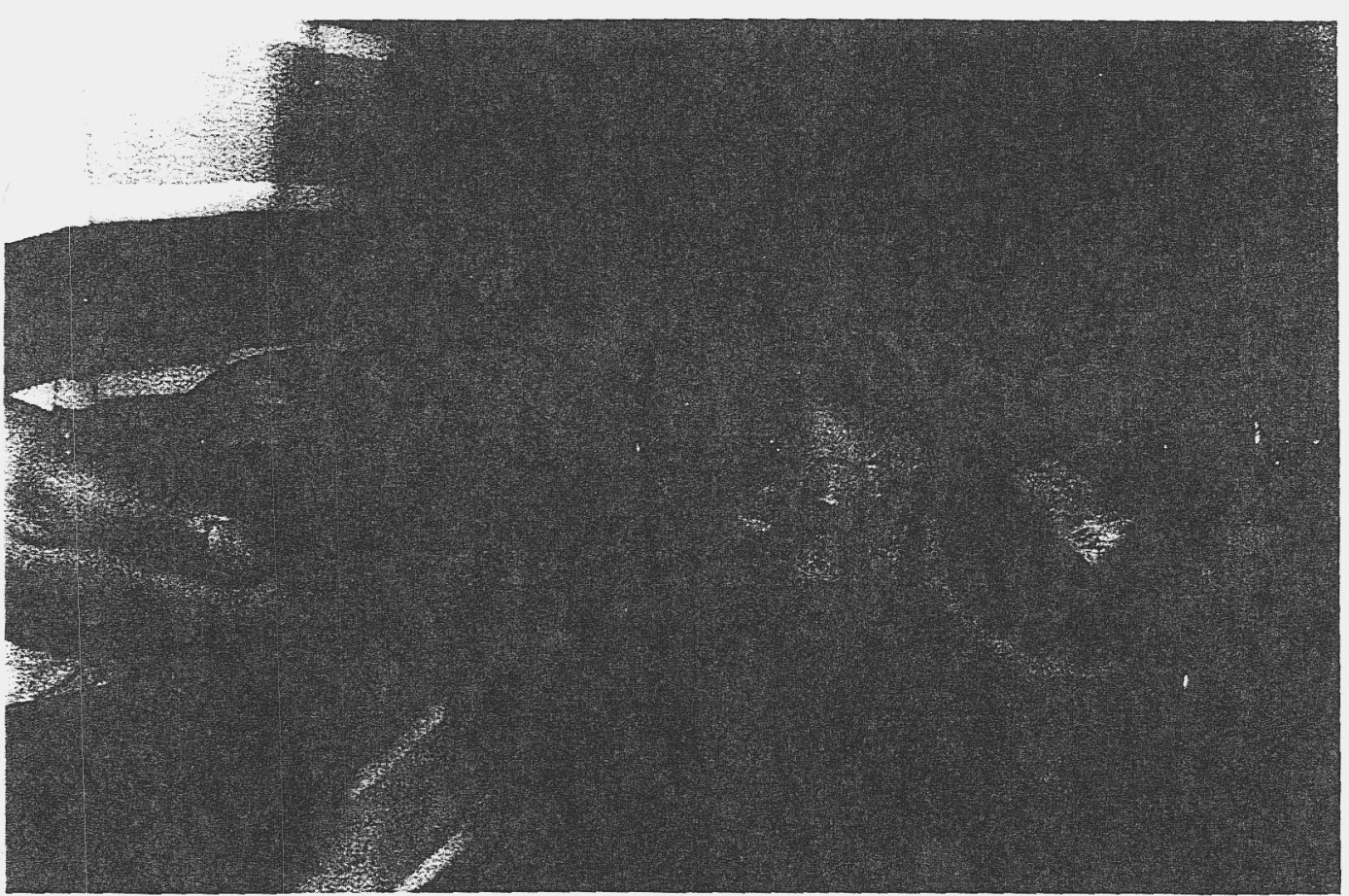


AP NM M14 Antipersonnel Mine
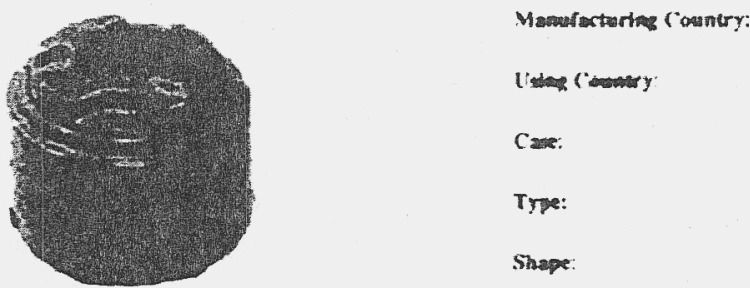

Inotia

Intia

Plasus:

Amtherwand

Cytinder

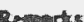

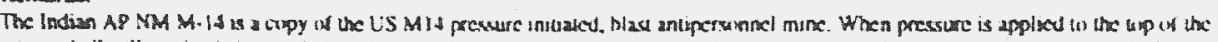

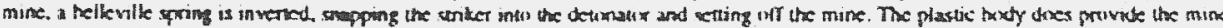

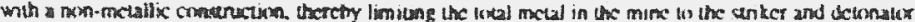

DETAREO TrOAMATOM

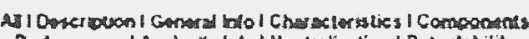

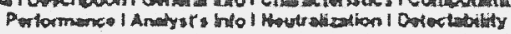

Saumons

Courhermane Solvtons

Return 10 Seren Results

Mine Highlighed

TS-50

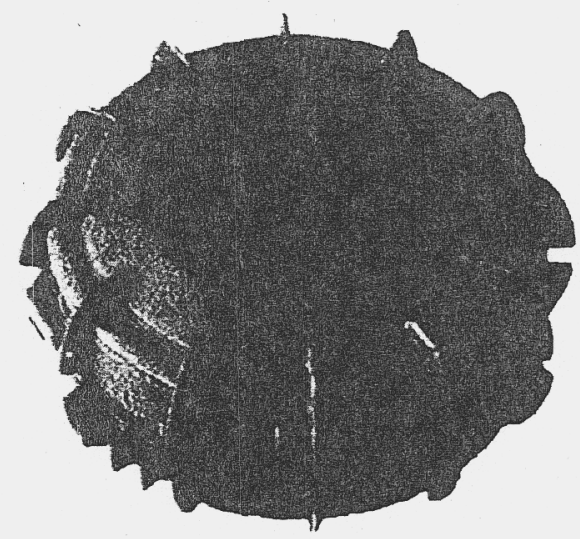

The TS.50 Na small. ante

proxmel hlas mine that on

sither he placed by hand o

vallered from helicoplers. The

nune is blast-fesistant and is

designed 11 deteat moss

explosive cixuntermeasures

This mut is found in Georgia.

iray. Lethanos and $R$ wanda 


\section{Existing mine detection R\&D programs are largely directed to the military mission}

\section{Military Countermine}

- Clearing and marking safe lanes

- Avoiding mined areas

- Requires rapid detection any time, weather, environment, enemy activity

- Some casualties accepted

- Mines not necessarily destroyed

- Objective taken; continue the battle

- Preserve the force
Humanitarian Demining

- Detect and destroy all land mines

- Post conflict endeavor

- Detection of individual mines more important than speed of movement

- Conducted by the host country organization or outside assistance

- Detection and safety are critical (approach 100\%)

- Promote mine awareness of returning refugees

- Reestablish economy and infrastructure

\section{Humanitarian demining goals and requirements} are rigorous

- Once a mineted is located

- $100 \%$ removat of minearuXo to ptough-depth $(20 \mathrm{~cm})$

- Do not make the mines more dangerous:

- Don't bury them deeper

- Don't leave any demaged mines

- Don's modity them ctremicaly etc.

- Locate eyery mine

- Approsen $100 \%$ detection

- Reduce false adarms

- Speed overall removal process

- Speed brush removal

- Whthour hitting iripwires or booby traps

- Clear each square meter quickly/reliably

- Plastic anti-personal (AP) mines are the main problem

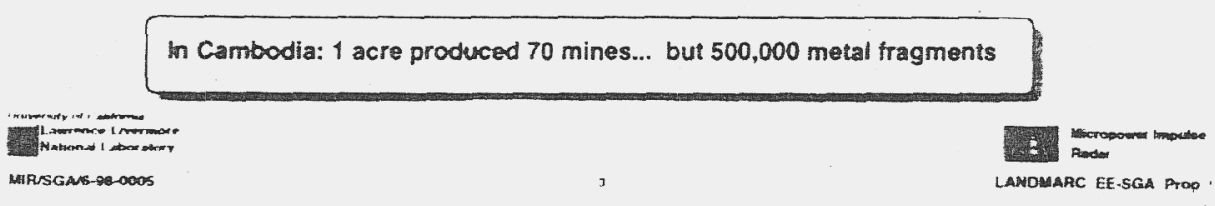




\section{Current demining operations are done with} simple tools

- Large-scale survey - Locate fields, prioritize them

- Narrow the search - Not yet done; will use dogs and mechanical systems

- Careful demining - Remove all metal from field to $20 \mathrm{~cm}$ depth

- Establish safe lanes

- Teams of 2 , in platoons of 30 (separated)

- Calibrate metal detectors (one per team)

- Over sections 1-by-0.5m:

- Clear brush (1-30 minutes)

- Pass detector over surface (15 sec.)

- Probe for objects (many minutes)

- When found, remove or detonate mine

- Validation - often none

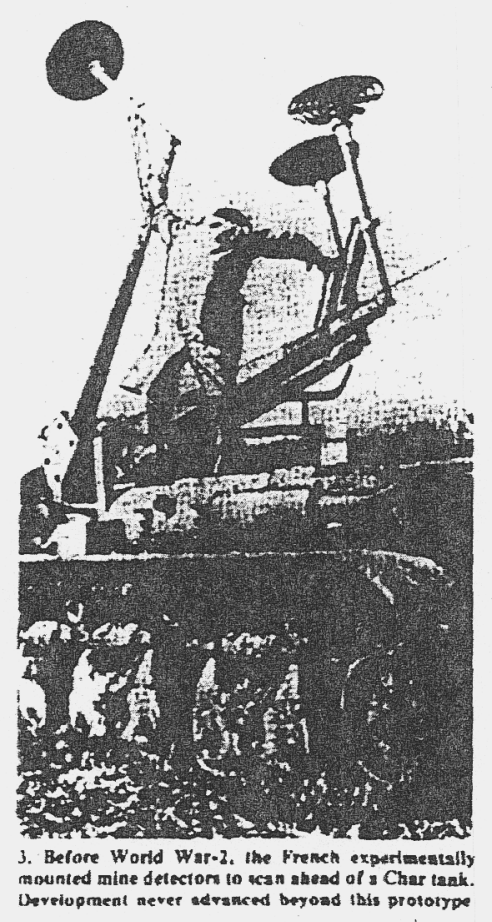




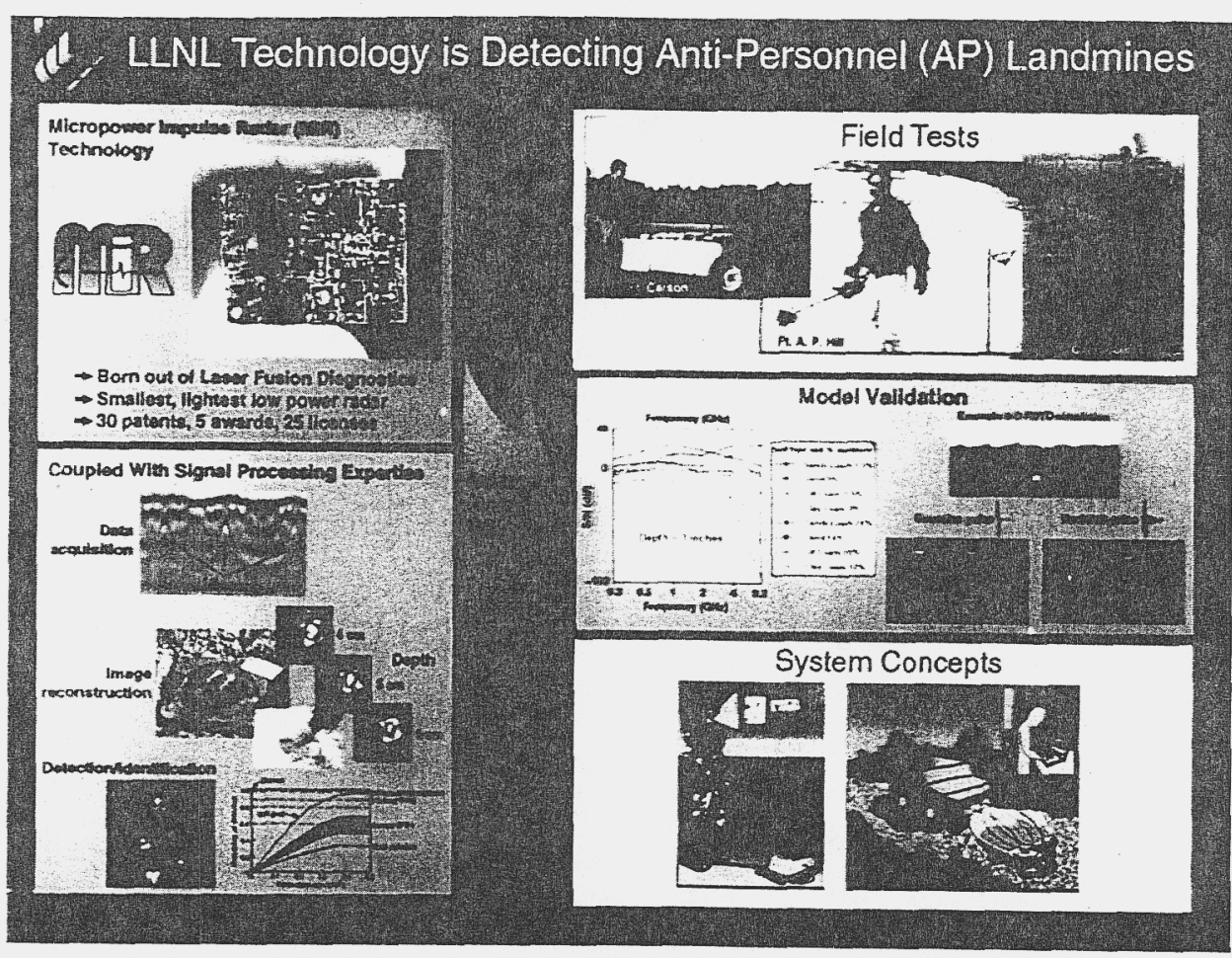

The MIR pulse is stable and wideband
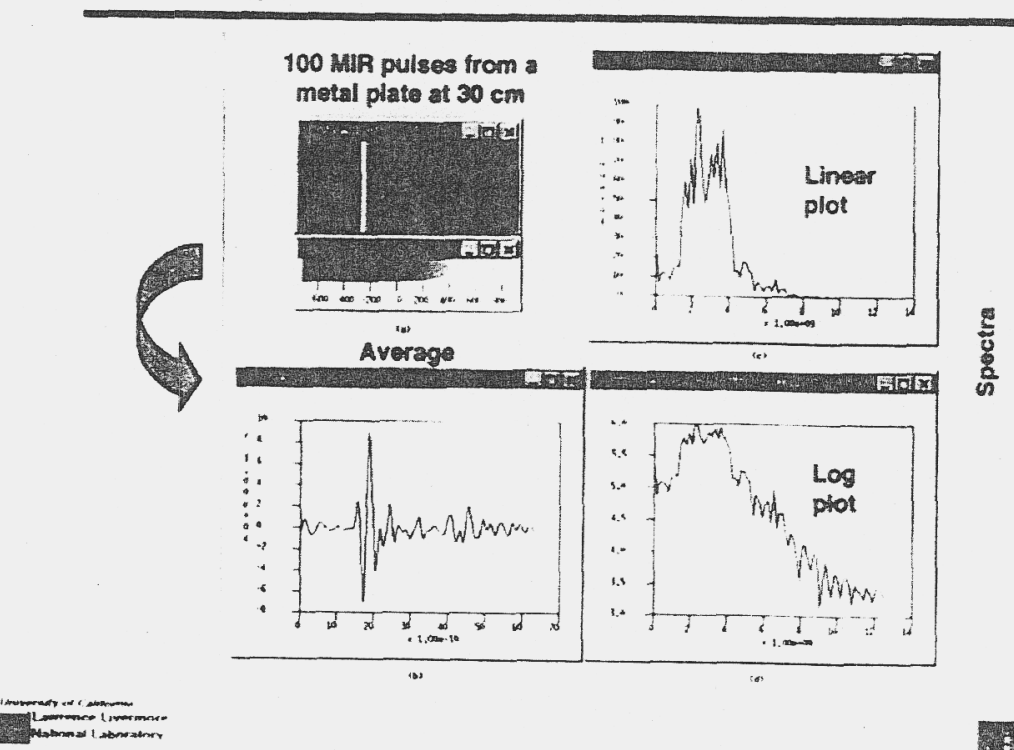

4ascen-0005

10

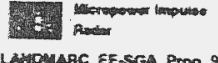


High Performance Robotic Radar Imaging System

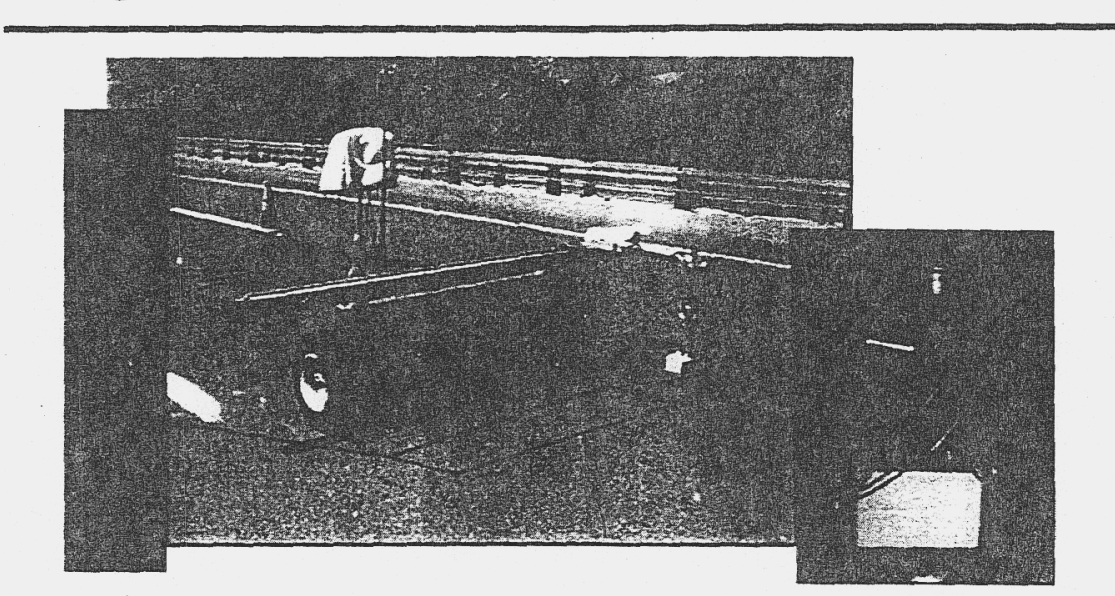

An MIR based NDE imaging system would be suitable for applications where a robotic vehicle is needed, e.g. inspection of large and tall vertical structures.

\section{Surface Removal is done in several steps}

\section{Raw Data}

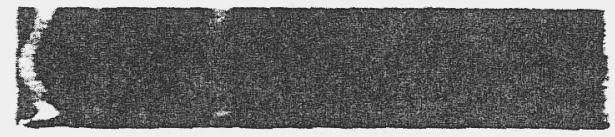

D
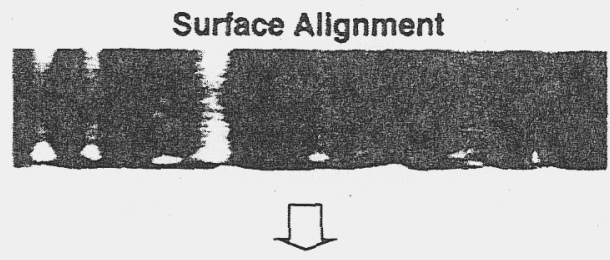

Surface Removed

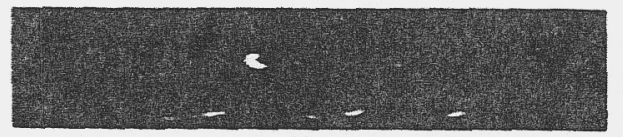

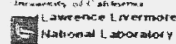


A rough surface is a significant source of clutter in mine reconstructions and can distort the image of the mine

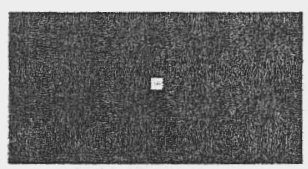
$d=5 \mathrm{~cm}$
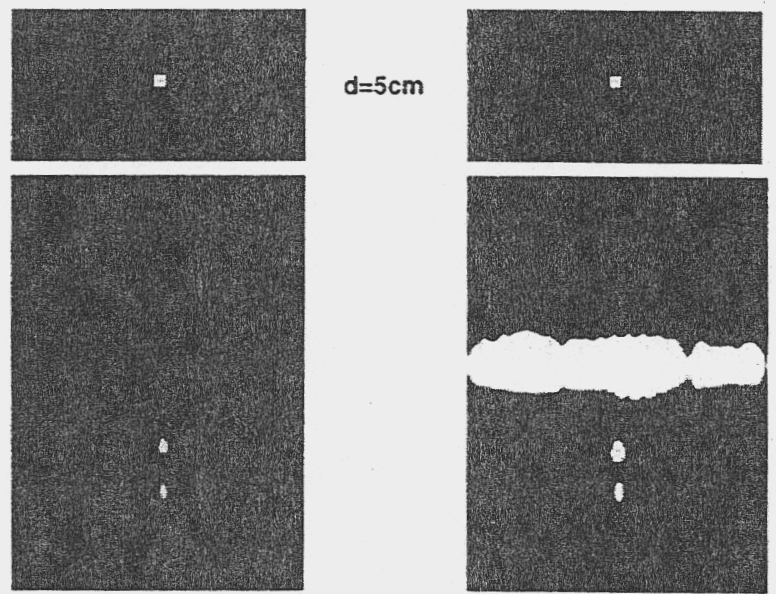

We will refine the simulation to reflect actual conditions based on real data

Surface clutter is dominant for shallow mines however attenuation is the primary problem for deeper mines

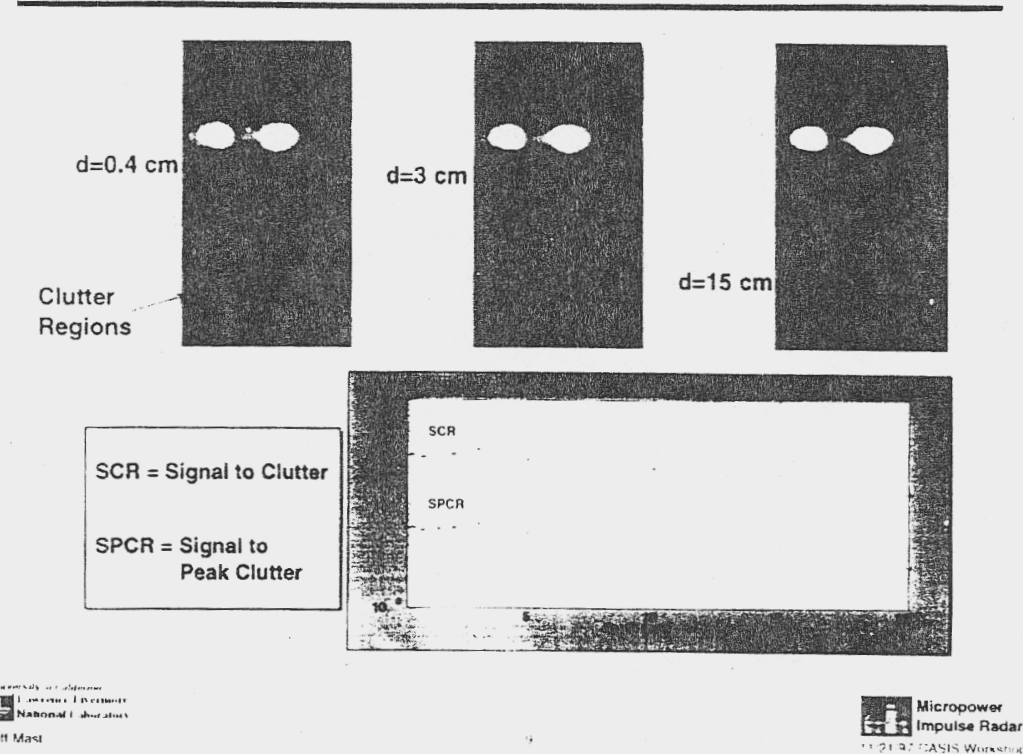


The DSWALLNL mine detection systern is based on needs of the humanitarian deminers
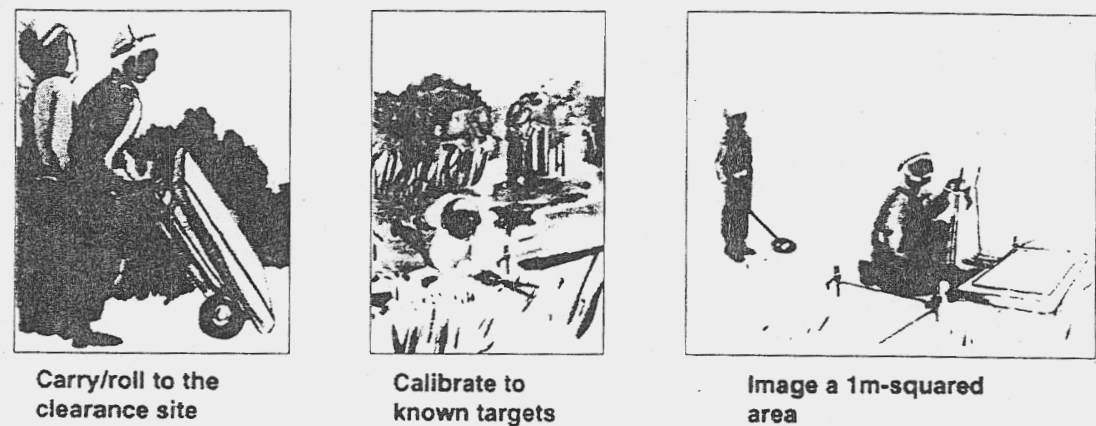

clearance site

known targets

Image a $1 \mathrm{~m}$-squared

area

Operational scenario:

- "Safe lanes" pre-marked

- Brushifoliage cleared to $5 \mathrm{~cm}$ in a $1 \mathrm{~m} \times .5 \mathrm{~m}$ area

- Detector brought into find subsurface mines

- Clear mines

- Move markers and repeat

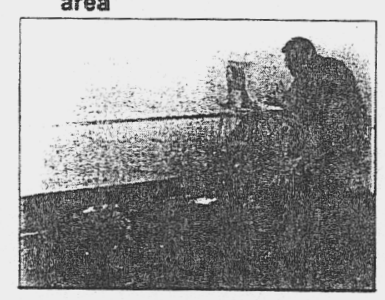

Lab prototype detector

Results to date (April 1998)

\begin{tabular}{|l|c|c|}
\hline Average of 18 tests & ANPSS-12 & MIR \\
\hline Detection rate & $44 \%$ & $85 \%$ \\
$\begin{array}{l}\text { False alarms (Per detected } \\
\text { mine) }\end{array}$ & 31.8 & 14.6 \\
\hline
\end{tabular}

Sponsors: LLNL, Defense Special Weapons Agency (DSWA), U.S. Army Humanitarian Demining R\&D 


\section{MIR Mine Detection Program Status}

\begin{tabular}{|c|c|c|}
\hline Completed & $\begin{array}{c}\text { Partially } \\
\text { Completed }\end{array}$ & Planned \\
\hline $\begin{array}{l}\text { MIR singlo-sensor operational } \\
30 \text { image displaylanalysis } \\
\text { Anomaly detection in soil } \\
\text { Precision 3D positioning } \\
\text { MIR scanning array operational } \\
\text { Mine detection in } 3 \text { soil types } \\
\text { Detection in presence of clutter }\end{array}$ & $\begin{array}{l}\text { EM model of mines and solls } \\
\text { Clutter discrimination } \\
\text { Surface roughness removal } \\
\text { Wider bandwidth ( } 1-10 \mathrm{GHz})\end{array}$ & $\begin{array}{l}\text { Automatic Target Recognition } \\
\text { Mine discrimination in: } \\
\text { Variety of soils } \\
\text { Heterogeneous soils } \\
\text { Various climates } \\
\text { Vegetation } \\
\text { Field ready: } \\
\text { Rugged, miniaturized } \\
\text { Man-machine interiace } \\
\text { Low-cost }\end{array}$ \\
\hline
\end{tabular}

10.

\section{Developmental MIR mine detection experiments were performed in April ' 98}

The experimental set up was as follows:

- MIR system: 4-element impulse radar array ridged-horn antennas ( 1 to $5 \mathrm{GHz}$ )

- 3 soil types: top soil, gravel, sand; homogeneous with flat surface

- Mines used: Extremely low metal-content mines (M-14's) with initiator removed and dielectrically filled $\left(-.75\right.$ mines per $\left.\mathrm{m}^{2}\right)$; mines laid within $1 \mathrm{~cm}$ of the surface

- Intentional clutter: Small metal pieces in known moveable locations $\left(-10\right.$ per $\left.\mathrm{m}^{2}\right)$

- Environment: From dry to rain-soaked

- Independent Test Control: Blind experiments with ANPSS-12 (metal detector) and the new MIR mine detector. Both with trained operators in identical lanes. 


\section{Signal processing approach for imaging detection}

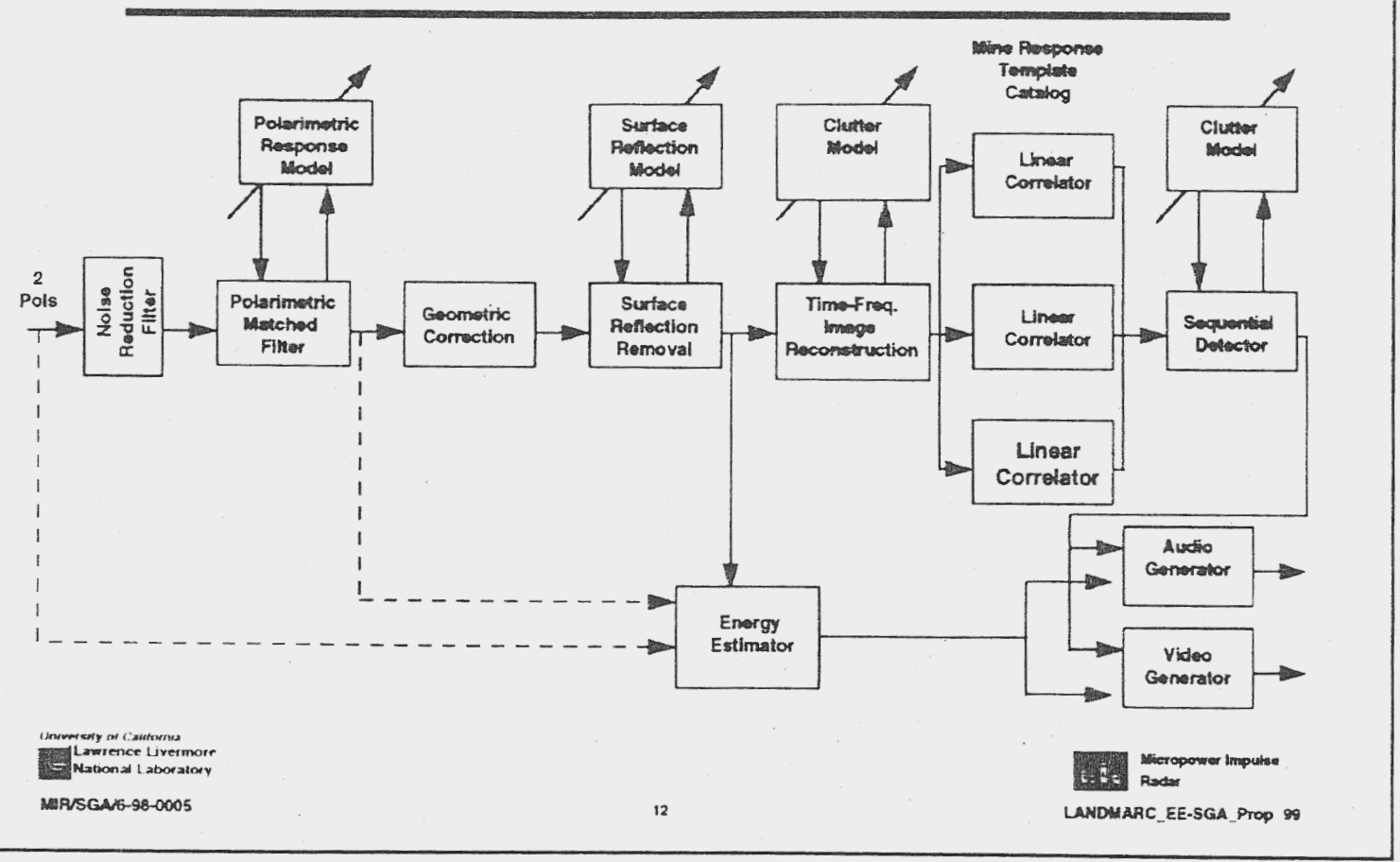

The goal is to develop a radar subsystem that has the best chance of finding buried mines

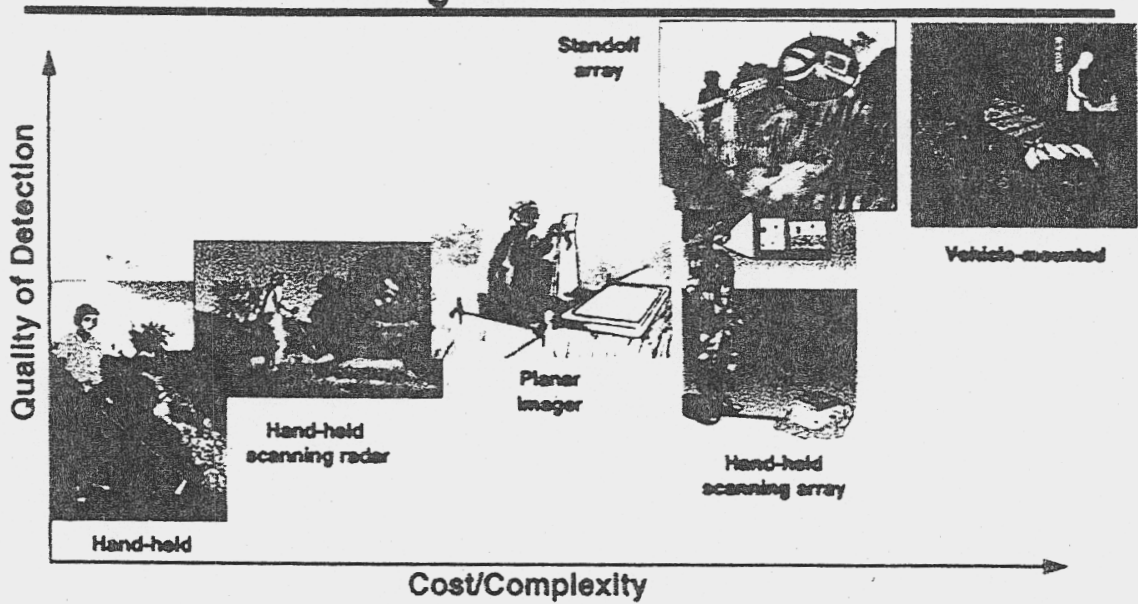

A series of trade-otfs determine the type of system and its performance

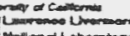


LANDMARC fins into the "big picture" of MIR mine detection

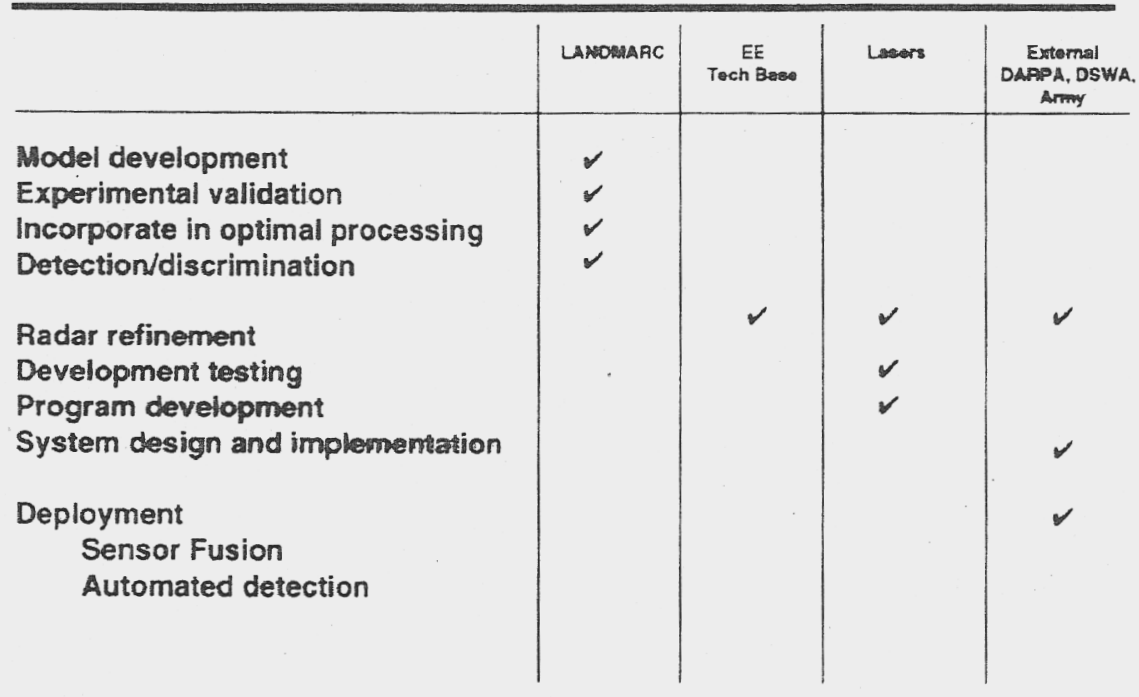

Summary: AP mine detection can be vastly improved with MIR technology

- Humanitarian demining and countermines are important global issues

- Detection in clutter is the key umesolved issue; MIR is an important enabling techmokogy for a high-resolution $3 \mathrm{D}$ imaging approach

- Laboratory technology and expertise can and should be directed to this problem

- MIR and systems

- Radar modeling \& simulation

- Imaging techniques and detection

- Plans for future support and deployment include

- U.S. Gov't sources and teaming with industry

- Japanese interactions with gov't and companies

- Non-governmental organizations

US-based technology will be directly applied to saving lives 


\section{HERMES Bridge Deck Inspection System Current Results and Future Research}

Holger E. Jones Lawrence Livermore National Laboratory

As a recent R\&D 100 award winner, HERMES (High-Performance Electromagnetic Roadway Measurement and Evaluation System) showcases the multidisciplinary expertise, which LLNL can bring to bear on complex projects. Having completed acceptance testing by FHWA officials, HERMES enters into a new phase of research that will tie radar phenomenology to physical understanding. FHWA will survey many different bridges over the coming year, and collaborative efforts will focus on supporting field experiments, performing correlation studies of deck samples, and implementing techniques to increase image quality. This talk will highlight some of these efforts.

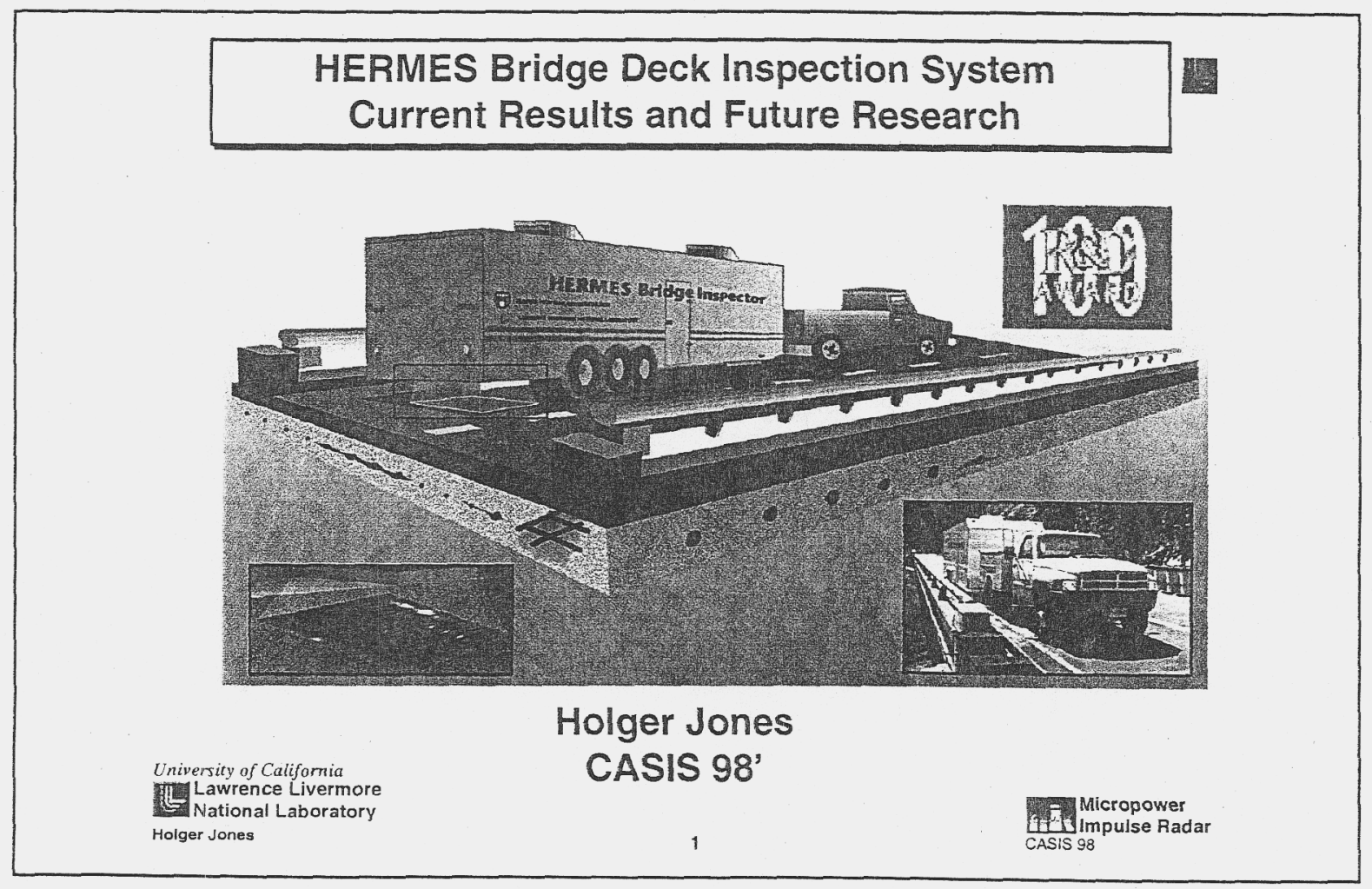




\section{LLNL has a wide range of capabilities for development of radar based NDE}

- Electromagnetic effects and modeling

- Modeling and simulation

- Source specifications and requirements

- Antenna and array design

- Experimental analysis

- Signal and image processing

- Algorithm development

- System specification and design

- Detection and identification

- Data analysis

- System development and integration

- Custom source development

- Custom software and data acquisition systems

- Examples

- LLNL patented Micropower Impulse Radar (MIR) technology

- GPR technology

- Bridge deck inspection (shallow depths)

- Surface to borehole imaging (deep depths)
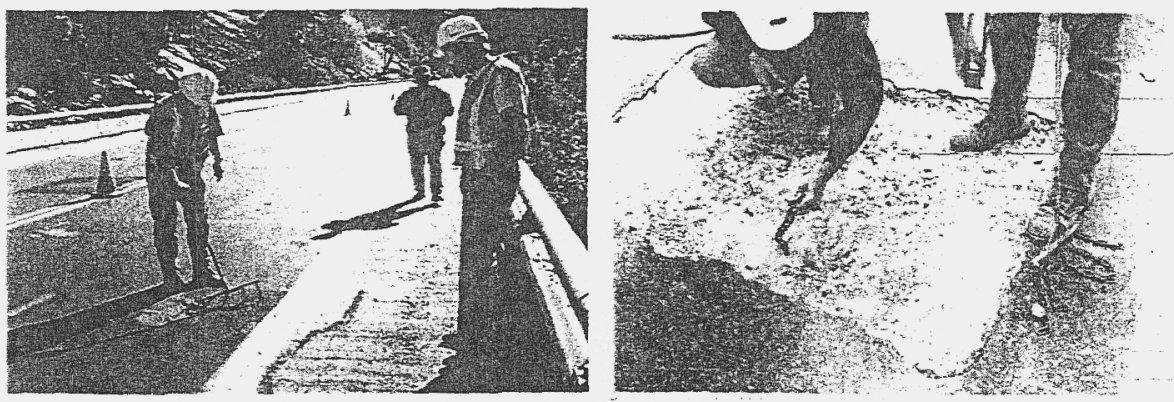
Hermes Array Implementation
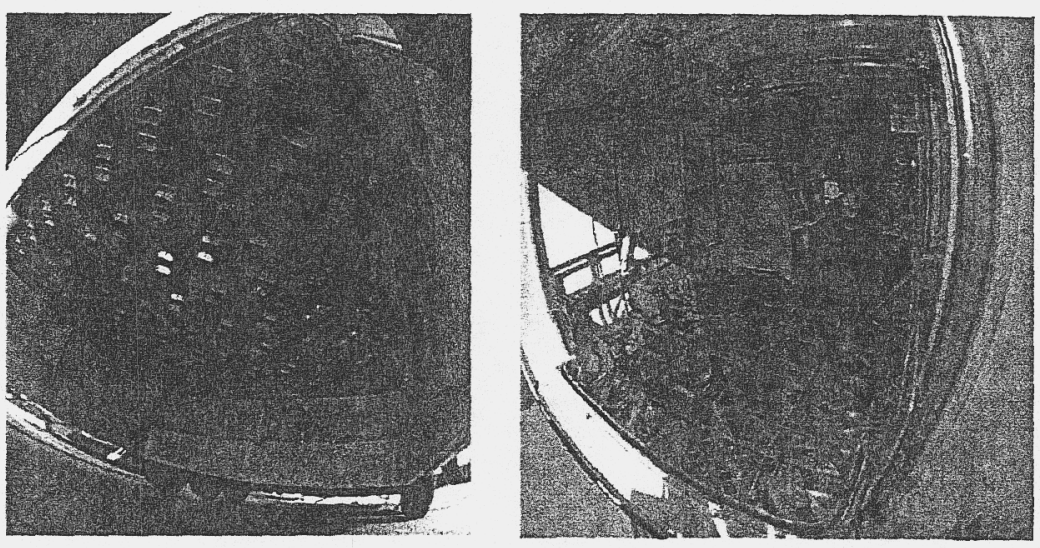

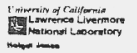

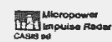

\section{Frequency Domain Technique: A flow diagram}

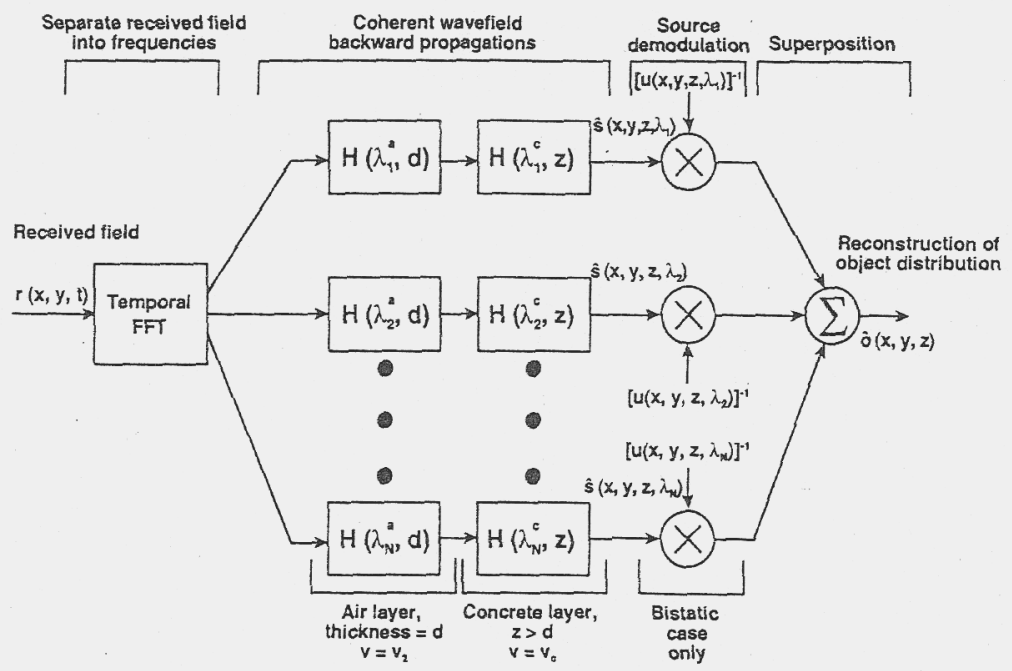

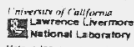

5

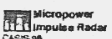



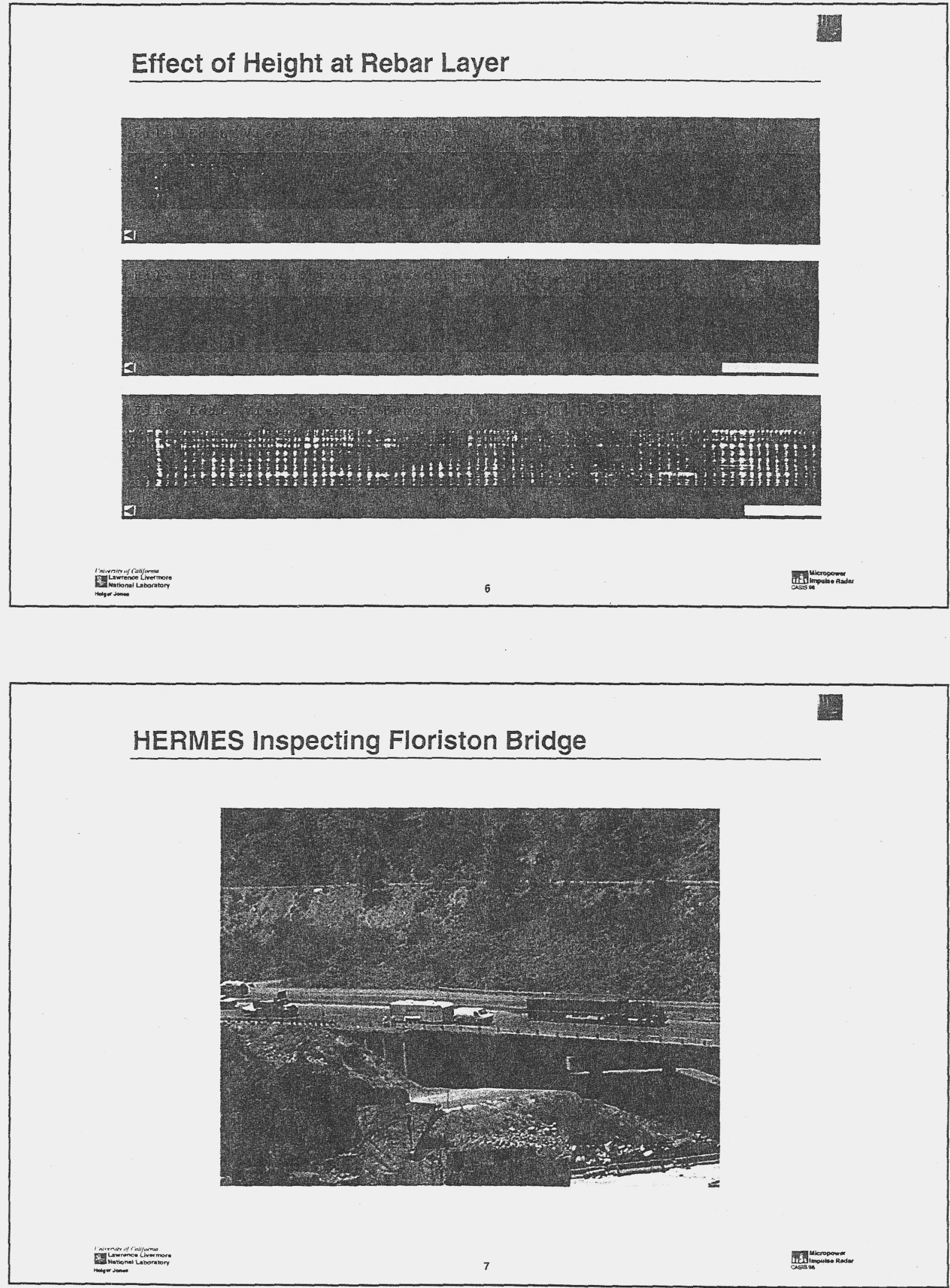


\section{Data Post-Processing Efforts}

- Surface Clutter is our worst problem

- Mitigate this with Advanced Surface Removal Algorithms

Cleaner Pulse Signature

Aggravated by platform motion

Ambiguity in depth cues

Use accelerometers for motion sensing

- Auto-focus Algorithm development

- Block-Processing Artifact Removal, Multi-lane montage

- 3-D Rendering Techniques, (opengl)

- Advanced Imaging Techniques

- iterative

- multi-monostatic correction

- feedback from core-sample dielectric and contaminant profile

- feedback from modeling efforts

Rebar Fade Phenomenon:
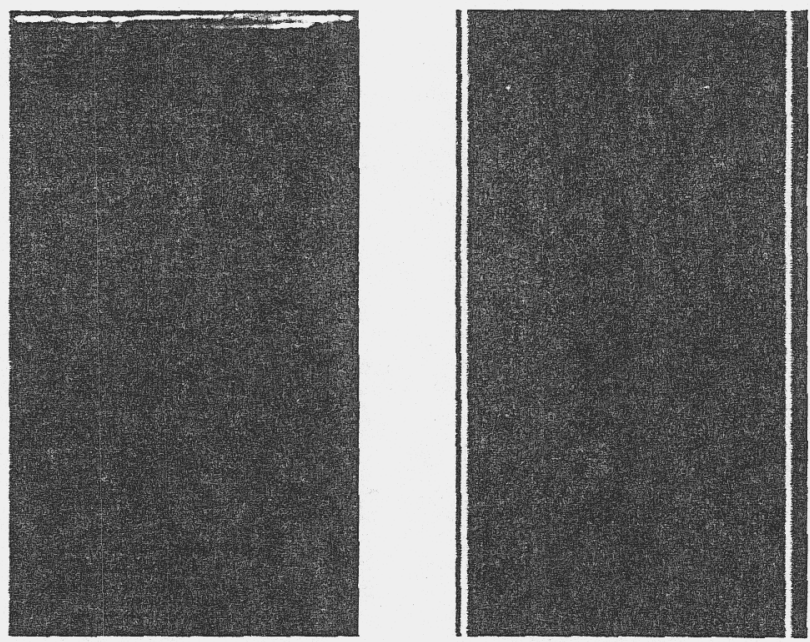


\section{Radar Phenomenology Studies}

- Collaborate with Universities

- Models (contaminant diffusion, corrosion chemistry, etc)

- PERES based controlled experiments

- Leverage deck-rehabilitation projects as data goldmines

- Before/After study of RCS during deck planing (hydro-demolition)

- Cores analyzed (dielectric/attenuation profiles, corrosion products, etc)

- High Power and Ground-coupled radar augmentation

\section{Features Obscured/Aliased by Surface Clutter Ringing}
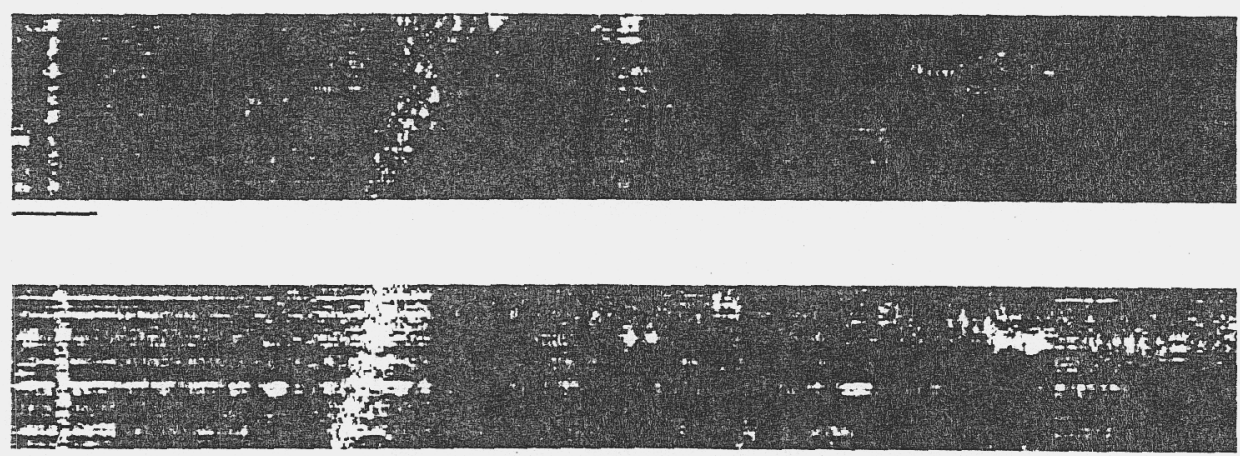
Effect of Permittivity Parameter

\section{调}

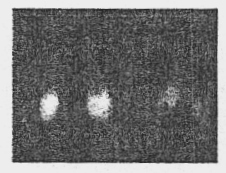

er $=1.0$

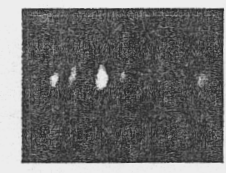

$e r=2.0$

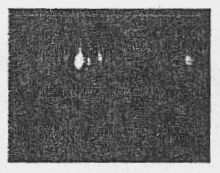

$e r=4.0$

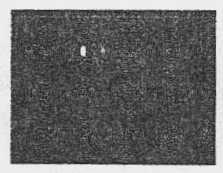

er $=8.0$

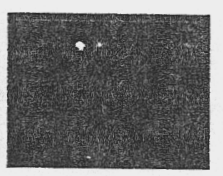

er $=16.0$

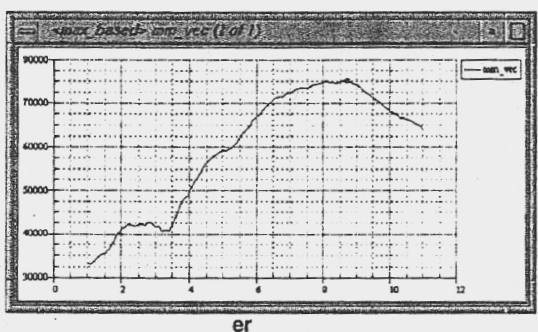

Peak Intensity

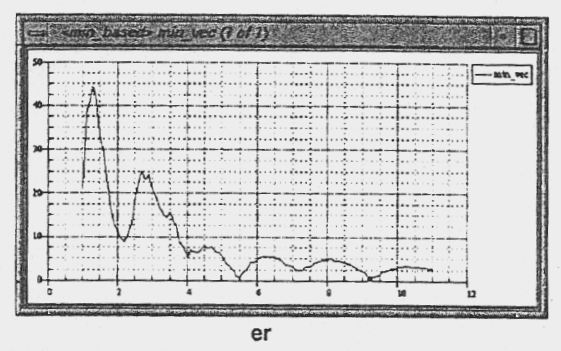

Normalized then summed

1.

12

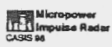

3D Alpha Rendered (BoB)

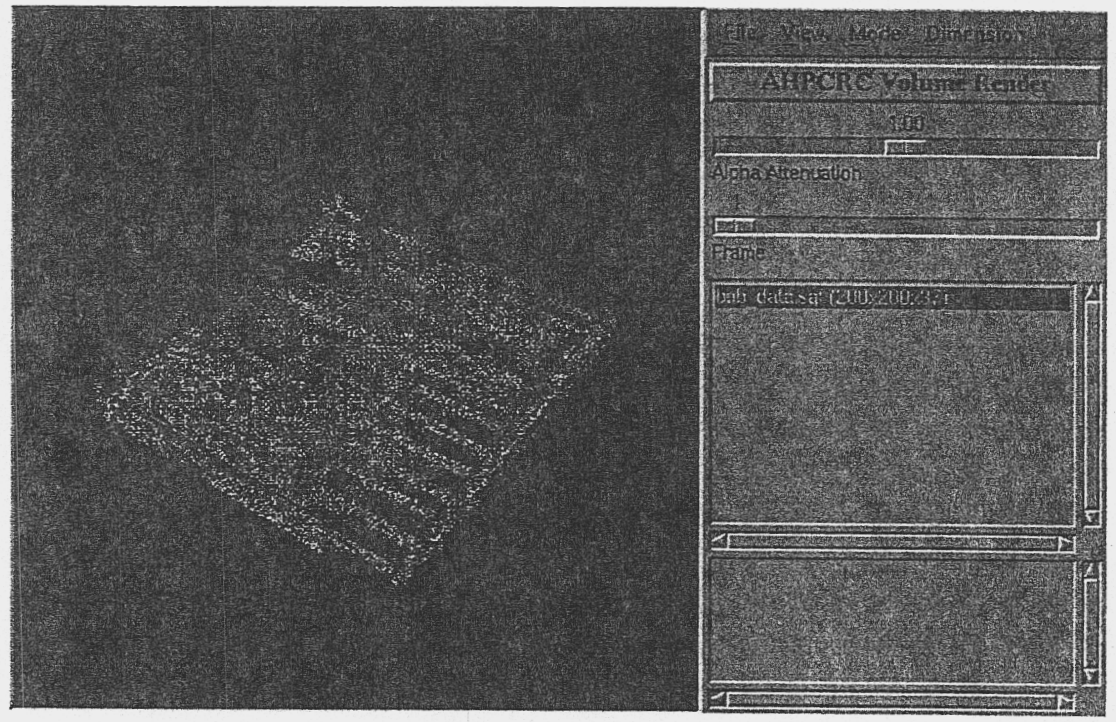

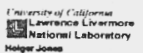

13

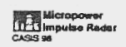




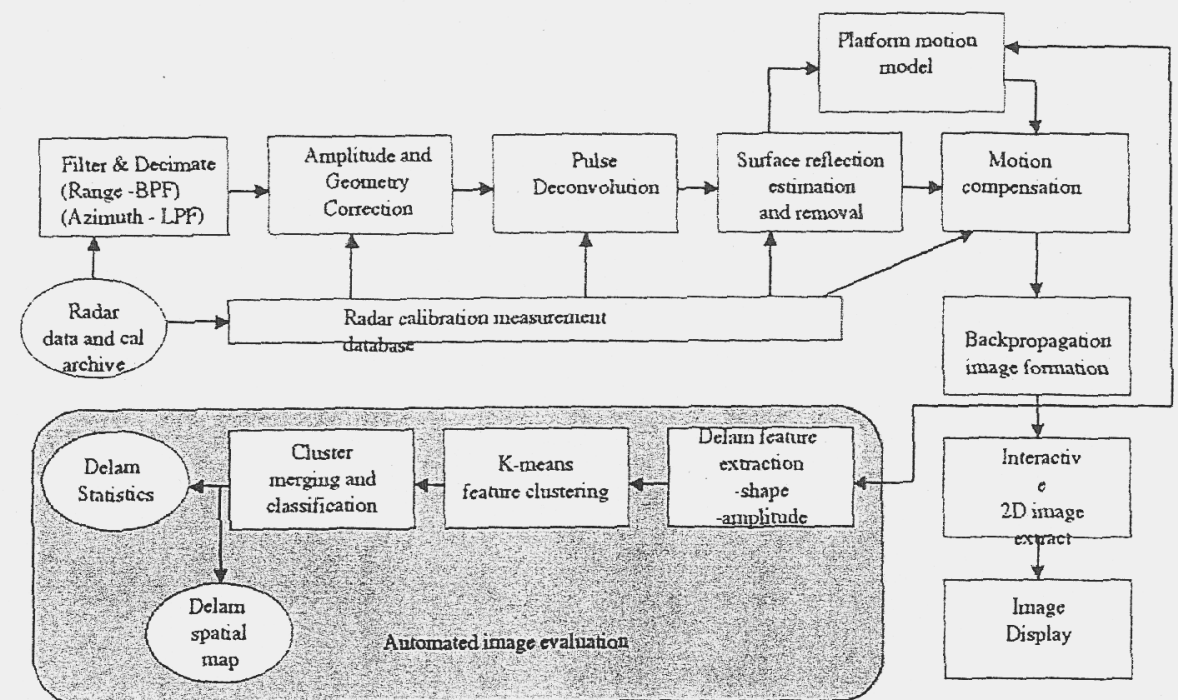

\section{Connected Displays}

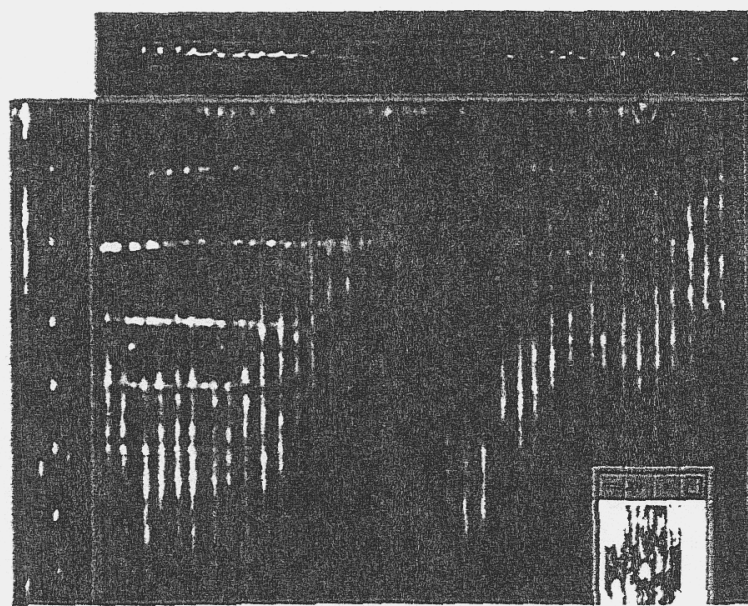




\section{Connected Displays (different depth)}

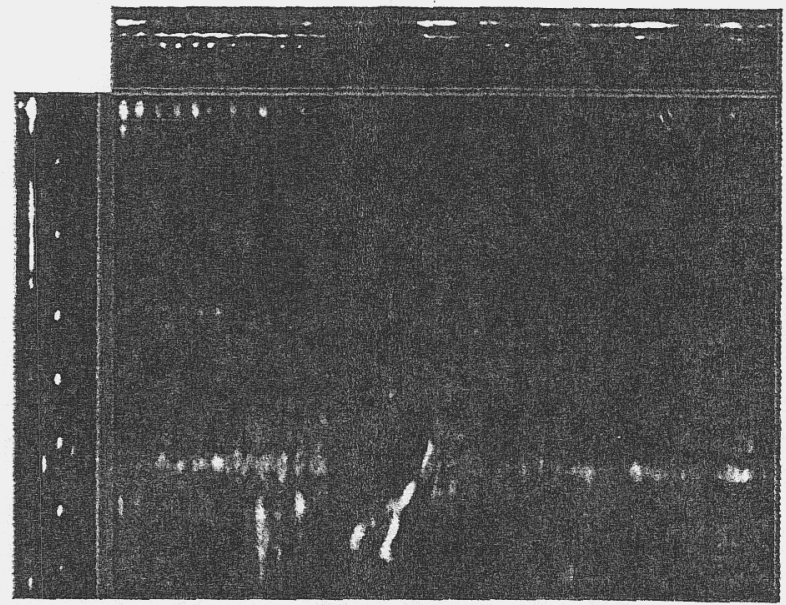

\section{Beowulf is a commodity parts based approach}

to cost-effective high performance computing

Highest performance computational components now arrive in $P C$ 's first .-.- this drives us to look as networked PC's for computationally dominated applications

Parallel compuning based entirely on commodity components:

$400 \mathrm{MHz}$ PII 256MB Ram 4GB EIDE

Dual Processor Capable Backplanes

100Mbit Ethernet

Point-to-point interconnect via switch

OS: Linux, GNU, X, PVM, MPICH

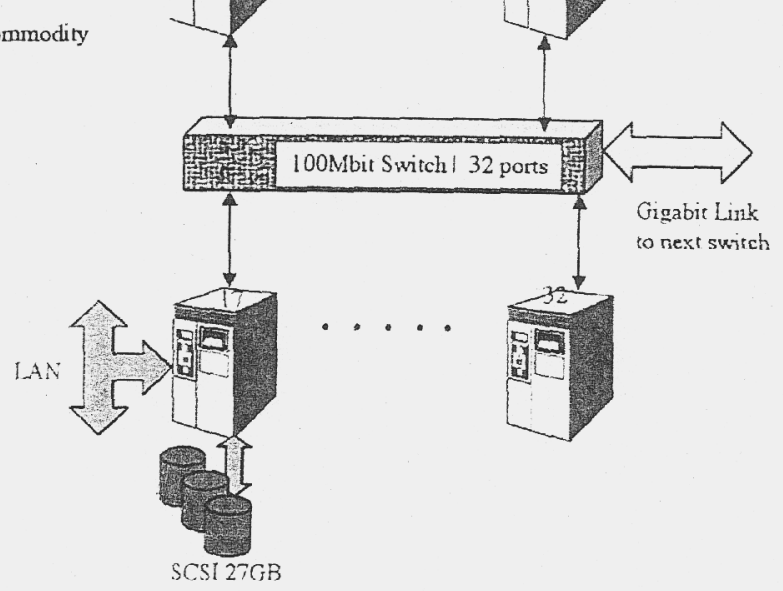




\section{Conclusion}

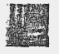

- HERMES is entering its experimental phase

- FHWA will undertake its own studies this fall

- Maintain LLNL as Center for Inspection Studies

- Start on 2nd Prototype?

- Commercialization is key 


\title{
ACTIVE AND PASSIVE COMPUTED TOMOGRAPHY WITH A CONSTRAINED CONJUGATE GRADIENT SOLUTION
}

\author{
By Jessie A. Jackson, Dennis Goodman,
}

An active and passive computed tomographic technique (A\&PCT) has been developed at the I awrence Livermore National Laboratory (LLNL). The technique uses an external radioactive source and active tomography to map the attenuation within a waste drum as a function of mono-energetic gamma-ray energy. Passive tomography is used to localize and identify specific radioactive waste within the same container., The passive data is corrected for attenuation using the active data and this yields a quantitative assay of drum activity.

A\&PCT involves the development of a detailed system model that combines the data from the active scans with the geometry of the imaging system. Using the system model, iterative optimization techniques are used to reconstruct the image from the passive data. Requirements for high throughput yield measured emission levels in waste barrels that are too low to apply optimization techniques involving the usual Gaussian statistics. In this situation a Poisson distribution, typically used for cases with low counting statistics, is used to create an effective maximum likelihood estimation function. An optimization algorithm, Constrained Conjugate Gradient (CCG), is used to determine a solution for A\&PCT quantitative assay. CCG, which was developed at LLNL, has proven to be an efficient and effective optimization method to solve limited-data problems. An overview of the algorithms used in developing the model and optimization codes will be given. 


\section{Active and Passive \\ Computed Tomography \\ with a Constrained Conjugate Gradient Solution}

Jessie A. Jackson, Dennis Goodman, G. Patrick Roberson, Harry E. Martz

November 12, 1998

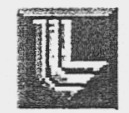

Lawrence Livermore National Laboratory

Agenda

- Background and Goals of A\&PCT Project

- Overview of the Passive Reconstruction Code

- System Model

- Cost Function

- Optimization

- Results

- Summary 


\section{Schematic Design of A\&PCT Data Processing}

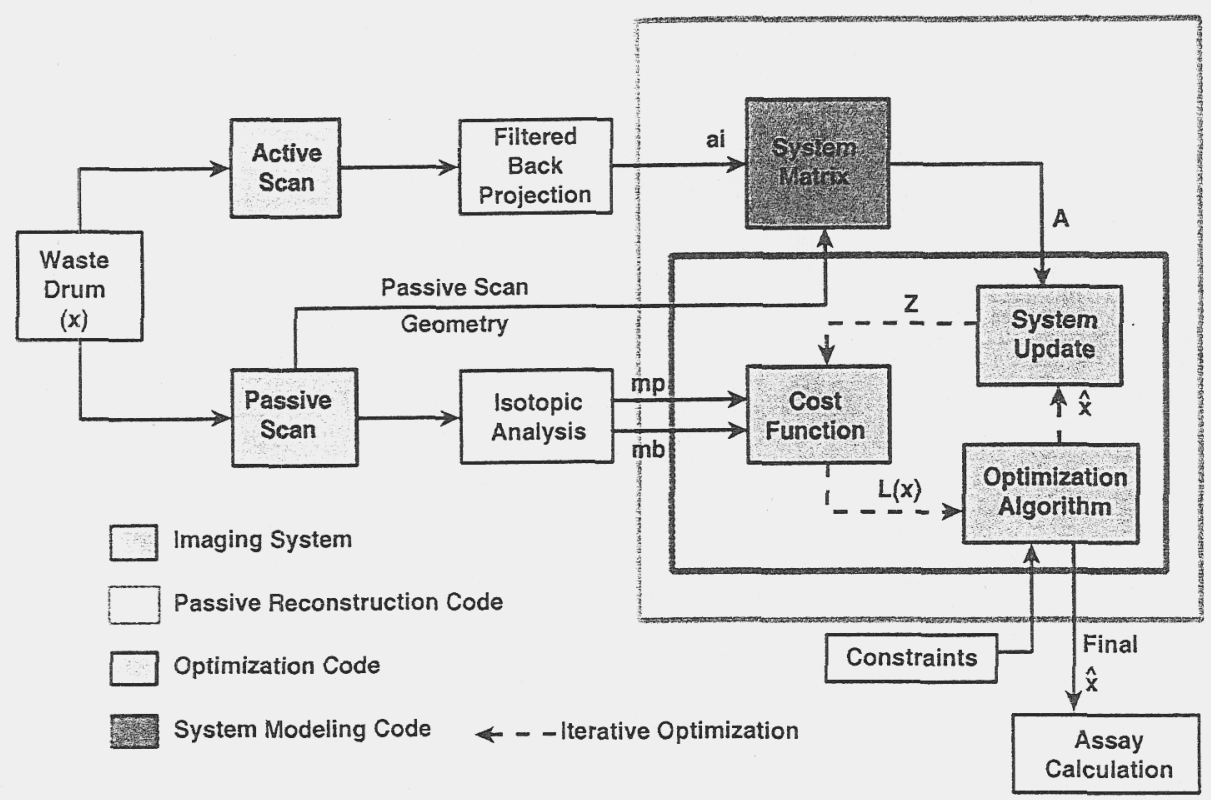

We combine active and passive computed tomography to more accurately assay wastes

Active CT (ACT)

ACT quantitallyely maps the attenuation of the drum's contents to a specinic volume elemen

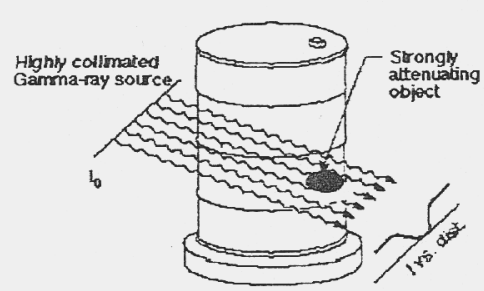

\section{Passive CT (PCT)}

PCT localizes and identifies the radioaclivity to a specific volume element in the drum

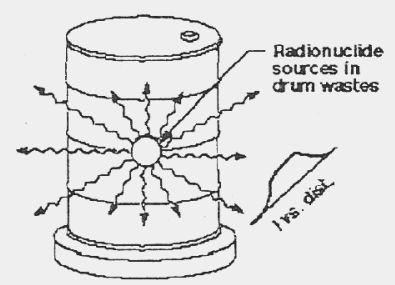




\section{A\&PCT Imaging System}

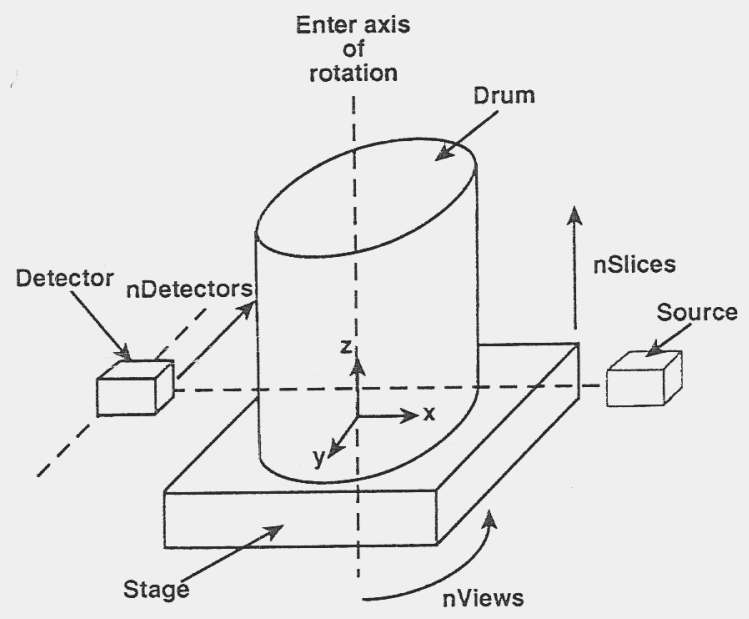

Typical Active Image

17 slices

each slice has

$14 \times 14$ voxels

Total Voxels $=3332$

Typical Passive Scan

7 detector positions

10 rotational views

17 slices

Total detectors $=1190$

\section{Spectra Sample from a Passive Scan}

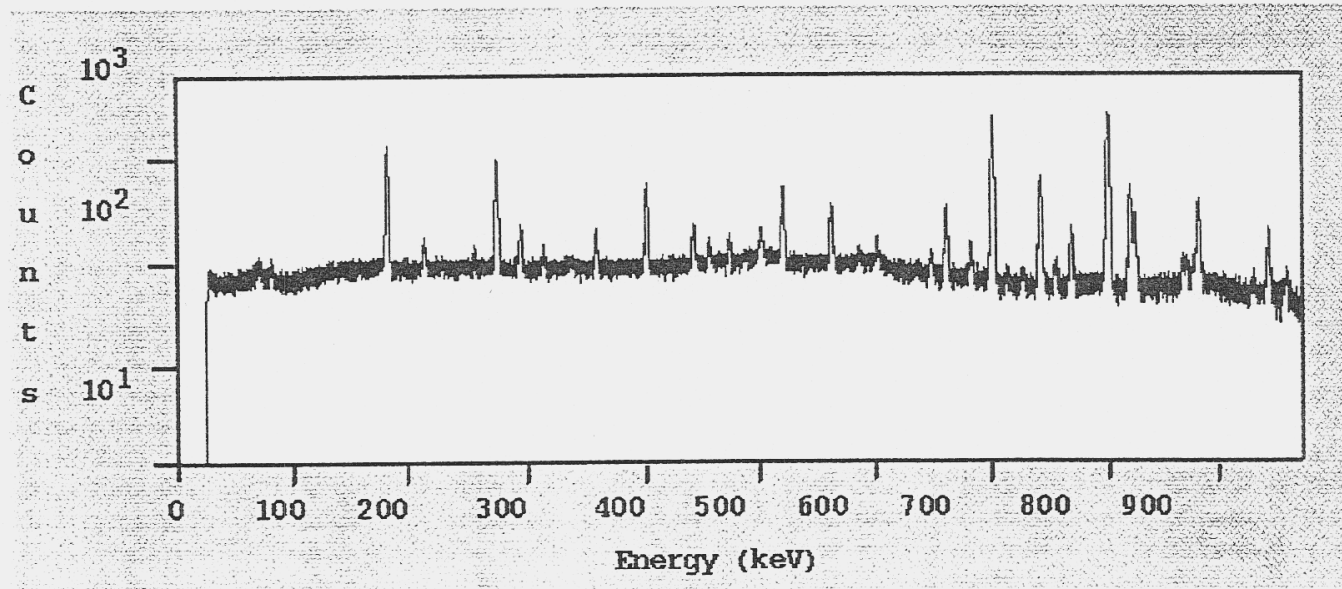




\section{A\&PCT Reconstruction Code Development}
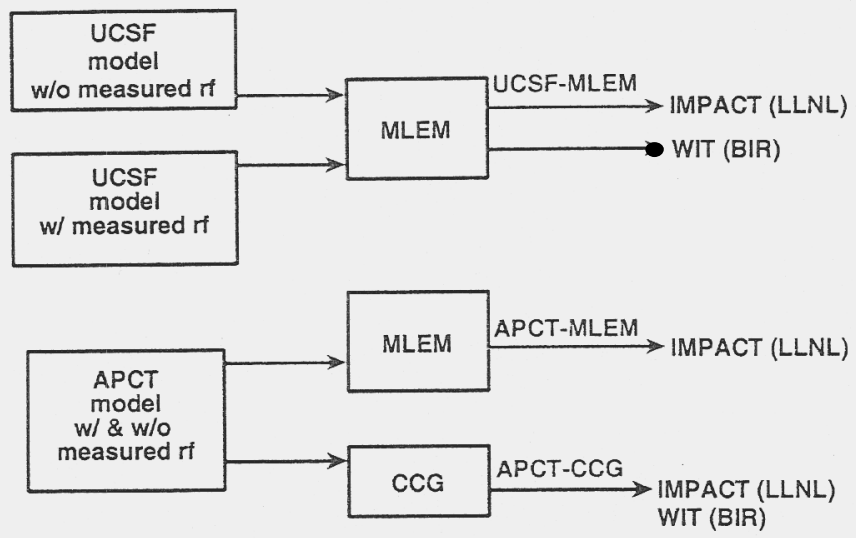

MLEM - Maximum Likelihood Expectation Maximizer

CCG - Constrained Conjugate Gradient

IMPACT - Isotope Measurements by Passive and Active Computed Tomography

BIR - Bio-Imaging Research Inc.

WIT - Waste Inspection Tomography

rf - response function of the detector-collimator

\section{Spectral Signal at a Detector}

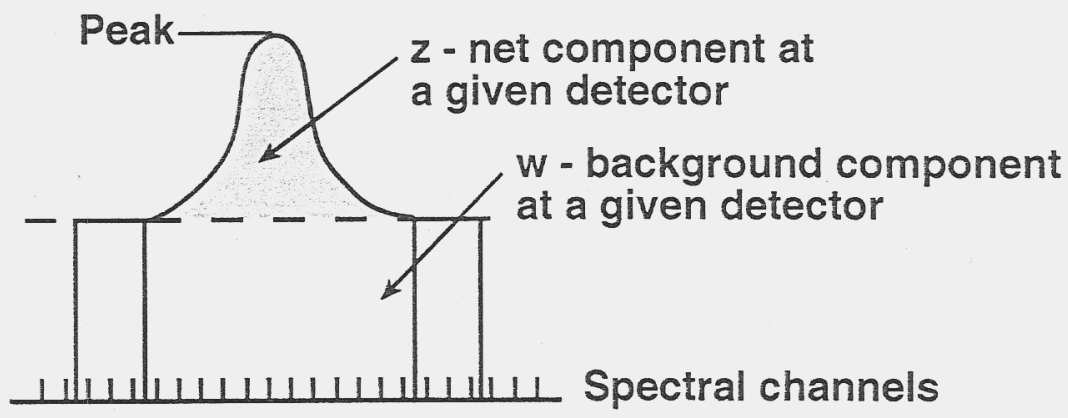

$$
z=A x
$$

\section{$z$ - vector of net counts per detector \\ $x$ - vector of emissions per image voxel \\ A - system Matrix}


General Iterative Solution Technique

$$
y=A x
$$

$y$ - vector of measured values

$x$ - vector of unknowns

A - system matrix

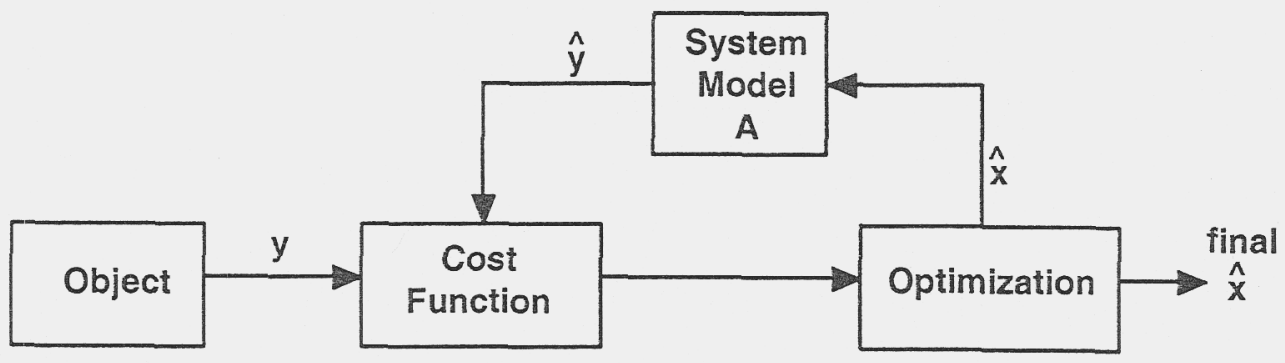

\section{Passive Scan Measurements}

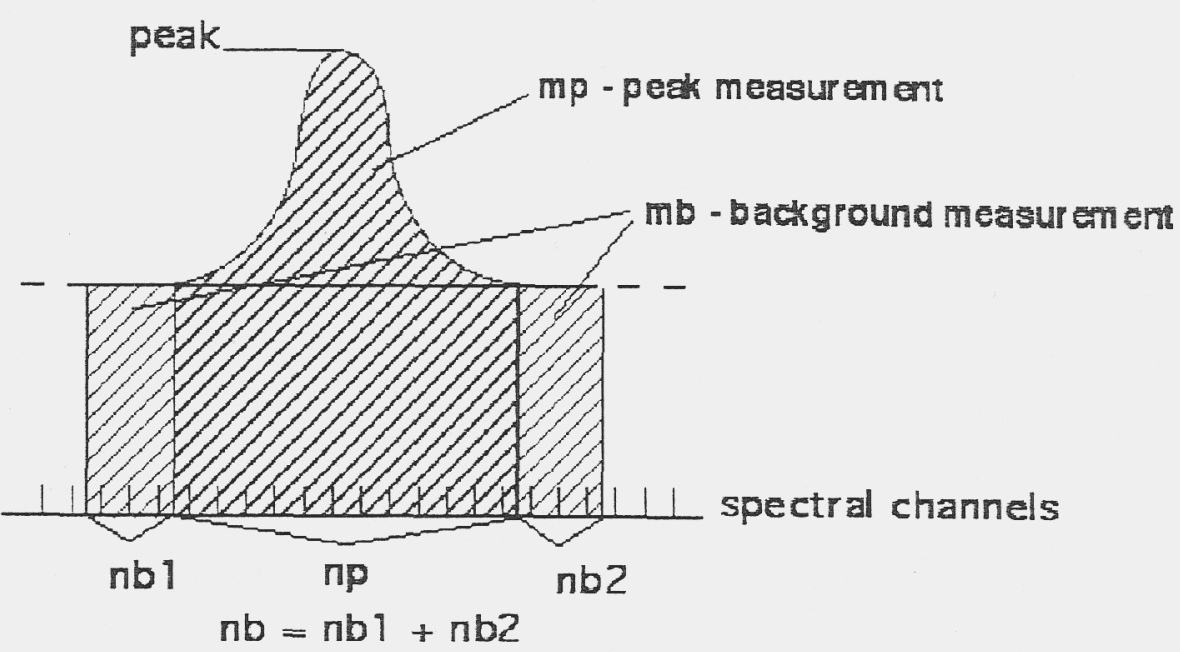




\section{Iterative Solution for A\&PCT}

$$
z=A x
$$

mp - vector of peak counts per detector mb - vector of background counts per detector

$z$ - vector of net counts per detector

x - vector of emissions per voxel

A - system matrix

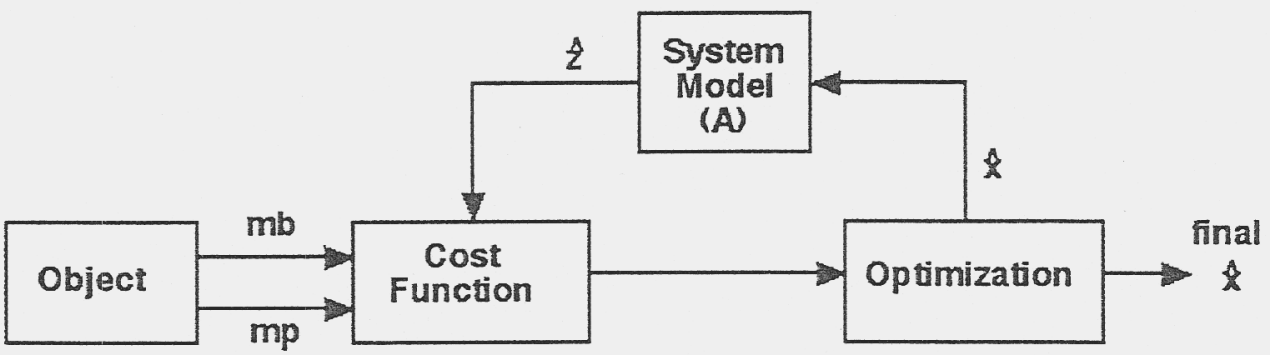

Cost Function - Poisson Distribution

$$
\operatorname{Pr}\{n\}=\frac{1}{n !} a^{n} \exp (-a)
$$

- observed value

$\operatorname{Pr}\{n\}$ - probabilty that is observed

- the expected value (mean) of $\operatorname{Pr}\{n\}$

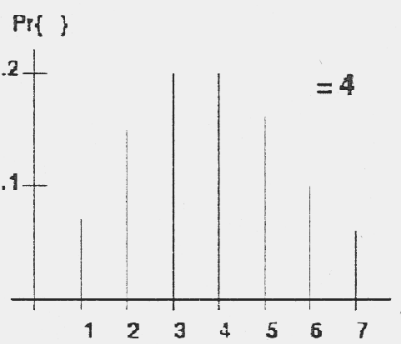

$$
\begin{aligned}
& \text { characterizes or defines a } \\
& \text { particular Poisson process }
\end{aligned}
$$




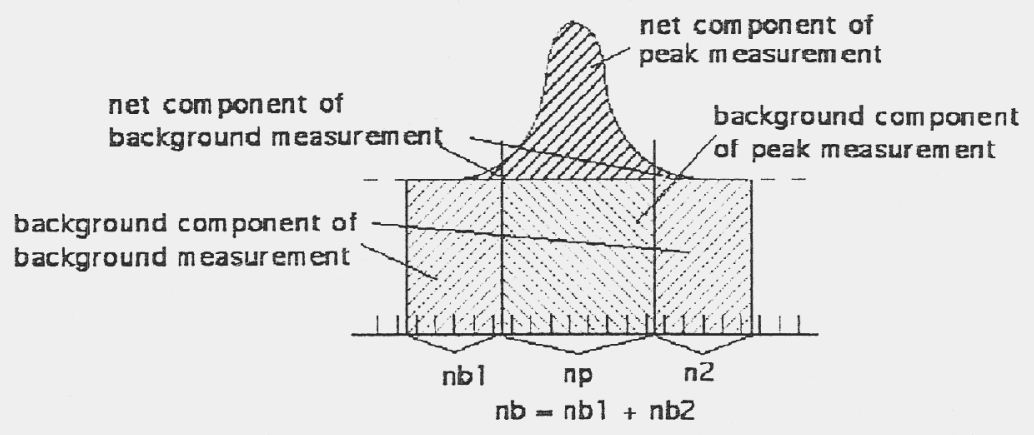

$$
\begin{aligned}
& E\{m b\}=\tau_{1} w+\tau_{g} z \\
& E\{m p\}=\tau_{2} w+\tau_{4} z
\end{aligned}
$$

\section{Comparison of Optimization Methods (MLEM and CCG) with Simulated Data}

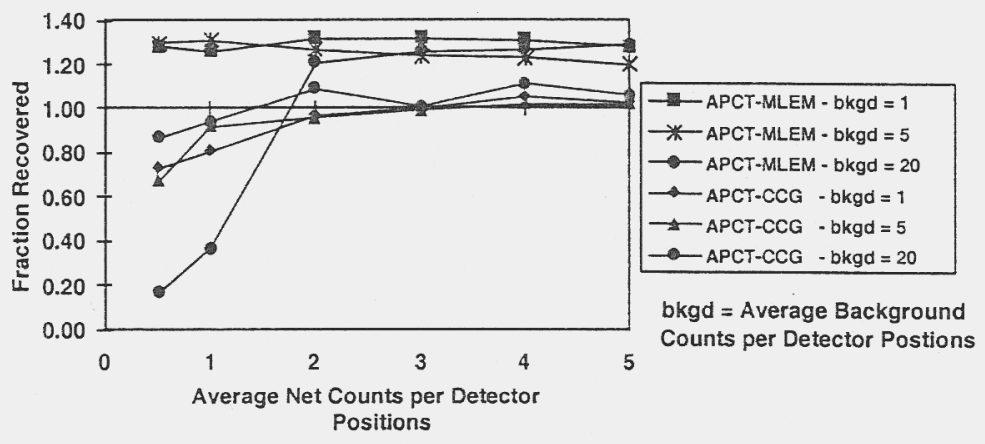


Comparison of Reconstruction Methods

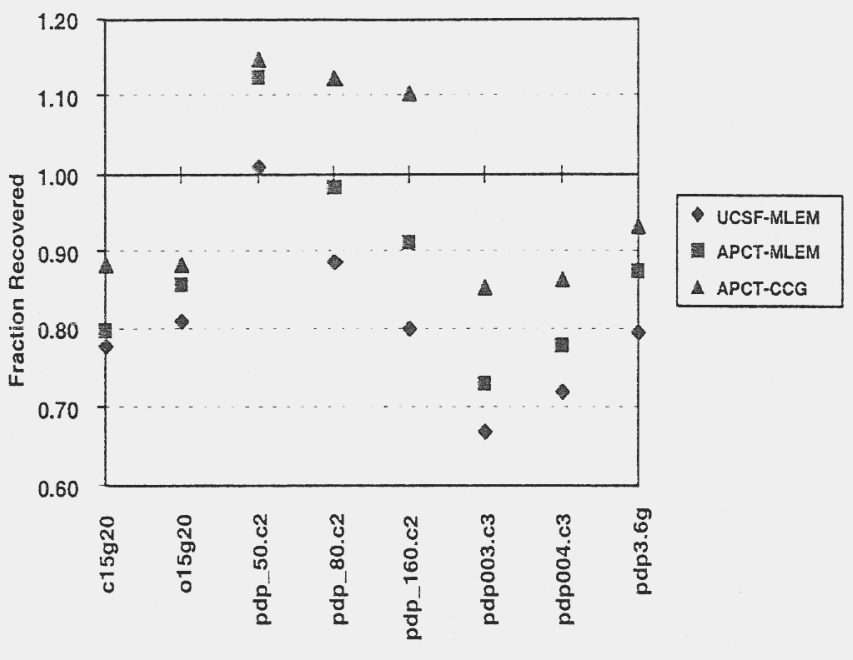

Simulated Comparison of MLEM and CCG

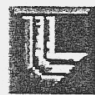

Simulation

no background

Average Net

$=2$ counts

Simulation with

Average Background

$=20$ counts

APCT-MLEM

Calculated

Sinograms Tomographic Images

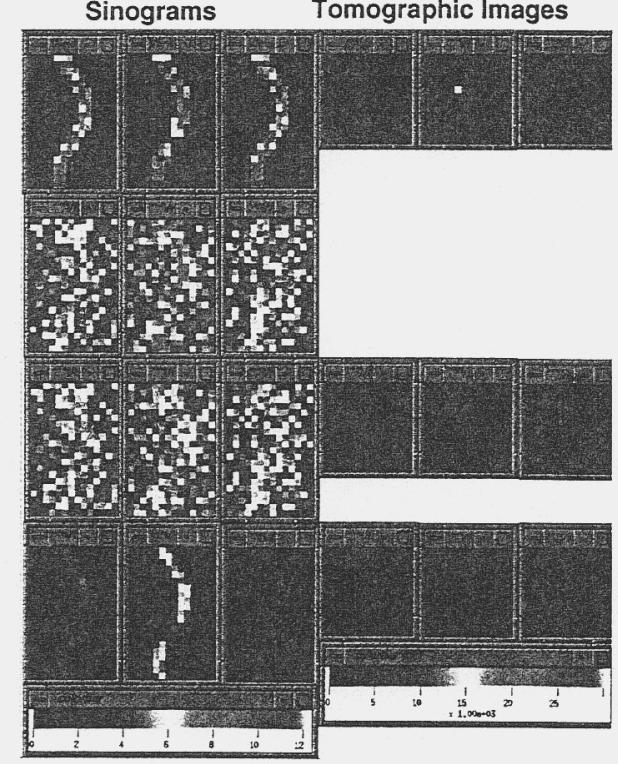

True Image

30350 counts

APCT-CCG

Calculated

APCT-MLEM

Reconstruction

36710 counts

$R=121 \%$

APCT-CCG

Reconstruction

30072 counts

$\mathrm{R}=99 \%$ 


\title{
Applying Optimization to Tomography Problems
}

\author{
Dennis M. Goodman
}

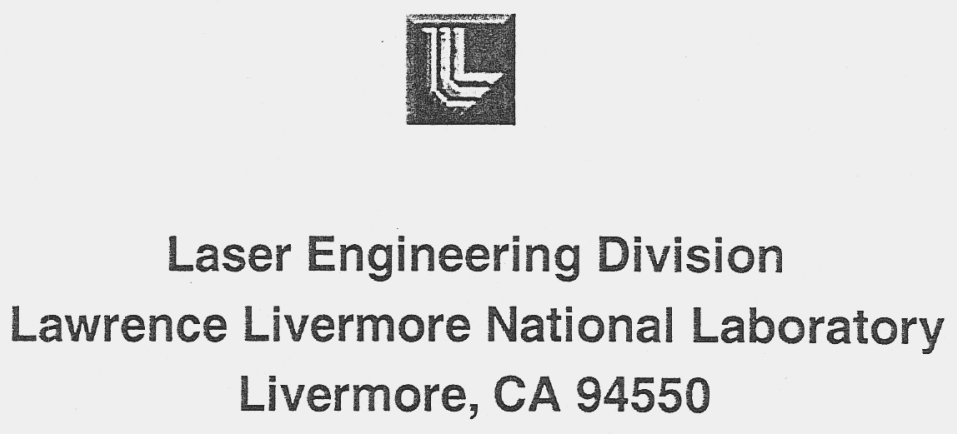

\section{Outline}

- Present basic ideas

- Show pretty pictures 
Basic Ideas:

- Develop flexible tools for new problems (avoid ad hoc algorithms).

- Linear attenuation (transmission, etc.) model: $\hat{y}=A \hat{x}$.

- Fitting function based on statistics: $f=(y, \hat{y})$.

- Data is $y$, Model of what data is $\hat{y}$.

- Optimize fit of model to data: minimize $L(y, \hat{x})=f(y, A \hat{x})$.

Usually $\underline{L}$ is Negative log-likelihood or a robust (outlier resistant) fitting function.

Basic Ideas (Contd.):

- Model described above is called Generalized Linear Model in statistics. Lots of applicable theory that should be investigated.

- Can apply our methods to more general models as well.

- Two optimization methods to solve: both use bending linesearch.

- Constrained Conjugate Gradients (CCG): if cols. of $A$ easy

- Constrained Limited Memory Quasi Newton (QN): if not 
Fitting function depends on statistics:

Our algorithms let you use whatever is appropriate (or try several) (L

- Gaussian data leads to least squares for max. likelihood:

$$
f(\mathrm{y}, \hat{\mathrm{y}})=\sum r_{i}^{2}, \text { where } r_{i}=y_{i}-\hat{y}_{i} \text { (residuals). }
$$

- Least squares sensitive to outliers, use robust technique, e.g.:

$$
f(\mathrm{y}, \hat{\mathrm{y}})=\sum g\left(r_{i}\right) \quad \text { where } g(r)= \begin{cases}r^{2} & \text { if }|r| \leq c \\ 2 c|r|-c^{2} & \text { if }|r|>c\end{cases}
$$

- For counting data (emission tomography), max. likelihood assuming

Poisson statistics leads to (we use n for vector of integer counts):

$$
f(\mathbf{n}, \hat{y})=\sum g\left(n_{1}, \hat{y}_{i}\right) \quad \text { where } g(n, \hat{y})=\hat{y}-n \log \hat{y}
$$

Both CCG and QN Algorithms solve:

$$
\dot{\mathbf{x}}=\underset{\mathbf{x} \in S}{\operatorname{Argmin}}\left\{L(\mathbf{x}, \mathbf{y})+\nu\|\mathbf{x}\|_{1}+\lambda\|\mathbf{x}\|_{2}+\delta g(\mathbf{x})\right\}
$$

- The set $S$ defines "Box" constraints: $S=\{\mathrm{x}: 1 \leq \mathrm{x} \leq \mathrm{u}\}$.

- These constraints are very useful in imposing prior information about the unknown object: nonnegativity, regions of known absorption voids, occlusions, etc.

- The $\hat{\ell}_{1}$ and $\hat{\ell}_{2}$ norm terms penalize size of unknown (regularization). - $\ell_{2}$ same as Gaussian prior; may oversmooth.

$\circ \ell_{1}$ same as Laplacian prior; good for "spiky" unknown.

- $g(x)$ arbitrary penalty term: entropy, Good's roughness, derivative penalties, etc. 
During an iteration CCG and QN pick a direction, then they use a linesearch to optimize the function along that direction.

- Let $\underline{h(x)}=$ function being minimized.

- CCG picks new direction: $d_{i+1}=-\Delta_{x} \underline{h}\left(x_{i}\right)+\beta_{i+1} d_{i}$ i.e.: Negative gradient plus scaled old direction.

- QN picks new direction: $d_{i+1}=-H \Delta_{x} \underline{h}\left(x_{i}\right)$ where $H$ is a low order approximation to inverse Hessian matrix. Hessian = second partial matrix of $\underline{h}\left(x_{i}\right)$.

- "Bending" linesearch does not stop when face of $\underline{S}$ is hit:

$$
\begin{aligned}
& \tilde{x}_{i+1}=\operatorname{Median}\left\{1, x_{i}+\alpha d_{i}, u\right\} \\
& \alpha_{i}=\operatorname{Arg} \min _{\alpha>0}\left\{h\left[\tilde{x}_{i+1}(\alpha)\right]\right\}
\end{aligned}
$$

\section{Bending linesearch advantages} over more common methods.

- Conventional optimization algorithms pick good search directions, but stop when hit first constraint: can hit only one face of box per iteration

- Common limited data CT methods (POCS, ART, EM, etc.) usually are steepest descent, project back to box with no linesearch.

- Our algorithms get good search directions. Uses bending to hit many faces of box per iteration. If cols. of $A$ available, bending linesearch is very fast, use CCG; else use QN to minimize function evaluations in linesearch. 
A bending linesearch approach is more efficient when many variables are bounded.

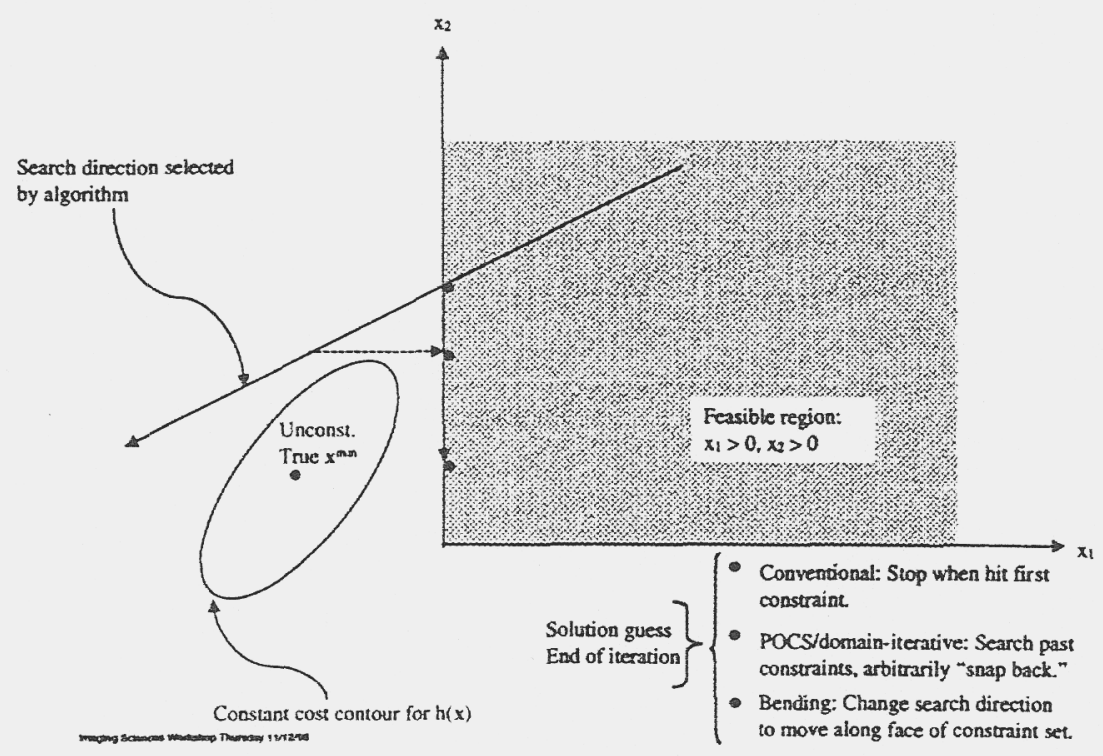

Now for the pretty pictures... We show six examples using CCG:

- Reconstruction from outlier-corrupted sinogram.

- Reconstruction from neutron CT data.

- Waste drum assay from counting (Poisson) data.

- Cone beam tomography for the Advanced Hydrotest Facility (AHF)

- Improved reconstruction using prior information.

- Pulsed photothermal tomography (invert heat equation). 
Robust Example

- Sinogram data corrupted Gaussian mixture noise.

- Outlier occurs with $1 \%$ probability

$-\sigma_{\text {outlier }}=100 \times \sigma_{\text {regular }}$

- Many problems at LLNL are outlier corrupted.

- CCG handled robust function very well.

- Reduced rms error by factors of 2-3.

Robust example: Sinogram data is corrupted with Gaussian mixture noise

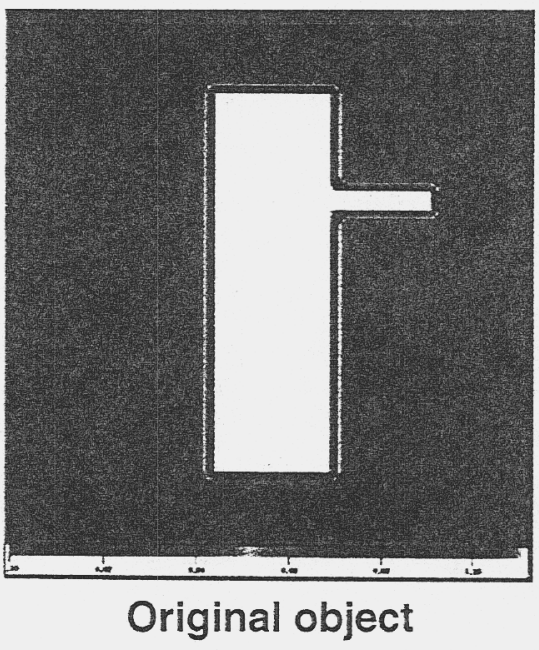


CCG reconstruction using the least-squares fitting function is very sensitive to outliers

\section{Least squares fit reconstruction}

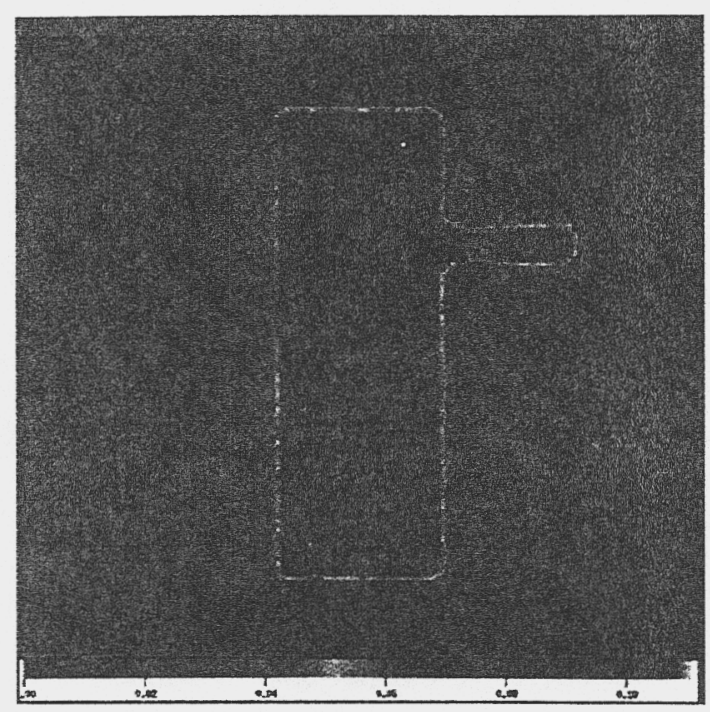

CCG reconstruction using robust fitting function reduces outlier sensitivity: lowers rms estimation error by factor of 2-3.

Robust (Huber Function) reconstruction

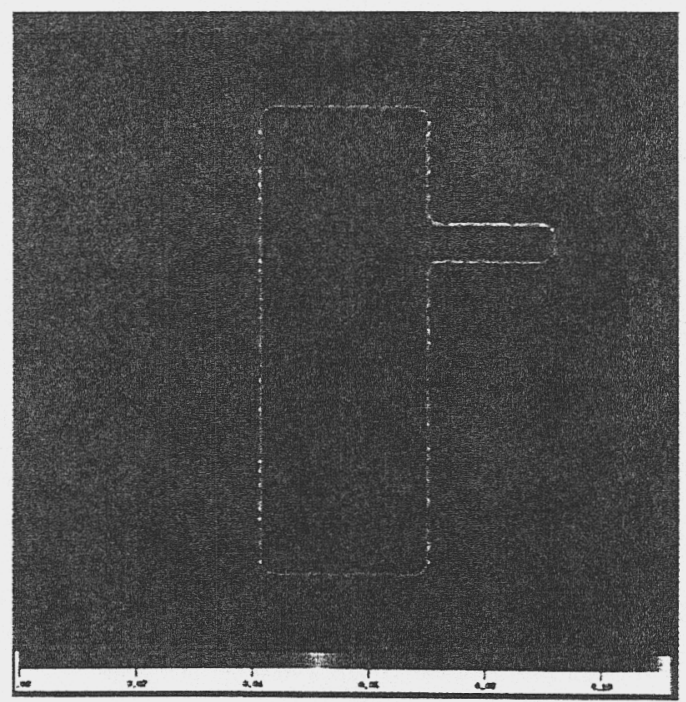


Neutron Imaging

- Important to ESP: image through dense stuff.

- CCG forced nonnegativity.

- Used least-squares, should have used counting statistics.

- Full of outliers due to external radiation.

- Outliers removed by preprocessing.

- Our robust model-based approach will be much better.

\section{Summary of the LANL/LLNL experiments at LANSCE}

- LANL/LLNL experiments at LANSCE first demonstrated the potential of neutron radiography for stockpile surveillance applications.

- Incident neutron energies up to $400 \mathrm{MeV}$

- Uranium and LiD test object assembly

- Tungsten converter with multiwire detector

- Sensitive to neutrons $40 \mathrm{MeV}$
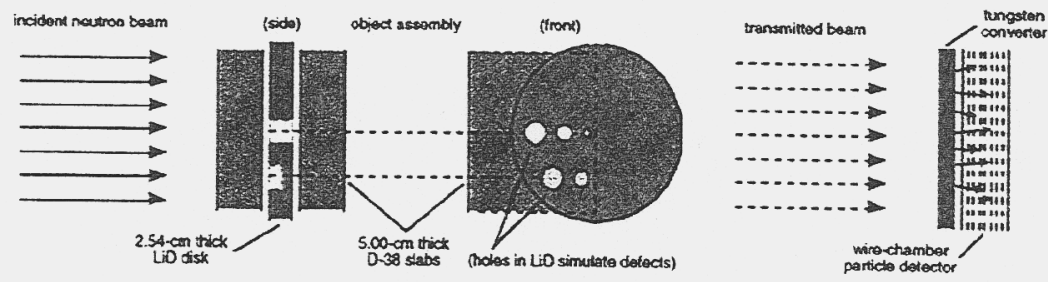


\section{Experimental setup used to acquire the neutron CT data}

- The lead/poly-cylinder phantom was imaged using $10 \mathrm{MeV}$ neutrons produced at the Ohio University Accelerator Laboratory

- 64 separate radiographic images

- 10 minute exposures

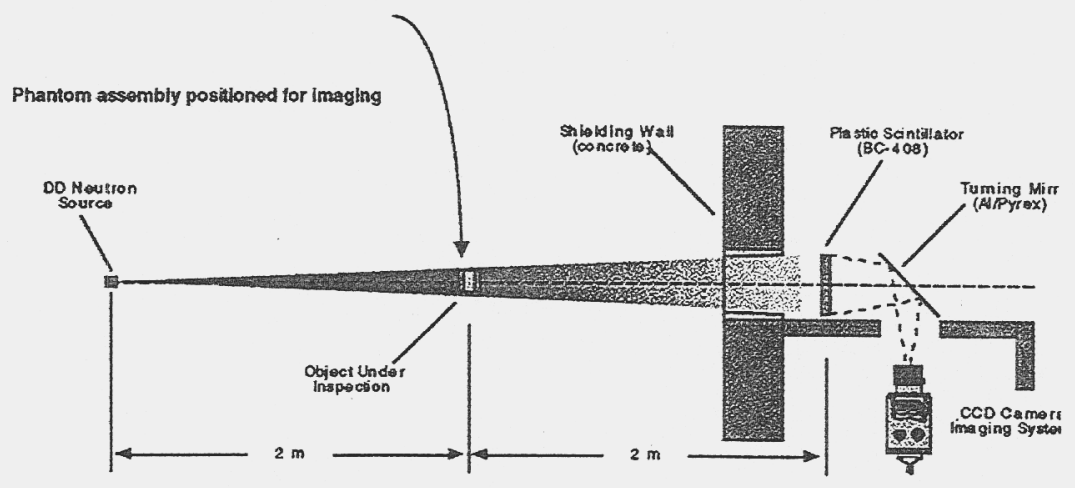

\section{Lead/poly-cylinder phantom $10 \mathrm{MeV}$ neutron imaging}
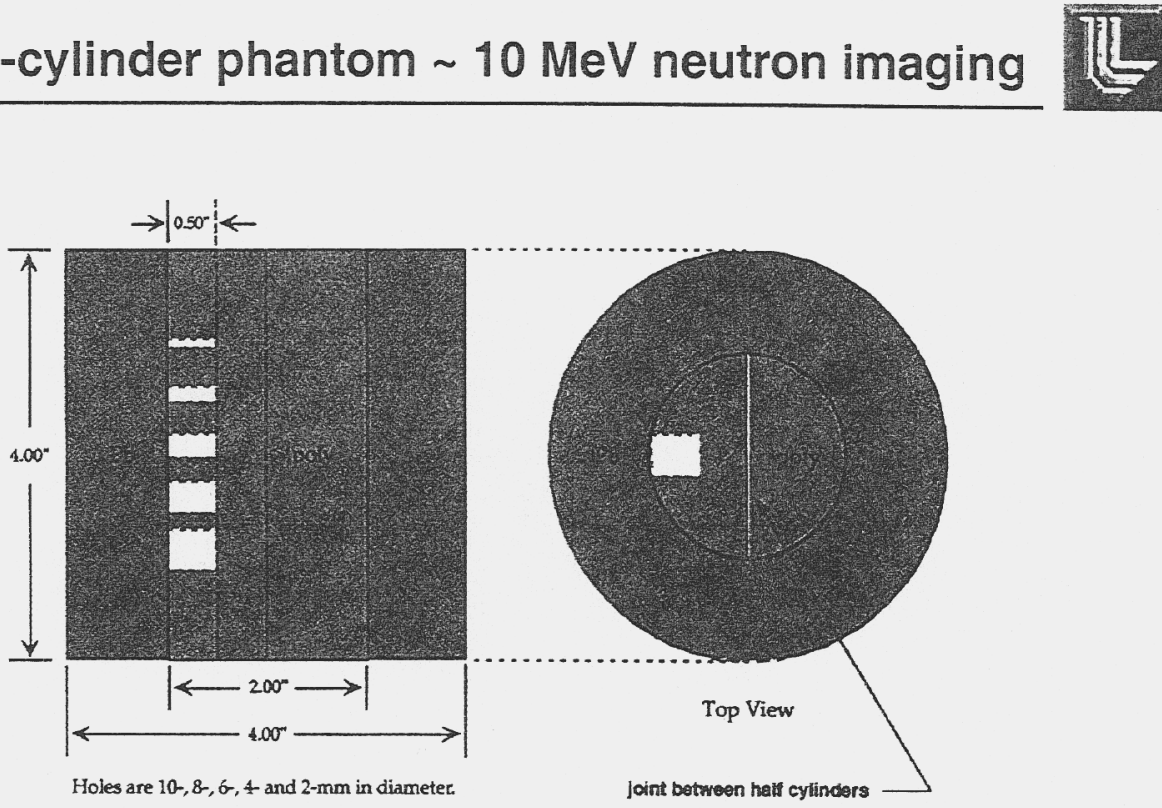


\section{CCG reconstruction gave better results than conventional FBP for $10 \mathrm{MeV}$ neutron imaging}
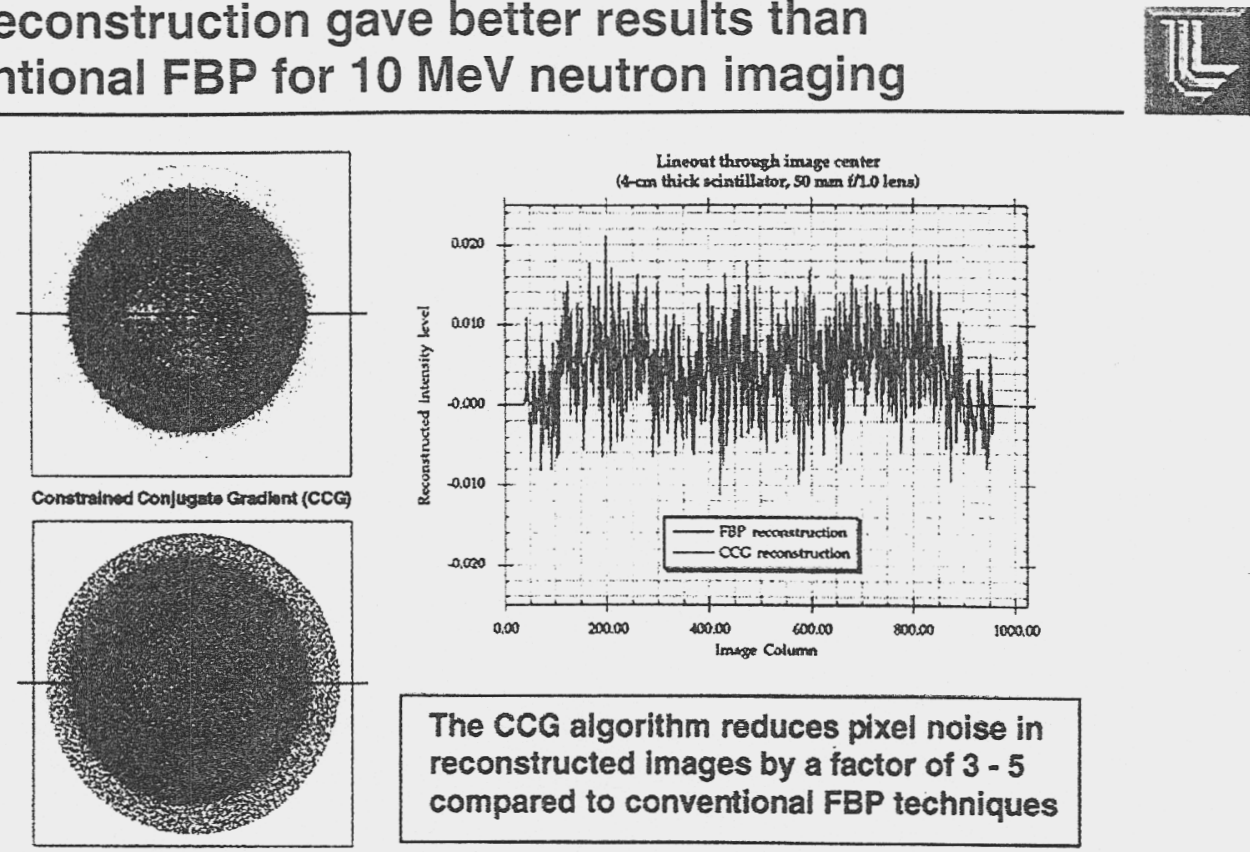

The CCG algorithm reduces pixel noise in reconstructed images by a factor of $3-5$ compared to conventional FBP techniques

Standard Fatered Black Protoction (FBP)

\section{Enlarged view of horizontal slice through $6 \mathrm{~cm}$ hole}

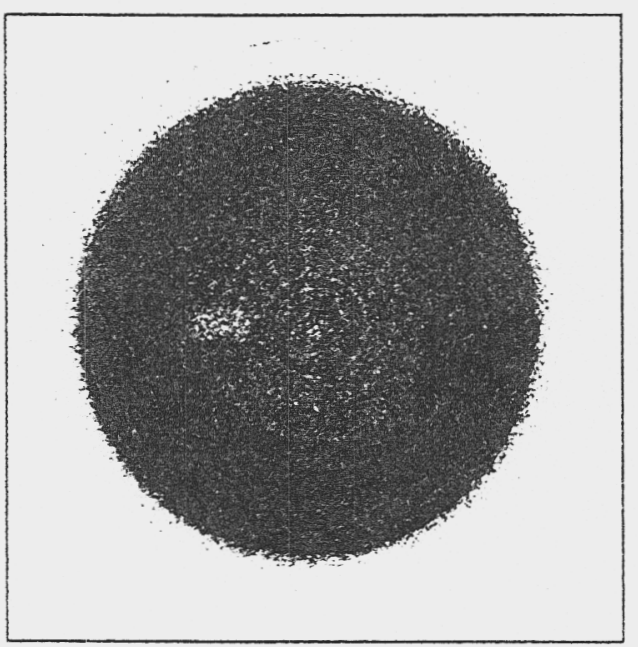

Constrained Conjugate Gradient (CCG) tomographic reconstruction

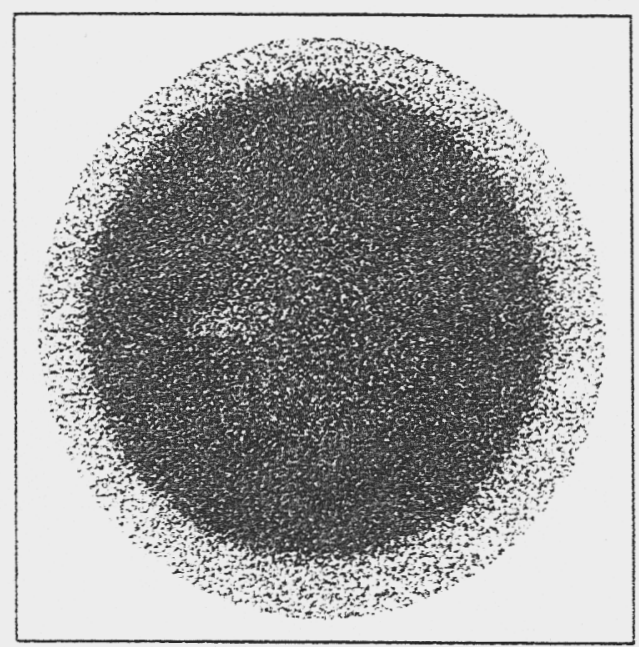

Standard Filtered Back Projection (FBP) tomographic reconstruction 


\section{Waste Drum Assay}

- Complicated model: two measurements at each location.

- Background.

- Spectral peak.

- MLEM not flexible enough: used net = peak - background.

- Statistically incorrect: net is not Poisson distributed.

- Physically incorrect: net count may be negative.

- CCG implemented true likelihood.

- Better assay.

- Better pictures.

\section{Comparison of CCG and MLEM reconstruction using simulated A\&PCT data}

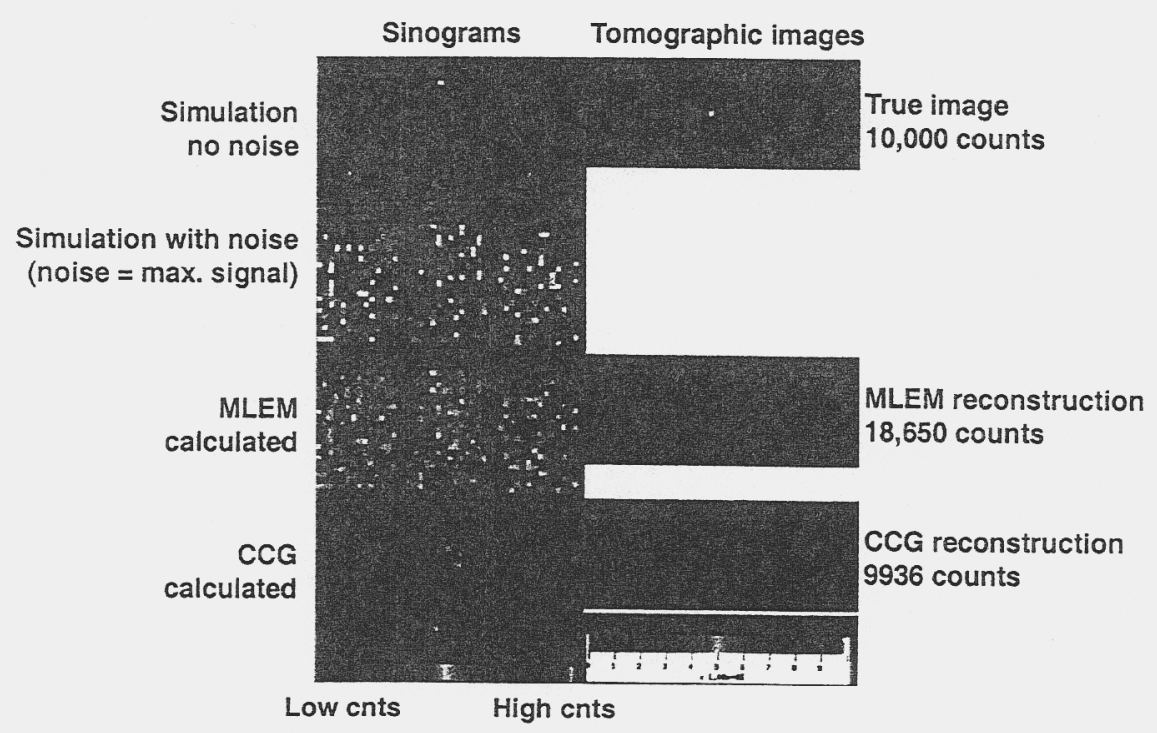




\section{Comparison of CCG and MLEM reconstructions from actual low count A\&PCT data}

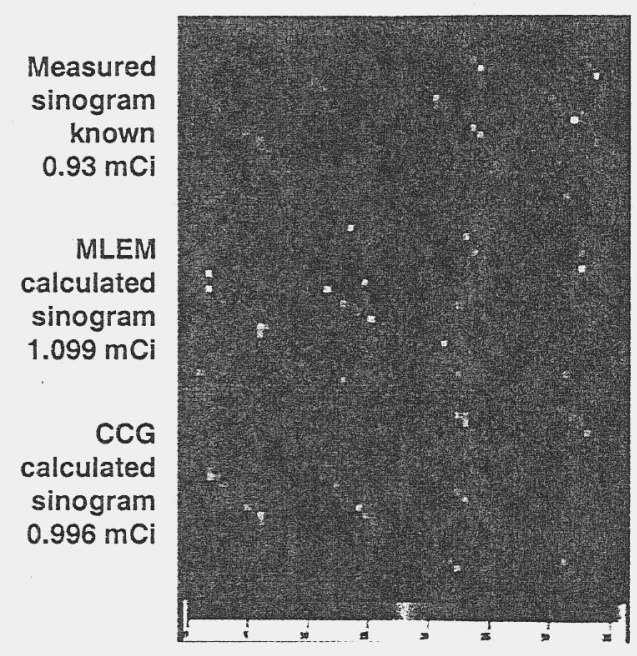

The 3D cone-beam CCG reconstructor shows significant improvement over conventional Feldkamp CT
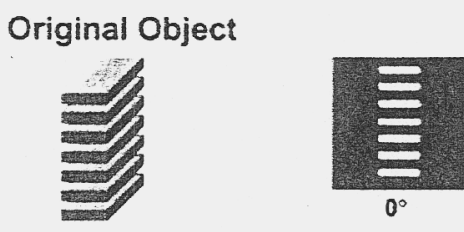

Cone Beam Projections

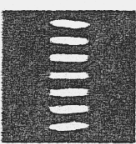

$45^{\circ}$

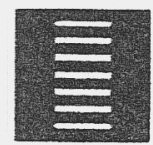

$90^{\circ}$

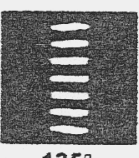

135

Orthogonal Slices

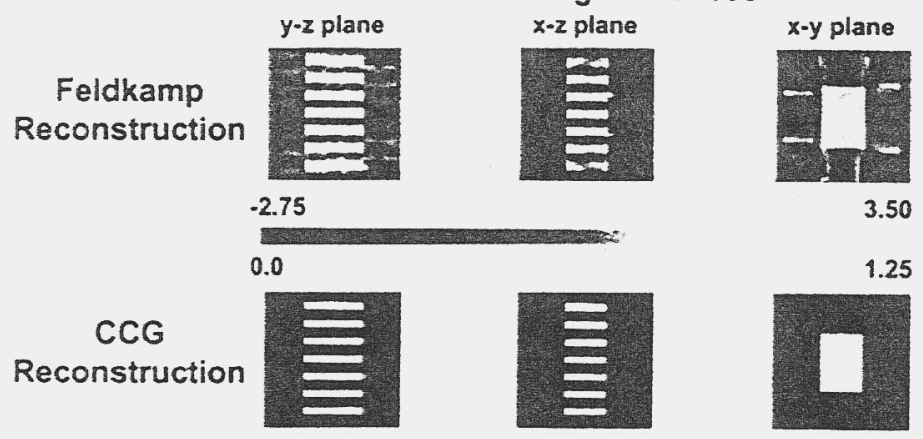


Applying prior information about known void and occlusion improved reconstruction quality outside these regions as well: total rms error reduced by factor of 3 .
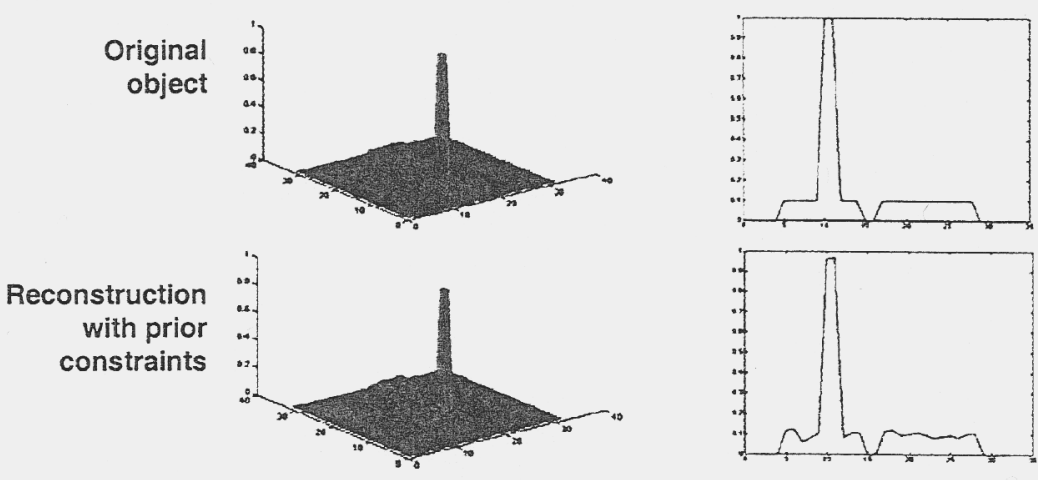

Reconstruction with no prior constraints
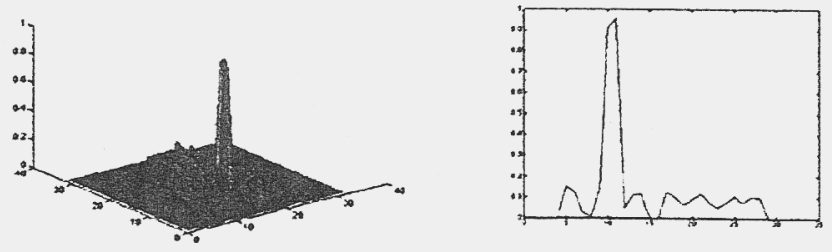

Pulsed photo-thermal tomography reconstructs 3-D absorption map of object from time-sequenced IR images of its surface heat following a laser pulse.

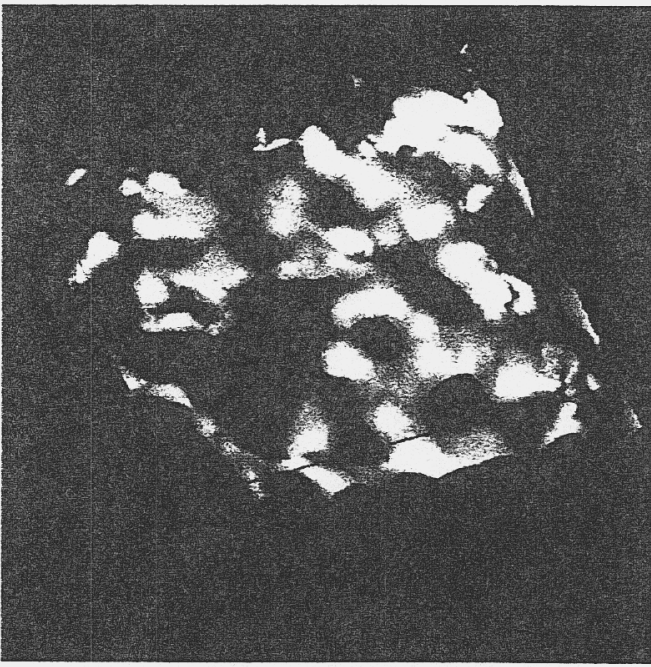

3-D reconstruction of

in vivo port wine

stain blood vessels

of human subject.

Image dimension is

$1.6 \times 1.6 \times .6 \mathrm{~mm}$.

Blood vessels are

$\sim 100$ microns in diameter 


\title{
Can We Cure Osteoporosis?
}

\author{
J.H. Kinney, Dept. of Chemistry and Materials Science \\ Lawrence Livermore National Laboratory; \\ Division of Biomaterials, University of California, San Francisco \\ D.L. Haupt, Center for Nondestructive Evaluation \\ Lawrence Livermore National Laboratory
}

Osteoporosis is a serious public health problem. It is known that fracture risk is strongly correlated with the loss of bone mass at menopause, so current treatments are targeted at bone mass maintenance. Estrogen replacement therapy and anti-resorptive bisphosphonates are the first line treatments for preventing bone loss. None of these treatments, however, are anabolic-that is, they do not restore bone mass or strength to premenopausal levels.

By taking advantage of the high intensity, collimation, and monochromaticity of synchrotron radiation, we have been able to image the three-dimensional trabecular bone structure in living rats, thus providing serial data on the earliest architectural changes that occur with estrogen loss.

Results from these in vivo animal experiments demonstrated that one of the earliest manifestations of estrogen loss, in addition to a decrease in the amount of trabecular bone, was decreased connectivity.

We hypothesized that estrogen, if administered in a cyclic manner, could increase the thickness of the remaining trabecular bone by suppressing osteoclast (bone resorbing) activity while stimulating osteoblast (bone forming) activity. Our experiments demonstrated that estrogen replacement therapy, when initiated in this cyclic manner, restored bone mass to baseline levels but did not recover the trabecular connectivity. Even without an associated recovery in trabecular connectivity, finite element calculations on the three-dimensional images suggested that cyclic estrogen can recover the original structural modulus of elasticity. We believe the recovery of the elastic properties is due to an increase in trabecular thickness above baseline values. This is the first demonstration of an anabolic behavior of estrogen replacement, and points the way to new treatments to restore lost bone mass, either with estrogen or the new class of selective estrogen receptor modulators. 
Can We Cure Osteoporosis?

I.H. Kinney and D. L Haupt

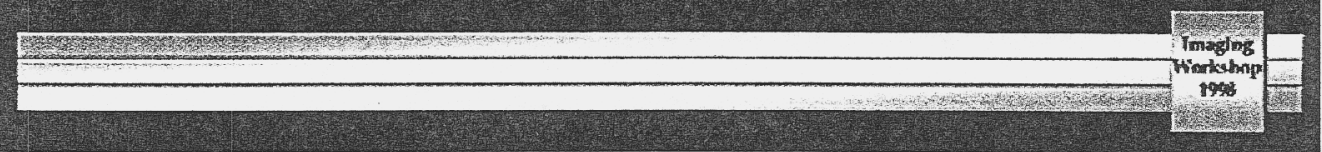

Osteoporosis is a major public health problem

- Among leading causes of death for women over 65

- All women over 65 are sufficiently at risk to justify bone mineral densily measurements

- Also affects men

- age related decline in estrogen receptor efficiency

- use of steroidal anti-inflamatories 
Bones form from endochondral growth
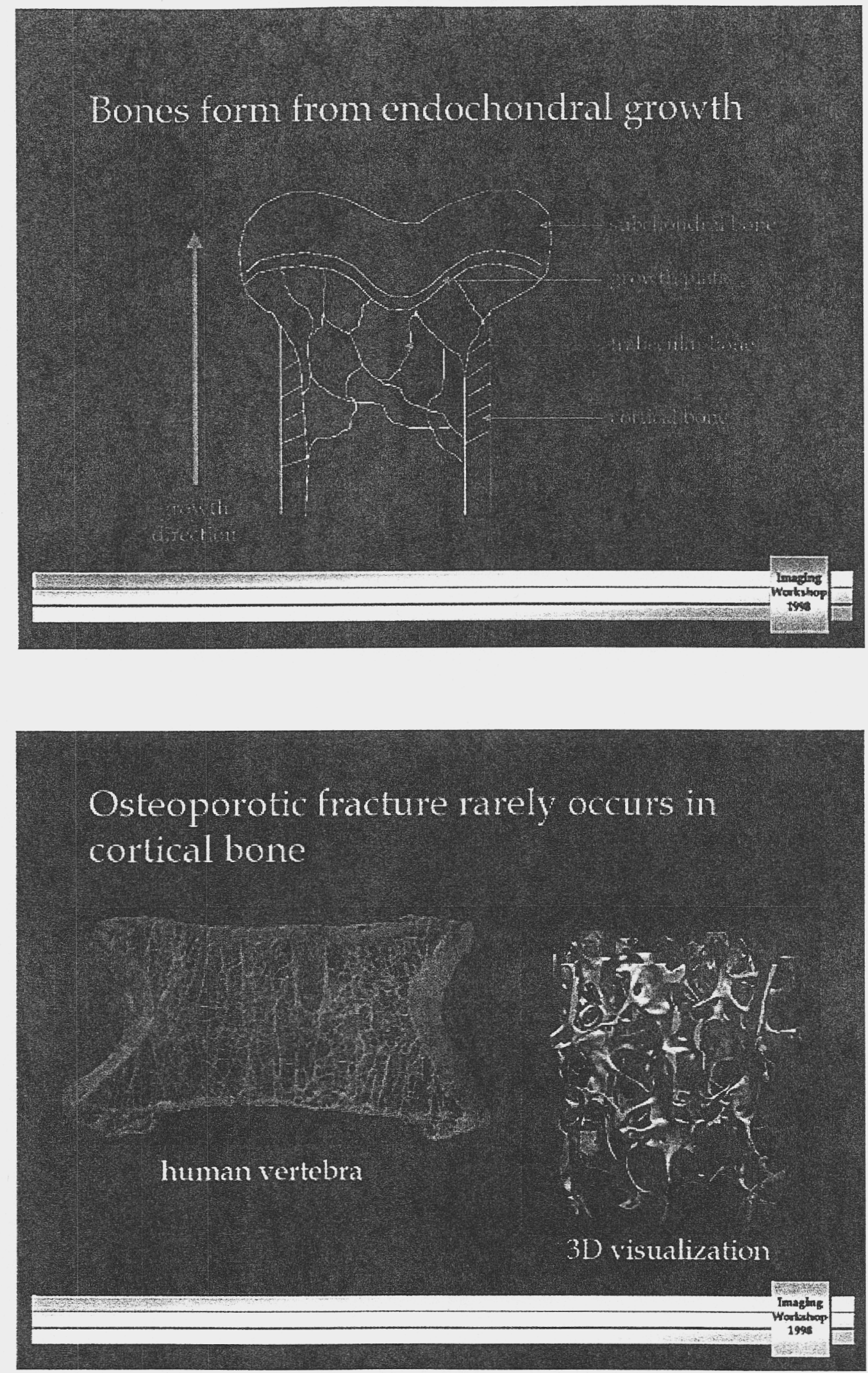
Femoral neck is also largely composed of trabecular bone
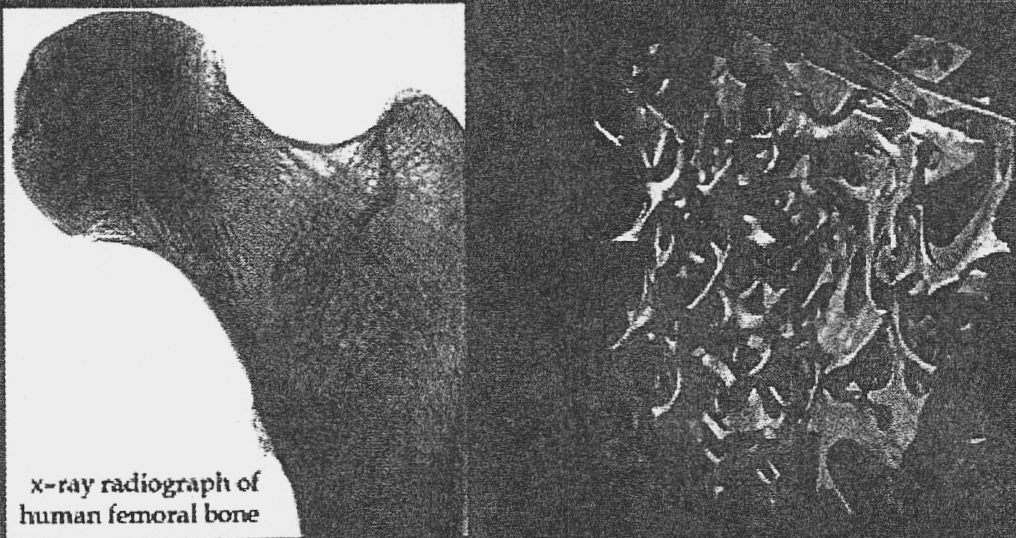

Understanding bone structure/properties is complicated by remodeling

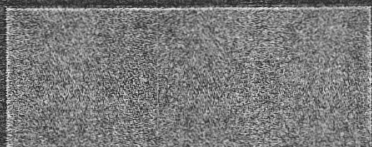

activation

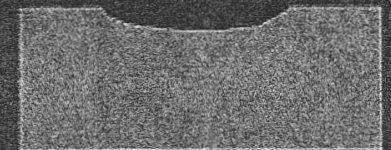

resorption

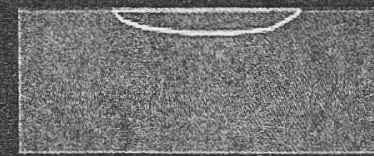

formation

In healthy bone, remodeling is controlled by the need to : 1) maintain mineral balance, 2) repair tissue damage, and 3) respond to mechanical usage. Trabecular bone does not follow simple scaling laws of regular cellular structures, nor the typical scaling laws of random networks. 


\section{What happens at menopause?}

- activation of remodeling increases

- bone formation is inhibited

o net loss of bone

- strength decreases with loss of bone mass

present treatments for osteoporosis attempt to suppress activation: estrogen, anti-resorptives, calcitonin.

How can imaging science help cure osteoporosis?

o In vivo imaging

- provides structural information

- provides mineral density information

- large scale finite element modeling

- images can be used to compute fracture risk 


\section{CT Imaging provides 3D structural information}
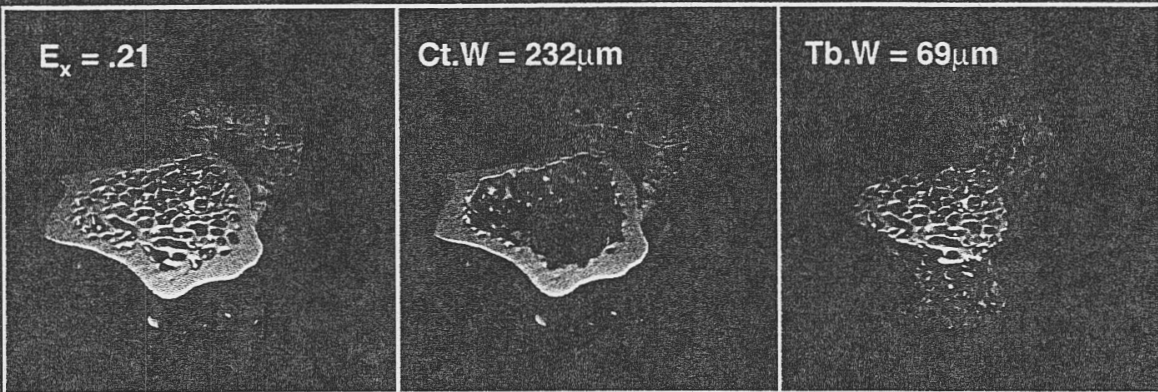

3D CT inages provide structural information such as trabecular bone volume, cortical/trabecular width, and connectivity. With finite-element method, the strength of the structure can also be determined.

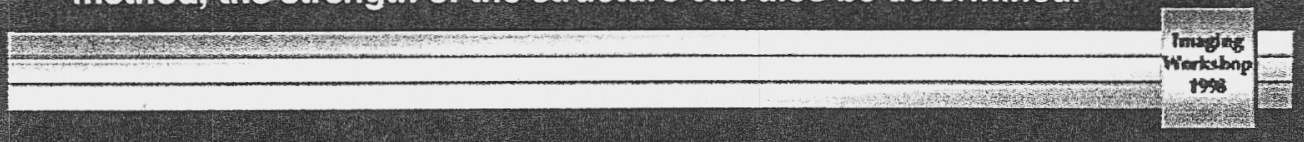

\section{Monochromatic synchrotron radiation} enables compositional analysis to be performed in vivo

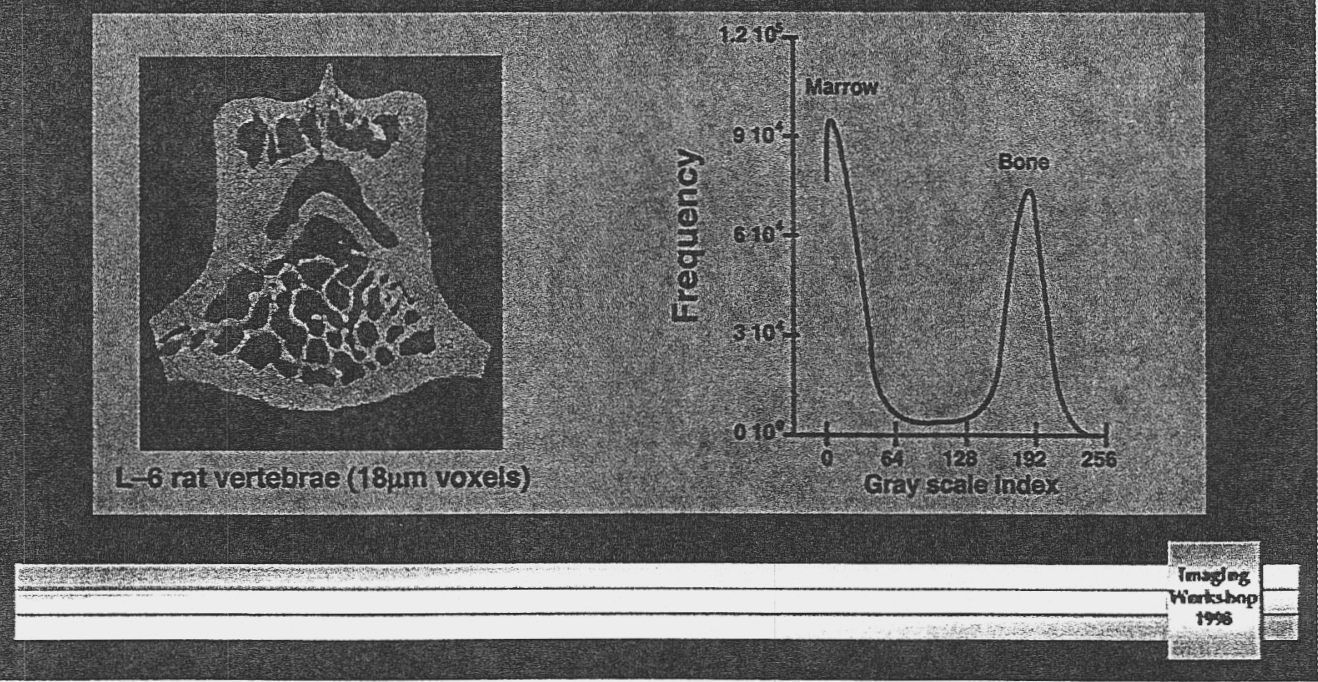


Mineral density distribution can be easily determined from the images

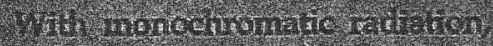
the concentrut tor oimineral erin te simply diated to we gray retile valle of the marg

$$
\begin{aligned}
& y-\frac{c+6 y^{2}-c}{m-c}
\end{aligned}
$$

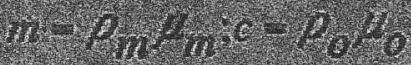
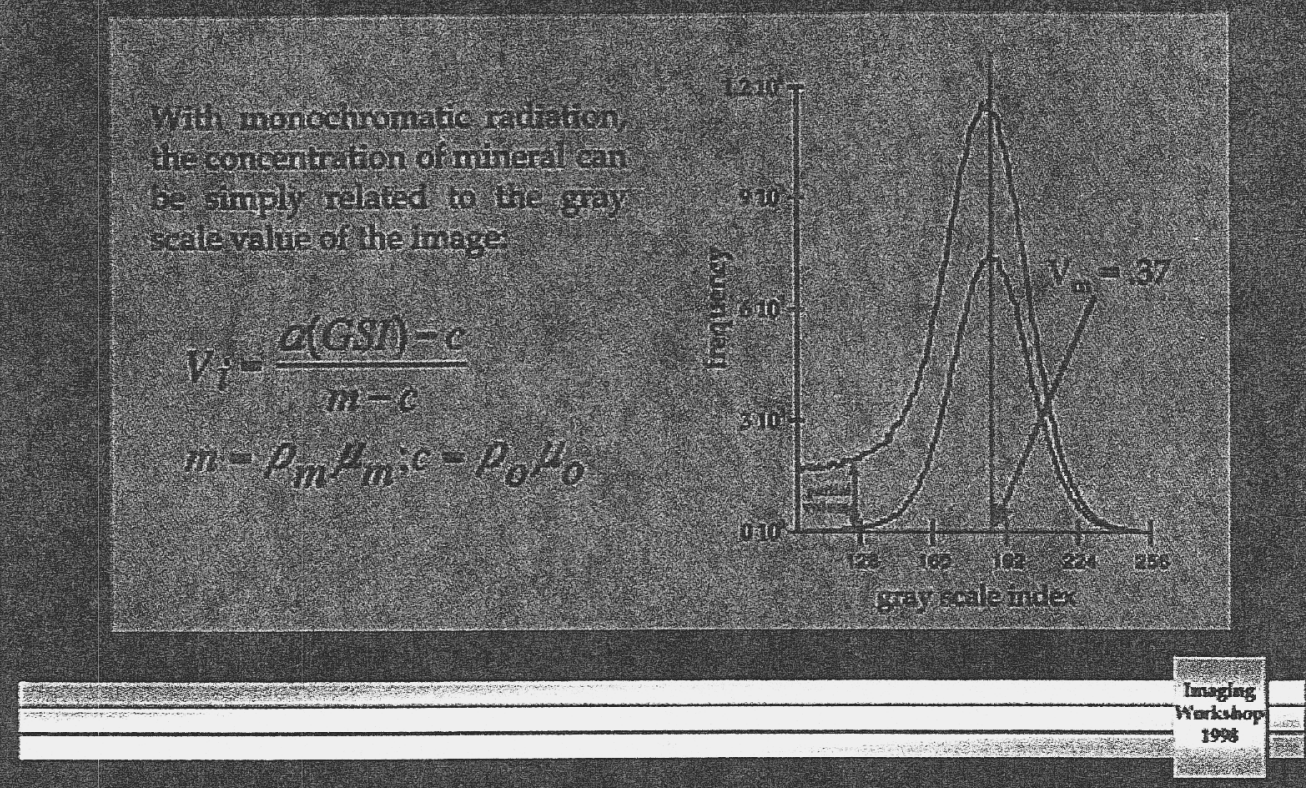

\section{In vivo characterization of trabecular bone}

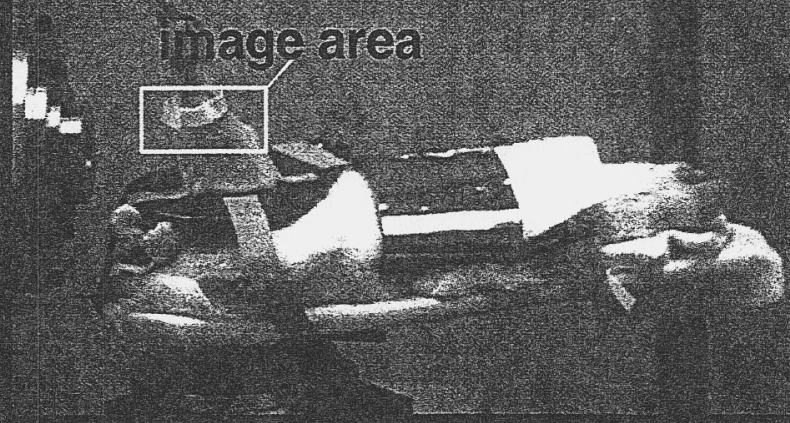

anesthetized 


\section{In vivo materials}

\section{characterization}

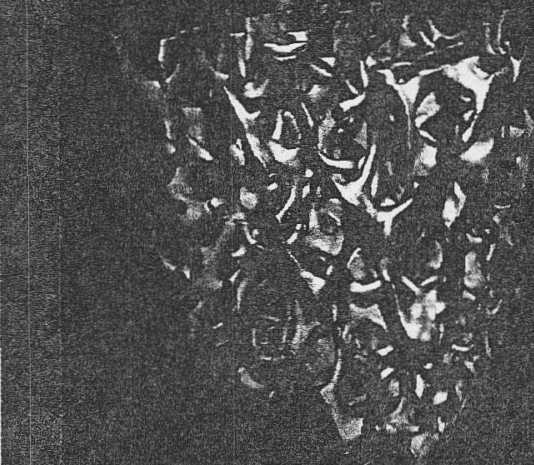

before OVX

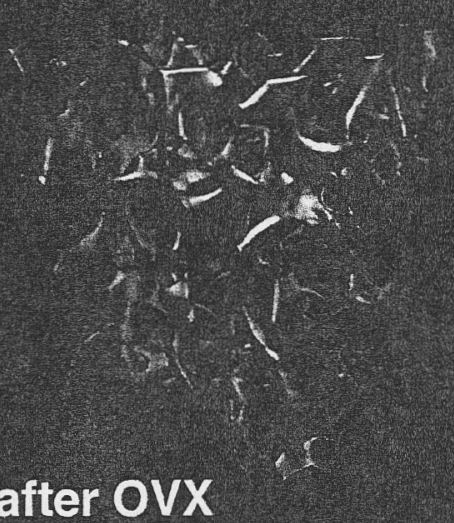

after OVX

Estrogen loss leads to rapid decline in trabecular bone volume and connectivity

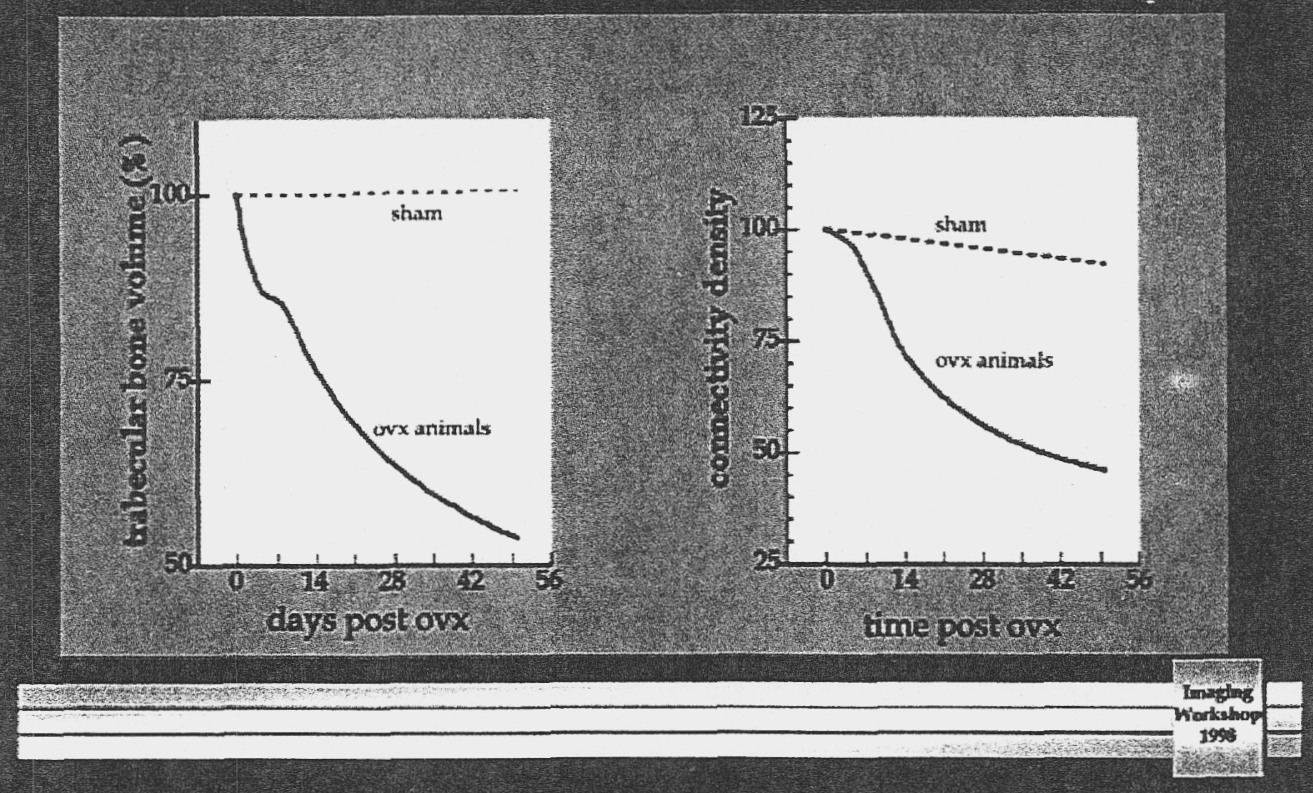


Element by element meshing
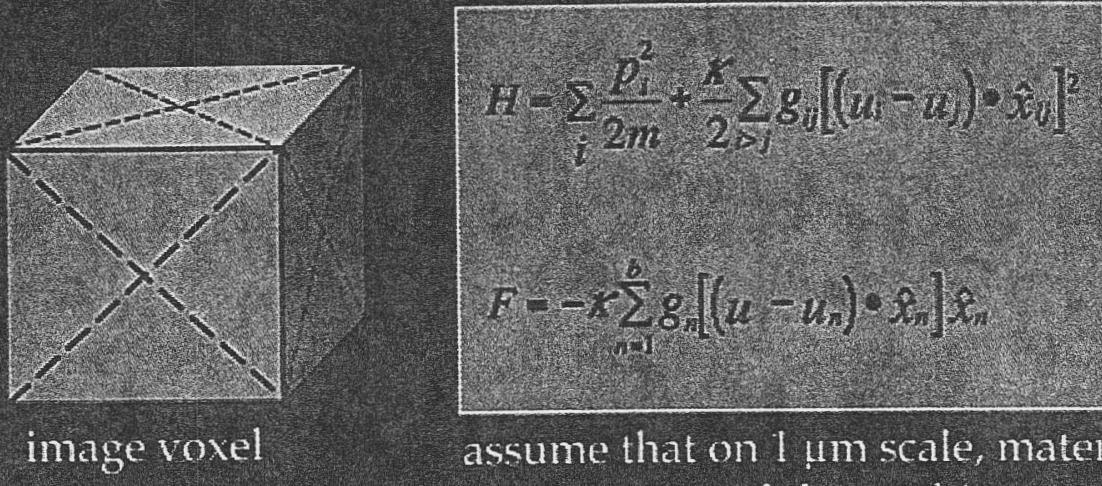

assume that on I im scale, material is isotropic, and that architecture dominates material behavior

FE modeling reproduces the elastic properties within experimental error

Numerous problems with testing bone:

- boundary conditions

- small specimen size

- non physiological
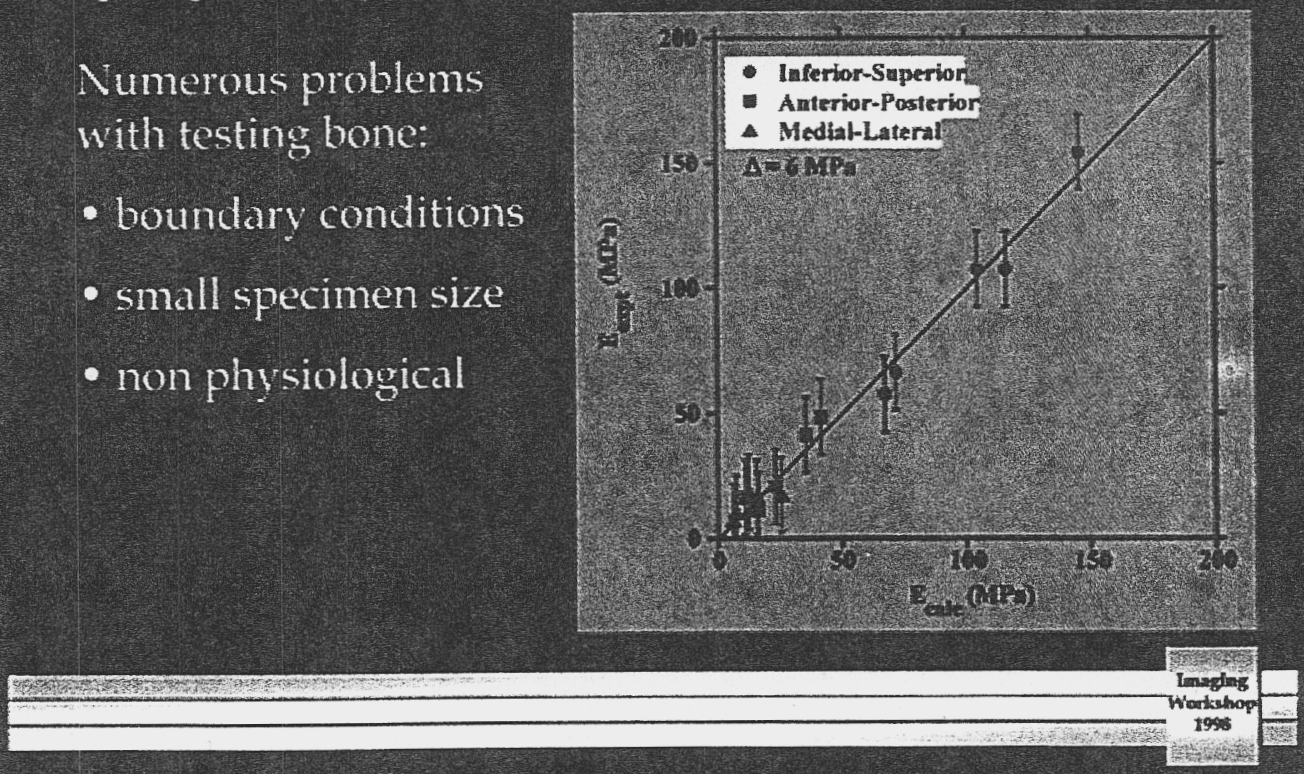
Hypothesis: cyclic administration of estrogen can reverse established OP

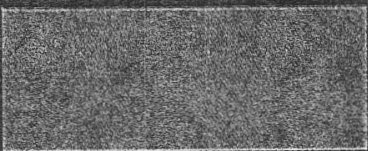

activation

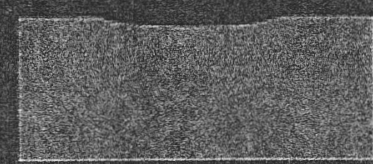

resurption

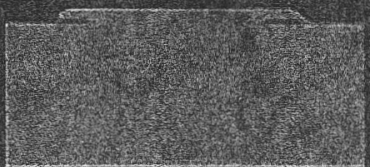

formation

low estrogen levels increase active remodeling sites elevated estrogen stops resporption, stimulates formation

net effect: increased trabecular bone volume

Mass is recovered, but connectivity is not
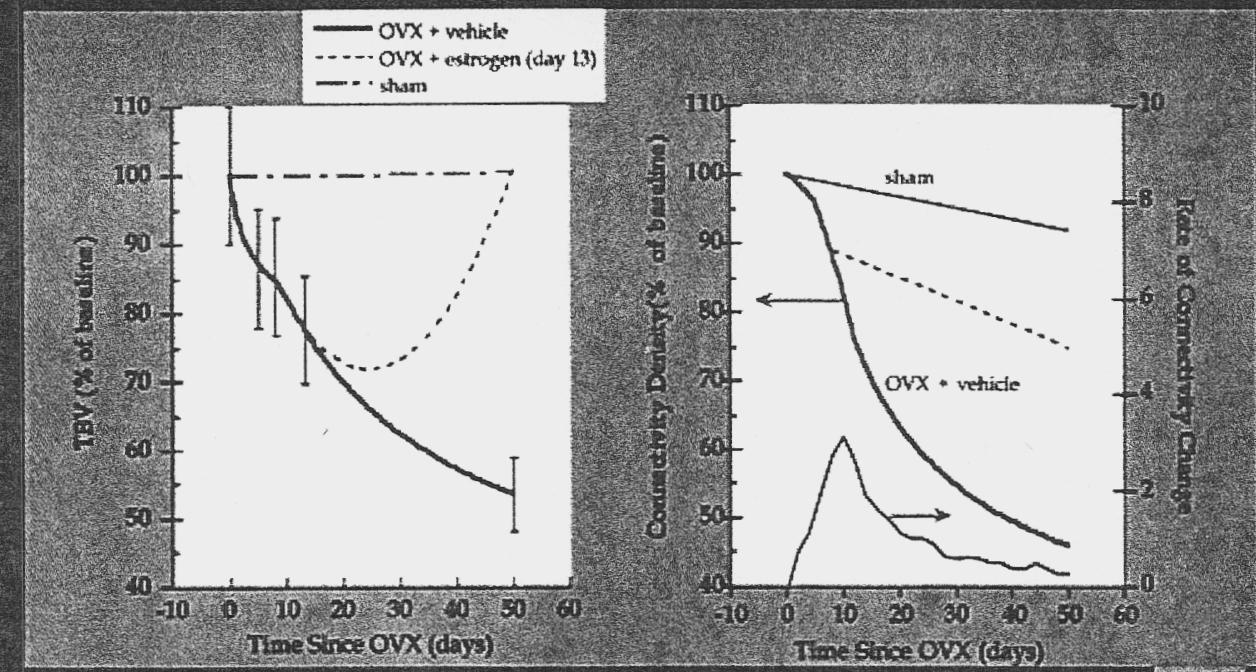

Thestice ovx (ats 
However, strength is recovered, even without restoring connectivity

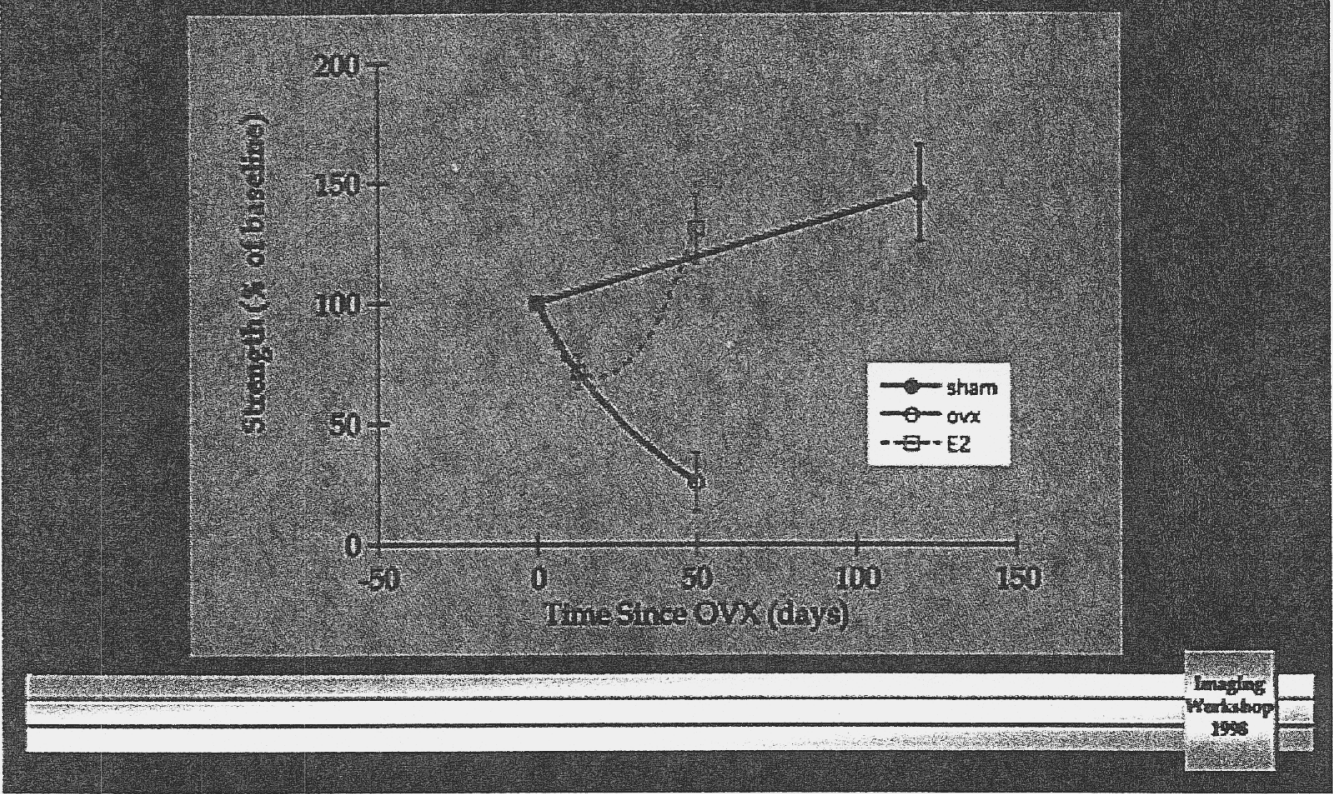

\section{Strength is recovered because thickness increased} beyond baseline

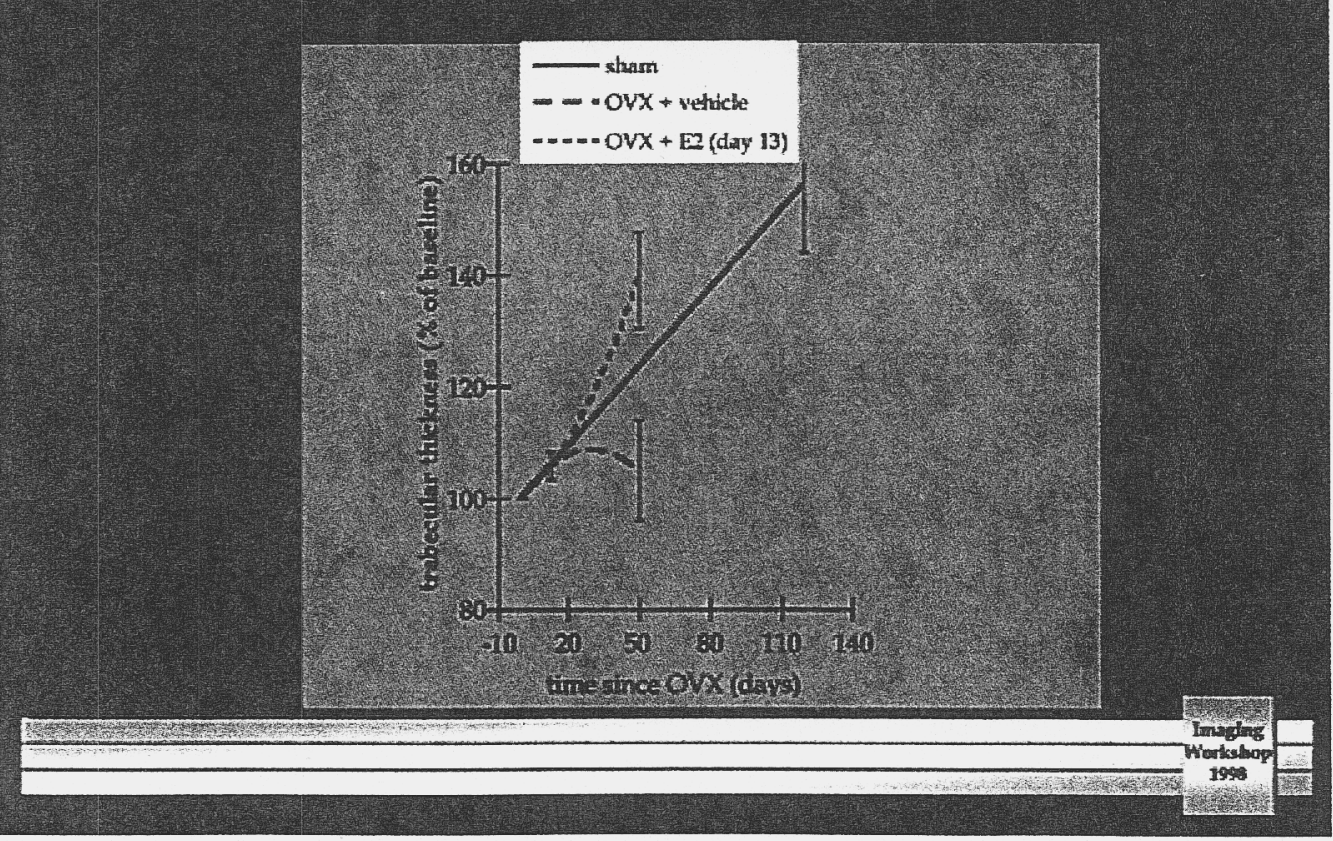


These are important results, suggesting potential monotherapies for treating established osteoporosis

- Estrogen replacement therapy.

- cyclic admistration to build bone mass

- continuous administration to mantain

- Selective estrogan receptor modulators

- cyclic administration lo build bone mass

- contiruous administration to mantain

There are important implications for treating osteoporosis

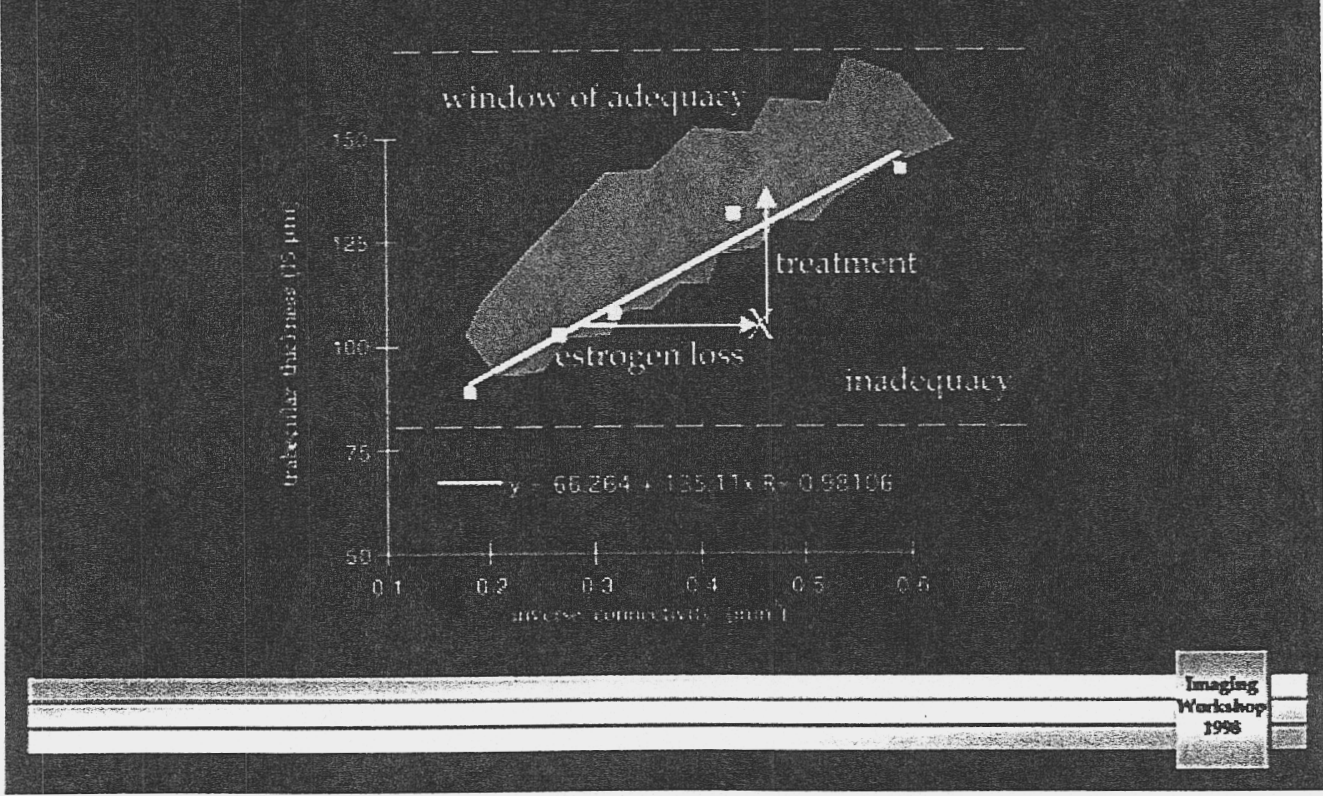




\section{Future work suggests focusing on severe} osteopenia

We are continuing to explore therapies for generating new (de novo) trabecular bone.

basic fibroblast growth factor (Tom Wronski, N I ane) bone morphogenic proteins (Hari Redili) 


\title{
SPECTRAL PARTITIONING OF THE OBJECT FUNCTION IN DIFFRACTION TOMOGRAPHY
}

\author{
By Sean K. Lehman
}

Tomography is a method of producing (reconstructing) two-dimensional slices of the internal structures of a solid object such as the human body, a mechanical part, or the layered earth, by the observation and recording of the transmitted and/or reflected wave energy, either electromagnetic or acoustic, impinging on those structures. We distinguish between projection tomography which is diffractionless, and diffraction tomography. In the former, the irradiating wavelengths are such that the fields pass through the structures without being scattered. The only effect the object under evaluation has on the waves is that of attenuation. In diffraction tomography the fields are both attenuated and scattered.

The scattering mechanism of diffraction tomography is described by the integral form of the Helmholtz equation. The goal of diffraction tomography is to invert this equation in order to reconstruct the object function from the measured scattered fields.

In performing this reconstruction, care must be taken in how the evanescent (non-propagating) fields are used. If not handled correctly, these information carrying fields will be considered noise terms and this will reduce the quality and resolution of the reconstruction.

The object spectrum can be partitioned into resolvable and non-resolvable parts based upon the cutoff between the propagating and evanescent fields. We present near-field data showing how much of the measured scattered field consists of evanescent energy (energy which most current reconstruction techniques consider noise), and how non-resolvable object spectral information is converted to the propagating part of the field spectrum which can be used for reconstructions.

This work was performed under the auspices of the Department of Energy by the Lawrence Livermore National Laboratory under contract W-7405-Eng-48. 


\section{Spectral Partitioning of the Object Function in Diffraction Tomography}

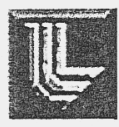

Sean K. Lehman

CASIS Workshop

November 12,1998

\section{Introduction}

- Definition of forward problem

- Projection vs diffraction tomography

- Operator development of diffraction tomography*

- Importance of evanescent information

- Partitioning of terms

- Partitioning of Helmhotz Equation

- Examples

- Conclusion

"Part of Ph.D. thesis, "Superresolution of Buried Objects in Layered Media by Near-Field Electromagnetic Imaging," at U.C. Davis, Dept. of Applied Science 


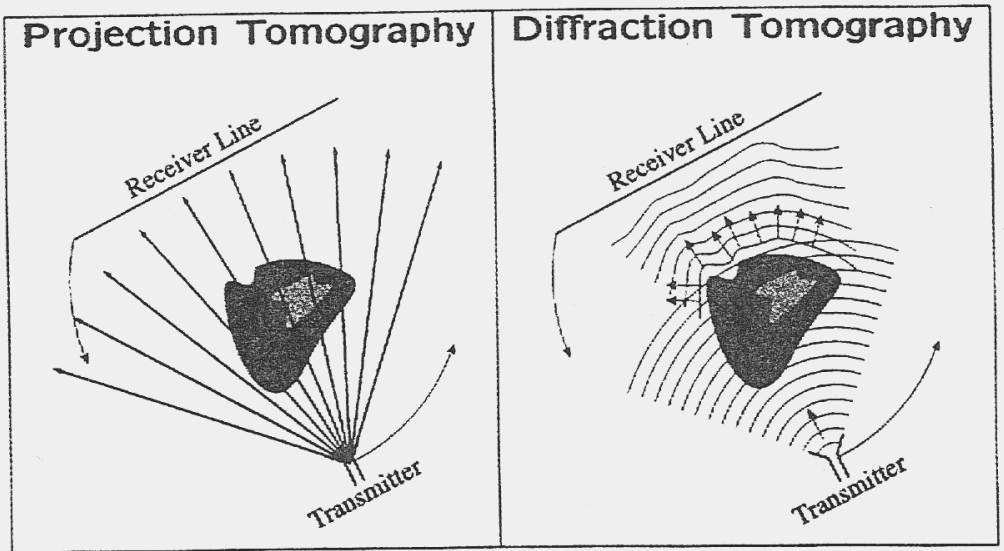

Projection vs Diffraction Tomography - I

Both Projection and Diffraction tomography fall under the category of Computed Tomography

\begin{tabular}{|c|l|}
\hline Projection Tomography & Diffraction tomography \\
\hline - Geometrical optics & - Physical optics \\
- Time delay \& attenuation & - Phase \& amplitude \\
- Diffractionless & - Diffraction \\
- Reconstruction through & - Reconstruction through \\
Backprojection & Backpropagation \\
\hline
\end{tabular}




\section{Projection vs Diffraction Tomography - II}

\section{Governing Equations}

Projection Tomography

$$
P_{\theta}\left(l, z_{0}, \omega\right)=U^{i}(\omega) \int d \mathbf{r} o(\mathbf{r}, \omega) d s(\mathrm{r}, l)
$$

Diffraction tomography (Integral form of Helmholtz equation)

$$
\begin{aligned}
& \mathrm{E}_{s c a t}\left(\mathrm{r}_{\perp}, z_{0}, \omega\right)= \\
& \quad k_{0}^{2}(\omega) \int d \mathrm{r}_{\perp}^{\prime} \int d z^{\prime} \stackrel{\leftrightarrow}{\mathrm{G}}\left(\mathrm{r}_{\perp}-\mathrm{r}_{\perp}^{\prime}, z_{0}-z^{\prime}, \omega\right) \cdot \mathbf{E}\left(\mathrm{r}_{\perp}^{\prime}, z^{\prime}, \omega\right) o\left(\mathrm{r}_{\perp}^{\prime}, z^{\prime}, \omega\right)
\end{aligned}
$$

\section{Operator Development of Forward Problem}

In operator notation, the Helmholtz equation can be expressed as:

$$
\begin{aligned}
\mathbf{E}_{\text {scat }}\left(\mathbf{r}_{\perp}, z_{0}, \omega\right) & =\mathcal{G}\left(\mathbf{r}_{\perp}, z, \omega\right) *\left[\mathrm{E}\left(\mathbf{r}_{\perp}, z, \omega\right) \circ\left(\mathrm{r}_{\perp}, z, \omega\right)\right] \\
\mathbf{E}_{\text {scat }} & =\mathcal{G} *[\mathrm{E} o]
\end{aligned}
$$

Computing a planar Fourier transform w.r.t. $\mathbf{r}_{\perp}$ :

$$
\tilde{\mathrm{E}}_{s c a t}=\tilde{\mathcal{G}}[\tilde{\mathrm{E}} * \tilde{O}]
$$




\section{Partitioning of the Field Spectra}

The spectrum of the wave operator, $\mathcal{G}$, and the spectra of the fields, $\tilde{\mathbb{E}}_{s c a t}$ and $\tilde{\mathbb{E}}$, are naturally partitioned into propagating and evanescent parts depending upon the wavenumber cutoff:

$$
k_{0}(\omega)=\frac{\omega}{c}
$$

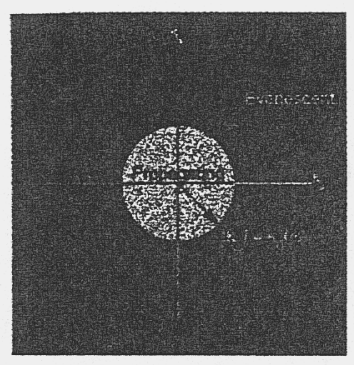

- Carries higher object spatial frequency information:

- Object spectrum is "smeared" across the propagating and evanescent regions via the convolution, even in the far field:

- Reconstruction schemes that take this into account will achieve superresolution and feature enhancements. 


\section{Partitioning of the Field Spectra - II}

We express the wave operator and field spectra terms as:

$$
\begin{aligned}
\tilde{\mathcal{G}} & =\tilde{\mathcal{G}}_{<}+\tilde{\mathcal{G}}> \\
\tilde{\mathrm{E}}_{s c a t} & =\tilde{\mathrm{E}}_{s c a t<}+\tilde{\mathrm{E}}_{s c a t>} \\
\tilde{\mathbf{E}} & =\tilde{\mathbf{E}}_{<}+\tilde{\mathrm{E}}_{>}
\end{aligned}
$$

where

$$
\begin{aligned}
& \tilde{F}_{<}\left(\mathbf{k}_{\perp}, z, \omega\right) \equiv\left\{\tilde{F}\left(\mathbf{k}_{\perp}, z, \omega\right)|| \mathbf{k}_{\perp} \mid \leq k_{0}(\omega)\right\} \\
& \tilde{F}_{>}\left(\mathbf{k}_{\perp}, z, \omega\right) \equiv\left\{\tilde{F}\left(\mathbf{k}_{\perp}, z, \omega\right)|| \mathbf{k}_{\perp} \mid>k_{0}(\omega)\right\}
\end{aligned}
$$

Note: For any field term $\lim _{z \rightarrow \infty} \tilde{F}_{>}=0$

\section{Partitioning of the Object Spectrum}

The object, however, not being a field, does not have propagating and evanescent parts. It does have resolvable and non-resolvable parts depending upon the wavenumber cutoff of the field measurement system.

Thus we write:

$$
\tilde{O}=\underbrace{\tilde{O}_{s}}_{\text {resolvable }}+\underbrace{\tilde{O}_{\geqslant}}_{\text {non-resolvable }}
$$




\section{Partitioning of the Helmholz Equation}

Combining the partitioned terms, the Helmholtz equation,

$$
\tilde{\mathbf{E}}_{s c a t}=\tilde{\mathcal{G}}[\tilde{\mathbf{E}} * \tilde{O}]
$$

becomes:

$$
\tilde{\mathrm{E}}_{\text {scat }}+\tilde{\mathrm{E}}_{\text {scat }}=\left(\tilde{\mathcal{G}}_{<}+\tilde{\mathcal{G}}_{>}\right)\left[\left(\tilde{\mathrm{E}}_{<}+\tilde{\mathrm{E}}_{>}\right) *\left(\tilde{O}_{<}+\tilde{O}_{>}\right)\right]
$$

Further partition this into far field and near field terms using the

$$
\lim _{z \rightarrow \infty} \widetilde{F}_{>}=0
$$

property of the field terms.

\section{Partitioning of the Helmholz Equation - Far Field}

$$
\begin{aligned}
& \text { Far Field }(z \rightarrow \infty) \\
& \tilde{\mathrm{E}}_{\text {scat }}=\tilde{\mathcal{G}}_{<}\left[\tilde{\mathbf{E}}_{<} *\left(\tilde{O}_{<}+\tilde{O}_{>}\right)\right] \\
& =\underbrace{\tilde{\mathcal{G}}_{<}\left[\tilde{\mathbf{E}}_{<} * \tilde{O}_{<}\right]}_{\text {current techniques }}+\underbrace{\tilde{\mathcal{G}}<\left[\tilde{\mathbf{E}}_{<} * \tilde{O}_{>}\right]}_{\text {currently treated as noise }} \\
& \tilde{\mathrm{E}}_{\text {scat }}>=0
\end{aligned}
$$




\section{Partitioning of the Helmholz Equation - Near Field}

$$
\begin{aligned}
& \tilde{\mathrm{E}}_{s c a t<}=\tilde{\mathcal{G}}_{<}\left[\tilde{\mathrm{E}}_{<} *\left(\tilde{O}_{<}+\tilde{O}_{>}\right)\right]+\tilde{\mathcal{G}}_{<}\left[\tilde{\mathrm{E}}_{>} *\left(\tilde{O}_{<}+\tilde{O}_{>}\right)\right] \\
& \tilde{\mathrm{E}}_{\text {scat }}=\underbrace{\tilde{\mathcal{G}}_{>}\left[\tilde{\mathbf{E}}_{<*}\left(\tilde{O}_{<}+\tilde{O}_{>}\right)\right]+\tilde{\mathcal{G}}_{>}\left[\tilde{\mathbf{E}}_{>} *\left(\tilde{O}_{<}+\tilde{O}_{>}\right)\right]}_{\text {currently ignored }}
\end{aligned}
$$

\section{Partitioning of the Helmholz Equation - Comments}

- There are two physical effects occurring:

- A "smearing" of object spectral information due to the convolution with the field;

- A conversion between propagating and evanescent information via the wave operator.

- Reconstruction algorithms which take these effects into to account will result in imaging enhancements and superresolution. 


\section{Partitioning of the Helmholz Equation - Far Field}
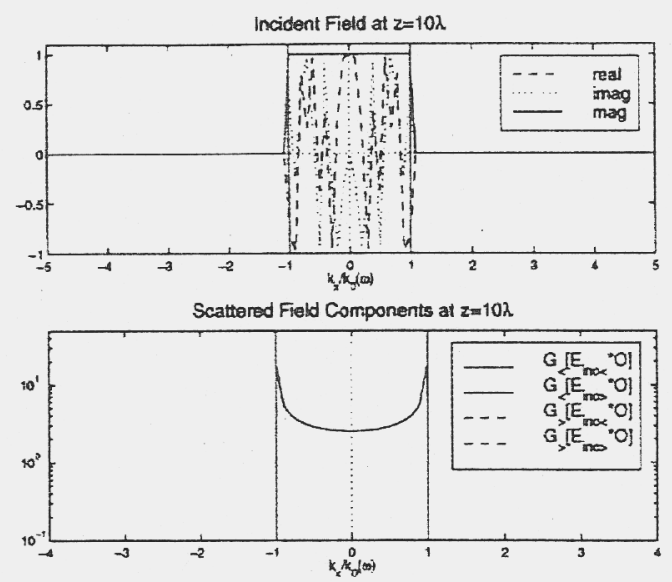

\section{Partitioning of the Helmholz Equation - Near Field}
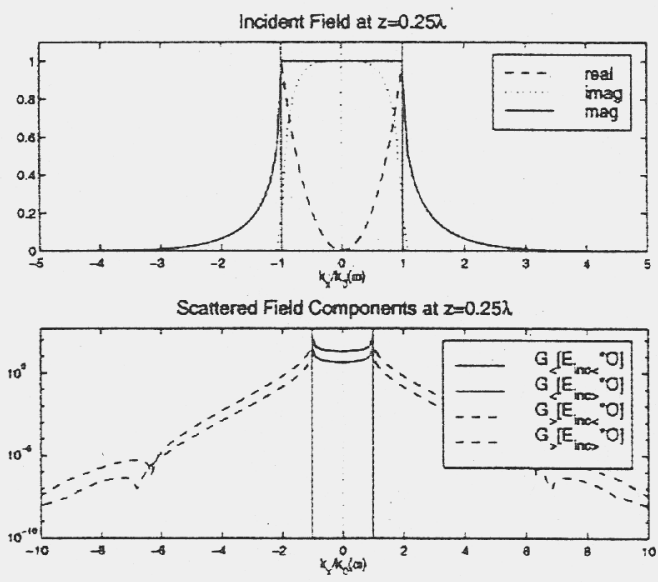


\section{Partitioning of the Helmholz Equation - Far Field Details}
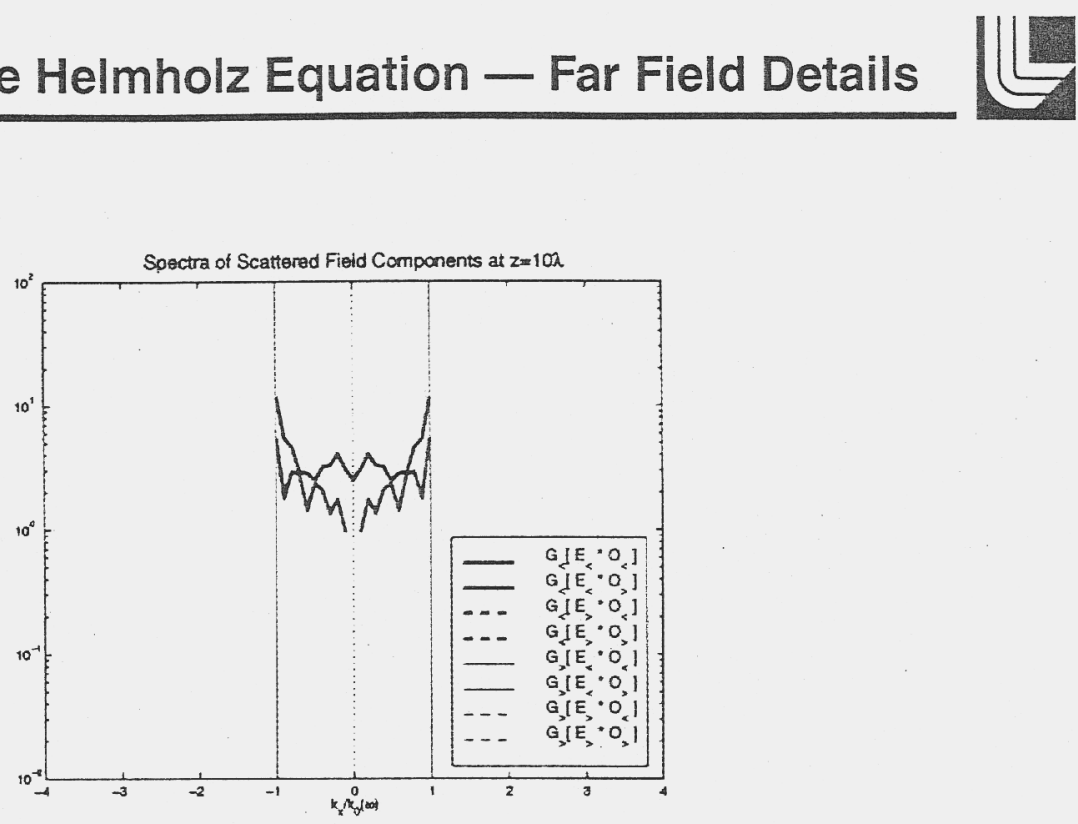

\section{Partitioning of the Helmholz Equation — Near Field Details}

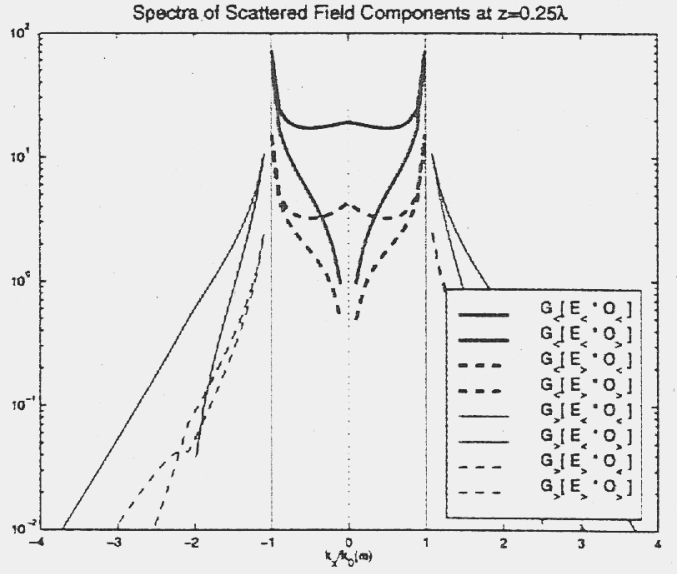




\section{FDTD Mine Simulation}
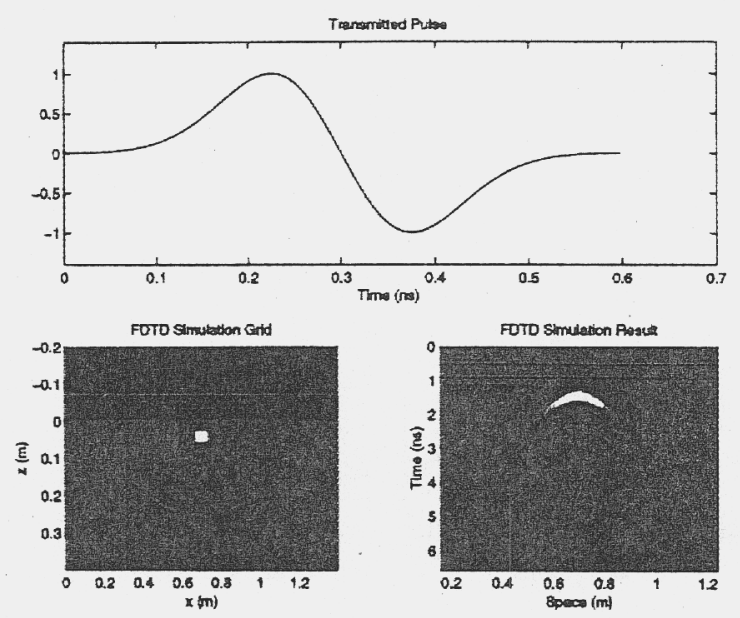

\section{FDTD Mine Simulation}
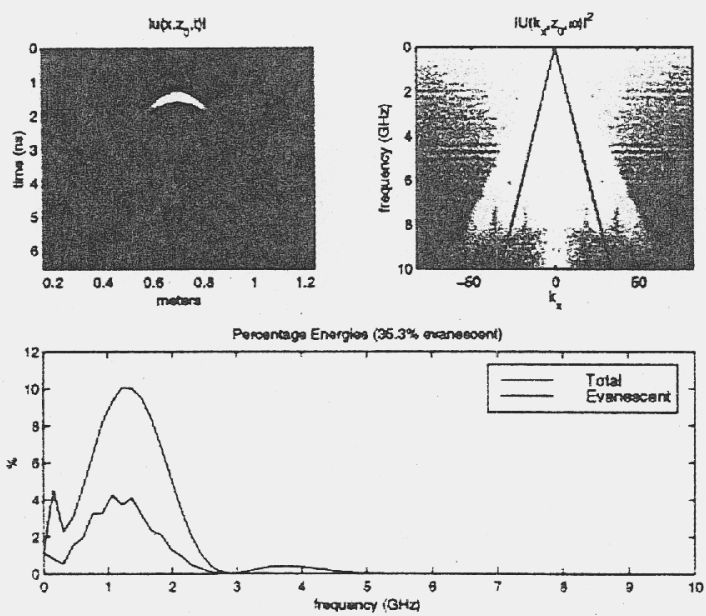


\section{Real Mine Data}
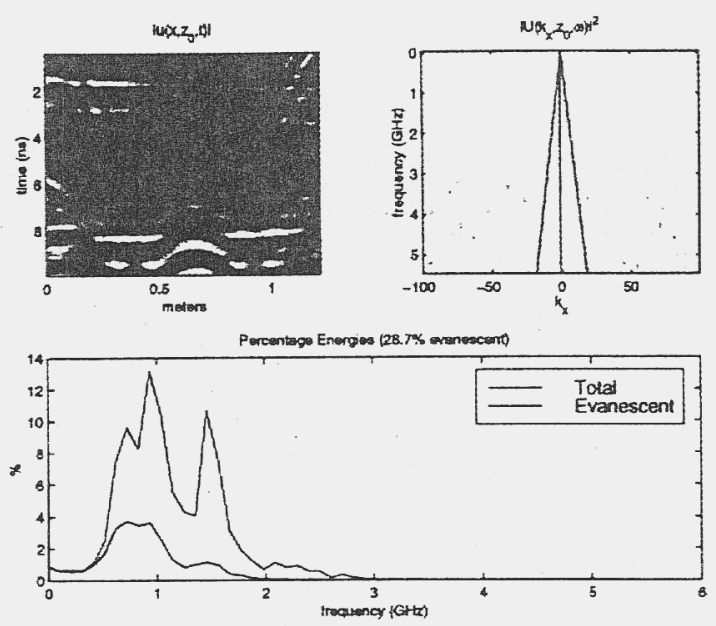

\section{Conclusions}

- Mine simulations show within the near field up to $30 \%$ of the measured energy is evanescent;

- The forward scattering operator shows there is a "smearing" of object spectral information across the measurement system's propagating and evanescent regions;

- Reconstruction schemes must take this into account. If not, object spectral information will be treated as noise and there will be a loss of detail and resolution. 
Radar-Acoustic

Applications 


\title{
Introduction to Low Power Radars for Acoustic Applications: Speech and Object Characterization
}

\author{
John F. Holzrichter
}

The use of radar (and related propagating wave Electro Magnetic wave devices) for acoustic research and applications has not been well developed. LLNL's suite of low power radars, e.g., the MIRs, are making possible many applications where low power, portability, and low cost are dominant factors. They make possible accurate amplitude measurements, ranging from centimeters to micrometers, of moving mechanical elements. The measurements can be performed at meter distances from the antenna, and through surrounding dielectric media such as skin and water. Several such examples relating to human speech recognition and coding (Burnett), to speaker verification (Gable), to pitch determination $(\mathrm{Ng})$, and to structural vibration detection and identification (Roberts) will be given in the accompany talks.

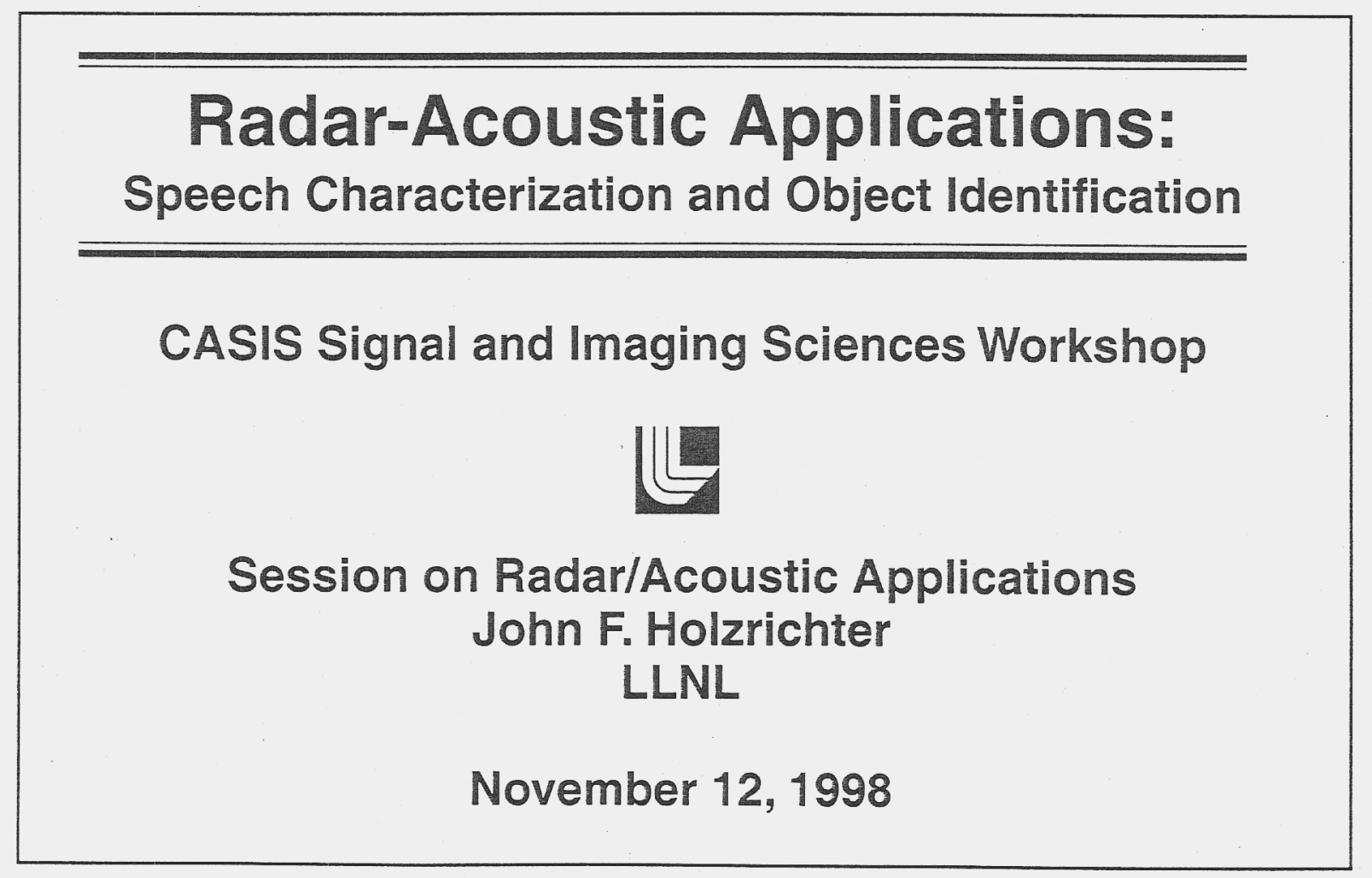




\section{Two applications of EM Sensors and Acoustics are human speech and object characterization}

Speaker Recognition and Verification

Anti-Personnel Mine detection
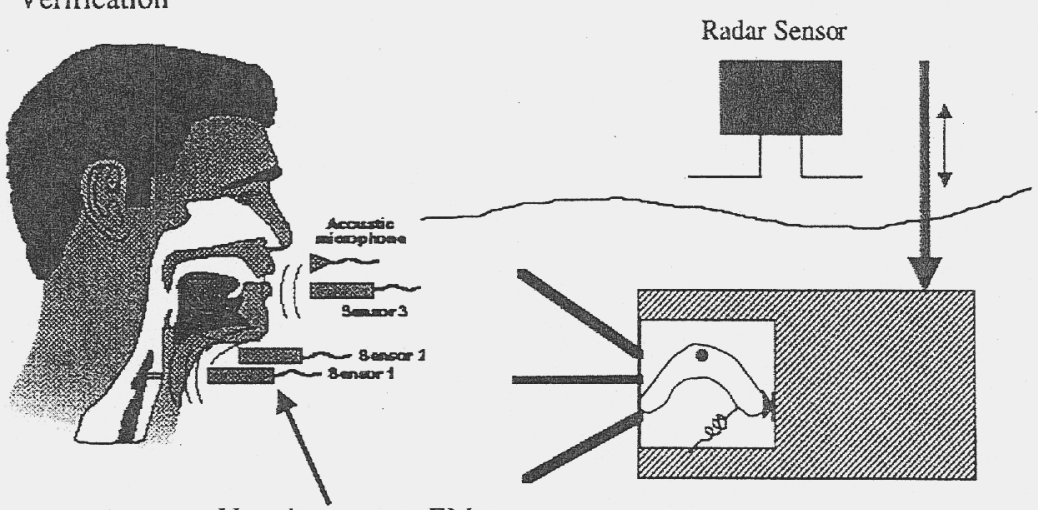

Very low power EM

radar sensors

\section{The Radar/Acoustic Session will include the following topics (see agenda for details)}

- The use of micro-power homodyne radar to measure in real-time, an excitation function of human speech

- Greg Burnett et al, LLNL, UCD/DAS

- Using excitation function information to obtain instant "pitch" of voiced speech and to enable "pole/zero" (e.g., ARMA) techniques to be used to estimate transfer functions

- Lawrence Ng et al, LLNL/EE

- Using human vocal tract articulator information, obtained with micro-power EM radar sensors, to verify the identity of a speaker

- Todd Gable et al, LLNL, UCD/DAS

- Using homodyne radar to measure the acoustic response of unknown objects (e.g., antipersonnel land mines) for purposes of identification

- Roger Perry/Randy Roberts et al, LLNL/EE 


\section{We would like to thank many LLNL, UC and other researchers for their assistance and support}

- Signal Processing:

- Drs. Greg Clark, Farid Dowla and Jim Candy

- Dr. Mark Duchaineau, LLNL/Comp. Dept.

- Micro-power EM Sensors:

- Dr. Stephen Azevedo, Tom Rosenbury, Doug Poland, Bob Stever, Greg Dallum, Pat Welsh - MIR Group

- Don Mullenhoff - NAI

- UC System Collaborations:

- Dr. Rebecca Leonard, UCD Medical Center, Sacramento

- Profs. R. Freeman and R. Vermuri - DAS

- Prof. N. Luhmann, C. Domier and Mr. C. Liang - UCD/EE

- Dr. Wayne Lea (consultant)

\section{The interpretation of EM Sensor information depends upon its use in near, intermediate or far field modes}
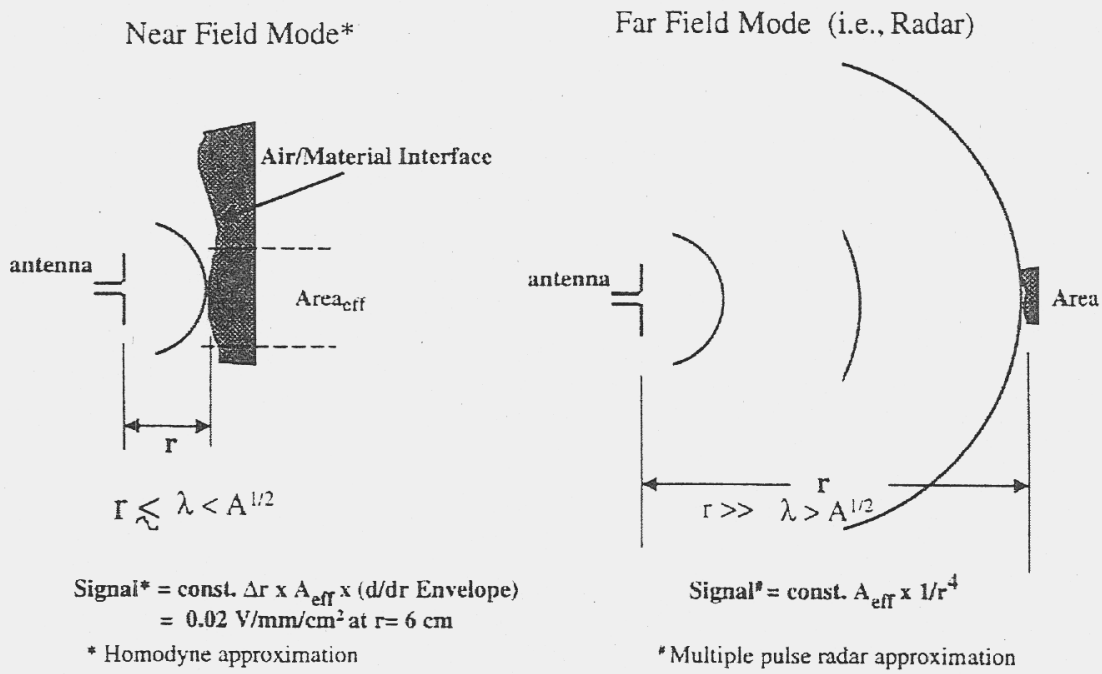
The human vocal tract has excitation source(s), E, followed by a sequence of tubes and resonators that can be described as $\mathrm{H}$, using linear equations

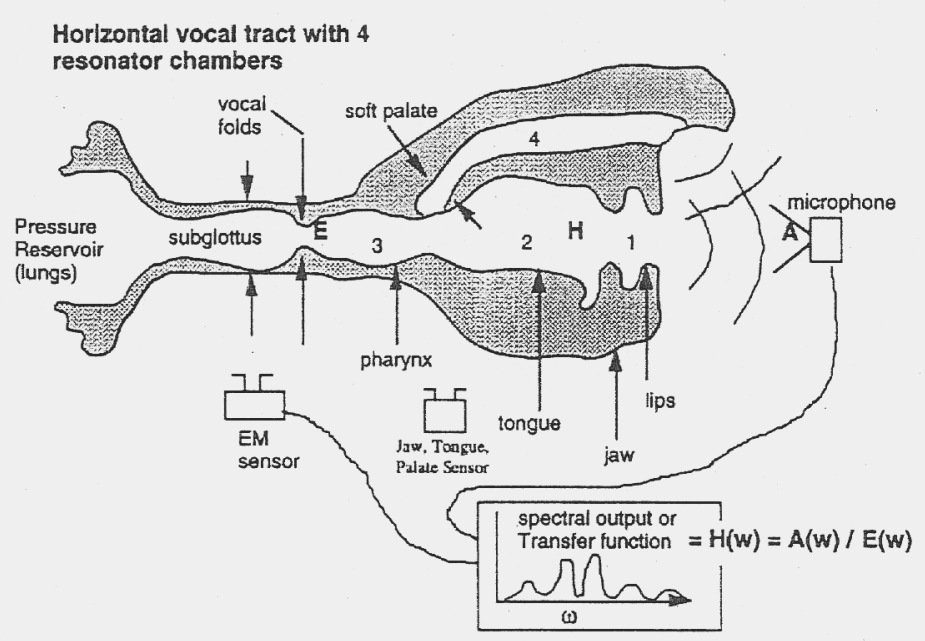

JFH:11/12/98:pt.6tlb

\section{One or more miniature micropower EM sensors (reducible to one or two $\$ 2$ chips) can be used}

This image shows the present MIR sensor, which costs about $\$ 15$ dollars in parts and about $\$ 200$ to replicate (margin costs). Most sensors for speech are used in the homodyne "filed disturbance" mode.

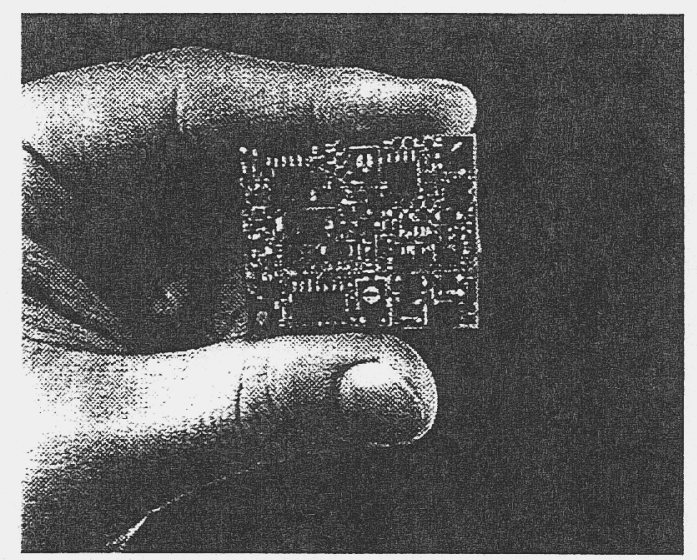




\section{Audio and EM sensors measure several speech articulator motions simultaneously, e.g. "print"}

\section{Why Radar and Acoustics?}

- Probe structural motions under unusual conditions

- Micron to centimeter amplitudes

- At a distance - centimeters to kilometers (i.e., non-contact)

- Extended area responses

- High data rates

- New EM sensors may make possible new applications: e.g., speech recognition, speaker verification, object identification, etc.

- Low cost (in principle)

- Multiple modalities - impulse radar, field disturbance, homodyne, etc.

- Human safe 


\title{
MIR Measurements of Human Glottal Activity and An Estimation of a Voiced Speech Excitation Function \\ Gregory C. Burnett \\ Lawrence Livermore National Laboratory
}

Special purpose, homodyne-mode, micro-power EM sensors, called GEMS, enable the measurement of tissue motions associated with the opening and closing of the human glottis (i.e., the vocal fold opening). These are directly linked to the modulation of the vocal tract air flow as the vocal folds open and close. We show that the "ballooning" of subglottal tracheal tissue, as pressure increases and decreases, is the predominant contributor to the GEMS signal. These relatively simple measurements lead to accurate speech pitch determination, quality of vocal fold contact, individual specific tissue motion patterns, and a voiced speech excitation function.

\section{Defining an Excitation Function of the Human Vocal Tract Using Glottal Electromagnetic Micropower Sensors (GEMS)}

\section{CASIS Signal and Imaging Sciences Workshop November 12, 1998}

\author{
Gregory C. Burnett
} UC Davis/ LLNL DSED 


\section{What is the GEMS?}

- Emits pulses of very low power (less that one-thousandth that of a cell phone) and short duration

- Center frequency is around $2 \mathrm{GHz}$, prf $2 \mathrm{MHz}$

- Uses homodyne detection method to sense the reflectivity of objects around it

- The change in that reflectivity is amplified to produce a signal approximating the velocity of the objects

- In essence an excellent motion and vibration detector

We want to determine what the GEMS is sensing and how that relates to the excitation function

Questions to answer:

- How does the GEMS sense motion?

- What is the physiological basis of the GEMS return?

- How can we relate the GEMS return to subglottal pressure? 


\section{Raw GEMS signal coming from neck}
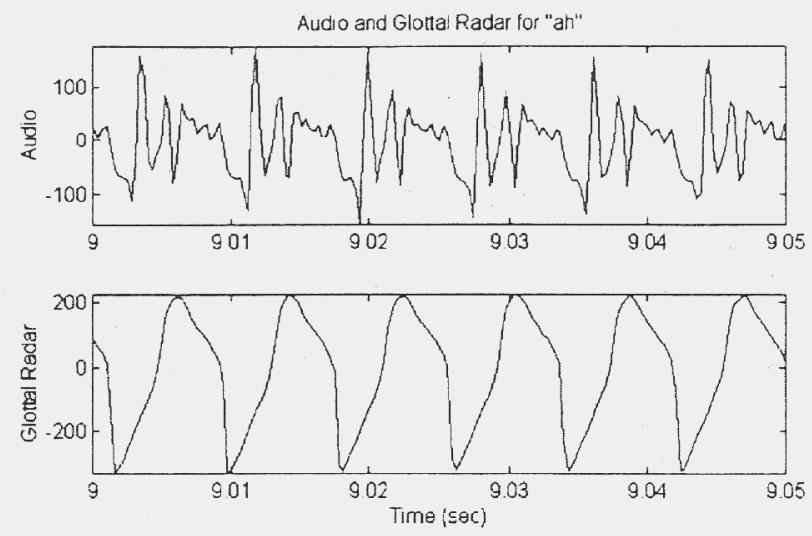

\section{The GEMS has built-in analog filters in order to} detect the frequencies of interest $(70-7000 \mathrm{~Hz})$

- The filter response was measured by injecting sinusoidal signals into the receive antenna of the radar

- An anticasual filter in conjunction with allpass filters was used to compensate for the phase and magnitude distortion

- As the GEMS measures velocity, position may be derived by approximating the integral with a cumulative sum 

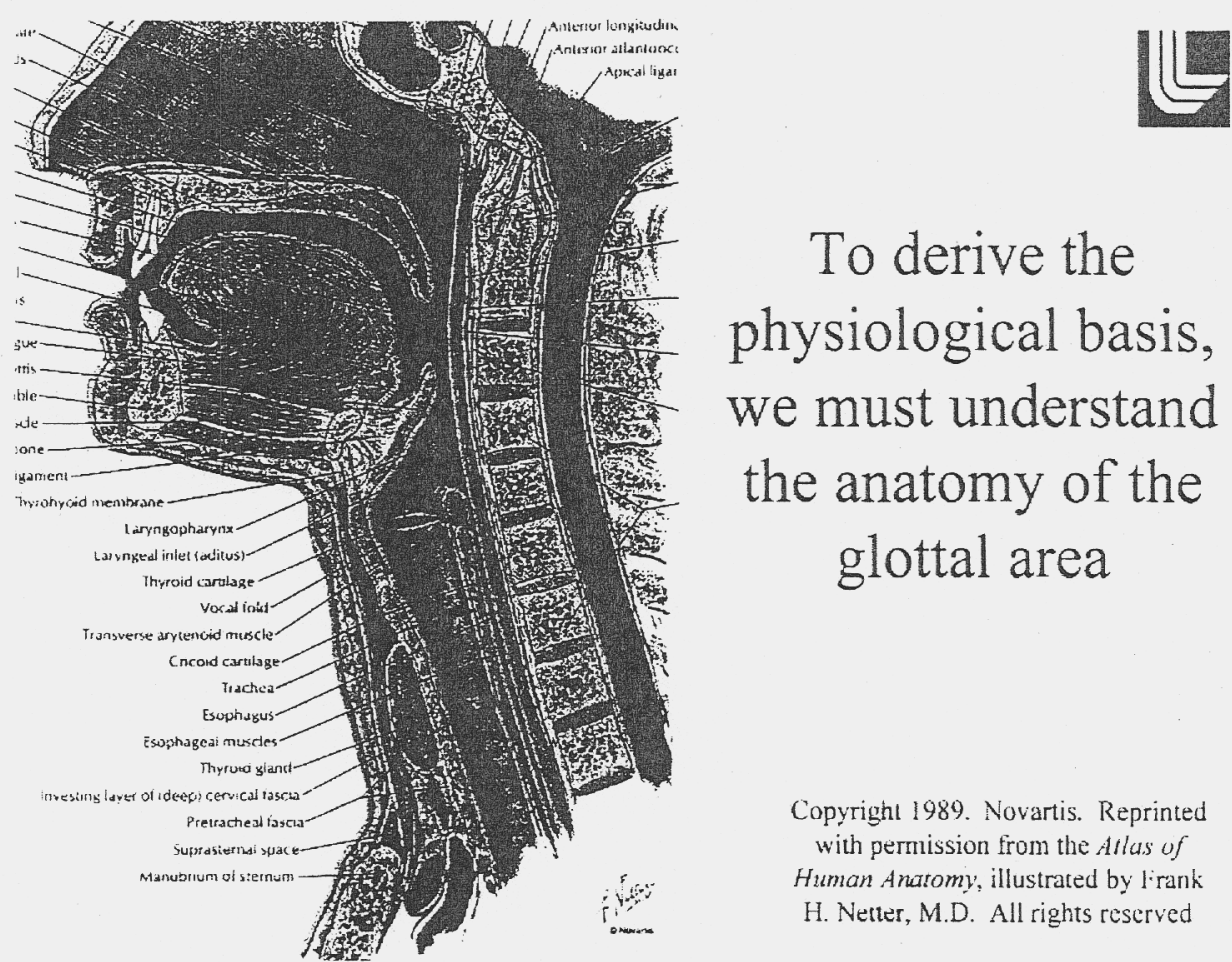

To derive the physiological basis, we must understand the anatomy of the glottal area

Copyright 1989. Novartis. Reprinted with permission from the Allas of Human Anatomy, illustrated by lirank

H. Netter, M.D. All rights reserved

\section{A cross-sectional view of the trachea suggests the posterior wall would be a better reflector}
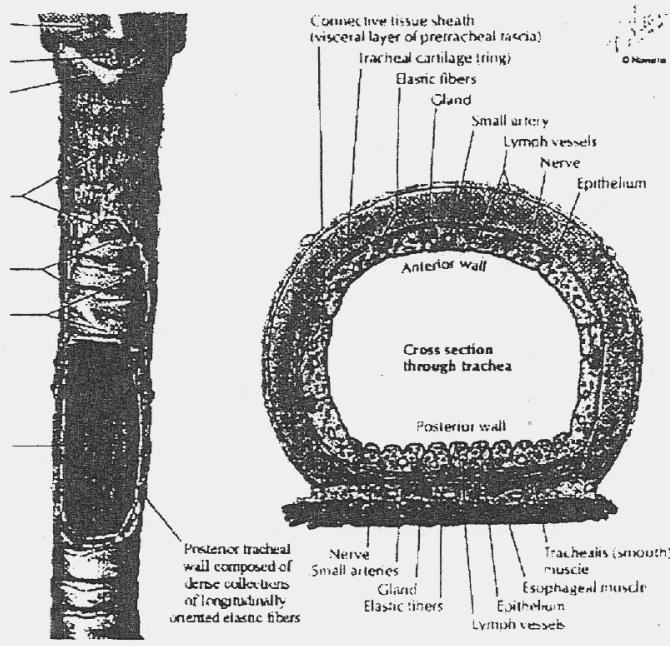

Copyright 1989. Novartis. Reprinted with permission from the Atlas of Human Anatomy, illustrated by Frank 11. Netter, M.I). All rights reserved 
Electromagnetic simulations using Jeff Kallman's TSARLITE indicate the trachea reflects far more energy than the max-open folds

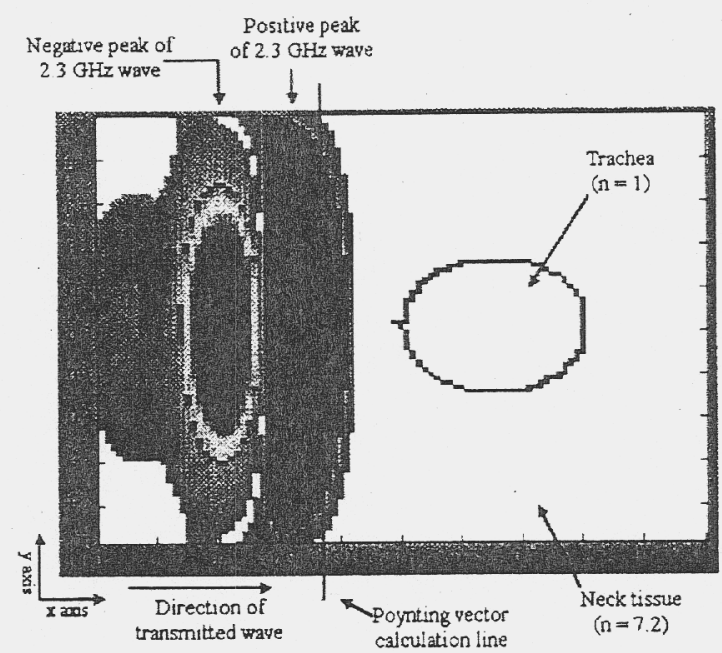

Trachea had a reflectivity of $15.2 \%$, more than $18 \mathrm{x}$ the max-open fold reflectivity of $0.8 \%$ (close to the noise level of the simulation)

High speed (1000 - $3000 \mathrm{fps}$ ) video experiments indicated fold motion did not correlate well with GEMS signal

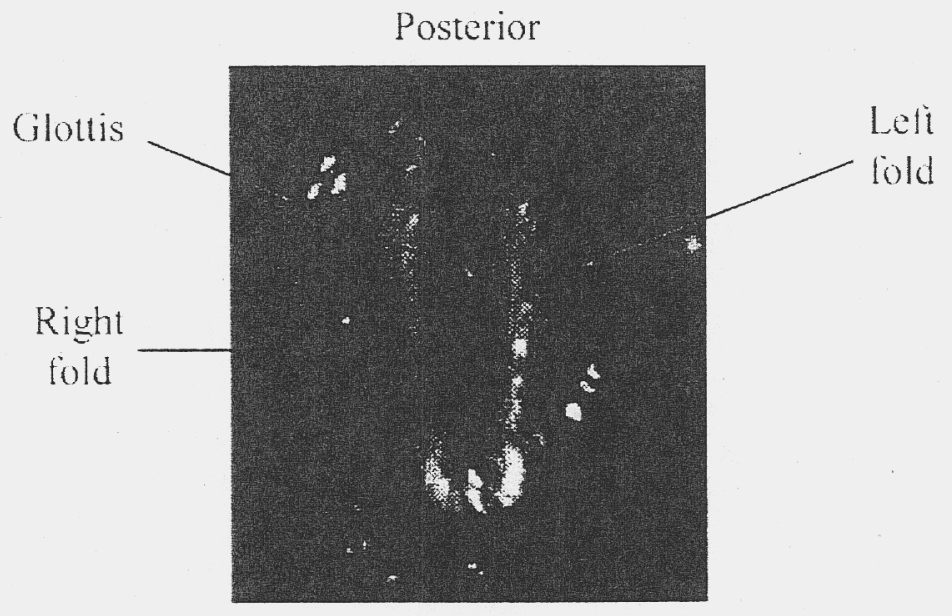

Anterior 
Quantifying the response of the GEMS to physical motion of a signal reflector

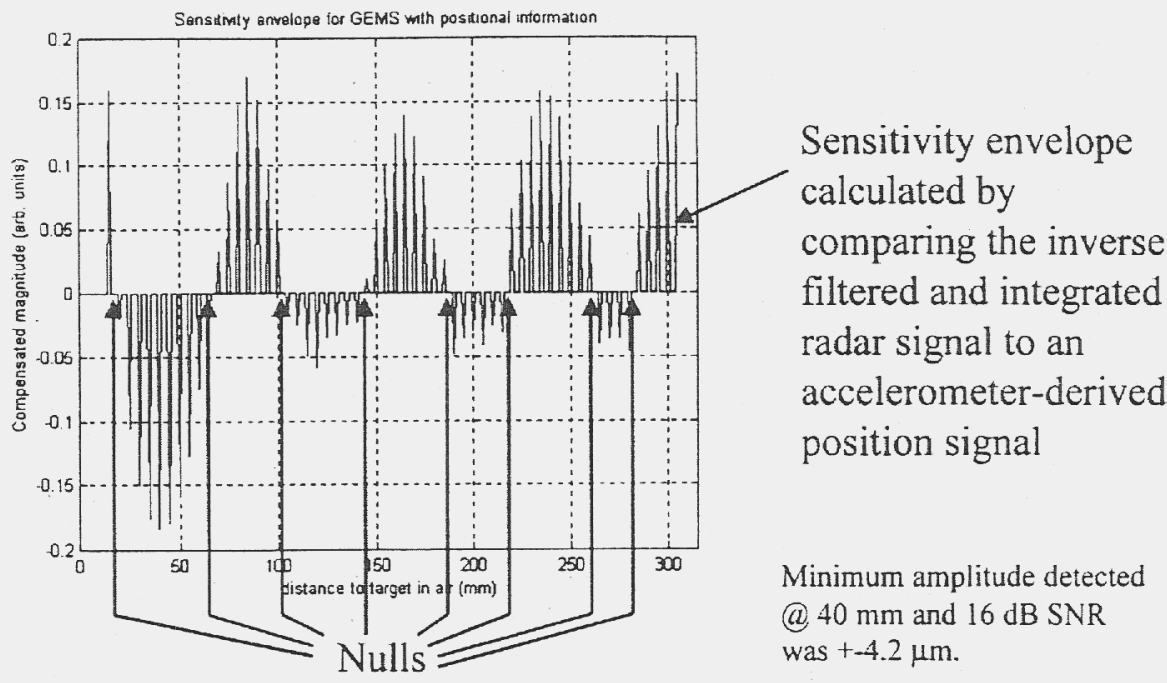

Nulls in GEM signal strength observed as GEMS is moved away from the glottis indicate the posterior wall is the basis for the reflection

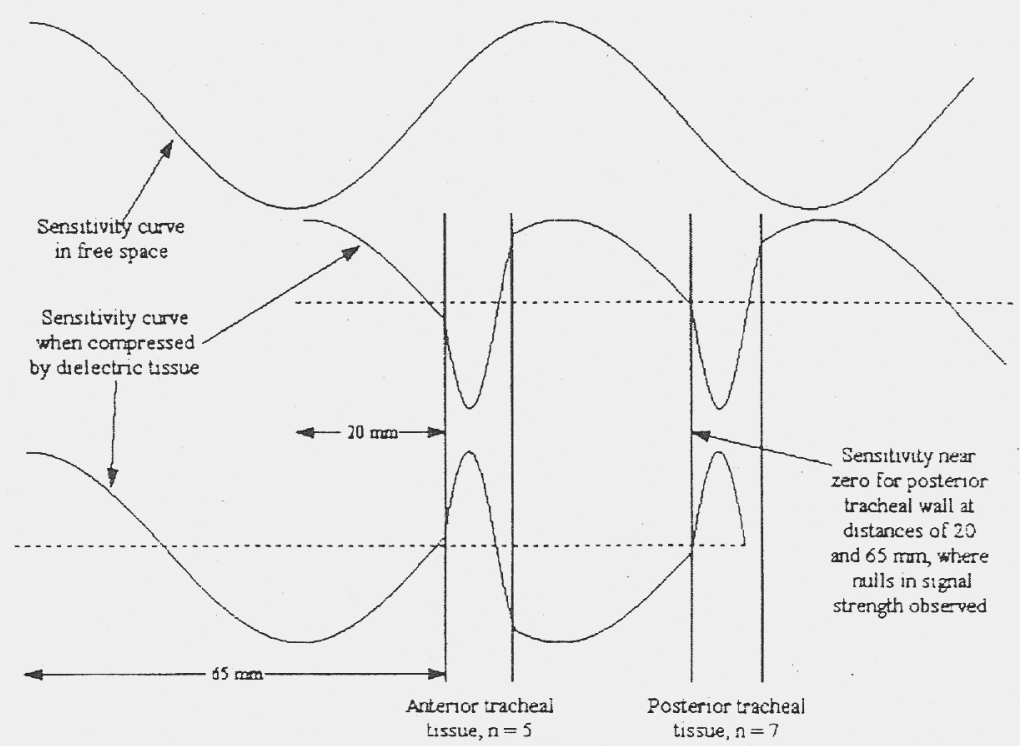


Conclusions about physiological basis of the GEMS return

- The object responsible for the detected vibration is the posterior tracheal wall

- Furthermore, we are detecting subglottal vibrations of the wall, as a phase change is observed when the GEMS is moved from its usual position to an area above the glottis

- Now, we must relate this tracheal motion to the driving subglottal pressure

\section{A lumped-element circuit model is used to quantify} the trachea's response to changing air pressure

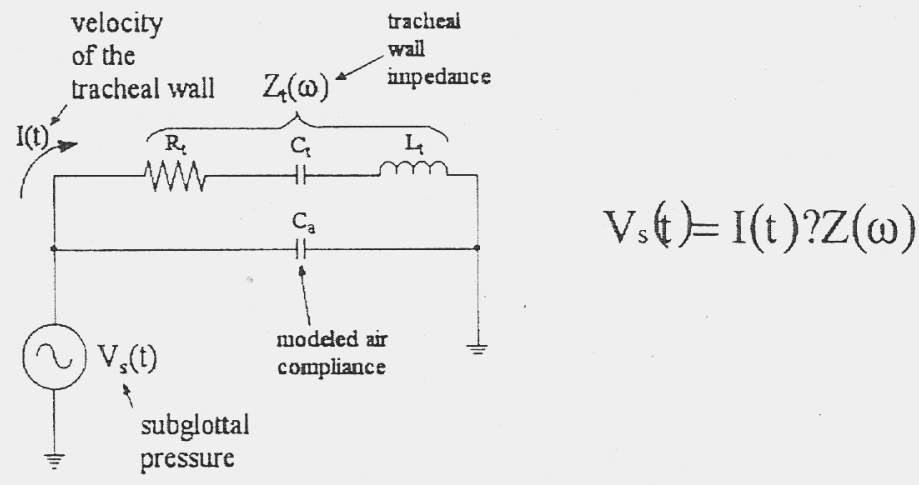


Thus, the inverse filtered, integrated radar signal gives us an approximation to the subglottal pressure (and thus the excitation function) of the vocal tract

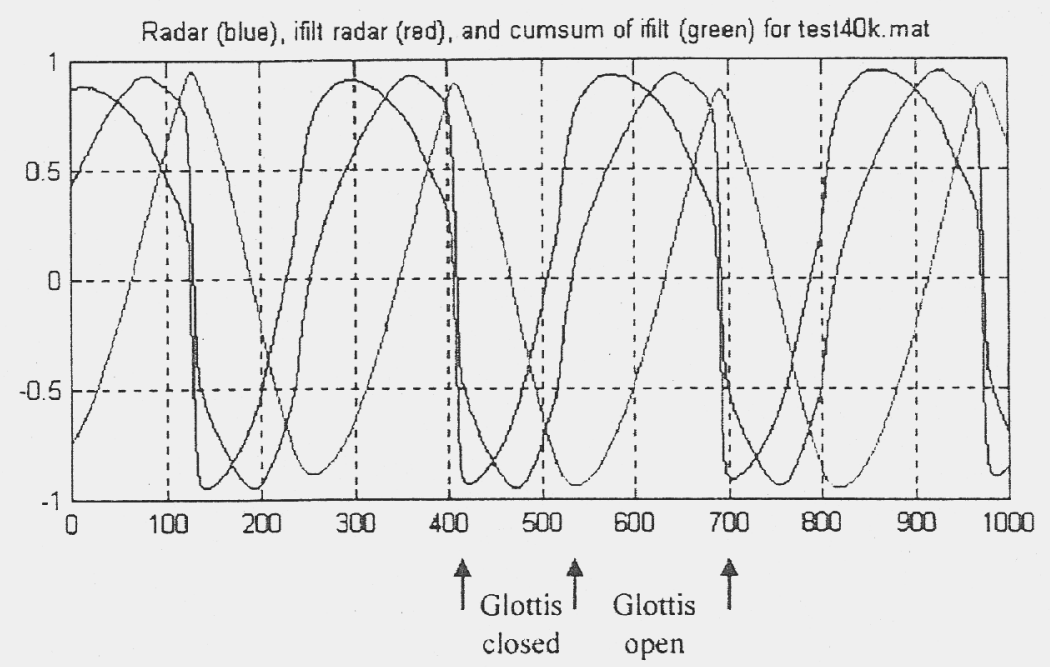

\section{Many thanks to ...}

- John Holzrichter, Larry Ng and Todd Gable

- Roger Perry, Randy Roberts and Tom Woehrle

- Jeff Kallman and Melinda Bass

- Rebecca Leonard and Kathy Krandall of the UC Davis Medical Center

- Jong An 


\title{
Accurate EM/Acoustic Pitch Estimation
}

\author{
Lawrence C. $\mathrm{Ng}$
}

Lawrence Livermore National Laboratory

This talk will discuss a new time domain approach in real-time estimation of pitch information for human speech using the small Glottal Electromagnetic Micropower Sensors (GEMS) - a Micropower Impulse Radar (MIR) developed at LLNL specifically for speech applications. GEMS operate in the microwave regime of the EM spectrum at a radiated average power of less than $1 \mathrm{~mW}$, and it uses a field-disturbance mode of reception in which signals are obtained only from moving tissue/air interfaces. The GEMS algorithm and its performance will be discussed in comparison to conventional pitch estimation algorithms. In particular, this talk will show that GEMS algorithm outperforms existing algorithms by a factor of 100 in computational speed and a factor of 20 in accuracy. This paper will also show the inherent advantage that accurate pitch estimation leads to a new improved method of pitch synchronized speech processing.

\section{Accurate EM/Acoustic Pitch Estimation}

November 12, 1998

Signal and Imaging Sciences Workshop

Lawrence Livermore National Laboratory

Presented by:

Lawrence C. Ng

DSED/EE

Engineering 
Glottal EM Sensors (GEMS) Tissue Measurements Show Strong Correlation with Vocal-Fold Electroglottalgraphy (EGG) Signal
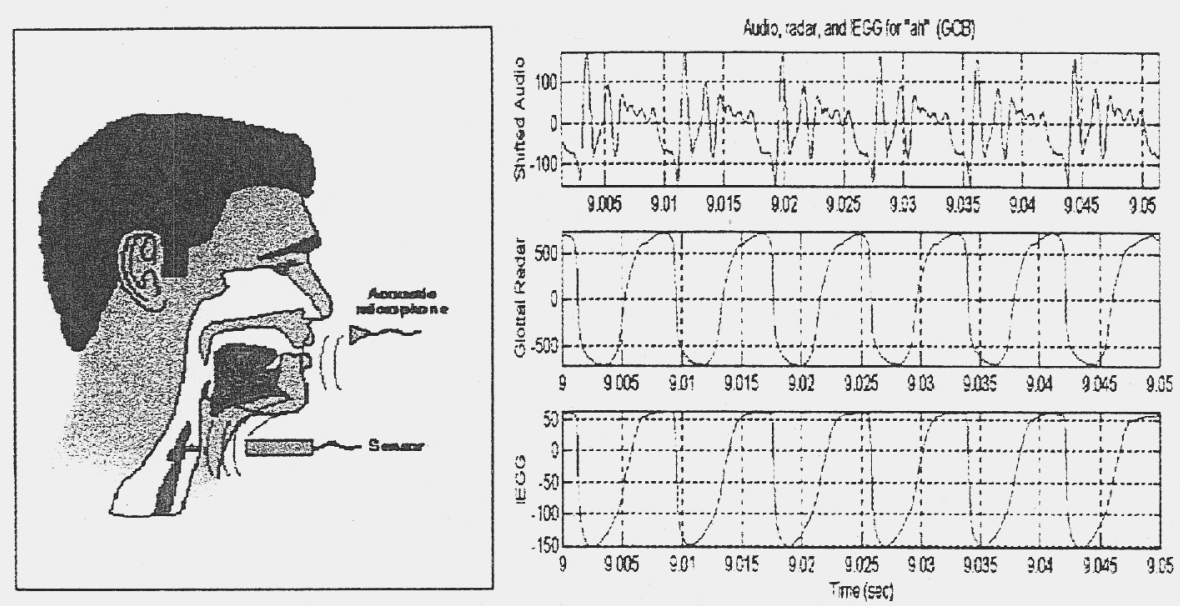

\section{Traditional Pitch Estimation} Approaches Using Acoustic Signal

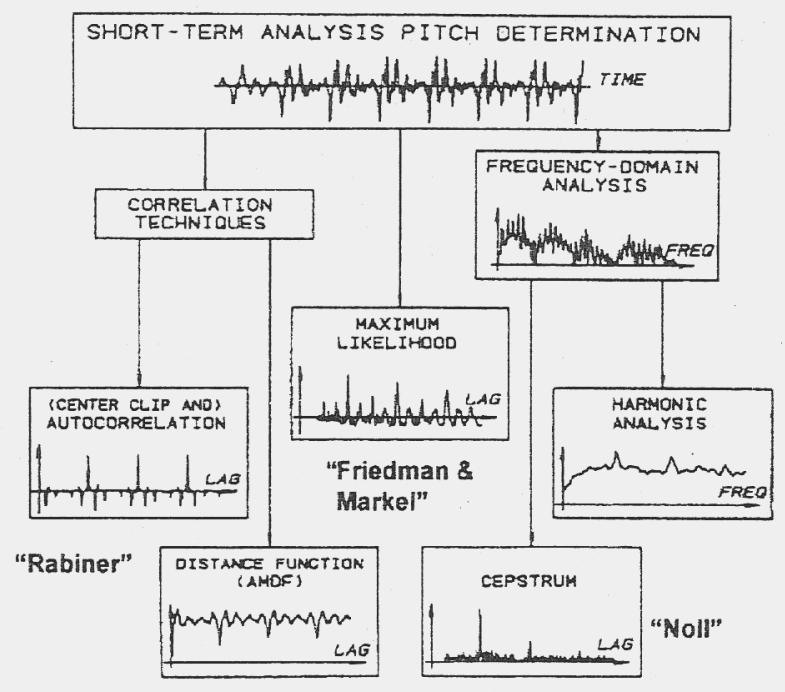




\section{Traditional Error Rate of Pitch Estimation is 1\% (for the Best Case) and Degrades Rapidly in Noise}

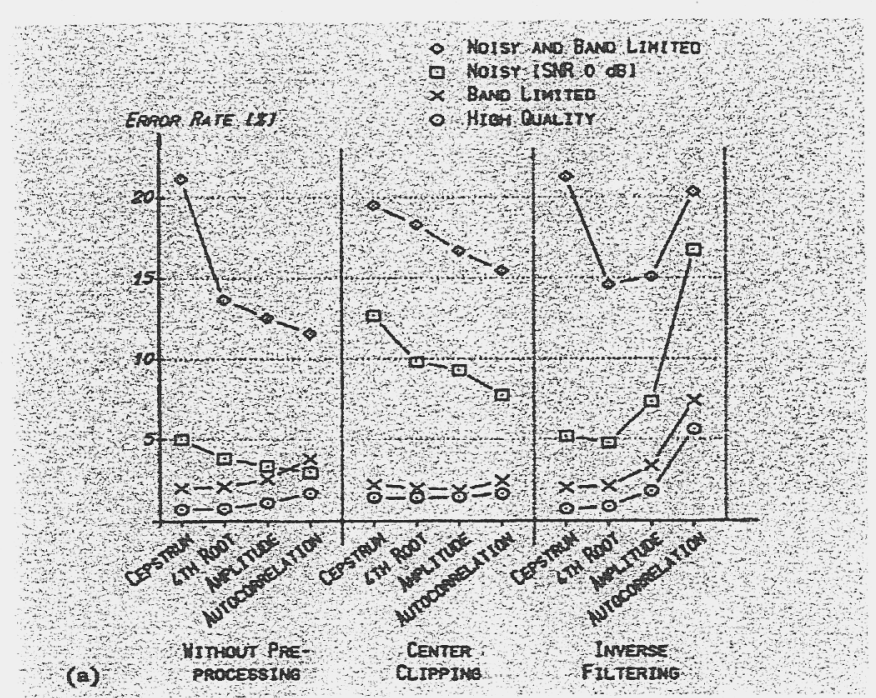

Taken from “Advances in Speech Processing," Furui and Sondhi, Merkker 92

\section{The Em Sensor Yield and Accurate, Robust, Single Glottal Period Pitch Estimate and Speech Onset}
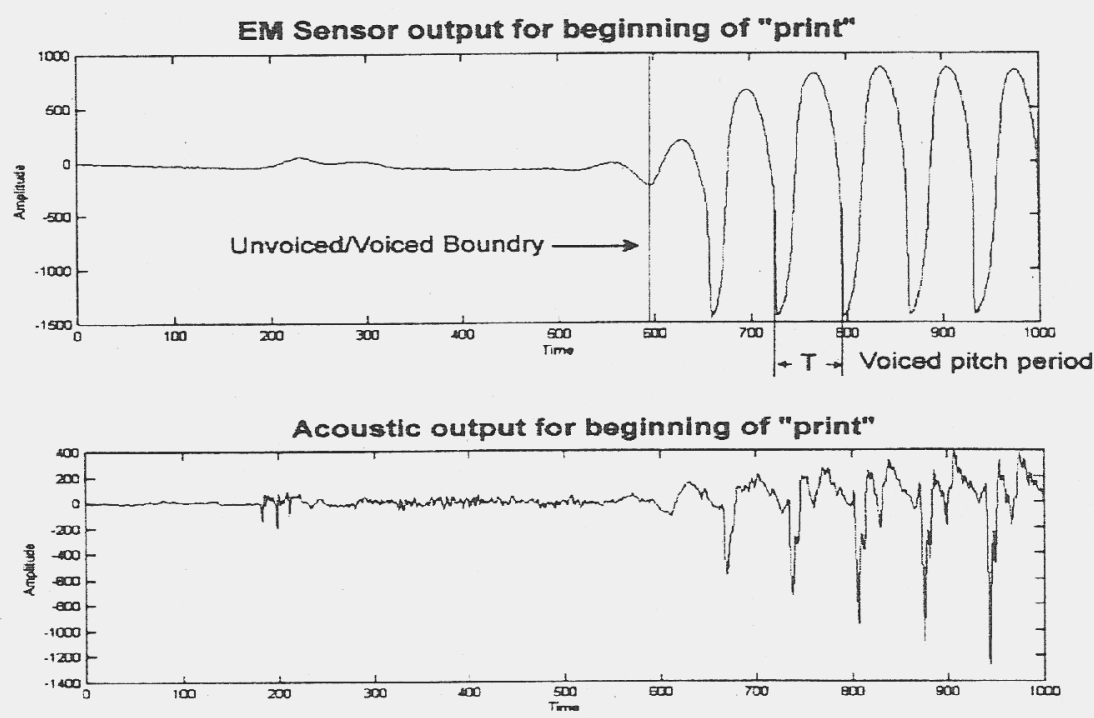
The Zero-Crossing Pitch Algorithm is a Simple, Fast, and Accurate Time Domain Calculation

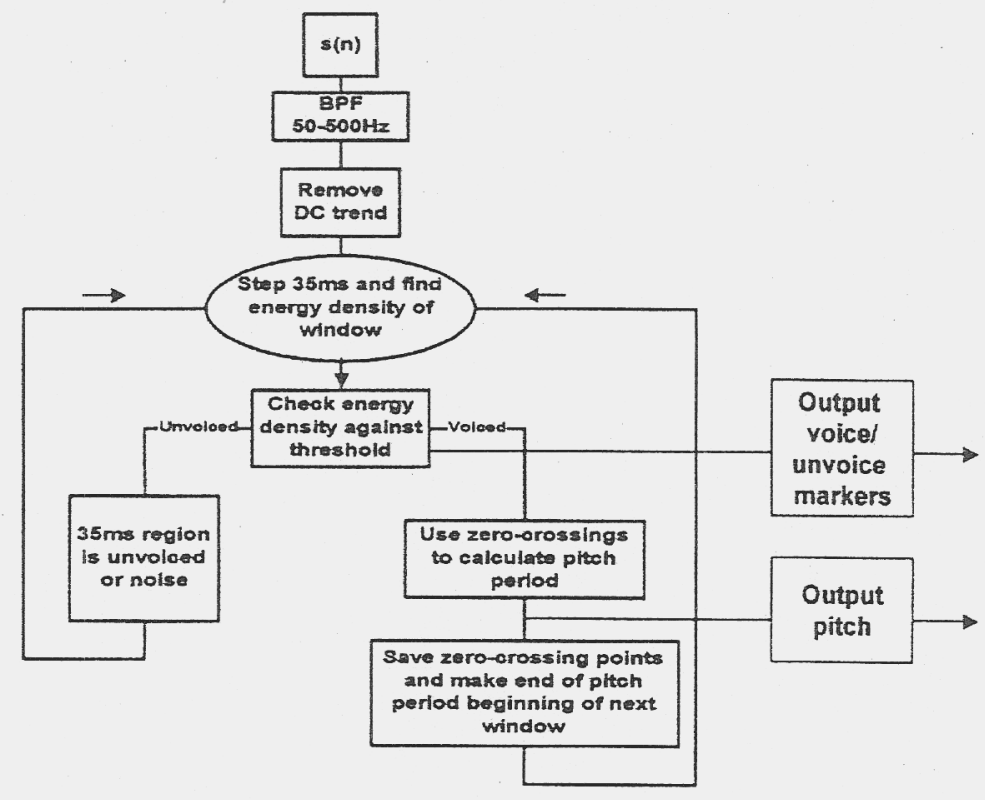

\section{Traditional Cepstral and Autocorrelation} Techniques were Used as a Basis for Comparison

\section{Cepstral Pitch Determination}

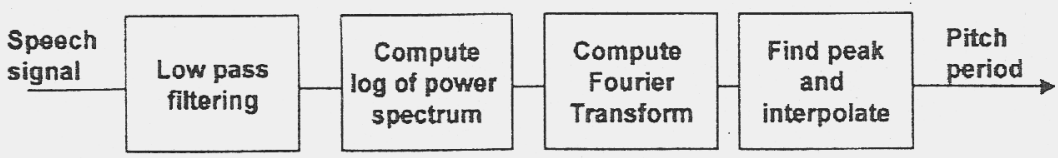

Autocorrelation Pitch Determination

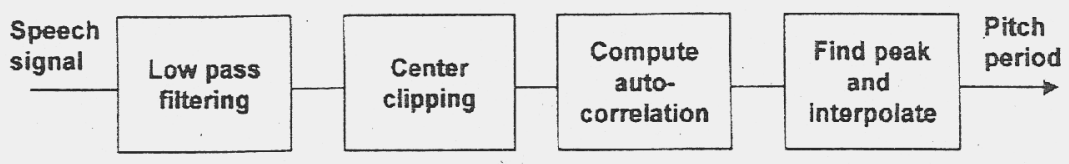




\section{Example of Pitch Estimation Using Correlation and Cepstral Techniques}
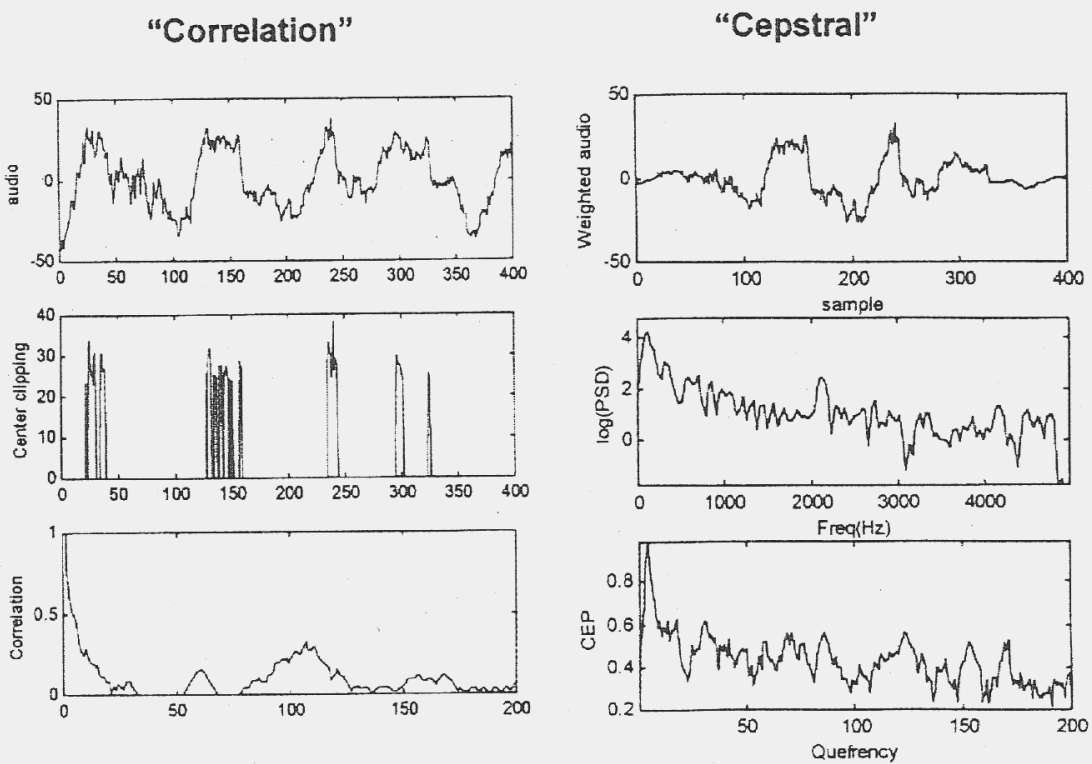

\section{The EM Sensor Gives Better Pitch Estimates} Than the Traditional Cepstral Method

Audio of fil ( $400 \mathrm{~ms})$
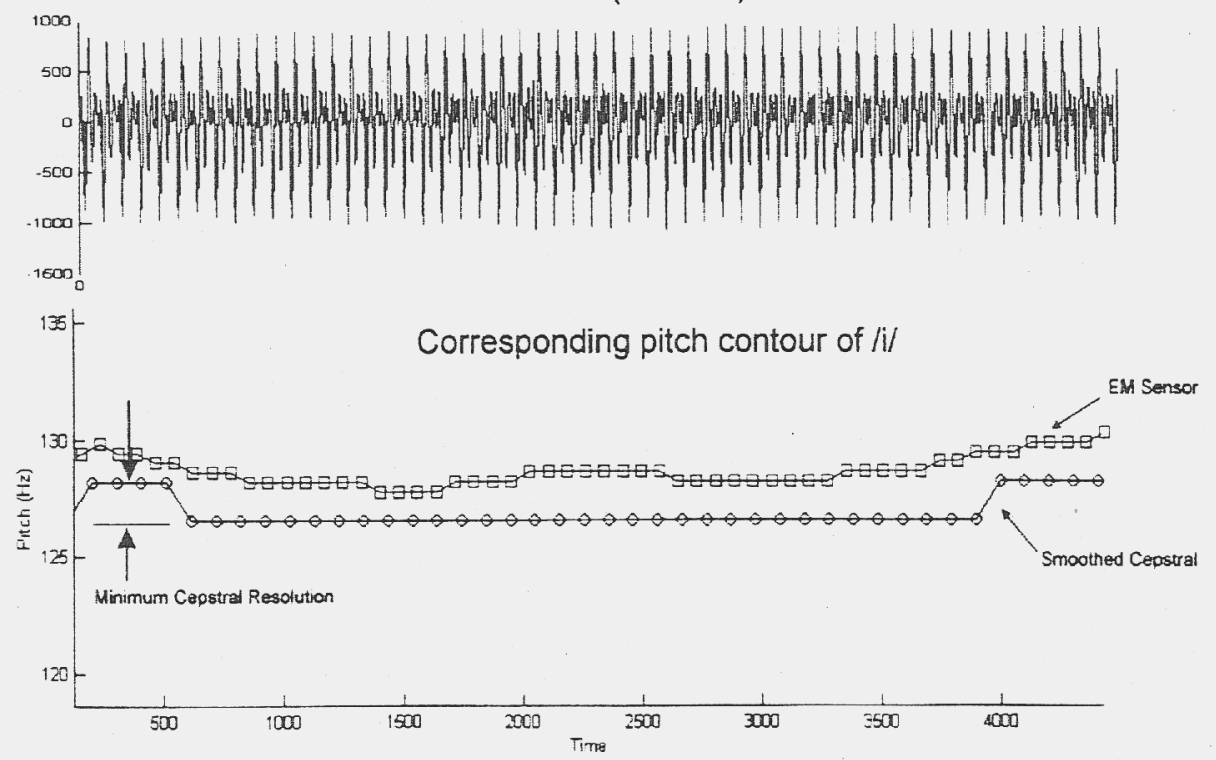


\section{The EM Sensor Yields Smoother, More Accurate} Pitch Contours Relative to Traditional Methods
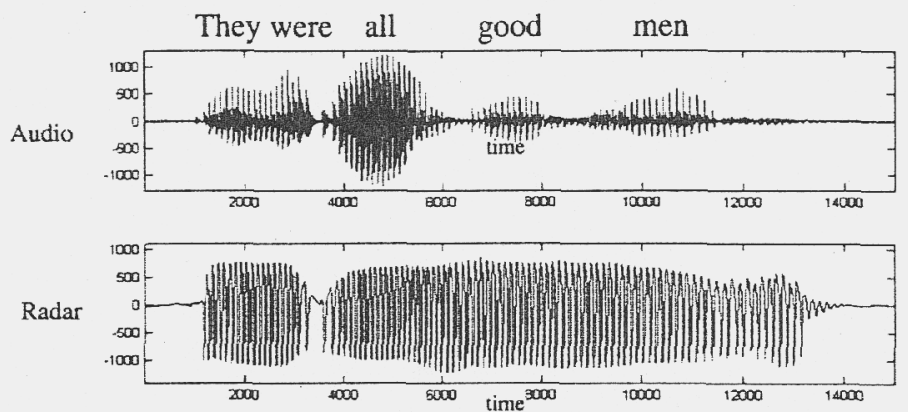

EM sensor
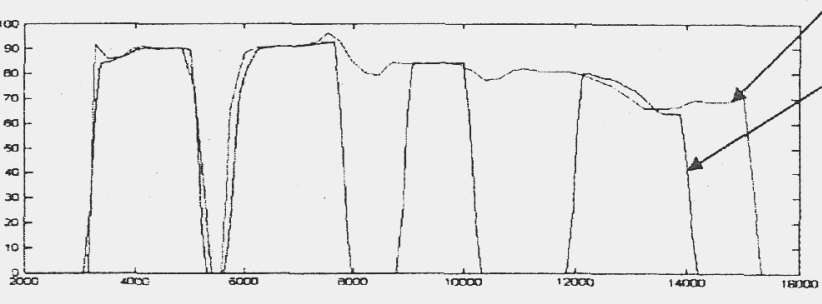

Audio

CEP

\section{Simulations With Synthetic Signals Confirm the} Accuracy of Our Zero-Crossing Pitch Algorithm

Pick $f_{k}$ at random to four decimal places

Synthetic signal $s(t)=\sum_{N=1}^{20} \frac{1}{N} \sin \left(2 \pi N f_{k} t\right)$

Relative Pitch Error

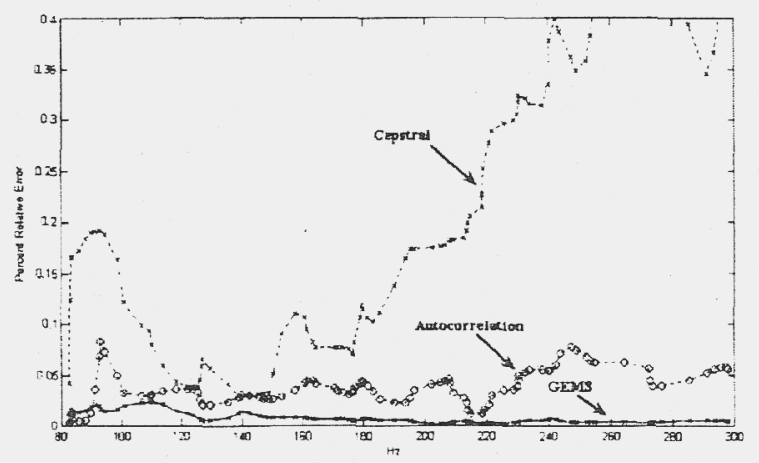




\section{From Tuning Fork Experiments We Determine Pitch Estimate Performance of GEMS}

- Generate known signals from tuning fork experiments

- Compute pitch estimate using cepstral, autocorr, and GEMS zero-crossing

- Results indicated that GEMS pitch estimate is:

- 100 times faster in computational efficiency

- 5 to 20 times smaller in error rates

- Conclusions

- GEMS is insensitive to acoustic noise

- Fast computation of pitch can provide real-time pitch synchronous speech processing

- Performance improve with higher sampling rate

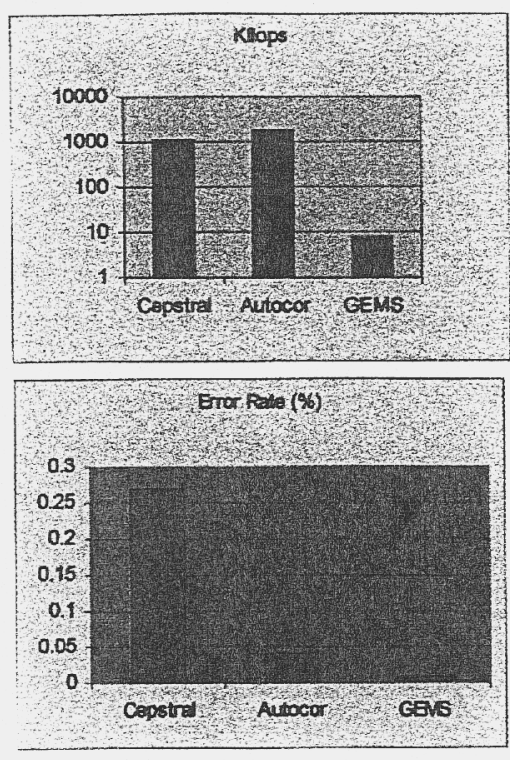

\section{Using a Synthetic Transfer Function We Validated the Improved Accuracy of ARMA Modeling}

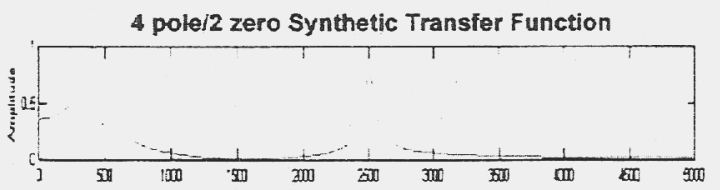

$X(z)$ EM Excitation
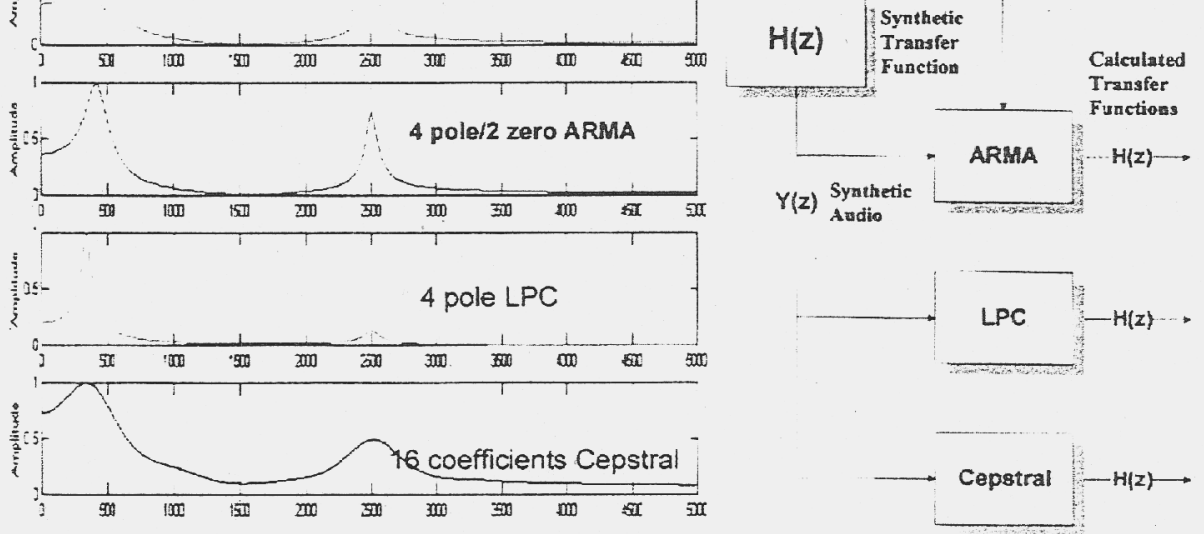

Frequency (Hz) 


\section{The ARMA Model Yields Accurate and Robust Transfer} Functions Which Compare Well to Traditional Models

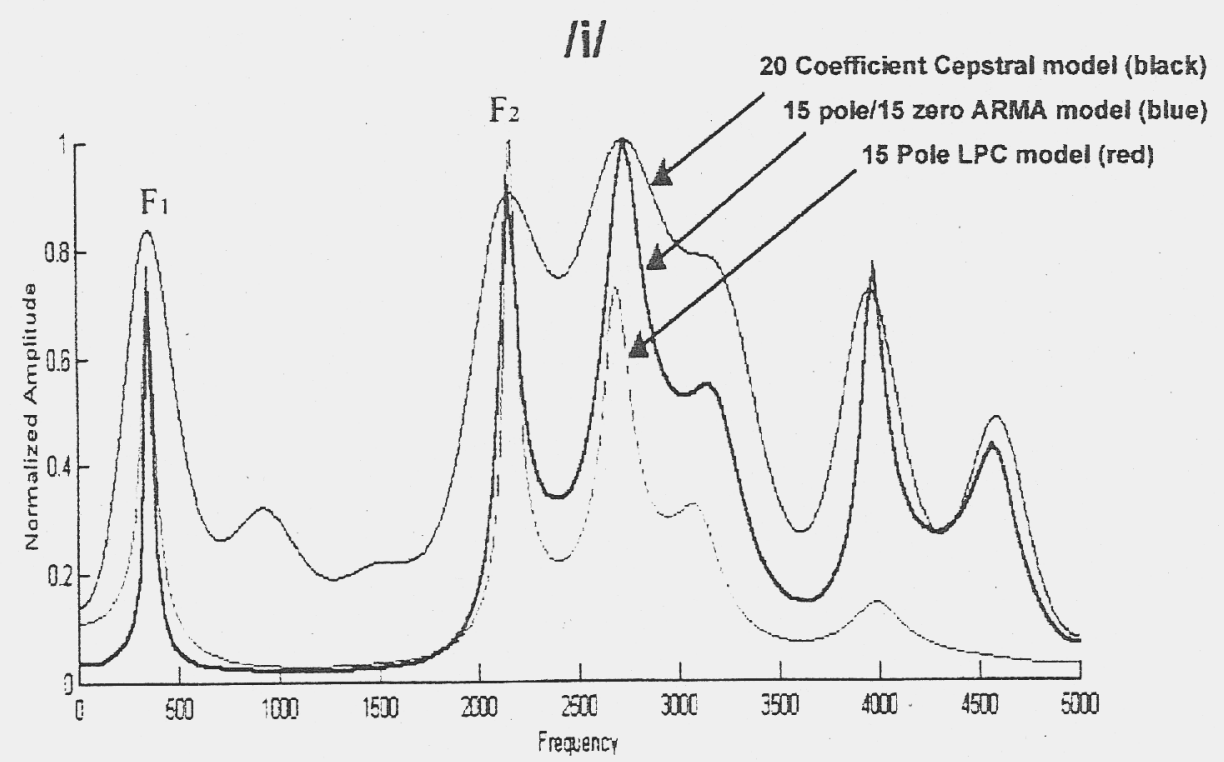

Access to the Voiced Excitation Signal Gives Pitch Synchronous Windows Used by the ARMA Model
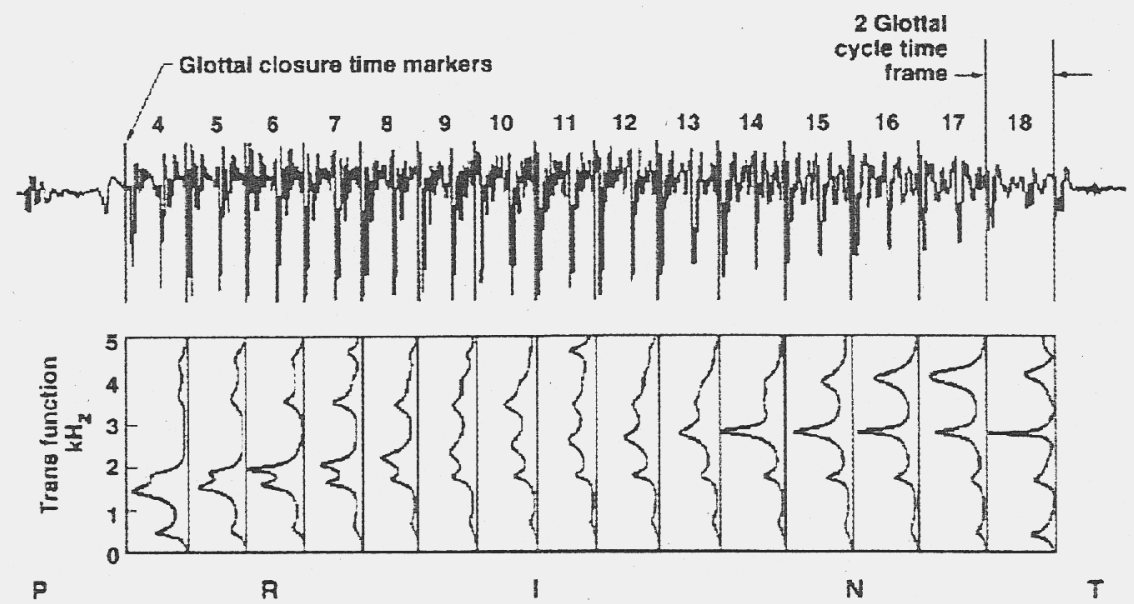


\section{Example of Showing That GEMS'Pitch Estimate is Unaffected by Acoustic Noise}
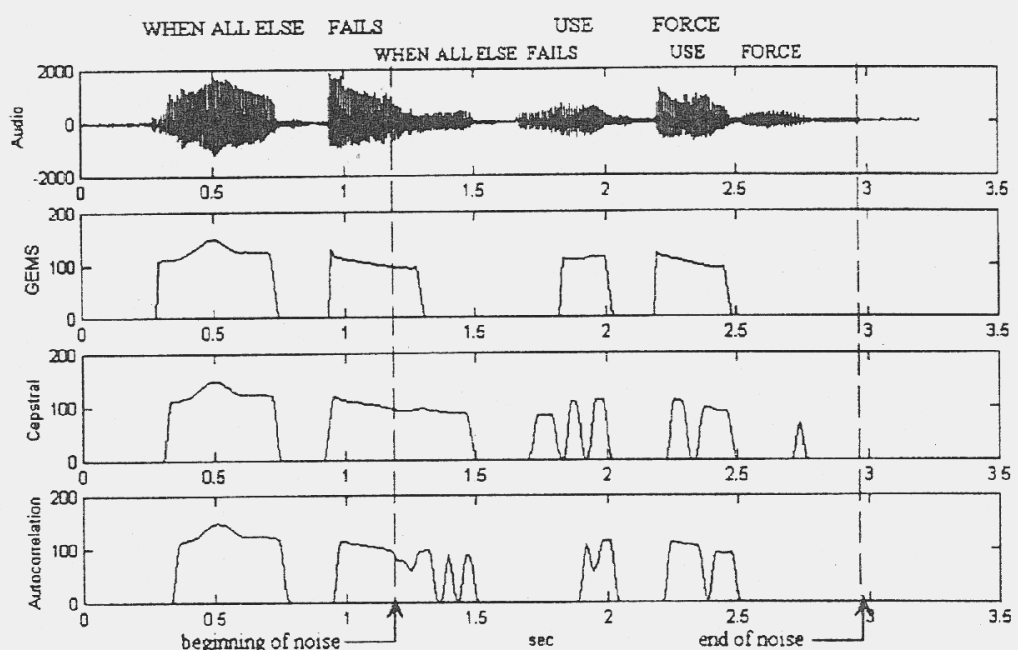

\section{Summary and Conclusions}

- Results indicated that GEMS pitch estimate is:

- 100 times faster in computational efficiency

- 5 to 20 times smaller in error rates

- GEMS is insensitive to acoustic noise

- Fast computation of pitch can provide real-time pitch synchronous speech processing

- Performance improves with higher sampling rate

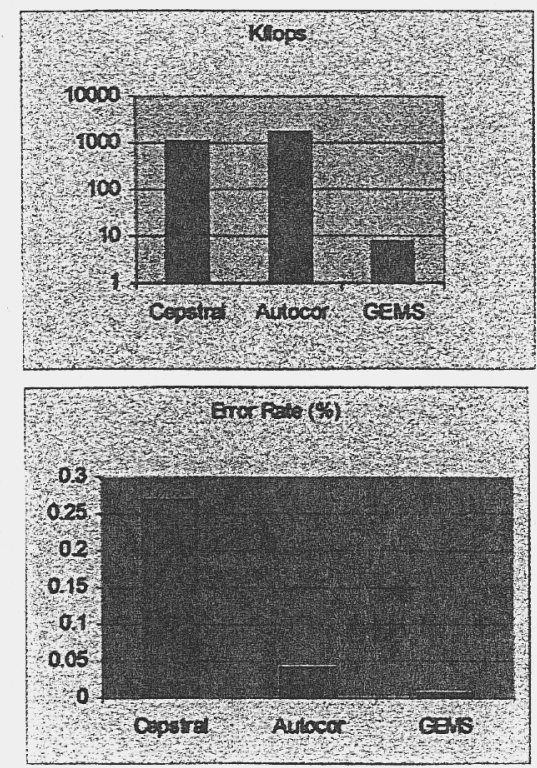




\section{EM-Microphone - A Potentially New Machine Interface for the 21st Century}
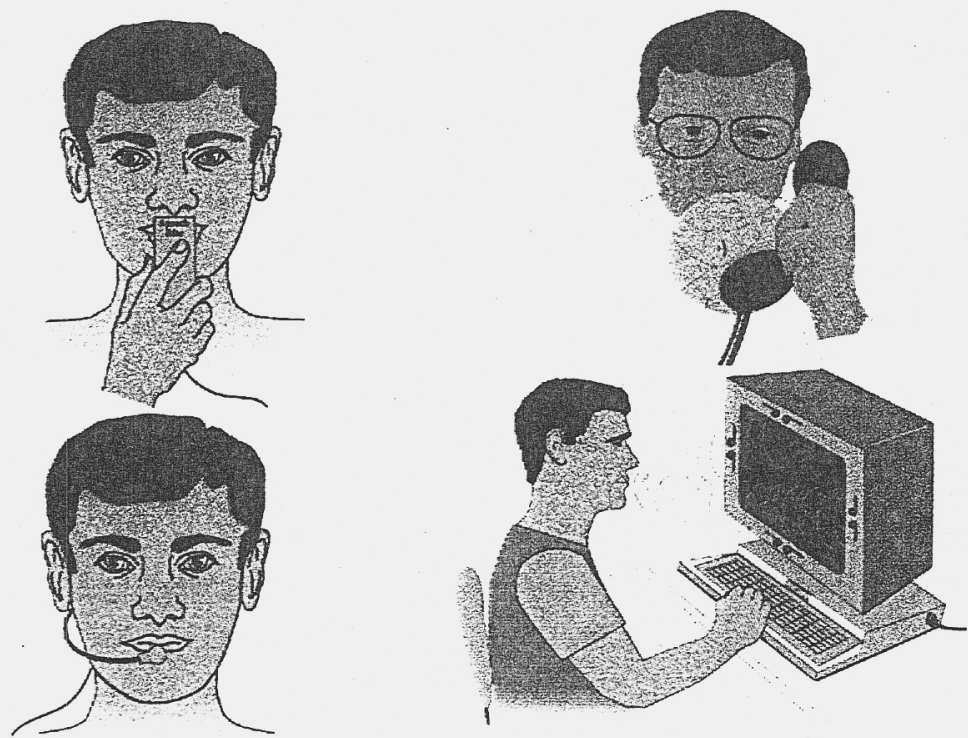


\section{Speaker Verification Using Acoustic and Glottal EM Sensors (GEMS)

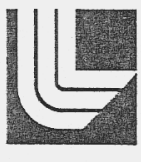

\section{CASIS: Signal and Imaging Sciences Workshop}

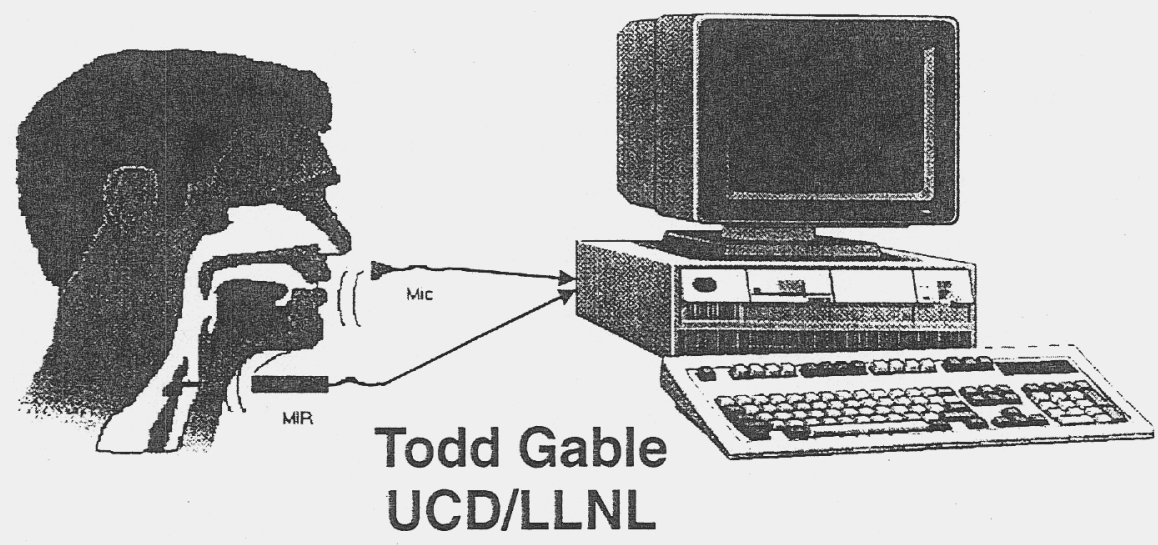

Overview - Why verification?

- The verification experiment is the first large scale test of the GEM Sensor applied to speech

- Verification involves many of the same types of problems found in other speech applications like speech recognition and speaker identification

- Speaker Verification can compete with or augment biometric security systems like retina scans and fingerprints

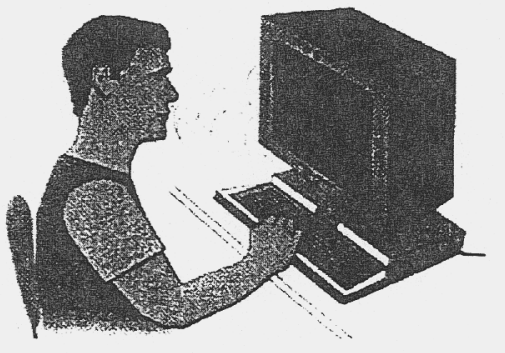


Speaker verification is the process of accepting or rejecting a persons identity claim based on parameters extracted from a test utterance

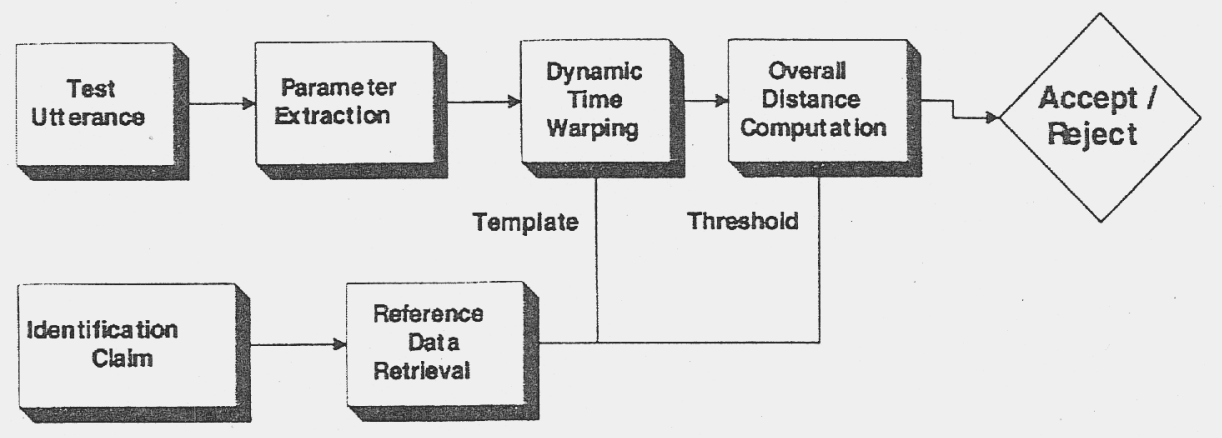

\section{GEMS provide enhanced parameters for verification}

Traditional Acoustic Parameters

- Pitch

- Spectral coefficients and their derivatives

- Energy

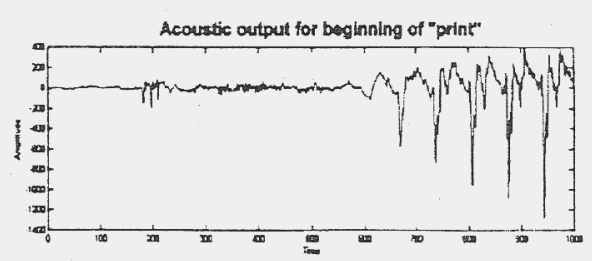

GEMS Enhanced Parameters

- GEMS extracted pitch

- Pitch synchronous spectral coefficients

- Energy

- Pitch synchronous ARMA coefficients

- GEMS signal parameters

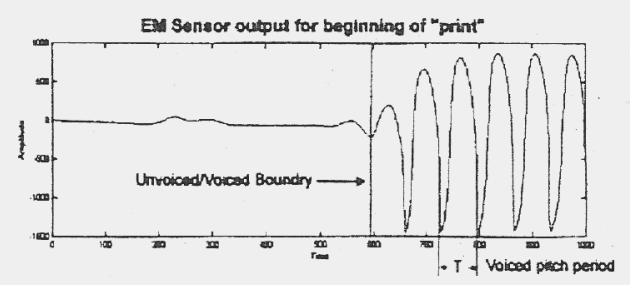




\section{The GEMS signal shows distinct differences among} different speakers - suitable for a glottal "fingerprint"

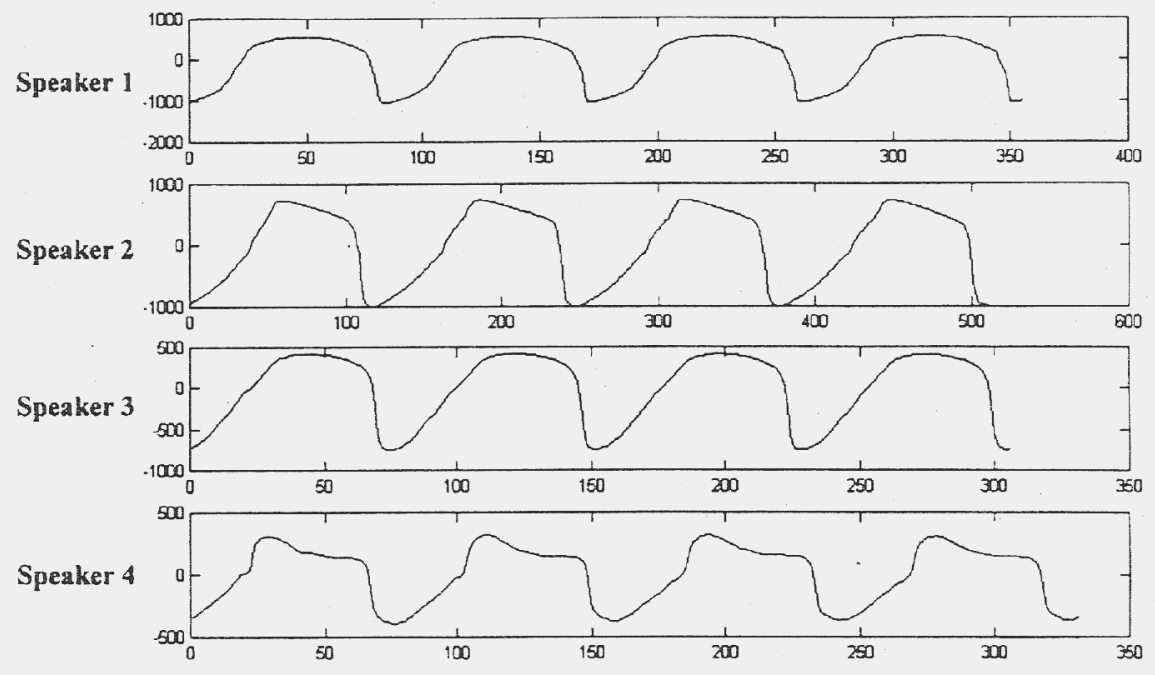

\section{The vocal tract is modeled as an LTI} system so we may use ARMA modeling
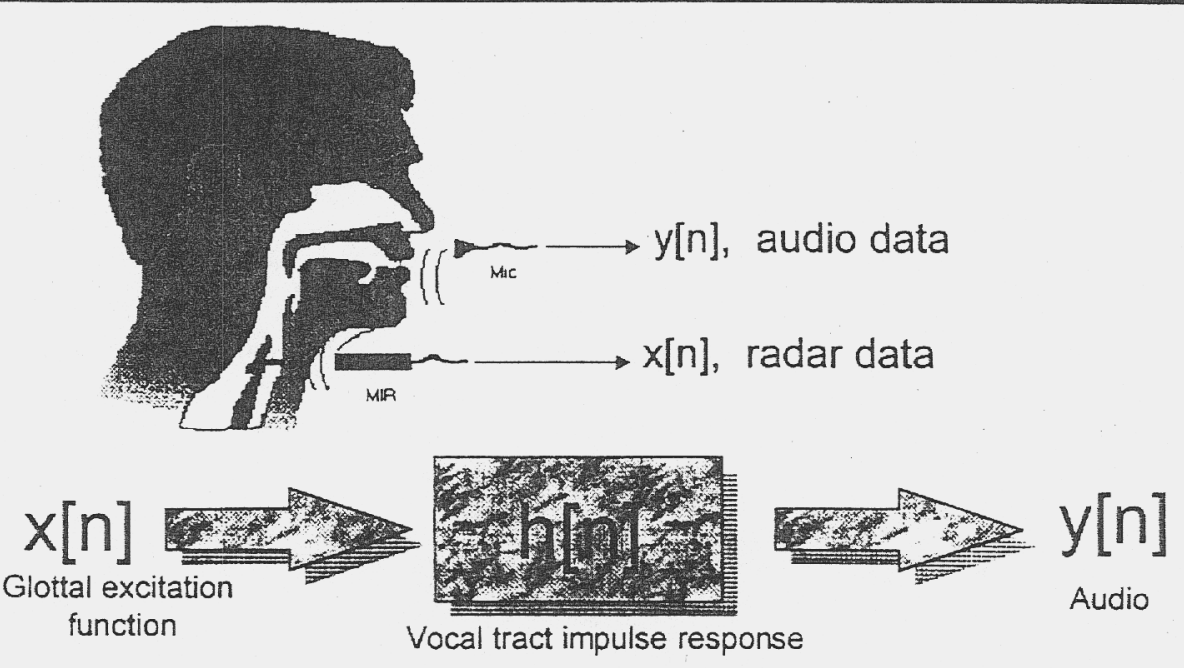

$$
\text { Transfer function } H(z)=Y(z) / X(z)
$$


Dynamic Time Warping (DTW) is the process of warping two vectors to the same length which yields a distance measure that mathematically tells us how similar they are
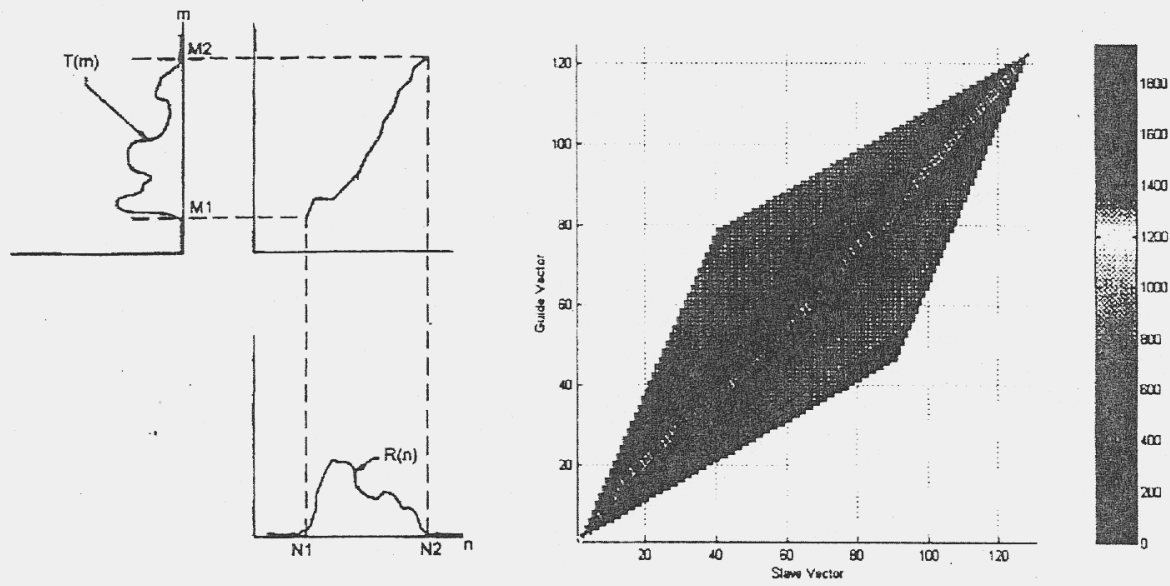

Example Warping

Pitch Contours before time warping
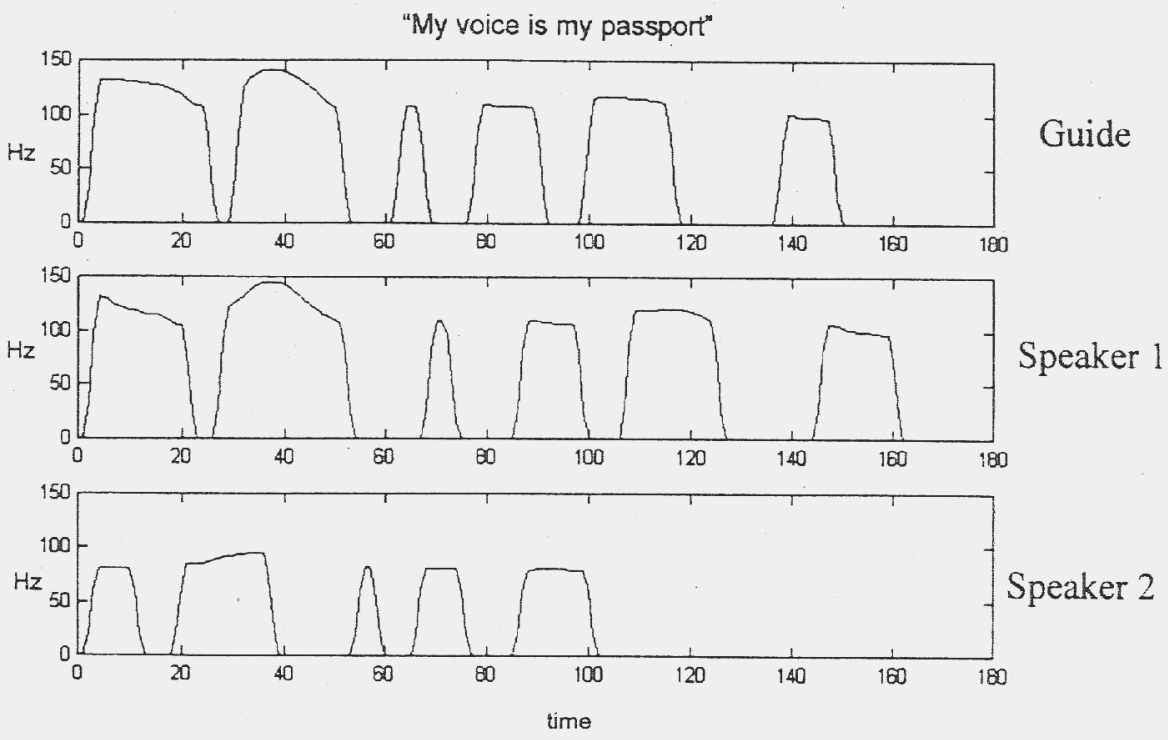


\section{Pitch Contours after time warping}
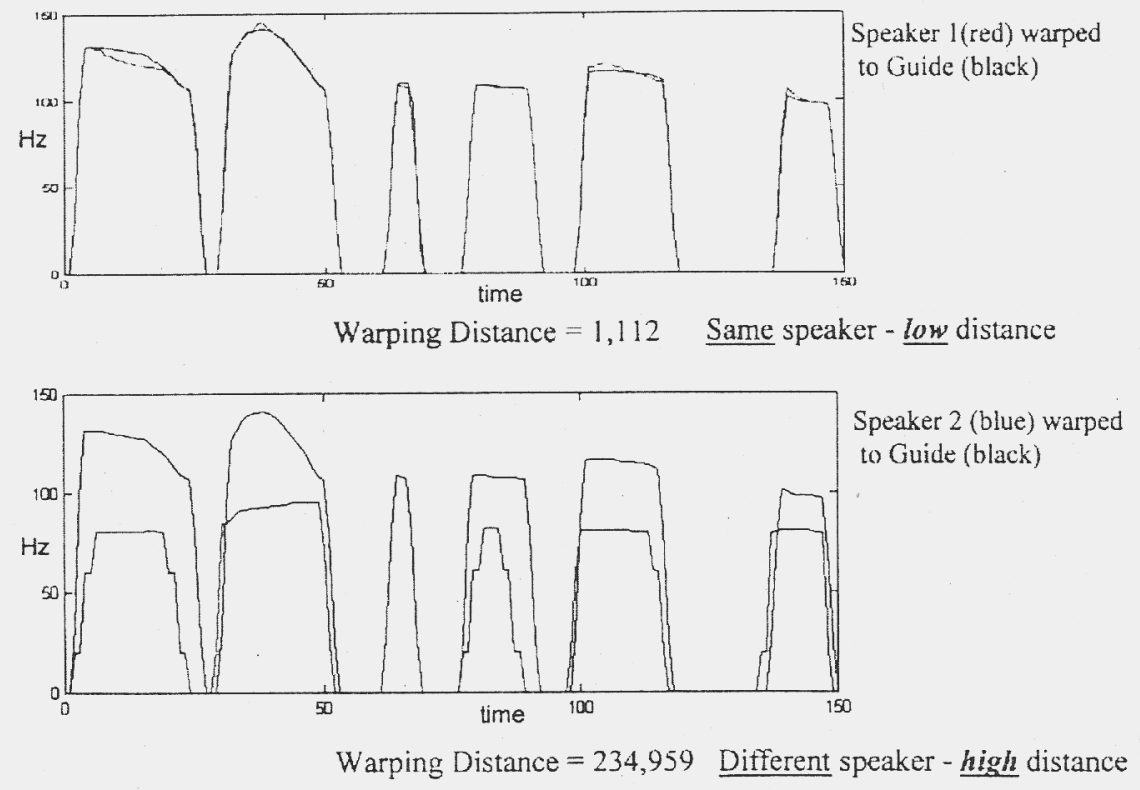

\section{Where we are now and what's in the future}

- We have audio and GEMS data for fifteen male speakers using standard sentences

- We have algorithms for extracting some verification parameters

- A Dynamic Time Warping algorithm is in place for time-registration and a distance measure

- We still need to finish the parameter extraction software and fine-tune the system

- With the software in place we can run the system for all fifteen subjects and generate statistics

- Eventually port the system to $\mathrm{C}_{++}$for demonstration purposes

Thank you 


\title{
Object Identification Using Low Power Radars
}

\author{
Randy Roberts
}




\section{Signal/Image}

Processing for

Non-Destructive

Measurement 


\title{
Explosive Detection and Discriminant Research for Civil Aviation Security $\dagger$
}

\author{
Harry E. Martz, Derrill Rikard, Earl Updike, Clint Logan, and Sailes Sengupta \\ Lawrence Livermore National Laboratory
}

$\mathrm{X}$ - and gamma-ray imaging techniques in nondestructive evaluation (NDE) and assay (NDA) have seen increasing use in an array of industrial, environmental, military, and medical applications. Much of this growth in recent years is attributed to the rapid development of computed tomography (CT). A new application area for $\mathrm{CT}$ is civil aviation security explosives detection. The only Federal Aviation Administration (FAA) certified explosives detection system is a linear-array based x-ray CT scanner developed by InVision, Inc. Unfortunately the system's airport false-alarm rate performance is higher than the certification rate. We are working with the FAA William J. Hughes Technical Center to help them advance their explosives detection and discriminant research efforts. We have applied three X-ray CT scanners in four different data acquisition configurations to characterize explosives, distinguish between explosives and $\mathrm{x}$-ray machine false-alarm items; and validate inert, non-hazardous materials that simulate the $\mathrm{x}$-ray properties of explosives. I will describe the X-ray CT scanner configurations, their results and our preliminary analysis of the data.

t This work is funded by the FAA William I. Hughes Technical Center and is performed under the auspices of the U.S. Department of Energy by the LLNL under contract W-7405-ENG-48.

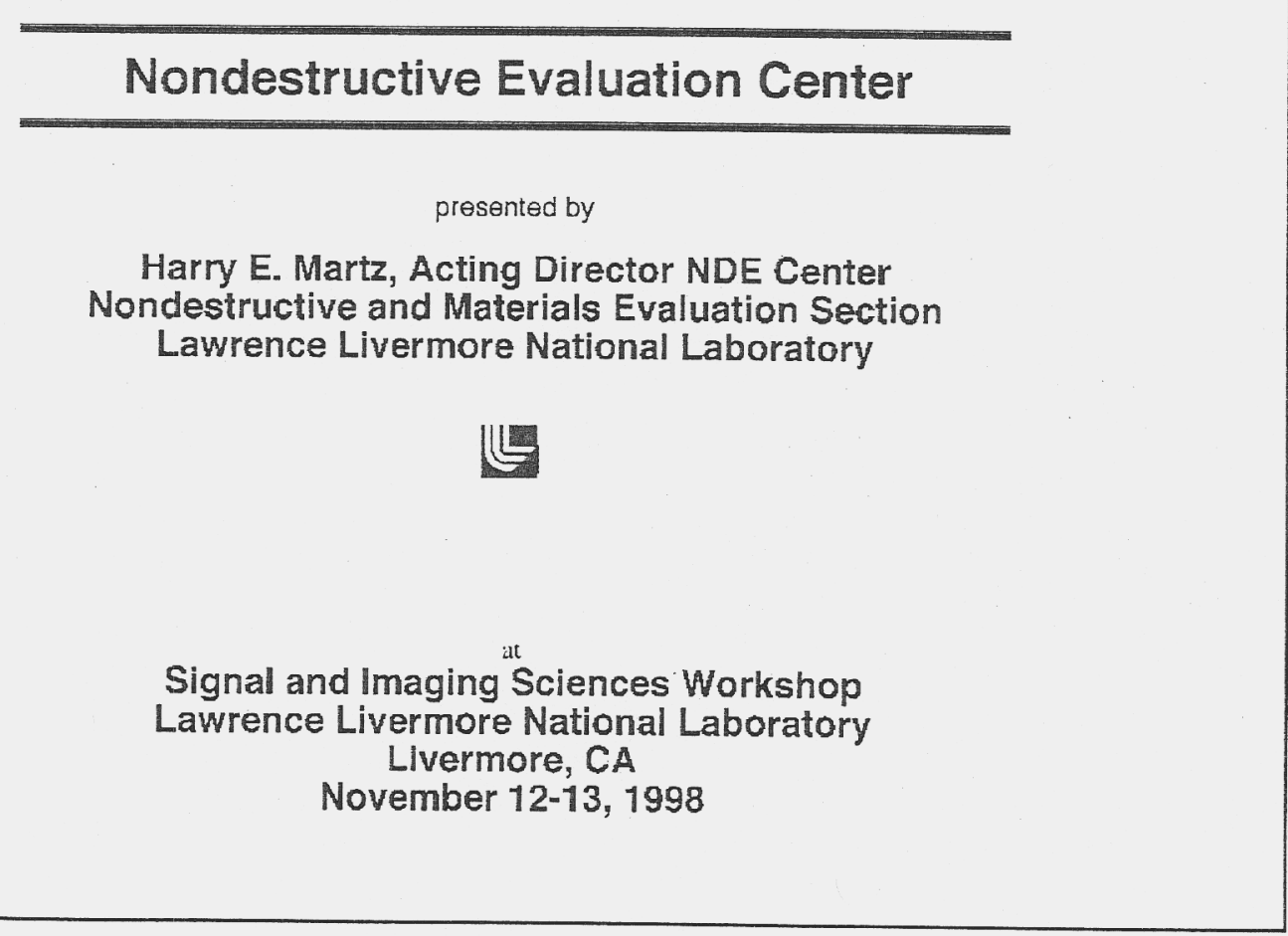


The NDE Center's role is to R\&D core technologies and competencies required to fulfill LLNL's mission

- Vision

- To be the World's leader in the research, development, and application of the nondestructive measurement technologies that enable successful LLNL and DOE programs

- Mission

- To provide LLNL and DOE programs with superior nondestructive measurement capabilities

- FY99 mission objectives are to advance nondestructive measurement core competencies and technologies needed for the following compelling problems

- Extraordinary laser systems, this includes advanced diagnostics, e.g., for fusion targets, and automated insitu optics damage inspection

- Weapon system performance, this includes three-dimensional measurements over $15 \mathrm{~cm}$ to better than $25 \mu \mathrm{m}$, advanced diagnostics, e.g., AHF, and ceramic properties measurements

- Science-based stockpile stewardship, specifically durability and life prediction

- Arms control, nonproliferation, and international security related to reducing the threat from weapons of mass destruction

The NDE Center's current strategic focus includes five objectives required to meet LLNL program needs

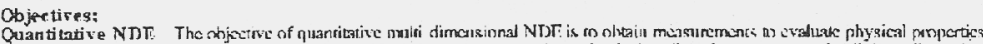
germetry. density, elementai \& molecular composition, then

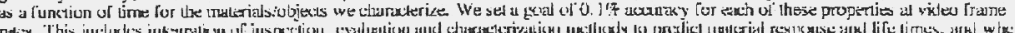

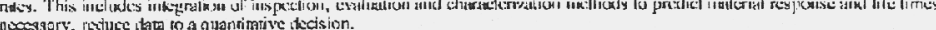

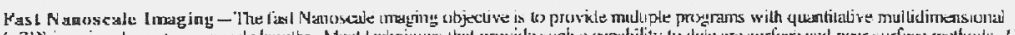

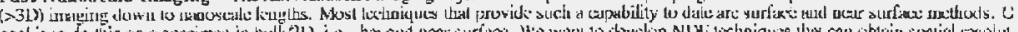

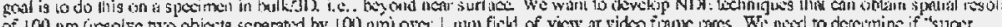

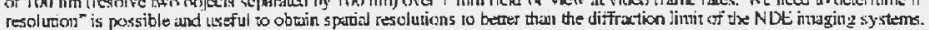

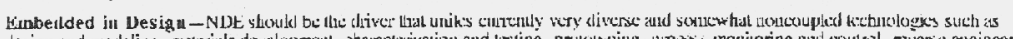

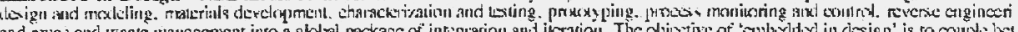

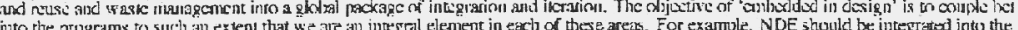

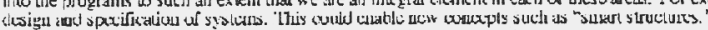

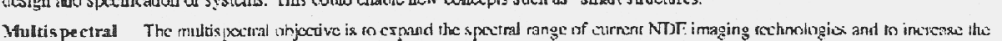

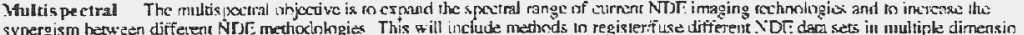

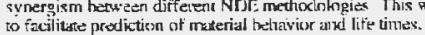

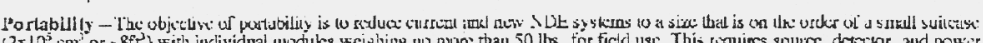

\begin{tabular}{|c|c|c|c|c|c|c|}
\hline Focus areas & $D \& N T^{t}$ & Lasars ${ }^{t}$ & $\mathrm{NAI}^{\dagger}$ & Energy/Env & Bloscience ${ }^{\dagger}$ & Other $\mathbf{r}^{\dagger}$ \\
\hline Quentitativa NDE & $\begin{array}{l}\text { H-Landram; } \\
\text { Oolan Kaliman i }\end{array}$ & & M & H & $\mathrm{H}$ & $\mathrm{H}$ \\
\hline Faal Nanoscate maging & 11 & Hluper & $M$ & $M$ & 11 & 11 \\
\hline Embedderd in Design & & 11 & $M$ & 11 & $M$ & II \\
\hline Mulitspectul & M & $\mathrm{H}$ & $H F \cdot 00=010$ & $\mathrm{H}$ & $\mathrm{H}$ & $M$ \\
\hline Ponability & $M$ & $M$ & $\mathrm{H}$ & $\mathrm{H}$ & M & $M$ \\
\hline
\end{tabular}




\section{Compelling NDE Center problems}

- Measurement Systems

- See inside objects beyond what NDE has seen before

$>$ Improve spatial resolution down to $\mathrm{nm}$ scale

$>$ Increased penetration/contrast to see inside materials not accessible before

$>$ Temporal (fast) volumetric imaging

- Analysis and Interpretation

- Multi-dimensional (>3D) signal and image processing

$>$ Speed, accuracy and nolse reduction in processing

$>$ Data interpretation-We see more and more things;

what does this mean to the customer

$>$ Fusion of different NDE measurement systems data

Measurement systems, and analysis and interpretation must be developed jointly to get optimal synergy

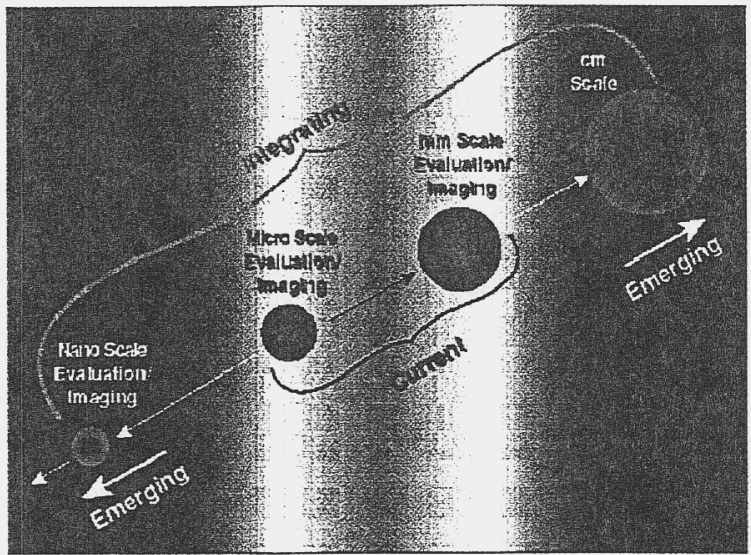

It is clear that one NDE challenge is to head in the nano-scale direction in addition to improving current scale capability 
Automated multi-dimensional data acquisition, processing and interpretation is the world of the future

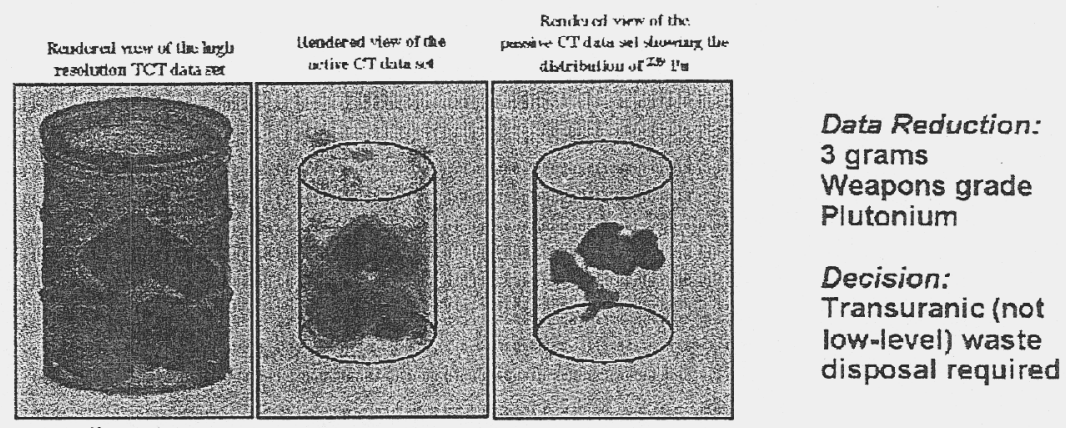

Typically multi-dimensional data are required to produce a single number to make a crucial decision

"GBytes to a Bit"

\section{"GBytes to a Bit"}




\section{Ultrasonic Compound B-scan Imaging \\ Co-authors: T. J. Gomm, J. A. Mauseth \\ Idaho National Engineering and Environmental Laboratory \\ Lockheed Martin Idaho Technologies Company}

The imaging of the internal contents of thin-wall tubes has led to the implementation of a little-used ultrasonic imaging technique that allows compounded images to be created from a series of simple B-scan images. These compound B-scans have distinct imaging advantages over standard ultrasound A-scan, B-scan, and C-scan modes, as well as some tomographic imaging methods.

Although this method is finding use in the medical world, there are issues pertaining to couplant homogeneity, reflection, and diffraction that must be addressed for application in the testing and imaging of metallic targets.

This presentation clearly defines the implementation of the Compound B-scan. The steps needed to convert each standard B-scan image to a dimensional image are discussed. The issue of non-homogeneity in the couplant is addressed. Because the time-to-spatial dimension conversion requires knowledge of the couplant velocity, use of non-homogenous couplant dictates that a different conversion algorithm be developed. Artifacts resultant in the compounded images are presented and discussed.

Test results are presented that show the ability of the Compound B-scan to image both concave and convex surfaces as well as tightly-packed clusters and phantoms in thin-wall tubes.

Keywords: Ultrasound, Compound B-scan, Tomography 

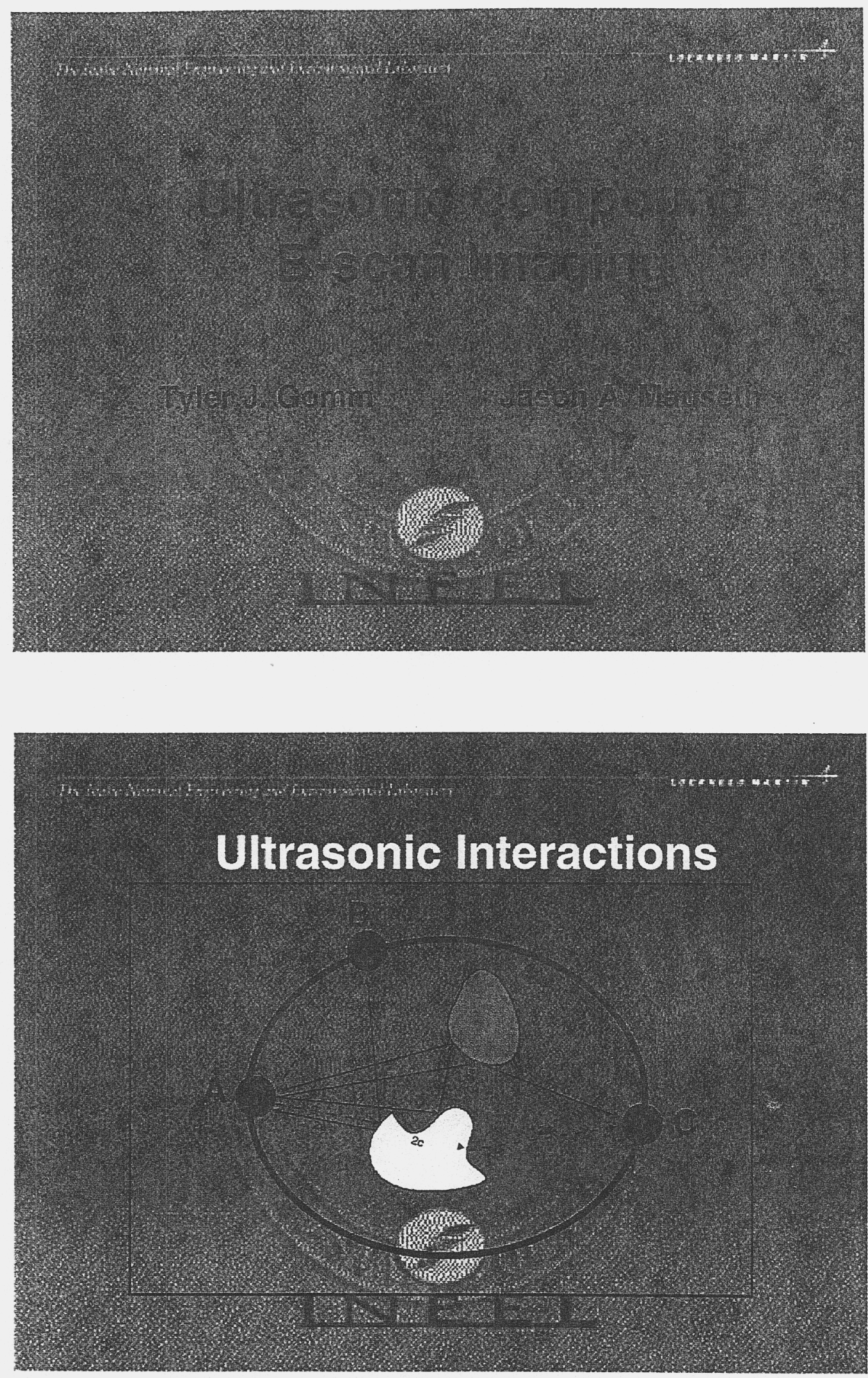

SIGNAL AND IMAGING SCIENCES WORKSHOP 1998 

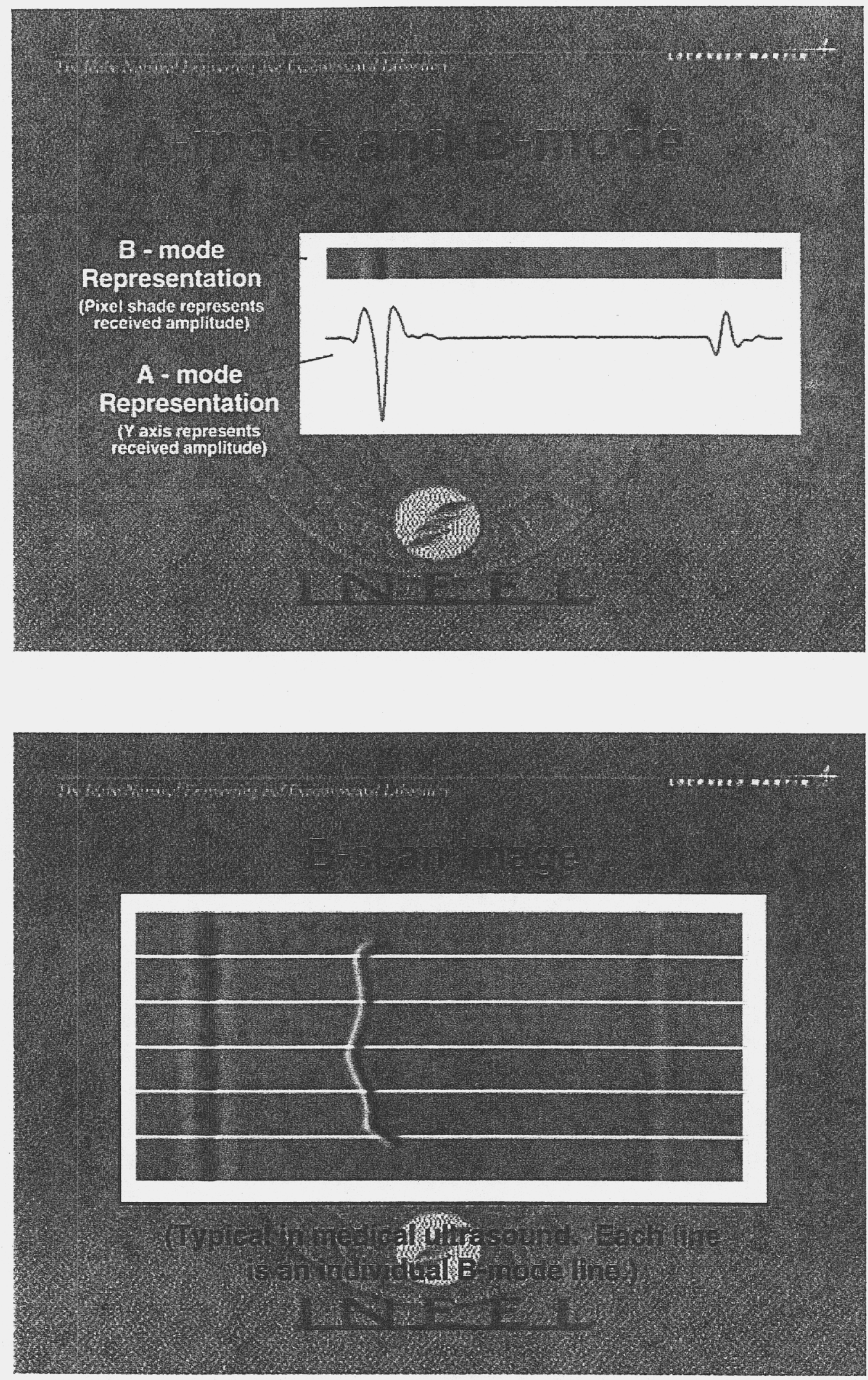

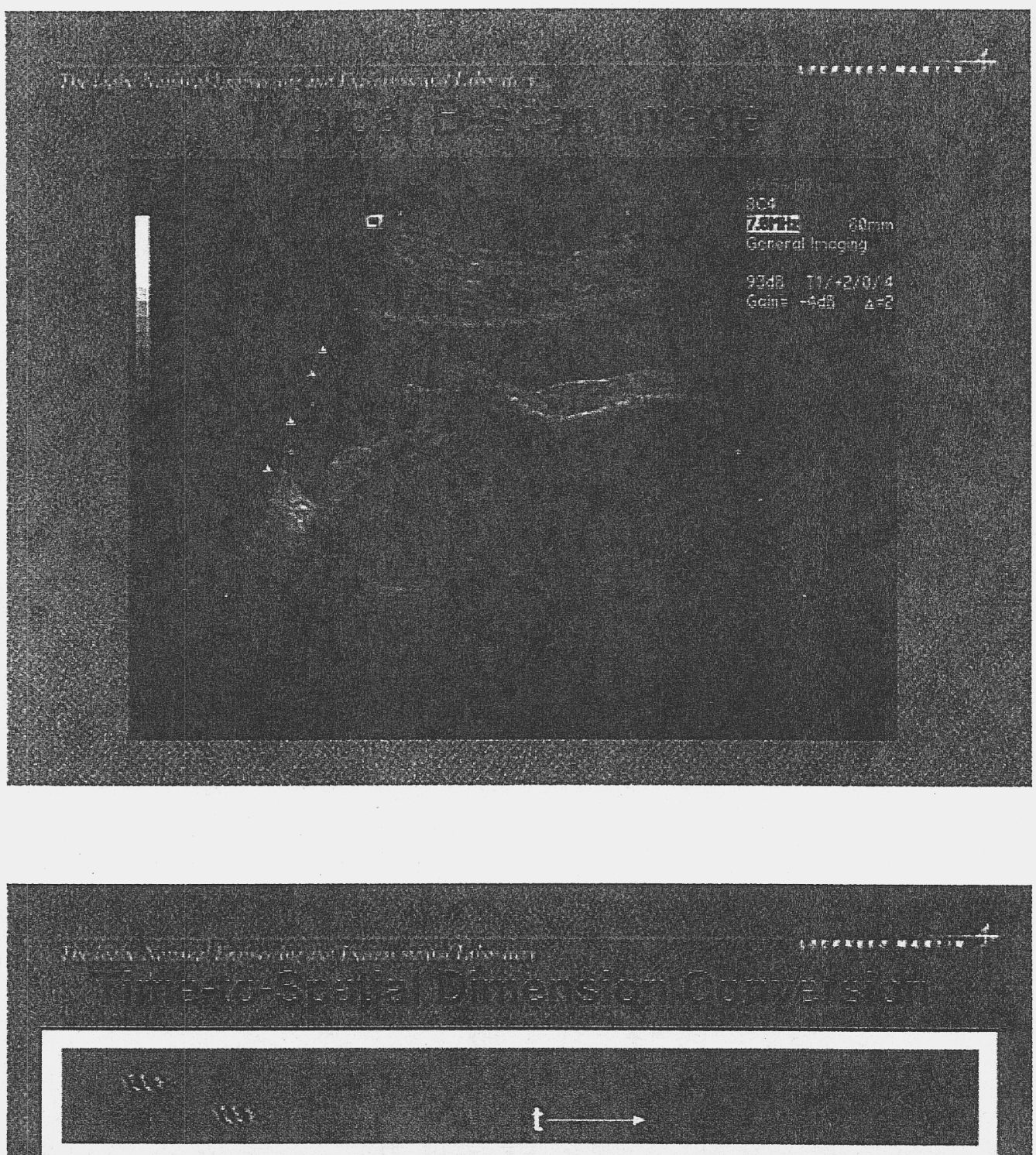

$$
X_{n e w}=\left(\frac{N_{x}}{S R}\right)\left(\frac{v}{2}\right)\left(\frac{1}{Y_{\text {step }}}\right)
$$

$x_{\text {nem }}$ Desired length of the $x$-axis is data $N_{x}$. Original number of data poins in tha $x$-axis S7. Acquisition sample rate v. couplant velooily Y.rep Step distance in the Yaxis

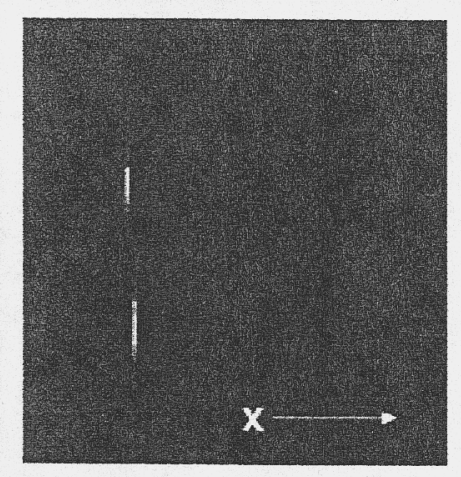



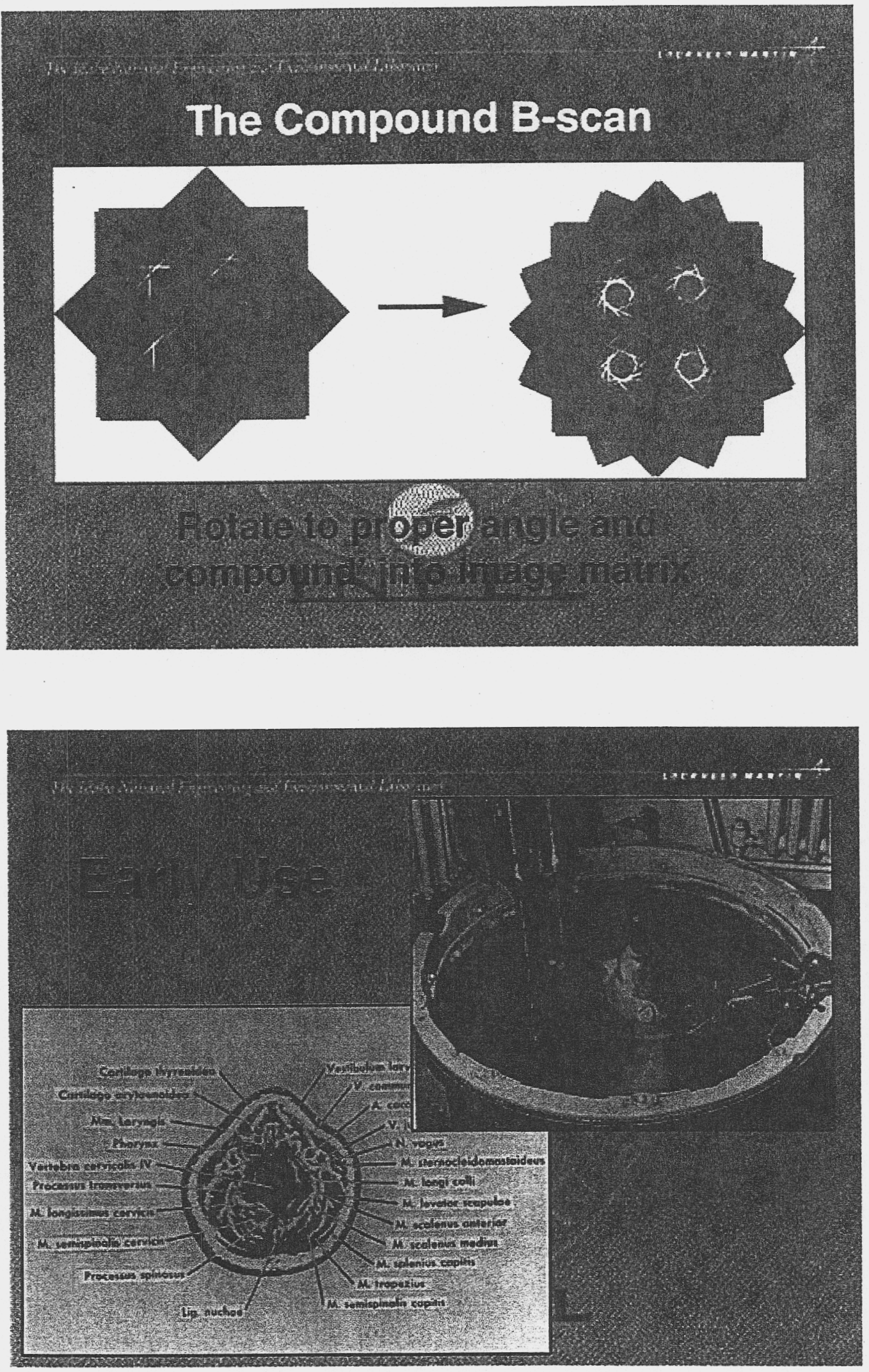

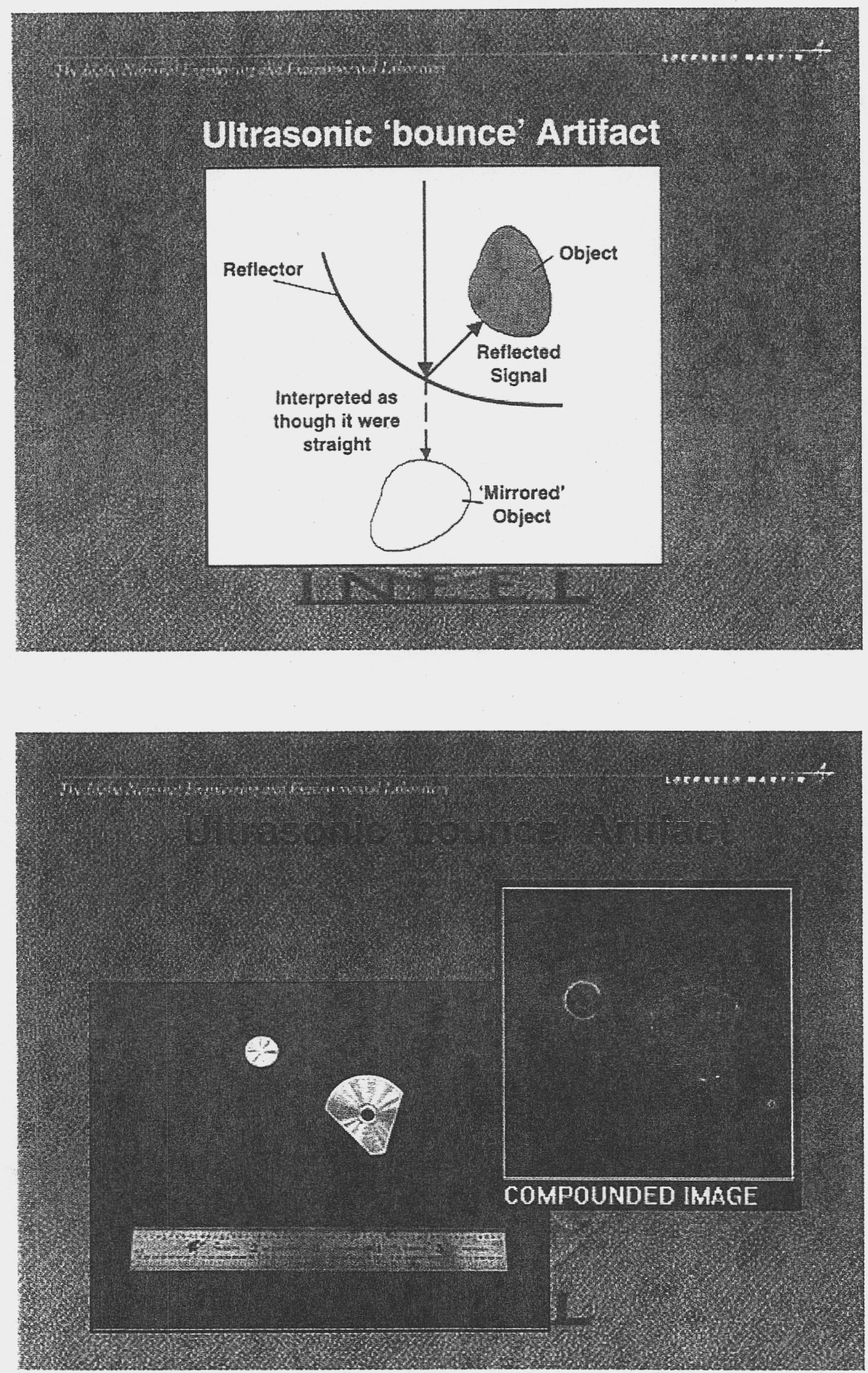

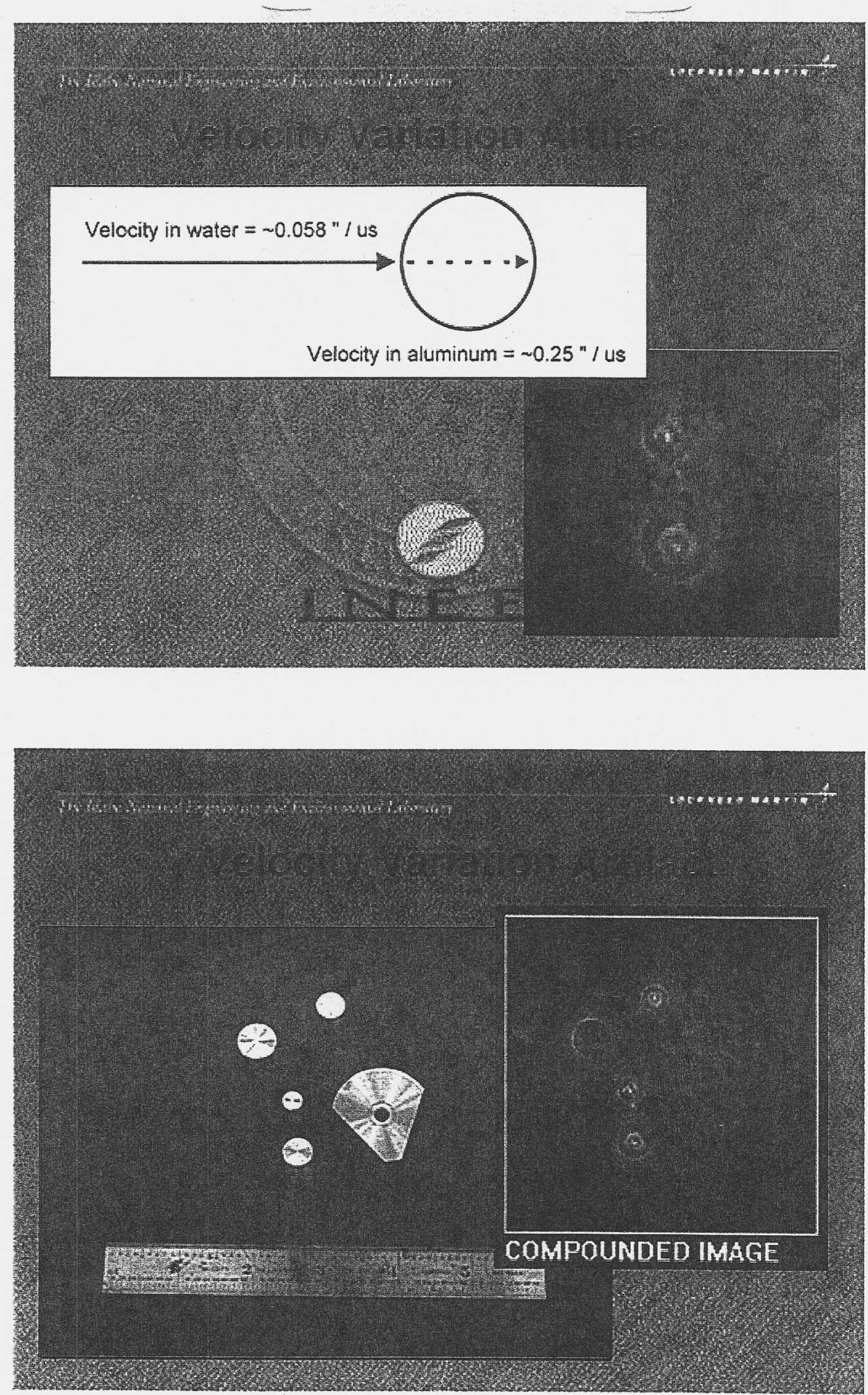

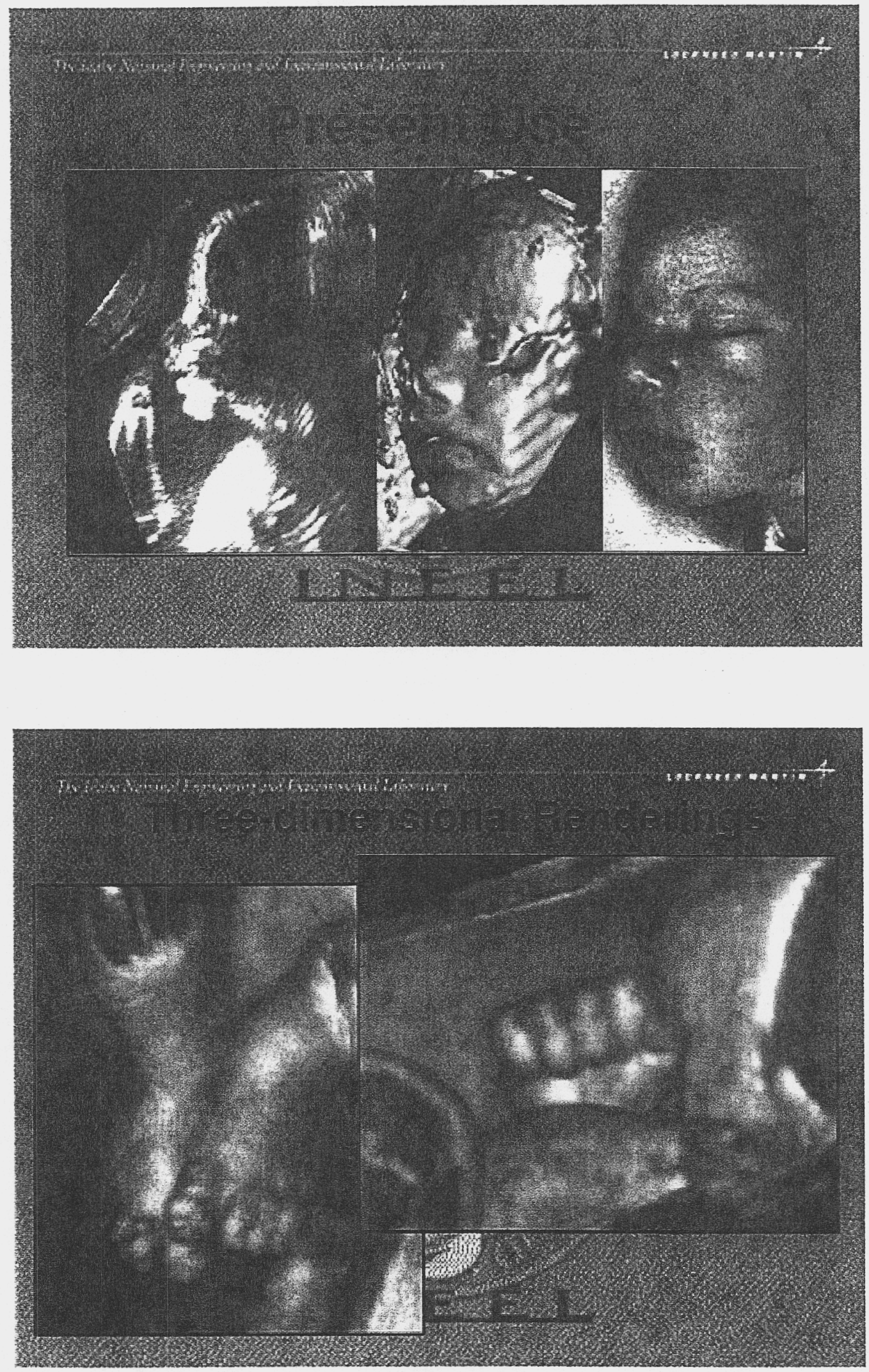

SICNAL AND IMAGING SCIENCES WORKSHOP 1998 


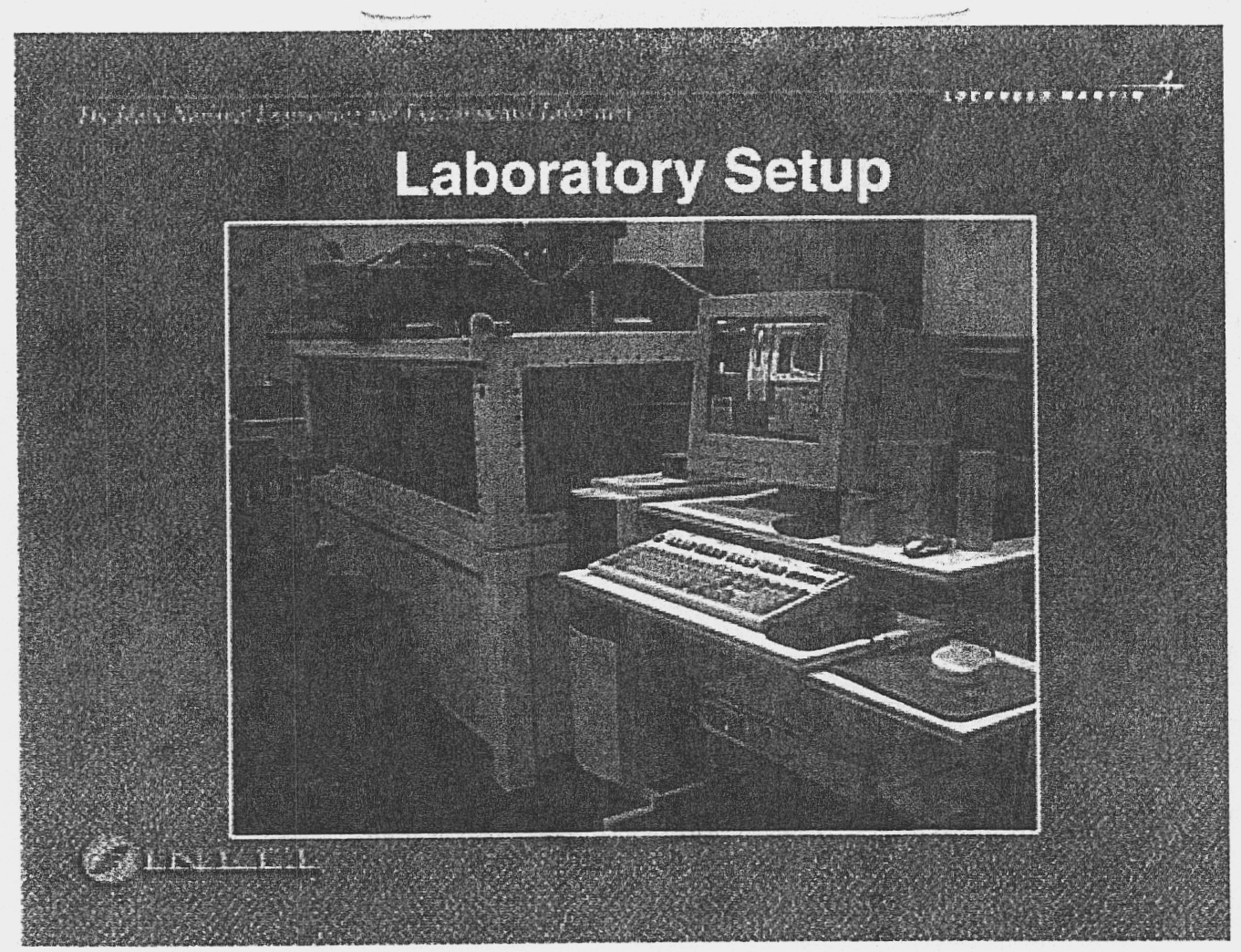

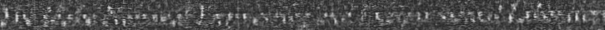

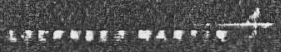

\section{Expected Reconstructions of Cluster Phantom}

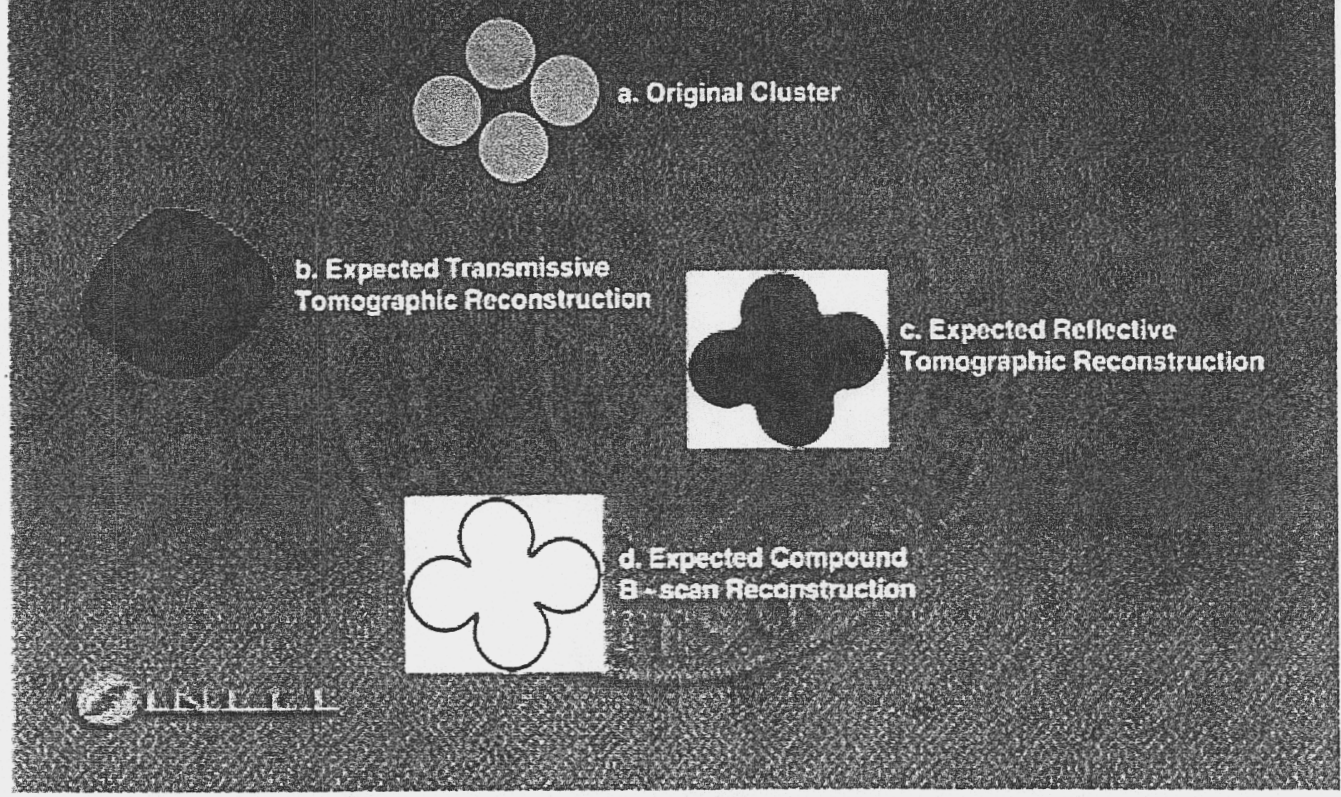



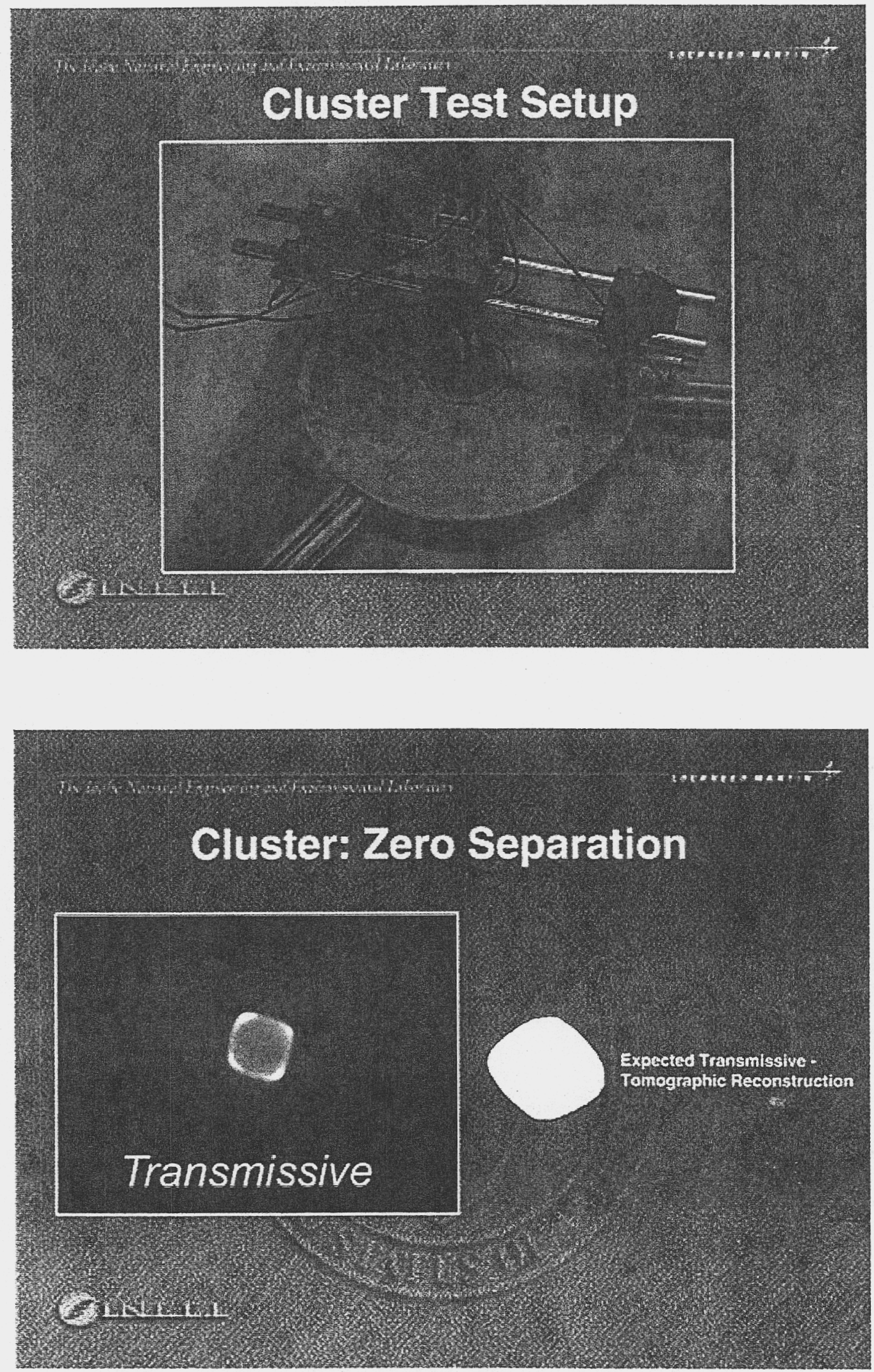

SIGNAL AND IMAGING SCIENCES WORKSHOP 1998 

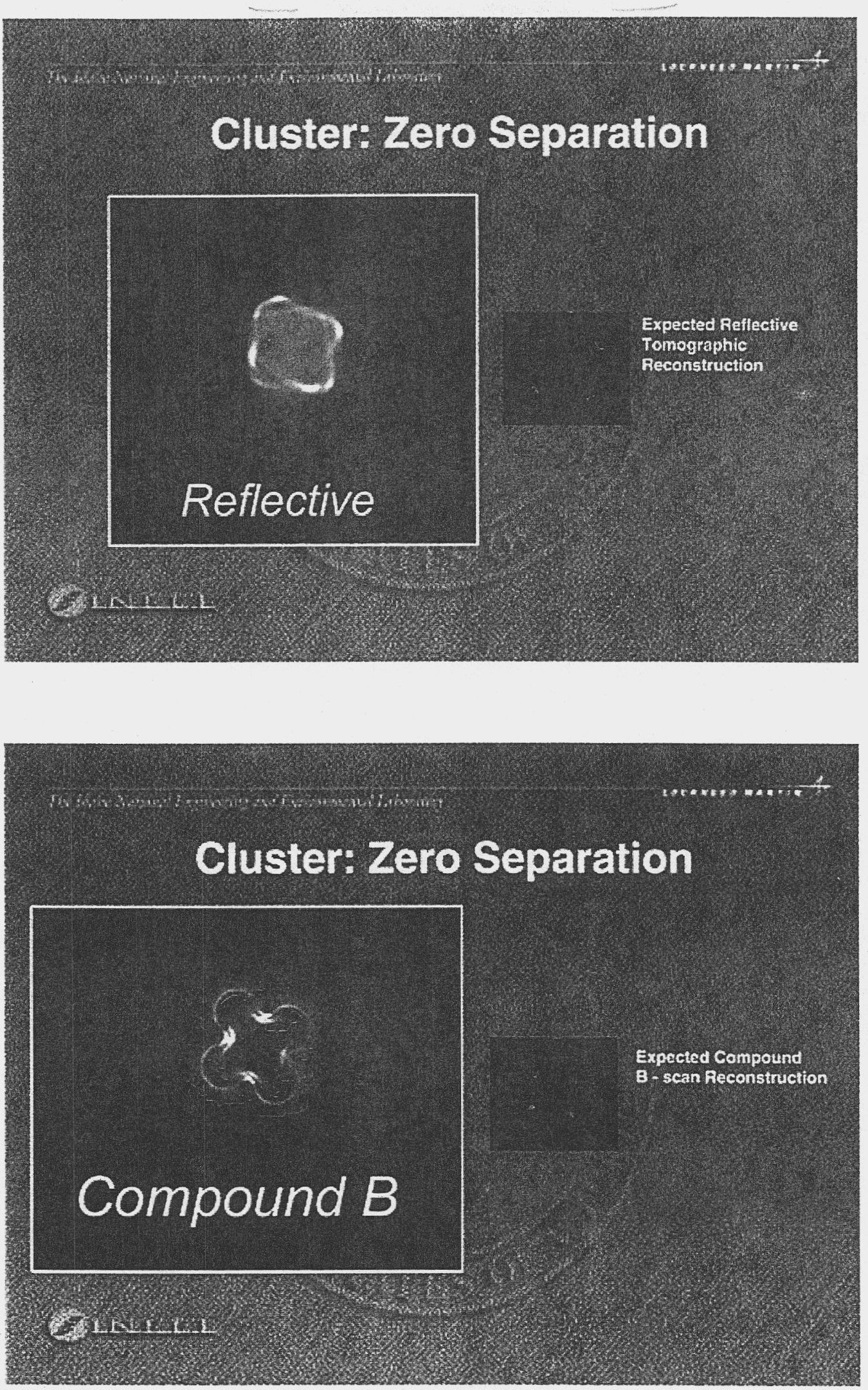

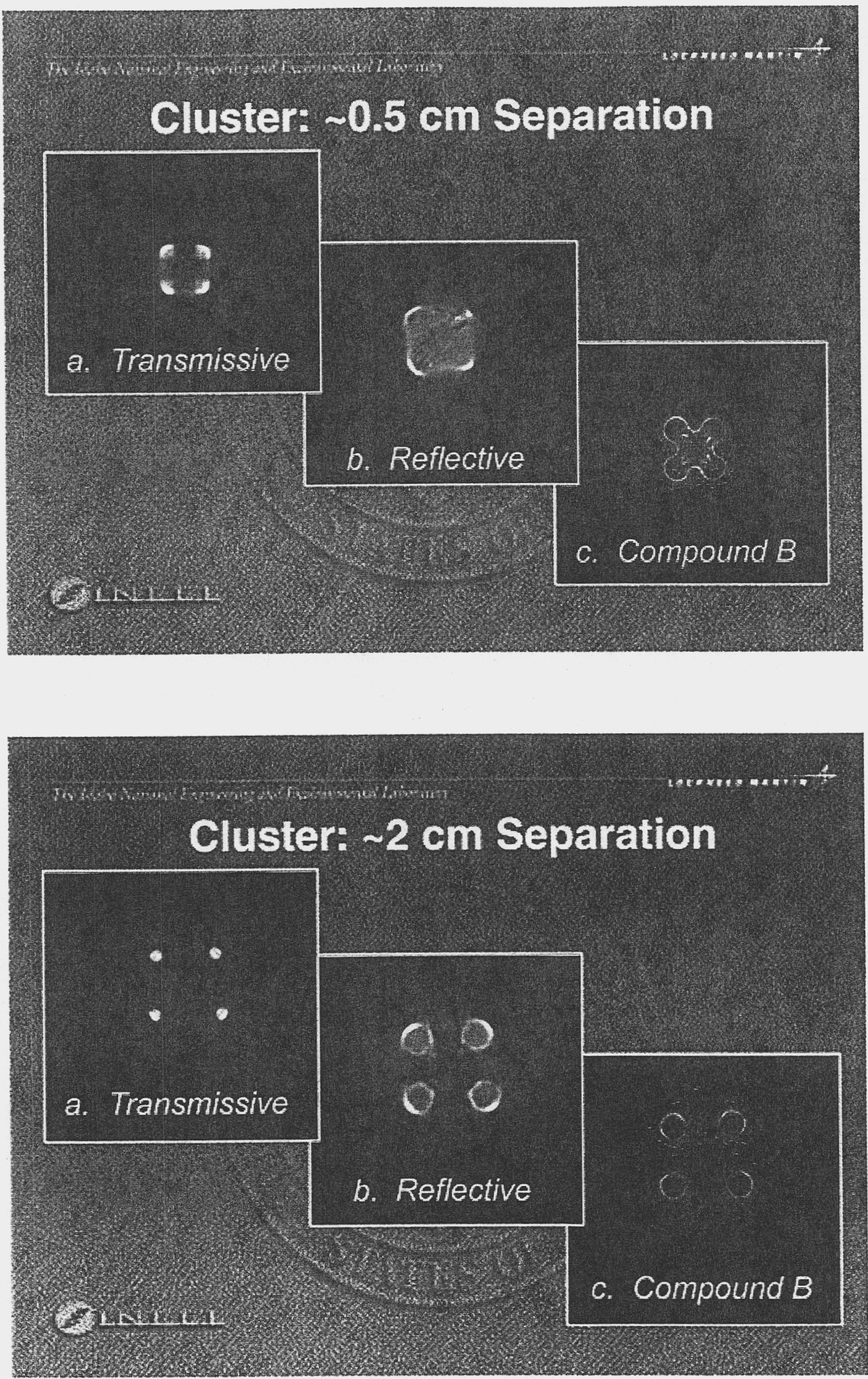

SIGNAL AND IMAGING SCIENCES WORKSHOP 1998 


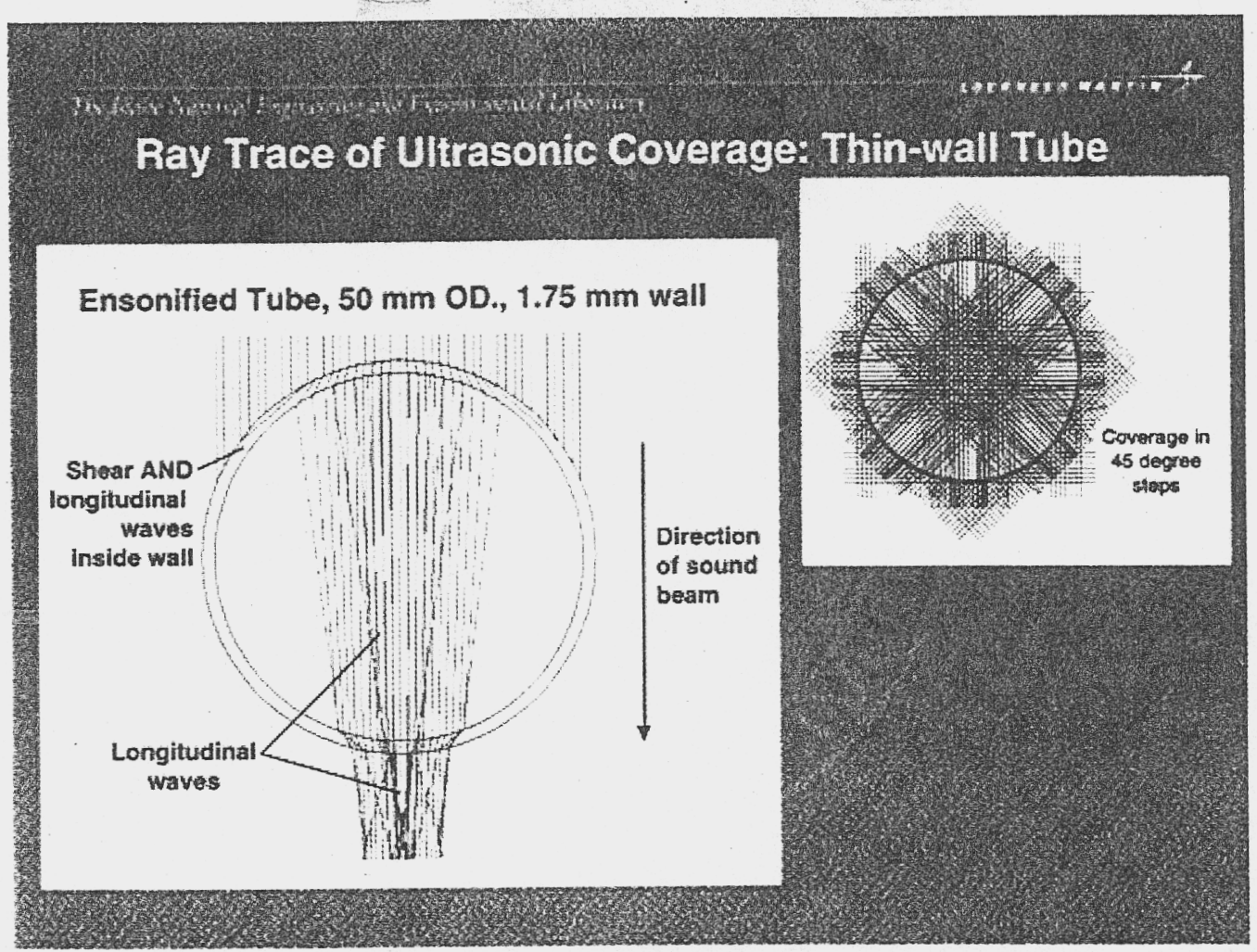

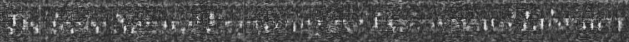

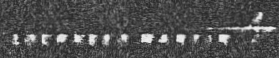

\section{Compound B-scan: Phantoms in Thin-wall Tube}

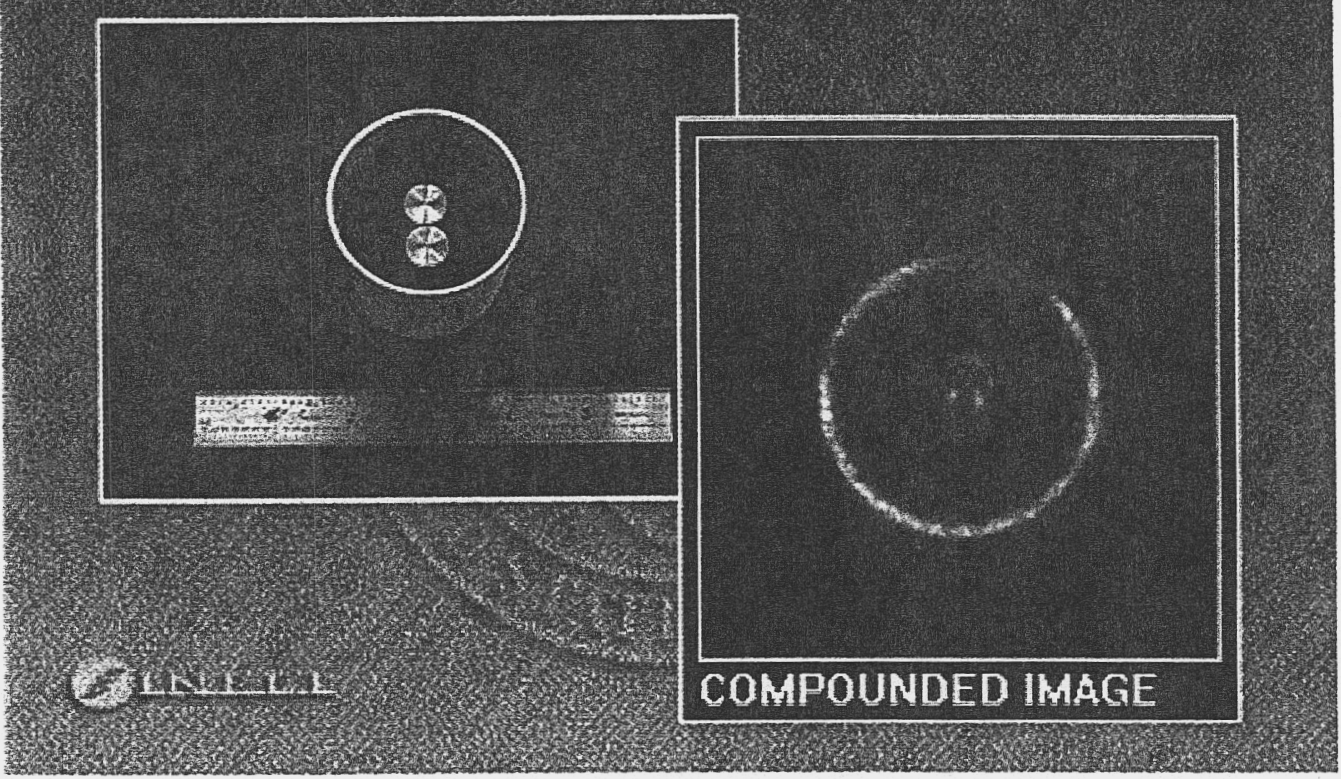



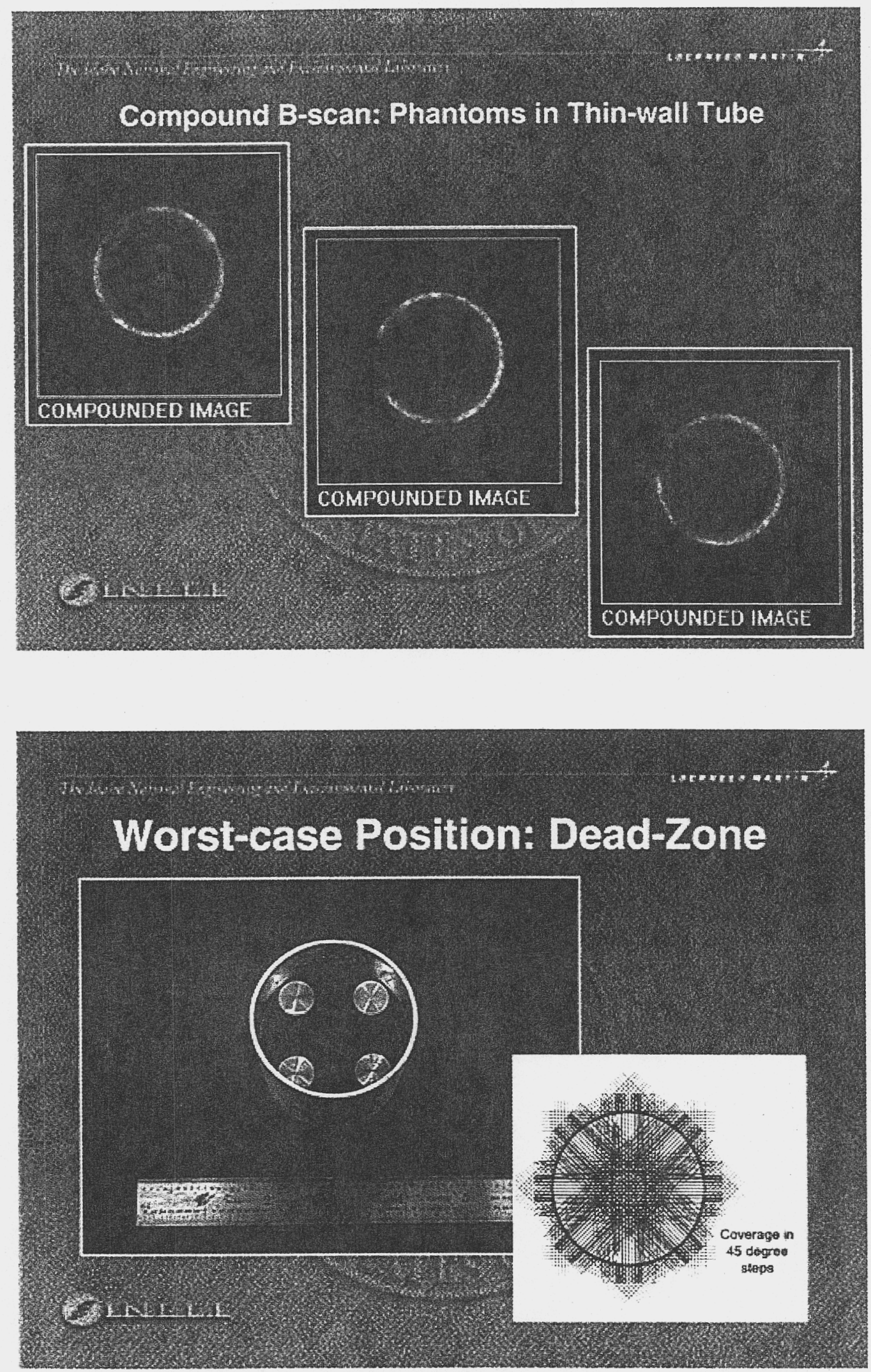

SIGNAL AND IMAGING SCIENCES WORKSHOP 1998 

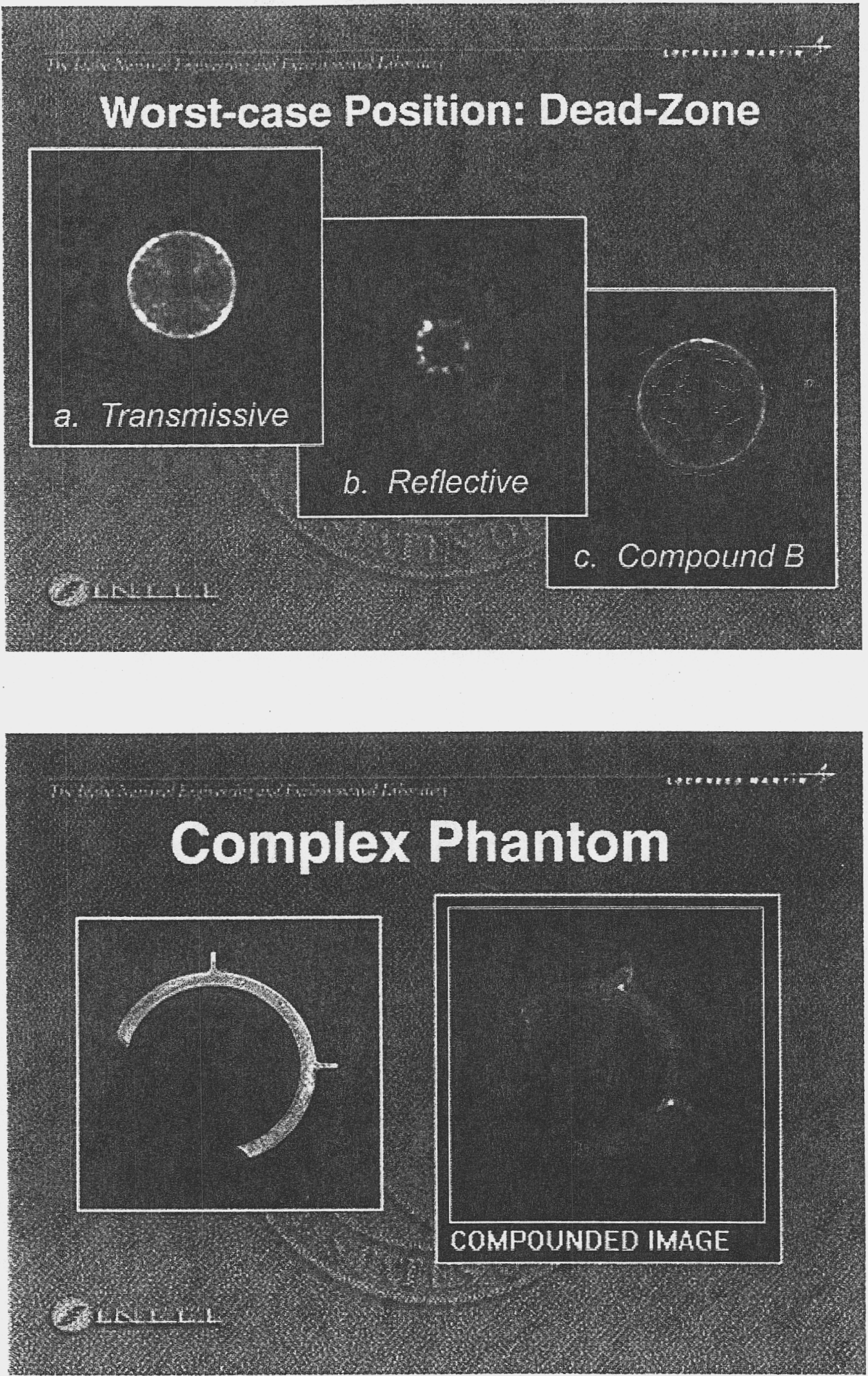


\section{Gigahertz Photodetectors: Faster, Smaller, Cheaper}

\section{$K E \&$ JS Froeschner}

Martin. Froeschner \& Associafes

14300 Mines Roud

Livermore, California 94550 USA

tel: 5204091121

fax: 9254494647

Prescritel an the

Signal and Imaging Sciences workshop

Sponsced by the Genter for Adunced Signat and Imagng Sciences

a lawrence Liwermore Vationil lahomon

12 Vorutiler $19 \%$

Lawrence Livermore National Laboratory

Adhesion assessed using shock-wave exfoliation

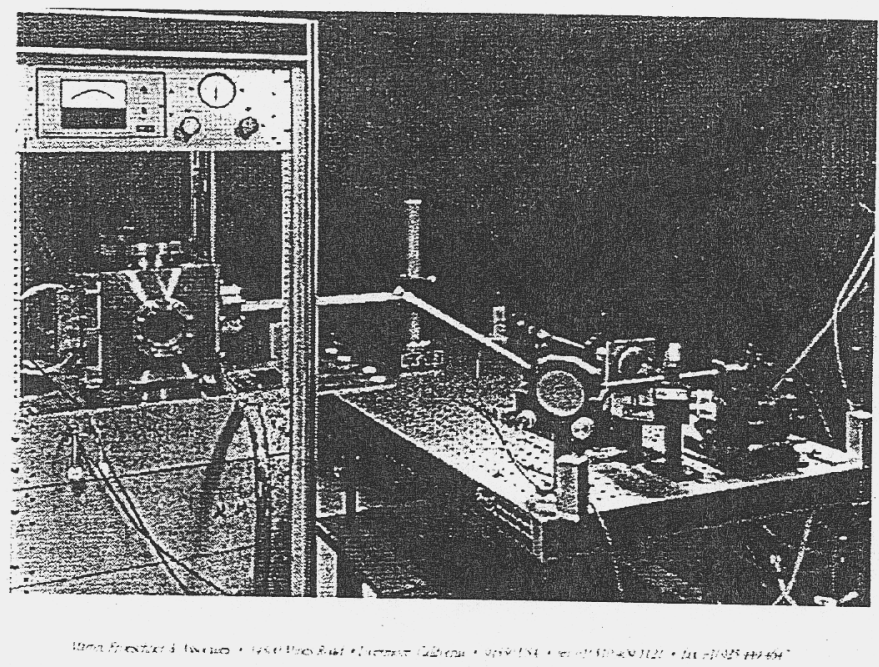




\section{Sub-nanosemond Velocimerry with a New Kind of VISAR}

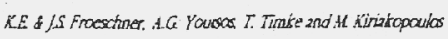

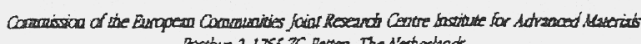

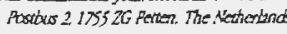

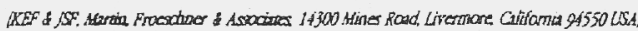

Abstrad

A fresh approach w the principles of the delay leg interferomener which was uhimatif developed by Barter, Hemsing and athers

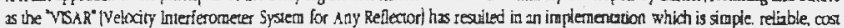
effecive and capable of yebocry measurements with sub-nanosecond lime resolution

Resulv from iaser induced shock exioliation experiments which measure the adhesion srength of hin films on cer amic subst ates are reported These experiments require resolution of the shodk sorucure produced by 20.3 or 8 nanosecond Nd. YAC baser pulke

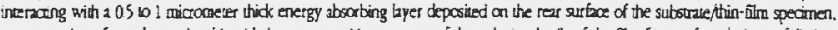

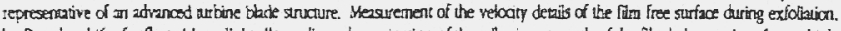

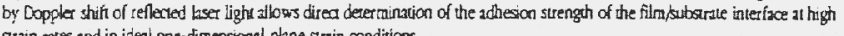
suan ates and in ideal one-dirensional plane srain conditions

The enremely small scale of the experinenl requires very high tine resolution. The Dopplet Yelociry Interieconacer developed

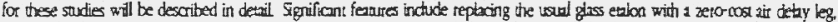

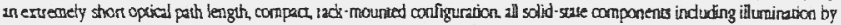
serniconductor diode laser and signal derection with sub-nanosenond PN photodiodios

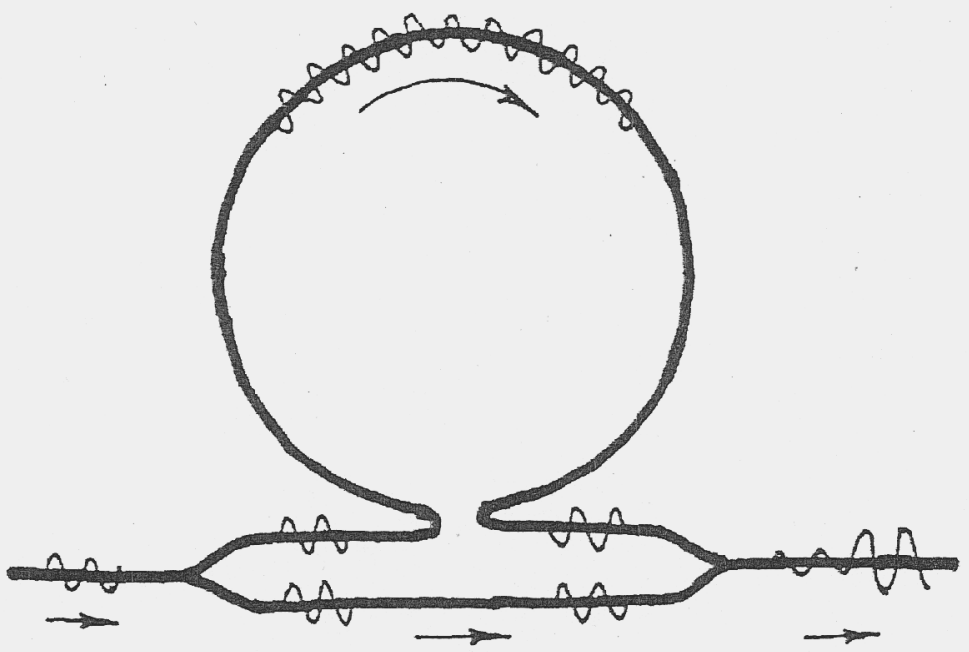

The Basic Delay leg Interferometer 


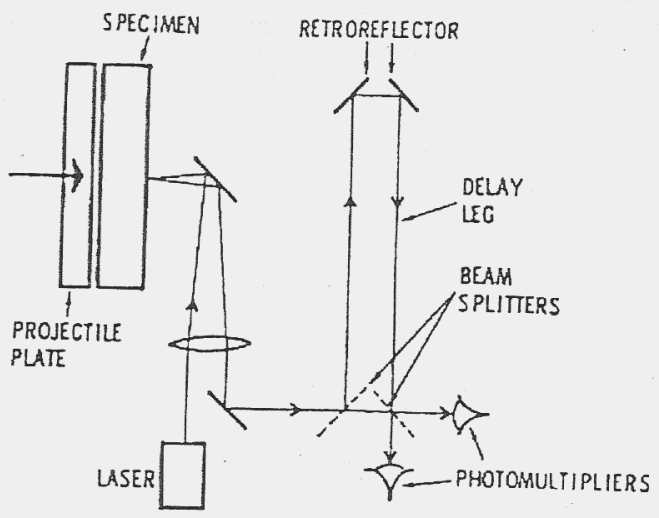

The Original Sandia Velocity Interferometer

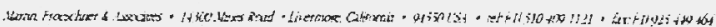

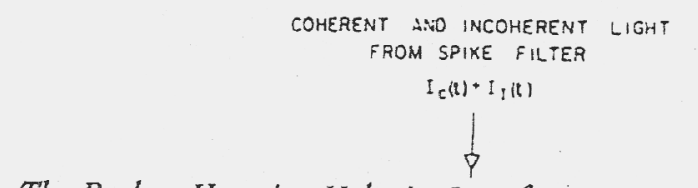

The Barker-Hemsing Velocity Interferometer System for Any Reffector, "VISAR," in Full-Quadrature, Push-Pull Configuration

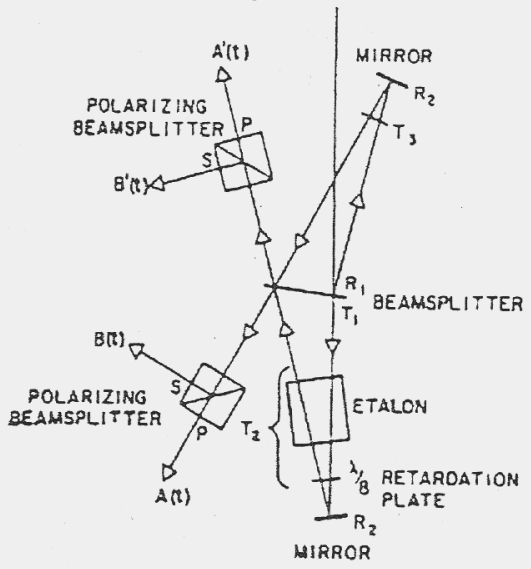

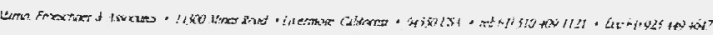



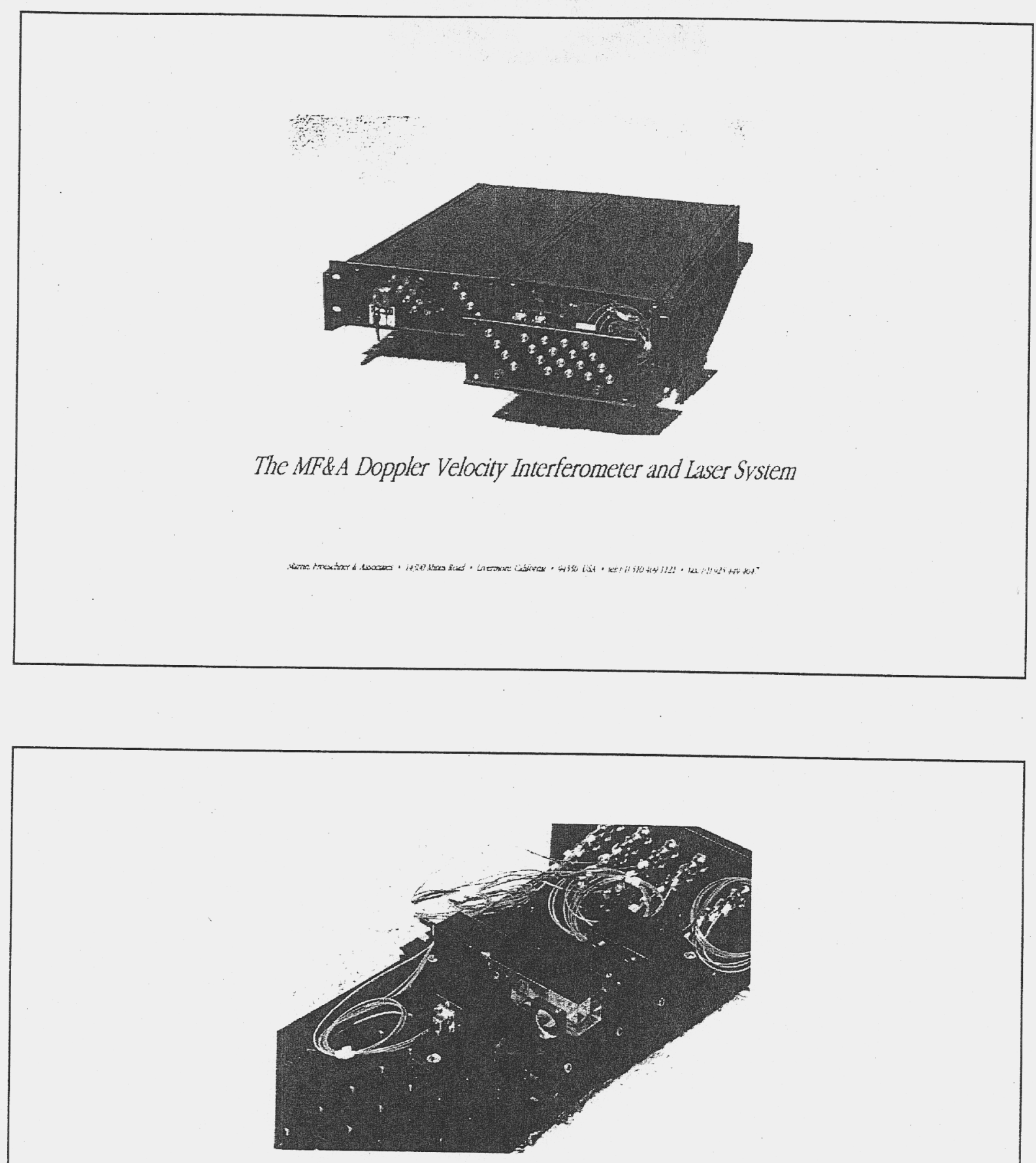

Inside the MF\&A Doppler Velocity Interforometer 


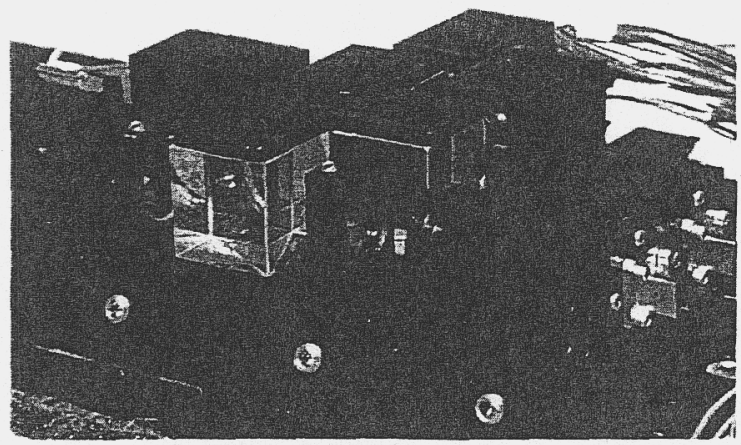

The Hcart of the Interferometer:

A Cemented Prism Assembly. That Nercr Needs Adjustment or Alignment

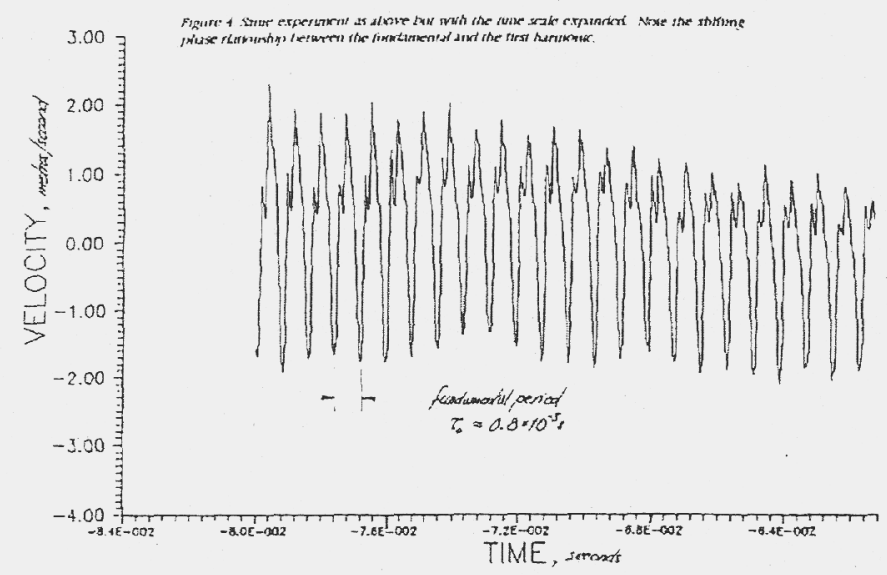

Vibration of Bond Paper Acoustically Driven at $1200 \mathrm{~Hz}$

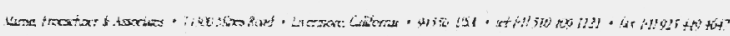




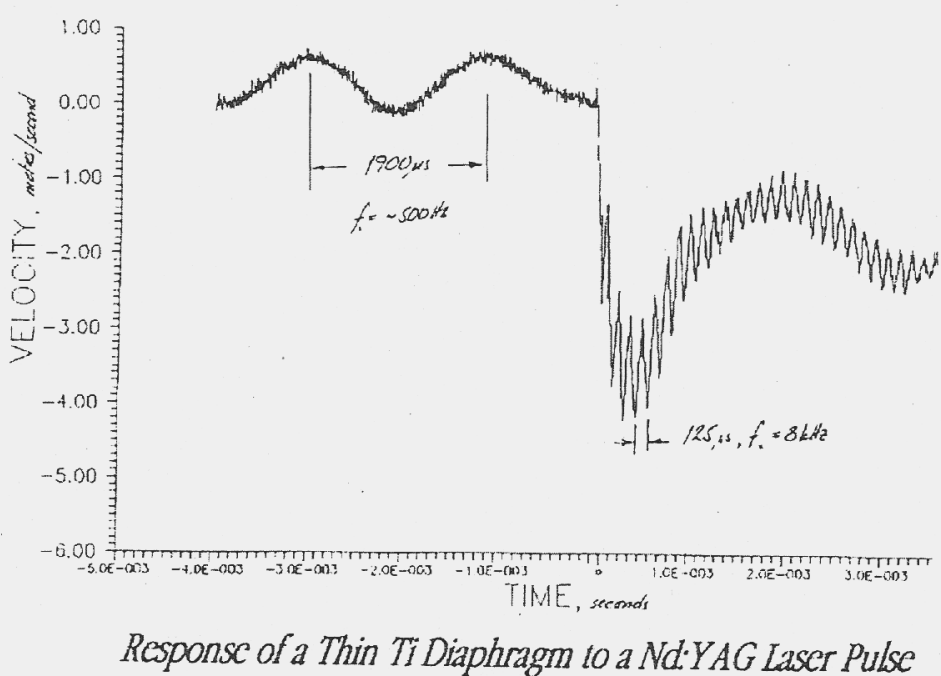

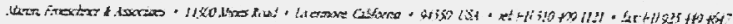

\section{Biased Detectors}

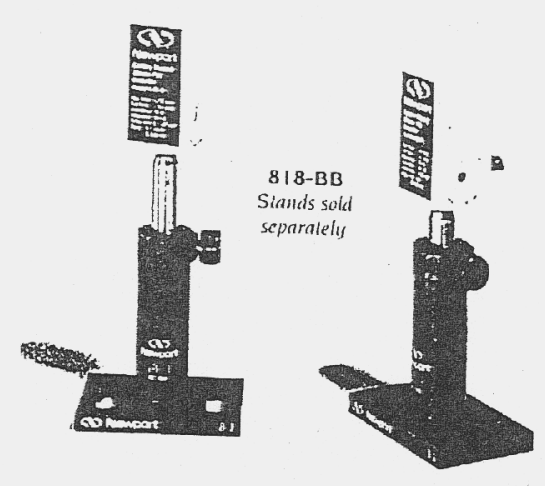




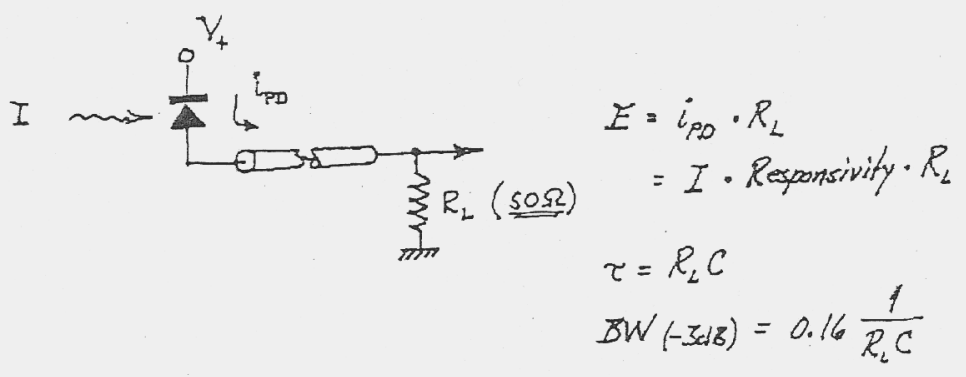

Biased Photodiode Circuit

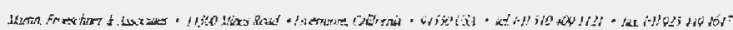

\section{Fermionics Opto-Technology}

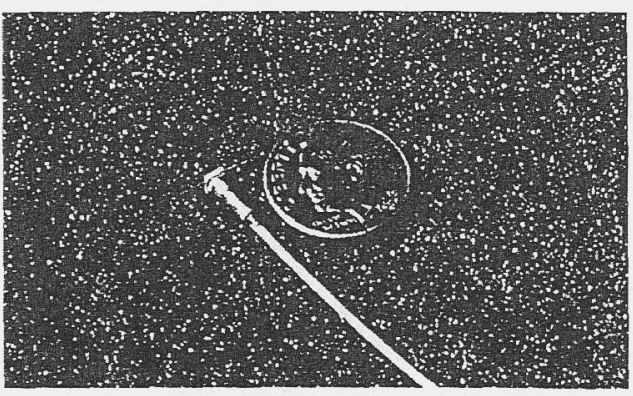

In GaAs Photodiode Bonded Directly To Optical Fibre End 


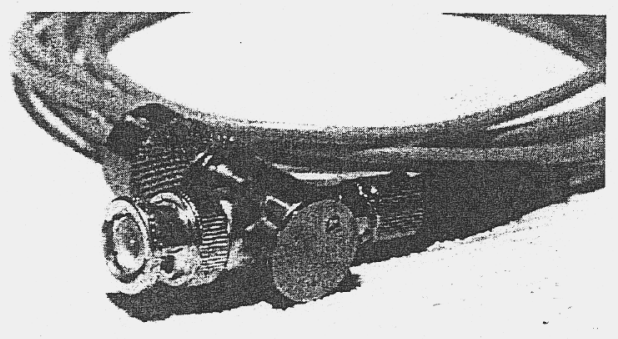

MF\&A Series 99 Biased Photodiode Module

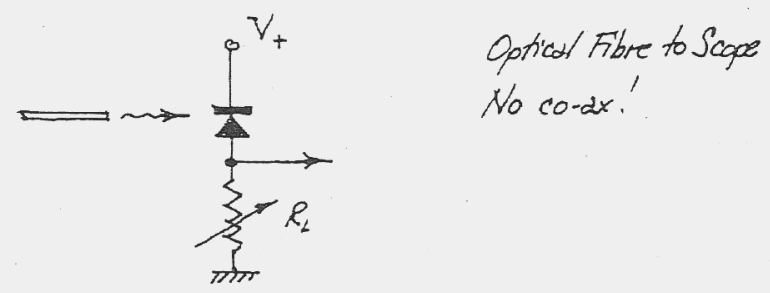

Series 99 Photodetector Module with Variable Load Resistance

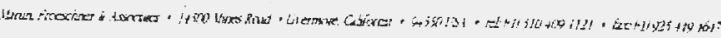




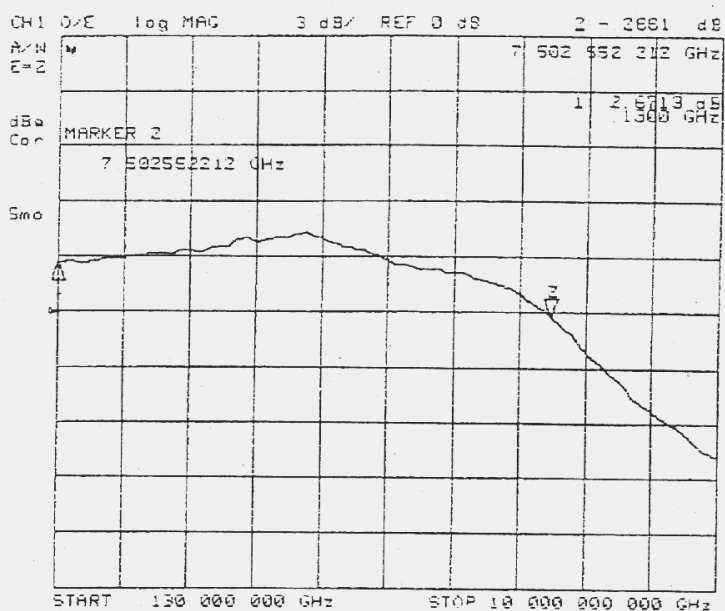

Frcquency Response for Series 99 InGaAs-2 Biased Photodiode

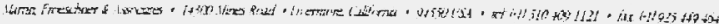

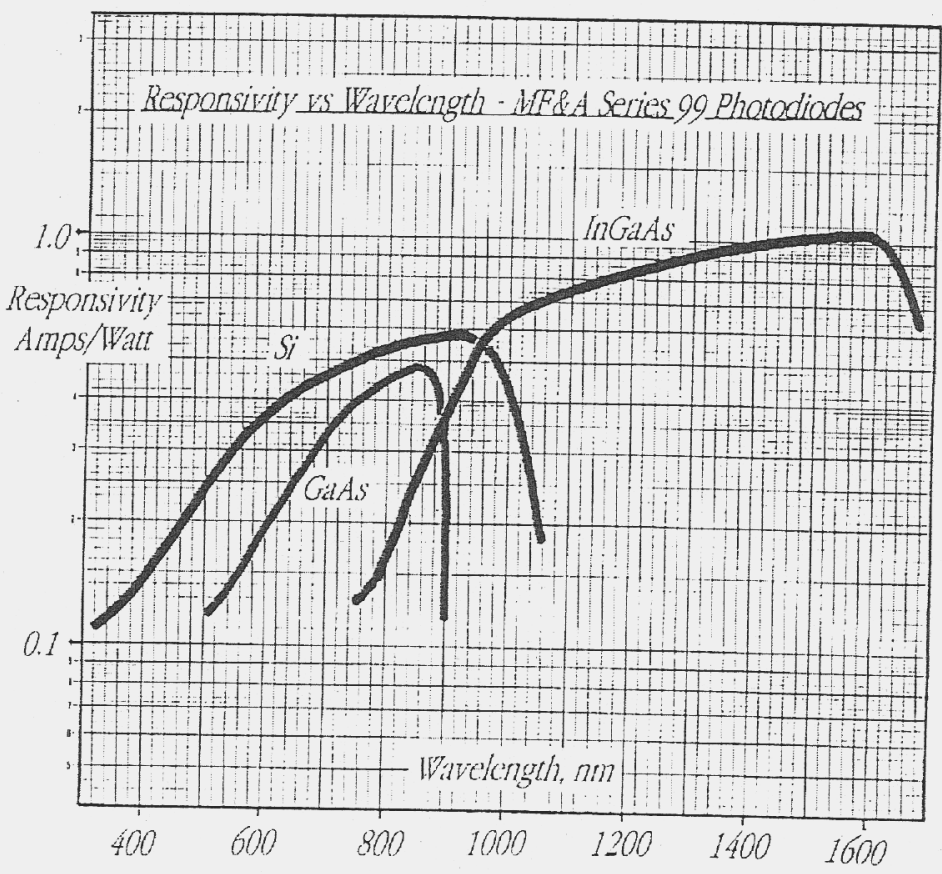

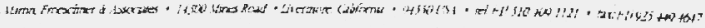




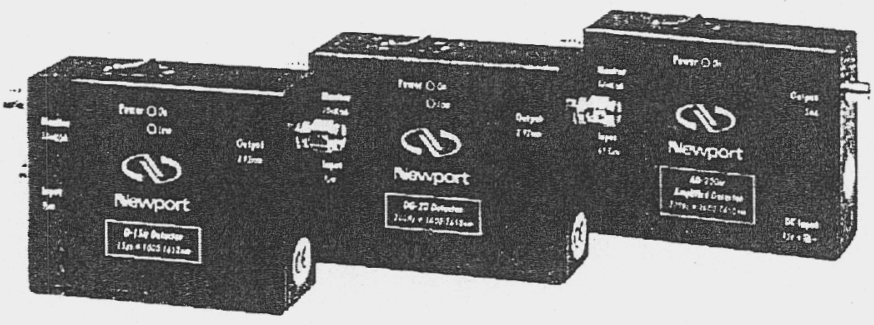

D Series Broadband Detectors $(400-1700 \mathrm{~nm})$

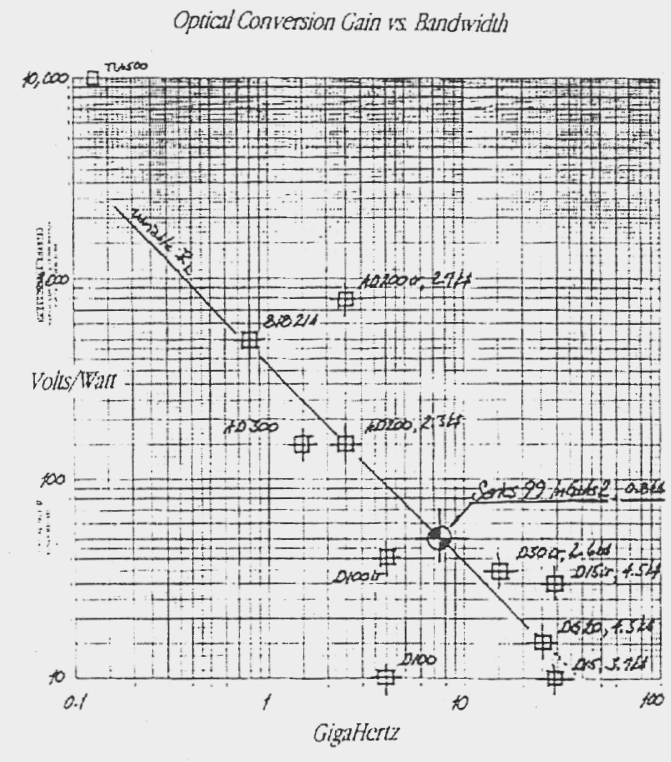



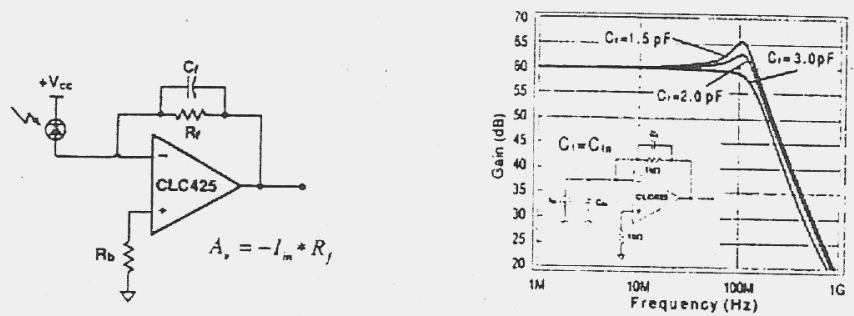

Classical Op Amp Transimpedance Amplificr

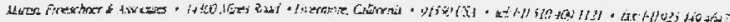

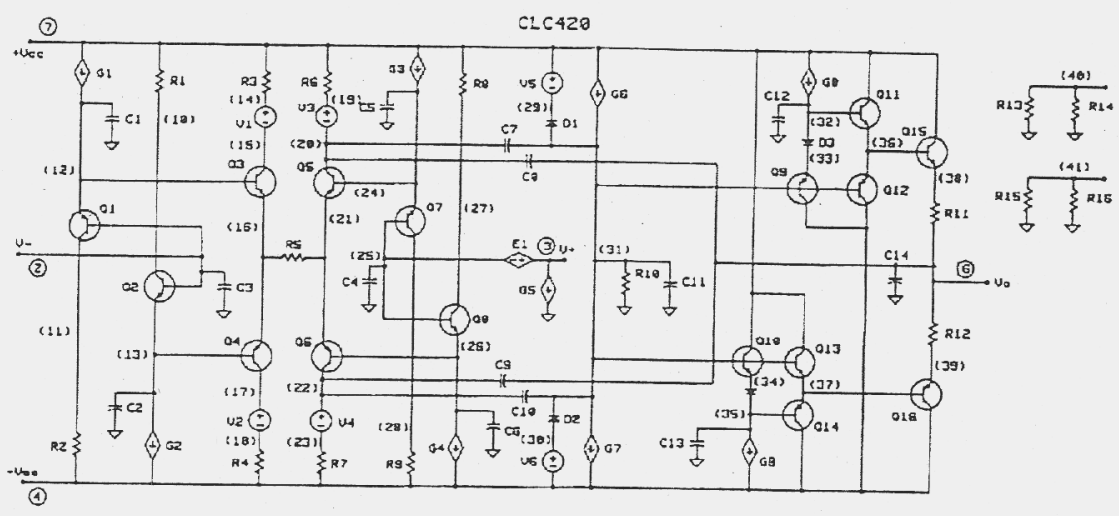

Typical Op Amp Internal Circuit Schematic

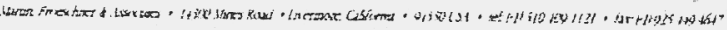



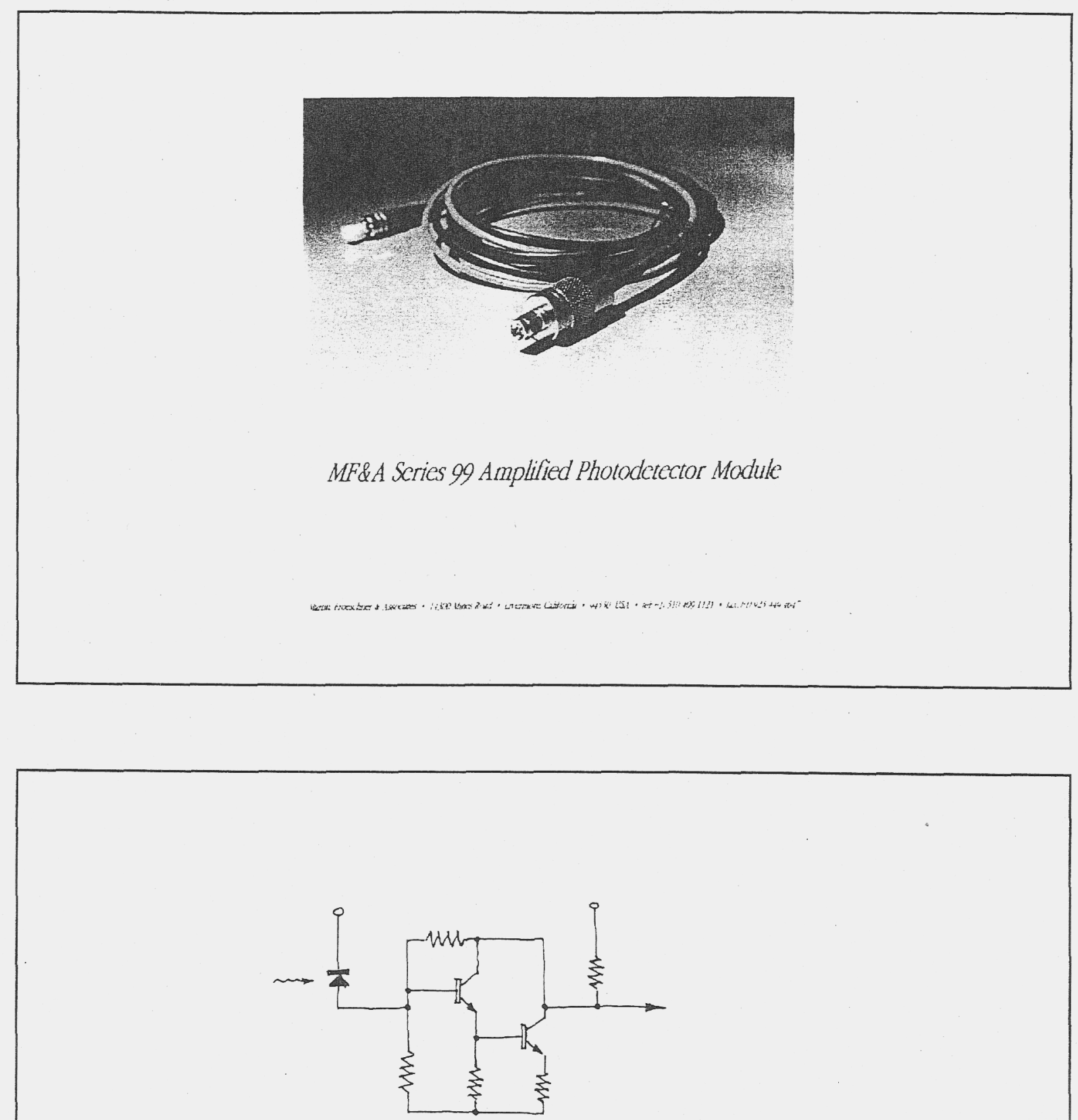

Hctcrojunction Bipolar Dariington Configuration used in MF\&A Series 99 Amplified Photodetector Modules

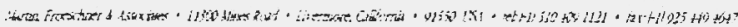




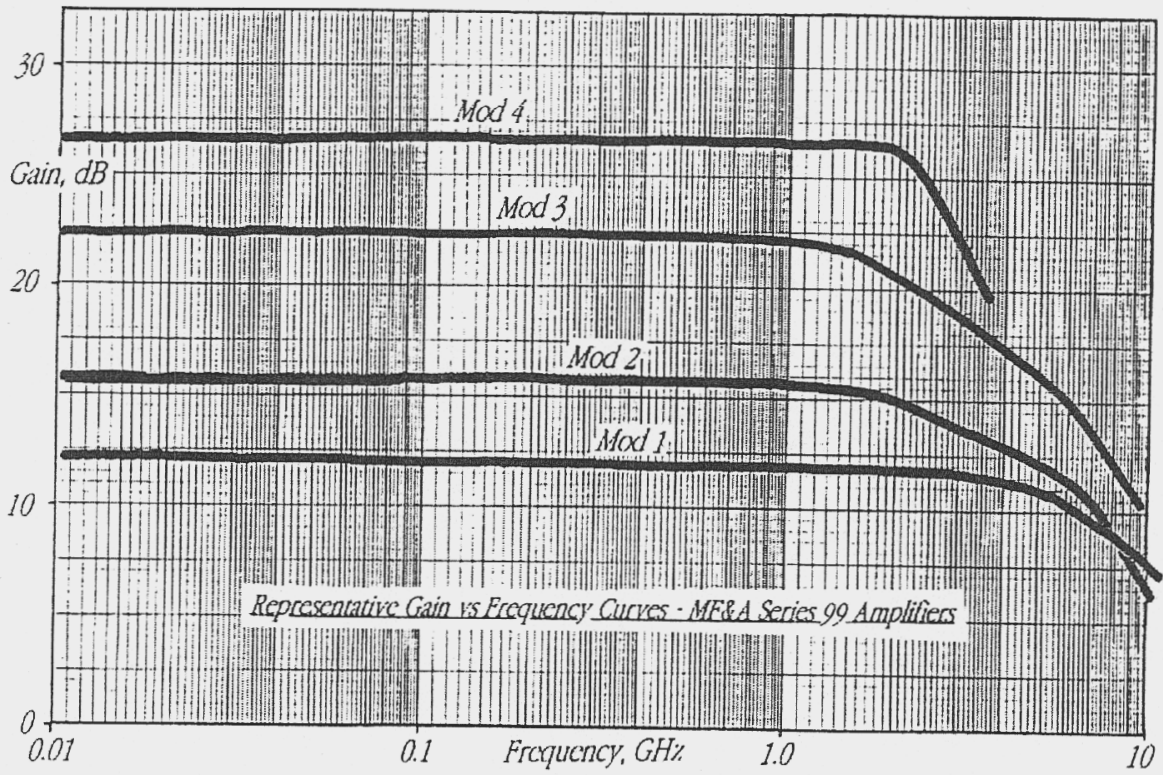

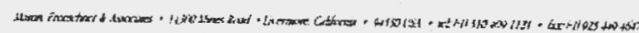

\section{Optical Conversion Gain ws. Bandwidth}

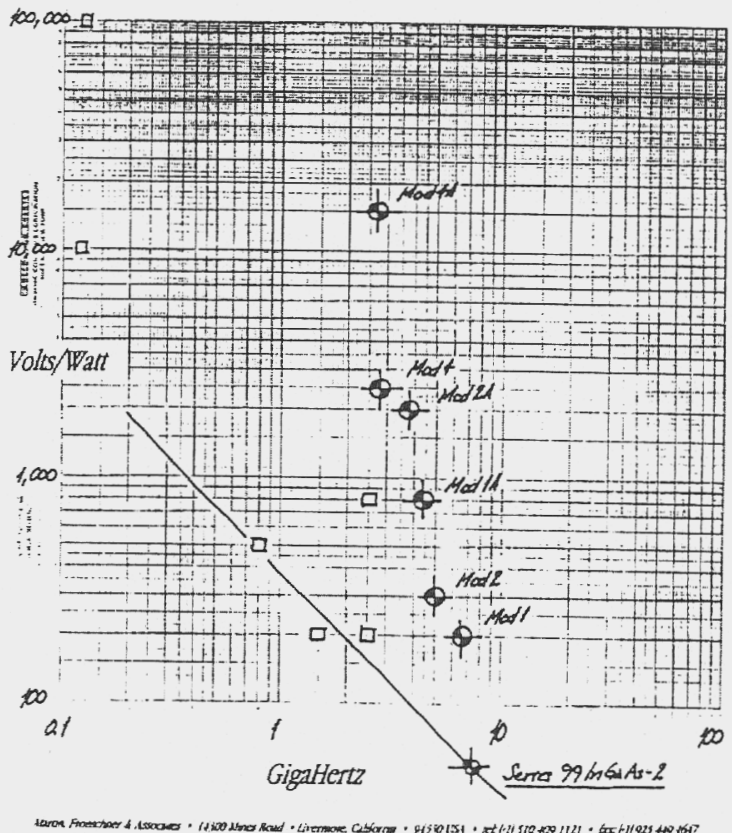




\begin{tabular}{|c|c|c|c|c|c|}
\hline & $\mathrm{Si}-1$ & $\mathrm{Si}-2$ & GaAs & $\ln (\mathrm{Ba} A \mathrm{As}-1$ & InGaAs-2 \\
\hline Speetral Range & $400-1000 \mathrm{~mm}$ & $320)-1000$ tun & $450 \cdot 870 \mathrm{lum}$ & $850 \cdot 1700 \mathrm{~nm}$ & $850-1700 \mathrm{~nm}$ \\
\hline Responsivity & $0.6 \mathrm{~A} / \mathrm{k}+850$ & $0.52 \times 1 \times 2760$ & $0.3 M N \times 850$ & (1) MNOP 550 & $0.9 \mathrm{~A} / \mathrm{Wa} 1550$ \\
\hline Bandwidtb $(-3 d b)$ & $350 \mathrm{M} 1 \mathrm{ll}$ & 1.5Gili & $10 \mathrm{GHz}$ & $3.5 \mathrm{Cl} / 2$ & $27.5 \mathrm{Gll} 2$ \\
\hline Rise/fiall Tüne & 517.5 & 231) $]^{x}$ & 40p & $100 \mathrm{ps}$ & $550 \mathrm{ps}$ \\
\hline Capacitance & $8.5 \mathrm{pF}$ & $1.5 \mathrm{pr}$ & $0.2 \mathrm{pF}$ & $0.8 \mathrm{pr}$ & $0.17 \mathrm{pl}^{\mathrm{F}}$ \\
\hline Adive Area & 254 mm dia. & 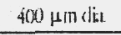 & $200 \mu \mathrm{nsq}$ & ton $\mu$ m diu. & $60 \mu$ нाn dis. \\
\hline Dark Current & slauk & $S(1.1 \mathrm{n} A$ & $\leq 0.3 \mathrm{M}$ & $50.4 \mathrm{nd}$ & $\sin 4 \mathrm{n}$ \\
\hline
\end{tabular}

\section{Photodiode Specifications}

\begin{tabular}{|c|c|c|c|c|c|c|}
\hline & Modl & Mokl2 & Mokl1h & Moxl2n & Moxl4 & $\operatorname{Mod}\{A$ \\
\hline Band widh $(-3 d B)$ & DK: in $10 \mathrm{Ghz}$ & [C.107 $7 \mathrm{Giz}$ & 7 Gillz & SGili & $\mathrm{DC}$ in $28 \mathrm{CHz}$ & $28 \mathrm{GH}$ \\
\hline Rise/fiall Time & $351 \times$ & 51110 & $50 \mathrm{ps}$ & $7(1) 10$ & $1251 \times$ & $125 p s$ \\
\hline Transimpedance & $200 \Omega$ & 3000 & $P(x) \Omega$ & $25 \% \times 1 \Omega$ & $2.5 \times 10$ & 15,0000 \\
\hline Noise Figurc & $5,1 \mathrm{~dB}=2 \mathrm{G} 1 \mathrm{lz}$ & $4.7 \mathrm{AlB} \otimes 2 \mathrm{C}_{1} \mathrm{~Hz}$ & 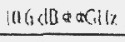 & 9) $4 \mathrm{AlB}: 2 \mathrm{CH} 2$ & $26 \mathrm{BB} \times 1.5 \mathrm{GH} 7$ & $7.3 \mathrm{~dB} \cdot 1.5 \mathrm{GHz}$ \\
\hline Coupling & IX:ar AC & $I X:$ is AC & Meraly & AC. (x)ly & DCor $A C$ & $A C$ only \\
\hline Oulput P'O & $11.3 \mathrm{c} 1 \mathrm{Bm}$ & $12+\mathrm{d} l \mathrm{~mm}$ & $11,3 \mathrm{dBm}$ & $12+\mathrm{dlllm}$ & 2 2 $d 13 m$ & $124 \mathrm{~dB} \mathrm{in}$ \\
\hline
\end{tabular}

Transimpedance Amplificr Specifications

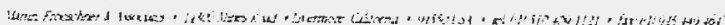




\section{Martin, Froeschner \& Associates Series 99 Photodetector Modules}

Comparison of Measurcd Risetimes to Specifications

\begin{tabular}{|c|c|c|c|c|c|c|}
\hline & Serial No. & $t_{\cos }$ & $t_{\alpha i}$ & $t_{\mathrm{mad}}$ & $t_{\text {amp }}$ & tak \\
\hline InGaAs2 Mod1 & 135 & 55ps & 550 & 35 & - & $61 p 0$ \\
\hline Siz Modf Hod s & 136 & $280 p s$ & 230 & 125 & 125 & $290 \mathrm{ps}$ \\
\hline InGAAsI $\operatorname{Mod} 3$ & 091 & 100ps & 100 & 120 & - & $156 p s$ \\
\hline InGaAs2 Mad2 & 105 & $62 p s$ & $\leq 50$ & 50 & - & $71 p s$ \\
\hline InGaAs2 HodIA & 148 & $80 p s$ & $\leq 50$ & 35 & 35 & $70,0 s$ \\
\hline
\end{tabular}

Risetimes are from $10 \%$ 10 90: measured by independent laboratories.

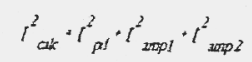

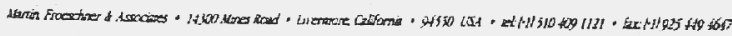

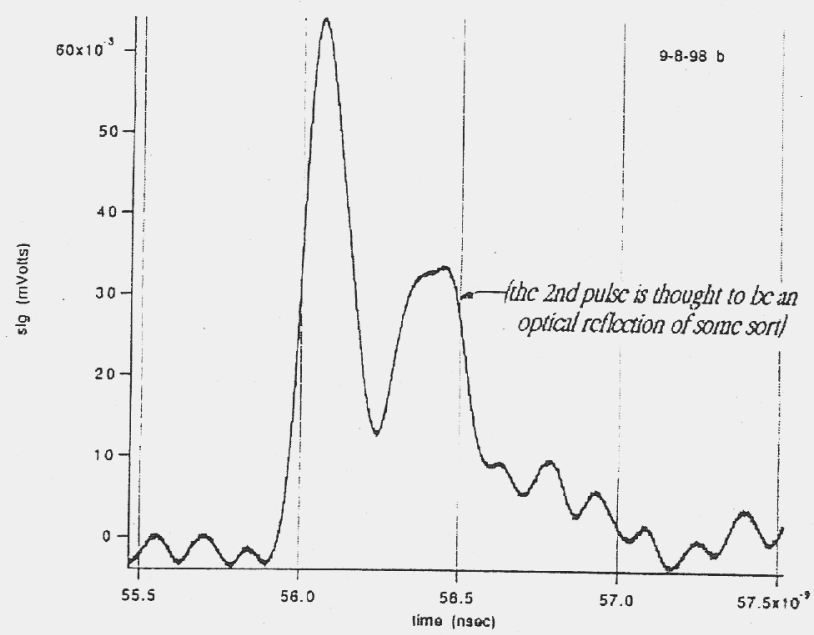

Response of Series 99 InGaAs-2 Mod 1 A Photodetector to 90 femtosecond $820 \mathrm{~nm}$ Pulsc 


\section{Noise Recorded by Independent Lab (CIENA Corporation)}

$$
N_{\text {snis } 9 g^{2}} \cdot \sqrt{1.98^{2} \cdot 1.24^{2}} \cdot 1.5 m \mathrm{rmms}
$$

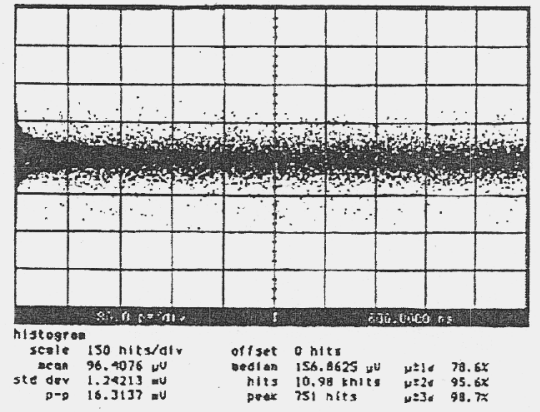

Baseline

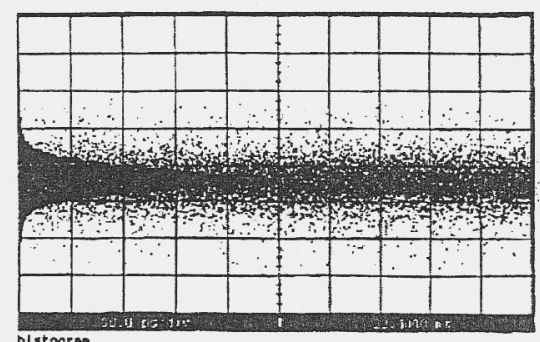

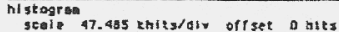

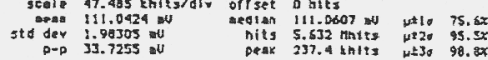

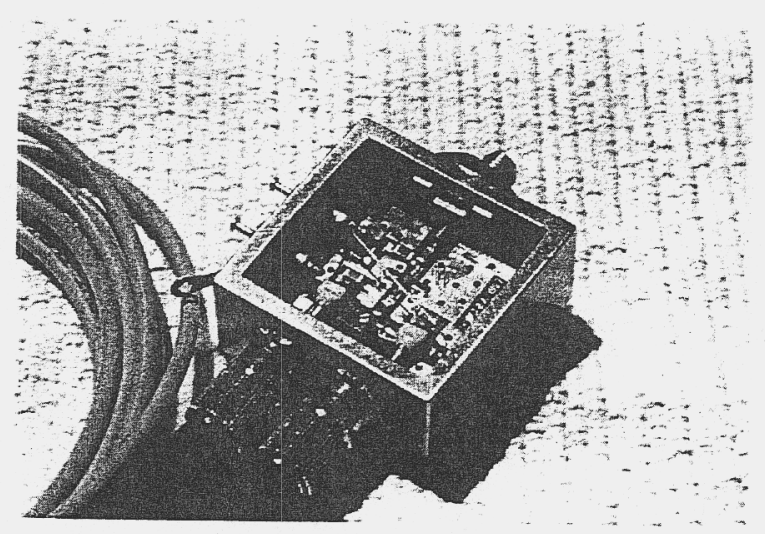

MF\& A $2.5 \mathrm{~Gb} / \mathrm{sec}$ Differential Optical Recciver 


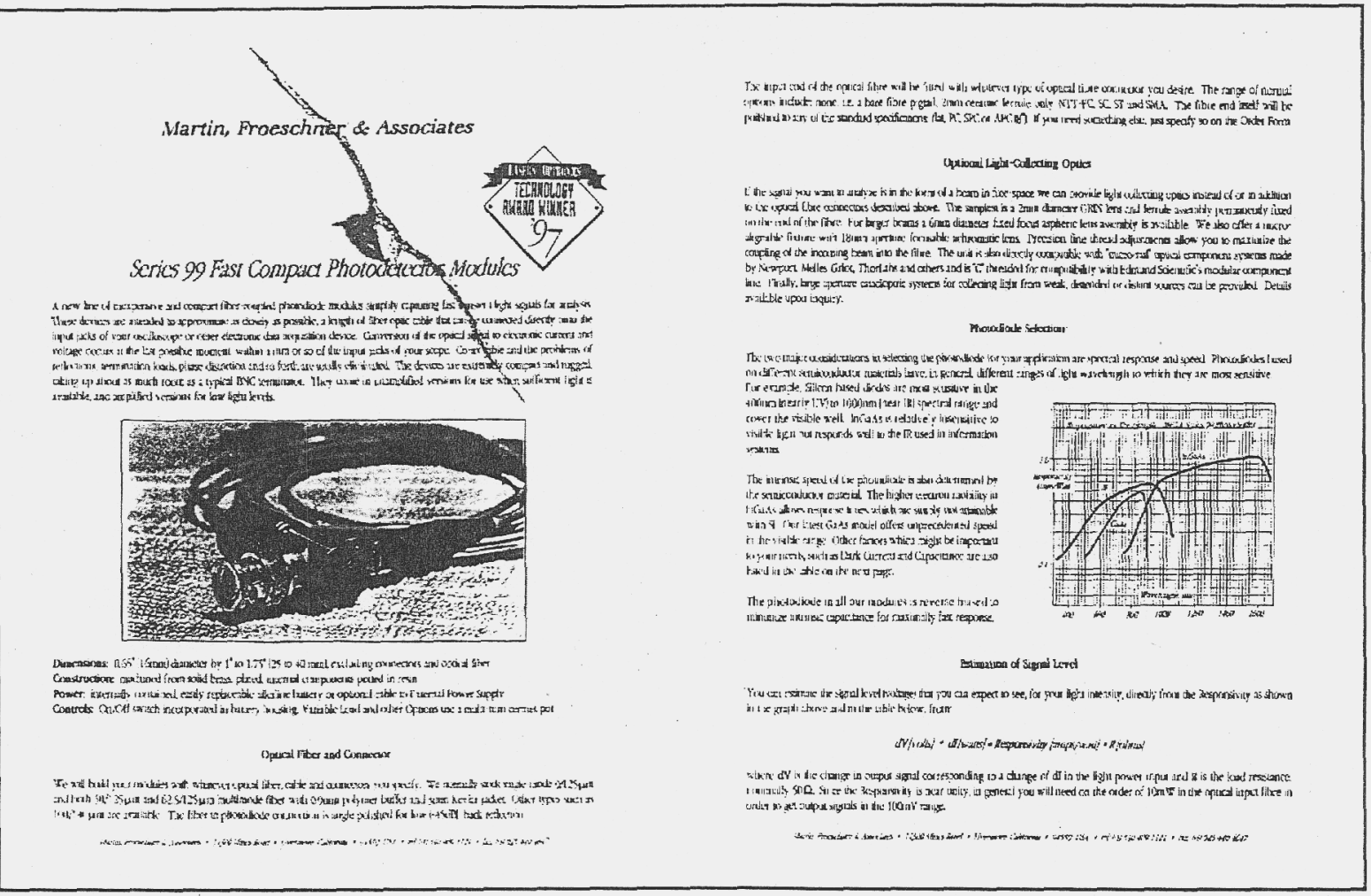

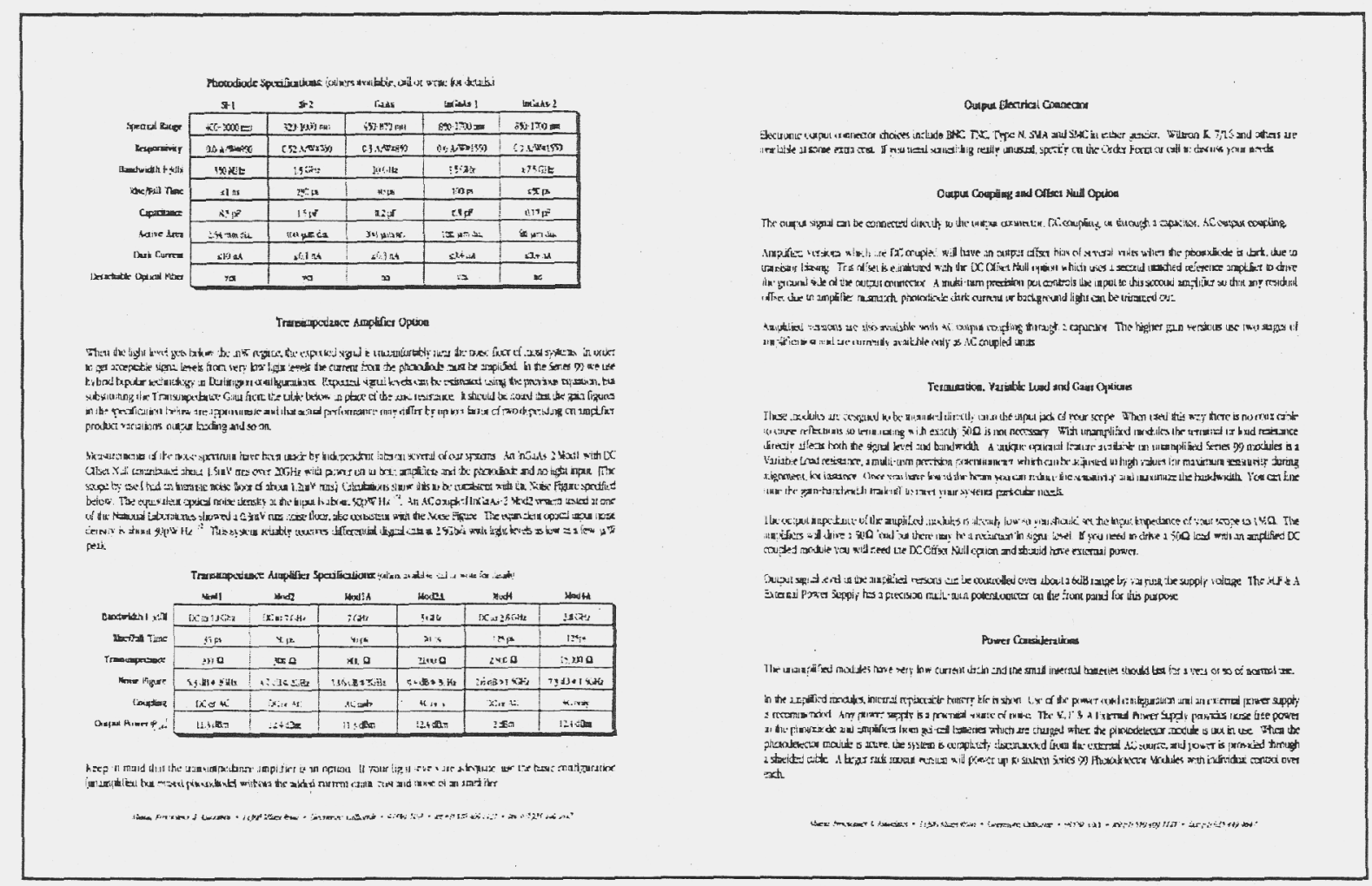


You can have a Series 99 PHOTODETECTOR HODULE built to your specificutions.

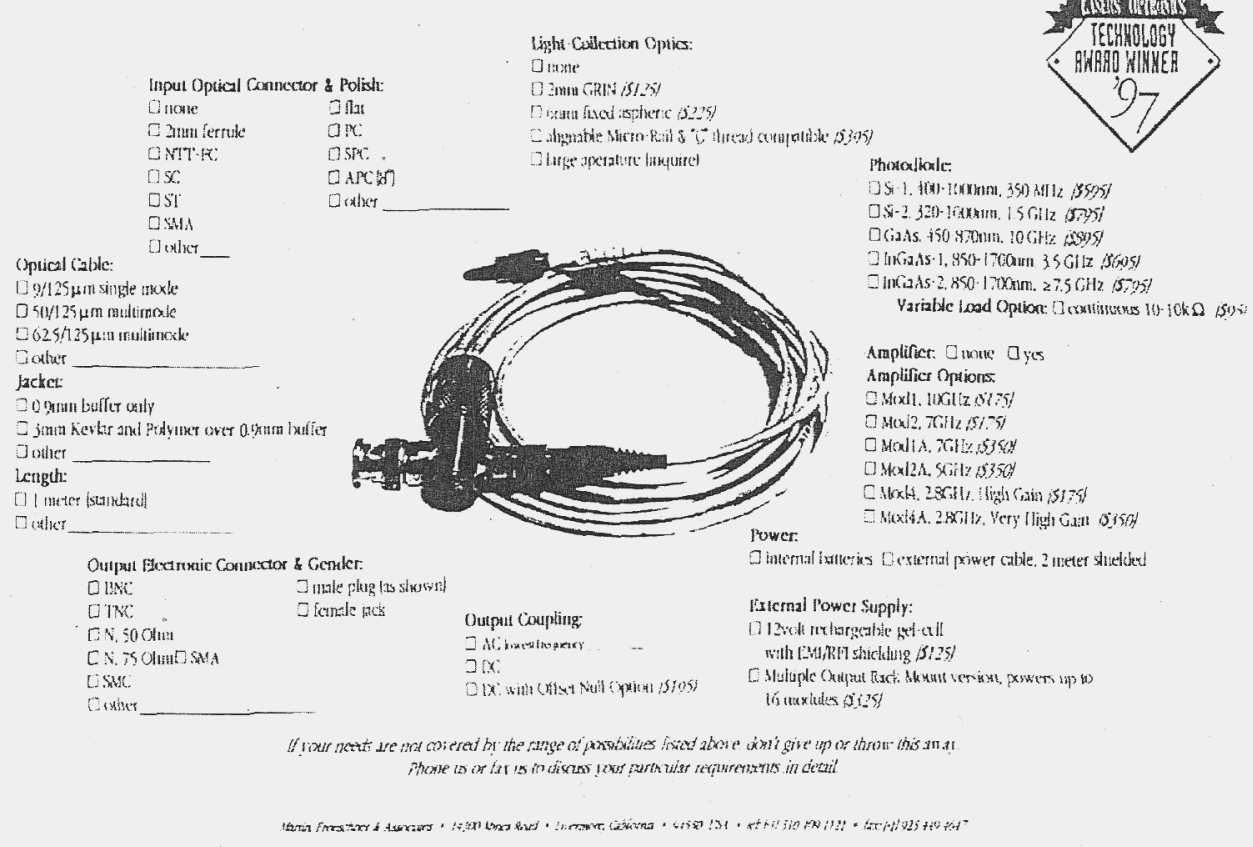




\title{
InGaAs Linescan Camera System for Paper Web Moisture Measurements
}

\author{
By Jose E. Hernandez
}

The latest results in applying InGaAs sensor technology for measuring moisture on paper will be presented. Traditionally lead sulfide sensors have been used for measuring moisture on paper at 1.9 microns. The lead sulfide detector is a photo resistive element, and requires complex electronics and temperature stabilization to work properly. InGaAs on the other hand operates in a photo voltaic mode, which is quite stable, however, standard InGaAs only operates to about 1.7 microns. We will present preliminary results of a system that has been tested on a paper web using a less optimum absorption band at 1.4 microns, but which benefits from using an InGaAs based linescan camera system. This system also has the main benefit of providing a continuos moisture measurement across the entire web, compared to current scanning systems which only sample a small percentage of the web. 


\title{
Evaluation of an Amorphous Selenium Array for Industrial X-Ray Imaging
}

\author{
Clint Logan, Lawrence Livermore National Laboratory
}

The large market for digital $\mathrm{x}$-ray imaging in medicine has driven the development of flat panel imaging devices. We evaluated one such device using the higher energy $\mathrm{x}$-rays typical of industrial applications. This imager is a $360 \mathrm{x} 430-\mathrm{mm}$ array employing amorphous selenium (amSe). It was developed and manufactured by Sterling Diagnostic Imaging. Liberty Technologies is developing a product for the NDE market. We obtained this array from Liberty Technologies.

The defining characteristic of this technology is that electrons produced by radiation interaction in the Se are directly collected and processed. There is no intermediate scintillation or other conversion process. We compare performance to existing LLNL technology. We found:

- Modulation transfer function remains high as spatial frequencies approach the limit imposed by the $139 \mu \mathrm{m}$ pixel size.

- Increasing x-ray energy degrades spatial resolution performance somewhat, but the amSe imager performs well in spectra as energetic as $450-\mathrm{kV}$ applied potential.

- The low-frequency response, $<0.1 \mathrm{~mm}$ - 1 , of this amSe imager is poor at energies of interest to NDE. Secondary radiation transport in material behind the $\mathrm{Se}$ is a significant contributor to this attribute.

- Required exposure for the spectra tested ranges from 10 to 100 mRoentgen. This is 15 to 100 times less than LLNL's home-built system employing scintillating glass and a CCD camera. Cycle time for CT applications will be dominated by read and refresh time of nearly 1 minute. This will allow for great flexibility in utilizing microfocus (low power) sources or large souce-todetector distances.

- Except for issues of peripheral electronics, which we did not address, Monte Carlo simulations suggest that this amSe imager could offer attractive performance at $\mathrm{x}$-ray energies of $3 \mathrm{MeV}$ or more.

This amSe imager shows great promise for digital radiography and CT. We encountered no problems in operating it in $\mathrm{x}$-ray spectra up to $450 \mathrm{kV}$ applied voltage. UCRL-JC-132315 Abs 


\section{Evaluation of an Amorphous Selonium Array tôt \\ Industrlal X-Ray Imaging}

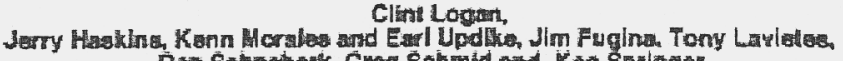
Don Sehneberi, Gres Schmid and Keo Springer

$$
\text { Peat Sortioni }
$$

Libery Technologies. ine

Conshohoction

Ken Siverta

Stonting Dlagnostic inagring

Mevanis, Dokgmara

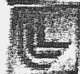

Slgnal and Imaging Setances Workahop November 12, 1000

amSe imager directly collects electrons with no intermediate conversion to light

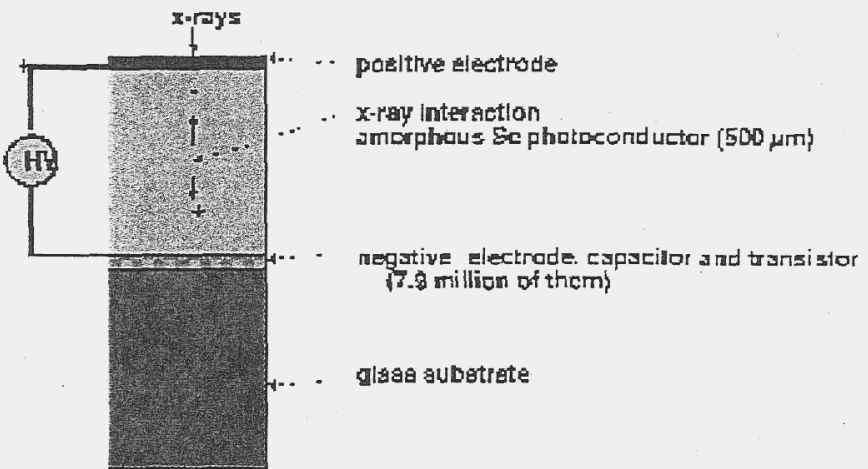

\begin{tabular}{|c|c|}
\hline ixel pitch & $139 \mathrm{~mm}$ (Nyqulst of 3.6 latmm) \\
\hline I factor & 86吸 \\
\hline & 14 bils \\
\hline a & 14 inch $x$ in inch $(2,560$ x 3,072 plxcis $)$ \\
\hline
\end{tabular}


We evaluated performance with three heavily-filtered spectra

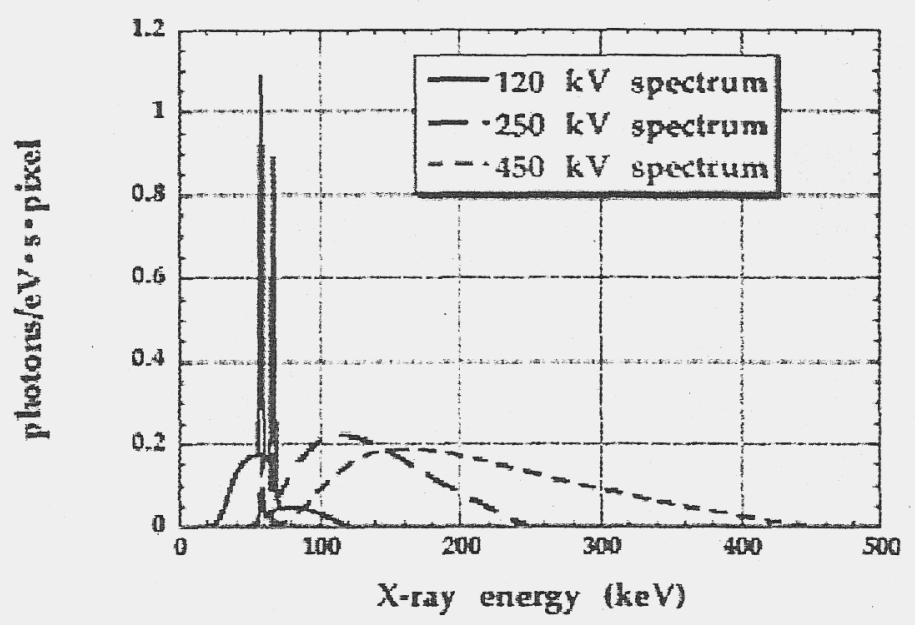


Monte Carlo results indicate degraded MTF as energy increases

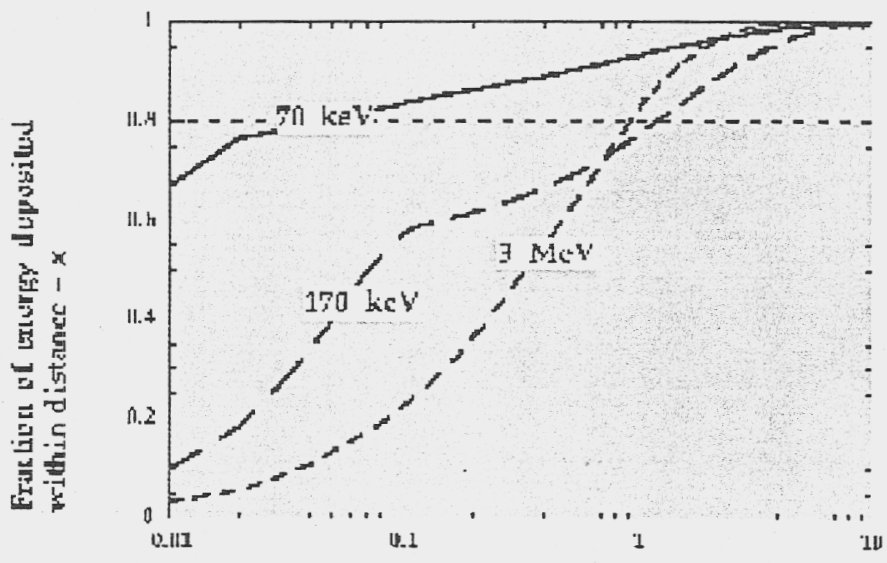

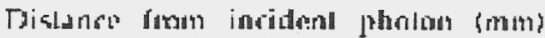

We used a 450-kV Philips source at $3 \mathrm{~m}$
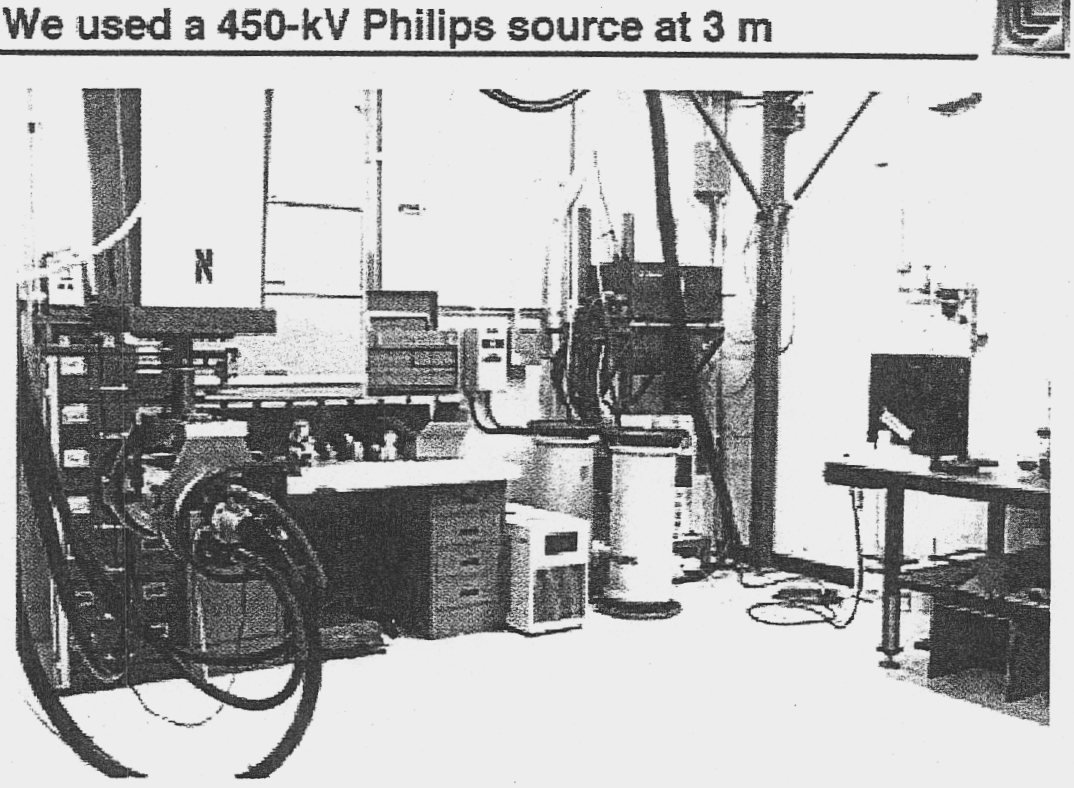
Test objects are: an edge, a slug, and a line-pair gage
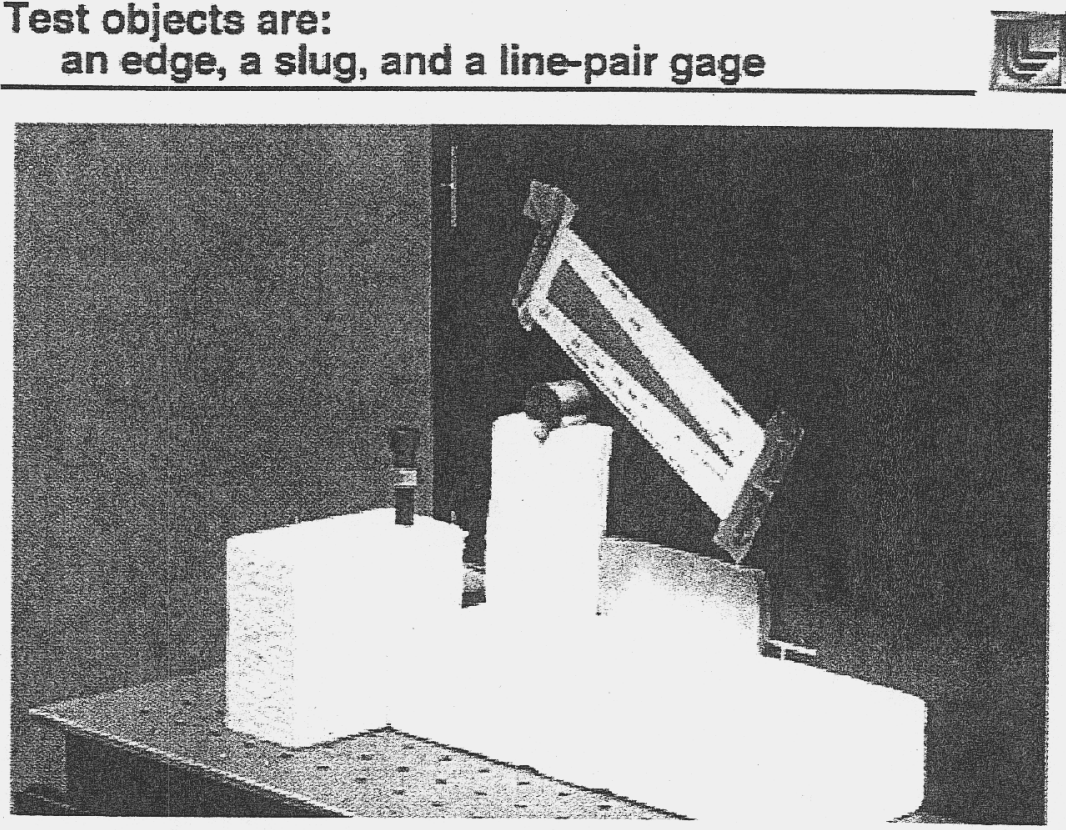

The amSe imager requires fewer $x$-rays than a lens-coupled CCD system
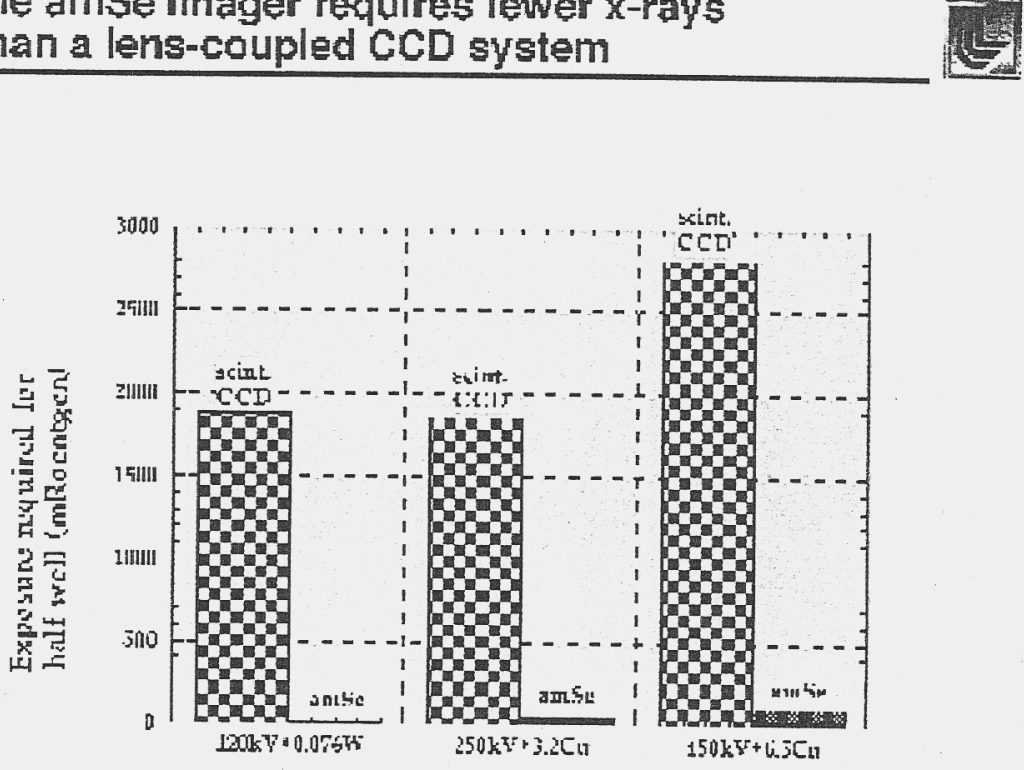


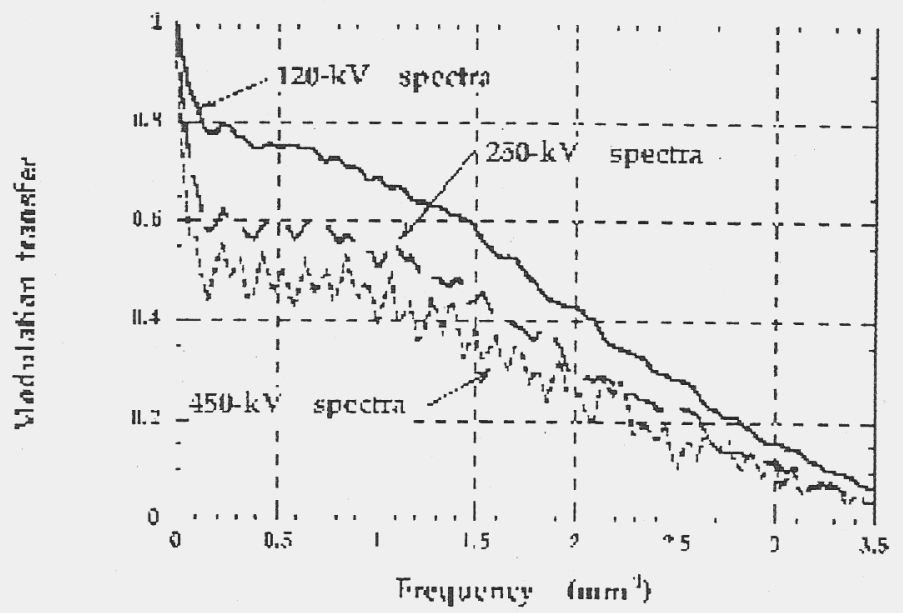

amSe is superior to glass/CCD at all but the lowest spatial frequencies

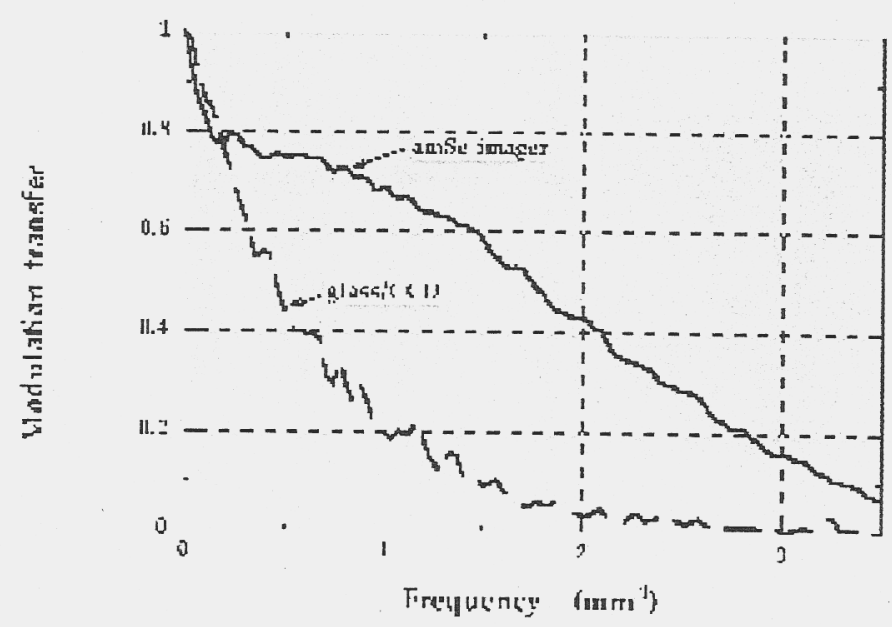


poor low frequency MTF for amSe imager is evident in image of a slug

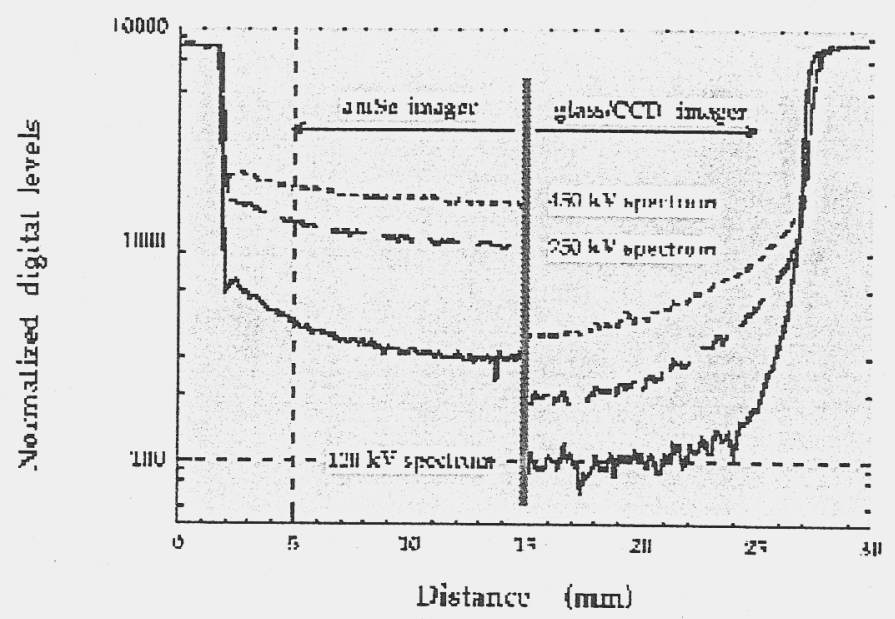

\section{Let the images speak}

The object is a $\mathrm{Pb}$ line pair gage $0.1 \mathrm{~mm}$ thick.

The $x$-ray spectrum is the $450-k V$ spectrum, so the object is very low contrast. Images are raw with no corrections.

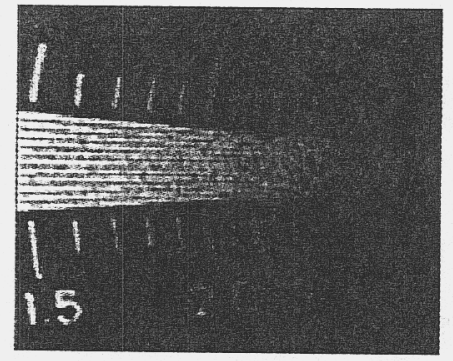

glass/CCD

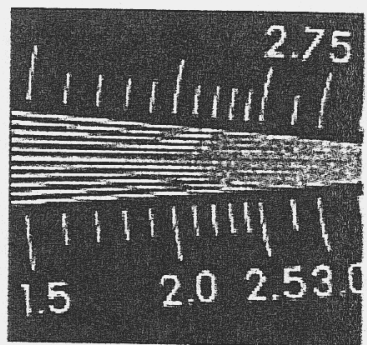

amse

The amse imager has extremely interesting attributes. 


\title{
Matched Field Imaging of Laser-Based Ultrasound Data
}

\author{
Robert Huber, James Candy \\ Lawrence Livermore National Laboratory
}

Laser-based ultrasonic nondestructive evaluation is a non-contact testing and characterization technique that extends the application of ultrasonic testing to a wider variety of testing situations. This includes materials at elevated temperature, parts with curved surfaces, components with highly polished surfaces, which would be damaged by contact or immersion techniques, and hazardous materials. Although, laser-based ultrasound offers several advantages over traditional piezoelectric transducer ultrasound, its lower sensitivity restricts its use. Signal processing offers a way of increasing the sensitivity of laser-based ultrasound. Matched field imaging has been applied to data obtained from laser-based ultrasound tests. This signal processing technique uses a novel correlation canceling approach to locate flaws in parts. This technique compares data obtained from a real part to data obtained from a flawless reference. Through this processing, information that correlates between the two data sets is eliminated, leaving information about any flaws present in the part. Data will be presented on tests run on an aluminum part before and after a drill hole was placed in the part. Images obtained using the matched-field technique will be shown.

This work was performed under the auspices of the Department of Energy by the Lawrence Livermore National Laboratory under contract W-7405-Eng-48. 


\section{Matched Field Imaging of Laser-Based Ultrasound Data}

Robert Huber

James Candy 


\section{Talk Organization}

- Introduction

- LU Problem (Defect detection and imaging)

- Signal Pre-processing

- Matched Field Imaging

- Summary 


\section{PZT or LBU}

Piezoelectric Transducers

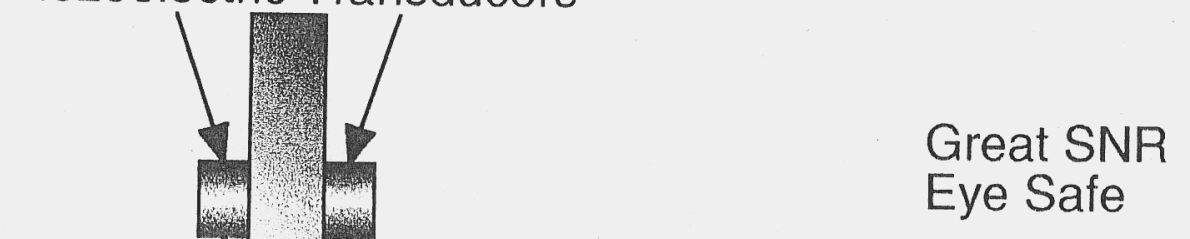

Eye Safe

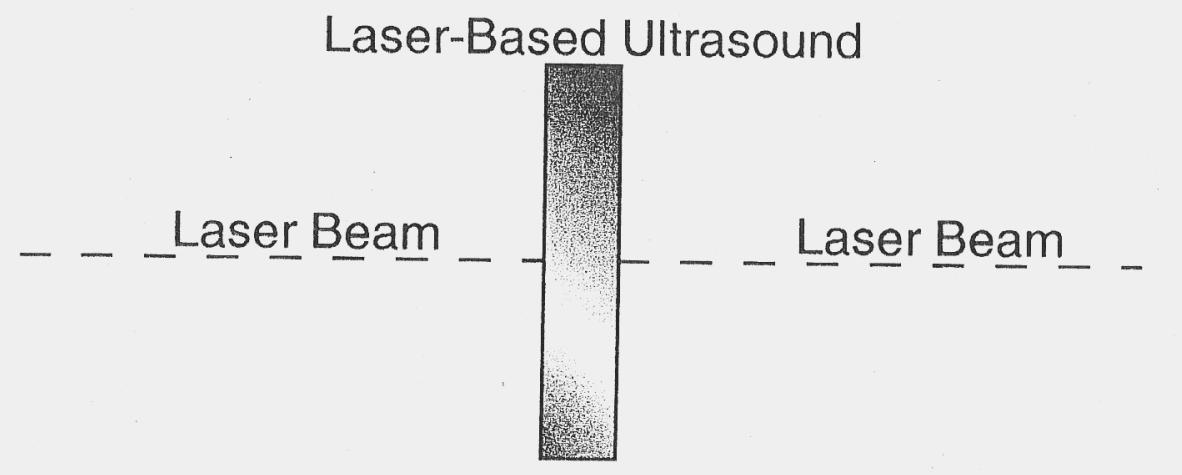

Non-contact

Remote

Small Footprint

Does not load or

damage surface 


\section{Signal Variation}
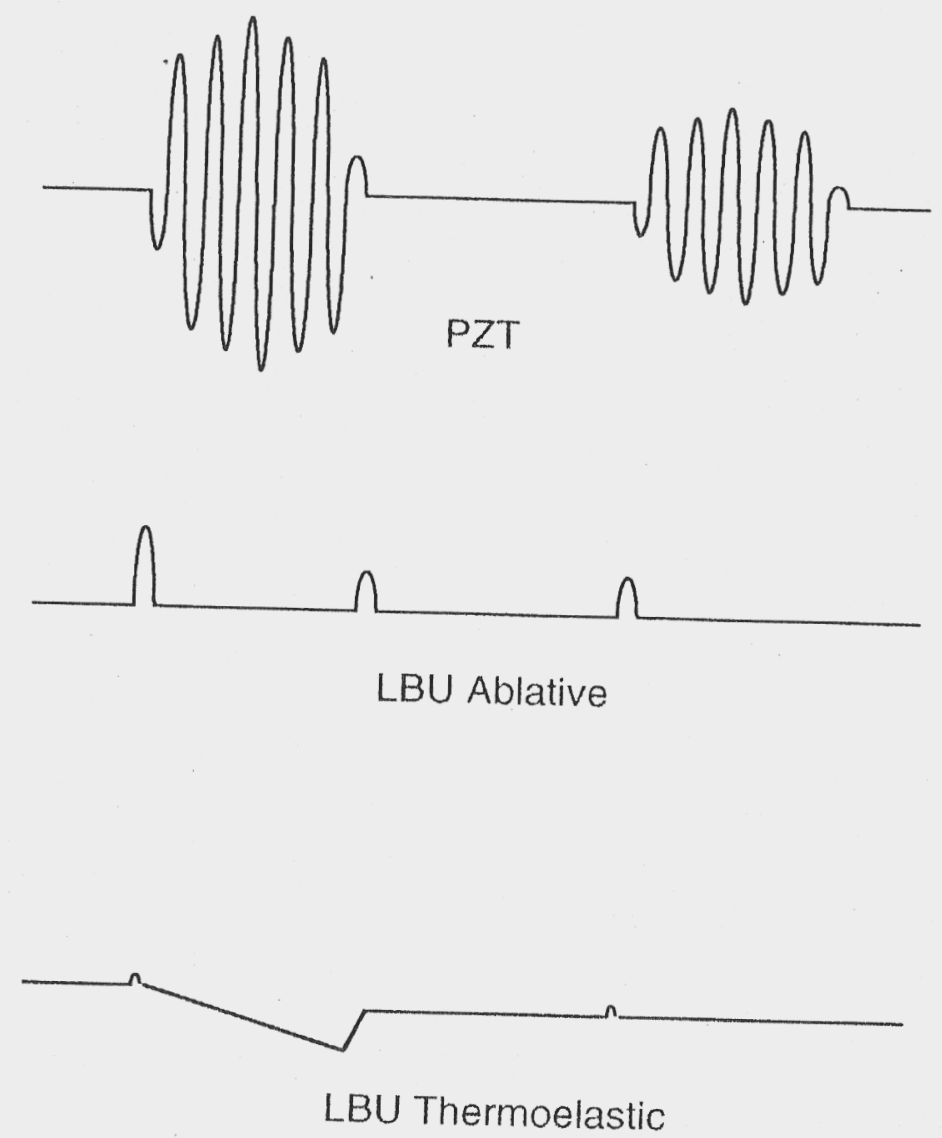


\section{Test Configuration}

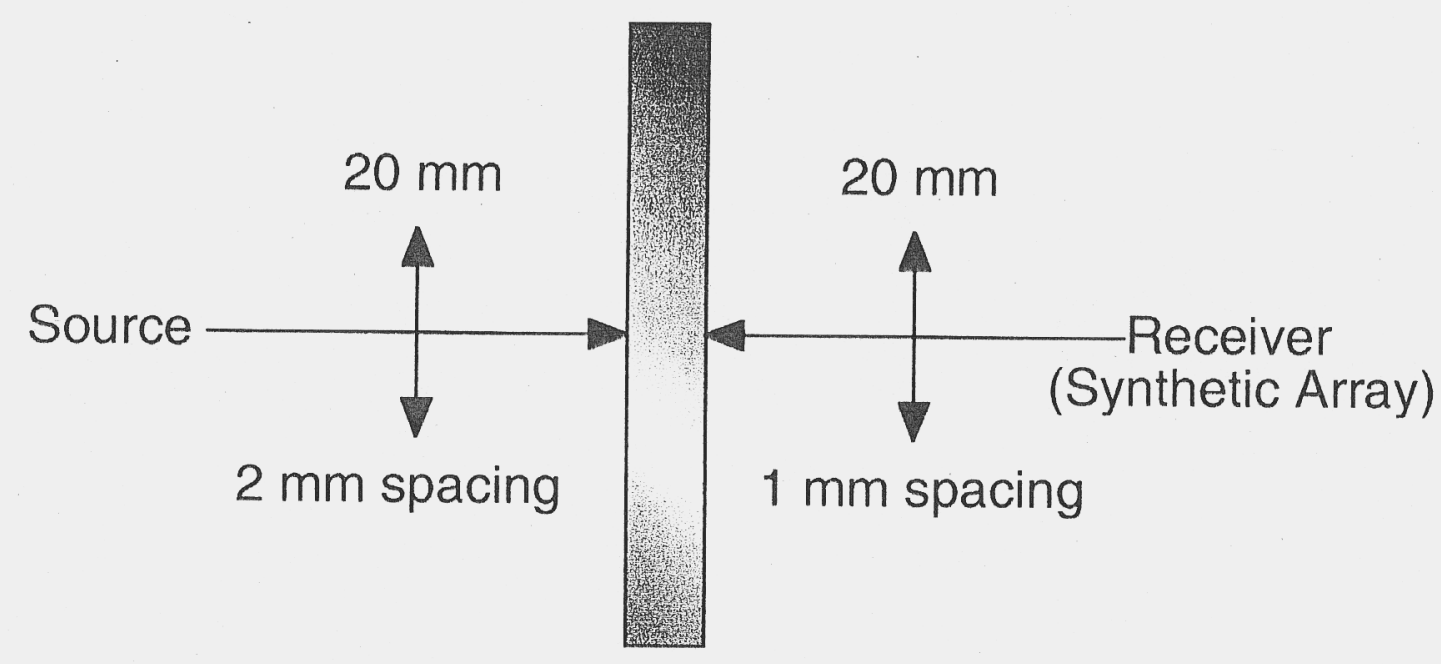

$9.5 \mathrm{~mm}$ thick aluminum plate

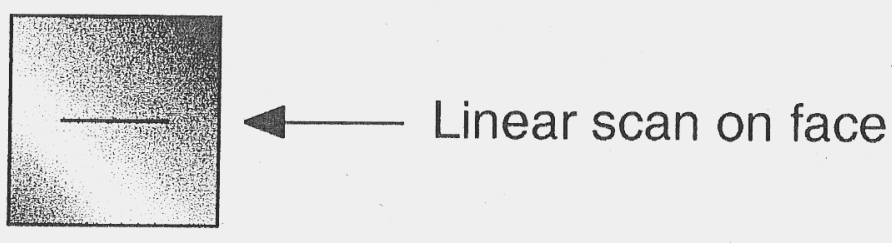




\section{Two Problems That Interest Us:}

- Defect Detection (Epicenter Only)

- Localization/Imaging (Synthetic Array)

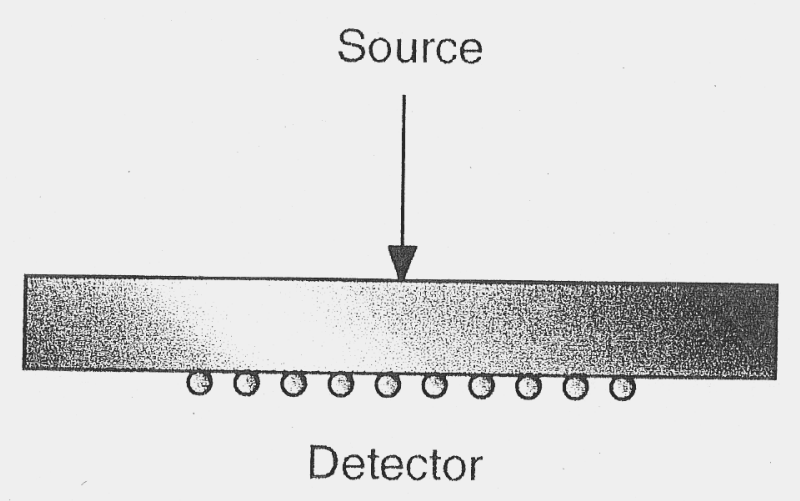




\section{Synthetic array data set for one source location.}

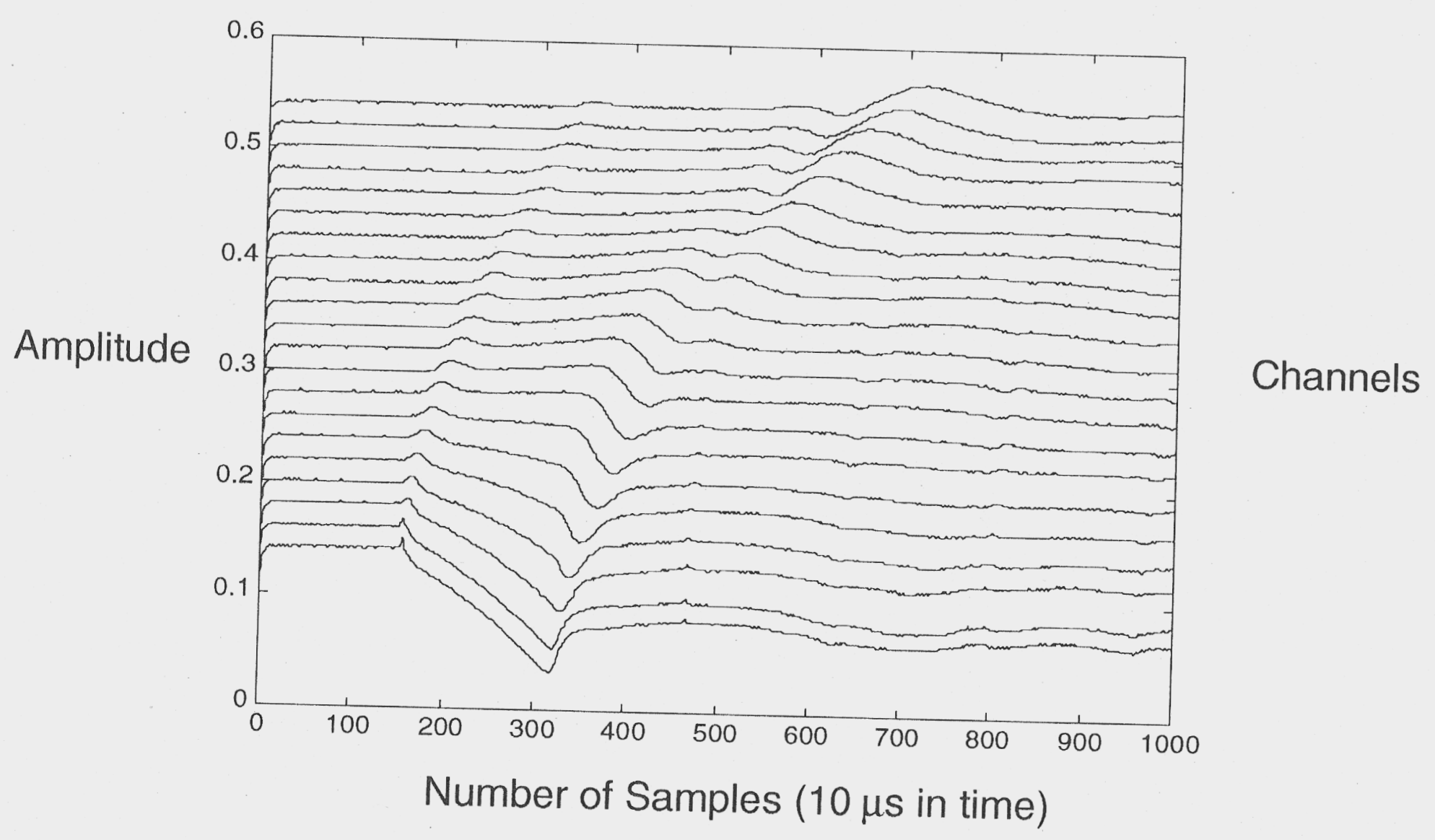




\section{Pre-Processing}

- De-mean/ de-trend

- Correlation Canceling 


\section{CORRELATIONNOISE PROBLEM}

GIVEN a set ff hoisy measurement data AND a referencesichal (correlated fo noise or disturbance)

FIND the "best" (mean-squared error) estimate of the noise/disturbanceito cancel is effect.

APPLICATIONS: signal processing, fetal heartbeat, cockpit communications, bedy function monitoring etc.

期 


\section{CORRELATIONNOISE CANCELLER STRUCTURE}

Measurement

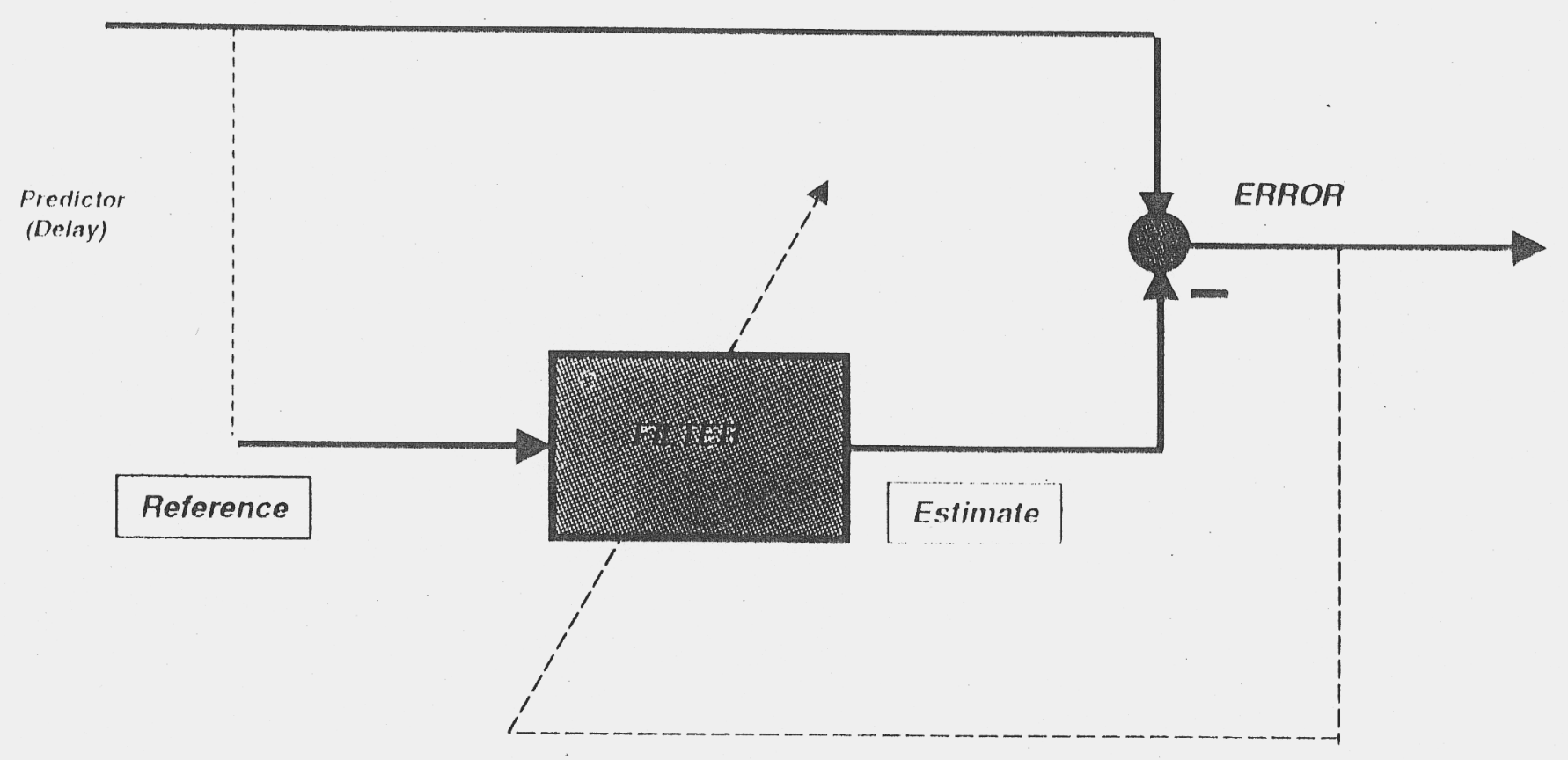

Adaptive

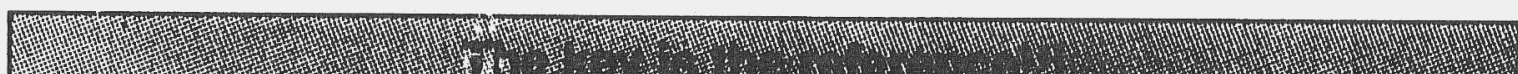




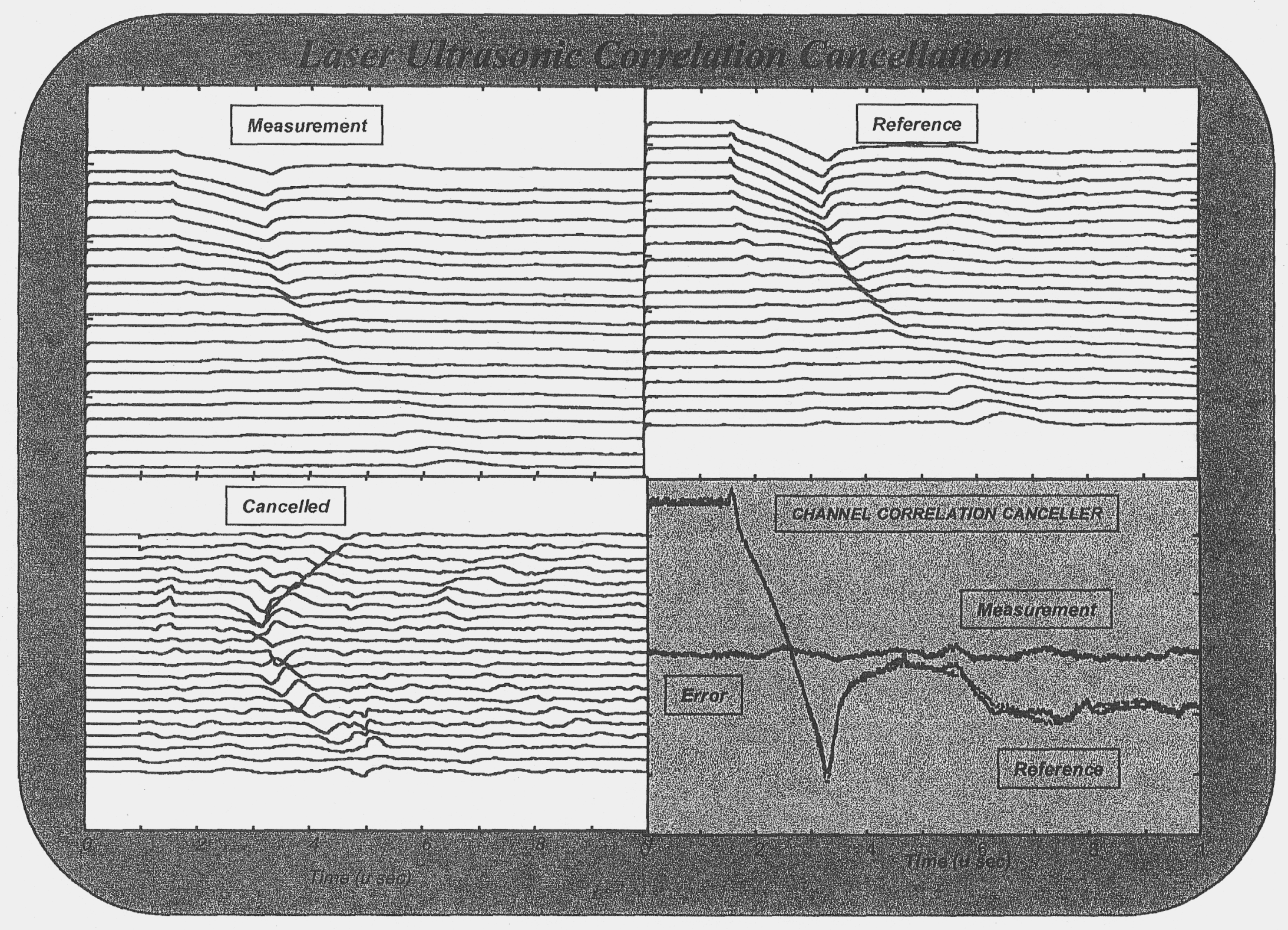




\section{We use a simple homogeneous model of the test specimen (medium) characterized by the Green's function:}

In the spatio-lemporal domain

$$
\left[g\left(r_{m}, r_{n} ; t\right)=\frac{1}{\left|r_{m}-r_{n}\right|} \delta\left(t-\tau_{m n}\right)\right]
$$

or in the temporal Fourier domain as:

$$
\left[G\left(r_{m}, r_{n} ; \omega\right)=\frac{1}{\left|r_{m}-r_{n}\right|} e^{-j \omega\left(1-\tau_{m n}\right)}\right]
$$

where the time delay is given by:

$$
\tau_{m}=\frac{\left|r_{m}-r_{n}\right|}{v}
$$

which is an outgoing spherical wavefront emanating from a point source 


\section{With our broadband source, we have that:}

- the displacement field measured by the array is simply the source convolved with the Green's function of the medium:

$$
f\left(r_{m}, r_{n} ; t\right)=g\left(r_{m}, r_{n} ; t\right) * s\left(r_{m}, r_{n} ; t\right)
$$

or for the homogenous medium

$$
f\left(r_{m}, r_{n} ; t\right)=\frac{1}{\left|r_{m}-r_{n}\right|} s\left(t-\tau_{m n}\right)
$$

- simply a delayed and attenuated replica of the source 


\section{Therefore, our approach to "Imaging"using this model is to:}

- assume a scatterer (source) location $\left(r_{i}, r_{j}\right)$ on the specimen or equivalently pixel position $(i, j)$

- using the homogenous medium model calculate the attenuation and required time delays

$$
\left\{\frac{1}{\left|r_{i}-r_{j}\right|}, \tau_{i j}\right\}
$$

- perform the required "beamforming" (shift and sum)

$$
b f(t)=\sum_{\ell=1}^{L-1} \alpha_{i j} f\left(t-\tau_{i j}\right.
$$

- extract the significant features from the resulting signal (beamformer output): peaks, power, etc. 


\section{Epicenter Source Location}

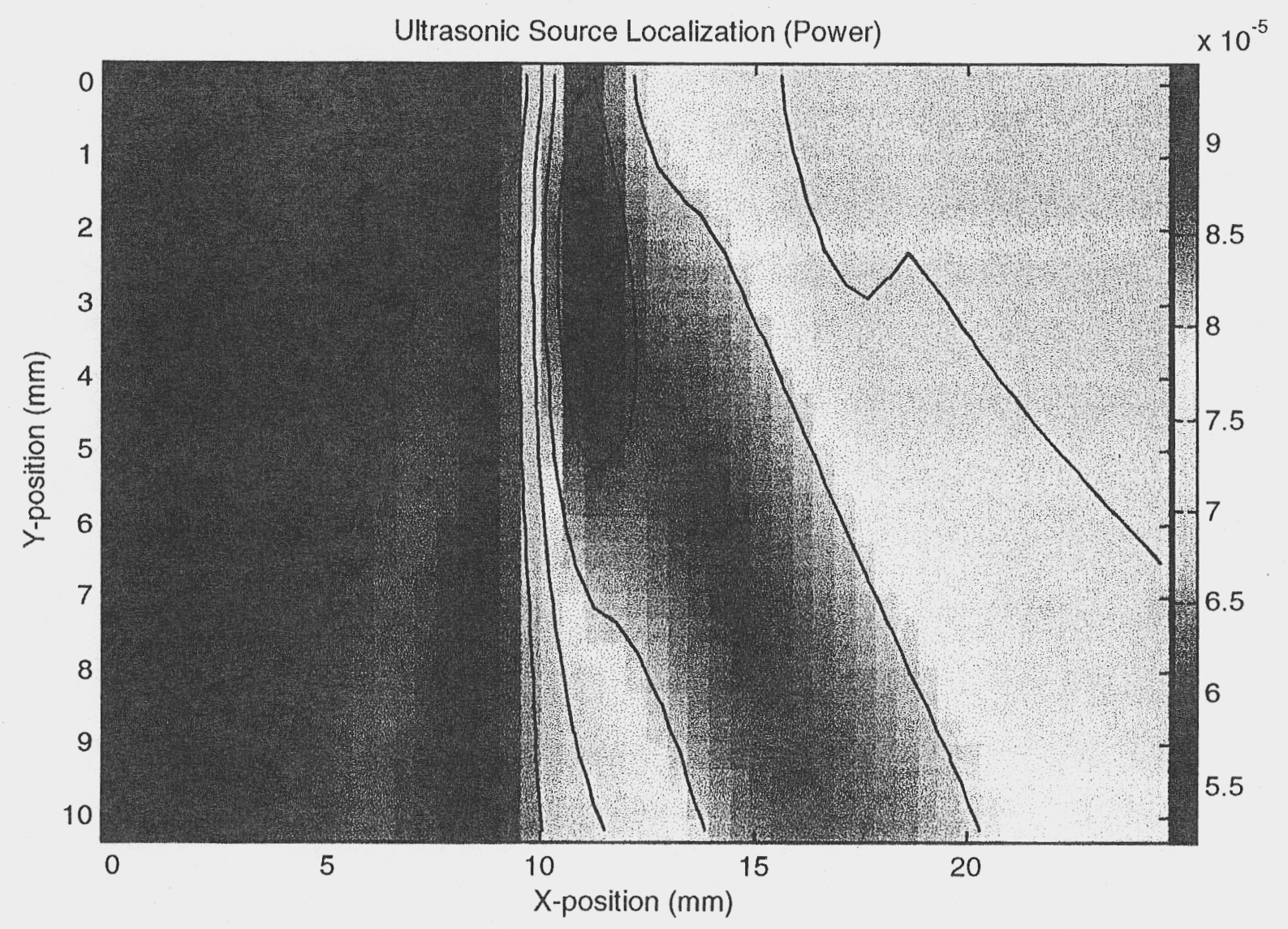



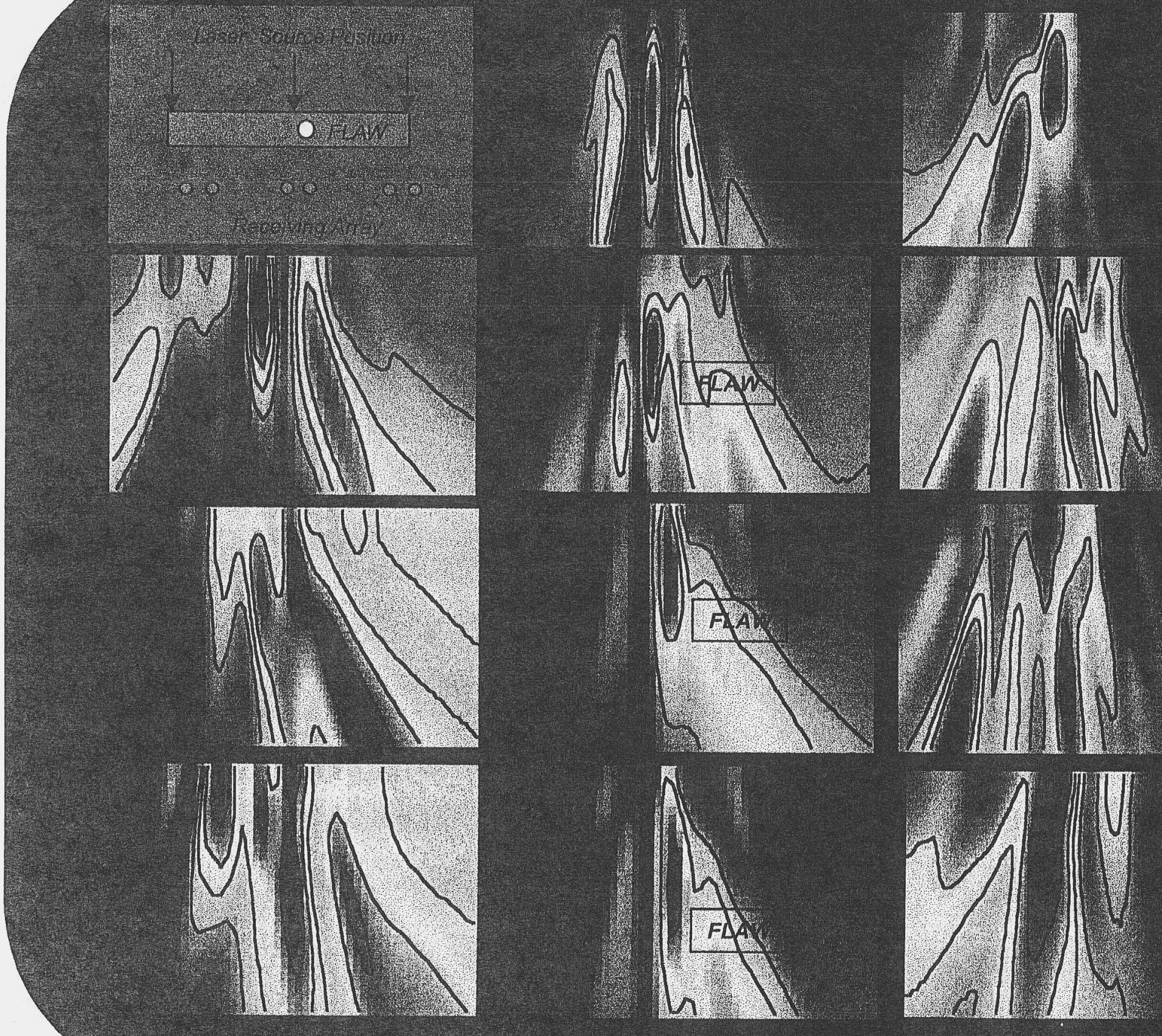


\section{We have applied this novel correlation canceling scheme to laser-based ultrasound signals to obtain images of a defect.}




\section{R.N. Bracewell}

NASA and the European Space Agency are planning to obtain images of nonsolar planetary systems, understanding that success would rally public support for continued space exploration.

The NASA Terrestrial Planet Finder, with a possible launch date of 2010, will go beyond simple detection of a massive planet, constructing an image of where the planets are and analyzing their atmospheres. In the event of success, imaging individual planets would follow (2020?).

Suppressing the blinding radiation of the central star, a main technical challenge, is to be met by an infrared interferometer in orbit near Jupiter. The technique will be explained and ground-based tests, both completed and planned, will be described. 


\section{STANDARD SYSTEM}

SUN AND JUPITER SEEN FROM 10 PC $\left(3 \times 10^{17} \mathrm{~m}\right)$

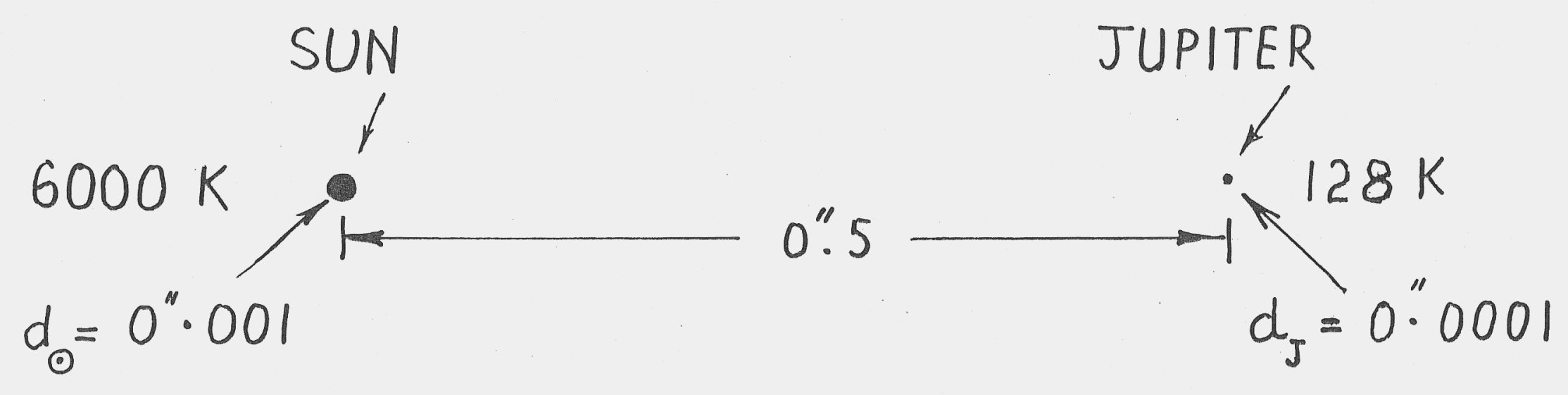


THE INFRARED ADVANTAGE: $50 \mathrm{db}$ See Nature 274, 780, 1978

$\log$ Power

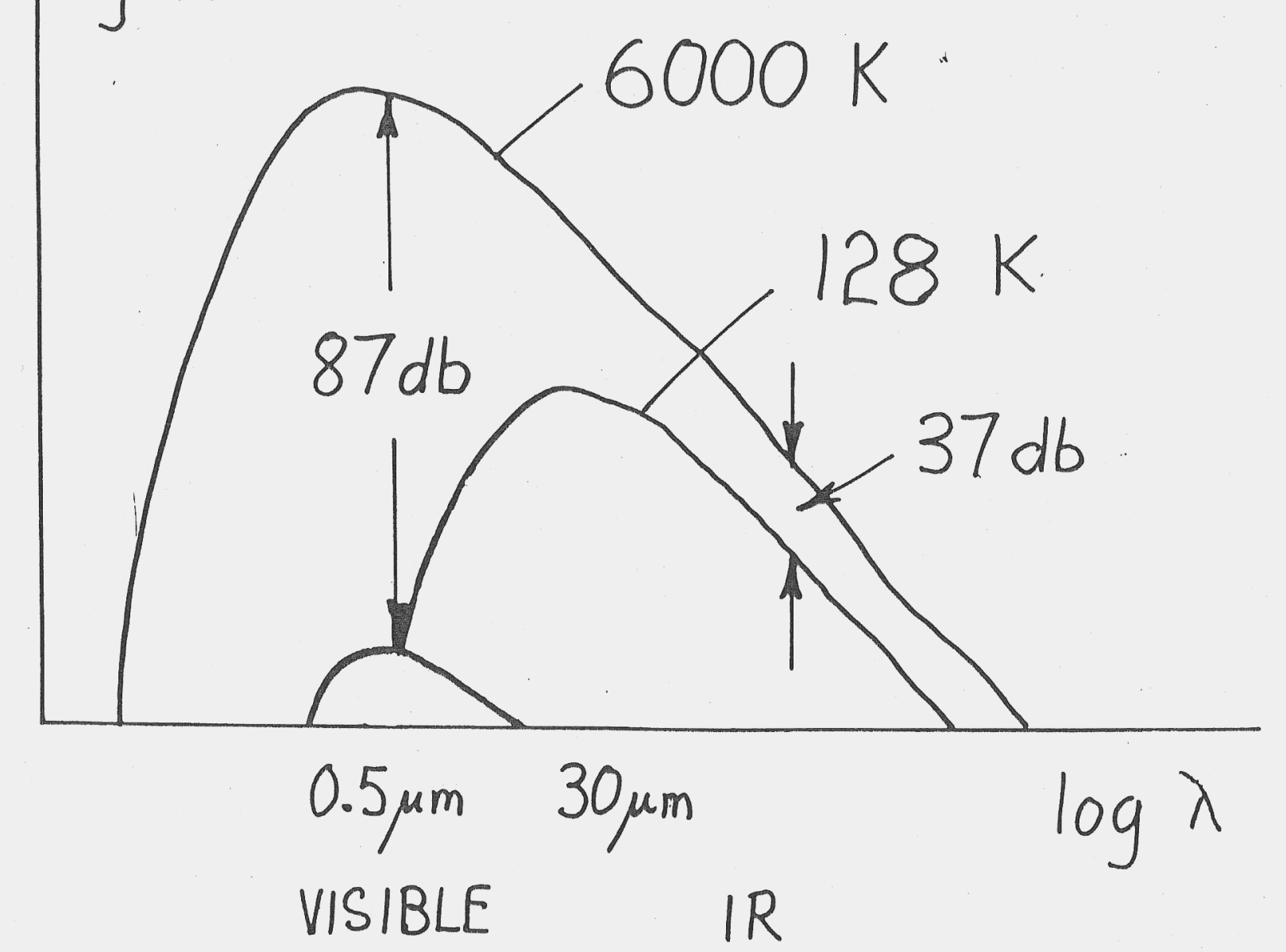


BRACE WELL AND : $2 A$ L

Icarus, 38, 136, 1979

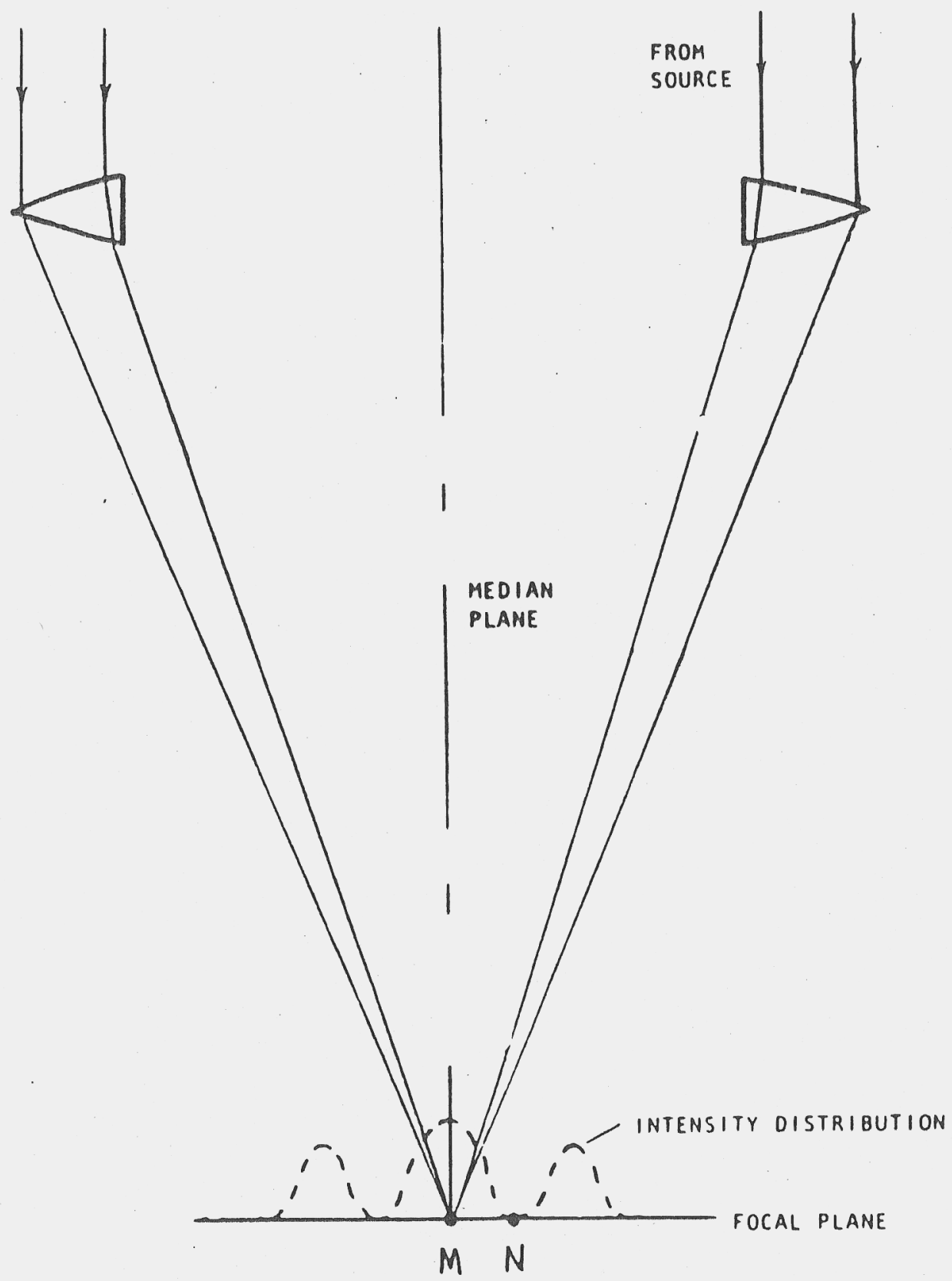

FiG. 4. Simple stellar interferometer. 


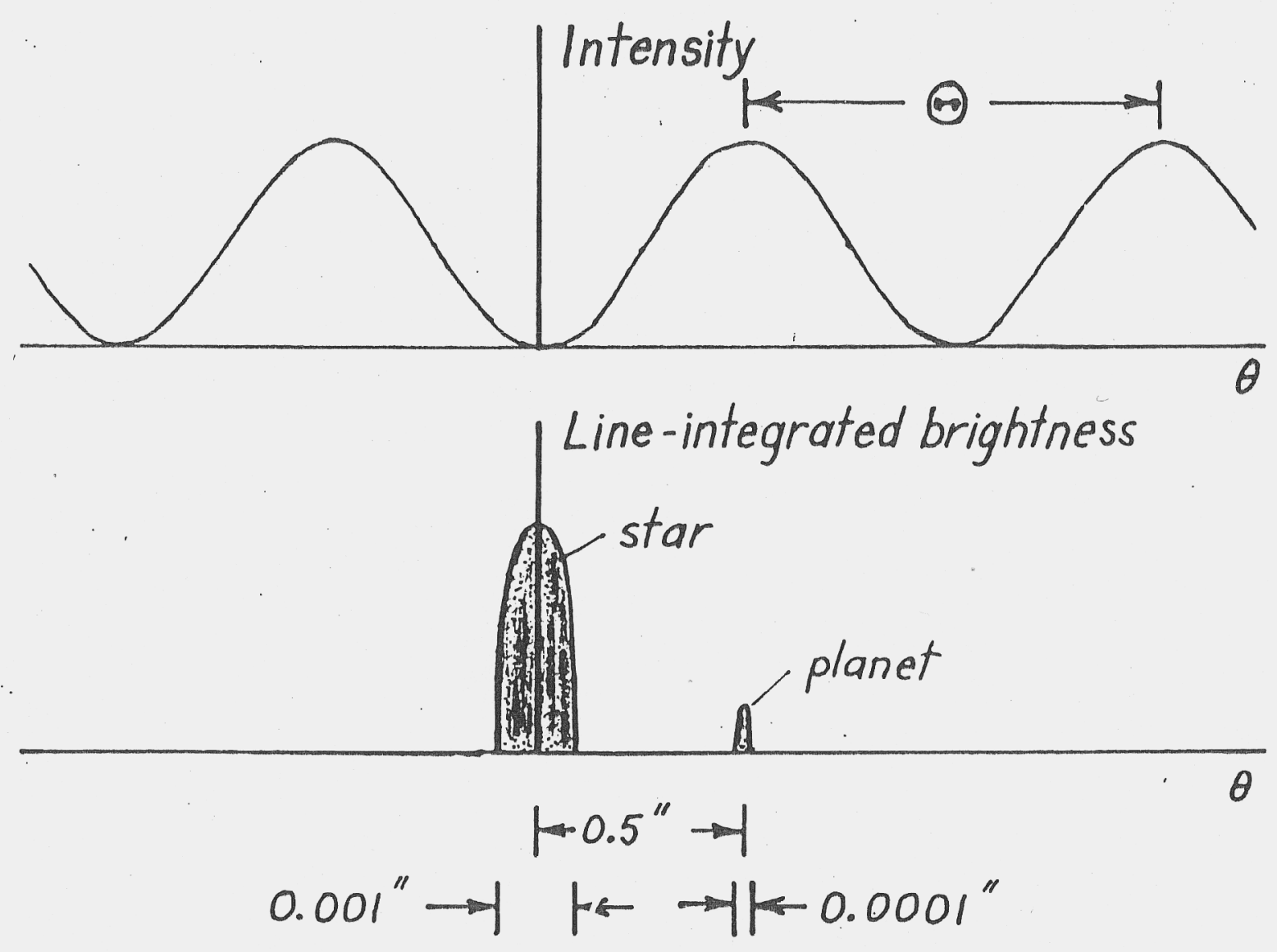

Fig. 2. Intensity of interference fringes and lineintegrated brightness ( $W \mathrm{~m}^{-2} \mathrm{~Hz}^{-1} \mathrm{rad}^{-1}$ ) of star and planet (not to scale). 
SEARCHING FOR NONSOLAR PLANETS

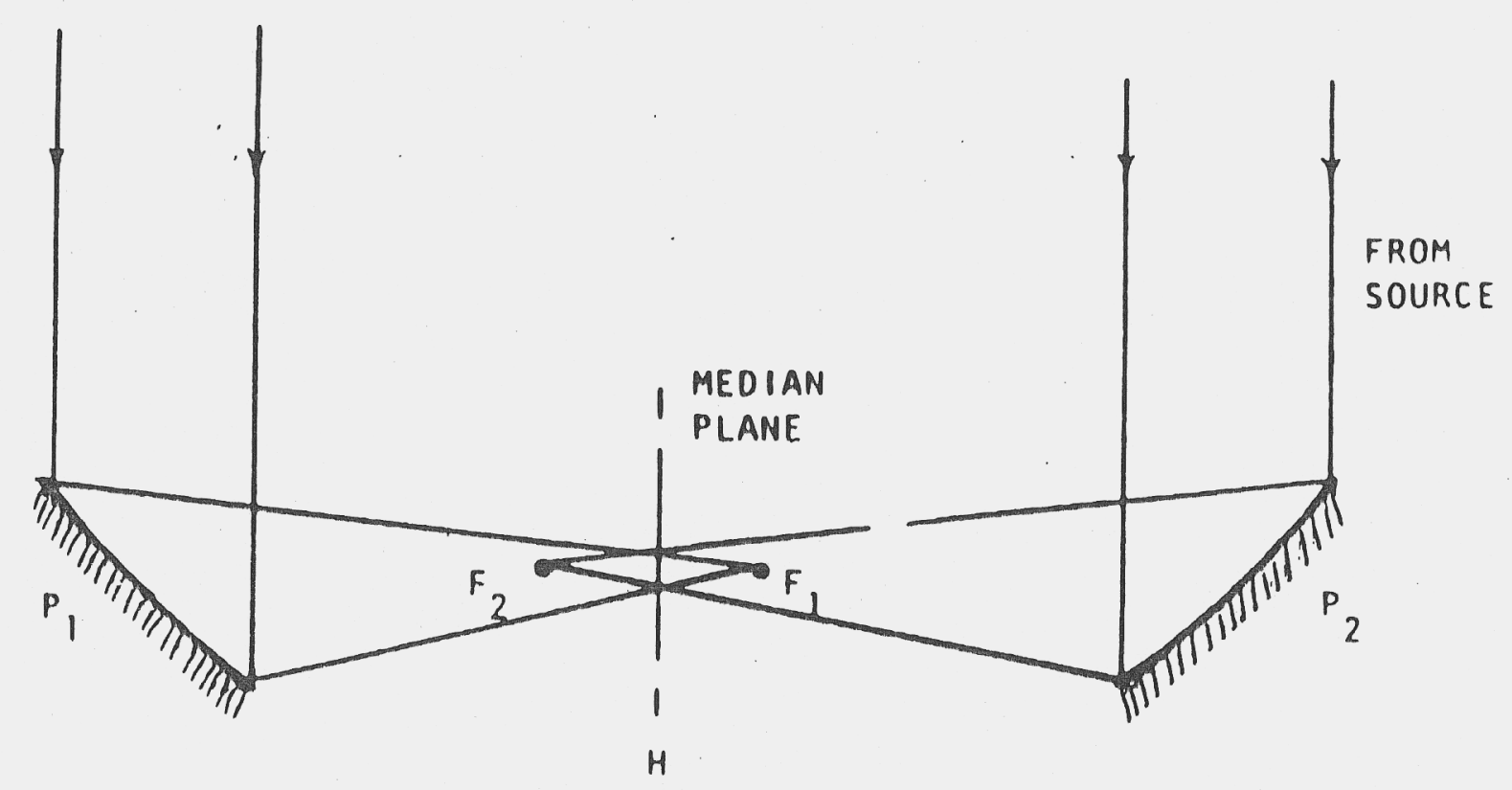

Fig. 5. Interferometer using reflection optics exhibits wideband null. 


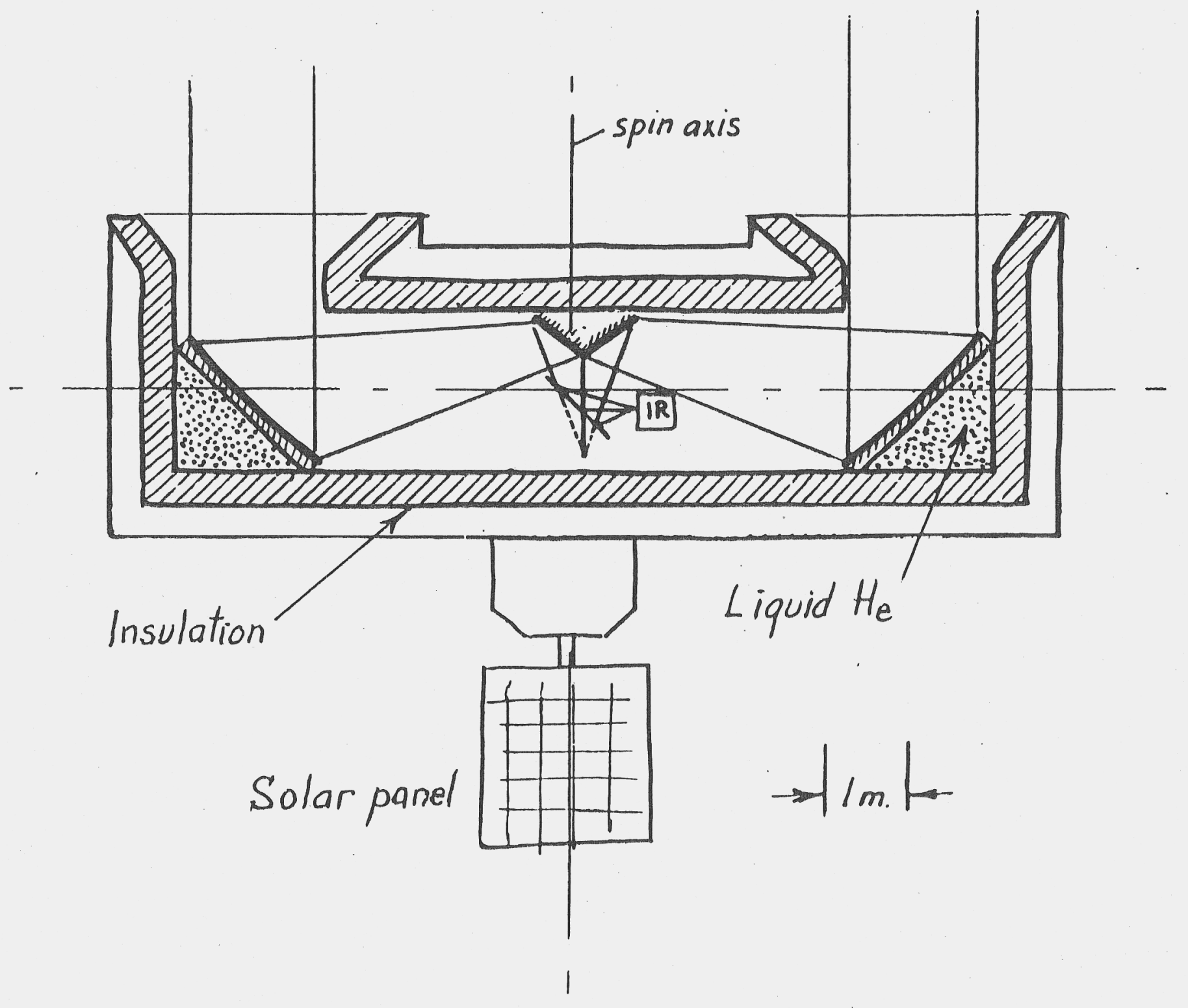




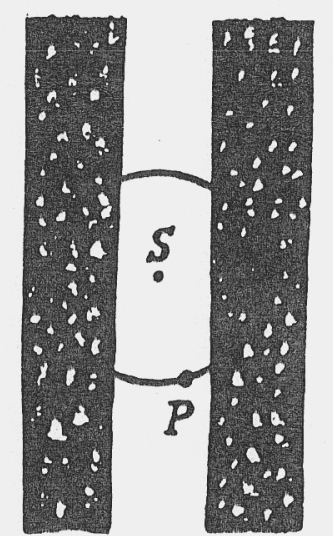

$k-\theta \rightarrow 1$

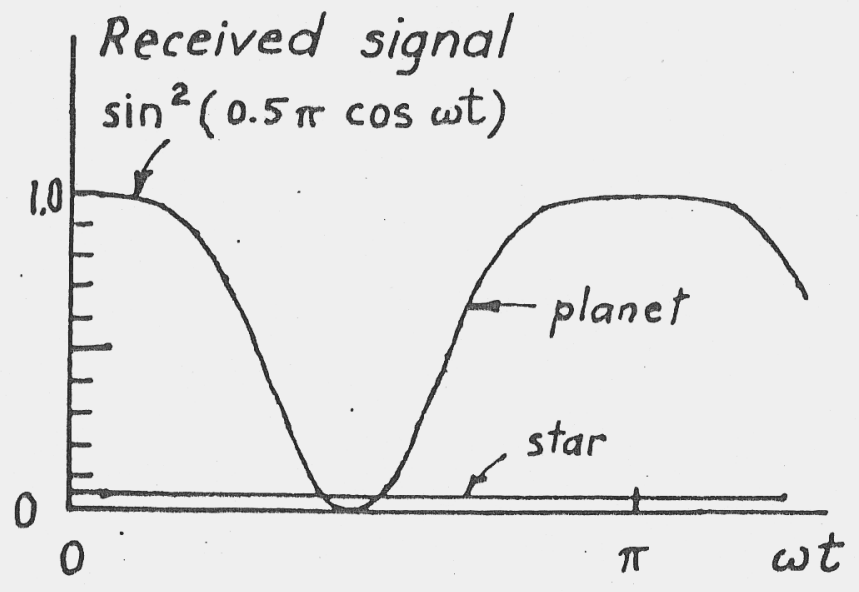

Fig. 3. As planet $P$ traces locus through fringes of spacing $\Theta$, flat topped signal rich in $4 \omega$ is received. Precisely centered star $S$ produces small constant signal 


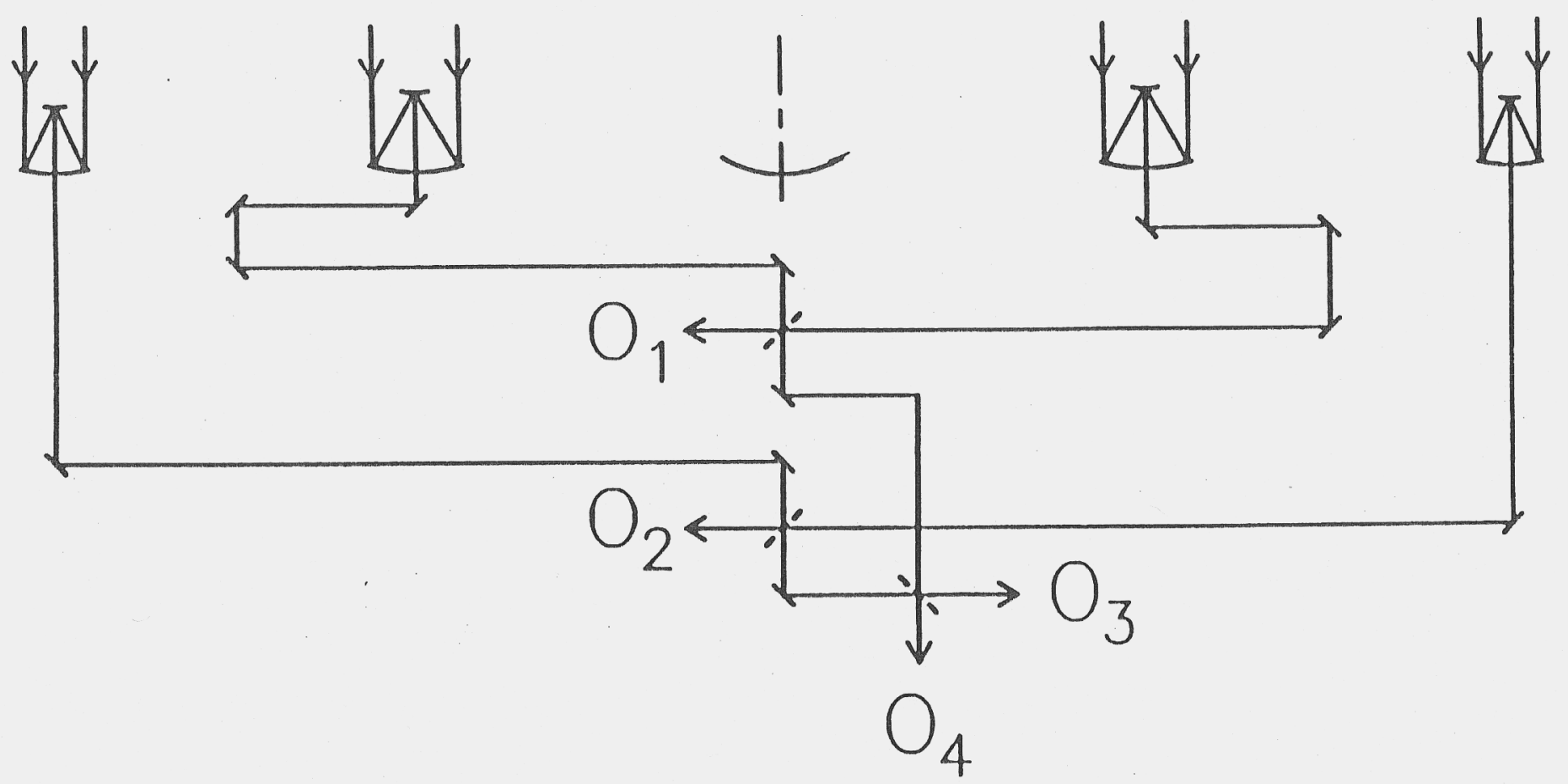

Figure 1. Schematic diagram of the 4-element linear nulling interferometer. The beam combiners feeding $\mathrm{O}_{1}$ and $\mathrm{O}_{2}$ have equal transmitted and reflected intensities. The final combiner is chosen to yield amplitudes in the ratio (inner pair/outer pair) $=0.504$. Figs. I- 7 from Angel \&. Woolf, Astrophys. J., 475, 373-379, Jan 1997 


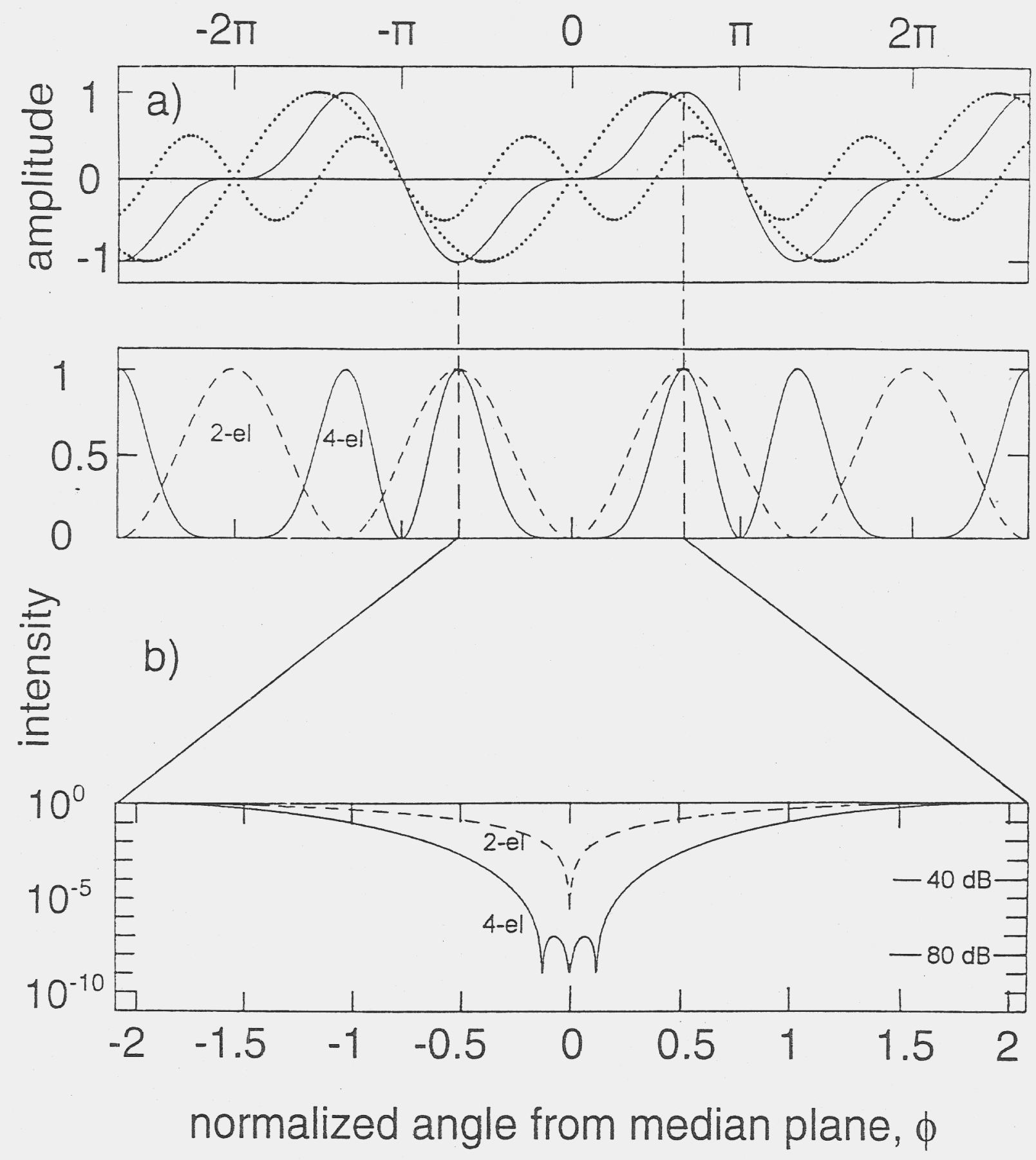

Figure 2. a) Dotted curves show separately the fringe amplitudes for the inner and outer Bracewell pairs of the 4 element interferometer (the two terms of Eq.3). For a source at the meridian plane, both amplitudes are zero, and the slopes are equal and opposite, leading to a summed amplitude with the zeroth, first and second order terms all zero (unbroken curve). b) The transmitted intensities of the 4 element interferometer and of a single Bracewell pair $3 / 8$ as long, chosen to have its first maxima at the same absolute angle from the meridian plane (broken line). The normalized angle (which depends on length) is appropriate for the 4 element interferometer. The central nulls are shown expanded and in logarithmic scale, to illustrate the much greater depth and width achieved by the 4 element configuration. 


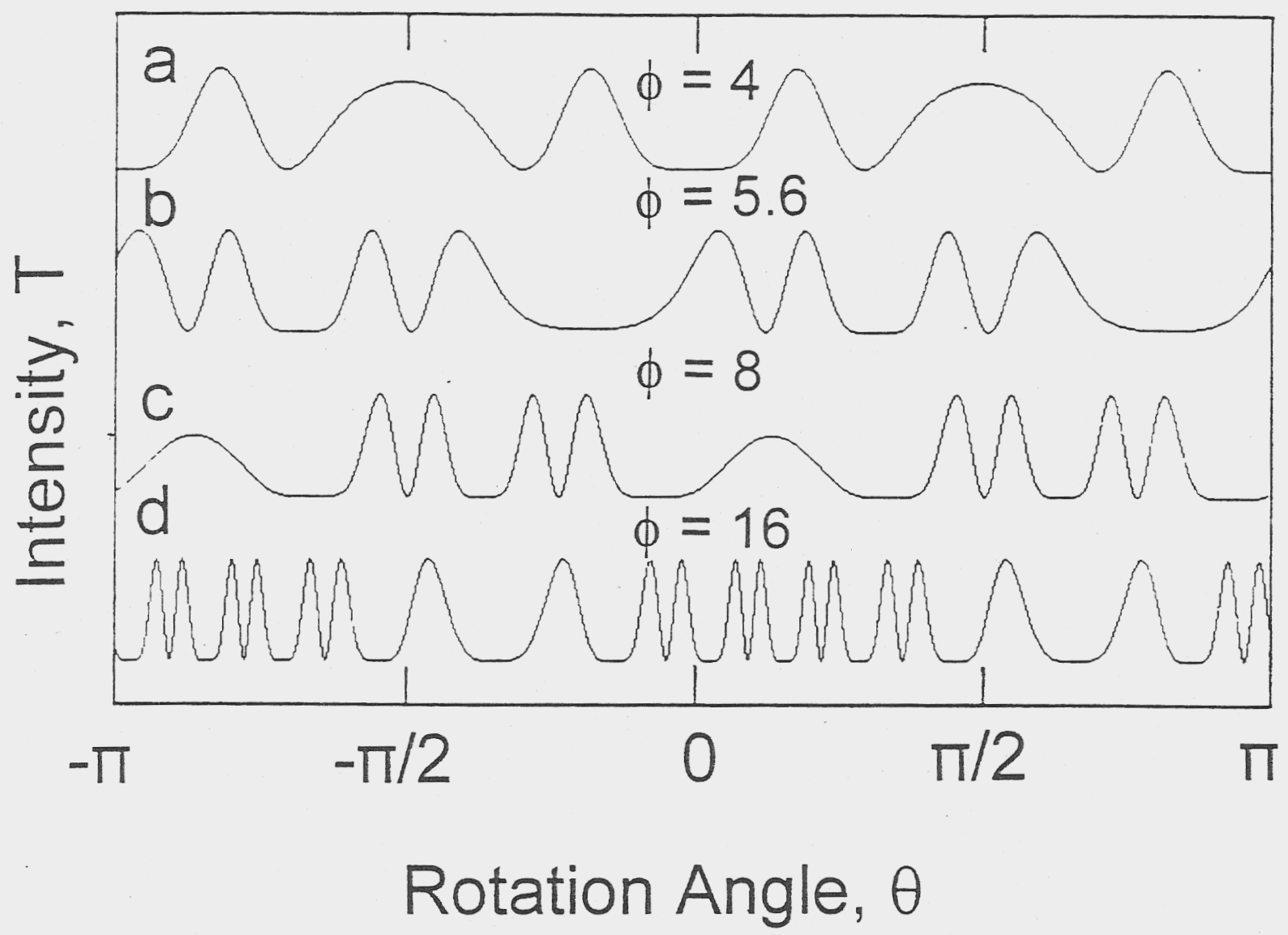

Figure 3. Transmitted intensities as a function of rotation angle $\theta$ for different maximum values of $\phi$ and position angle $\beta$. 


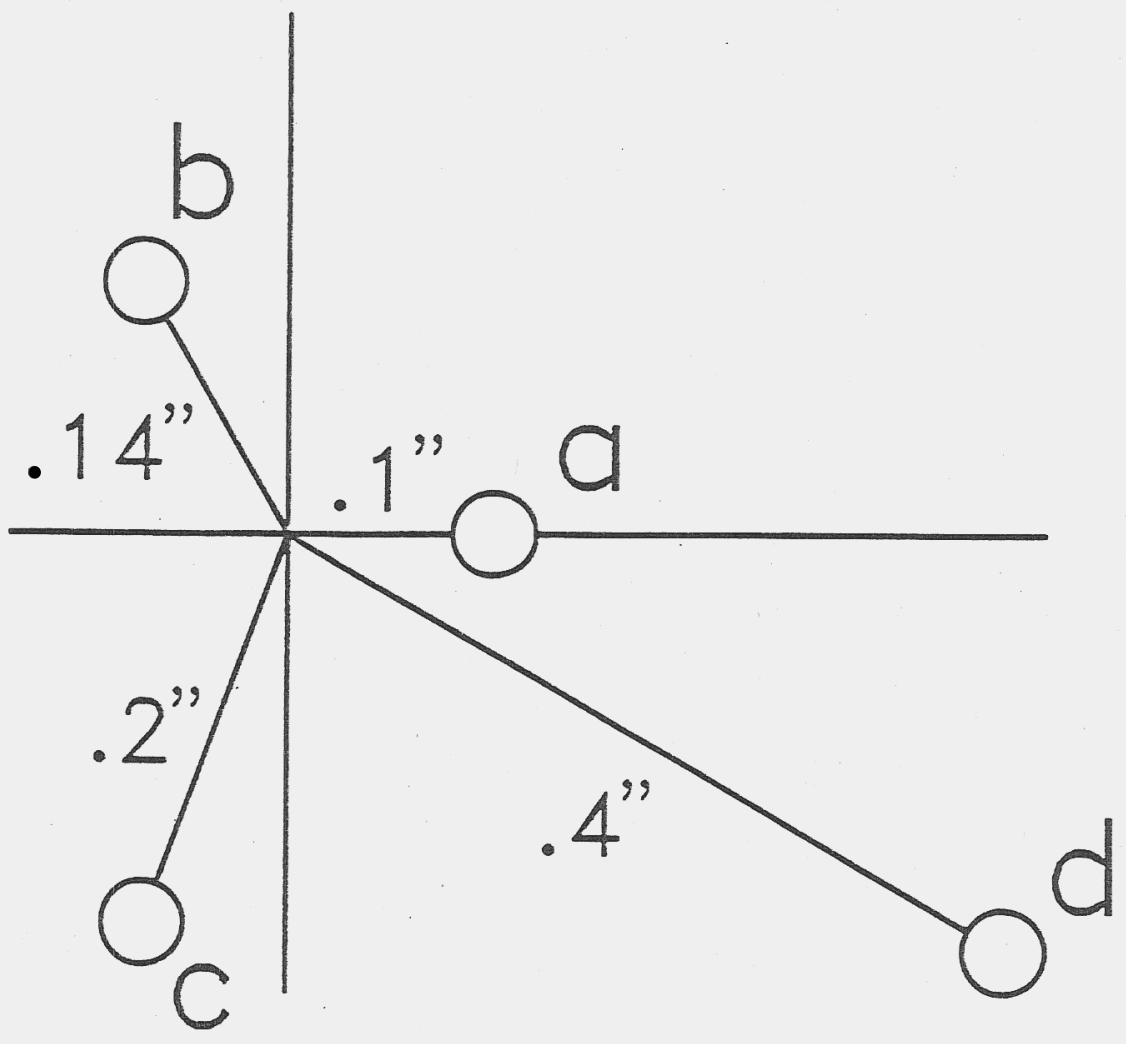

Figure 4. Locations of planets used to model interferometer response. Separations are given in arcseconds, and correspond to projected separations 1 - 4 AU from a star seen from $10 \mathrm{pc}$. 


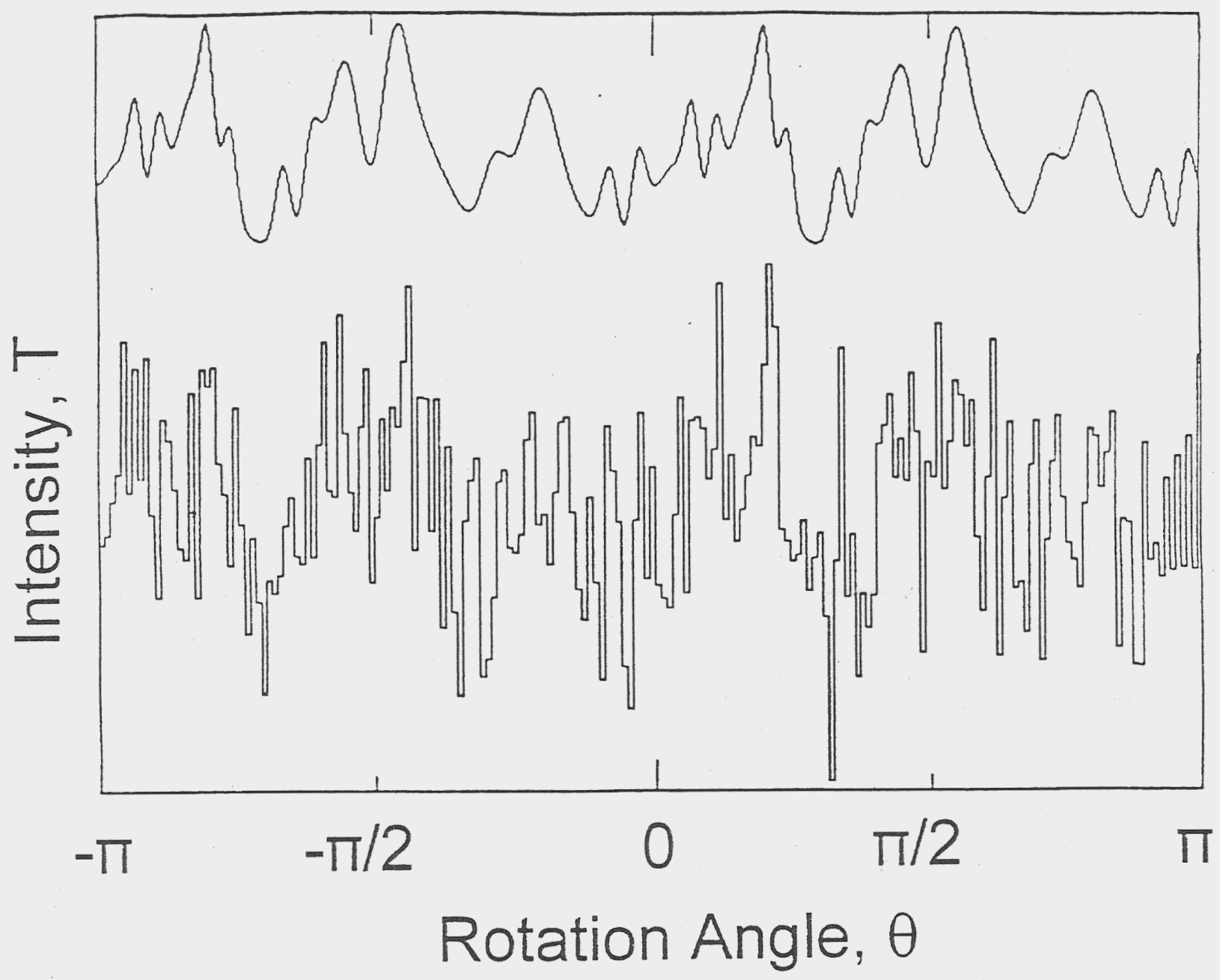

Figure 5. a) is the combined $10 \mu \mathrm{m}$ signal for all 4 planets in figure 4 (b) shows the same signal as with the addition photon noise, as projected for a 10-hour integration and $1 \mu \mathrm{m}$ bandwidth. Each planet is projected to be as bright as the Earth seen from $10 \mathrm{pc}$. The photon noise level is appropriate for a stellar zodiacal cloud having the same luminosity as the sun's. 


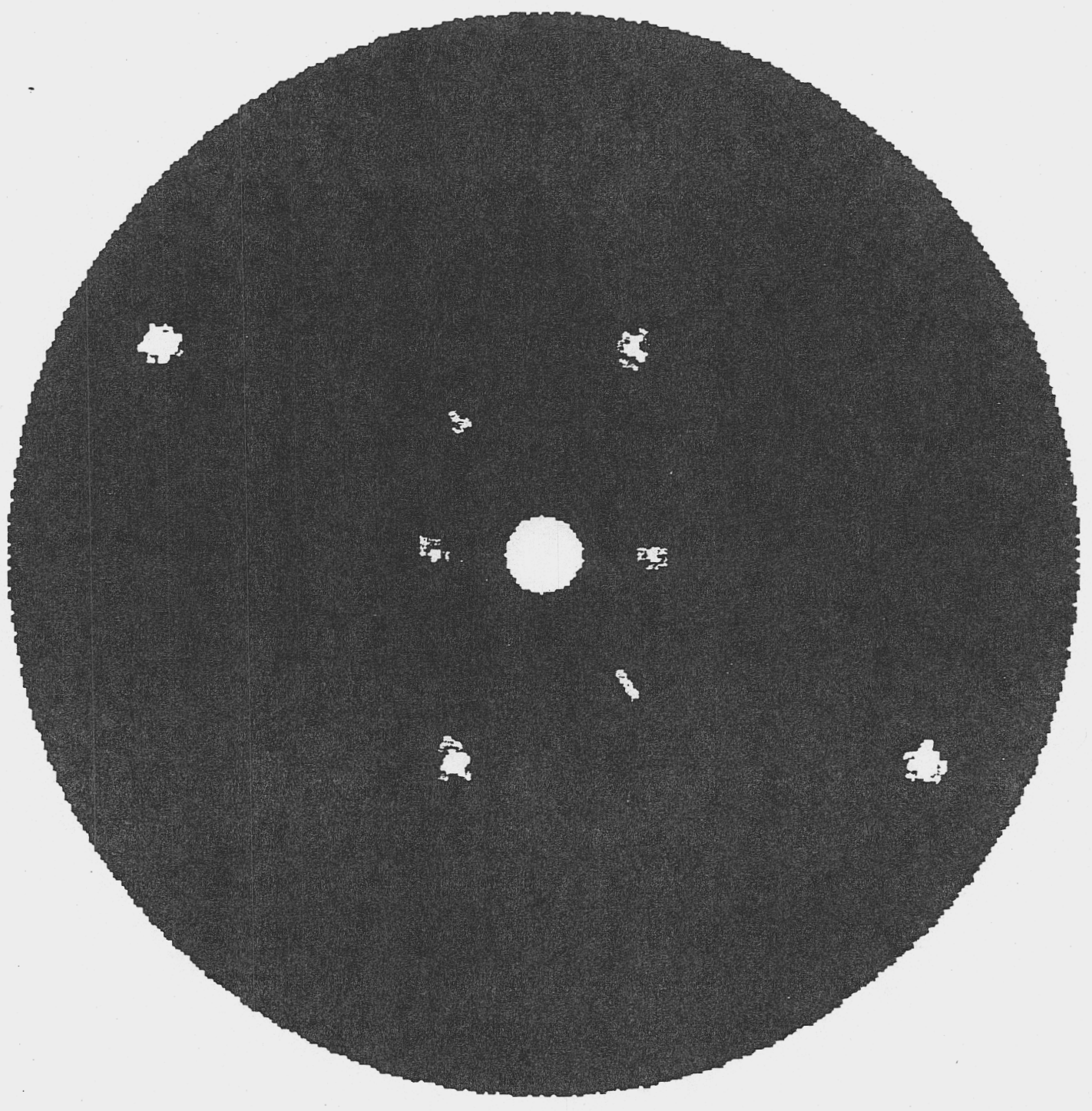

Figure 6. Image of 4 planets recovered by cross correlation from synthesized noisy interferometer data like fig $5 b$, over 10 wavelength bands from $7-17 \mu \mathrm{m}$. The image doubling could be removed by extended observations. 


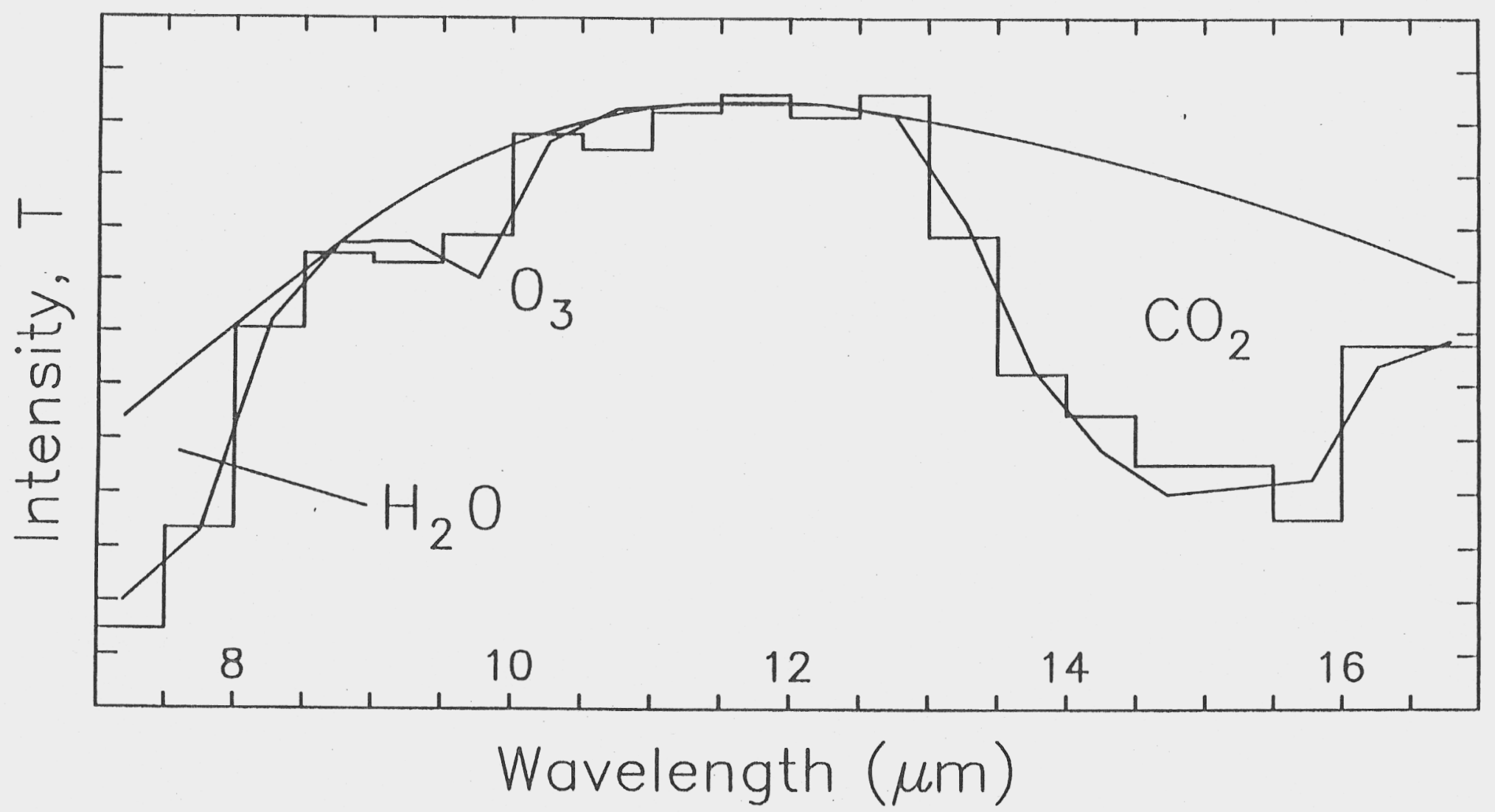

Figure 7. A spectrum of the Earth at a resolution of $0.5 \mu$ and a signal/noise ratio of $20: 1$, as would be obtained from 80 days of observation of a solar system-like set of planets at $10 \mathrm{pc}$. The circles give the true spectrum. The smooth line shows a blackbody curve. 


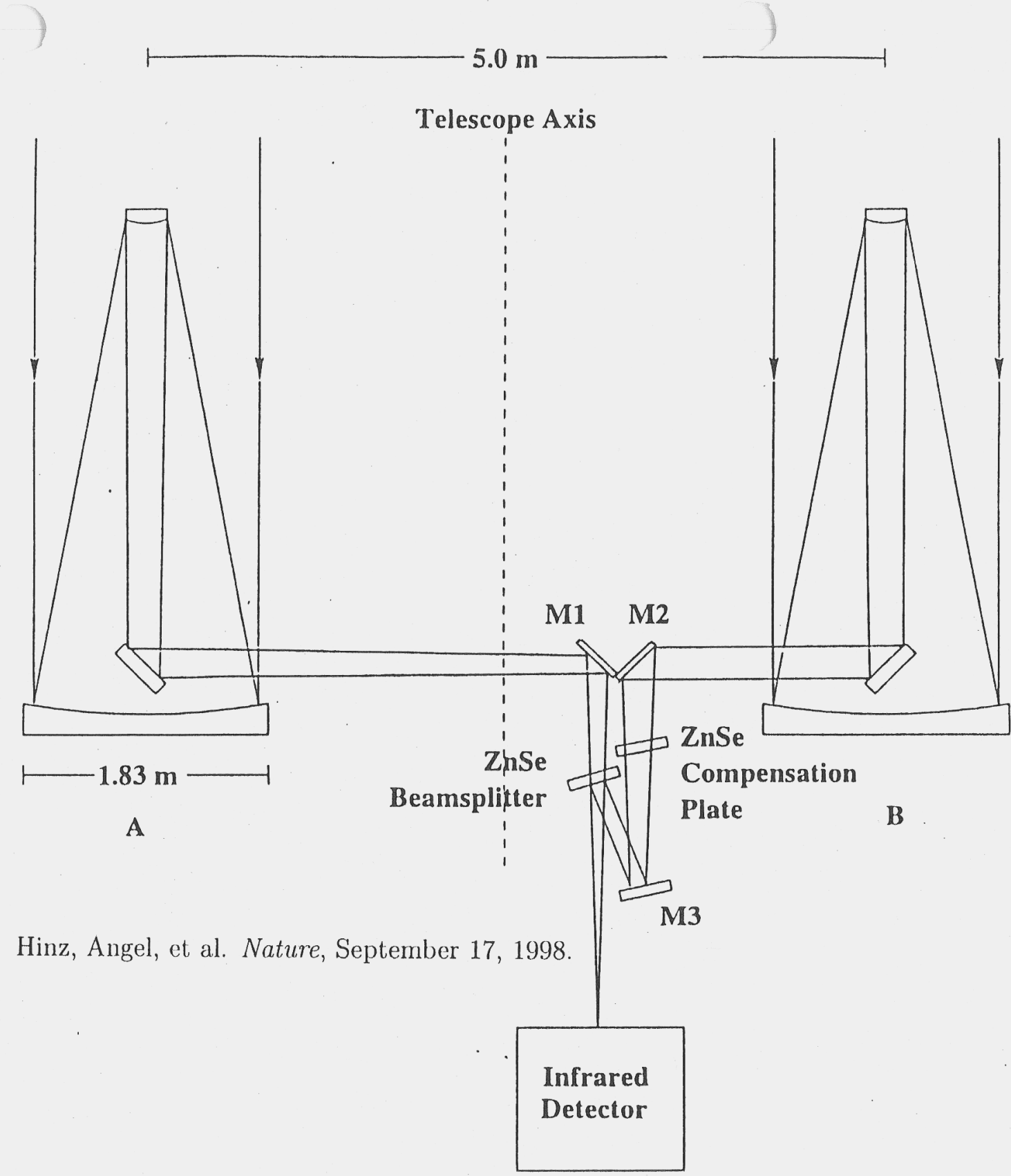

Fig. 1.- Schematic of our nulling interferometer at the MMT. The beam path-lengths are equalized by translating the beamsplittter vertically. 


\title{
Novel Interferometer-Spectrometer for Sensitive Doppler Planet Detection
}

\author{
David Erskine, Jian Ge \\ Lawrence Livermore National Lab
}

\begin{abstract}
A saturn-like planet at saturn-like distances creates a wobble in the velocity of a star of about 3 $\mathrm{m} / \mathrm{s}$, and Earth-like planets even less, about $0.1 \mathrm{~m} / \mathrm{s}$. Current spectrometers have velocity resolution of $3-10 \mathrm{~m} / \mathrm{s}$, which is insufficient to detect Saturn-like or smaller planets. Secondly, these spectrometers are not light efficient, allowing only bright stars to be measured. Thirdly, these instruments are not portable or space-launchable, and their highly individual character makes comparison of data during 10-30 year orbits problematic. We have built a novel instrument (called a fringing spectrometer) which is a hybrid of an interferometer and spectrometer, which is particularly well suited for accurately measuring small Doppler shifts in a light efficient manner, is portable and space-launchable, and was constructed from off-the-shelf components on hand. Other advantages include a 200x larger field of view compared to the Lick Observatory spectrometer, stability, and independence from slit vignetting vagaries. We are testing it on sunlight by seeking the $12 \mathrm{~m} / \mathrm{s} @ 27$ day effect of the moon tugging the Earth. (Jupiter on the sun is $12 \mathrm{~m} / \mathrm{s}$ ). We have built an algorithm to unwrap the phase of the interferogram to $1 / 10000$ wave (corresponding to $\sim 1 \mathrm{~m} / \mathrm{s}$ Doppler velocity). This is a $20 \mathrm{x}$ improvement in the art over monochromatic interferograms and may lead to other applications in high precision metrology.
\end{abstract}

Keywords: Spectrometer, Doppler shift, Interferometer

This work was performed under the auspices of the Department of Energy by the Lawrence Livermore National Laboratory under contract W-7405-Eng-48. 


\title{
Deconvolution of images from the Lick Observatory adaptive optics system
}

\author{
J. M. Brase \\ J. Kolman \\ B. A. Macintosh
}

CASIS 1998 


\section{Deconvolution of images from adaptive optics systems}

- Current AO systems provide partial correction of images in the near infrared (1-2.2 $\mu \mathrm{m})$

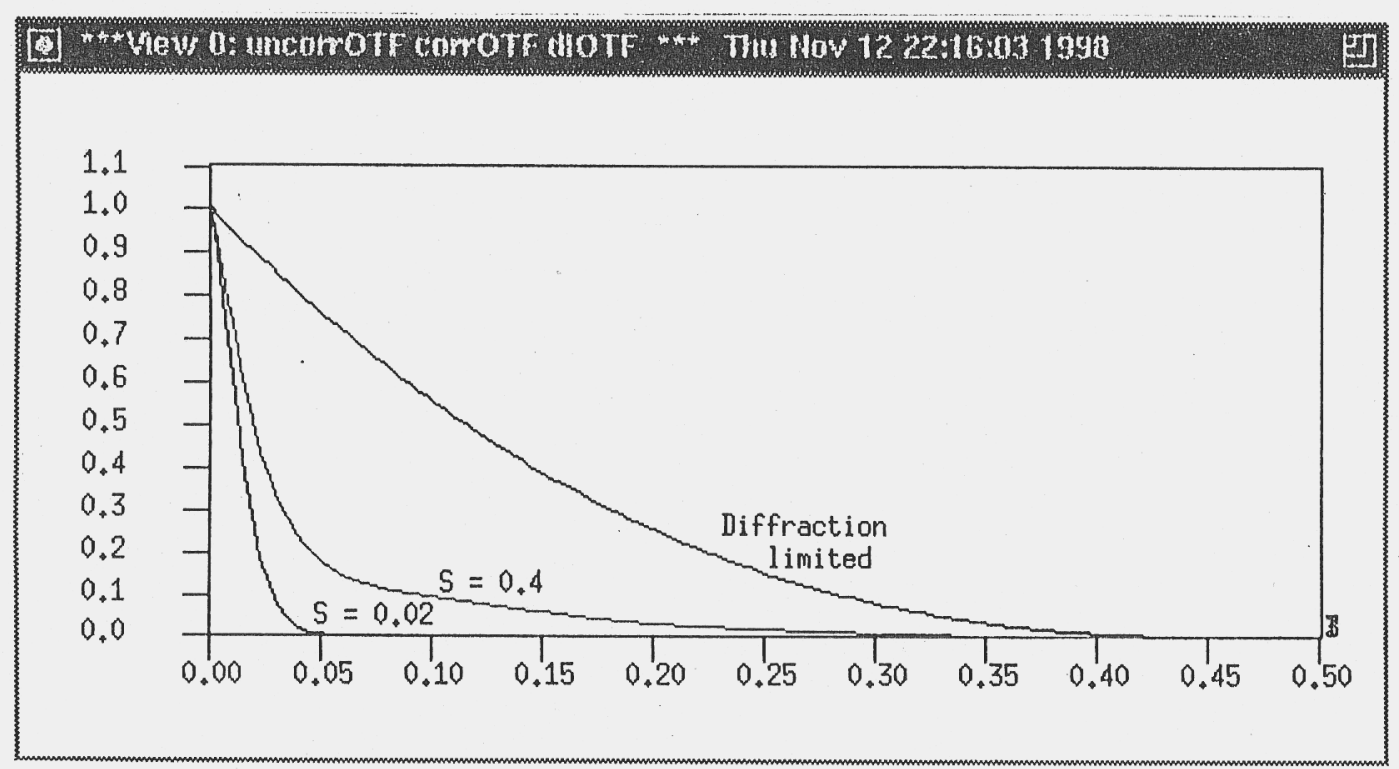

- Because adaptive optics corrected images contain frequency components out to the diffraction limit (at Strehl > 0.1), deconvolution should work significantly better than for uncorrected images 


\section{Stochastic deconvolution algorithms for $\mathrm{AO}$ applications are based on} Poisson image models

The image measurement $\mathrm{g}(\mathrm{j})$ is a Poisson distributed random variable with mean

$$
\overline{g(j)}=h(j) * f(j)
$$

where $\quad \mathrm{j}$ is the spatial index

$\mathrm{h}$ is the point spread function

$\mathrm{f}$ is the object

The MAP estimate of the object $\mathrm{f}$ is given by

$$
\underset{f}{\arg \max } p(f \mid g)=\underset{f}{\arg \max } \frac{p(g \mid f) p(f)}{p(g)}
$$

If we assume a uniform prior probability $\mathrm{p}(\mathrm{f})$, the MAP estimate reduces to the maximum likelihood estimate

$$
\underset{f}{\arg \max } p(g \mid f)
$$




\section{Formulation for Poisson measurement and prior probability}

Assuming Poisson statistics for $\mathrm{p}(\mathrm{f})$ and $\mathrm{p}(\mathrm{glf})$ and the above measurement model, the MAP estimate is given by

$$
f(j)=\overline{f(j)} \exp \left\{\left[\frac{g(j)}{h(j)^{*} f(j)}-1\right] * h(j)\right\}
$$

Where $\overline{f(j)}$ is the expected value of the object $\mathrm{f}$.

The maximum likelihood estimate is given by

$$
\left[\frac{g(j)}{h(j)^{*} f(j)}\right] * h(j)=1
$$

Both estimates are nonlinear sets of equations that must be solved iteratively. 
MAP:

$$
f_{n+1}(j)=f_{n}(j) \exp \left\{\left[\frac{g(j)}{h(j)^{*} f(j)}-1\right] * h(j)\right\}
$$

ML:

$$
f_{n+1}(j)=f_{n}(j)\left\{\left[\frac{g(j)}{h(j)^{*} f(j)}\right] * h(j)\right\}
$$

Note that the ML algorithm is generally referred to as Lucy-Richardson deconvolution.

The algorithm is initialized with $\mathrm{f}_{0}(\mathrm{j})=\mathrm{g}(\mathrm{j})$. The iteration is stopped when

$$
\frac{1}{N} \sum_{j}\left|\left(f_{n}(j) * h(j)\right)-g(j)\right|^{2}<\operatorname{var} g
$$


Comparison between ML and MAP restorations

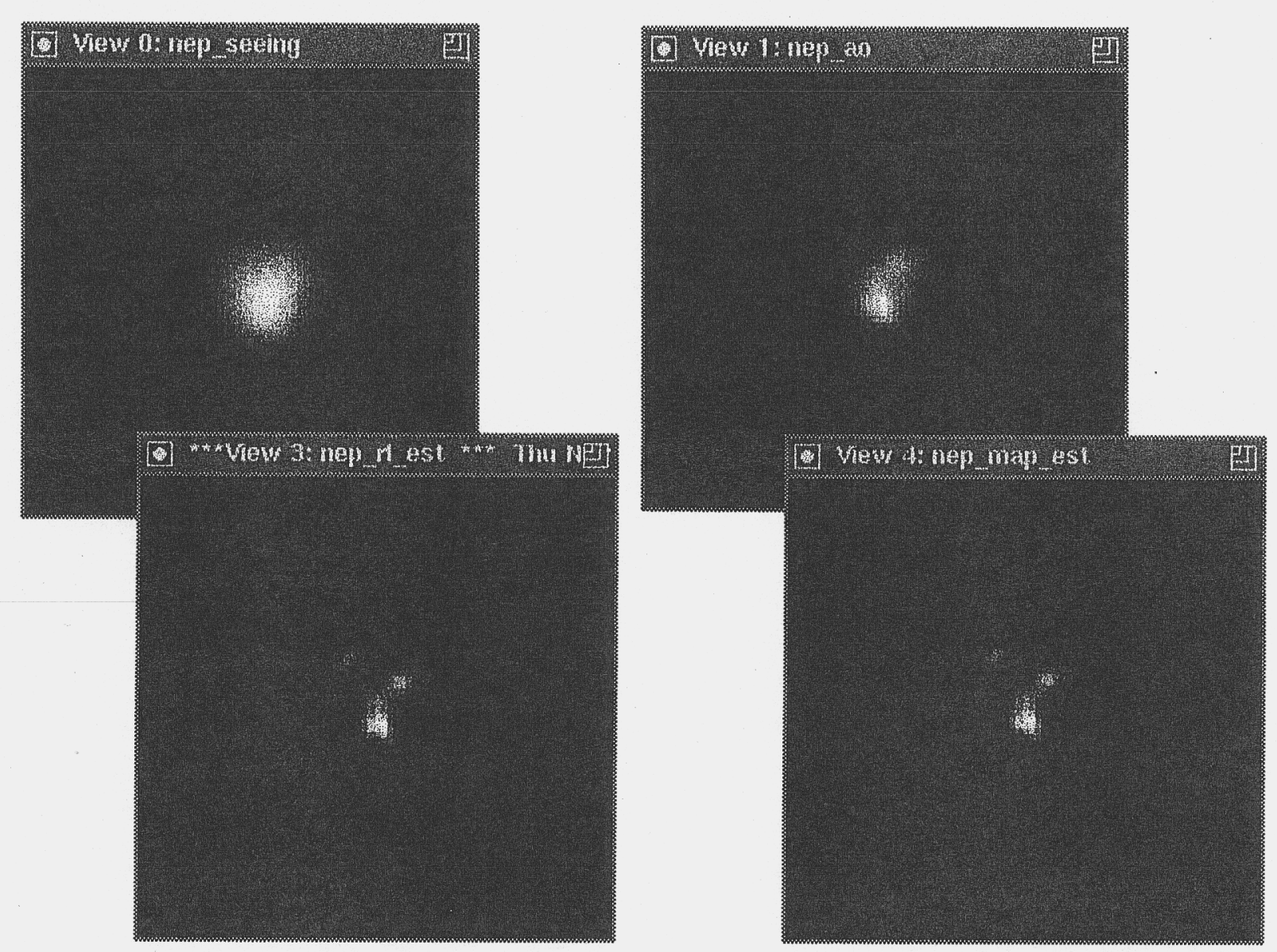



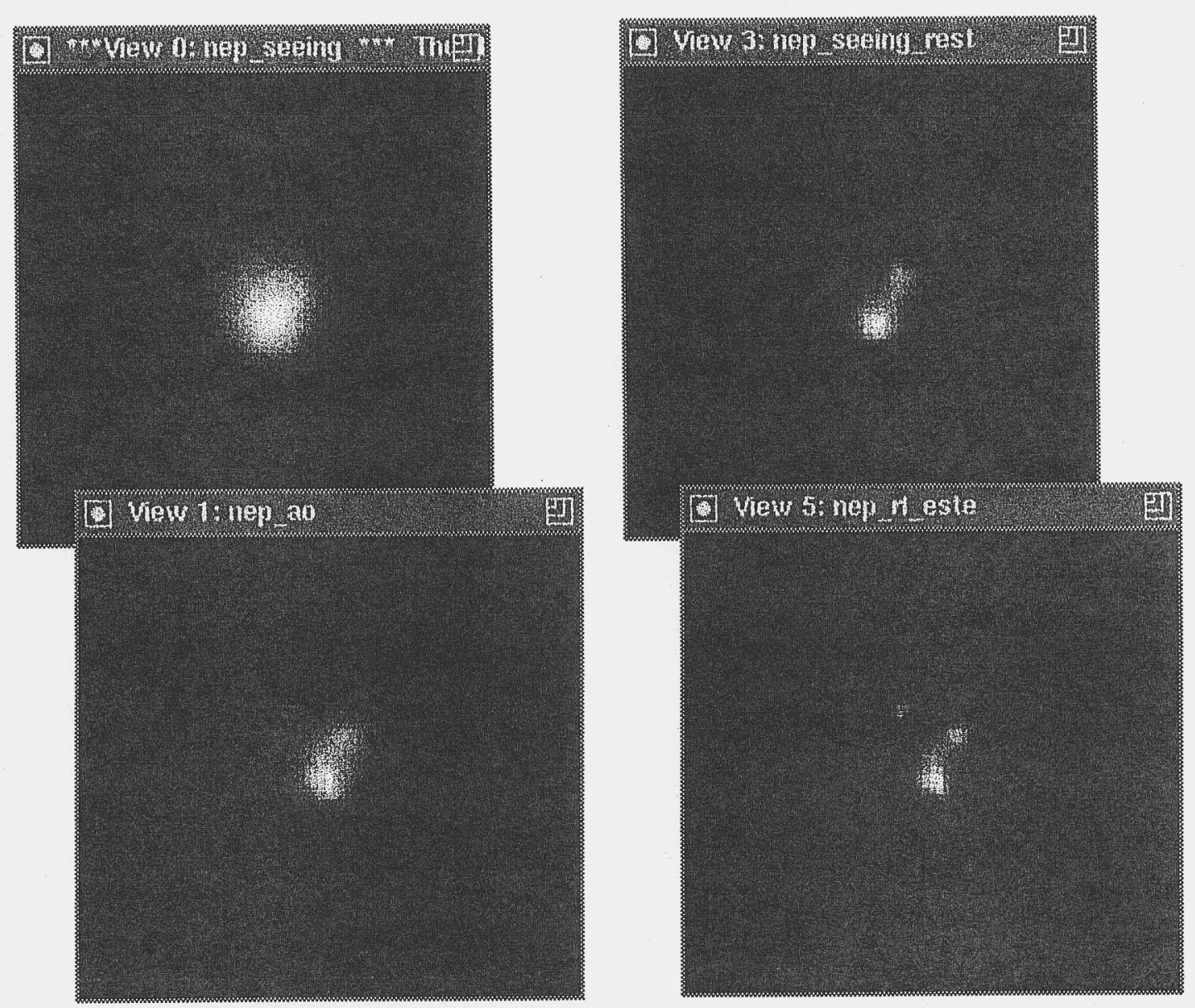


\section{Much remains to be done on restoration of $\mathrm{AO}$ images}

Point-spread-function estimation is the most serious problem. The psf varies in time as the atmosphere changes which makes the use of calibrator stars very difficult.

Two approaches are being investigated:

- estimation of the psf from AO system real-time diagnostic data - joint estimation of the object and psf (blind or myopic deconvolution)

The psf also varies with angle from the AO guidestar. This will require spatially variant deconvolution techniques. Not a big problem right now because IR array FOV is within the isoplanatic angle (to first order). 


\title{
LBNL/Lick Observatory Fully-Depleted CCD Image Sensor Development
}

\author{
S. Holland, D. Groom, A. Karcher, M. Levi, N. Palaio, S. Perlmutter \\ Lawrence Berkeley National Laboratory \\ R. Stover, W. Brown, K. Gilmore, and M. Wei

\section{University of California Observatories, Lick Observatory}

We are developing CCD image sensors for astronomy and astrophysics applications. The devices are derived from high-energy physics detector technology. The back-illuminated CCD's are fabricated on ultra-pure, high-resistivity silicon substrates [1-2]. A bias voltage applied to the back-side contact results in full depletion of the $300 \mu \mathrm{m}$ thick substrate. The depletion voltage is relatively low due to the high resistivity of the starting silicon, about $10,000 \Omega$-cm, which corresponds to a purity level of about 1 part in $10^{11}$.

This device has several advantages over conventional thinned, back-illuminated CCD's. Because of the large absorption depth of near-infrared photons in silicon, thinned CCD's typically have poor response in the $\lambda=800 \mathrm{~nm}-1 \mu \mathrm{m}$ region. In addition, fringing results from multiply-reflected waves when narrow-band, long-wavelength light is imaged. The much thicker device described here has greatly improved near-infrared response and negligible fringing. In addition, we have demonstrated good quantum efficiency in the short-wavelength range for back-illuminated devices. This is accomplished with a simple back-side window consisting of a thin layer of in-situ doped polycrystalline silicon with an antireflection coating of indium tin oxide.

[1] S.E. Holland et al, "A 200 x 200 CCD image sensor fabricated on high-resistivity silicon," IEDM Technical Digest, pp. 911-914, 1996.

[2] R.J. Stover et al, "Characterization of a fully depleted CCD on high resistivity silicon," Solid State Sensor Arrays: Development and Applications, Proceedings of SPIE, vol. 3019, pp. 183-188, 1997.

Keywords: Charge coupled device, back illuminated, fully depleted

Author contact: Steve Holland

Lawrence Berkeley National Laboratory

Mail Stop 50B-6208B

Berkeley, CA 94720

510-486-5069 (5401 FAX)

seholland@lbl.gov 


\section{Extrasolar Planet Search with an Adaptive Optics Spectrometer \\ with $R=200,000$}

Jian Ge

Lawrence Livermore National Lab

Roger Angel, Nick Woolf, Mike Lloyd-Hart

Steward Observatory, The University of Arizona

Robert Fugate

Starfire Optical Range, Air Force Phillips Lab

A breakthrough in extrasolar planet searches has been made in recent years using precision Doppler techniques. About 10 extrasolar planets have been detected at typical precision of 15 $\mathrm{m} / \mathrm{s}$. However, these previous techniques have not had the sensitivity to detect solar system analogs, which should represent most planet populations.

The new adaptive optics spectrometer, being developed by us at Steward Observatory, is capable of providing much improved sensitivity to routinely detect Jupiter and Saturn-like planets. The spectrometer takes full advantage of adaptive optics corrected images in the wavelength range between 0.4 to 1.0 microns from the Starfire Optical Range $3.5 \mathrm{~m}$ telescope to provide spectral resolution of $\mathrm{R}=200,000$, a factor of four times higher than previous spectrometers used in planet searches.

It will well resolve absorption line profiles from late-type stars, which are the main targets for present planet searches. With a large format back-illuminated $2 \mathrm{kx} 4 \mathrm{k}$ CCD detector, a wavelength of $400 \mathrm{~nm}$ is covered in a single exposure. This permits the selection of a large number of stellar absorption lines for simultaneous study of line profiles from different stellar atmospheric layers. Precise measurements of the variations in the shapes of different line profiles would help distinguish small Doppler perturbations with amplitude of around $1 \mathrm{~m} / \mathrm{s}$ caused by sub-Jupiter mass planet companions from that caused by stellar activities.

This work was performed under the auspices of the Department of Energy by the Lawrence Livermore National Laboratory under contract W-7405-Eng-48. 


\title{
Extrasolar Planet Search with Adaptive Optics Spectrometer with $\mathbb{R}=\mathbf{2 0 0 , 0 0 0}$
}

\author{
Jian Ge \\ Lawrence Livermore National Lab
}

Roger Angel, Nick Woolf, Mike Lloyd-Hart

Steward Observatory, Univ. of Arizona

Robert Fugate

Starfire Optical Range, Air Force Phillips Lab

\author{
Outline
}

- Overview

- Novel Adaptive Optics Spectrometer

- Sub-Jupiter Mass Planet Searches 


\section{Overview}

\section{5-Present}

- A dozen of extra-solar planets discovered with precision Doppler technique

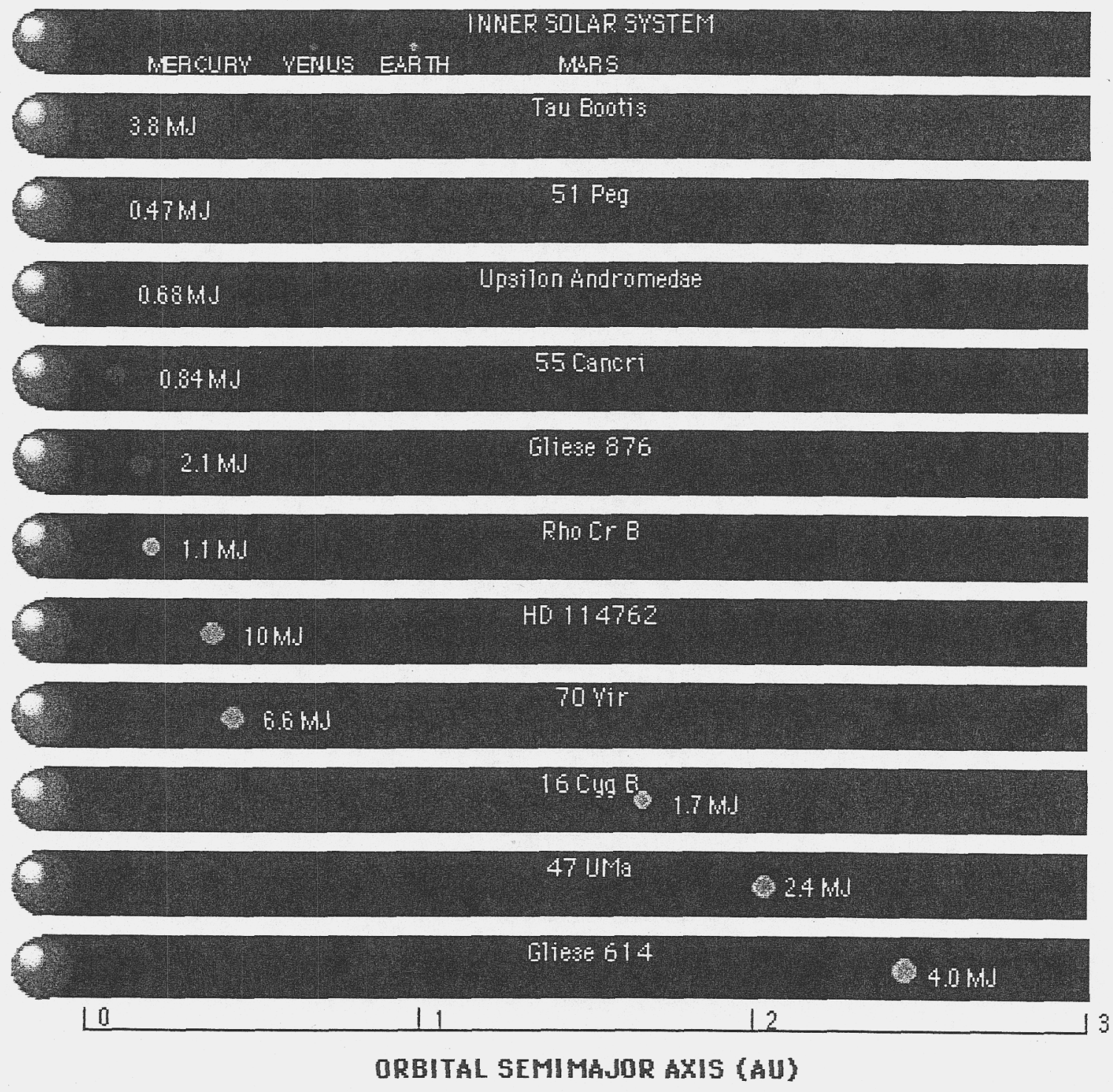

From http://cannon.sfsu.edu/ gmarcy/planetsearch 


\section{Extrasolar Planets Properties}

- Radius < 3 Astronomical Unit vs. 5 AU for Jupiter

- Mass $\sim 1-10 \mathbf{M}_{\mathrm{Jupiter}}$

- Period $~ 3.3$ - 1092 days

- Eccentricity $\sim 0.0-0.7$

\section{Current Technology Limits}

- Doppler precision $\geq 3 \mathrm{~m} / \mathrm{s} \Rightarrow 4 \sigma$ detection limit, Jupiterlike planets $(12.5 \mathrm{~m} / \mathrm{s}$ variation on the Sun)

- Spectral resolution $\mathrm{R} \sim \mathbf{5 0 , 0 0 0}$, limited by

ATMOSPHERIC SEEING, no line profile information

- Stellar activity including photospheric granulation, turbulence, magnetic cycling, stellar oscillation etc could mimic small Doppler perturbation, $\sim 3 \mathrm{~m} / \mathrm{s}$, caused by smaller planets 


\section{What is Next for Doppler Planet Search?}

- Improve precision to $1 \mathrm{~m} / \mathrm{s}$ or less for sub-Jupiter mass planet search

- Saturn-like planet, $3 \mathrm{~m} / \mathrm{s}$ over 30 years

- Earth-like planet, $0.1 \mathrm{~m} / \mathrm{s}$ over 1 year

Solutions to New Challenges

- Higher spectral resolution with adaptive optics (Ge et al.):

- Resolve line profiles

- Simultaneously trace stellar activity \& planet perturbation

- Reduce wavelength calibration errors

- Combination of spectrometer and interferometer (Erskine \& Ge):

- very stable point spread function (PSF) over broad wavelength coverage 
Solar Spectrum at Resolution $R=550,000$

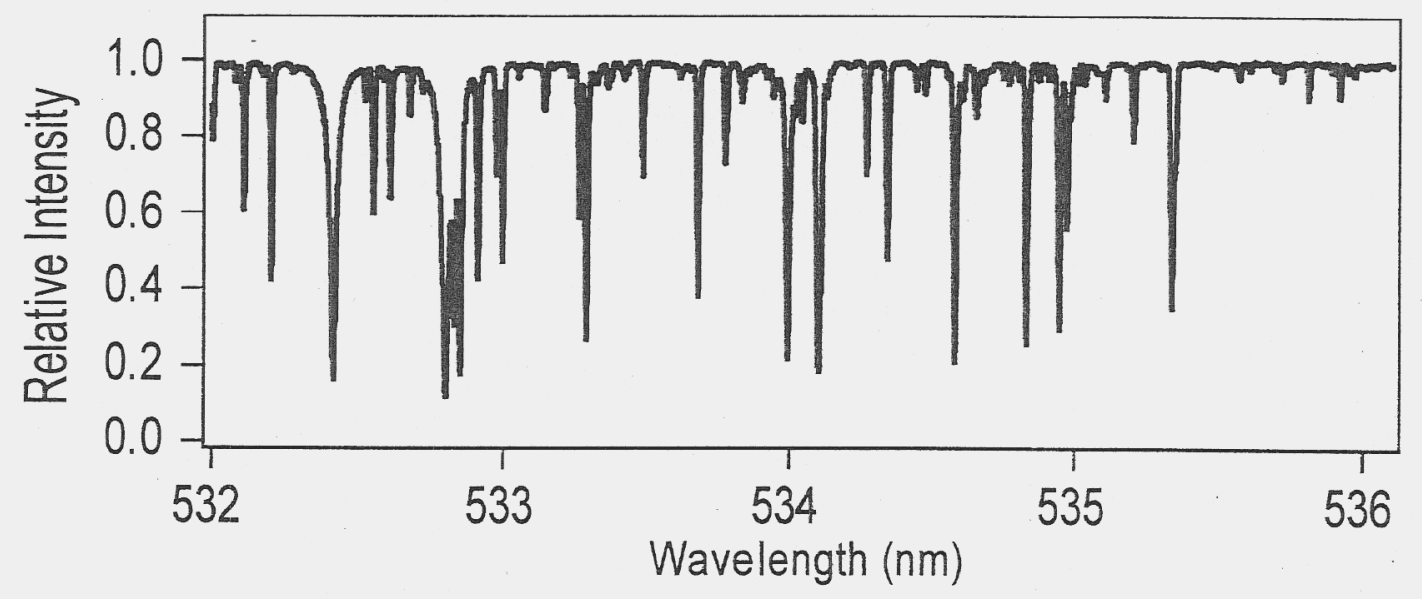

Solar Spectrum at Different Spectral Resolution

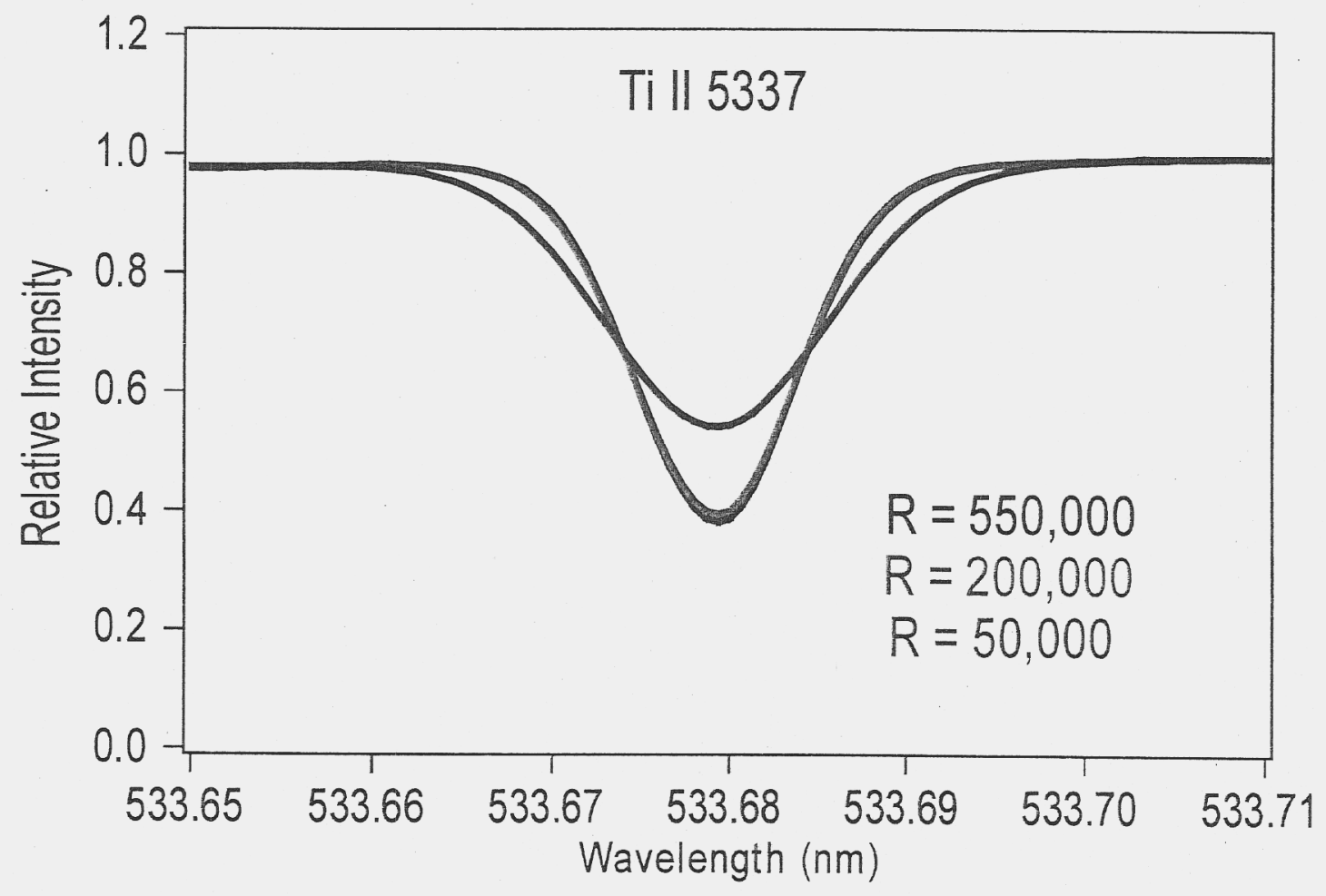


Bisector of Solar Spectral Line, Ti II 5337, with $R=550,000$

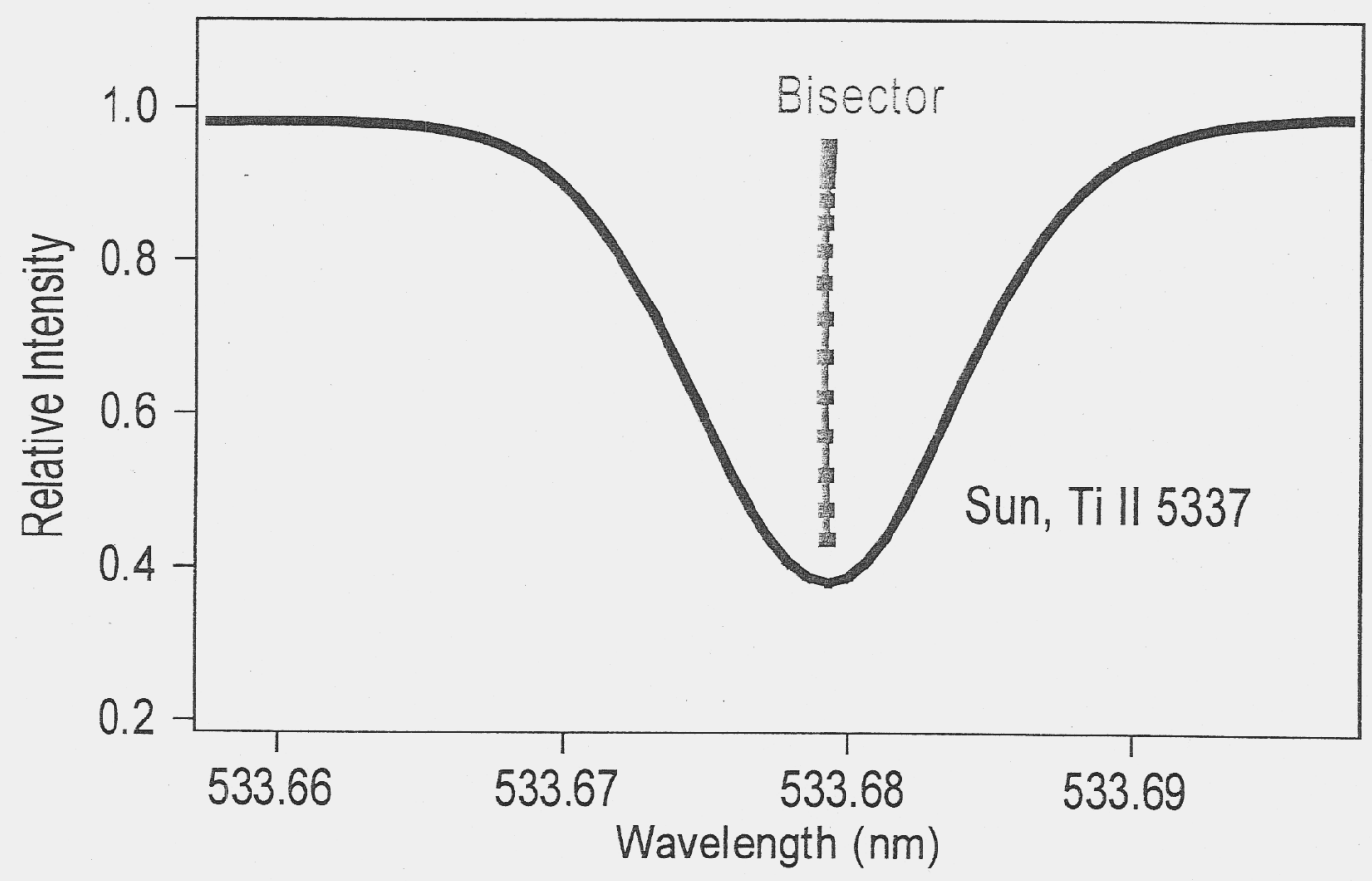

Expanded Part of Bisector: Distorted "C" Shape

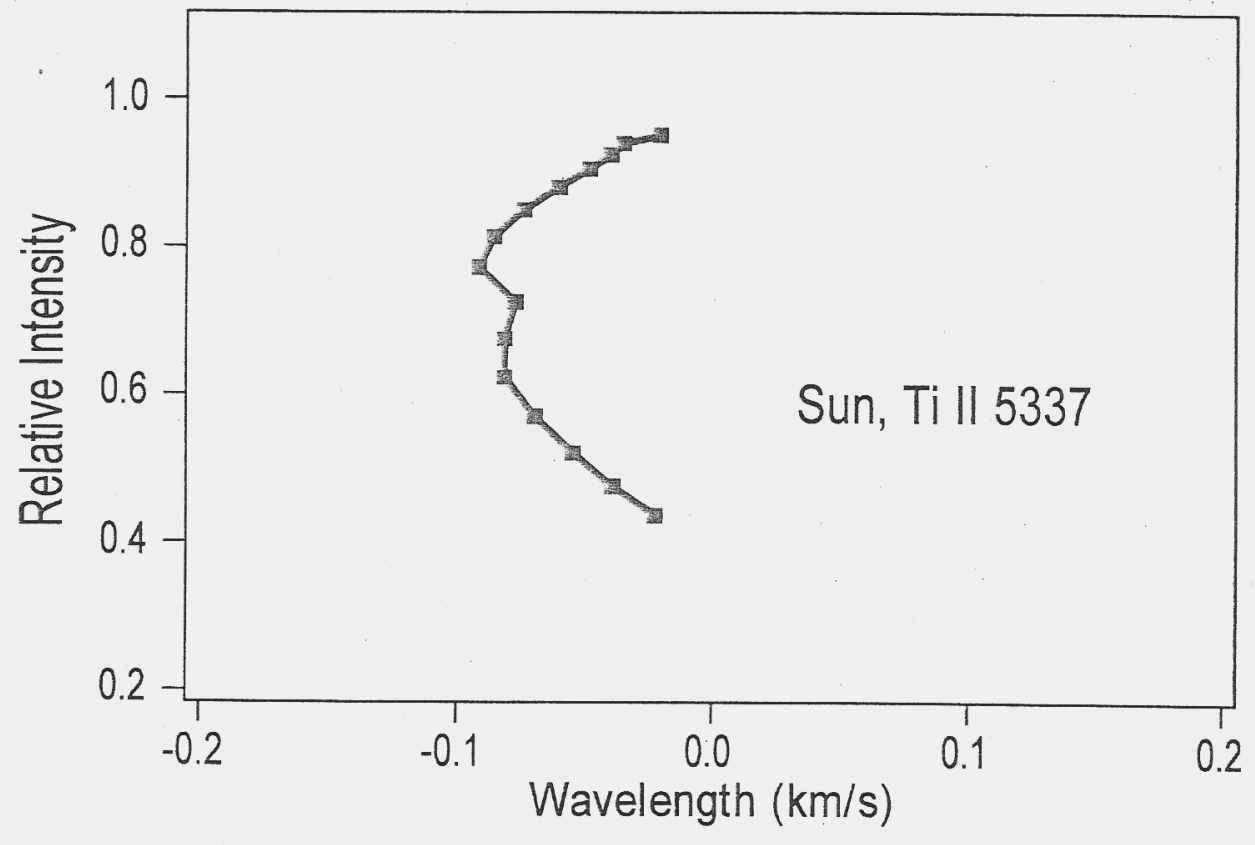




\section{Comparison of Bisectors from Different Resolution}

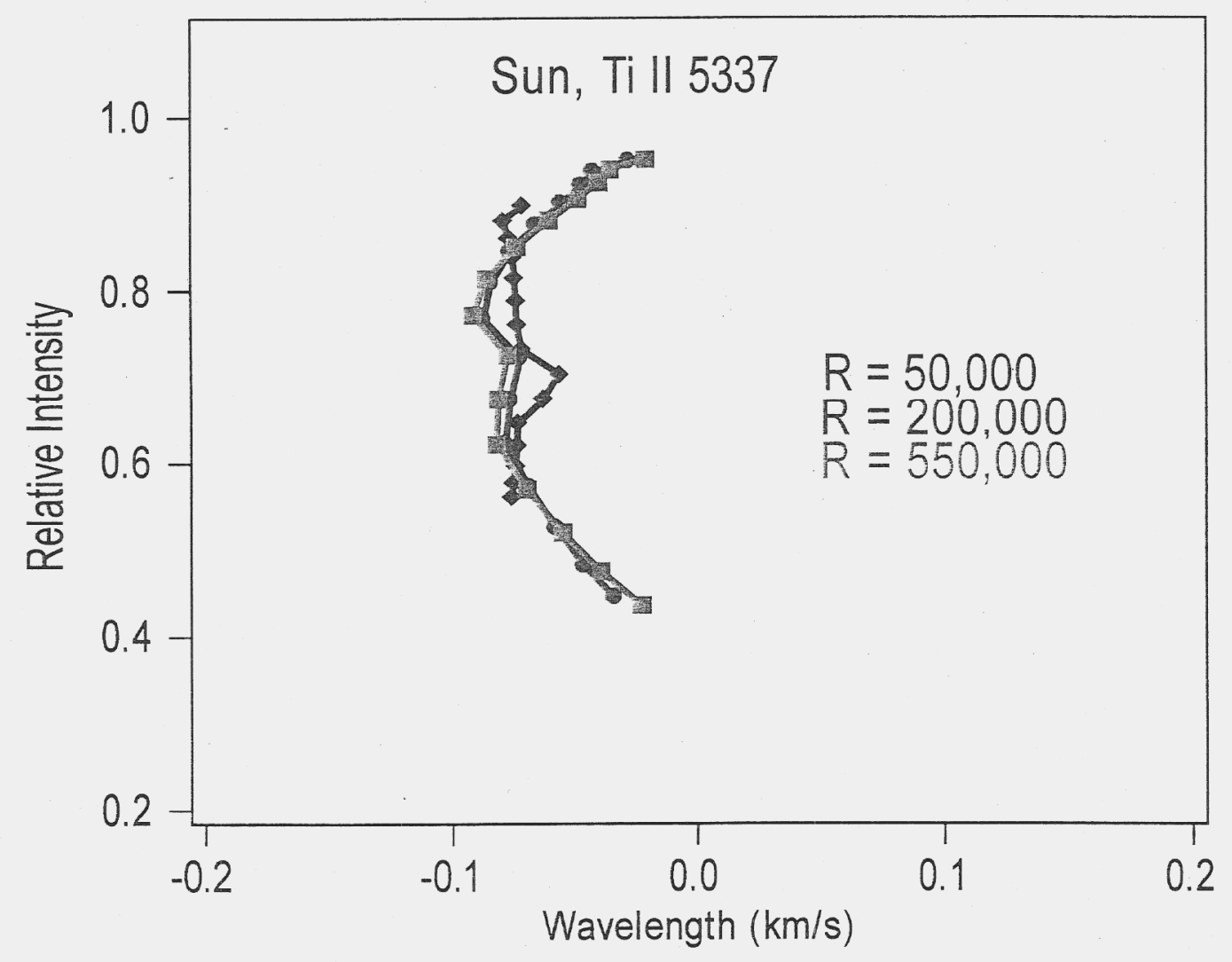

$R=50,000$ cannot preserve line profile information $R=200,000$ required for line profile measurements 


\section{Novel Adaptive Optics Spectrometer}

\section{I-band $(0.9 \mu \mathrm{m})$ Image at Mt. Wilson 100 inch}

Seeing-limited image, 1 arcsec

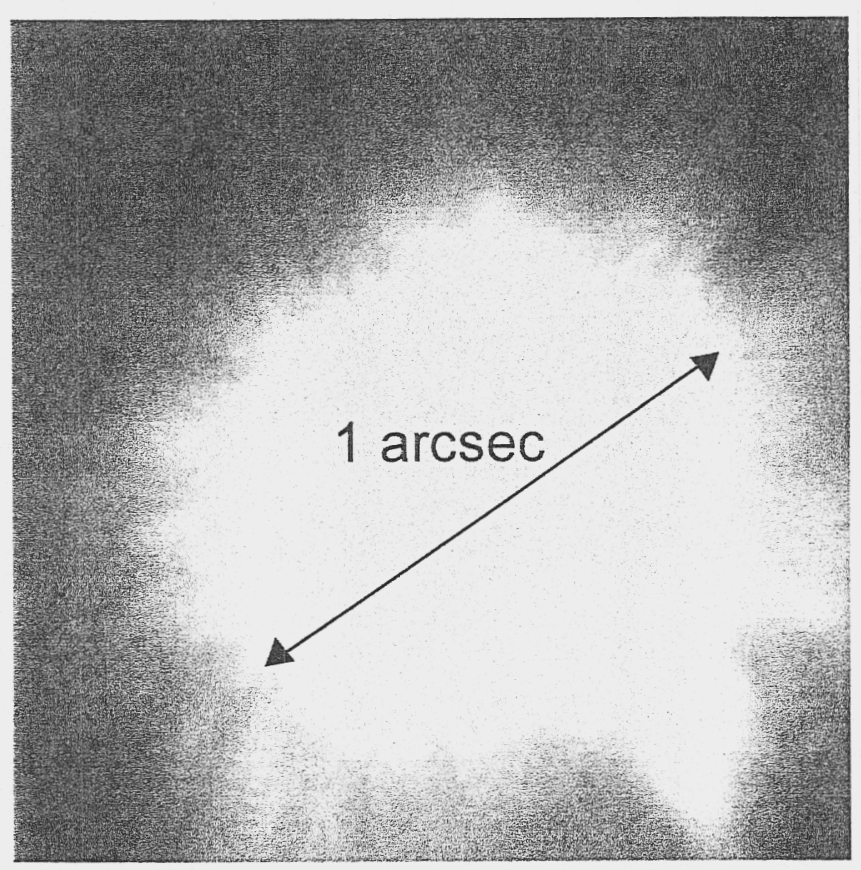

Adaptive Optics corrected image: 0.071"

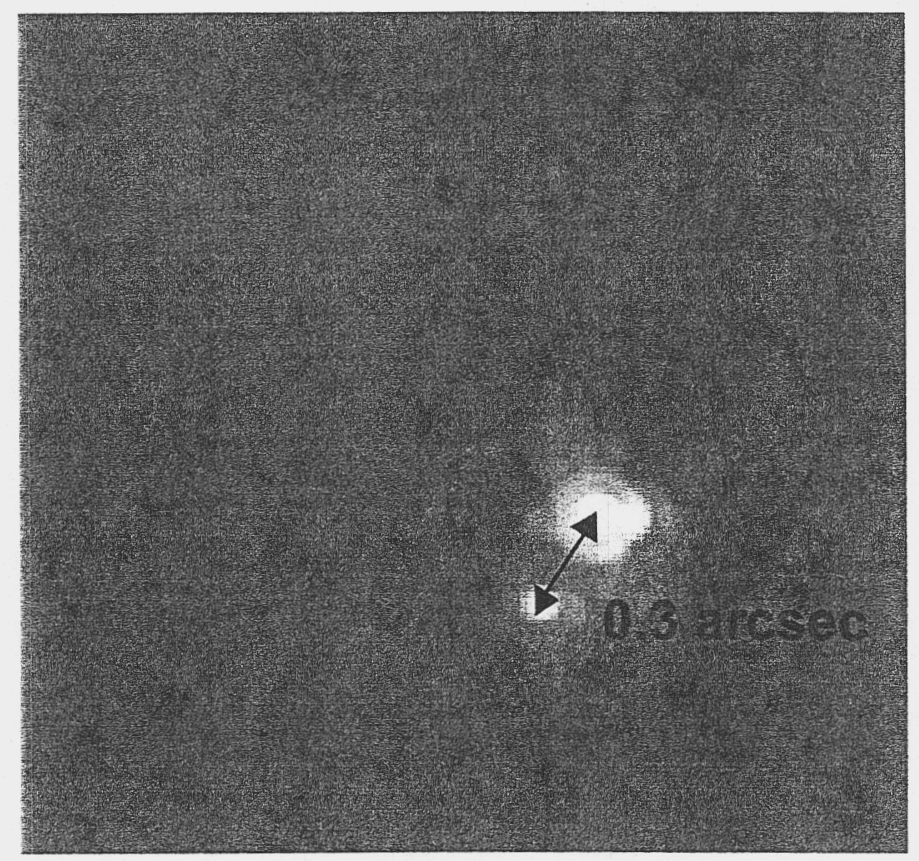




\section{Advantages of adaptive optics spectroscopy:}

- Smaller slit width without large photon loss $\Rightarrow$ higher resolution, high efficiency

- Smaller image size in the spatial direction $\Rightarrow$ packing more cross-dispersed orders on a detector $\Rightarrow$ large wavelength coverage

- Smaller image size $\Rightarrow$ higher spatial information

- Optics small and slow $\Rightarrow$ low cost

New era of ultra-high resolution AO spectroscopy

$\mathrm{R} \sim 200,000-\mathbf{5 0 0 , 0 0 0}$ with $\mathrm{AO}$ vs. $\mathrm{R} \sim 50,000$ w/0 AO

Test of a novel prototype AO spectrometer at Starfire Optical Range $1.5 \mathrm{~m}$ telescope, Air Force Phillips Lab and at Mt. Wilson 100inch telescope 
Setup of AO spectrometer at Mt. Wilson 100 inch telescope, Nov. 1997

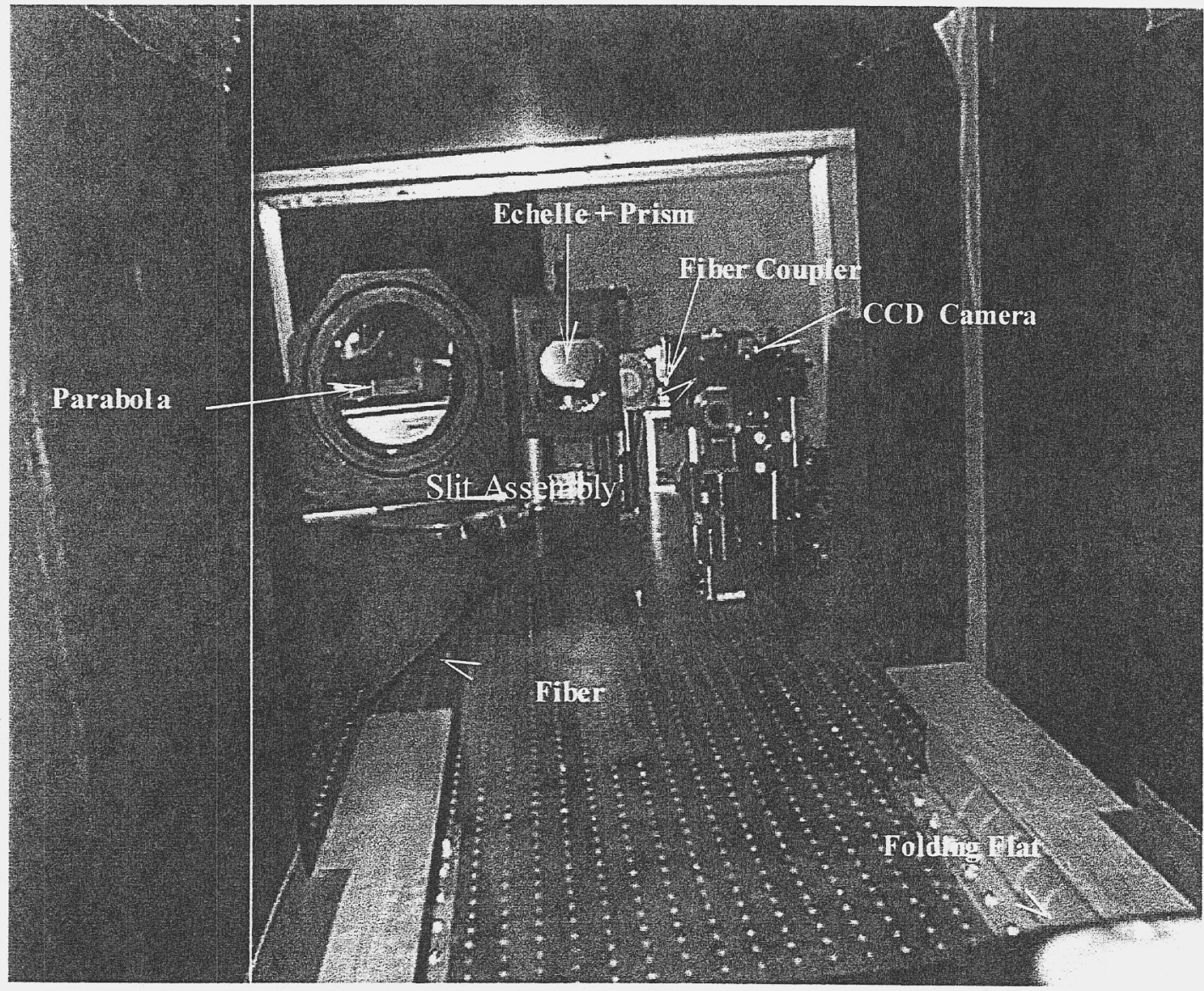

- Off-axis parabola focal length $=6 \mathrm{~m}$

- $\mathrm{F} / \#=55$

- Grating: $250 \times 125 \mathrm{~mm}^{2} \mathrm{R} 2$ echelle

- Cross-disperser: $160 \times 160 \mathrm{~mm}^{2} 8 \mathrm{deg}$ wedge prism

- Beam diameter: $125 \mathrm{~mm}$

- Working wavelength: 0.4-1.0 $\mu \mathrm{m}$ 


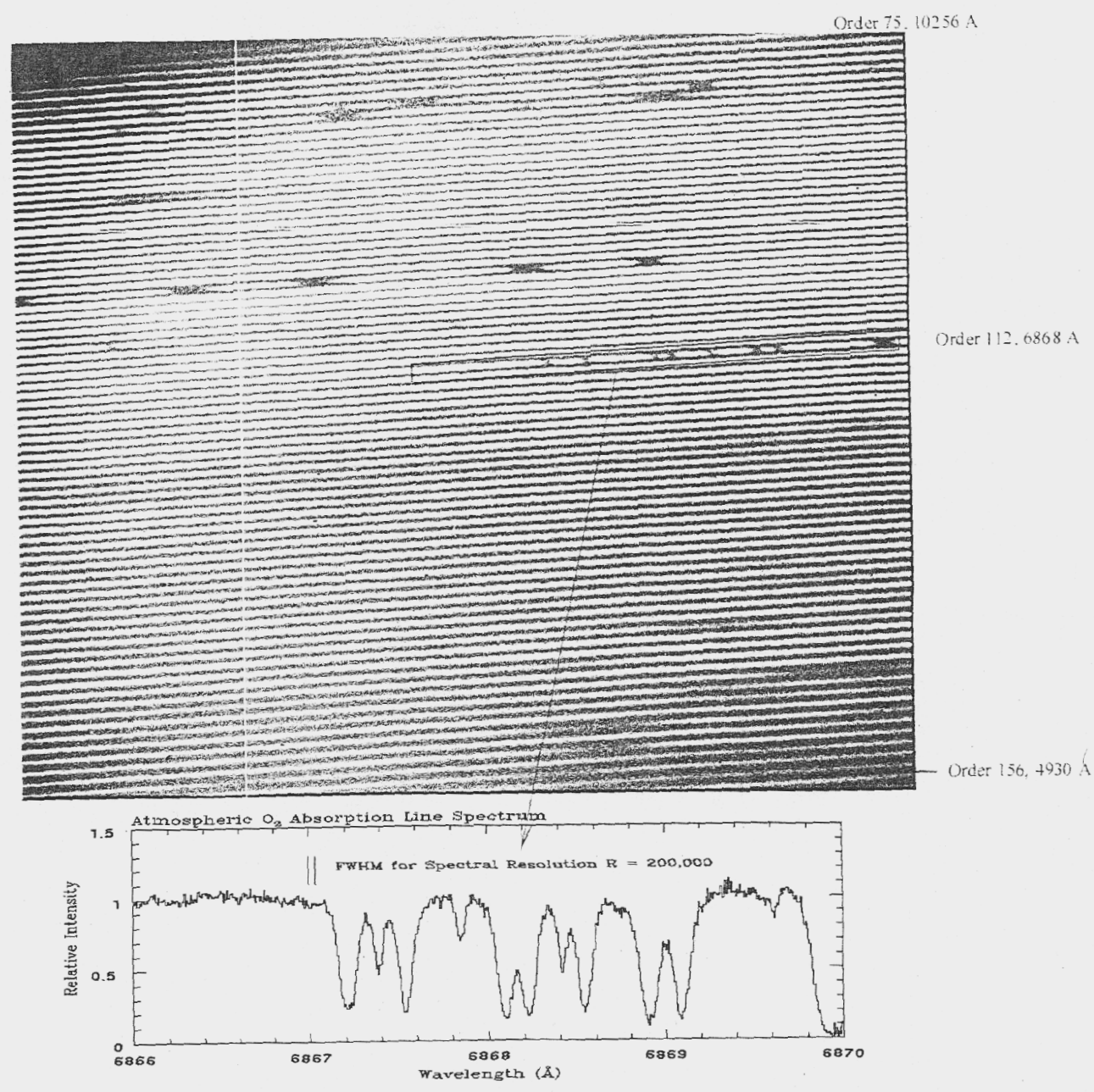

Fiber-fed spectrum of $\alpha$ Ori at Mt Wilson 100 inch telescope, Nov. 1997. A near single mode fiber with only $10 \mu \mathrm{m}$ core size

- Spectral Resolution, $\mathrm{R} \sim 200,000,0.5-1.0 \mu \mathrm{m}$

- 80 cross-dispersed orders covered by a $25 \times 25 \mathrm{~mm}^{2} \mathrm{CCD}$ detector

- $\sim 650 \AA$ wavelength coverage 


\section{Adaptive optics spectroscopy of interstellar pottasium

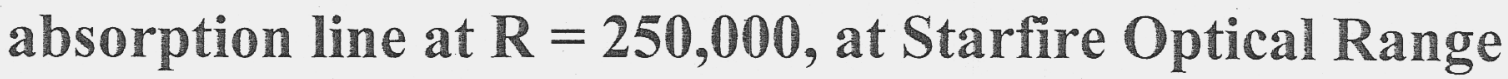 $1.5 \mathrm{~m}$ telescope, Nov. 1995}
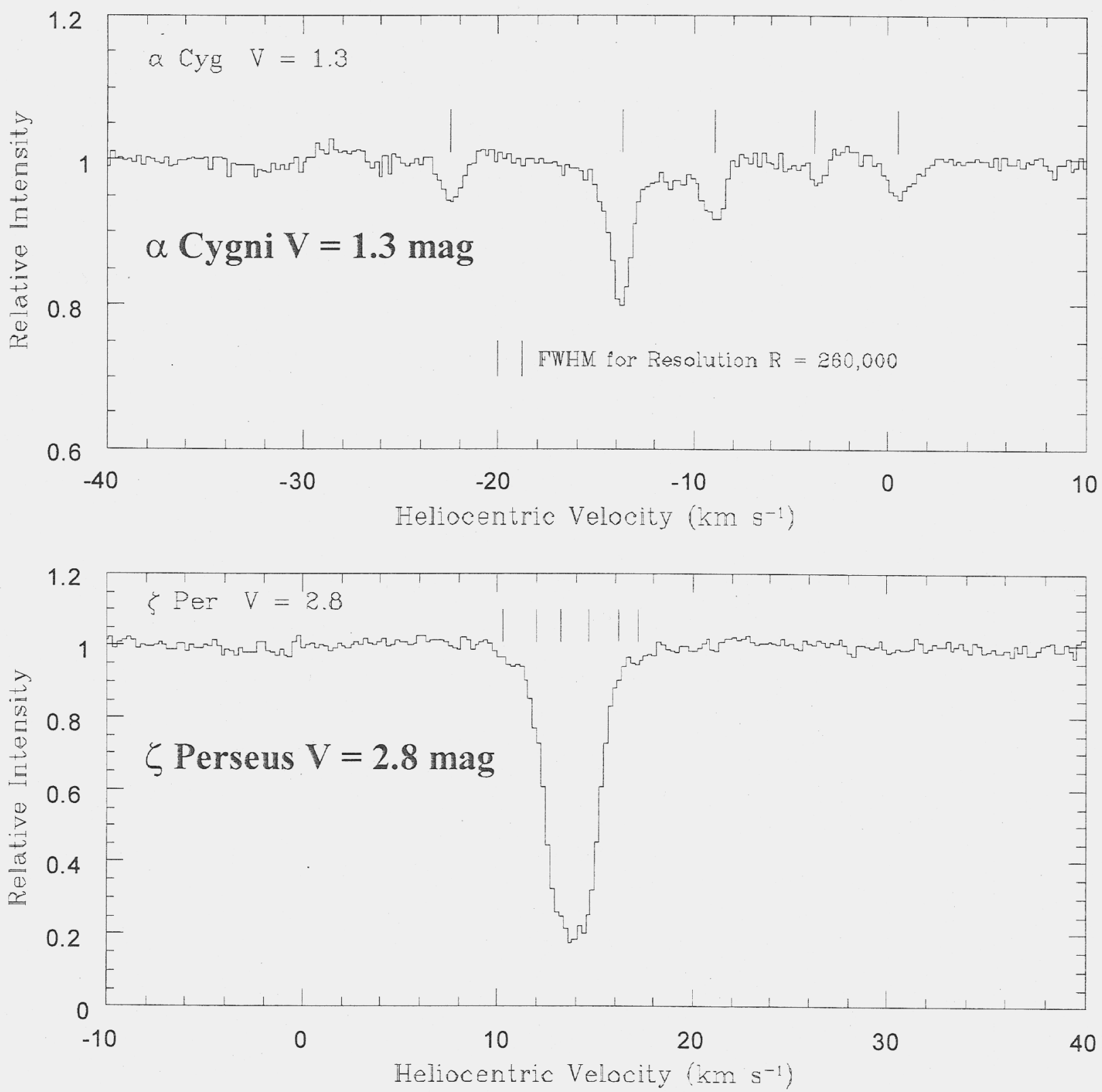


\section{New Optimized AO Spectrometer Design}

Maksutov-type design, all spheres

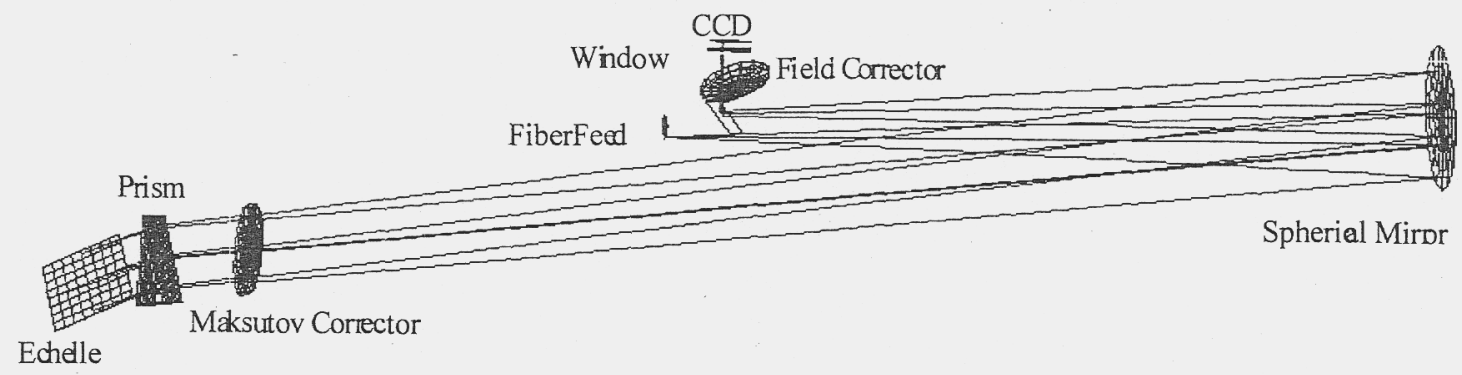

3 meter

Near single mode fiber-fed optical cross-dispersed echelle spectrometer

- Focal length: $1900 \mathrm{~mm}$

- Focal ration: $\mathrm{F} / \#=16$

- $\sim 10 \mu \mathrm{m}$ core size fused silica fiber, preserve AO system etendue

- Separated from telescope, more stable

- Portable, easily matched with different optical telescopes with AO system

- Much less scattering light 


\section{Spectral format on a $2 \mathrm{kx} 4 \mathrm{k} \mathrm{CCD}$ detector with $30 \times 60 \mathrm{~mm}^{2}$}

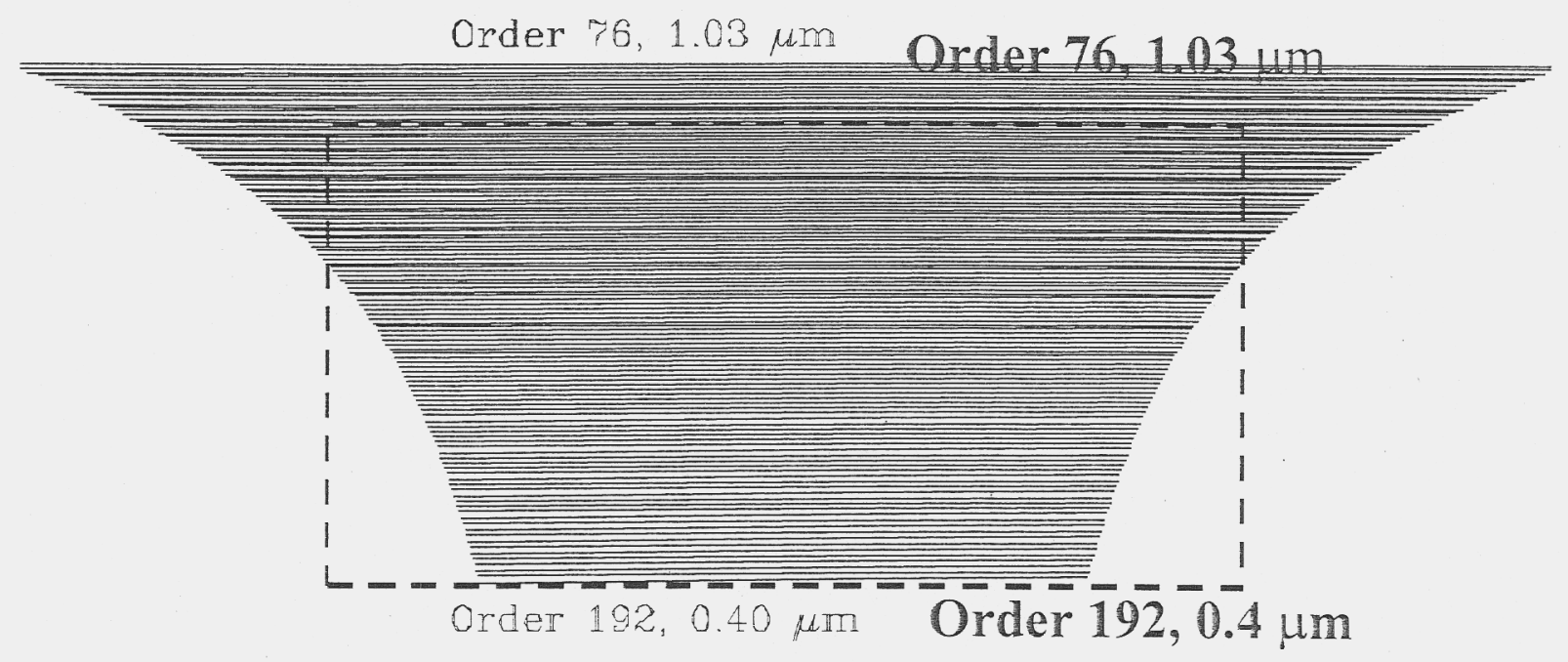

- Spectral resolution: $\mathrm{R}=\mathbf{2 0 0 , 0 0 0}$

- Total detection efficiency: 4\% (including telescope, AO, spectrometer and $\mathrm{CCD}$ detector)

- Wavelength coverage per exposure: $4000 \AA$ 


\section{Sub-Jupiter Mass Planet Searches}

* Signal-to-noise ratio per CCD pixel: $\mathrm{S} / \mathrm{N} \sim 1000$ At SOR $3.5 \mathrm{~m}$ telescope, $R=200,000,0.5$ hour exposure, $V=5$ mag.

* Doppler shift error per pixel: $\sigma_{V} \sim 18 \mathrm{~m} / \mathrm{s}$ (photon statistics)

* Doppler shift error per absorption line: $\sigma_{\mathrm{V}} \sim 4 \mathrm{~m} / \mathrm{s}$ for $\sim 20$ pixel to sample typical lines

* For $\sim 700$ strong stellar absorption lines covered by iodine absorption $(0.5-0.6 \mu \mathrm{m})$, mean error: $\sigma_{\mathrm{V}} \sim 0.2 \mathrm{~m} / \mathrm{s}$ - ultimate precision! 
Saturn Signal Observed with a Precision of 1 and $3 \mathrm{~m} / \mathrm{s}$
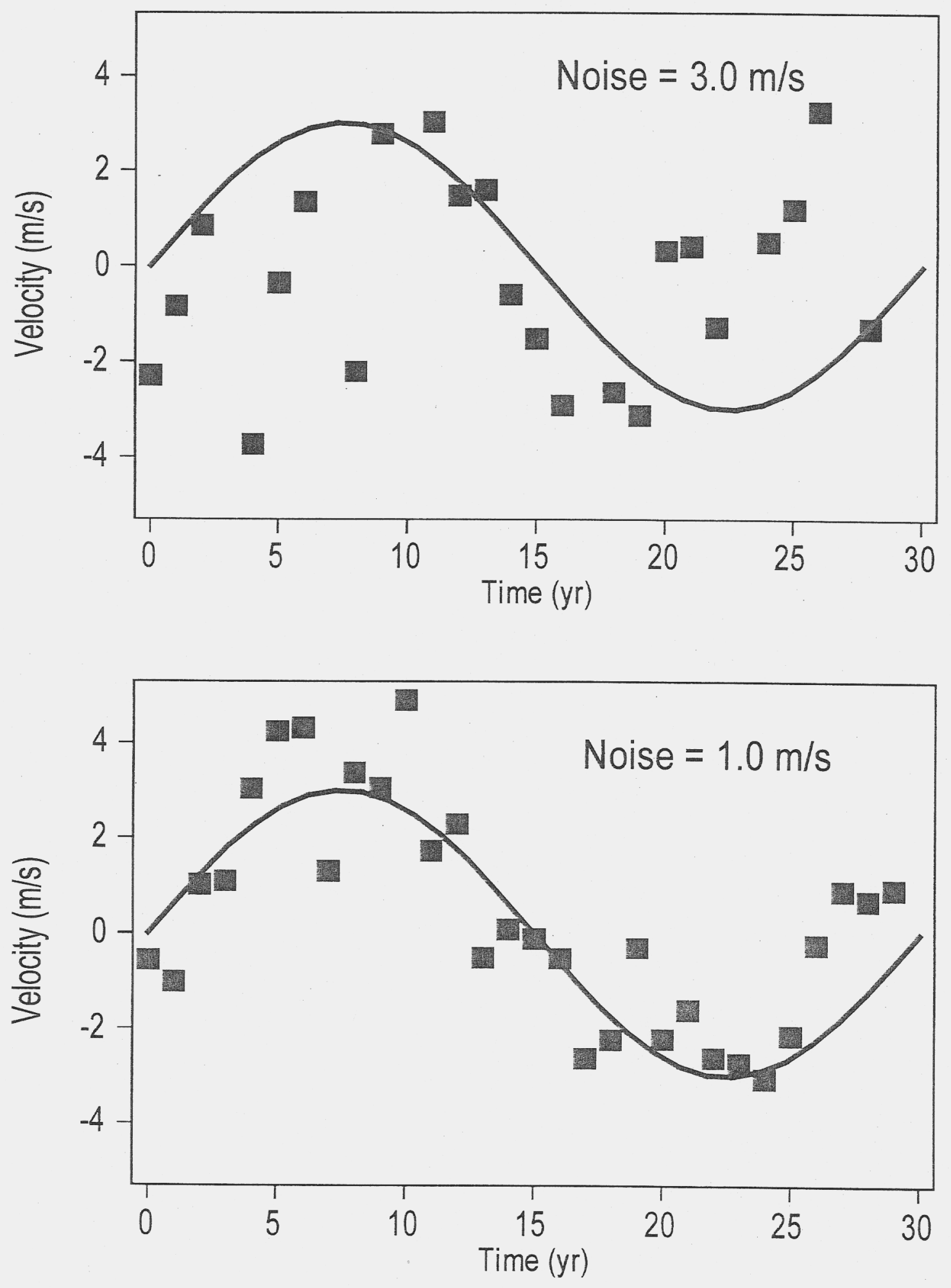
- First time of large wavelength coverage, $\sim 4000 \AA$, with $R=\mathbf{2 0 0 , 0 0 0}$

- Simultaneously monitor different stellar absorption lines of different ionization and excitation, which respond differently to different atmospheric heights, and to hotter and cooler patches of surface

- Simultaneously monitor perturbations from small extra-solar planets and stellar activity, initiate the study with $\sim 1 \mathrm{~m} / \mathrm{s}$ accuracy 


\section{Micro-Satellite Technology for the $21^{\text {st }}$ Century Lawrence C. $\mathrm{Ng}$ Ng6@1lnl.gov}

This paper will examine the development of micro-satellite technology for use in space. It is envisioned that potential missions for the micro-satellites may include: rendezvous with a satellite, conduct proximity inspection, land on a satellite, and perform rescue or other logistic mission. The critical enabling technology for this capability is the development of an agile micro-satellite or MicroSat. A MicroSat is a small satellite weighing only a few tens of kilograms but carrying onboard a host of lightweight sensors and actuators, inertial navigation instruments and avionics. When equipped with a propulsion system and smart guidance, navigation and control software, the MicroSat has the ability to maneuver precisely in space in both orientation and translation. When further equipped with smart vision based image analysis software and repair tools, the MicroSat can autonomously perform many of the functions mentioned above. Ground command and control support can therefore be minimized and used only to augment and enhance performance by providing ground truth knowledge, such as emphemeris data. The MicroSat can also function in a telerobotic mode, with a man-in-the-loop, to more effectively execute complex (less predictable) maneuvers and to provide a manned presence (in surrogate form) when and where one is required. This technology will help define the critical pathfinder capability for future operations in near Earth space.

UCRL-ID-129880 
Date: 29 Oct 1998 14:12:05 -0700

\section{Detecting Massed Troops with the French SPOT Satellites \\ By Vipin Gupta and George Harris}

This study is a technical assessment on the utility of the French SPOT satellites for detecting troop concentrations in a desert environment. The capabilities of the SPOT satellites for this specific application were tested empirically using two archived SPOT images of the Saudi-Iraqi border acquired shortly before and during the 1991 Gulf War. Change detection analysis was done to detect, locate, and identify troop deployments. The results were then extrapolated to forecast the feasibility of using medium resolution, broad swath imagery to detect and track future offensive attack preparations. The presentation concludes with a discussion on the security implications associated with the future deployment of SPOT-type systems as well as an appendix on the impact of using SPOT-type imagery to support conventional and unconventional weapons strikes on imaged troop concentrations. 


\section{Detecting Troop Concentrations using the French SPOT satellite}

A Case Study from the 1991 Gulf War

Vipin Gupta

Systems Research Dept.

Sandia National Labs

Livermore, $C A$
George Harris

NAI Directorate

Lawrence Livermore Lab

Livermore, $C A$

Initiated by SNL/CA and LLNL

Sponsored by Sandia Cooperative Monitoring Center (NN-40) 


\section{What's the question and why answer it}

Q: Can one use fuzzy ( $\sim 10 \mathrm{~m}$ resolution) satellite images to detect the massing of troops?

Q: Can one use such imagery for early warning and targeting?

A: Yes and Yes 


\section{How we did it...}

1. Selected a real war situation (1991 Gulf War)

2. Selected a division to observe (the US 24th Mech)

3. Obtained "before" and "after" images

4. Conducted change detection analysis

5. Compared results with ground truth 


\section{Implications}

- Moderate resolution, broad swath imagery is widely available to all countries

- Countries can utilize this technology to monitor borders and DMZs

* Technology can be used for long-range targeting 


\section{Deployed and planned commercial satellites with SPOT-type sensors}

\begin{tabular}{|c|c|c|c|c|}
\hline Satellite & Country & Resolution of & Swath & Launch Date \\
\hline & & SPOT-type Sensor & Width & \\
\hline SPOT 1-4 & France & 10 meter panchromatic & $120 \mathrm{~km}^{*}$ & $\begin{array}{l}1986,1990, \\
1993,1998\end{array}$ \\
\hline $\begin{array}{l}\text { COSMOS series } \\
\text { TK-350 }\end{array}$ & Russia & 10 meter panchromatic & 300 km & $1987-1998$ \\
\hline IRS-1C, IRS-1D & India & 6 meter panchromatic & $141 \mathrm{~km} *$ & 1995,1997 \\
\hline Priroda & Russia & 6 meter panchromatic & $40,80 \mathrm{~km}$ & n 1996 \\
\hline ADEOS & Japan & 8 meter panchromatic & $80 \mathrm{~km}$ & 1996 \\
\hline OrbView 3 & USA & 8 meter hyperspectral & $5 \mathrm{~km}$ & 1999 \\
\hline KOMPSAT & S. Korea & 10 meter multispectral & $40 \mathrm{~km}$ & 1999 \\
\hline RESOURCE21 & USA & 10 meter panchromatic & $205 \mathrm{~km}$ & 2000 \\
\hline ALOS & Japan & 10 meter multispectral & $70 \mathrm{~km}$ & 2000 \\
\hline
\end{tabular}




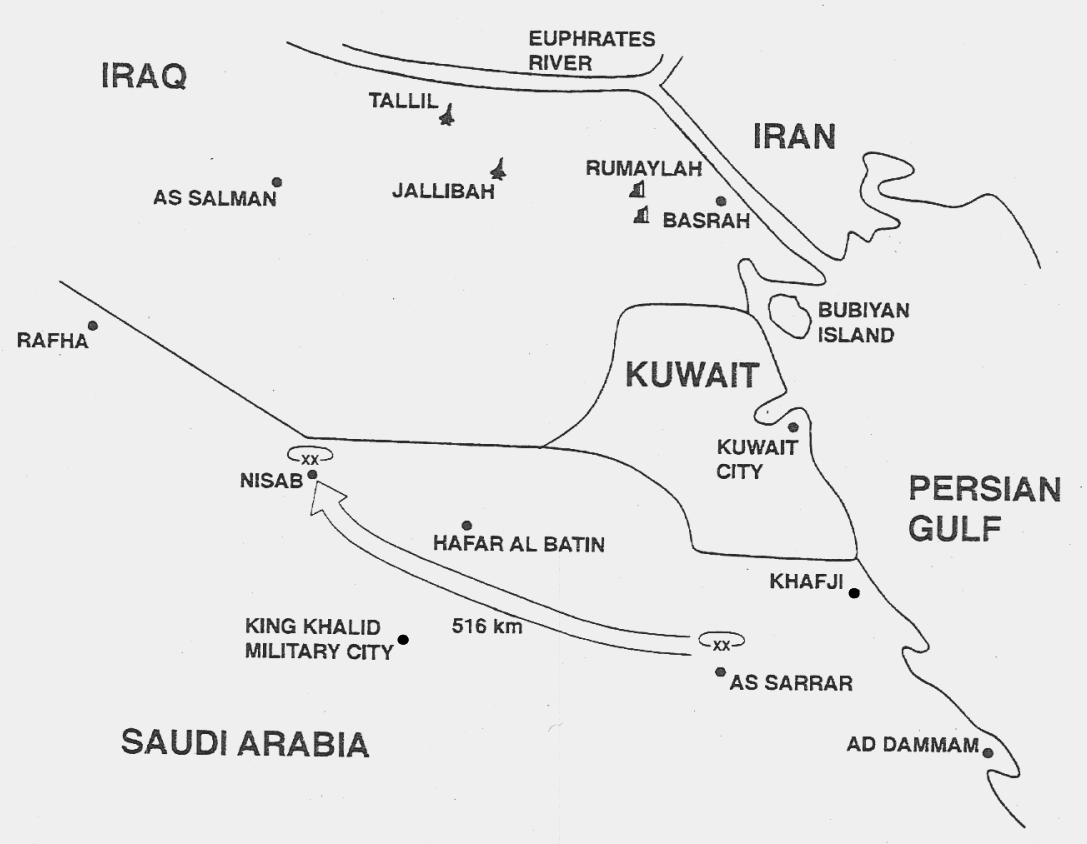




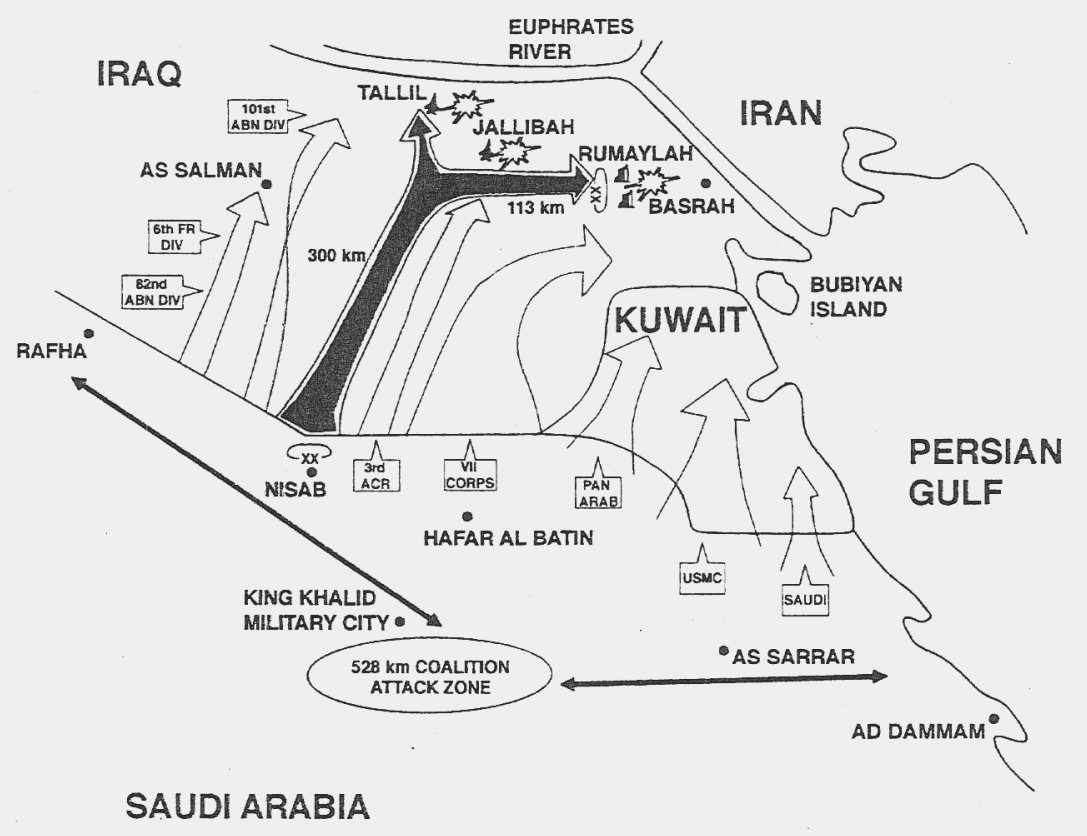

Figure 1 


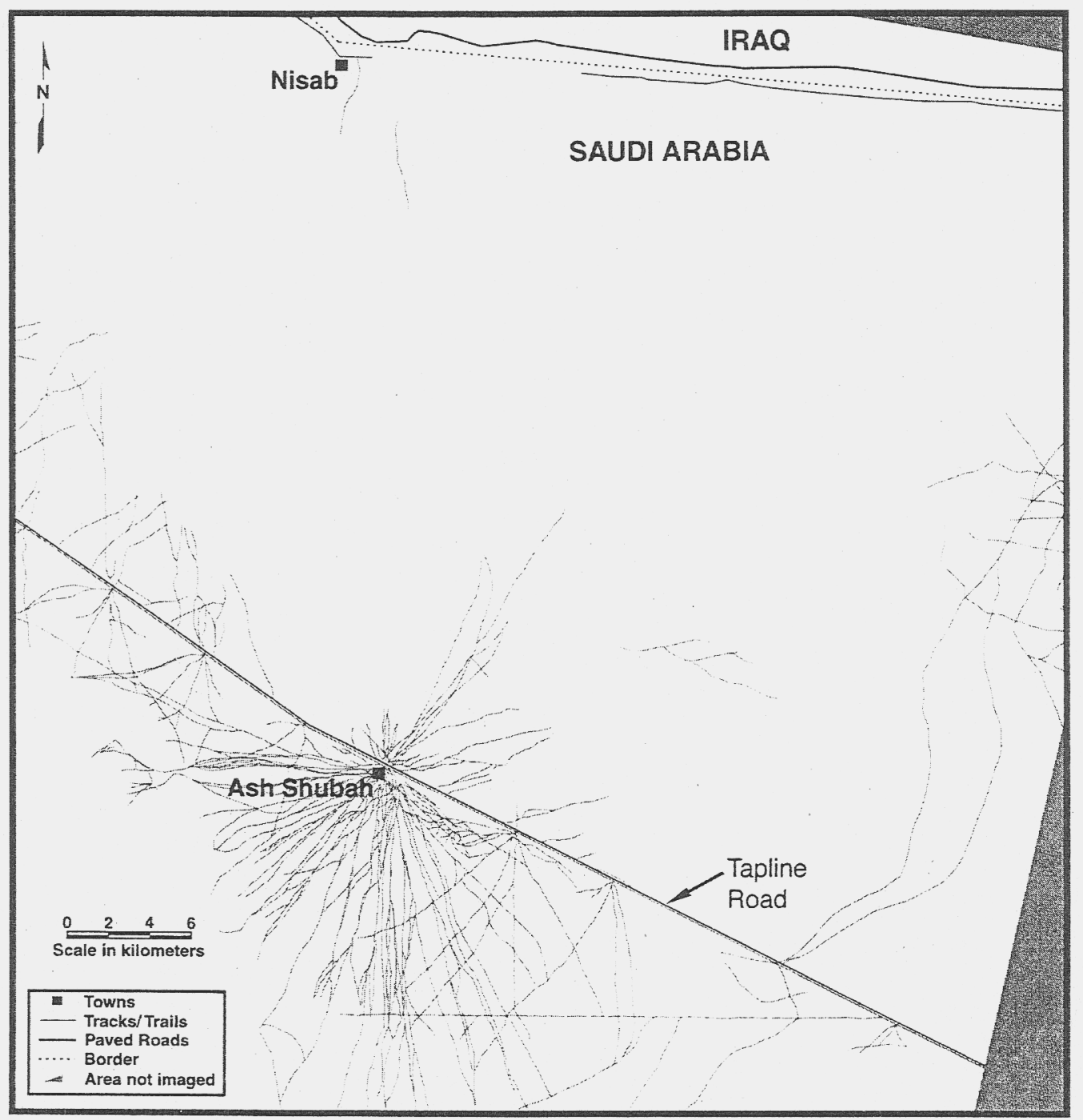




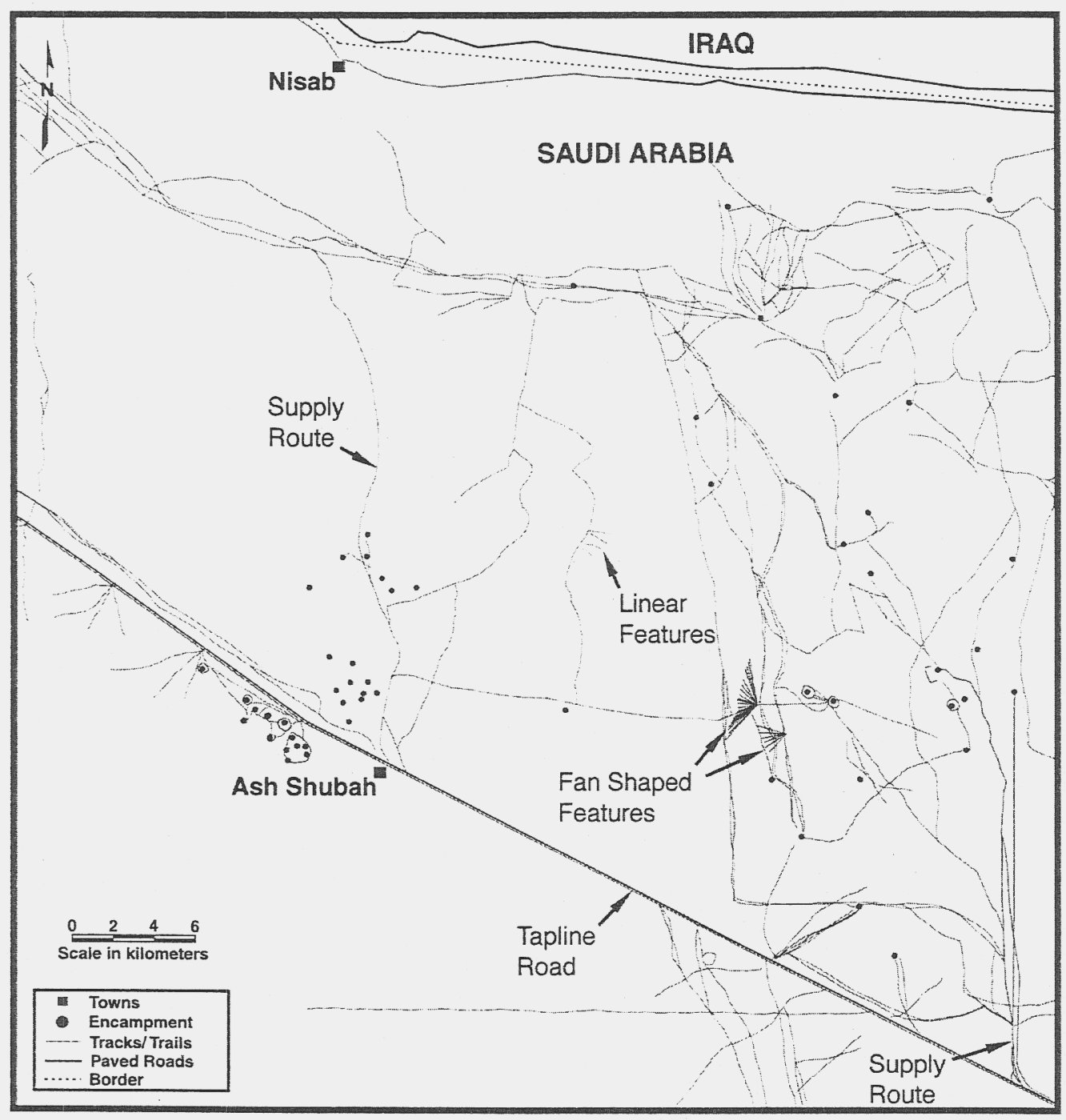




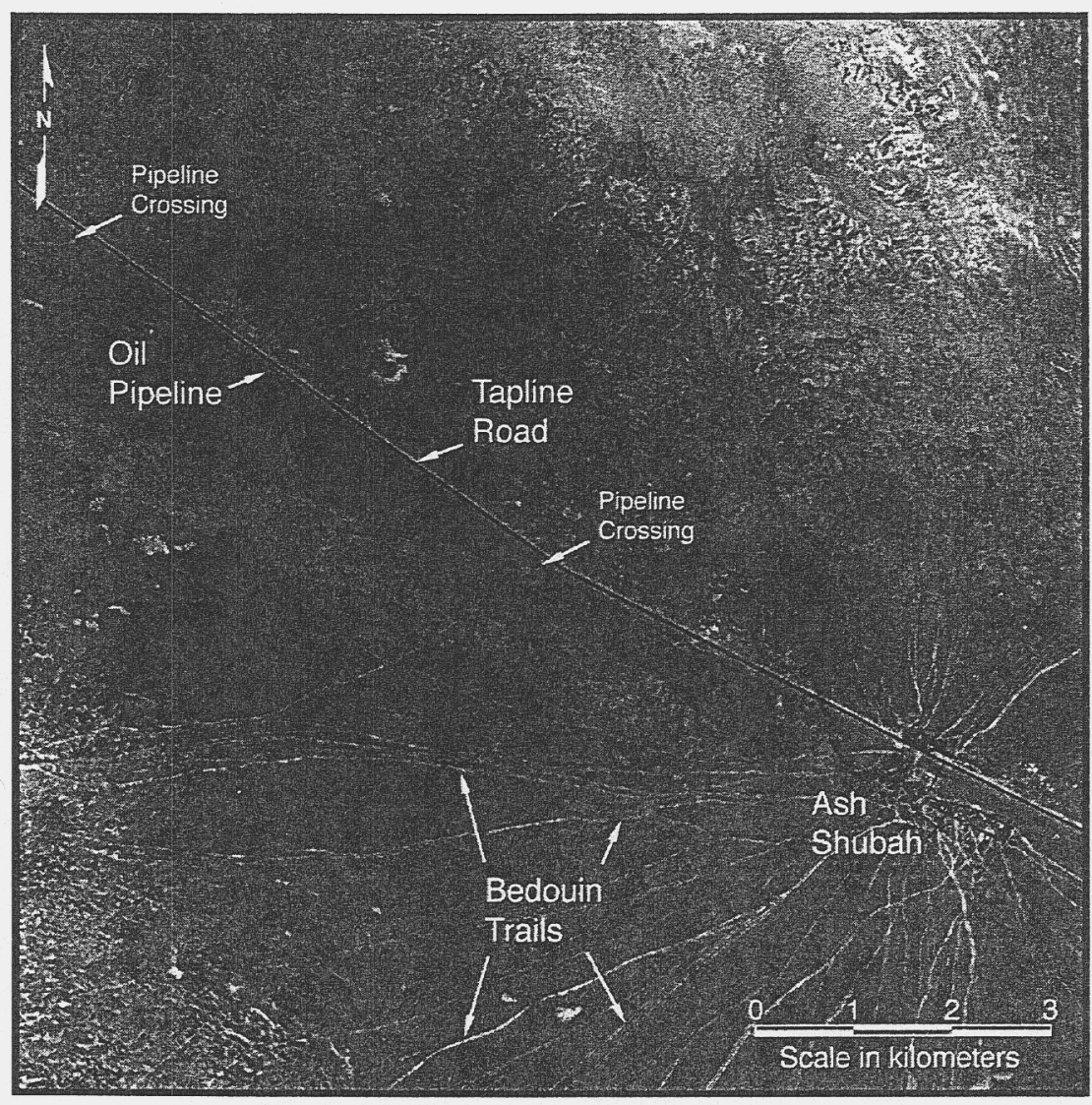




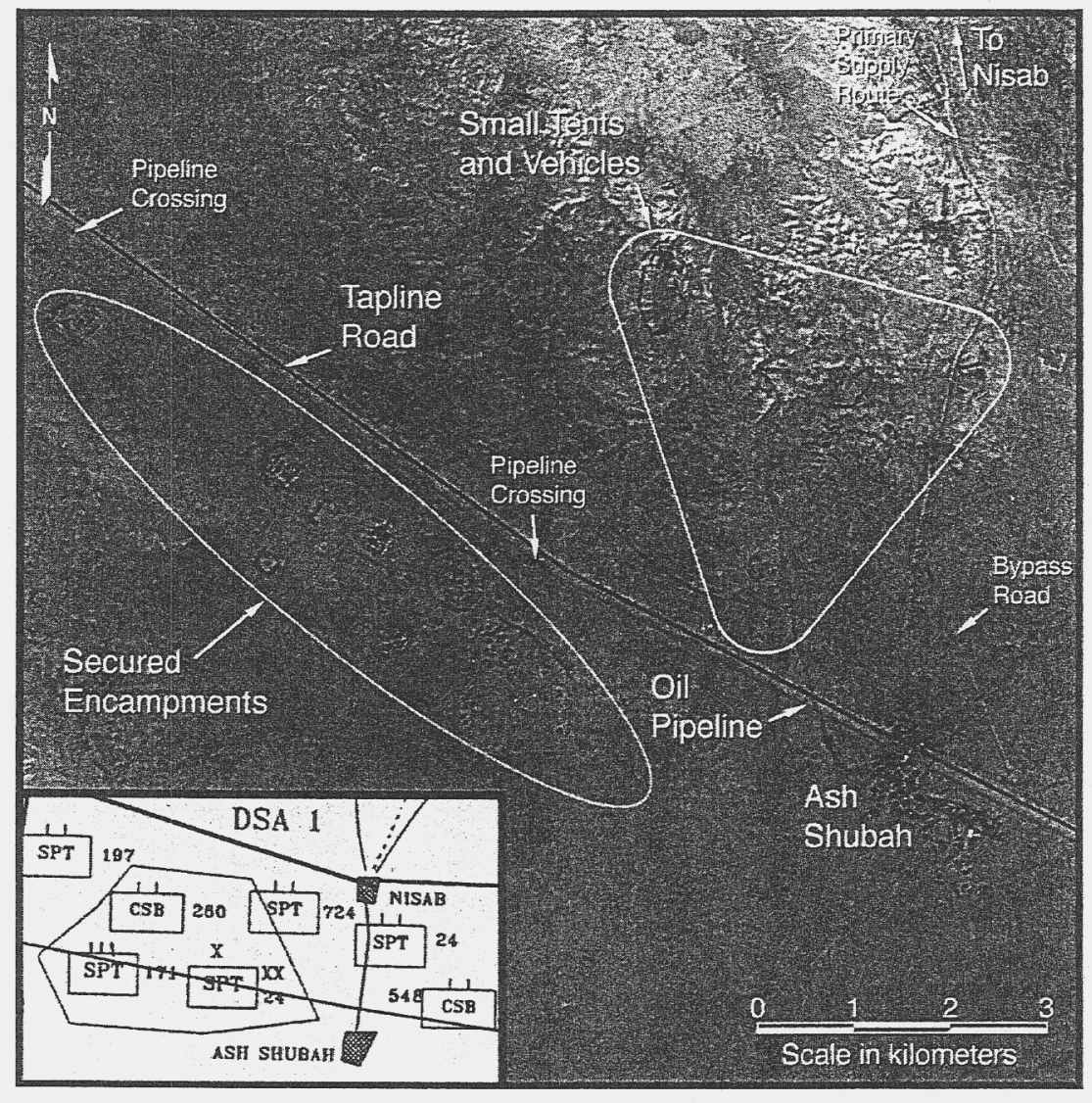




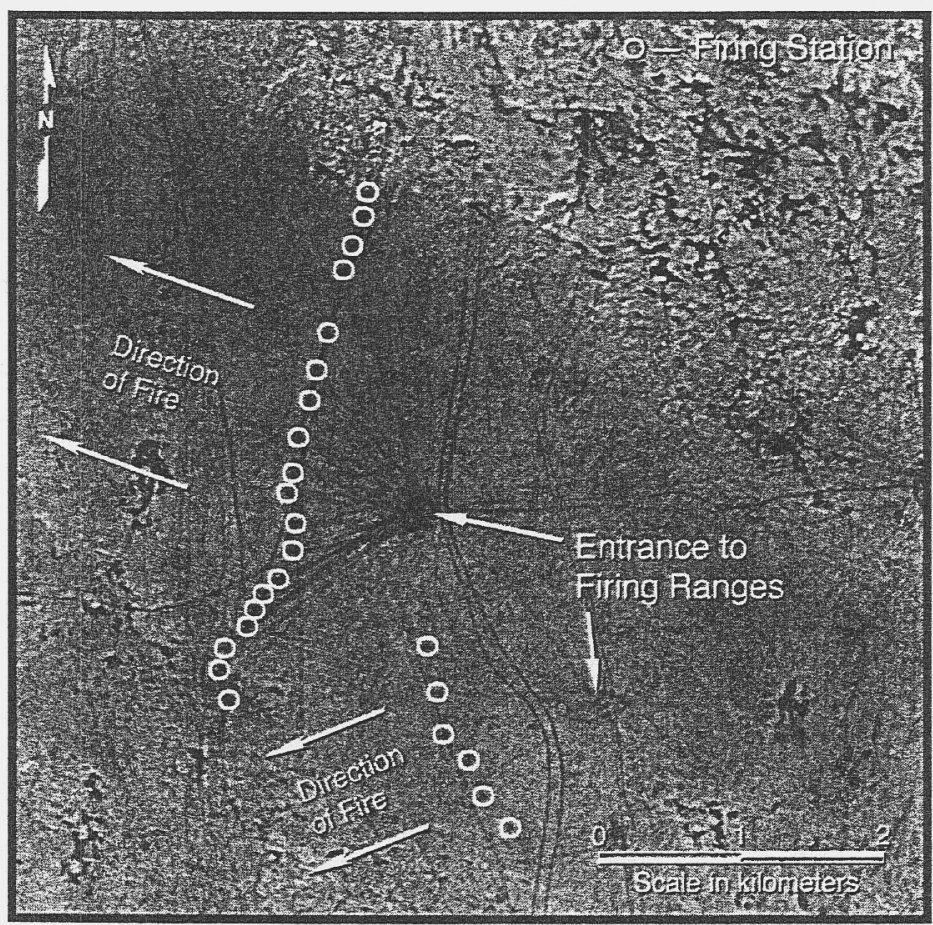




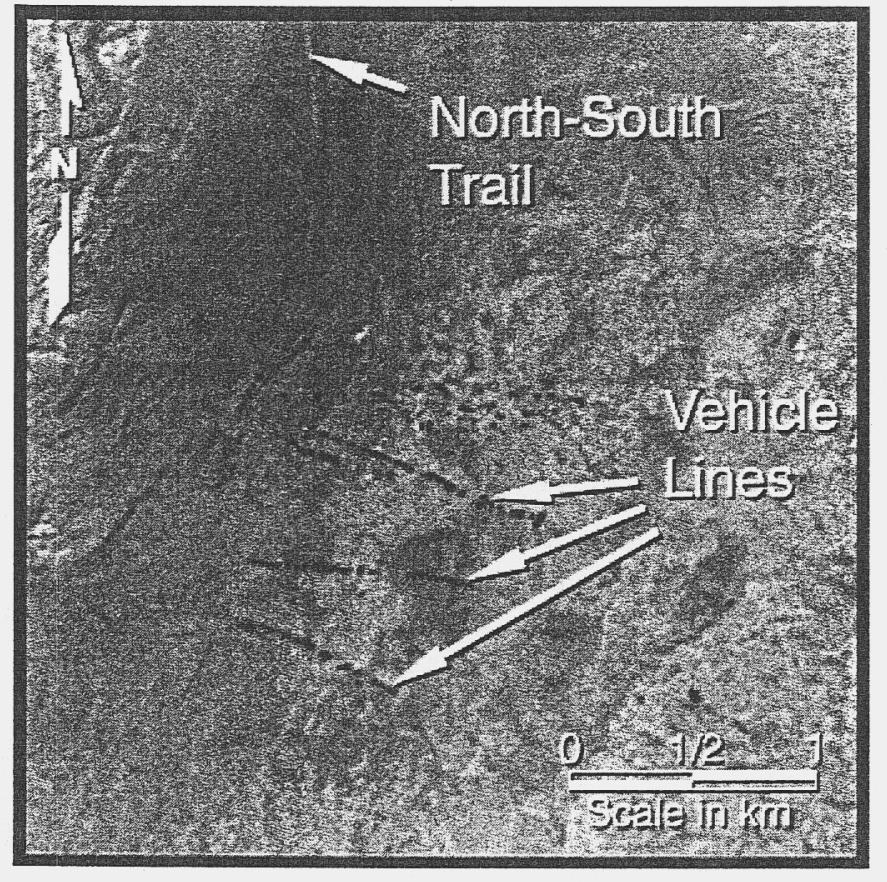




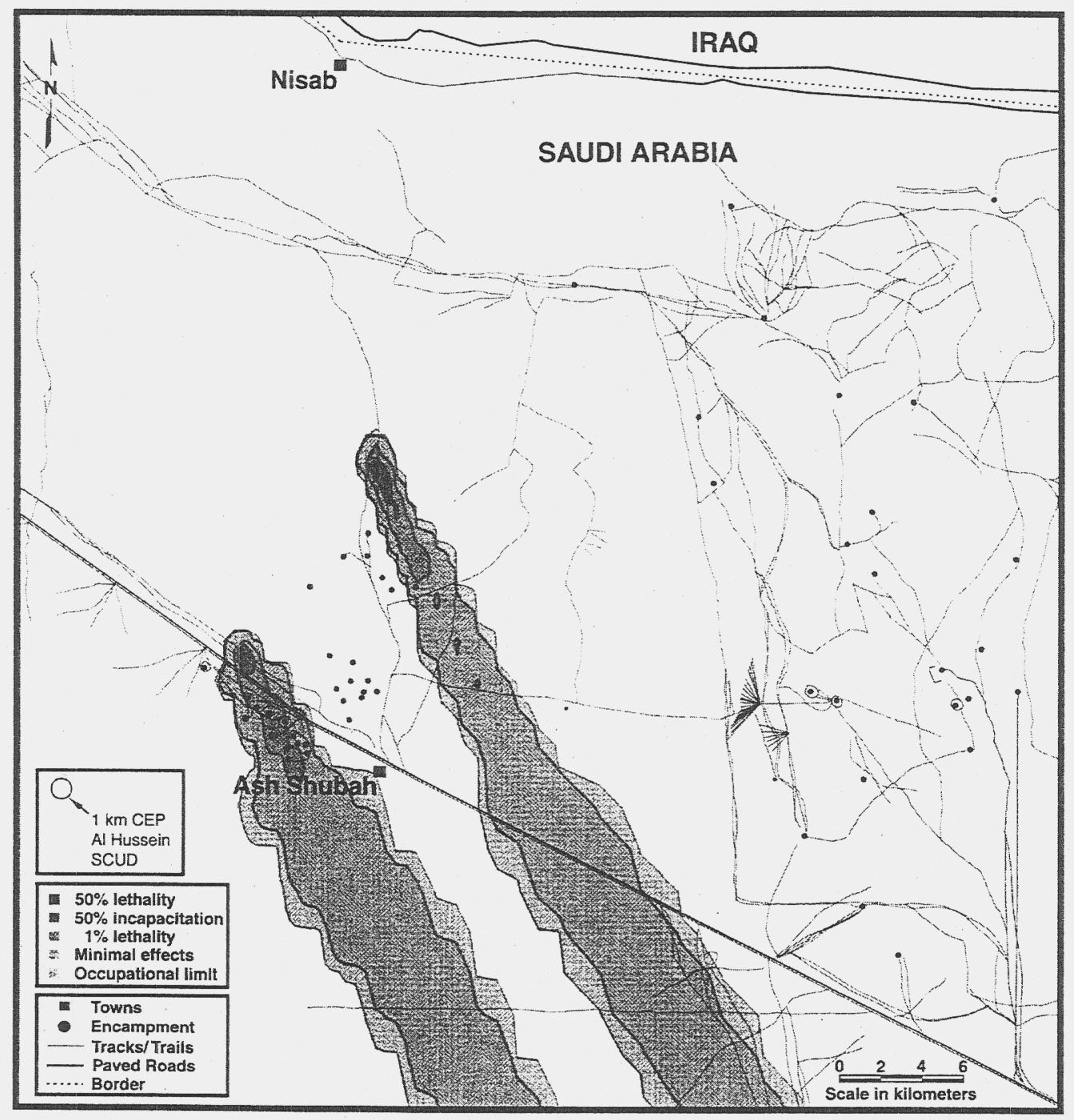




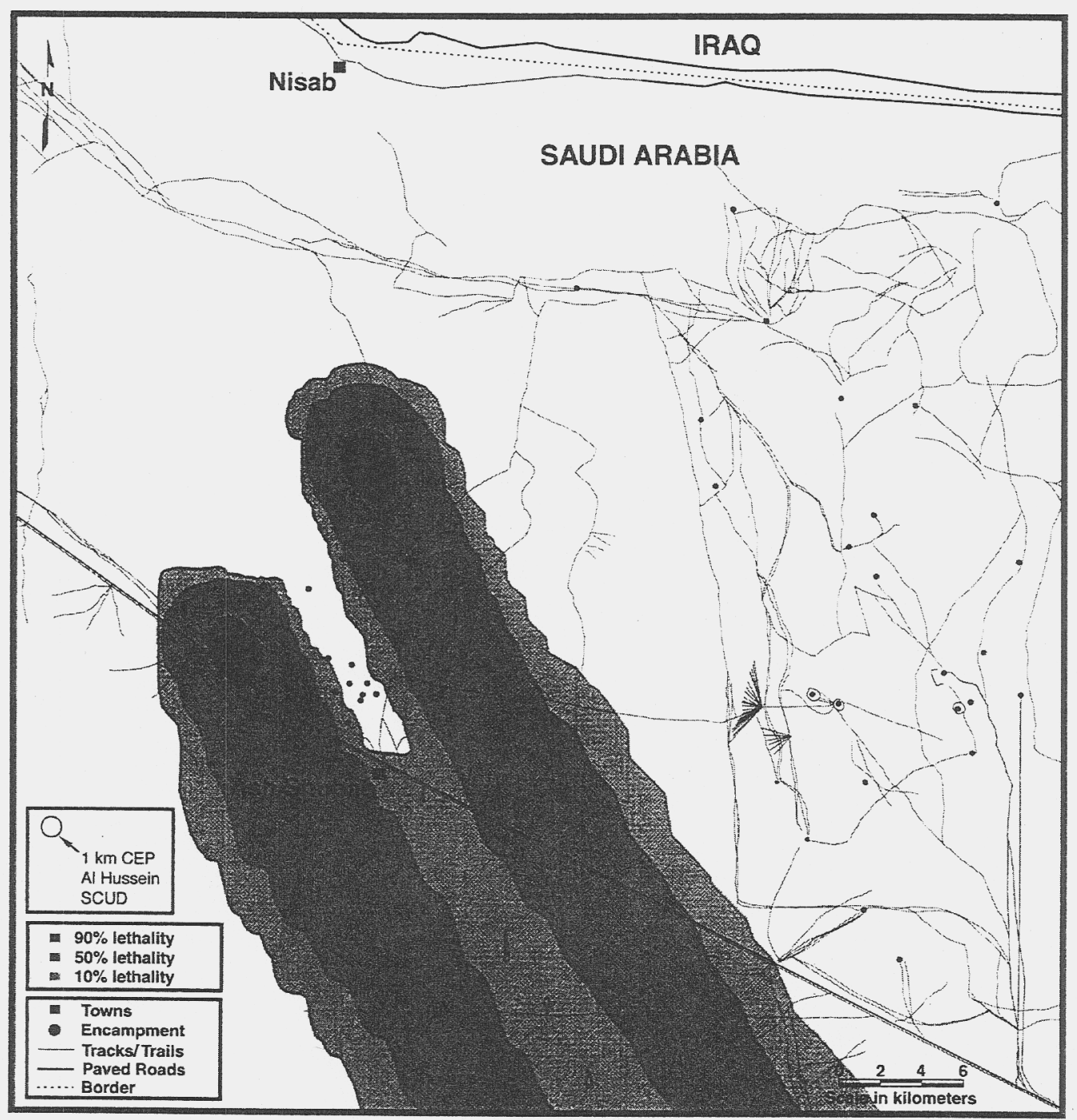




\title{
Combined Sensing and Simulation for Enhanced Evaluation of Large Structures
}

\author{
David McCallen \\ Structural and Applied Mechanics Group \\ Lawrence Livermore National Laboratory
}

\begin{abstract}
With the proliferation and advancement of sophisticated numerical simulation software tools over the past twenty years, computational simulation of large structural systems has been a subject area experiencing rapid growth. Large-scale computer simulation is an essential tool in the design and analysis of modern structures and engineers are now highly reliant on large-scale structural simulations to design and evaluate the performance of critical new structures, and to establish the performance of expensive retrofits on existing structures. With the enormous cost and construction effort associated with many large structures, it is imperative that computer simulations provide an accurate picture of structural performance spanning a multiplicity of loading environments - for example bomb blast, earthquakes and ambient vibrations. Despite the advances in computational methods, there remains a significant degree of uncertainty in predicting the field performance of many large-scale structural systems. These uncertainties are rooted in our inability to precisely quantify the phenomenological behavior of certain aspects of structural excitation and structural response - e.g. uncertainties in the precise deformation characteristics of complex structural element interconnections or uncertainties in estimation of the actual loads a structure will be subjected to. In order to advance our ability to accurately and confidently simulate the response of structures, use must be made of measured structural response characteristics and measured excitation functions.

In the current study, techniques are being investigated which allow evaluation of simulation model accuracy with the possibility for subsequent enhancement of the simulation model. The multidisciplinary LLNL tools being employed include finite element based structural simulation, model based signal processing, and remote sensing and data communication. The overall objective of this research is to symbiotically couple numerical simulation with field measurement of structural behavior through model based signal processing. The essential links between simulation and measurement are indicated in the Figure. As indicated in the Figure, information from a numerical structural model and data from field measurements of structural response are fed into a model-based signal proces-
\end{abstract}


sor. The signal processing toolbox is used to evaluate whether the model and as-built structure are in agreement, or if there is a discrepancy between the dynamical behavior of the model and the actual structure. In addition, research on the signal processing toolbox will attempt to discriminate the source of existing discrepancies between the simulation model and the as-built structure. Once discrepancies can be identified, the basis for damage detection in a structural system has been established. Experimental measurements before and after an event can be employed to identify changes in the structural system and identification of the source of the differences with a damage detector can be performed in order to investigate where damage has occurred in a large distributed structure. The result

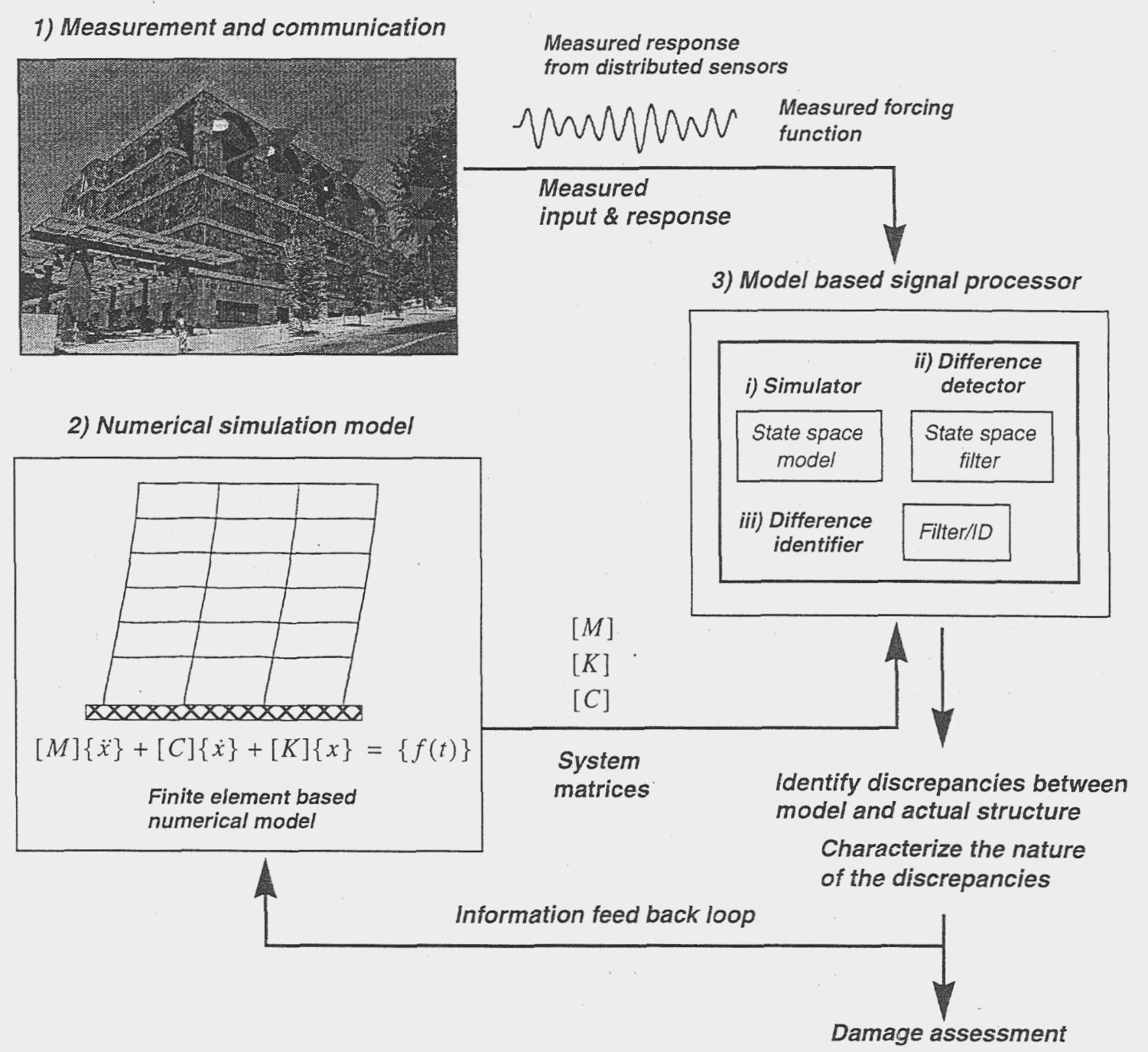

will be enhanced accuracy and reliability of numerical simulation of structural response, and the ability to monitor fundamental changes in complex structural systems, a prerequisite to health monitoring and damage detection. 


\title{
Failure Detection for Mechanical Structures: A State-Space Model-Based Approach
}

\author{
Gregory A. Clark \\ Lawrence Livermore National Laboratory
}

This paper describes early applied research to develop signal processing algorithms for detecting failures (flaws) in mechanical structures using vibrational measurements and a state-space modelbased detection approach. Throughout this study, an example 5-story building model is used to simulate the structure and demonstrate the signal processing/detection algorithms. Future project plans involve applying these algorithms to stressed monumental structures in California, including the Oakland-San Francisco Bay Bridge, the Bixby Creek Bridge and the National Ignition Facility (NIF) structure at LLNL.

The first step is the development of dynamic equations of motion used to model linear mechanical structures of the commonly-used "M-C-K" form. Once the state-space model is formulated, its dynamic behavior is validated with the $\mathrm{M}-\mathrm{C}-\mathrm{K}$ model developed by our mechanical engineering colleagues. A Gauss-Markov model of the linear system with noise is developed and used as the basis for a Kalman filter for estimating the system states and measurements. Finally, a detector consisting of a statistical whiteness test on the innovations (estimation errors) is used to detect any structural failures.

The work herein focuses on simulated linear structures and the detection of structural failures. The problems of real data from controlled experiments with actual structures, failure identification, failure location, nonlinear systems, large-scale structures will be addressed in future work.

This paper describes results from the LLNL Joint ME/EE LDRD Tech Base Project, known as "Combined Sensing and Modeling for Enhanced Evaluation and Safety of the Built Environment." and "Determining the Vulnerabilities of Stressed Monumental Structures."

This work was performed under the auspices of the Department of Energy by the Lawrence

Livermore National Laboratory under contract W-7405-Eng-48. 
Date: Tue, 20 Oct 1998 15:45:21 -0700

Parametric Time-Frequency Estimation of Prosthetic Heart Valve Sounds. By Perkins D. E., Thomas G. H., and Clark G.

Prosthetic heart valves have been responsible for extending and improving the quality of life of many people with serious heart conditions. Even though the heart valves are extremely reliable they are susceptible to the long-term fatigue and structural defects expected for mechanical devices operating over long periods. In this paper we describe the application of parametric time-frequency estimation techniques to acoustic measurements of prosthetic heart valve sounds under test in an anechoic tank. The analysis reveals interesting results when comparing fractured to normal valve sounds. The emphasis of this work is the application of recursive parametric techniques to "track" changes in propagation sounds as it is captured by a sensitive omnidirectional hydrophone. 


\title{
Near Field / Far Field Transition Distance for Broad Band Acoustic Pulses
}

\author{
David H. Chambers and D. Kent Lewis \\ Lawrence Livermore National Laboratory
}

\begin{abstract}
Acoustic pulses emitted from a planar array can be designed to produce a very narrow beam of energy in the near field of the array. In the far field the array behaves like a point source and the energy spreads accordingly. For single frequency pulses the near field / far field transition distance is predicted by the familiar formula for the Rayleigh range. However, this formula has no meaning when the band width of the pulse is very wide. In this talk we present alternative formulas which predict the near field / far field transition distance for pulses with arbitrary band. width. These reduce to the classical Rayleigh range expression in the narrow band width limit. Comparisons between the predicted transition distance and simulations for broad band acoustic pulses emitted from a ring array are shown.
\end{abstract}

Key words: diffraction, pulse, radiation 


\title{
Near Field / Far Field Transition Distance for Broad Band Acoustic Pulses
}

\author{
David H. Chambers \\ Strategic Systems Support Program \\ Lawrence Livermore National Laboratory
}

CASIS Workshop, Nov. 12-13, 1998 


\section{Radiation of acoustic potential}

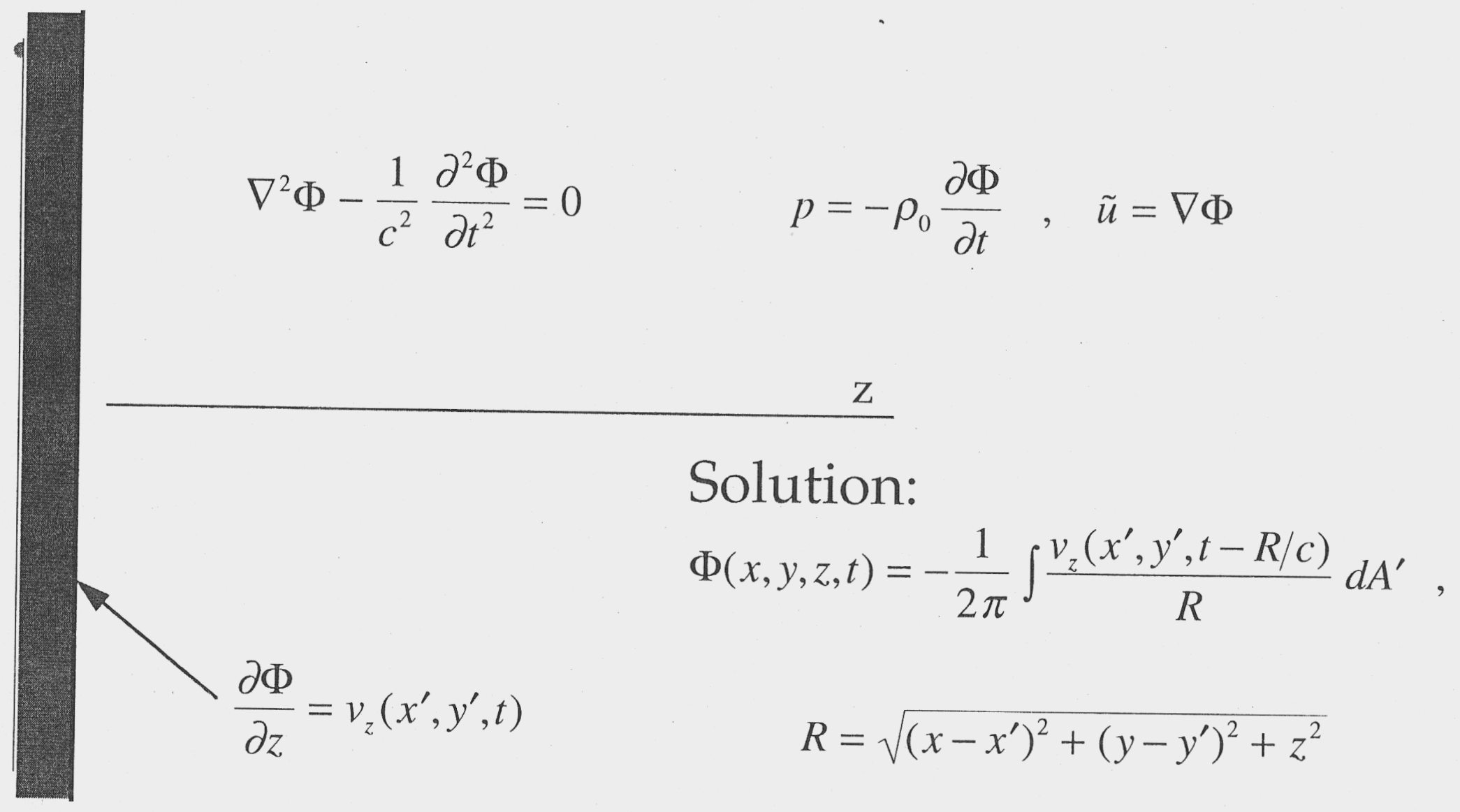




\section{Acoustic radiation from an oscillating piston}

- Let normal velocity in a circular region of radius "a" be $\exp (i \omega t)$

- Potential is given by

$$
\begin{gathered}
\Phi(r, \vartheta, t)=-\frac{\exp (i \omega t)}{2 \pi} \int_{-\pi}^{\pi} d \varphi \int_{0}^{a} d \rho \frac{\rho}{\boldsymbol{R}} \exp (-i k \boldsymbol{R}) \\
\boldsymbol{R}=\sqrt{\boldsymbol{r}^{2}+\rho^{2}-2 r \rho \sin \vartheta \cos \varphi}
\end{gathered}
$$

- On axis $(\vartheta=0, r=z)$ we obtain

$$
\begin{gathered}
\Phi(z, t)=-\frac{2}{k} \exp \left(i \omega(t-\bar{z} / c) \sin \left[\frac{1}{2} k z\left(\sqrt{1+a^{2} / z^{2}}-1\right)\right]\right. \\
\bar{z}=\frac{1}{2} z\left(1+\sqrt{1+a^{2} / z^{2}}\right)
\end{gathered}
$$




\section{Behavior of amplitude factor}

- Plot amplitude factor $\frac{2}{k} \sin \left[\frac{1}{2} k z\left(\sqrt{1+a^{2} / z^{2}}-1\right)\right]$ for various ka

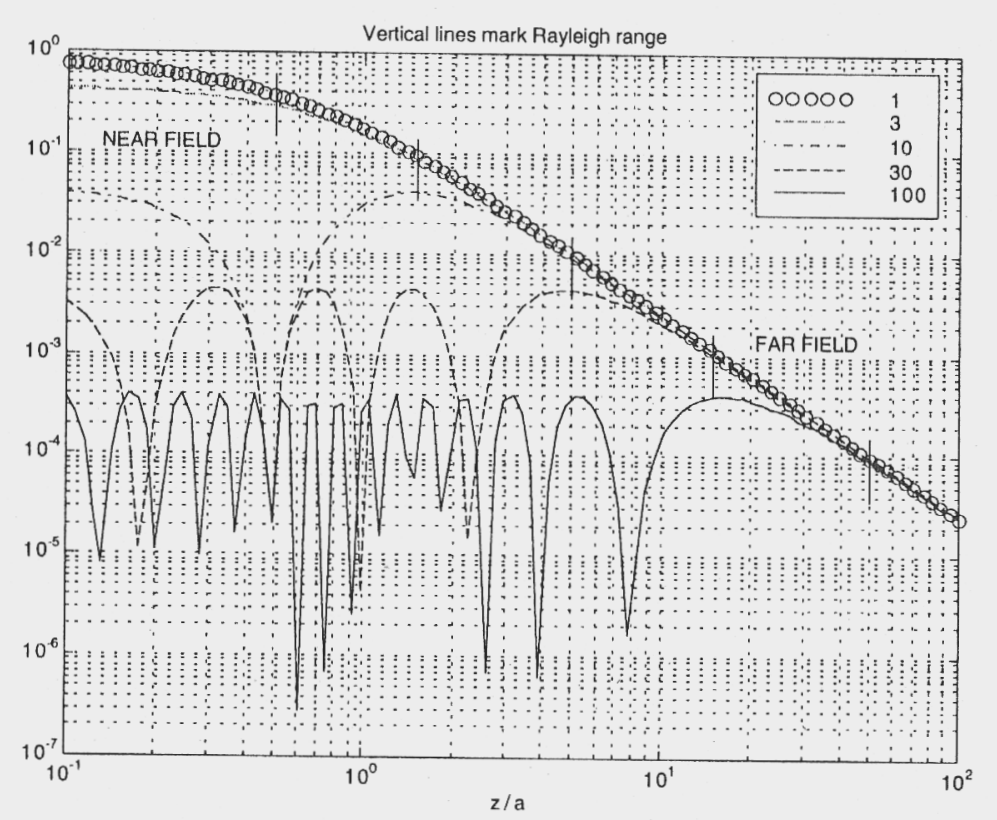

- For z/a > ka/2 (Rayleigh range) amplitude decreases as 1/r 


\section{Comparison between near field and far field behavior}

- Near field:

- complicated behavior

- high degree of control (e.g. focusing)

- finite size of array is important

- amplitude variation is arbitrary (can be constant on axis)

- Far field:

- field behavior depends only on angle from axis and range

- low degree of control

- array is equivalent to a point radiator

- amplitude falls off in all directions as $1 / \mathrm{r}$ 


\section{Prediction of near field / far field transition}

- For single frequency piston radiator, transition distance is well defined by the Rayleigh range

- Rayleigh range is useful for arrays with elements radiating in phase with constant amplitude at a single frequency (narrow band)

- No Rayleigh range is defined for narrow band radiation with phase differences between elements or for broad band radiation

- Expect transition for band-limited radiation to lie between Rayleigh ranges of lowest and highest frequencies 


\section{Heuristic estimate for short pulses}

- Consider a piston radiator emitting a short pulse (broad band)

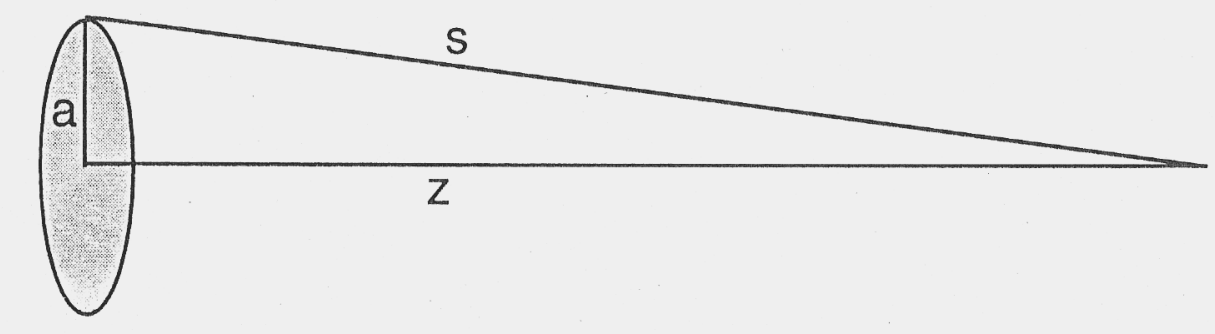

- Let $\tau$ be the pulse width, then fractional time delay between paths is

$$
\frac{\Delta t}{\tau}=\frac{s-z}{c \tau}=\frac{\sqrt{z^{2}+a^{2}}}{c \tau}
$$

- By setting $\Delta t / \tau=\beta<<1$ at $z=z_{R}$ we obtain the estimate

$$
z_{R}=\frac{a^{2}}{2 \beta c \tau}
$$




\section{Transition distance for general axisymmetric source distributions}

- Heuristic estimate does not consider amplitude shading or variation of excitation within circular aperture

- Use radiation formula for acoustic potential as basis for estimating transition distance:

$$
\Phi(z, t)=-\int_{-\infty}^{\infty} d \omega \exp (i \omega t) \int_{0}^{a} \frac{d \rho \rho}{\sqrt{\rho^{2}+z^{2}}} V_{z 0}(\rho, \omega) \exp \left(-i \frac{\omega}{c} \sqrt{\rho^{2}+z^{2}}\right)
$$

where $V_{z 0}(\rho, \omega)$ is the Fourier transform of the normal velocity

- In the far field this becomes

$$
\Phi_{F F}(z, t)=-\frac{1}{z} \int_{-\infty}^{\infty} d \omega \exp (i \omega(t-z / c)) \int_{0}^{a} d \rho \rho V_{z 0}(\rho, \omega)
$$




\section{Define transition distance}

- Transition distance is the distance where the difference between the actual potential and its far field approximation $\Delta \Phi=\Phi-\Phi_{F F}$ is less than a prescribed value $\beta$

- More precisely: $\frac{\left\|\Delta \Phi\left(z_{R}, t\right)\right\|}{\left\|\Phi_{F F}\left(z_{R}, t\right)\right\|}=\beta$

where $\|f(z, t)\|^{2} \equiv \int_{-\infty}^{\infty}|f(z, t)|^{2} d t=2 \pi \int_{-\infty}^{\infty}|F(z, \omega)|^{2} d \omega$

- In case of multiple values, choose largest 


\section{Approximate integral to calculate distance}

- Integral for $\Delta \Phi$ is

$$
\Delta \Phi\left(z_{R}, t\right)=\int_{-\infty}^{\infty} d \omega \int_{0}^{a} d \rho \rho V_{z 0}(\rho, \omega)\left[1-\frac{z_{R}}{\sqrt{\rho^{2}+z_{R}^{2}}} \exp \left(-i \frac{\omega}{c} \sqrt{\rho^{2}+z_{R}^{2}}\right)\right]
$$

- Make approximation for large z:

$$
1-\frac{z_{R}}{\sqrt{\rho^{2}+z_{R}^{2}}} \exp \left(-i \frac{\omega}{c} \sqrt{\rho^{2}+z_{R}^{2}}\right) \approx \frac{\rho^{2}}{2 z_{R}^{2}}\left(1+i \omega z_{R} / c\right)
$$

- This allows one to solve for $z_{R}$ 


\section{Formula for transition distance}

- Transition distance is given by

$$
z_{R}^{2}=\frac{1}{8 \beta^{2} c^{2}} \frac{M_{22}}{M_{00}}\left[1+\sqrt{1+16 \beta^{2} c^{4} \frac{M_{02} M_{00}}{M_{22}^{2}}}\right]
$$

where

$$
M_{m n} \equiv \int_{-\infty}^{\infty} \omega^{m}\left|\int_{0}^{a} \rho^{1+n} V_{z 0}(\rho, \omega) d \rho\right|^{2} d \omega
$$

- Due to approximation of integral, we expect this to be accurate when transition distance is much greater than radius 


\section{Special case: single frequency}

- Let $V_{z 0}(\rho, \omega)=f(\rho) \delta^{1 / 2}\left(\omega-\omega_{0}\right)$, then

$$
\begin{aligned}
& M_{m n}=\omega_{0}^{m}\left|F_{n}\right|^{2}, \quad F_{n} \equiv \int_{0}^{a} \rho^{1+n} f(\rho) d \rho \\
& z_{R}^{2}=\frac{\omega_{0}^{2}}{8 \beta^{2} c^{2}} \frac{\left|F_{2}\right|^{2}}{\left|F_{0}\right|^{2}}\left[1+\sqrt{1+\frac{16 \beta^{2} c^{4}}{\omega_{0}^{4}} \frac{\left|F_{0}\right|^{2}}{\left|F_{2}\right|^{2}}}\right]
\end{aligned}
$$

- For $f(\rho)=1$ we have

$$
z_{R}=\frac{k_{0} a^{2}}{4 \beta \sqrt{2}}\left[1+\sqrt{1+64 \beta^{2} / k_{0}^{4} a^{4}}\right]^{1 / 2} \approx \frac{1}{2} k a^{2} \text { for } \beta=\frac{1}{2}
$$




\section{Piston pulse case}

- Let $V_{z 0}(\rho, \omega)=V_{0}(\omega)$ for $\rho \leq a$ then

$$
\begin{aligned}
& M_{m n}=\mu_{m} \frac{a^{2(n+2)}}{(n+2)^{2}}, \quad \mu_{m} \equiv \int_{-\infty}^{\infty} \omega^{m}\left|V_{0}(\omega)\right|^{2} d \omega \\
& z_{R}=\frac{a^{2}}{4 \beta} \sqrt{\frac{\mu_{2}}{2 \mu_{0}}}\left[1+\sqrt{1+\frac{64 \beta^{2} c^{4}}{a^{4}} \frac{\mu_{0}^{2}}{\mu_{2}^{2}}}\right]^{1 / 2}
\end{aligned}
$$

- Gaussian pulse: $v_{z 0}(t)=-\frac{2 t}{\tau^{2}} \exp \left(-t^{2} / \tau^{2}\right)$

$$
z_{R}=\frac{1}{4 \beta} \sqrt{\frac{3}{2}} \frac{a^{2}}{c \tau}\left[1+\sqrt{1+\frac{64 \beta^{2}}{9}\left(\frac{c \tau}{a}\right)^{4}}\right]^{1 / 2}
$$




\section{Compare prediction with transition distance determined by numerical simulation}

- Calculate on-axis pressure field for a 24 ring array (equally spaced) driven with a prescribed velocity distribution

- Determine on-axis behavior of $\int_{-\infty}^{\infty} p^{2}(z, t) d t$

- Set transition distance at point where on-axis fall off deviates by $5 \%$ from far field rate

- Compare with prediction using $\beta^{2}=1 / 8$ 


\section{Comparison with two classes of pulses}

- Gaussian piston pulses with varying durations: $0.01 \leq c \tau / D \leq 1.0$

- Ziolkowski's MPS pulses with varying waists

$$
\begin{gathered}
v_{z 0}(\rho, t)=\left.\frac{\partial \Phi_{M P S}}{\partial z}\right|_{z=0}, \quad s(\rho, z, t)=\frac{\rho^{2}}{z_{0}+i(z-c t)}-i(z+c t) \\
\Phi_{M P S}(\rho, z, t)=\operatorname{Re}\left[\frac{2 R_{m} z_{0}}{z_{0}+i(z-c t)} \frac{\exp \left(-z_{0} s(\rho, z, t) / w_{0}^{2}\right)}{s(\rho, z, t)+2 R_{m}}\right] \\
\boldsymbol{R}_{m}=1000, \quad z_{0} / D=0.0064, \quad 0.1 \leq w_{0} / D \leq 10
\end{gathered}
$$




\section{Decay curves for Gaussian piston pulses}

\section{Decay curves}

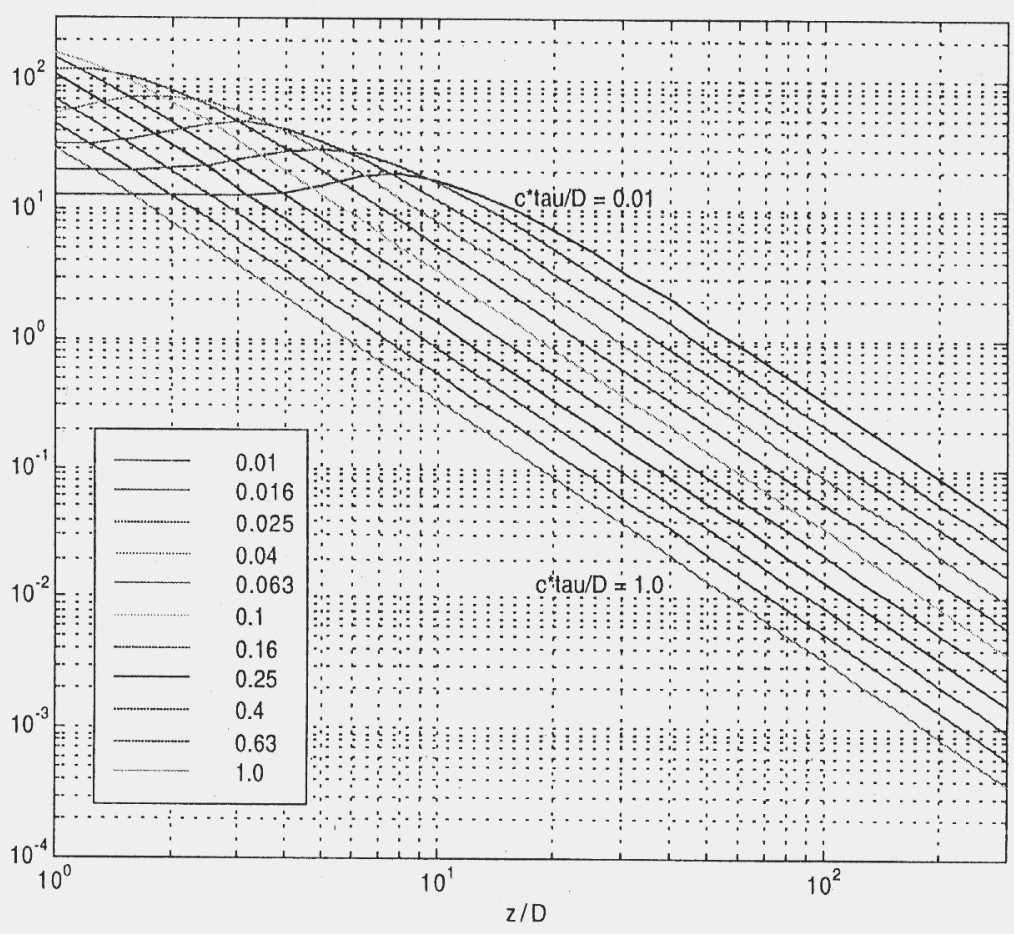

Deviation from far field

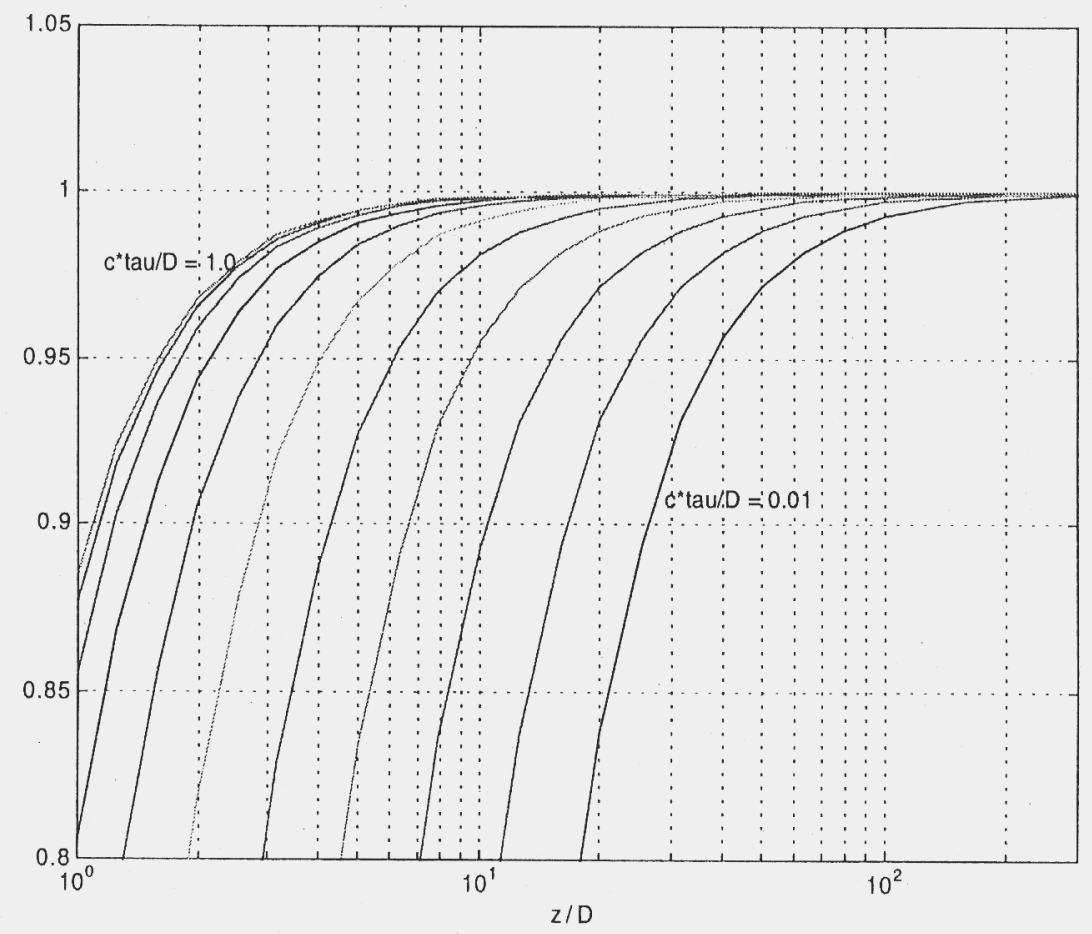




\section{Transition distances for Gaussian pulses}

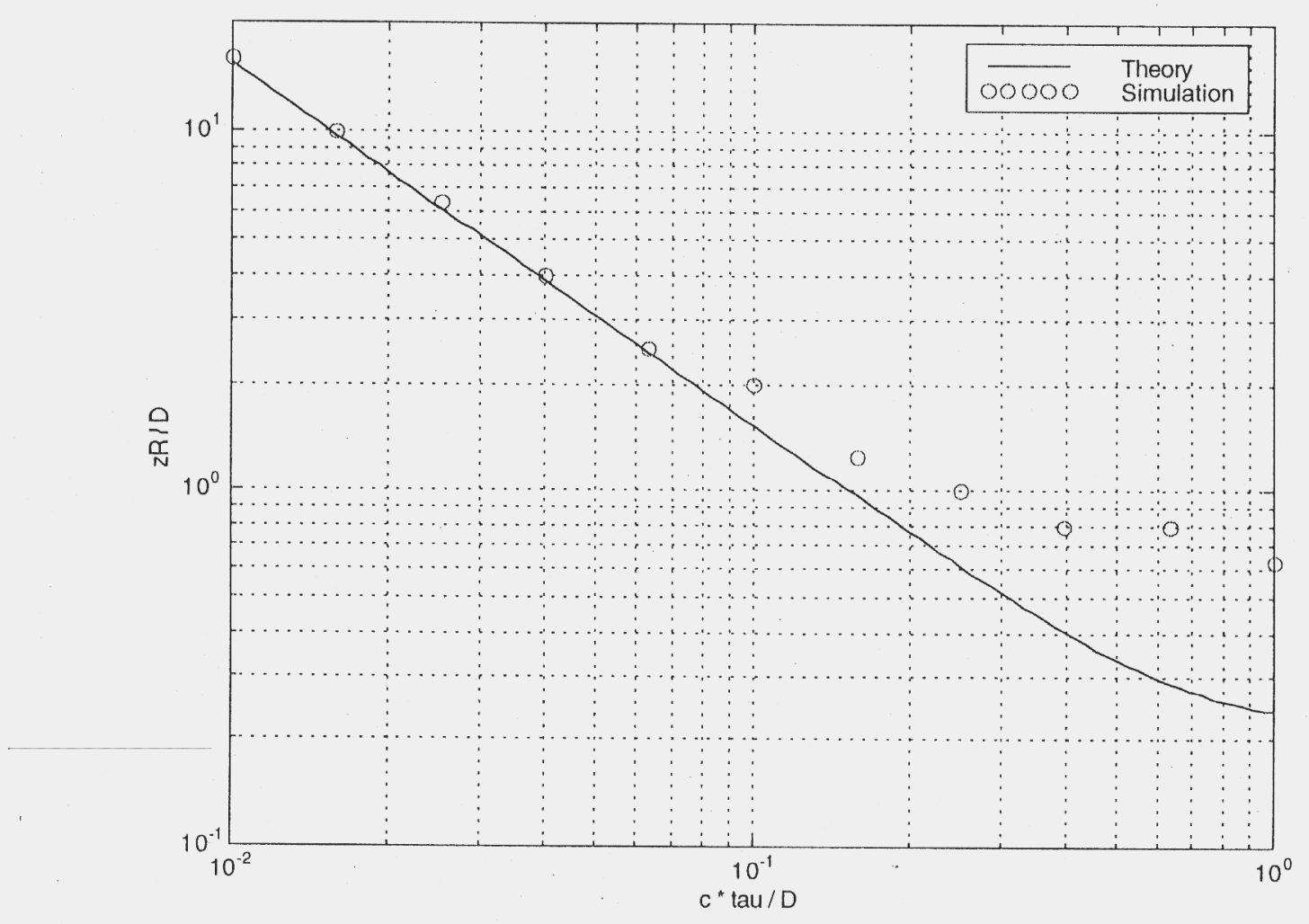




\section{Velocity distributions for MPS pulses}

Plotted as functions of $c^{\star} t / D$, largest amplitude is at center of array

$$
w_{0} / D=0.01
$$

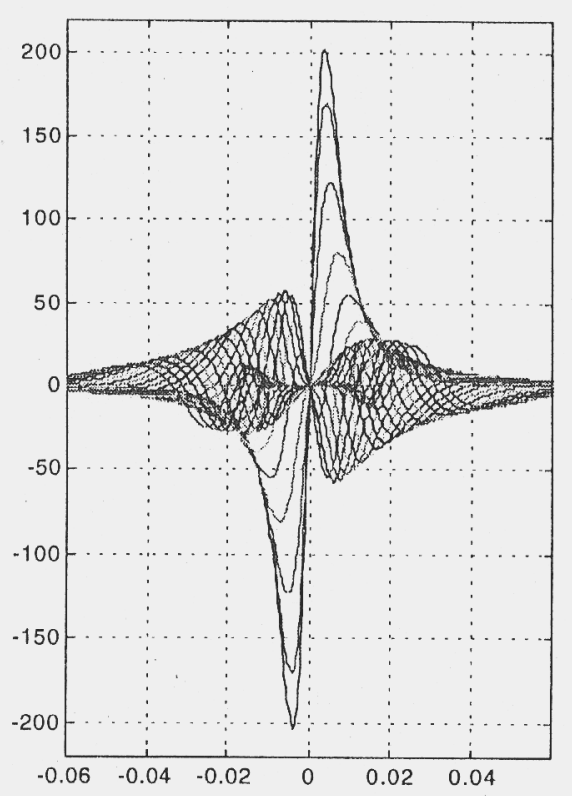

$$
w_{0} / D=1.0
$$

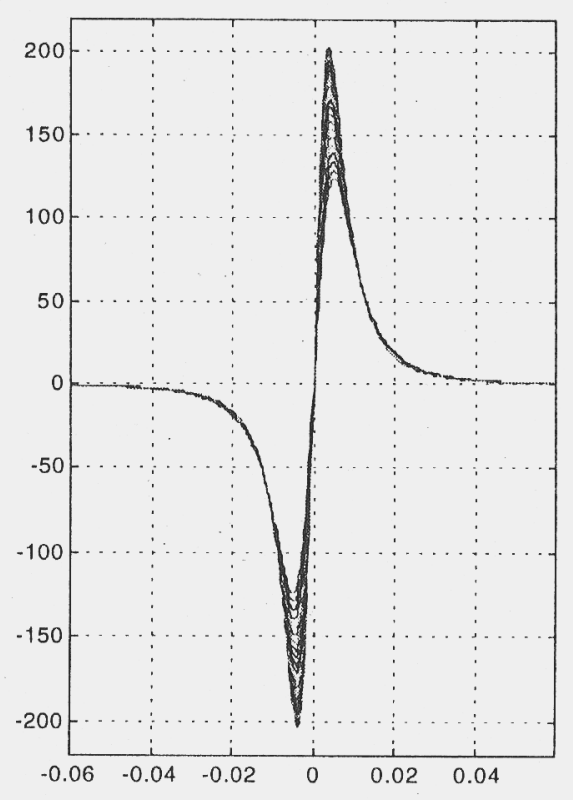

$$
w_{0} / D=10
$$

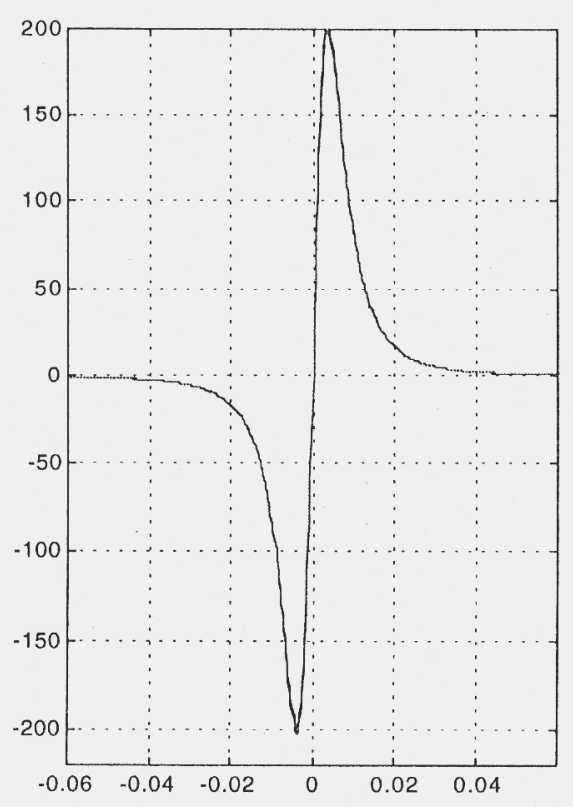




\section{Decay curves and deviation from far field}

Decay curves

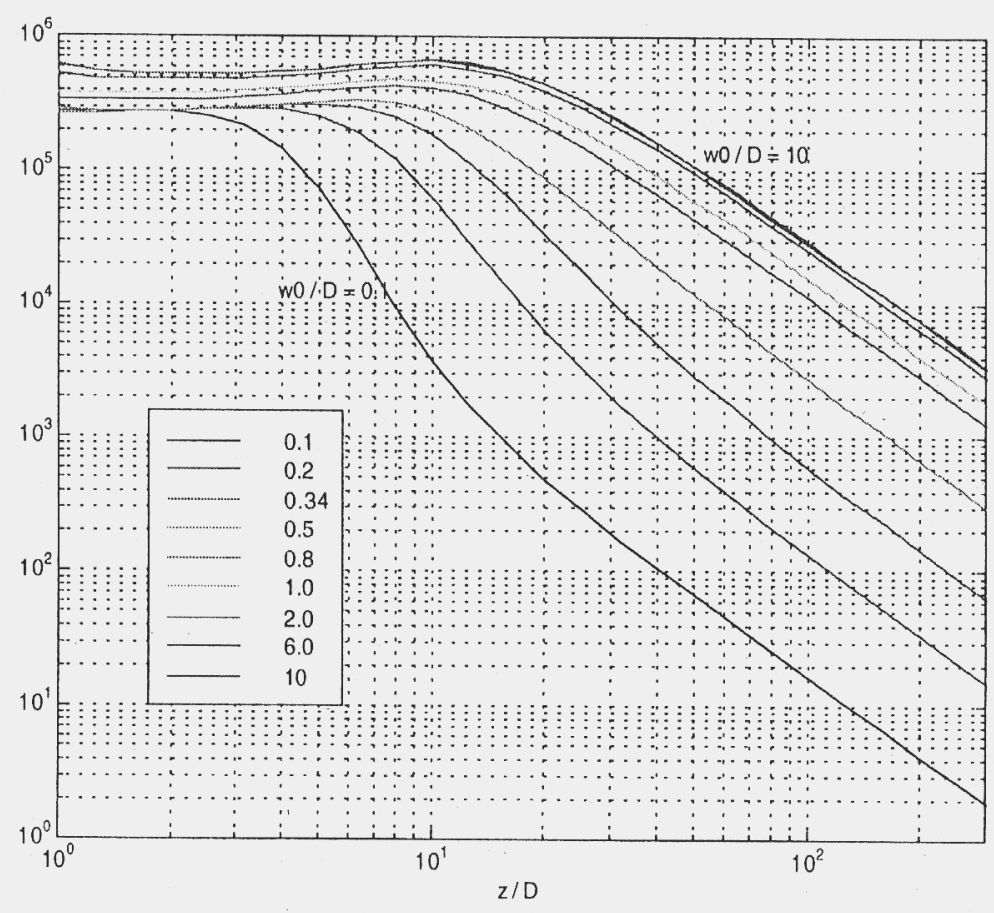

Deviation from far field

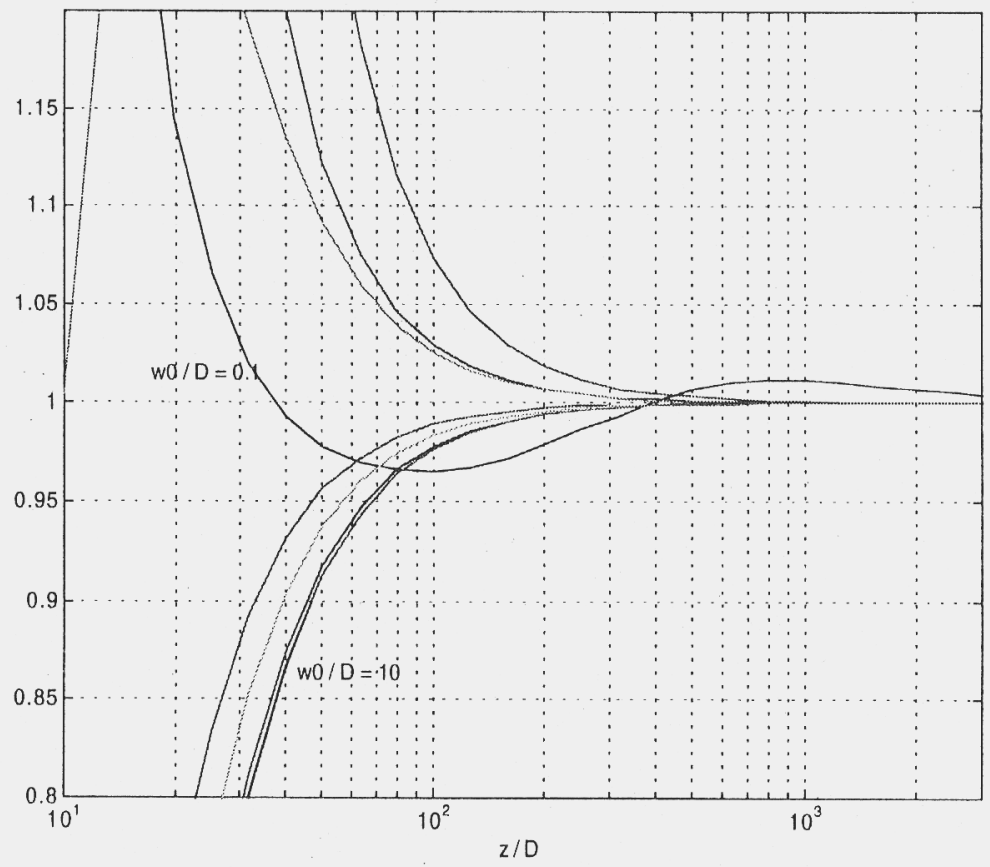




\section{Transition distances for MPS pulses}

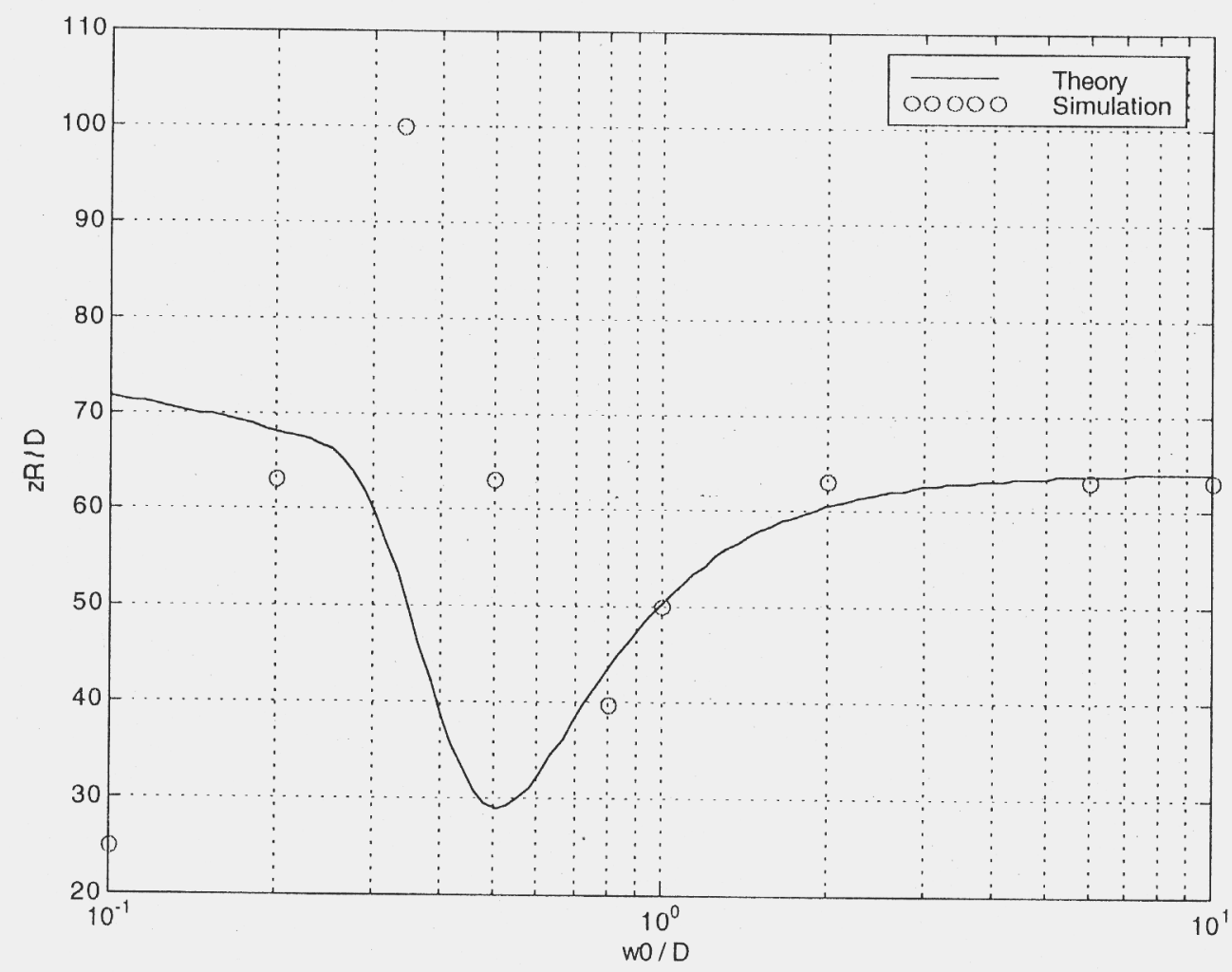




\section{Conclusions}

- An estimate of near field / far field transition distance has been formulated for general broad band axisymmetric velocity distributions

- Estimate agrees with Rayleigh range formula for single frequency piston radiation

- Estimates of transition distance from simulation data depend on which field is being calculated

- Transition distance estimate works well for pulses whose initial velocity distributions do not vary greatly within the aperture

- Further analysis is required to determine true range of validity of the estimate 


\title{
Optimization of Array Generated Fields for Broad Frequency Band Width
}

\author{
Kent Lewis \\ Lawrence Livermore National Laboratory
}

Accurate active beam forming methods depend on putting the right signal at the right place at the right time. Modern arrays are increasing dependant on individual source signals for each element or set of elements in a larger array. This capability allows generation of beams, which are optimized both spacially and temporally.

LLNL has pioneered this technology for more than a decade, and is presently using its capability in several projects, among them the Karmanos Breast Cancer Detection Initiative, and the Localized Wave project. This talk outlines the background of our present work, and shows a field application of the pre-processing capability. 
Optimization of array generated fields for broad frequency band width

D. Kent Lewis

Strategic Systems Support Program

Accurate active beam forming methods depend on putting the right signal at the right place at the right time. Modern arrays are increasing dependant on individual source signals for each element or set of elements in a larger array. This capability allows generation of beams which are optimized both spacially and temporally.

LLNL has pioneered this technology for more than a decade, and is presently using its capability in several projects, among them the Karmanos Breast Cancer Detection Initiative, and the Localized Wave project. This talk outlines the background of our present work, and shows a field application of the pre-processing capability. 
UNCLASSIFIED

\section{Background for Individually addressable array}

Quantitative NonDestructive Evaluation Project

'85-88 LLNL funded

Signal Pre-Processing to generate arbitrary linear fields

Spectral extrapolation post processing for sharpening

Localized Wave Project

89-present LLNL and Navy funded

Multi-channel source electronics

Individually addressable array materials

Multi-channel beam forming and simulation

Alternate materials and fabrication

PVDF and associated co-polymers

PZT-N extruded and sintered arrays

Si "drum-head" array elements

Flexible piezo-electric "wires" 


\section{Quantitative NonDestructive Evaluation}

Pre-processing of source waveforms

Linear inverse filtering

Correction for non-idealized element response

Optimization for non-idealized media

Correction of array structural resonances

Spectral extrapolation of received signals

Similar to analytical continuation in complex plane

Model based, part of the spectrum is "believed"

Parameters control continuation area

and time signal threshold 


\section{Localized Wave Pulse Beam}

Localized Wave Pulses are wide band width, low diffraction pulse beams

The Localized Wave ( $\mathrm{LW}$ ) pulse is created by linear superposition of a well characterized choice of basis functions.

It is not parametric generation or solitons

- Based on solid theoretical foundation spanning 1981 - 1998

- Focused Wave Modes and Modified Pulse Spectrum

- Graussian packets optimized for each frequency

- FWMs are solutions to the wave equation, a complete basis set, an orthogonal set

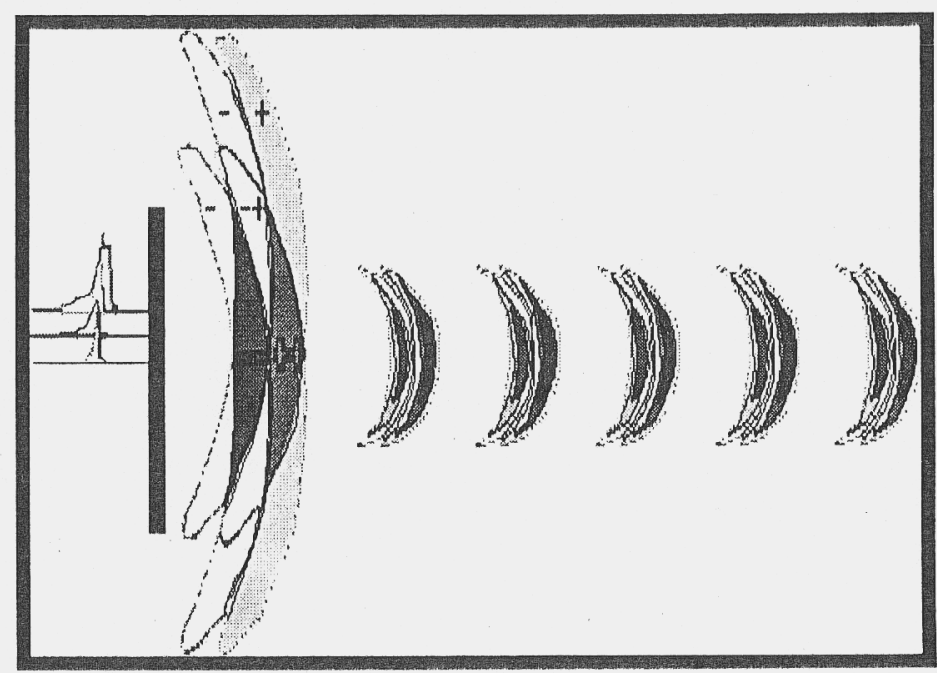

- Supported by a solid body of theory development coupled with experiment

- First LW acoustic pulses launched, 8 experiments to date

- First LW microwave pulses launched, planned for fall 1998

- Proven record of accomplishments

- Discovered 2 of the first Low Diffraction, broad band width beams

- Designed and performed original multi-channel proof of principle work, 8 to date

- Designed and conducted several proof of principle experiments with contractors

NUWC Keyport AdCap array, 1987

UTx ARL 24 ring array, 1998

- LW beam requires individually addressable array elements

and programmable, broad band width source generators 


\section{Time Line Summary, Localized Wave Pulse}

'81 Brittingham finds the Focus Wave Modes (FWM)

'84 Ziolkowski builds the Modified Pulse Spectrum (MPS) Localized Wave

'87 Lewis and Cook launch LW waveforms, acousto-optics

'88 Lewis and Cook simulate 441 element 2D array

'89 Ziolkowski and Lewis create first real time LW pulse, 25 element array

'90 Ziolkowski and Lewis use 11 ring array for LW pulse

'91 Lewis sabbatica at U. Paris 6\&71, Ziolkowski moves to Tucson

'95 Work begins on ocean bottom penetration

'96 Work begins on microwave simulation

'96 Chambers builds the Superposed Gaussian Localized Wave

'96 Candy and Chambers preliminary resolution enhancement results

'97 Lewis and Chambers launch Localized Waves with 8X8 tonplitz head in Keyport, WA

'97 Planning for microwave experiment at Baltimore approved

'97 Plan for 1-3 composite array tests in Lake Travis approved

'98 Array design and experiments at Lake Travis

'98 Microwave linear antenna LW generation experiments

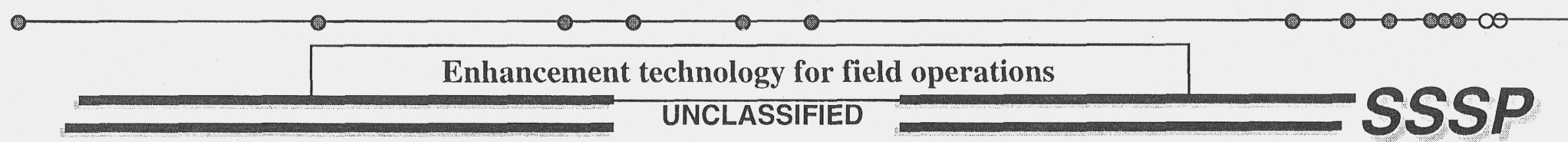




\section{Localized Wave Ocean Applications}

LW pulses are very broad frequency bandwidth, typically 3 or more octaves wide Beam energy is highly localized on the axis, very low to no side lobe creation

LW capability enhances both active and passive beam forming

\section{LW technology has proven useful in enhancing current Navy systems}

Active:

- Highly directional, covert, broad band communication and location

- Covert broad band width scanning

- Tailored spectrum to optimize penetration of difficult media, e.g.. the ocean floor

- Improved clutter rejection in shallow water

Passive:

- Applied as a matched filter front end for enhanced sensitivity

- Improved noise rejection off axis, better directivity

- Higher spacial and temporal resolution

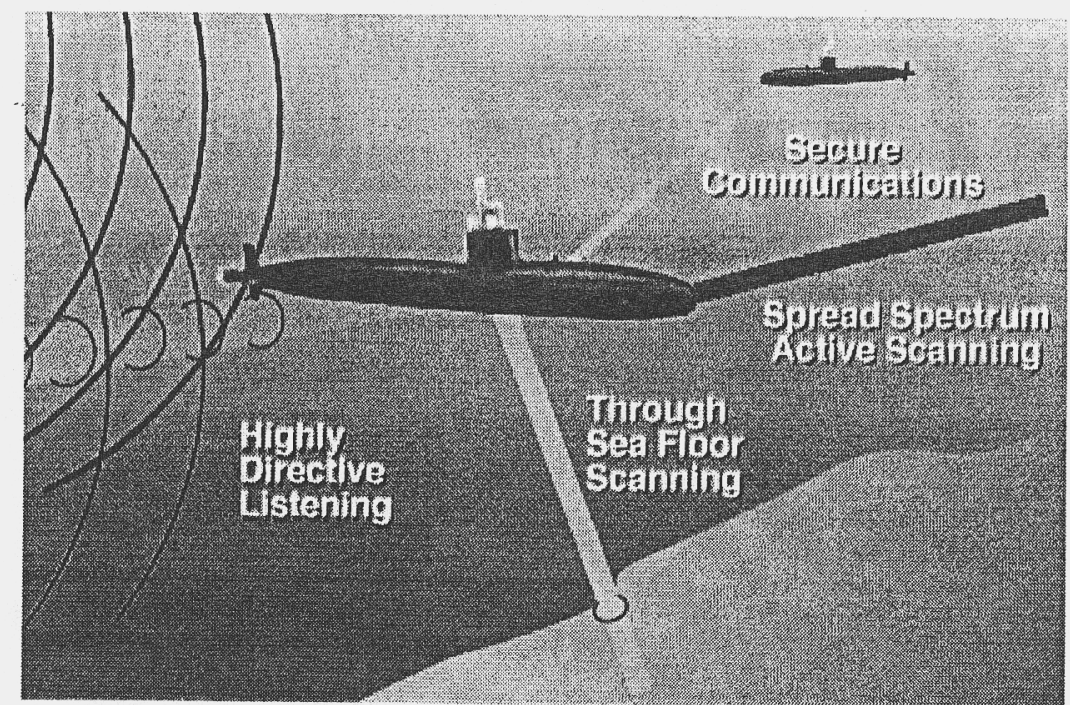




\section{Localized Wave Passive Sensitivity}

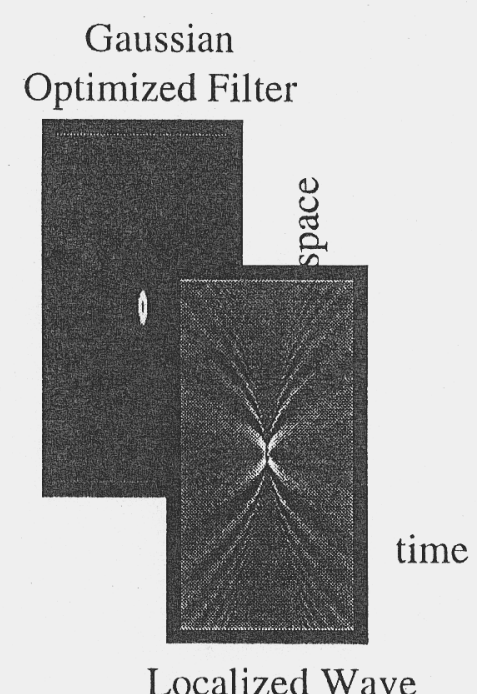

For Optimized Space-Time Filters

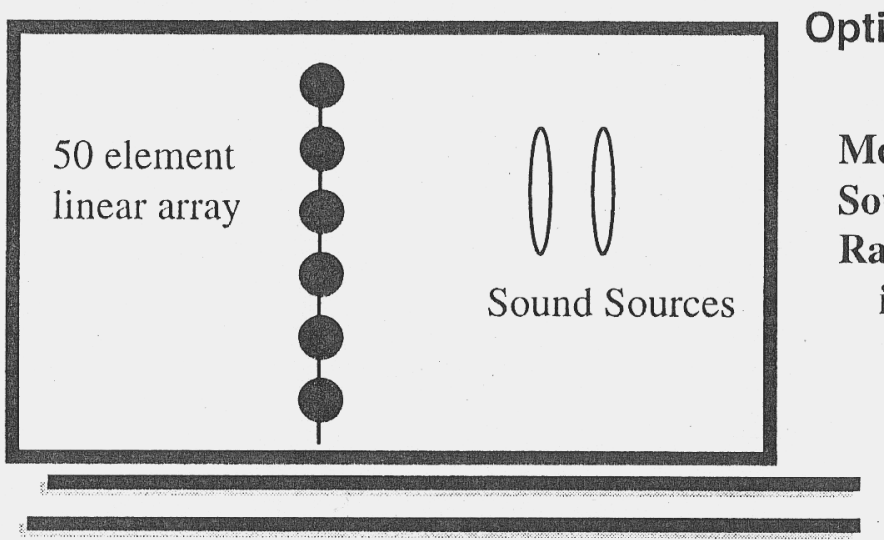

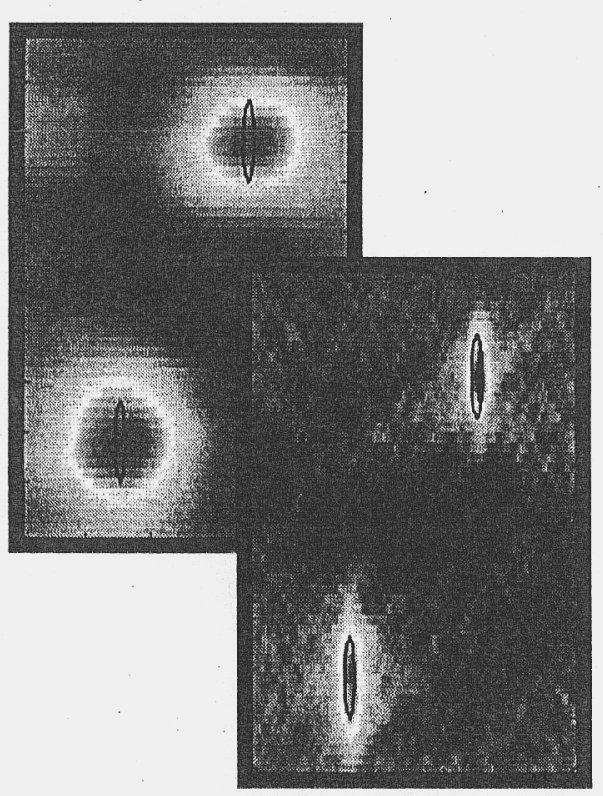

Spatial and Temporal Localization is as accurate as

Optimized Gaussian Methods

Model is a $\mathbf{5 0}$ element linear, broad band width array

Sources are separated in space and time

Range is vanishingly small, no range information

is obtainable from this simulation

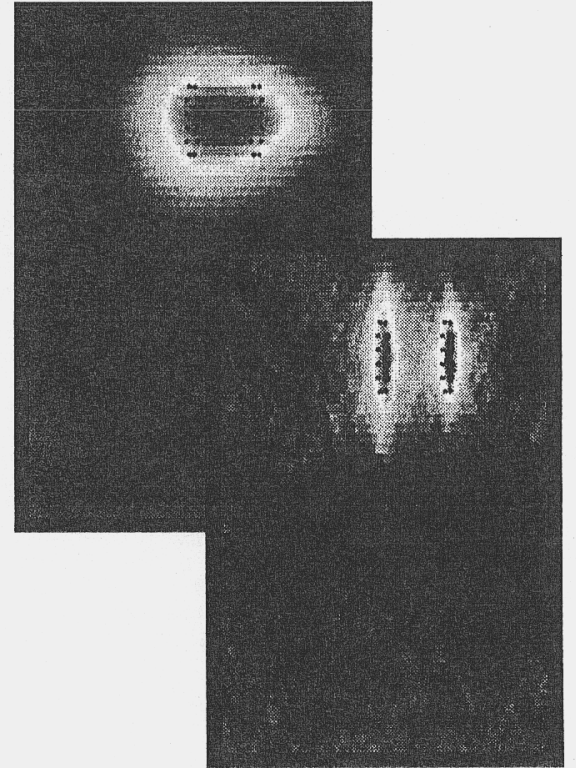

Resolution is enhanced using LW methods 
UNCLASSIFIED

\section{Array Selection candidates}

Requirements dictate multiple, individually addressable, broad band width elements

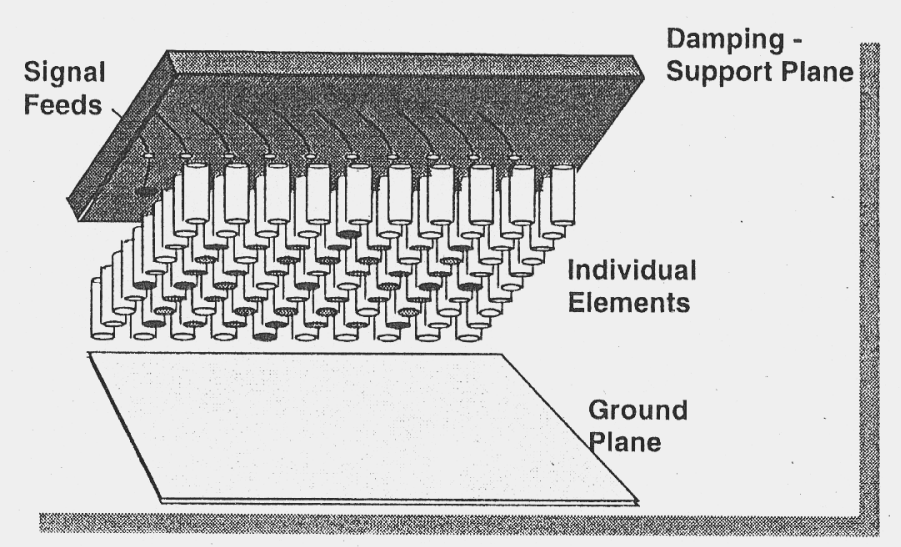

As a first cut, flat array panels will be used for PZT-N, Si, LiNb, etc.

Issues are bandwidth, radiation efficiency, detection sensitivity, cross talk suseptability, etc. Goals are simplicity, economy, resolution, and contrast in mass production components.
Natural geometry selection is spherical (or spheroidal). Avoids most beam forming problems, and addresses the breast at normal incidence

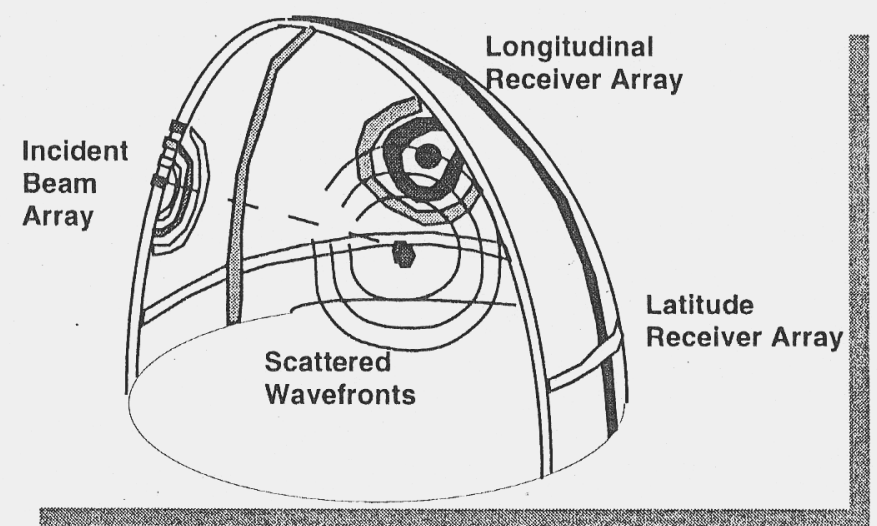

Individual addressability allows beam forming in any configuration. Perhaps overkill, it becomes a testbed for simulation, measurement, and imaging techniques. 


\section{Simple model of transducer system}

Gaussian pulse input becomes first derivative (or velocity)in the water
Disk to disk coupling described by Rhyne yields a derivative

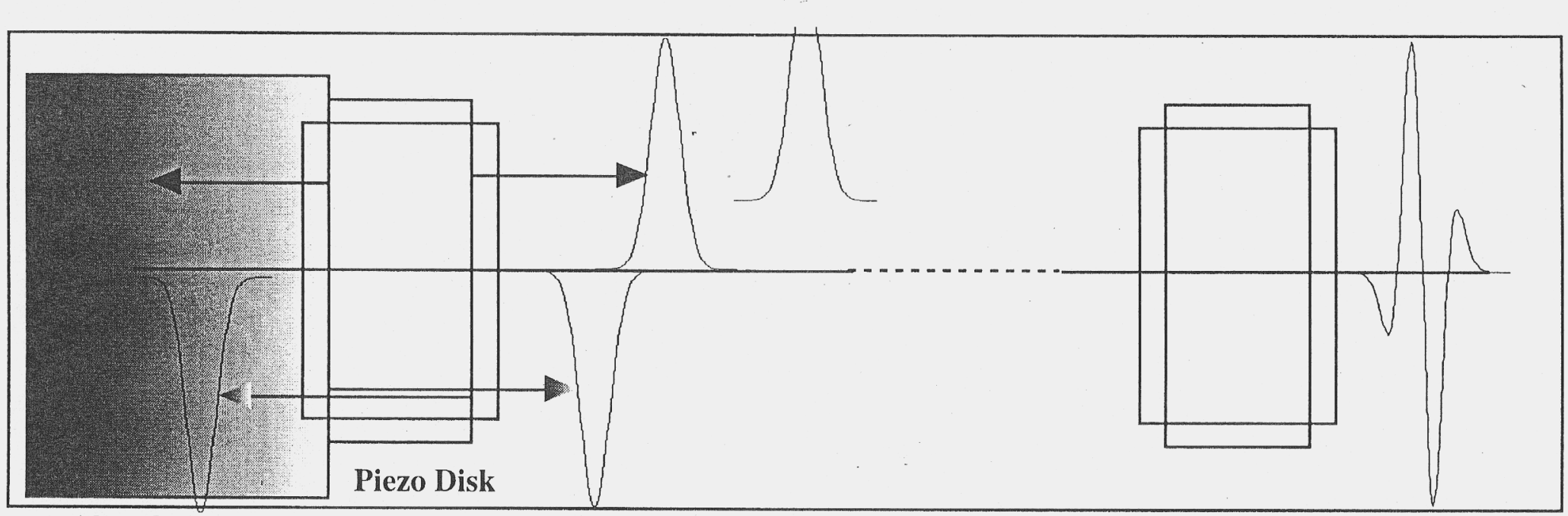

Absorber

For far field measurements, we expect the third derivative of the input

Receiver yields another derivative 
UNCLASSIFIED

\section{First cut showed array problems}

Our imput signal is a broad band width Gaussian pulse, covering the frequency range of interest

Comparing shows multiple signals overlap, and a frequency filtering

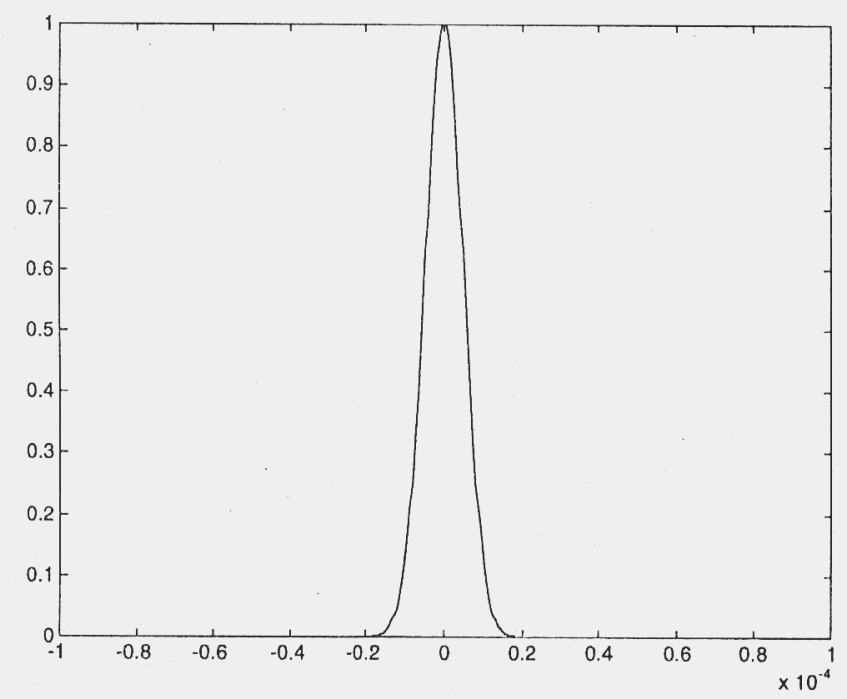

We expect to measure the 3rd derivative of this input signal response of system to Gaussian pulse
Full received
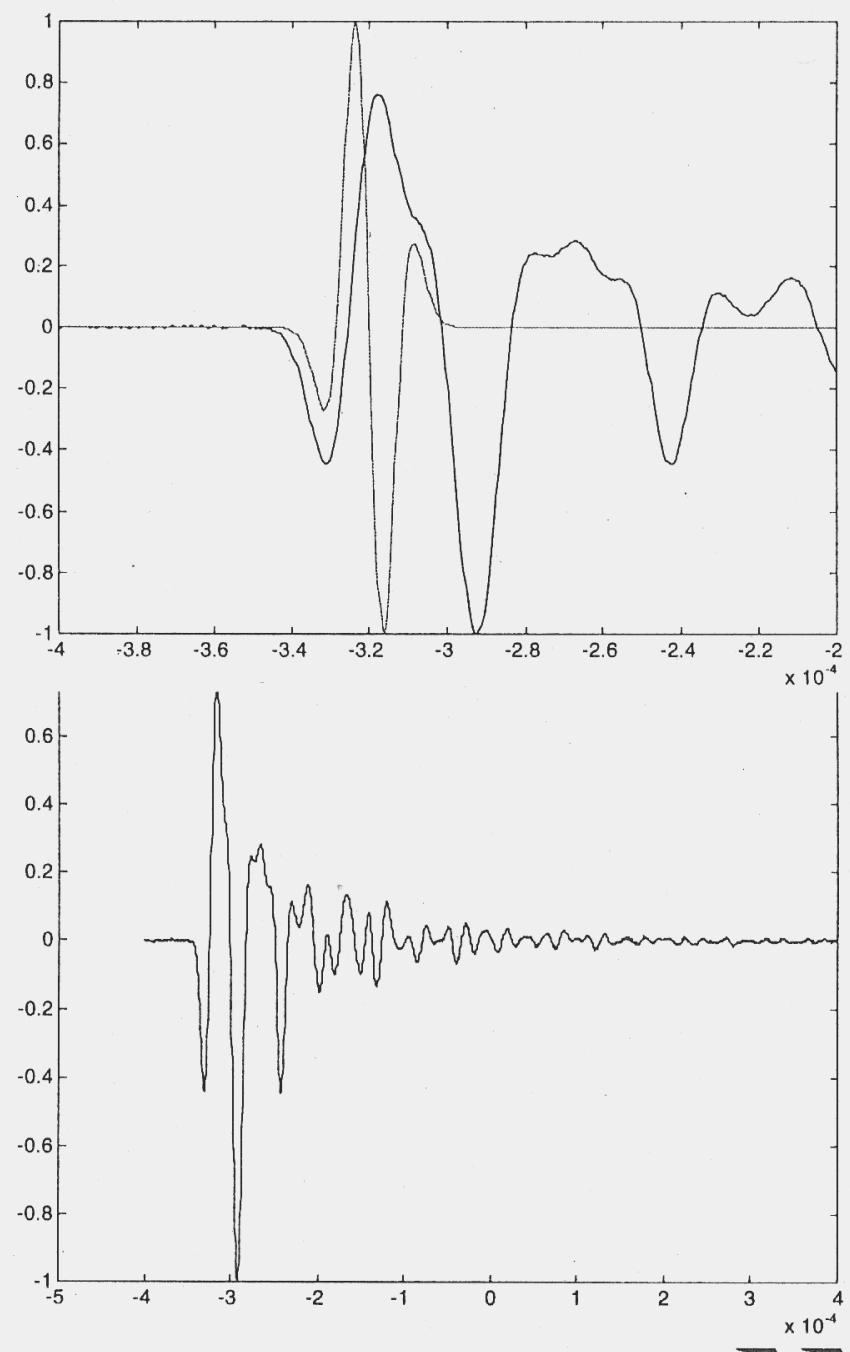
UNCLASSIFIED
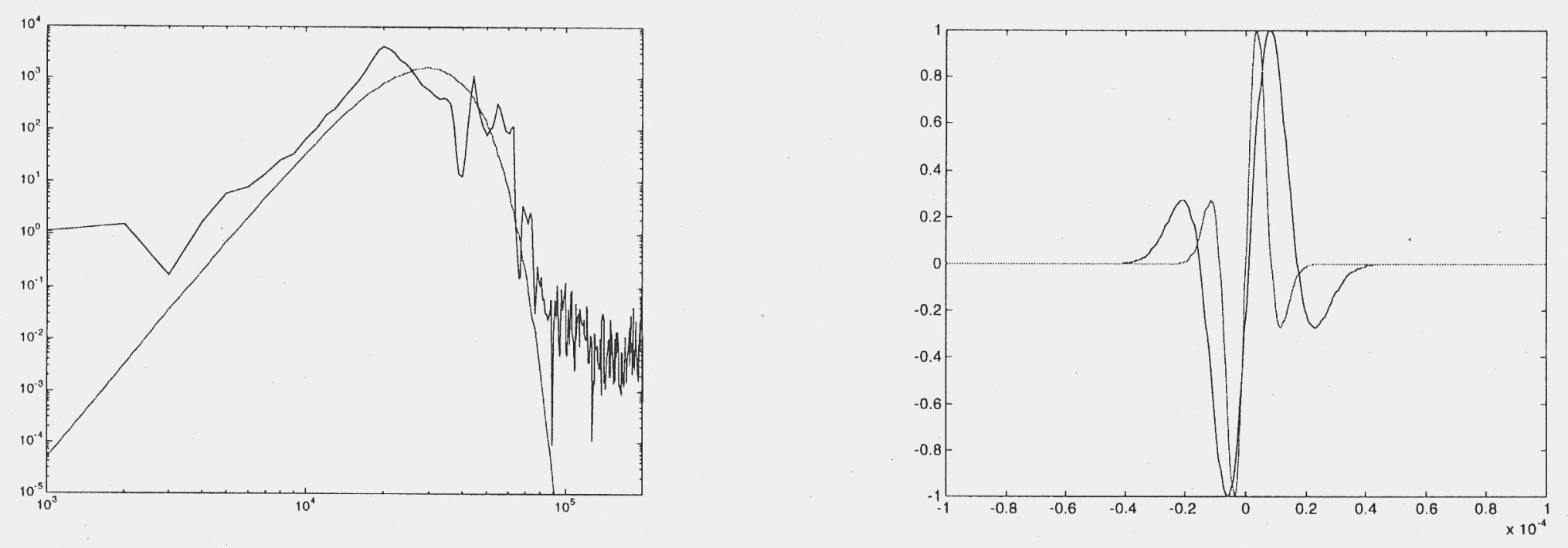

UNCLASSIFED $=$ SSSP 


\section{The system inverse was inefficient but effective}

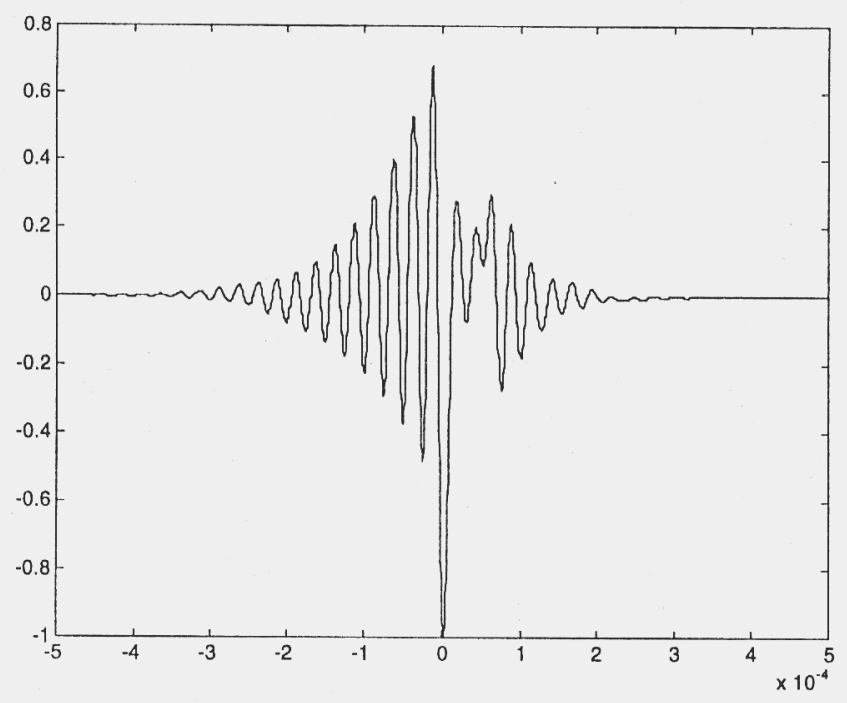

The system inverse (in time) was convolved with all the succeeding source signals

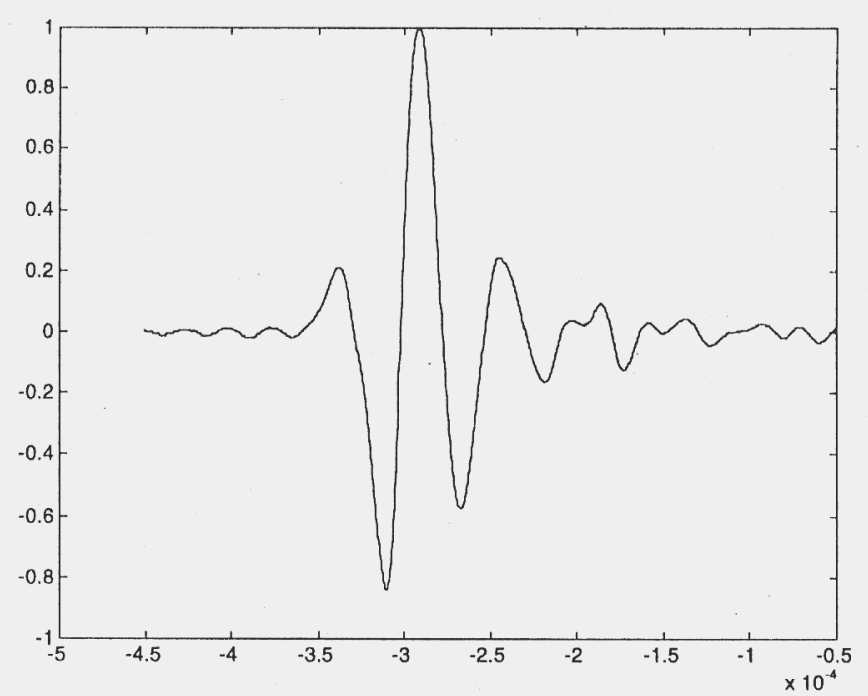

The resultant system response was cleaner, but not ideal. Further work was deemed too costly. 


\section{The data was acquired at 15 locations from 44" to 15'}

Absolute value of the time records on axis

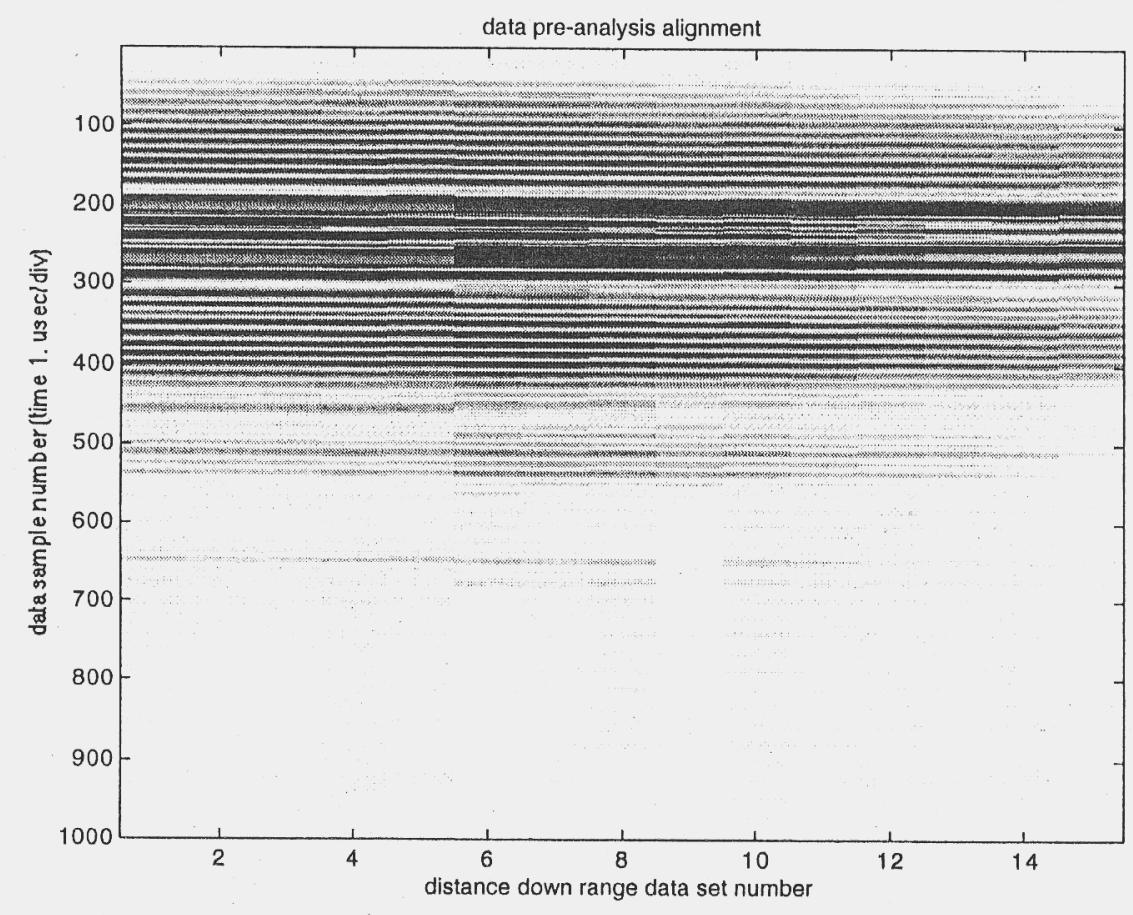

Time signals were lined up in relative time. The characteristic double peak structure in the absolute value time plot.
Representative time record for

LW pulse on axis

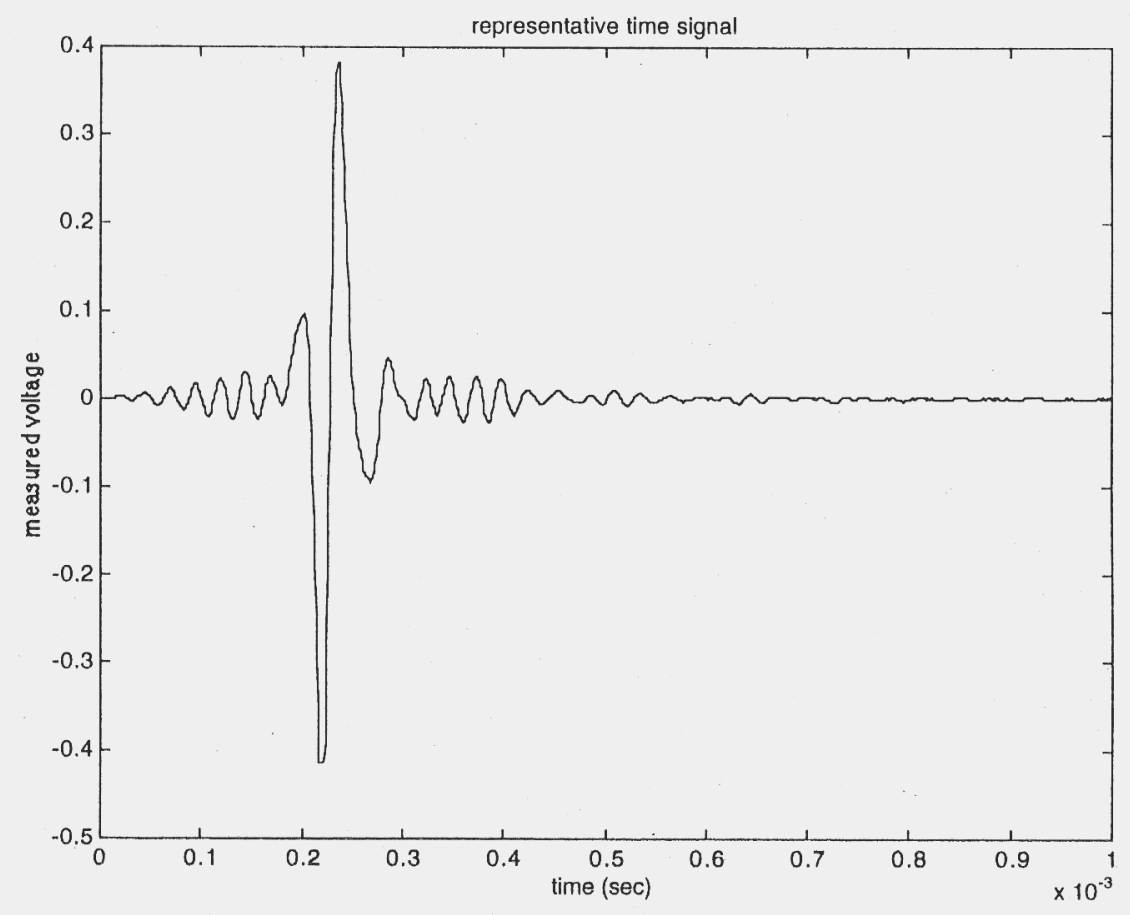

A measurement of the on axis time signal was made for each of the pulse sets at each of the down range positions 
UNCLASSIFIED

\section{Time window for time-frequency analysis}

Time record and candidate Hanning window

Signal windowed at one step prepared for processing
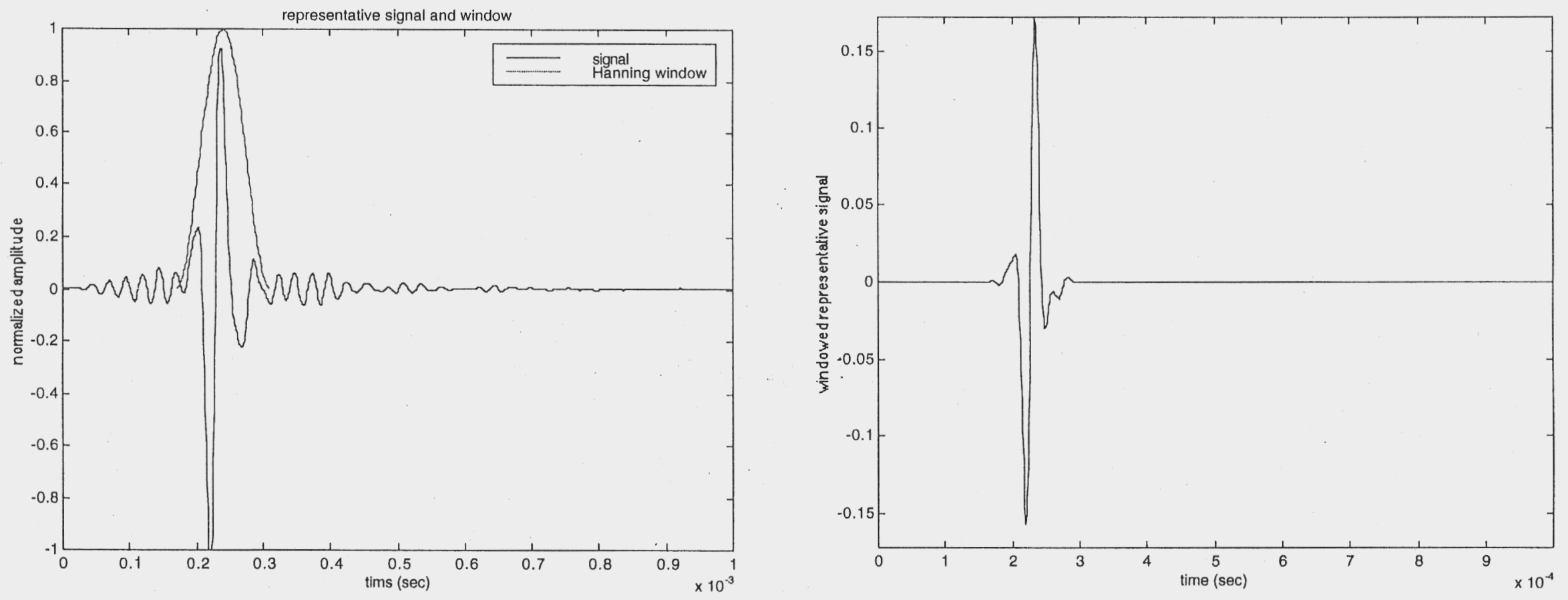

The Hanning window was chosed wide enough to allow a good representation of the main signal to pass relatively unchanged

The chosen window was stepped through each data record by $1 / 20$ of the window width. This choice was arbitrary. 
UNCLASSIFIED

\section{Time-frequency analysis shows uniform arrival of energy}

The windowed data is Fourier transformed without further processing

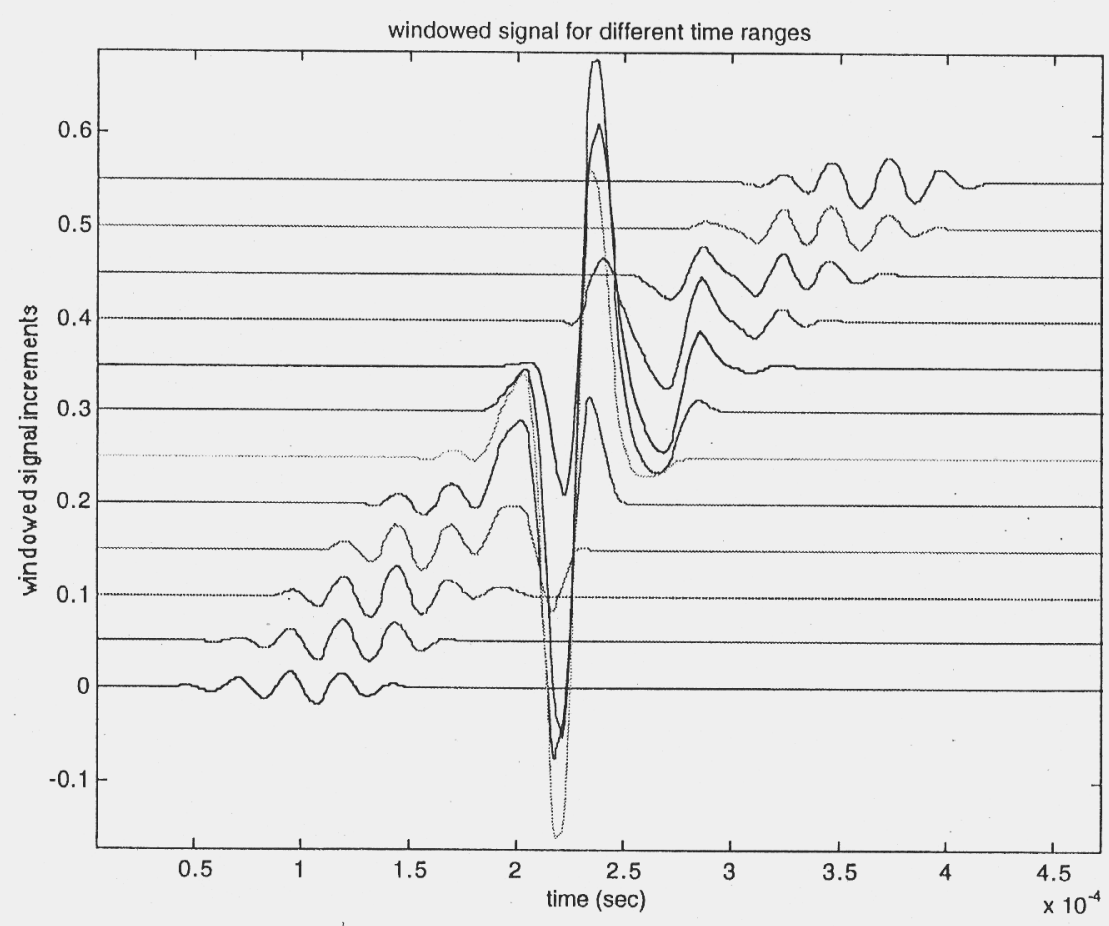

The compensation signal lead-in is clearly visible in the time signal segments
The power spectrum for time and frequency shows a broad bandwidth in the pulse

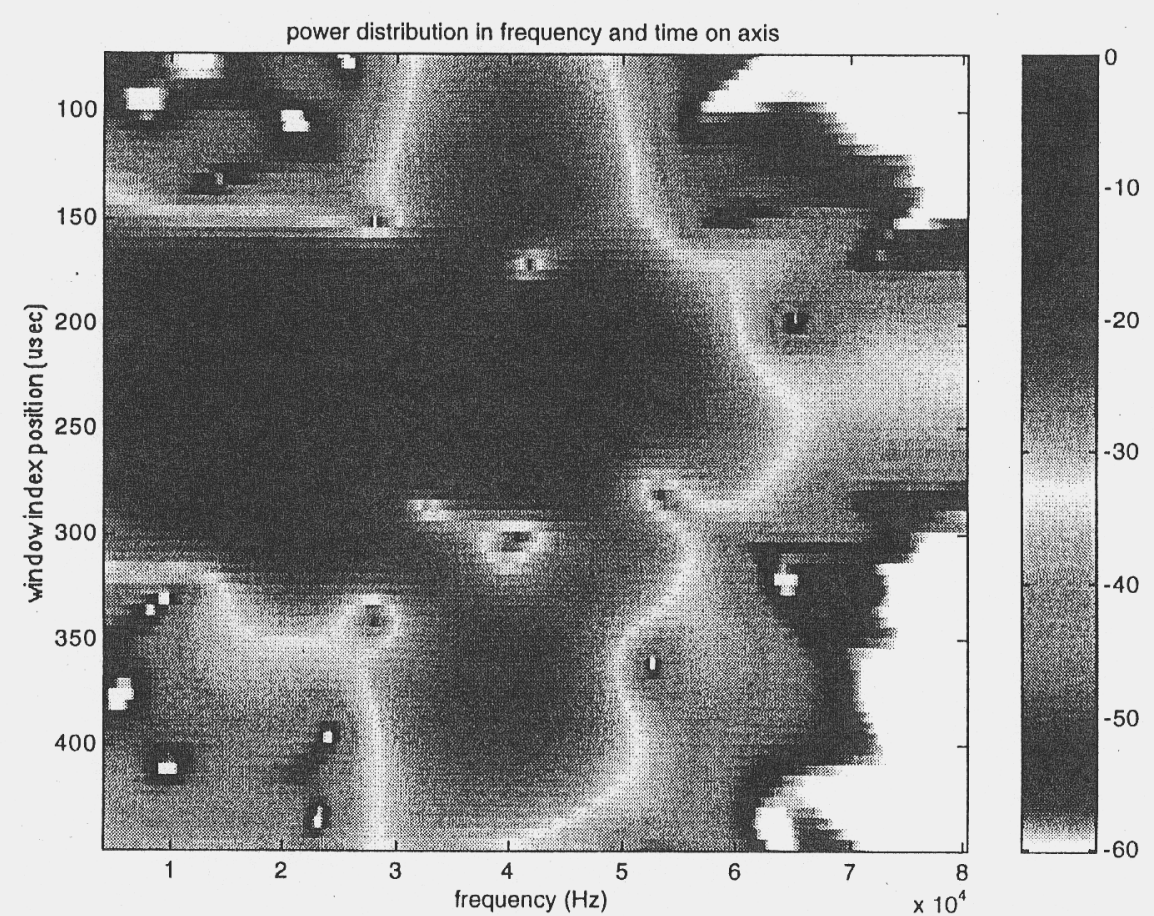

The time-frequency picture shows the lead-in and trailing signals are nearly single frequency 
UNCLASSIFIED

\section{Amplitude and phase surfaces vrs. time and frequency}

The power spectrum for time and frequency shows a broad bandwidth in the pulse
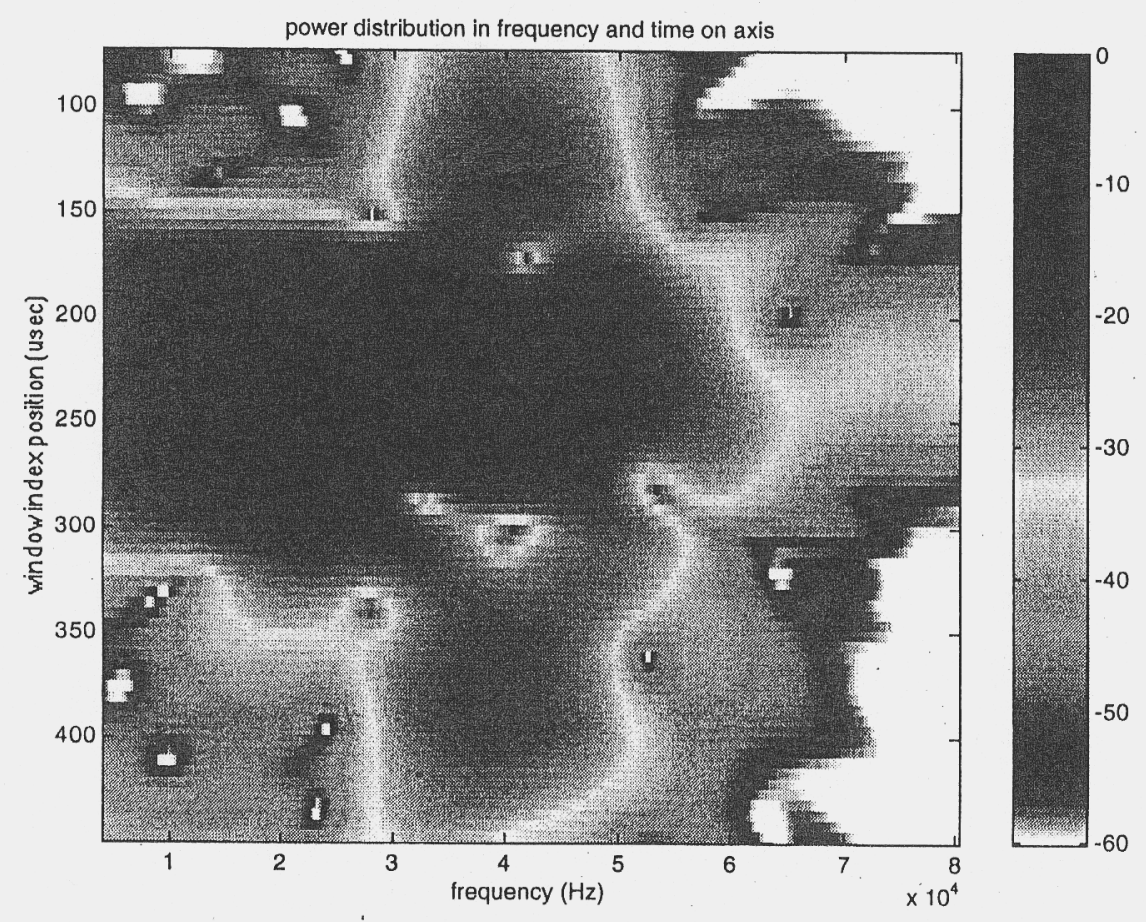

The time-frequency picture shows the lead-in and trailing signals are nearly single frequency
The phase surface for the region of pulse power (150 - 300 usec)

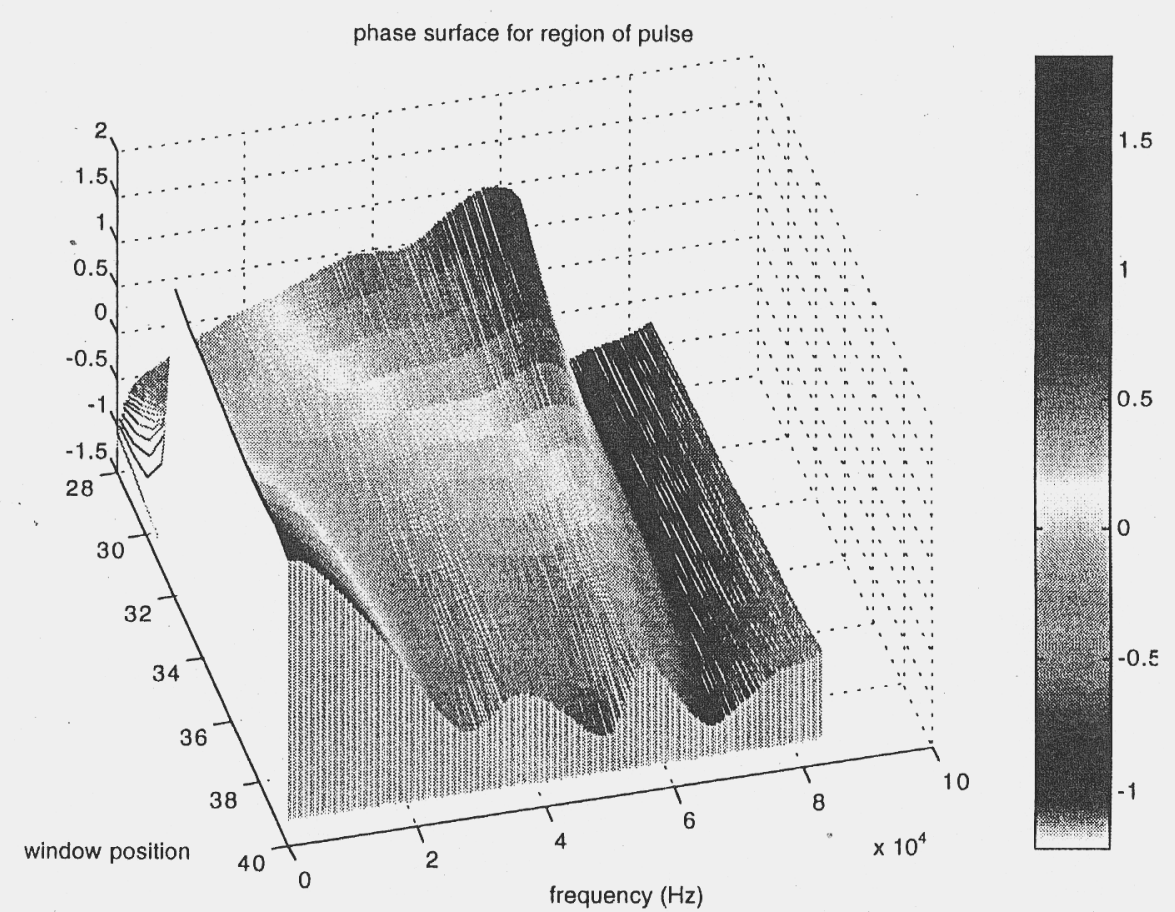

The phase surface has been unwrapped and detrended. It is presented for the region of significant power only 


\title{
Automatic Event Picking for Seismic Oil Exploration
}

\author{
Peter K.-Z. Cheng (U.C. Davis) \\ Gregory Clark (LLNL), and Gary Ford (U.C. Davis)
}

\begin{abstract}
Velocity estimation is currently a "bottleneck," or barrier to progress in oil exploration, because the required seismic event picking step must be carried out manually at great cost in time and money. The goal of this research is to create $2 \mathrm{D}$ and $3 \mathrm{D}$ algorithms for automating the process of picking seismic events in pre-stack migrated gathers arranged in common reflection point (CRP) panels. The work has been funded through the DOE/NGOTP (National Gas and Oil Technology Program) and Shell E\&P Technology Company. The approach uses supervised learning and statistical classification / neural network algorithms along with signal / image processing algorithms.
\end{abstract}

The most significant accomplishments during the first 2 years of work include the following:

(i) Delivery of MATLAB code implementing the $2 \mathrm{D}$ automatic picking algorithms and a preliminary 3D algorithm. (ii) Excellent performance in picking events correctly in CRP panels, both during training and testing with a real data set. (iii) Excellent performance in reducing or eliminating the difficult loop skipping problem. (iv) Reduction of the time required to pick a data set from approximately one day (24 hours) to about 6 minutes, using a Shell implementation of the algorithm. Shell anticipates that with the $3 \mathrm{D}$ algorithms, the cost of picking a full seismic survey will be reduced from about 12 weeks and $\$ 75,000$ to about 1 week and $\$ 6,000$, for a factor of 12 savings. (v) Most recently, we have achieved significant results for the $3 \mathrm{D}$ problem of picking and tracking events from CRP panel to CRP panel to produce picked common offset panel (COP) displays of the full data set. 


\title{
Explosive Detection and Discriminant Research for Civil Aviation Security ${ }^{\dagger}$
}

\author{
Harry E. Martz, Derrill Rikard, Earl Updike, Clint Logan, and Sailes Sengupta \\ Lawrence Livermore National Laboratory
}

\begin{abstract}
$\mathrm{X}$ - and gamma-ray imaging techniques in nondestructive evaluation (NDE) and assay (NDA) have seen increasing use in an array of industrial, environmental, military, and medical applications. Much of this growth in recent years is attributed to the rapid development of computed tomography (CT). A new application area for CT is civil aviation security explosives detection. The only Federal Aviation Administration (FAA) certified explosives detection system is a linear-array based $\mathrm{x}$-ray CT scanner developed by InVision, Inc. Unfortunately the system's airport false-alarm rate performance is higher than the certification rate. We are working with the FAA William J. Hughes Technical Center to help them advance their explosives detection and discriminant research efforts. We have applied three x-ray CT scanners in four different data acquisition configurations to characterize explosives, distinguish between explosives and $\mathrm{x}$-ray machine false-alarm items; and validate inert, non-hazardous materials that simulate the $\mathrm{x}$-ray properties of explosives. I will describe the $\mathrm{x}$-ray $\mathrm{CT}$ scanner configurations, their results and our preliminary analysis of the data.
\end{abstract}




\title{
Grain Identification and Analysis of Shock Induced Damage of Sandstone
}

\author{
Carl Hagelberg \\ Los Alamos National Laboratory
}

Oil and gas wells are "completed" by sending a gun tool into the pipe to a particular pay-zone depth where shaped charges are used to perforate the well casing to allow the fluid to flow into the well for extraction. We are studying the microstructure damage and dynamics of what occurs when the rock is perforated in such a fashion. Gas gun recovery experiments are used to understand how rock is damaged under short duration shocks. The recovered samples are analyzed using a Scanning Electron Microscope (SEM). We also have an undamaged sample volume that has been imaged using X-ray Computed Micro Tomography (XCMT) providing a volume data set.

Image and volume analysis algorithms are being explored and developed to extract boundary and surface representations from the XCMT data and to characterize the SEM images. One of our goals is to use the data to create representations in two or three dimensions of a grain structure (e.g. sandstone) that can then be used in numerical simulations that model a shock applied to the material. Another goal is to develop analysis procedures that allow comparison of the experimental data and output from numerical simulations. Measures that can be used for comparison include a characterization of fractures in the rock using fractal analysis and grain size distributions.

The x-ray attenuation data is a (noisy) representation of a three-dimensional grain structure of a small core ( $1 \mathrm{~mm}$ diameter) of sandstone. The process of extracting geometric information is essentially a two-tiered task: a multi-partition segmentation into individual grains, and a geometric representation of each grain. The segmentation into individual grains is the most difficult in that it involves categorizing each voxel into grain and non-grain material (relatively easy due to image contrast) and into distinct grains (relatively difficult due to ambiguity of the grain boundaries). Various approaches to segmenting the image data from a slice have been explored including edge finding, wavelet analysis, two-dimensional entropic segmentation, and region growing. A better understanding of how to manage ambiguities in grain boundaries in the CMT data is still needed. It seems evident that humans deduce structures from the images using multi-scale information suggesting that multi-scale analysis may be a necessary characteristic to include in algorithm designs. 


\title{
Signal Design for Lithography
}

\author{
Jesse Kolman \\ Lowrence Livermore National Laboratory
}

We have developed an algorithm for designing input signals for a lithography system with the goal of printing prescribed patterns on semiconductor substrates. The input signal can represent various types of static masks and spatial light modulators. The forward model employed by the algorithm describes a partially coherent imaging system as well as a nonlinear photoresist. The imaging system is modeled by a bilinear transformation, which accounts for both the condenser and projection optics. Our algorithm attempts to work backwards, constructing an input signal, which yields the desired printed pattern, by evaluating the quality of the pattern and iteratively adjusting the input. The forward model is used to do the evaluation, and a constrained conjugate gradient optimizer solves the iterative problem.

Recent developments have included techniques for greatly speeding up the required calculations and extensions of the model. The fast computational techniques allow practical sized problems to be solved in a reasonable amount or time, with no reduction in quality. The model extensions consist of the consideration of arbitrary complex objects and constraints, which allow certain portions of the input signal to be fixed in value. This latter extension may be useful for modeling defects in multilayer masks. Another important avenue of future research is the development of an algorithm, which produces a binary input signal. 


\title{
Fluoro Immuno Sensors for Biological Warfare Agents Detection and Identification
}

\author{
Kodumudi S. Venkateswaran ${ }^{I}$ Fred P. Milanovich ${ }^{2}$, Raymond P. Mariella Jr. ${ }^{1,3}$ and \\ Richard G. Langlois ${ }^{l}$ \\ ${ }^{1}$ Biology and Biotechnology Research Program, ${ }^{2}$ Nonproliferation, Arms Control and \\ International Security Directorate, ${ }^{3}$ Center for Microtechnology \\ Lawrence Livermore National Laboratory
}

Advanced biodetector systems are of paramount importance for reducing the threat of biological weapons and also for containing the devastating effects of any incidence involving pathogenic microorganisms. Biological Defense Program at Lawrence Livermore National Laboratory is focussed in the development of rapid and sensitive detector/ identifier systems useful both in the battlefield and the urban settings. Under this program, pioneering development was made in the real time monitoring of nucleic acid amplification. Non-nucleic acid based fluoro immuno sensors are also being developed for simultaneous identification of multiple microbial agents in the sample. Fluorescent-labeled antibodies were used for detection of the microbial agents by flow cytometry. Blue or red laser was used as light source for excitation and sensitive photo detectors were used for fluorescence and light scatter measurement. Assays were standardized for the specific detection of simulants of biological warfare agents and tested using LLNL patented miniFlo flow cytometer and also with two different commercial flow cytometers. Four simulants used in this study were Bacillus globigii (Bg) for bacterial spore, Erwinia herbicola (EH) for vegetative bacteria, Ovalbumin (Ov) for protein toxin and bacteriophage MS2 (MS2) for virus. Flow cytometric detection is a sensitive, specific and reliable method and the fluoro immuno sensor will be very valuable for the rapid, simultaneous detection of different biological warfare agents.

Key Words: Fluoro immuno sensor, biological weapons, flow cytometry

This work was performed under the auspices of the Department of Energy by the Lawrence Livermore National Laboratory under contract W-7405-Eng-48. 
Date: Mon, 26 Oct 1998 11:40:12 -0800 (PST)

UCRL-JC-131209 Abs

\title{
Preliminary results from an environmental geophysics project for improving geophysical imaging of fluid distribution in the shallow subsurface
}

\author{
By Patricia A. Berge, James G. Berryman, \\ Brian P. Bonner, Jeffery J. Roberts, Dorthe Wildenschild
}

The purpose of this project is to develop a computer code for joint inversion of seismic and electrical data to improve underground imaging for site characterization and remediation monitoring. The computer code developed in this project will invert geophysical data to obtain direct estimates of porosity and saturation underground, rather than inverting for seismic velocity and electrical resistivity or other geophysical properties.

This should improve the state-of-the-art of underground imaging significantly, so that interpretation of data collected at a contaminated site will be much less subjective. Potential users include DOE scientists and engineers responsible for characterizing contaminated sites and monitoring remediation of contaminated sites. In this three-year project, we use a multi-phase approach consisting of theoretical and numerical code development, laboratory investigations, tests on available laboratory and borehole geophysics data sets, and a controlled field experiment, to develop practical tools for joint electrical and seismic data interpretation. We are now in the second year of the project. We will present results to date from this work. Results from controlled laboratory experiments on sand-clay mixtures improve our understanding of the connections between measured geophysical properties and the parameters that control fluid distribution and flow (porosity, saturation, and permeability). Results from theoretical work include determining how to use appropriate effective medium theories and available physical properties information in algorithms for relating geophysical measurements to porosity, saturation, and permeability.

Other results include new guidelines for planning and carrying out geophysical field experiments for improved site characterization.

For more information on this project, please see the world-wide web page at: http://www-ep.es.llnl.gov/www-ep/esd/expgeoph/Berge/EMSP/

This work was performed under the auspices of the U.S. Department of Energy by the Lawrence Livermore National Laboratory under contract No. W-7405ENG-48 and supported specifically by the DOE Office of Energy Research within the Office of Basic Energy Sciences, Division of Engineering and Geosciences. This project is funded as part of the Environmental Management Science Program (EMSP), a pilot program managed jointly by the Office of Energy Research and the Office of Environmental Management. 


\title{
Surface and Borehole Electromagnetic Imaging of Conducting Contaminant Plumes
}

\author{
Authors: \\ James G. Berryman, H. Michael Buettner, Nathan J. Champagne II, \\ J. Brian Grant, Robert M. Sharpe \\ Lawrence Livermore National Laboratory \\ Co-Authors: \\ Oliver Dorn, George Papanicolaou \\ Mathematics Department, Stanford University
}

Electromagnetic induction tomography is a promising new tool for imaging electrical conductivity variations in the earth. The EM source field is produced by induction coil (magnetic dipole) transmitters deployed at the surface or in boreholes. Vertical and horizontal component magnetic field detectors are deployed in other boreholes or on the surface. Sources and receivers are typically deployed in a configuration surrounding the region of interest. The goal of this procedure is to image electrical conductivity variations in the earth, much as x-ray tomography is used to image density variations through cross-sections of the body. Although such EM field techniques have been developed and applied, the algorithms for inverting the magnetic field data to produce the desired images of electrical conductivity have not kept pace.

One of the main reasons for the lag in the algorithm development has been the fact that the magnetic induction problem is inherently three dimensional; other imaging methods such as X-ray and seismic can make use of two-dimensional approximations that are not too far from reality, but we do not have this luxury in EM induction tomography. In addition, previous field experiments were conducted at controlled test sites that typically do not have much external noise or extensive surface clutter problems often associated with environmental sites. To use the same field techniques in environments more typical of cleanup sites requires a new set of data processing tools to remove the effects of both noise and clutter. The goal of this project is to join theory and experiment to produce enhanced images of electrically conducting fluids underground, allowing better localization of contaminants and improved planning strategies for the subsequent remediation efforts. The poster first explains the physical context in more detail and then summarizes the progress made so far on this project: (1) on code development, (2) on field tests of these methods, and (3) data inversion methods.

This work was performed under the auspices of the Department of Energy by the Lawrence Livermore National Laboratory under contract W-7405-Eng-48. 
Date: Tue, 20 Oct 1998 10:53:11 -0700 (PDT)

\title{
Clinical Access of PEREGRINE Dose Calculations
}

\author{
By Sarita S. May
}

The goal of the PEREGRINE Program is to improve the effectiveness of radiation therapy in the treatment of cancer, using Monte Carlo photon transport, by ensuring wide-spread rapid distribution of this technology to every cancer center that uses radiation therapy. In order for PEREGRINE to have the highest impact on the maximum number of patients, our program has the following objectives:

- Demonstrate that Monte Carlo dose calculations are fast and accurate

- Design an affordable package that is compatible with existing treatment systems

- Address intellectual property and copyright issues

- Prepare validation and verification package for FDA submission

- Develop and implement licensing strategy

Through these activities we believe we can fulfill the expectations of the medical community now and continue to advance the field of radiation therapy well into the 21st century.

UCRL-JC-132169 Abs

Clinical Access of PEREGRINE Dose Calculations

S. S. May

Sarita May

External and Regulatory Affairs

PEREGRINE Program Office

Lawrence Livermore National Laboratory (L-174)

7000 East Ave, Livermore, CA 94550

email: may1@llnl.gov

Phone: (925) 422-7473

Fax: 925-423-1447 
Date: Mon, 26 Oct 1998 11:59:40 -0800 (PST)

UCRL-JC-131116 Abs

\title{
Direct observation of fluid-clay interactions with implications for mechanical and electrical properties
}

\author{
By C. Aracne-Ruddle, D. Wildenschild, B. Bonner, and P. Berge
}

When measuring sound speed in dilute sand-clay mixtures, observed changes in energy transmission have indicated that the pore fluid composition, as well as the amount of clay, may influence the acoustic properties of the medium. We devised an experiment to directly observe how sand and clay particles interact on the grain scale. An optical microscope was used to observe changes in clay morphology as a function of the chemistry of the pore fluid. We used a pure silica sand with grain sizes between 74-420 microns and a median diameter (d50) of 273 microns, mixed with 1, 3, and 10 weight-percent of sodium montmorillonite, a swelling clay. The wetting fluids were deionized water and a $0.1 \mathrm{~N} \mathrm{CaCl} 2$ solution. For t5he dry sand-clay mixture, we observed that the clay particles would electrostatically cling to the sand grains, tending to bridge the gaps and thus influence the acoustic and electrical properties of the combined medium. As expected, due to the chemical interactions between the clay and the water, the clay particles swelled to occupy the available pore space between sand grains when wetted with deionized water. Subsequently, when wetted with $\mathrm{CaCl} 2$, the clay particles settled and clumped together to form larger clusters or flocs by a process called flocculation. The flocculation process depends mainly on the charge that may be present on the particles in solution. The charge on each particle may repel the other particles and keep the material in suspension, or it may cause the particles to be attracted to each other and form clusters (or flocs). The visual observation of these phenomena verifies our initial assumption that fluid-clay interactions can play a major role when making acoustic and electrical measurements on natural soils.

For more information, please visit our web-site at: http://wwwep.es.llnl.gov/www-ep/esd/expgeoph/Berge/EMSP/

This work was performed under the auspices of the U.S. Department of Energy by the Lawrence Livermore National Laboratory under contract No. W-7405ENG-48 and supported specifically by the DOE Office of Energy Research within the Office of Basic Energy Sciences, Division of Engineering and Geosciences.

This project is funded as part of the Environmental Management Science Program (EMSP), a pilot program managed jointly by the Office of Energy Research and the Office of Environmental Management. 


\title{
Ultrasonic Immunoassay for Detection of Breast Cancer Antigens*
}

\author{
By Amy Wang ${ }^{1}$, Radwan Kiwann ${ }^{2}$, Richard White \\ ${ }^{1}$ Lawrence Livermore National Laboratory \\ ${ }^{2}$ Cancer Research Fund of Contra Costa \\ ${ }^{3}$ University of California, Berkeley
}

\begin{abstract}
Patients with active breast cancer exhibit elevated blood serum levels of breast epithelial mucin antigens (BrE-Ags). Currently, serum levels of BrE-Ags in breast cancer patients are monitored during follow-up of surgery or chemotherapy treatment, where a rise in blood antigen levels indicates the presence of residual tumors.

We present a silicon-micromachined immunosensor package that has been designed to detect a specific breast epithelial mucin antigen, non-penetrating glycoprotein (NPGP). We couple an acoustic sensor, the flexural plate wave (FPW) device, with a novel mass-amplifying label that increases sensor signal from that of previous acoustic immunoassays. The use of a gravimetric immunoassay provides an alternative to radioimmunoassay, eliminating the need for radioactive labels that require costly disposal. In addition to gravimetric sensing, the FPW device is also capable of providing a source of ultrasonic agitation, which results in localized mixing near the active immunoglobulin binding area of the device. We have observed increased antibody binding using ultrasonic agitation during immunoglobulin incubation periods. Consequently, with ultrasonic agitation, we are able to reduce the length of time necessary to complete the immunoassay, and also affect the amount of antibody binding.
\end{abstract}

Keywords: acoustic, breast cancer, immunosensor, ultrasonic, MEMS, micromachining

Amy Wang is an electrical engineer in the Center for Microtechnology at LLNL. She is also an adjunct professor in the Department of Applied Science at the University of California at Davis. Her research interests include the application of micro electro-mechanical systems (MEMS) to biological and medical applications. Amy received her

$\mathrm{Ph}$. D. from the University of California at Berkeley.

* This work was performed at the University of California at Berkeley and the Cancer Research Fund of Contra Costa. 